\title{
Changes IN THE STRUCTURE OF EMPLOYMENT AND JOB QUALITY IN EUROPE 1995-2007
}

\author{
ENRIQUE FERNÁNDEZ-MACÍAS \\ Tesis dirigida por: \\ Rafael Muñoz de Bustillo Llorente \\ Modesto Escobar Mercado \\ Departamento de Sociología y Comunicación \\ Universidad de Salamanca
}





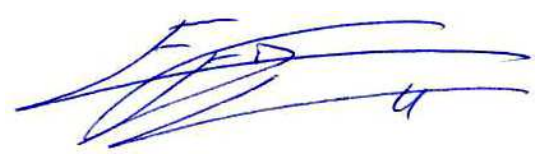

Fdo. Enrique Fernández-Macías

Fdo. Rafael Muñoz de Bustillo Llorente

Fdo. Modesto Escobar Mercado

Madrid, February 2010 



\section{Table of Contents}

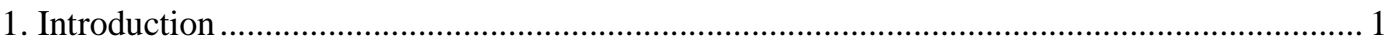

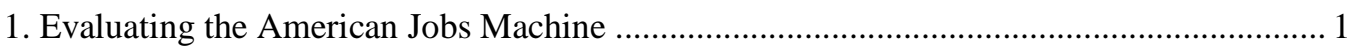

2. More and better jobs? Evaluating the quality of jobs created in the European Union

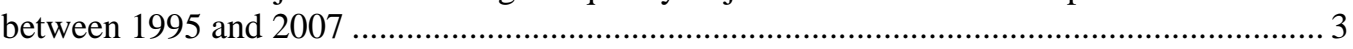

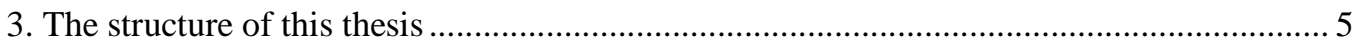

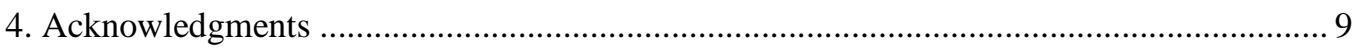

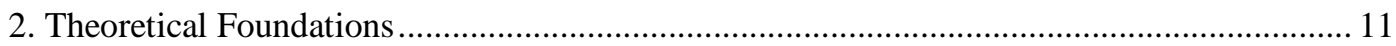

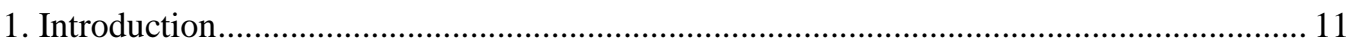

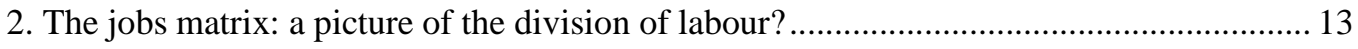

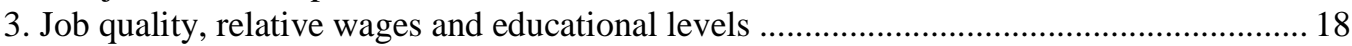

3.1. The mainstream economic approach to job quality ...................................................... 19

3.2. The subjective approach: job satisfaction as an indicator of job quality ...................... 23

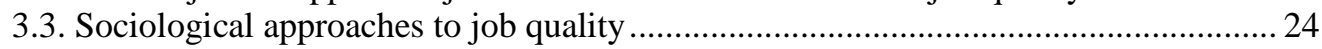

4. The structural evolution of the division of labour and job quality..................................... 30

4.1 The pessimists: the evolution of the division of labour and the degradation of work. 34

4.2. The optimists: more and better jobs in a post-industrial economy ……....................... 40

4.3 A more nuanced view: job polarization theories ........................................................... 44

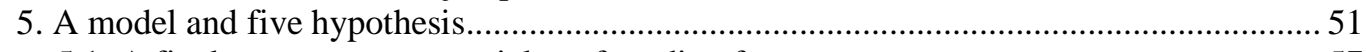

5.1. A final note on two potential confounding factors ..................................................... 57

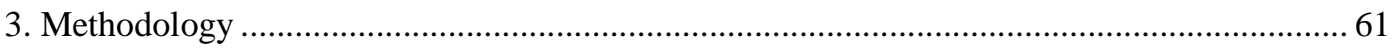

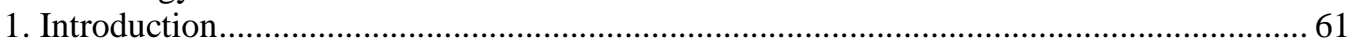

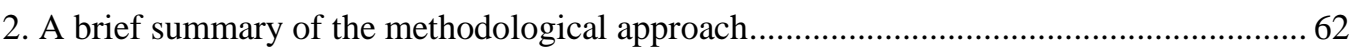

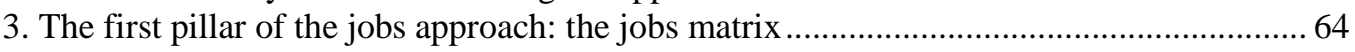

3.1. The data source of the jobs matrix: the European Labour Force Surveys .................... 64

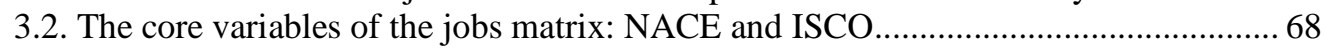

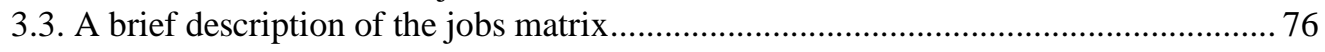

4. The second pillar of the jobs approach: the job quality rankings ...................................... 79

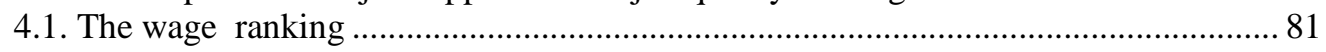

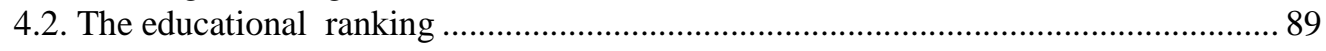

4.3. The amenities (multidimensional) ranking ............................................................. 91

4.4. The correlation between the three job quality rankings........................................... 110

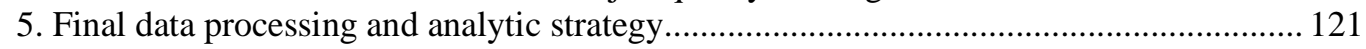

5.1. The target population: should we include self-employment and part-time work? .... 121

5.2. Merging the quantitative and qualitative job matrices .............................................. 123

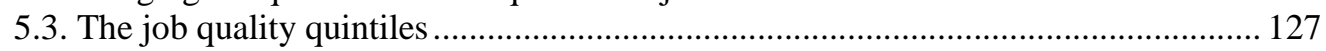

5.5. General strategy of analysis for the following chapters ........................................ 137

4. Division of labour and job quality across the EU - a static approach................................. 139

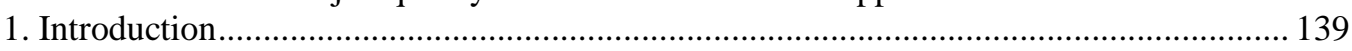

2. The division of labour across Europe: sector and occupational structures ........................ 141

2. 1. Employment by broad categories of economic activity ......................................... 146

2.2. Employment by broad occupational categories ...................................................... 152

3. The correlations of the educational and wage rankings across countries ........................ 157

3.1. Exploring the correlations of the wage and educational hierarchies across countries 158

3.2. Exploring the correlation patterns by sector using principal component analysis..... 168

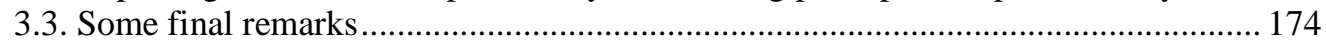

3.4. A small illustration of the ranking variability at the job level across countries ........ 175

4. A static comparison of job quality structures across Europe …….................................... 179

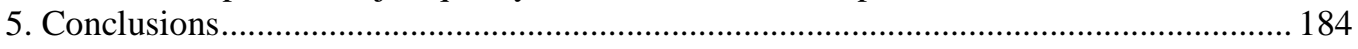




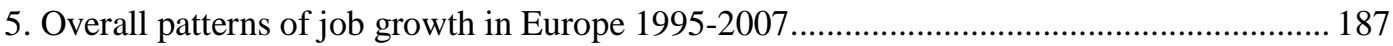

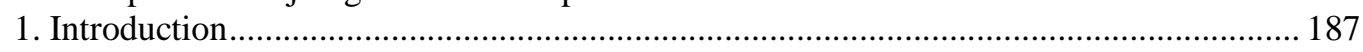

2. The transformation of job quality structures across Europe 1995-2007: identifying and

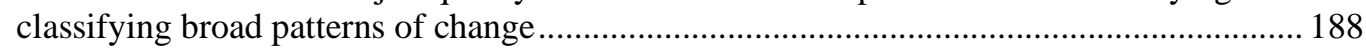

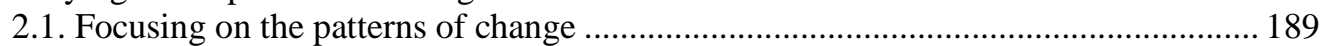

2.2. Putting change in its proper scale ...................................................................... 197

2.3. Job polarization in Europe? A response to Goos, Manning and Salomons ............... 200

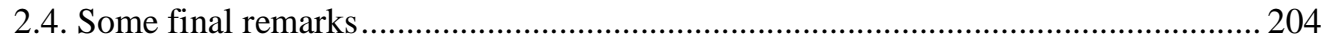

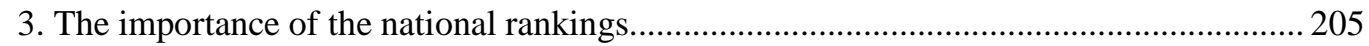

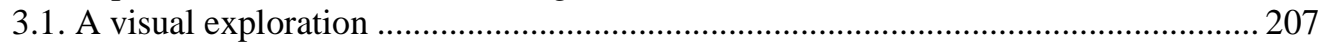

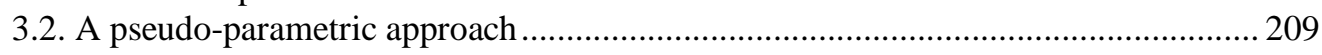

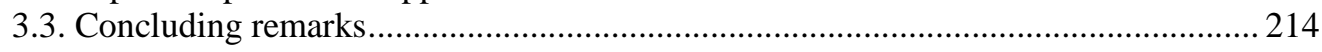

4. Convergence or parallel evolution of European job structures........................................ 215

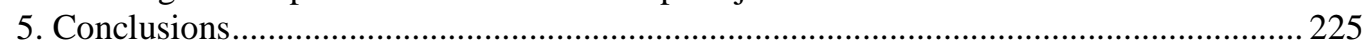

6. Understanding the patterns: decomposing the patterns of job growth by sector, occupation

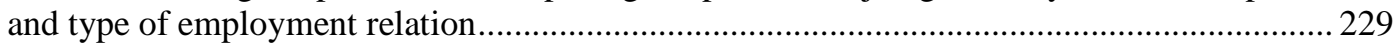

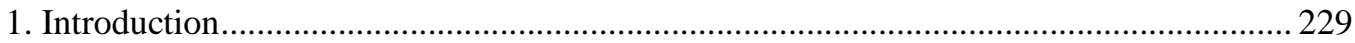

2. What drives the change in the quintiles: sector or occupation? ....................................... 232

3. Decomposing the patterns of job growth by occupation and sector .................................2238

3.1. A decomposition of the patterns of job growth by 9 broad occupational levels ....... 238

3.2. A decomposition of the patterns of job growth by 9 broad sectors .......................... 245

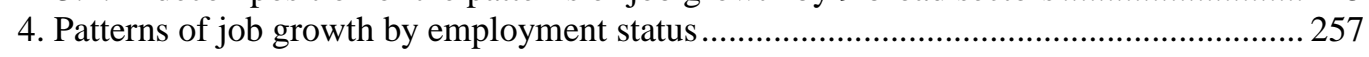

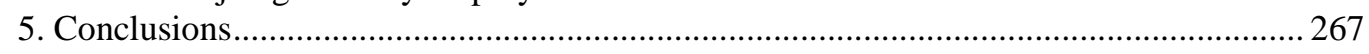

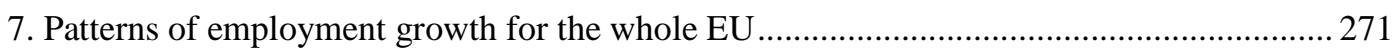

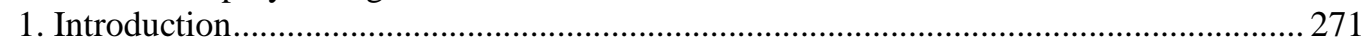

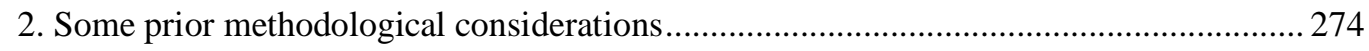

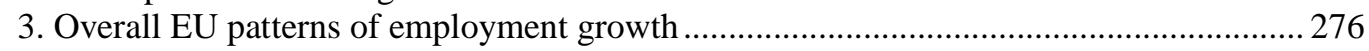

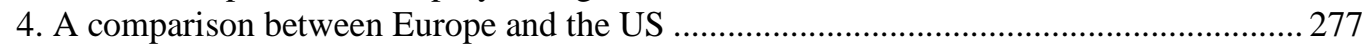

5. Decomposing the EU patterns of job growth by regions .................................................. 280

6. Decomposing the EU patterns of job growth by sectors and regions ................................228

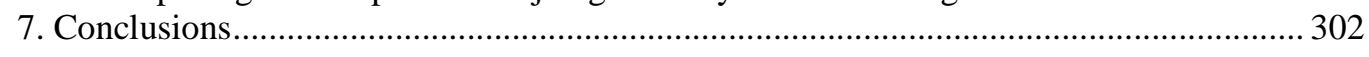

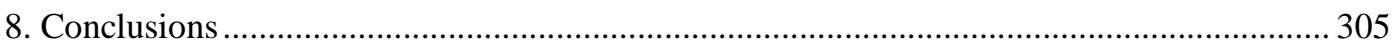

1. The debate on the effects of the changing division of labour on job quality .......................305

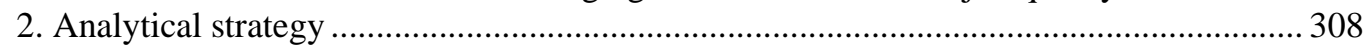

3. The hypothesis of the degradation of work..................................................................... 311

4. The upgrading hypotheses: the coming of the post-industrial society and skill-biased

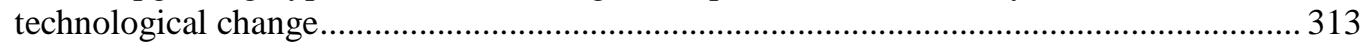

5. The polarization hypotheses: trade and technological explanations ................................... 316

6. Accounting for the plurality of patterns of employment growth ..................................... 320

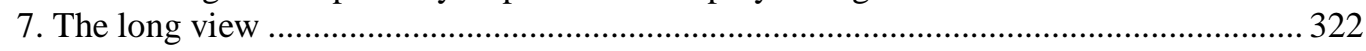

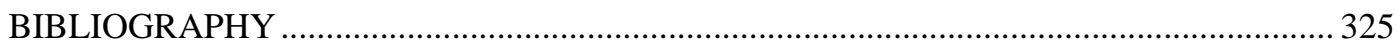




\section{Index of figures}

Figure 2.1: The jobs matrix in practice

Figure 2.2: Combinations of wages and amenities leading to the same level of well-

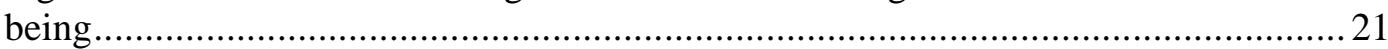

Figure 2.3: A synthetic model of the determinants of the changing structure of job

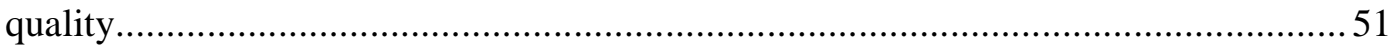

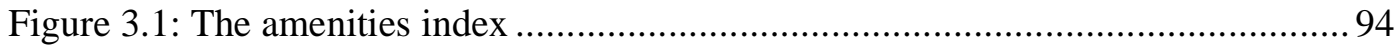

Figure 3.2: Relative wage intervals behind the quintiles....................................... 131

Figure 3.3: Year-on-year change in employment by quintile, UK ........................... 134

Figure 3.4: One versus two rankings, UK.......................................................... 137

Figure 4.1: Share of employment in the "most advanced" sectors and occupations .... 144

Figure 4.2: Share of employment in the "most advanced" sectors and occupations vs.

GDP pc

Figure 4.3: Counterfactual distribution of educational quintiles, using Germany as

reference

Figure 4.4:Counterfactual distribution of wage quintiles, using Germany as reference

Figure 4.4: Counterfactual distribution of amenities quintiles, using Germany as

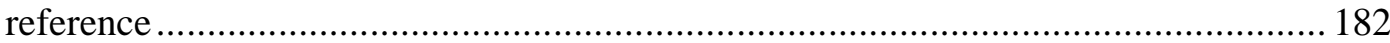

Figure 5.1: Absolute change in the wage quintiles (different periods)...................... 192

Figure 5.2: Absolute change in the educational quintiles (different periods) ............... 193

Figure 5.3: Absolute change in the amenities quintiles (different periods) ................. 193

Figure 5.4: Initial, middle and final size of the wage quintiles (different periods) ...... 198

Figure 5.5: Initial, middle and final size of the educational quintiles (different periods)

Figure 5.6: Initial, middle and final size of the amenities quintiles (different periods) 199 Figure 5.7: Counterfactual (Germanic) vs. real wage quintiles (different periods) ..... 208 Figure 5.8: Counterfactual (Germanic) vs. real educational quintiles (different periods)

Figure 5.9: Counterfactual job quality structures (wages), 1995 (white bar) to 2007

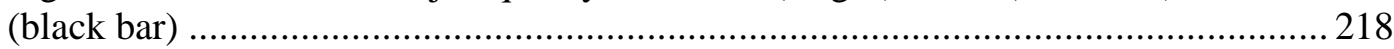

Figure 5.10: Counterfactual job quality structures (education), 1995 (white bar) to 2007

(black bar)

Figure 5.11: Counterfactual job quality structures (amenities), 1995 (white bar) to 2007

(black bar)

Figure 5.12: Convergence in the three job quality quintiles vs. original dissimilarity. 223

Figure 5.13: Convergence in the three job quality quintiles to Germany 1995 vs. original

dissimilarity

224

Figure 5.14: Convergence in the three job quality quintiles to Germany 1995 vs. GDP

$\mathrm{PC}$ in 2000

Figure 6.1: Patterns of job growth using only ISCO, ISCO and NACE (original), and only NACE

Figure 6.2: Counterfactual (keeping constant one dimension) vs. original patterns of job

growth

Figure 6.3: The contribution to job growth of managers, professionals and technicians

Figure 6.4: The contribution to job growth of clerks and service workers 
Figure 6.5: The contribution to job growth of skilled agricultural workers

Figure 6.6: The contribution to job growth of craft workers, operators and elementary

occupations

Figure 6.7: The contribution to job growth of the primary sector and construction .... 246

Figure 6.8: The contribution to job growth of low and high technology industries..... 247

Figure 6.9: The contribution to job growth of low and high knowledge intensive

services

Figure 6.10: The contribution to job growth of public administration, education and

health.

Figure 6.11: Breakdown of job growth by type of employment relation ................... 260

Figure 6.12: Breakdown of job growth by full-time or part-time status...................... 260

Figure 6.13: Breakdown of job growth by employment status .............................. 261

Figures 7.1-7.3: Patterns of job growth by job quality, EU(23) 1998-2007 ............... 276

Figure 7.4: EU vs. US patterns of job growth (wage quintiles) ................................. 278

Figure 7.5: Patterns of employment growth in the EU, broken up by regions............. 282

Figure 7.6: Patterns of employment growth in the EU, broken up by 9 sectors .......... 288

Figure 7.7: Contribution of PRIMARY SECTOR to quintile growth by region ......... 294

Figure 7.8: Contribution of CONSTRUCTION to quintile growth by region............. 295

Figure 7.9: Contribution of LOW TECH INDUSTRIES to quintile growth by region 296 Figure 7.10: Contribution of HIGH TECH INDUSTRIES to quintile growth by region

Figure 7.11: Contribution of LESS KNOWLEDGE INTENSIVE SERV. to quintile

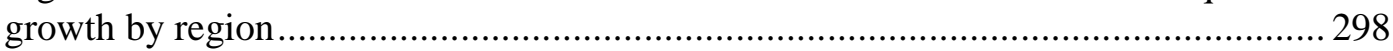

Figure 7.12: Contribution of KNOWLEDGE INTENSIVE SERVICES to quintile growth by region 


\section{Index of Tables}

Table 2.I: Dimensions of job quality suggested by the different traditions .................. 30

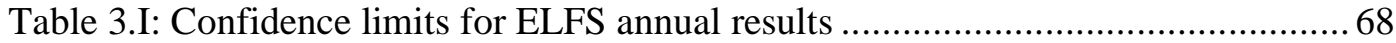

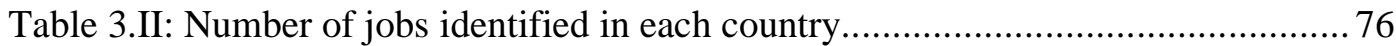

Table 3.III: Share of employment in the biggest $\mathrm{X}$ jobs......................................... 77

Table 3.IV: Distribution of jobs not available in all countries ................................. 78

Table 3.V: Correlations between the index, dimensions and indicators .................... 107

Table 3.VI: Scores of the index, dimensions and indicators for 1 digit sectors and

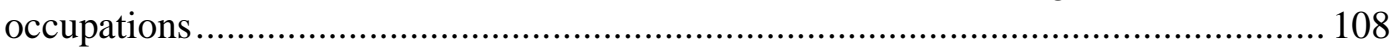

Table 3.VII.1: Analysis of Variance of the overall amenities index by NACE and ISCO

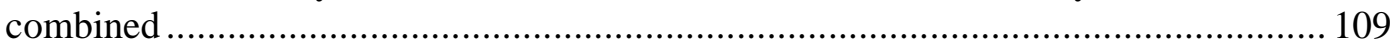

Table 3.VII.2: Analysis of Variance of dimension 1 by NACE and ISCO combined 109

Table 3.VII.3: Analysis of Variance of dimension 2 by NACE and ISCO combined 109

Table 3.VII.4: Analysis of Variance of dimension 3 by NACE and ISCO combined 109

Table 3.VII.5: Analysis of Variance of dimension 4 by NACE and ISCO combined 110

Table 3.VII.6: Analysis of Variance of the overall amenities index by country .......... 110

Table 3.VII.7: Analysis of Variance of the overall amenities index by NACE and ISCO combined, and country

Table 3.VIII: Structure of correlation between the rankings (jobs weighted by employment)

Table 3.IX: A job-based, positional test of the theory of compensating differentials.. 119

Table 3.X: Correlation between job structures of the different surveys .................... 124

Table 3.XI: Share of employment in jobs with no amenities ranking, 2000............... 127

Table 3.XII: Time periods covered, breaks and adjustments .................................. 133

Table 4.I: Distribution of employment by aggregated categories of sector and occupation

Table 4.II: Breakdown of manufacturing industries (2-digit NACE codes) into high- and low-tech 148

Table 4.III: Breakdown of private services into Knowledge Intensive (K.I.S.) and the

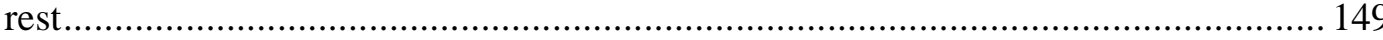

Table 4.IV: Unweighted correlations between the educational rankings for pairs of countries (observations vary)

Table 4.V: Unweighted correlations between the wage rankings for pairs of countries (observations vary)

Table 4.VI: Weighted correlations between the educational rankings for pairs of

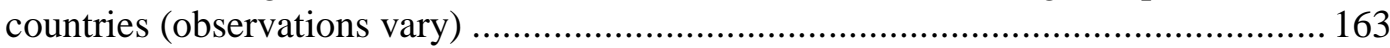

Table 4.VII: Weighted correlations between the wage rankings for pairs of countries

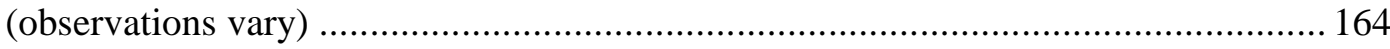

Table 4.VIII: Factor analysis of the educational rankings (weighted) ....................... 169

Table 4.IX: Factor analysis of the wage rankings (weighted) ................................. 173

Table 4.X: The quintile position of the ten largest jobs .......................................... 178

Table 5.I: Uneven vs. even groups of jobs in the GMS 2009 paper.......................... 203

Table 5.II.1: Pseudo-parametric comparison between counterfactual and actual patterns

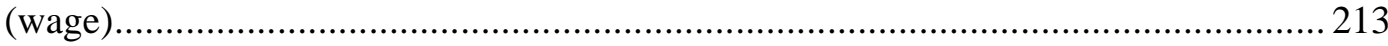

Table 5.III.1: Convergence towards Germany, wage quintiles .............................. 221

Table 5.III.2: Convergence towards Germany, educational quintiles ........................ 221 
Table 5.III.3: Convergence towards Germany, amenities quintiles .......................... 222

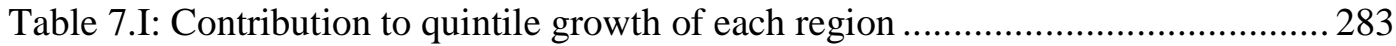

Table 7.II: Contribution to quintile growth of each region and country ......................2285

Table 7.III: Contribution to quintile growth of each sector .....................................28 288

Table 7.IV: Decomposition of sector contributions to quintile growth by region ....... 293 


\section{Country codes}

$\begin{array}{ll}\text { AT } & \text { Austria } \\ \text { BE } & \text { Belgium } \\ \text { CY } & \text { Cyprus } \\ \text { CZ } & \text { Czech Republic } \\ \text { DE } & \text { Germany } \\ \text { DK } & \text { Denmark } \\ \text { EE } & \text { Estonia } \\ \text { ES } & \text { Spain } \\ \text { FI } & \text { Finland } \\ \text { FR } & \text { France } \\ \text { GR } & \text { Greece } \\ \text { HU } & \text { Hungary } \\ \text { IE } & \text { Ireland } \\ \text { IT } & \text { Italy } \\ \text { LT } & \text { Lithuania } \\ \text { LU } & \text { Luxemburg } \\ \text { LV } & \text { Latvia } \\ \text { NL } & \text { Netherlands } \\ \text { PT } & \text { Portugal } \\ \text { SE } & \text { Sweden } \\ \text { SI } & \text { Slovenia } \\ \text { SK } & \text { Slovakia } \\ \text { UK } & \text { United Kingdom } \\ \text { EU } & \text { European Union (23) } \\ & \end{array}$





\section{Introduction}

The main objective of this thesis is to evaluate the quality of the jobs created in 23 European countries during the economic expansion that took place between 1995 and 2007, using a relatively new method for studying structural change in labour markets, which we call the "jobs approach". As a secondary objective, we will also try to contribute to developing the theoretical foundations of this approach, anchoring it in the Social Sciences literature on the implications of change in the structural division of labour. To introduce this thesis, we will briefly recount its origins.

\section{Evaluating the American Jobs Machine}

During the 1990s, the US experienced its longer period of sustained economic growth for the whole of the 20th Century, and the simultaneous expansion of employment was so large that it became known as the "American Jobs Machine". Unemployment fell to levels which were among the lowest of the OECD, and the employment rate reached its highest level in history (Freeman and Rodgers 2004: 22). But there was a heated debate on the nature and quality of the 20-odd million jobs that were created between 1991 and 2000. Some argued that most of these new jobs were low-paid, dead-end jobs in services, while the well-paid jobs in manufacturing were being destroyed by the combined forces of globalization and technical change. Others argued that on the contrary, this unprecedented employment expansion was associated with the creation of jobs with higher than average skill and pay levels, especially in professional and managerial occupations.

Part of the reason why there were so widely different views on this issue is because the contenders used different types of evidence to support their views. The gloomy perspective on the American Jobs Machine was often based on the evolution of wage 
inequalities, which clearly expanded over this period. The rosy perspective was often sustained by broad analyses of the sectors and occupations that had expanded most over this period, which suggested that the majority of new jobs were of a relatively good quality (managerial and professional occupations, financial services and information technology). But surprisingly, most of this evidence was only indirectly related to the key issue in this debate: the actual quality of the jobs created (Wright and Dwyer 2003: 290). The evolution of wage inequality measured at the individual level is obviously related to the type of jobs created over the expansion, but it simply could not provide a clear answer to the underlying question: different patterns of employment expansion by job quality levels can lead to similar outcomes in terms of wage inequalities. And the analysis of changes in the levels of employment by broadly defined sectors and occupations was clearly too rough for providing a clear answer either.

In a 1996 report of the US Council of Economic Advisers (chaired by Joseph E. Stiglitz), a simple yet powerful method for evaluating more directly the quality of the jobs created in the expansion was used for the period 1994-1996. This method consisted in the sorting of all full-time workers into 45 detailed occupations within 22 industries, and then calculating the median weekly earnings of each of the resulting industry/occupation cells. These cells were then ranked by their median wage levels, and split in two groups, each holding half of total employment in 1994. The group that contained the industry/occupation cells whose median wage was below the overall median could be considered as the one holding the "bad jobs", and the other one the remaining "good jobs". Then, the report found that $68 \%$ of the total net expansion of employment had taken place in the "good jobs" group of industry/occupation cells.

This simple idea provided a direct and convincing way for evaluating the quality of the jobs created during the 1990s US employment expansion, and other researchers started applying it to longer periods and with more complex analytic tools (Ilg 1996; Farber 1997; Ilg and Haugen 2000; OECD 2001). Erik Olin Wright and Rachel Dwyer, within their project on "Patterns of Change in the Jobs Structure", published two papers in which they refined the method originally proposed by Stiglitz by using a more detailed list of sector and occupations, covering a longer time span (going back to the 1960s), using five or ten rather than two job quality tiers and decomposing the patterns of job growth by third variables or different combinations of sector and occupation (Wright and Dwyer 2000, 2003). These two papers developed and consolidated the jobs 
approach, and widened its scope considerably. Wright and Dwyer found that between 1992 and 2000, the employment structure of the US transformed in an asymmetrically polarized way: the expansion of the top of the jobs structure was much larger than that of the bottom and middle, but the bottom expanded more than the middle. This development was contrasted with previous periods of employment expansion in the 60s, 70 s and 90s, in which there was a much more unambiguous upgrading evolution with hardly any sign of polarization.

This dissertation was directly inspired by the work of Wright and Dwyer. In 2001, I spent a few months in the Department of Sociology of the University of WisconsinMadison, where I could learn about the method and start applying it to Spain. It looked like a perfect methodology for studying the quality of jobs created in the expansion of employment that most European economies experienced over the same period, but it seemed completely impossible to try to do it in more than one country at a time because of the lack of comparable data at the European level.

\section{More and better jobs? Evaluating the quality of jobs created in the European Union between 1995 and 2007}

After 2001, I got involved in other projects, so I kept on delaying the application of the jobs approach to Spain or other European countries. Then, in 2005 I joined the European Foundation for the Improvement of Living and Working Conditions, a tripartite EU Agency that conducts research "to contribute to the planning and design of better living and working conditions in Europe". This seemed like the perfect opportunity to try an European-wide analysis of the quality of the employment created since the nineties using the jobs approach: it fit perfectly in the Foundation's mandate, which as an EU institution had much better chances of getting the necessary data, and recent economic and political developments in Europe had made such an analysis even more interesting than it would have been five years earlier.

In 2000, the bursting of the dot.com bubble interrupted the long cycle of economic growth of the 1990s in the US. Although real GDP growth recovered relatively quickly, the American Jobs Machine had disappeared: the period that started in 2001 (and finished abruptly in 2007) has been in fact described as the "jobless recovery" in the US 
(Freeman and Rodgers 2004). In Europe, on the contrary, the expansion of employment after 2001 was even larger than between 1995 and 2000.

Behind this diverging evolution of the US and Europe there was a very significant reversal of historical trends in labour productivity at the two sides of the Atlantic. Between 1975 and 1995, employment rates decreased in Europe slowly but steadily, from $64 \%$ to $60 \%$ of the working-age population, while in the US it increased from $62 \%$ to $72 \%$, yet during this period the gap between US and Europe in GDP per head at PPP remained more or less constant. What this means is that until 1995 labour productivity increased much faster in Europe than in the US: "by the 1990s the U.S. advantage in per capita income was being maintained largely through higher labour input supplementing its much reduced superiority in productivity" (Gregory, Salverda and Schettkat 2007: 4). But between 1995 and 2007, the period we will study here, this was completely reversed: the US employment rates remained more or less stagnant ${ }^{1}$, while the EU rate went from $60 \%$ to $67 \%$. Since the late 1990 s, the US expansion was mostly due to productivity growth (hence the jobless recovery), whereas in Europe it was mostly due to higher levels of labour utilization. Between 1995 and 2007, the European Jobs Machine outpaced the American one.

The large expansion of employment in Europe between 1995 and 2007, and the simultaneous slowdown of productivity growth, made the possibility of applying the jobs approach to evaluate the quality of jobs created in Europe in that period especially timely. What kinds of jobs were created in the 1995-2007 European expansion? Was the slowdown of productivity related to the creation of low-quality jobs? Furthermore, there is a crucial difference between Europe and the US which makes the European analysis much more complicated (as we will see later) but also more interesting. Whereas the US functions as a single labour market, the EU is in practice a collection of many different national labour markets, with often rather different characteristics and developments. This makes the EU a fantastic natural laboratory for studying social and economic change, because it allows to test the sensitivity of these developments to institutional and structural differences. In fact, the above mentioned European developments in terms of labour utilization and productivity were not at all pervasive across Europe: between 1995 and 2007, there was an increasing heterogeneity in the levels of labour utilization and productivity across Europe (Dew-Becker and Gordon 2008: 6). Did this

\footnotetext{
${ }^{1}$ In fact, it increased slightly between 1995 and 2000, but went back to 1995 levels after 2001
} 
mean that the patterns of employment growth by job quality were also divergent across Europe?

The policy debate also shifted in Europe around the turn of the century. Throughout the nineties, the top priority of the European Employment Strategy had been simply to create more jobs (see European Commission 1993). But when EU governments met in Lisbon in Spring 2000 to discuss the overall aims of the EU for the following decade, they emphasized the need to create not only more, but also better jobs. Thereafter, the phrase "more and better jobs" became the motto of the European Employment Strategy, and there were many attempts of evaluating the evolution of employment not only from a quantitative but also from a qualitative perspective, mainly through the development of indicators on job quality. But the quantitative and qualitative perspective had rarely been integrated into these evaluations. The jobs approach provided precisely such integration.

So between 2006 and 2008, the European Foundation for the Improvement of Living and Working Conditions financed a research project for evaluating the transformation of the employment structure across the EU between 1995 and 2007 (called the JOBS project). This project was carried out by an international research group that I coordinated. ${ }^{2}$ In 2008 and 2009, the main results of this project were published as two policy-oriented research reports (see Fernández-Macías and Hurley 2008; Stehrer, Ward and Fernández-Macías 2009). The project is still ongoing, currently on a second phase in which we are doing in-depth analysis of specific aspects of the 1995-2007 jobs expansions (such as its gender dynamics, the role played by migration, etc.). ${ }^{3}$

\section{The structure of this thesis}

This thesis draws directly from the European Foundation JOBS project which I coordinated for the last 4 years. The previous publications from this project (FernándezMacías and Hurley 2008; Stehrer, Ward and Fernández-Macías 2008) were mostly

\footnotetext{
${ }^{2}$ This research group was composed by Terry Ward from Applica, a research consultancy based in Brussels; Robert Stehrer and Hermine Vidovic, from the Vienna Institute for International Economic Studies; and John Hurley, Branislav Miculic, Donald Storrie and myself from the European Foundation for the Improvement of Living and Working Conditions.

${ }^{3}$ Participants in this group are Jose Ignacio Anton, Hugo Figueiredo, John Hurley, Francis Green, Damian Grimshaw, Jean-Marie Jungblut, Mark Keese, Sangheon Lee, Rafael Muñoz de Bustillo Llorente, Phillip O'Connell, Robert Stehrer, Matias Scaglione, Donald Storrie, Terry Ward, Erik Olin Wright and myself.
} 
policy-oriented reports, which did not pay much attention to the theoretical basis of the approach or its links to existing Social Sciences literature: they were mostly aimed at a very practical evaluation of the quality of the jobs created (and destroyed) in the 19952007 job expansion, with a view to the formulation of policy-relevant conclusions. This thesis constitutes an attempt to bring the results of the JOBS project to the scientific and academic field, by developing its theoretical foundations, providing a more exhaustive account of the methodology followed, adding two extra job quality indices to increase the robustness of the results, making a deeper and more careful analysis of the data, and discussing the wider implications of our findings within the context of the Social Sciences literature on the effects of technical and organizational change in the structure of employment. The chapter structure of this thesis can be summarized as follows.

In chapter 2, I try to contribute to developing the theoretical foundations of the jobs approach. I argue that this approach can be anchored in the Marxian argument about the two-dimensional nature of the division of labour: the basic element of this approach, the jobs matrix (basically a big table defined by crossing occupation and sector at a relatively detailed level) can be thus understood as a structural snapshot of the state of the division of labour in a particular country and point of time. Then I review the literature on job quality, providing a justification for the three job quality indices used in this thesis (one based on wages, the other on education, and the third one on nonpecuniary job attributes). Finally, I discuss the literature on the implications of the changing division of labour on job quality, arguing that three visions have dominated this debate over the last 200 years: a pessimistic one (dominant in classical political economy), an optimistic one (associated to theories of Post-Industrialism and SkillBiased Technical Change), and a more recent polarizing one (associated to theories of the "declining middle" and Task-Biased Technical change). These three visions, transformed into testable hypotheses, guide the empirical analysis of the rest of this thesis.

In chapter 3, I discuss the methodology used in this thesis. This methodology has two pillars: the jobs matrix and the job quality rankings. The jobs matrix was constructed by extracting data from the European Labour Force Surveys by sector and occupation (NACE and ISCO). The use of these international standard classifications is crucial for this thesis, because it ensures the comparability of the analysis across countries, and allows linking different information sources at the job level. Therefore, these 
classifications and their comparability across countries and over time are discussed in detail. The second section of the methodological chapter explains the construction of the three job quality rankings, which draw from information of several different surveys (again, taking advantage of the possibility of linking information at the job level). Previous research from the jobs approach had only used a wage-based job quality ranking: in this thesis, we have added two further rankings, one based on the educational level of job-holders and another on a multidimensional index of nonpecuniary job attributes. We believe that this addition increases considerably the scope and robustness of the analysis. Finally, the third section briefly explains the strategy followed for analysing the quantitative and qualitative jobs matrices generated in the two previous steps. The analytic methods used in this thesis are relatively simple, because they mostly consist in a graphical analysis of the evolution of employment by job quality quintiles (although we occasionally use other techniques as well). This simplicity is not only an advantage in terms of the intelligibility of results, but it is in fact a way to ensure that the inevitable problems of comparability and inconsistencies in the data used do not bias our conclusions (throughout this thesis, we will try to stick to a non-parametric, eagle-eye view analysis, which is perfectly adequate for the type of broad trend analysis that we intend to do).

Chapter 4 discusses the differences in the structures of employment and job quality hierarchies across Europe from a static perspective, setting the background against which the dynamic analysis of the rest of the thesis will be projected. The first section presents a broad overview of the distribution of employment for one-digit sectors and occupations, which reveals an association between economic development and employment structure that coincides broadly with a Northwestern-Southeastern regional axis in Europe. The second section discusses the similarity of the job quality hierarchies across Europe: we estimate this similarity to be between $50 \%$ and $70 \%$, which is on the one hand large enough as to prove conclusively that the jobs matrices are comparable across countries (otherwise, it would be impossible to find such a high level of correlation in the position of jobs in terms of their wages and educational levels across 23 European countries), but on the other hand not large enough as to suggest that the differences in job quality hierarchies are irrelevant (a degree of national specificity between $30 \%$ and $50 \%$ means that the same structural developments can have rather different implications in different countries). Finally, the last section merges the 
structures and the rankings constructing a set of counterfactual quintiles that allow to compare directly job quality structures across Europe: these results show that the same axis mentioned earlier is associated to differences in the relative shares of good and bad jobs. In particular, Southern and Eastern European countries have a larger share of employment in the lowest quintiles than Northern and Western European countries.

Chapter 5 constitutes the core of the whole thesis. It presents the broad patterns of employment expansion by job quality tiers across Europe, which show a relatively high degree of plurality that contradicts any pretence of universalist explanation, and suggests the influence of institutional factors endogenous to each country. Once assumed this fundamental plurality, it seems clear that the upgrading hypothesis is the one that fits more cases, although there are some important cases of polarization, especially in Continental European countries. These differences across Europe are mostly the result of different structural transformations, rather than the result of the existence of different job quality hierarchies, which means that European countries have created different types of jobs, not the same jobs but with different quality levels. And these differences did not seem either to be associated to the different initial positions, as would have been the case if there was some process of convergence or lagged parallel evolutions. The plurality of patterns seemed to be, therefore, genuine.

Chapter 6 analyses the employment dynamics which are behind the patterns of job growth identified in the previous chapter, by decomposing them in terms of broad occupations, sectors and status of employment. This decomposition provides further support for the upgrading hypotheses, showing that the relatively pervasive expansion of good jobs was primarily related to the expansion of Knowledge-Intensive Services and the Health sector, whereas the bottom and middle positions of employment were dragged down by the secular decline of the primary and secondary sectors. The expansion of the bottom, where it did happen (primarily in the polarizing countries), was associated to the expansion of personal and distributive services. But what differentiated the polarizing countries from the rest was that these countries suffered a specially intense process of destandardization of employment, which suggests an institutional explanation for these pattern of job growth, rather than the technological or trade arguments that predominate in the literature.

Chapter 7 shifts the level of analysis to the overall EU (for the 23 countries with data available), making a kind of what-if exercise (what if the EU was a single labour 
market?) that helps answering some specifically transnational questions. The overall EU patterns of job growth, which can be characterized as asymmetrically polarized (or polarized upgrading) look remarkably similar to the patterns of the US for a recent comparable period of employment expansion analysed by Wright and Dwyer in the study that inspired this thesis, and the underlying dynamics were also analogous. In this chapter, we also analyse the relative contribution of the different regions to the overall EU patterns of job growth, evaluating the relevance of the usual institutional country clusters for this area. Our results show that in general terms the country clusters were not associated to different patterns of job growth, except in the case of Continental Europe, which was clearly linked to the polarization pattern (and to a more intense destandardization of employment). Finally, in this chapter we look for evidence of the impact of European economic integration on job quality structures, only finding a significant impact in the case of high technology industries.

Chapter 8 presents the overall conclusions of this thesis, discussing its main results in the context of the existing literature on the implications of the structural division of labour on job quality.

\section{Acknowledgments}

It is obvious from the previous pages that this thesis has been enormously benefited from the support and collaboration of many different people. First of all, I would like to thank the European Foundation for the Improvement of Living and Working Conditions for funding and supporting the research project on which this thesis is based. I would like to thank Greet Vermeylen and Agnes Parent-Thirion (then coordinators of the Working Conditions Unit in which I worked) for believing in my original proposal and supporting it in the beginning. The Employment and Competitiveness Unit and especially its coordinator, Donald Storrie, who adopted this project when the Working Conditions Unit disappeared, were absolutely crucial for its success. The directors of the Foundation between 2005 and 2008, Willy Buschak and especially Jorma Karppinen were always supportive and encouraging, so I would also like to thank them. Probably the best librarian at any EU institution, Jan Vandamme provided invaluable help with the literature review. But I would like to acknowledge especially the excellent work and support of my Eurofound colleagues in this project (and friends): John Hurley, Branislav Mikulic and Donald Storrie. From outside the Foundation, Terry Ward and 
Robert Stehrer brought their enormous experience with European statistics and a lot of common sense, which were essential for the success of this project (especially, for the compilation of the JOBS dataset, which involved truly challenging methodological difficulties).

Of course, I have to give very special thanks to Erik Olin Wright and Rachel Dwyer, who developed the US study which served as main inspiration for this thesis, and who were so generous in sharing their knowledge while I stayed for a few months in Madison. This thesis has also benefitted enormously from the debates within the group of researchers carrying out the second phase of the JOBS project, formed by Jose Ignacio Anton, Hugo Figueiredo, John Hurley, Francis Green, Damian Grimshaw, JeanMarie Jungblut, Mark Keese, Sangheon Lee, Rafael Muñoz de Bustillo Llorente, Phillip O'Connell, Robert Stehrer, Matias Scaglione, Donald Storrie, Terry Ward and Erik Olin Wright.

I would like to thank my advisers, Modesto Escobar Mercado and Rafael Muñoz de Bustillo Llorente for all their help and support over the last few years. Most of my knowledge of quantitative analysis I owe to Modesto Escobar Mercado: from him, I learnt that statistics do not need to be unnecessarily complicated to contribute to the progress of knowledge, but honest and carefully thought. Rafael Muñoz de Bustillo Llorente has been more than an excellent PHD adviser: he has been my support and friend over the last few years. My intellectual development over this period owes more to him than to anyone else.

Finally, I would like to thank my parents Enrique and Victoria, and my brother Gonzalo, for their never-ending support with this PHD and with everything else. No words can make justice to the encouragement and support given to me by my wife Judit: she has listened my quandaries with patience, has helped me overcome them with her wonderful common sense, and has given me all the support I needed and much more. I also want to thank my still unborn daughter Elena, who with her coming to this world gave me the strongest motivation, and the strictest timetable, one can have to finish a dissertation. 


\section{Theoretical Foundations}

\section{Introduction}

In recent years, a number of academic papers and policy reports have used a methodology which we can call the jobs approach to study patterns of change in employment structures of advanced capitalist economies (US Council of Economic Advisors 1996; Ilg 1996; Ilg and Haugen 2000; Wright and Dwyer 2000; OECD 2001; Wright and Dwyer 2003; OECD 2003; Goos and Manning 2007; Fernández-Macías and Hurley 2008). There are two key elements to this approach:

1. The main unit of analysis is jobs rather than individuals, with jobs being defined as specific occupations within specific sectors. The whole set of jobs thus defined constitutes a jobs matrix, which is a simplified representation of the labour market.

2. Those jobs are then characterised by some indicator of job quality: normally, but not necessarily, wages. This "characterisation" is used for ranking the jobs and grouping them in a number of "job quality segments" (for instance: bad jobs, middling jobs, good jobs). Then, the evolution of employment ${ }^{4}$ in each of those segments for the period being studied is analysed, and maybe decomposed by third variables such as gender or employment status, etc.

The objective of most of the papers using the jobs approach has been descriptive and empirical, driven and oriented by policy rather than theory (with the possible exception of Goos and Manning 2007). In most cases, the aim was simply to evaluate the quality of the employment generated (or destroyed) within a particular period of economic expansion.

\footnotetext{
${ }^{4}$ This evolution is studied in terms of the net change in the stocks of individuals in jobs.
} 
In this chapter, I will try to contribute to the construction of a theoretical foundation for this jobs approach. Some of the theoretical arguments discussed in this chapter have been hinted at in some of those earlier papers using the jobs approach, and some of the arguments are new, even if derived from intuitions that were always implicit although not systematically developed in the mentioned papers.

In the following section of this chapter, I will try to provide a theoretical justification for the idea of using jobs (defined by occupation and sector) as the main unit of analysis. My main argument will be that the resulting matrix of jobs is a stylized representation (or a snapshot) of the existing division of labour within the production boundaries ${ }^{5}$ of contemporary capitalist economies. But the jobs approach can be also justified from a micro-perspective: from the perspective of workers, the cells in the jobs matrix represent relatively stable positions within the labour market, which require specific skills and correspond to specific employment trajectories, earnings and working conditions, and therefore, access to different life chances.

In the third section, I will discuss the state-of-the-art with respect to measuring job quality, providing a theoretical justification for the indicators that we will use in this thesis for characterizing jobs. If there is something clear in the specialized literature, it is that job quality is a multidimensional concept that requires multiple measurements. In this thesis, we will use three measures of job quality to evaluate the implications of structural change in the labour market: one based on wages, another based on the educational level of people in the different jobs and a third one based on a multidimensional measure of non-pecuniary job attributes.

In the fourth section, I will review the recent social sciences literature on the implications of the evolution of the division of labour on job quality. After reviewing the most important theoretical contributions, I will present a broad analytical model and some key hypothesis on the patterns of change in the composition of the division of labour that we expect to find in the following empirical analysis.

\footnotetext{
${ }^{5}$ Our concept of division of labour is circumscribed by the market boundaries as defined by the UN System of National Accounts (UN 1993: 6.18), which explicitly excludes all non-market production of services: most importantly, it excludes all domestic work which is not produced by paid domestic staff. The exclusion of domestic work has been criticized for years, with good reason, as it excludes half of all valuable work done in the total economic system (see Ironmonger 1998: 3). But even though we agree with such criticisms, we must stick to the UN definition for practical and theoretical reasons: practical, because all our analysis is based on statistics which follow the UN standards; and theoretical, because our approach derives from the Social Sciences literature on the division of labour and job quality, which concerns mainly work within the production boundary.
} 
Finally, I will discuss the potential implications of two confounding factors that can complicate the analysis and interpretation of our results: the initial structural differences between the countries covered in this thesis, and the time limits that the available data imposes on our analysis.

\section{The jobs matrix: a picture of the division of labour?}

The methodological pillar of the jobs approach is the jobs matrix, which is a representation of the structure of paid employment in a society at a specific point in time. In practical terms, this jobs matrix is simply a big table classifying all employment by sector and occupation, each cell being a job (see figure 2.1). The principle behind the existence of such a structure of sectors and occupations is the division of labour. This principle simply means that dividing tasks and assigning them to different specialized labourers increases greatly the overall efficiency of production. The application of this principle, at a societal level, implies that overall labour is divided into separated tasks and activities: the resulting structure of employment is itself called the division of labour in the literature. Therefore, we can understand the jobs matrix as a picture of the structure of employment by sector and occupation, which itself is another name for the division of labour. In this section, we will discuss the theoretical foundations of this idea.

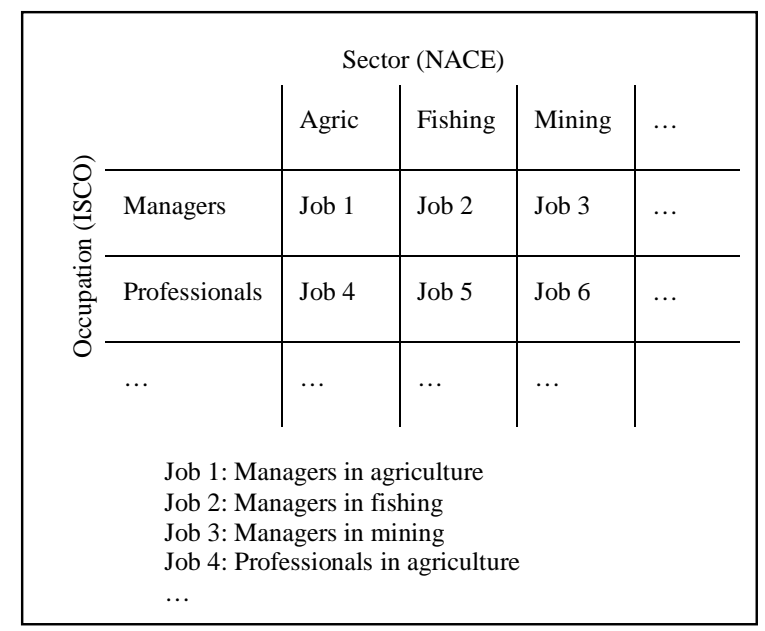

Figure 2.1: The jobs matrix in practice

Any cooperation between human beings aimed at providing with the means for subsistence and reproduction, in which there is separation and specialized allocation of activities to different individuals, is a form of division of labour. Almost by definition, 
therefore, some form of division of labour exists in all human labour. "All empirical evidence shows that labour or work always entails some specialization [...]. This does not, however, imply that it is caused by natural differences (biological differences between women and men, for example). Division of labour is always human-made, its forms are socially shaped." (Littek 2004: 8221). Human beings have to toil in order to survive in a hostile environment, but they have to toil together, they have to cooperate in one form or another. Dividing and specializing work increases greatly the possibilities for surviving, which is why this non-hardwired feature of human activity is pervasive in all known human societies.

The idea of increasing returns to specialization is one of the oldest in Western social thinking, going back as far as the Republic of Plato (for Plato, the principles of specialization and exchange were at the origin of the State itself; Sun 2005: 6). But it is, of course, Adam Smith who formulates the contemporary concept of division of labour. Smith considered the division of labour as the ultimate source of economic progress: even technological progress is a by-product of the division of labour for the founder of modern economics. Smith famously explained this principle with the example of a pin factory: dividing labour into 18 distinct operations carried out by specialized workers, the productive capacity of these workers can be multiplied several times. This is because the subdivision of tasks and specialization of labour increases the dexterity of workers, reduces coordination times and costs, and sows the seeds of technological innovation. The last point is specially important:

"[...] In consequence of the division of labour, the whole of every man's attention comes naturally to be directed towards some one very simple object. It is naturally to be expected, therefore, that some one or other of those who are employed in each particular branch of labour should soon find out easier and readier methods of performing their own particular work, wherever the nature of it admits of such improvement. A great part of the machines made use of in those manufactures in which labour is most subdivided, were originally the inventions of common workmen, who, being each of them employed in some very simple operation, naturally turned their thoughts towards finding out easier and readier methods of performing it" (Smith 1776: I.1.8).

Smith considered that the same general principle of subdivision of tasks and specialization of labour that he exemplified with the pin factory was behind all forms of 
division of labour, both within factories (division of labour between workers cooperating in the same production process) and within the society at large (division of labour between trades and companies producing different goods to be exchanged in the market). Marx would later argue that there is a fundamental distinction between the division of labour within factories and within society, so fundamental that it is necessary to speak about two different forms or principles of division of labour, despite the analogies and links connecting them.

"But what is it that forms the bond between the independent labours of the cattle-breeder, the tanner, and the shoemaker? It is the fact that their respective products are commodities. What, on the other hand, characterises division of labour in manufactures? The fact that the detail labourer produces no commodities. It is only the common product of all the detail labourers that becomes a commodity. Division of labour in society is brought about by the purchase and sale of the products of different branches of industry, while the connexion between the detail operations in a workshop, is due to the sale of the labour-power of several workmen to one capitalist, who applies it as combined labour-power. The division of labour in the workshop implies concentration of the means of production in the hands of one capitalist; the division of labour in society implies their dispersion among many independent producers of commodities [...]. Division of labour within the workshop implies the undisputed authority of the capitalist over men, that are but parts of a mechanism that belongs to him. The division of labour within the society brings into contact independent commodity-producers, who acknowledge no other authority but that of competition, of the coercion exerted by the pressure of their mutual interests" (Marx, 1867: chapter 14, section 4).

Smith did not see any fundamental difference between these two forms of the division of labour: first, because he saw it primarily as a technical principle for organizing production (and therefore, the social coordinating mechanism was for him a secondary concern), and second (most importantly), because he considered the labour relation as a market exchange like any other, with labour itself being the commodity traded (and therefore its coordination is handled by the market). In contrast, one of the cornerstones of Marx's thinking is the idea that labour is a commodity only in a metaphorical sense: what the capitalist buys from the worker is not labour, but labour power (not work, but 
the potential for work embodied in the worker), and therefore he must ensure the realization of this labour power into labour input in the production process by mechanisms other than the market (mechanisms involving some form of authority and control). This implies that the division of labour within producing units is fundamentally different from the division of labour between producing units: the former entails power relations and conscious coordination, whereas the latter entails a decentralized and spontaneous coordination based on price signals.

Of course, the two forms of division of labour are strongly related. For Marx, the manufacture division of labour originated historically from within the social division of labour, and the manufacture division of labour is constantly expanding the social division of labour in different ways (Marx, 1867: chapter 14, section 4; see also Ricoy 2003: 64). The limits between one and the other are porous, coinciding with the shifting limits of capitalist firms (or in more general terms, of productive organizations): whenever an activity moves outside the boundaries of a firm (for instance, because of a subcontracting decision by the company), it moves from the realm of the manufacturing to the social division of labour, and vice versa. But they remain two distinct principles for coordinating specialized productive activity, and despite their analogies and connections, they have to be considered as distinct forms of division of labour.

Modern orthodox economics remains as blind to the distinction between the division of labour within and between firms as Adam Smith was more than two hundred years ago: from such perspective, the labour exchange is a market exchange as any other (Putterman and Kroszner 1996), and therefore transactions within firms are necessarily (axiomatically) coordinated by prices as in any market (the fact that this is so patently at odds with the way work is organized within firms does not seem to be a problem for this school of thought). But in modern institutional economics, this distinction has become widely accepted, albeit in a slightly different flavour and coming from a different formulation. It was Ronald Coase, in his classic article on the nature of the firm, who reintroduced (without acknowledging it as such) the distinction originally made by Marx (Coase 1996). Coase started by asking himself why do firms exist at all: if the price mechanism is the most efficient coordinating mechanism for all types of transactions within market economies, why is this mechanism not present in the organization of production within firms? The answer given by Coase is that, for some 
types of transactions, the costs of using the price mechanism are simply higher than those of coordinating by direct authority:

"The operation of a market costs something and by forming an organization and allowing some authority (an "entrepreneur") to direct the resources, certain marketing costs are saved. The enterpreneur has to carry out his function at less cost, taking into account the fact that he may get the factors of production at a lower price than the market transactions which he supersedes, because it is always possible to revert back to the open market if he fails to do this." (Coase, 1996: 94).

Therefore, according to Coase there are two different principles for the coordination of transactions within capitalist economies: the market, which coordinates transactions between firms (or between producing units) and authority, which coordinates production within firms. This is exactly the same idea which is behind the distinction made by Marx between social and manufacture division of labour, even though the starting points of Marx and Coase are totally different.

This distinction between manufacture and social division of labour is the link between the jobs approach and the social sciences literature on the division of labour and its effects. In modern capitalist economies, two distinct forms of division of labour exist, one which applies to the division of labour between workers within producing units (manufacturing division of labour, vertical for brevity) and one which applies to the division of labour between producing units within an economy (social division of labour, for brevity horizontal). These two forms of division of labour are the basis for two classification systems that have become standard statistics for describing how labour is structured in modern economies: sectors and occupations. Using both principles simultaneously (occupations and sectors, or better: occupations within sectors), we can describe the current state of the division of labour within an economy along the two dimensions described earlier. This is exactly the idea behind the jobs approach.

An alternative way to justify or understand the jobs matrix is that it is a detailed list of all existing "jobs" within an economy, defining jobs as relatively stable and homogeneous positions, requiring specific skills, within the hierarchies of productive organizations. From this perspective, the jobs approach would consist in an evaluation of what types of such "jobs" are created or destroyed in a specific period of time: the 
relevance of such evaluation can be justified by the fact that such "jobs" are relatively stable categories within the economic and social structure, categories in which most people stay for a long time or even forever, and categories which imply differential working conditions as well as earnings potentials (this is the justification proposed by Wright and Dwyer 2003: 295). In my opinion, nevertheless, such a justification "from the bottom" of the jobs matrix is simply a reformulation of the earlier justification "from the top". There are "jobs" because there is a division of labour. In fact, we can understand the "jobs" in the jobs matrix as the smallest units in the structure of employment which derives from the current state of the division of labour within society and organizations.

\section{Job quality, relative wages and educational levels}

In this project, we want to study how the evolution of the structural division of labour has affected job quality in different European countries since the mid-nineties. In the previous section, we clarified what do we mean by division of labour, but what do we mean by job quality? In most of the papers which have used this jobs approach, a relative measure of wages has been used as the main indicator of job quality: we will do basically the same in this thesis, although we will use a secondary indicator based on average educational level, and as we will see, we will construct a tentative multidimensional index of job quality to test the robustness of the relative wages approach. Most of the papers using the jobs approach have provided mainly a pragmatic justification for the use of wages as the indicator of job quality: the ideal indicator would be a multidimensional job quality index, but the lack of adequate data makes it necessary to use relative wages as a proxy. In this section, we will briefly review the understanding of job quality and the role played by wages in the economic and social sciences literature, to try justify the use of a positional measure of wages as a job quality indicator and to discuss what implications may this have for our analysis.

Despite its importance in contemporary social sciences, job quality is a very elusive concept, for which there are no agreed definitions. We will start this discussion with a very broad definition to operationalize the concept ${ }^{6}$ : the quality of a job refers to the impact that having and doing such job implies for the well-being of the worker. This

\footnotetext{
${ }^{6}$ This broad definition and the distinction between work and employment quality derives from my previous work with Muñoz de Bustillo, Antón and Esteve (2009).
} 
broad definition emphasizes a couple of things: first, that our concept of job quality is worker-centered, and second, that it not only refers to the performing (doing) of the job (that is, physically carrying out the tasks associated with the job) but also to the contractual conditions associated with having that job. The impact of the doing element of the job can be called work quality (understanding work as the exertion of physical or intellectual activity in exchange for an economic compensation), while the impact of the having element of the job will be called employment quality (understanding employment as the contractual relation binding worker and employer). The broad concept of job quality, therefore, can be decomposed into two elements: work quality, referring to the impact of labour activity, and employment quality, referring to the impact of the contractual relation, on workers' well-being.

\subsection{The mainstream economic approach to job quality}

Mainstream economics have never paid much attention to the issue of job quality. The closest thing to a theory of job quality in this tradition is the theory of compensating differentials. The formulation of this theory comes from none other than Adam Smith himself, in the same Wealth of Nations (book 1, chapter 10) where he formulated the modern concept of the division of labour which we discussed in the previous section. In fact, after 200 years of economic literature, Adam Smith's formulation remains the canonic model of job quality in this tradition, as well as the canonic theory for explaining differences in wages for workers with similar skills and capacities (see Cahuc and Zylberberg 2004: 248).

The theory of compensating differentials argues that the utility derived from a job (which can be equated to our concept of job quality) depends on the combination of two separate but substitutive elements: on the one hand, the disamenities associated with such job (disamenities would be the characteristics of the job which negatively affect the well-being of the worker), and on the other hand, the monetary compensation that the worker receives for doing such job (wage). If the labour market is open and free, and agents have perfect information, these two elements (wages and disamenities) will tend to compensate each other, so that the overall level of utility derived from each job will be roughly the same for workers with the same skills and capacity.

Although the theory of compensating differentials is usually argued from the perspective of workers, it has its correlate on the employer side as well. Both for workers and employers these two elements have opposite impacts on their utility and 
benefits, and therefore, for both sides they will have to compensate each other: the worker will want a monetary compensation that counterbalances the decrease in wellbeing resulting from the performance of work (or the other way around: he will be happy to receive a lower wage for a job full of amenities); and for the employer, reducing the level of disamenities of a job will require higher costs, and therefore will reduce the wage offered (or the other way around: a job full of disamenities would be much cheaper for the employer, so he could increase its wage proportionately). Employers will offer jobs with different levels of disamenities (and therefore, wages) depending on the way they organize production and cost considerations. Employees will accept different levels of disamenities depending on their preferences for a higher wage or a more pleasant job. Therefore, the specific salaries for similar jobs or workers with similar skills and abilities may vary (depending on the specific combination of wages and disamenities that they prefer), but their overall level of utility derived from work will be the same.

The idea of compensating differentials can be represented graphically with indifference curves, as shown in figure 2. The vertical axis represents wages, and the horizontal axis, the (dis)amenities of different jobs. Each curve represents possible combinations of both elements open for workers with the same level of skills and abilities (this is very important, as we will see later). All the points within one curve provide the same overall level of utility to the worker, even if they vary in the specific level of wages or amenities that they imply. Each worker may select the specific combination of both elements that fits best his preferences with respect to the wage/disamenities trade-off. Workers with different skills and abilities (and therefore, with different productivities) will have different curves: those with a higher productivity will get higher amenities for the same wage or higher wages with the same amenities. This is shown by the distance between line Q1 and Q2 (the latter representing workers with higher skills and abilities). The theory of compensating differentials is one of the reasons why job quality has received so little attention in mainstream economics. This is because a corollary of this theory is that for a given level of skills and abilities (that is, within the constraints imposed by the fact that workers cannot cost more than their marginal productivity), and in the absence of external interventions, workers will have the maximal utility they can reach, with the specific combination of amenities and wage that they prefer. There is, therefore, no potential problem with job quality within a capitalist economy unless there 
are distortions resulting from external interventions: to increase job quality, therefore, we just have to ensure that markets operate freely and that agents have enough information. ${ }^{7}$

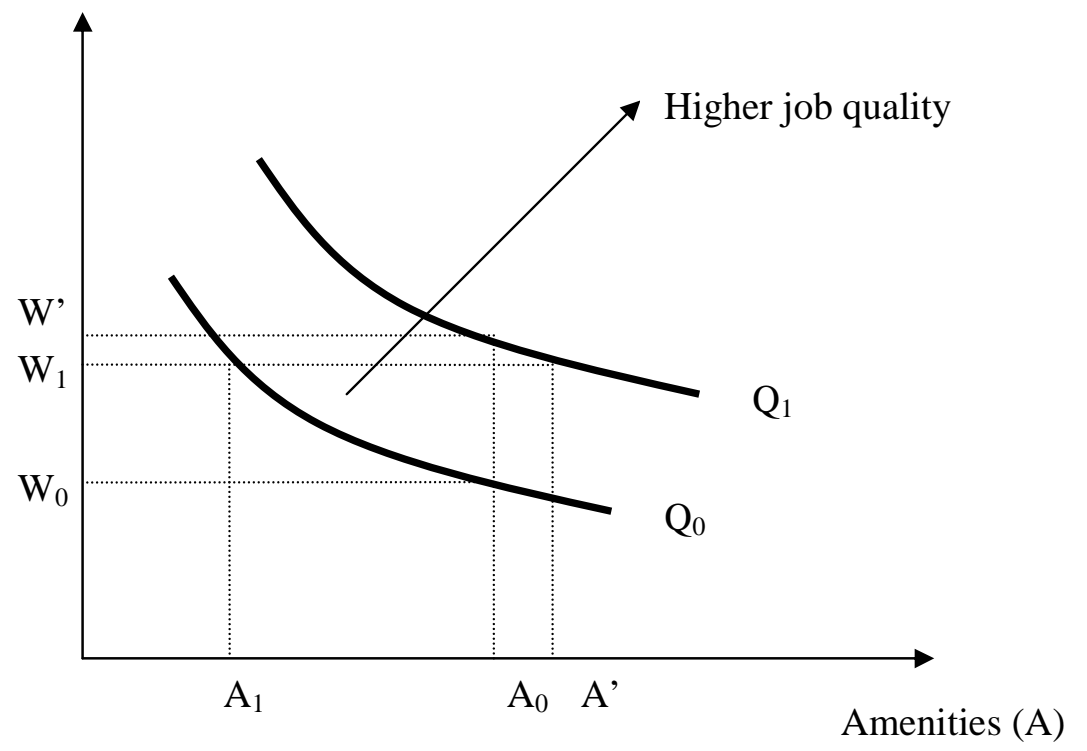

Figure 2.2: Combinations of wages and amenities leading to the same level of well-being (source: Muñoz de Bustillo, Fernández-Macías, Antón and Esteve 2009)

A crucial element of this theory for our purposes is that the compensating mechanism only applies to workers with similar levels of skills and abilities. This is because in mainstream economics wage levels (again, assuming a competitive labour market) are determined by the marginal productivity of labour - and the same goes for amenities-. Because amenities and wages are substitutive goods for employers, the bundle of wages and amenities that each worker can get is determined by productivity (therefore, the difference between Q1 and Q2 will be proportional to the productivity difference between both types of workers). So the theory of compensating differentials does not say that all workers will get the same level of utility from their jobs (that is, that all jobs will be of the same quality), but that all differences will reflect the different productivities of workers with different skills and abilities (which again, is the optimal outcome: a wage above marginal productivity of labour would be unsustainable, a wage

\footnotetext{
${ }^{7}$ In fact, from this perspective, any government attempt to regulate working conditions (for instance, regulating health and safety), will either reduce wages below the optimum level or generate unemployment.
} 
below would be exploitative and below the social optimum). All the differences that exist beyond those based on productivity are only apparent, different combinations of wages and amenities chosen by workers themselves. Job quality is, again, simply not problematic from this perspective.

Mainstream economic theory would probably not agree with the structural characterization of labour markets presented in the previous section, according to which markets and organizations structure the labour market into sectors and occupations, which together give rise to a labour market composed by jobs. But it is interesting to discuss what would be the implications of the theory of compensating differentials for our jobs approach. It seems quite reasonable to argue that the logic of compensating differentials would mostly apply within jobs (the level of skills and abilities required for the same job should be, in principle, very similar across different productive organizations, and therefore, differences in wages within a job would mostly derive from differences in preferences with respect to the wage-amenities trade-off), whereas across jobs there would be absolute differences in the bundles of wages and amenities because of differences in their relative productivities. Therefore, each job in the jobs matrix would correspond to a single line in figure 2.1, with internal wage differentials being different points within the same line, and the distance between the lines being the differences in job quality across jobs. ${ }^{8}$

Therefore, from this perspective the best way to study job quality would be to measure directly the skill and ability levels of each job (or productivity), because this variable determines the bundle of wage and amenities (and hence, job quality) associated to each particular job. Our variable of average educational level of workers within each job can be understood as a proxy of the skill level of jobs, and therefore, as a proxy of job quality from this perspective.

In principle, the use of wages or amenities as indicators of job quality would require evaluating both simultaneously. The logic of compensation between them implies that failing to take both into account simultaneously would lead to an incomplete assessment of job quality. But on the other hand, if the preferences of workers for wages and

\footnotetext{
${ }^{8}$ There are other potential mechanisms behind within-job differences in wage levels, such as career effects and personal abilities. Career effects involve within-person differences in wage across time (equalized at the average level throughout the lifetime) and therefore, it is irrelevant for job quality. Personal abilities are strictly the result of personal (endogenous) differences, and therefore it cannot have anything to do with job quality. In any case, these type of mechanisms should not affect the average level of job quality within a job, but its dispersion.
} 
amenities are roughly similar across jobs (assuming that they would vary within jobs), there would be no problem in taking only one of the two elements as the single indicator of job quality. There is nothing in this theory that says that the preferences vary across jobs, although it seems plausible that such a thing would happen. Because of the nature of the different types of productive activities, some jobs may be naturally more pleasant than others, irrespective of their relative productivities. Naturally more pleasant jobs may be associated with lower wage levels (ceteris paribus), because most workers would be positioned towards the lower right side of line Q1-and the opposite could easily happen to naturally unpleasant jobs-. If the effect of these job-biased combinations of wage and amenities would be large enough as to generate a variation in wage levels higher than the variation due to different productivities across jobs, then looking at only wages or amenities would lead to a wrong evaluation of the quality of jobs. From our point of view, although this potential source of bias must be taken into account (as we will see later, we will try to empirically test it), it is unlikely that the systematically biased differences in the wages-disamenities bundles across jobs are large enough as to distort the differences resulting form different productivity levels. Especially, if we consider that our approach will be ordinal rather than cardinal (this source of bias may affect the absolute level of wages, but it is unlikely that it is large enough as to change significantly the relative position of a job in the national wage hierarchy).

In any case, we will construct an alternative measure of job quality based on a multidimensional measure of disamenities to try to test these possible biases. This multidimensional measure will be based in the review of sociological approaches to job quality to be discussed later. Unfortunately, because of data availability issues, this multidimensional job quality indicator allows for a much less detailed analysis of the labour market, so we will only use it for testing the validity of wages and educational levels.

\subsection{The subjective approach: job satisfaction as an indicator of job quality}

An alternative approach for measuring job quality which has been gaining ground in recent times, both within economics and sociology, is to try to measure directly job quality (or the utility derived from work) by asking workers how satisfied they are with their jobs. Such an approach has the important advantage of simplicity, it avoids making a priori assumptions about what makes a good job (it is workers themselves who make 
that evaluation, on the spot of being asked about their job satisfaction) and produces a single straightforward index which is supposed to reflect an overall evaluation of job quality (workers are supposed to weight all the pros and cons of their jobs, and provide with an overall evaluation very close to the idea of utility of a job).

But as we have argued in previous work (see Muñoz de Bustillo and Fernández-Macías 2005; see also Green 2006: 10) this approach has the very important problem of being mediated by endogenous factors related to the subjectivity of the workers replying to the question. This alien element, which has nothing to do with job quality, contaminates the whole measure making it useless for our purposes. Two workers with exactly the same type of job (with the same bundle of wages and amenities, to say it in the economics terminology) may answer differently to the question of job satisfaction depending on such strictly psychological traits as adaptability, optimism or conformism. And the opposite: workers with very different conditions of work and employment may derive the same satisfaction from their jobs because of the simple fact that people tend to adapt their expectations to their objective situation. ${ }^{9}$ The strong endogenous bias of job satisfaction, therefore, makes it an inadequate measure of job quality for our purposes.

\subsection{Sociological approaches to job quality}

In the sociology of work literature, job quality has always been a central issue. On the one hand, because this tradition does not assume (as neoclassical economics) that salaries will automatically compensate for any unpleasant working condition, nor that wages reflect the full contribution of each worker to the productivity of his firm. In fact, in sociology the assumption tends to be the opposite: much research has focused on the mechanisms (such as exploitation or discrimination) that are behind the existence of unfair remunerations, or uncompensated disamenities. On the other hand, because with or without compensation, researchers within this tradition have tried to understand the implications of existing conditions of work on the well-being of workers. The fact that harsh working conditions are willingly accepted by workers in exchange for a high salary does not eliminate the negative implications of such conditions for the worker, which have to be studied and if necessary regulated. In a way, this second point derives from the first one, because once we reject the assumption of fair pay, once we accept

\footnotetext{
${ }^{9}$ This is, in fact, what usually happens with job satisfaction: in the most disparately different conditions of work and employment, most workers tend to give a remarkably similar answer to this question (job satisfaction tends to be implausibly high across nations, conditions of work and employment and time: see Muñoz de Bustillo and Fernández-Macías 2005).
} 
that workers may be forced by whatever reasons to accept otherwise unacceptable conditions of work, those conditions by themselves become a central social issue.

a) The traditional sociological approach: alienation and intrinsic quality of work

The sociological concern with job quality, as many other key sociological themes, goes back to the work of Karl Marx. In particular, two core concepts of Marxist theory have important implications for the sociological analysis of job quality. First, the concept of exploitation, which links an initial inequality in endowments between capitalists and workers to an intrinsically unfair compensation for work. This theory goes explicitly against the orthodox theory of wage determination of classical economics: contrary to classical economists, Marx argues that pay and job quality will always be suboptimal in capitalism (from the perspective of workers, of course). Capitalism as an economic system depends on labour exploitation, which is the ultimate source of profits, and for this reason, capitalism needs to ensure that workers are in a situation which force them to accept exploitative conditions of work. It will therefore tend to generate high levels of unemployment (Marx's reserve army of the unemployed) and de-skill work through the principle of the division of labour and mechanization, to keep workers easily substitutable and willing to accept almost any job for any salary (Marx 1867). The second key Marxist concept for the sociological literature of job quality is alienation, a philosophical concept that involves an anthropological interpretation of the nature of labour. For Marx, productive work is the essence of the human condition: it is through work that humans achieve realization and fulfilment. The capitalist labour relation, by separating workers from the means and fruits of their work, alienates them from their very nature and impedes their realization as human beings (Marx 1932). As exploitation, alienation is consubstantial to capitalism. Exploitation implies that workers never receive a full compensation for their work, and alienation that the nature of work under capitalism is inherently dehumanizing. The first concept implies a turn towards the conditions that maintain wages low: deskilling and power relations in the labour market and at the workplace. The second concept implies a turn towards the intrinsic qualities of work and the social conditions in which it is realized (autonomy, skills, the social environment).

Drawing on these Marxist concepts (and updating them), Braverman mounted his theory of the degradation of work in the $20^{\text {th }}$ Century (Braverman 1974). Braverman's arguments are more relevant for the study of the evolution of the division of labour than 
for the measurement of job quality, and therefore we will discuss them mainly in the following section. But it is important here to note his emphasis on skills and autonomy as the key elements of job quality (thus, primarily drawing from the Marxist concept of exploitation). This would have a lasting influence in the sociology of work literature, especially in the labour process tradition that derives directly from Braverman's magum opus.

The first important attempt at an empirical operationalization of Marx's concept of alienation in the social sciences was Blauner's study of "Alienation and Freedom" (Blauner 1964). Blauner proposed four dimensions of alienation, each of which could be measured through survey questions: powerlessness, meaninglessness, social alienation and self-stranglement. Blauner's approach has been criticised for resembling too much the standard job satisfaction analysis (Edgell 2006: 36). It was workers themselves who evaluated their own feelings of alienation (in terms of Blauner's four dimensions) through a questionnaire, something which is probably at odds with Marx's original idea, which referred to an objective state of deprivation of possibilities for self-fulfillment. Nevertheless, Blauner's approach was also very influential in the analysis of job quality, initiating a subjectivist tradition which has been applied to different countries and work environments (see, for instance, Shepard 1977; Hull et al 1982; Vallas 1988).

\section{b) Segmentation theory}

A different but equally important strand of sociological analysis of job quality is segmentation theory. This approach also departs from a rejection of the neoclassical model of the labour market and wage determination. The neoclassical model of competitive labour market, according to this theory, may be a useful description of some segments of the labour market, but not of the whole of it (Peck 1996: 47). The real-life labour market is split into different segments which function according to different rules and organizational principles (the competitive model being one of them, but not the only one).

The original formulation of this theory by Doeringer and Piore, two institutional economists, argued that advanced capitalist labour markets are dual in nature, with a primary and a secondary sector (Doeringer and Piore 1979). The primary sector is associated with the economic activities which make use of advanced technologies and highly skilled labour: work in this sector is coordinated by internal labour markets, in which employers and employees develop long-term relations (workers are protected 
from competition from outsiders), and wages and working conditions are above the standard. The secondary sector is associated to more backward economic activities which require relatively low-(or general-)skilled workers: this sector functions much more as the standard model of competitive labour market (with intensive competition between workers and salaries and conditions determined by supply and demand), employment relations are much more short-term and unstable, and working conditions and remunerations are considerably worse than in the primary sector. The key mechanism behind this dualism in Doeringer and Piore's model is the organizational response to the dilemma of how to keep up with technological advances and increase productivity while maintaining flexibility to deal with market fluctuations. The primary sector responds to the first necessity, while the secondary sector responds to the second.

Doeringer and Piore's theory sparked a whole strand of research and further theoretical development: segmentation theory remains one of the most important fields of sociology of work and heterodox labour economics. An important derivation of segmentation theory was initiated by radical political economists Gordon, Reich and Edwards, who challenged the technological/organizational explanation given by Doeringer and Piore arguing that the real mechanism behind segmentation was a conscious strategy by management to divide and rule (Gordon, Reich and Edwards 1982). Following Braverman's hypothesis of the fundamental tendency of capitalist industrial development to degrade work, these authors argued that it was the increasing levels of discontent which resulted from this tendency (which exploded in the restless 1970s) which led employers to a segmentation strategy, exploiting (or expanding) the divisions in the working class to undermine its solidarity and therefore to deflate workers' mobilization. Later developments of segmentation theory have become more multidimensional: segmentation is understood as a multiple process with multiple mechanisms behind, including social reproduction, discrimination, industrial relations or state policies (for a review, see Peck 1996: 56-79).

Overall, the segmentation approach implies that there is no automatic compensation between wages and working conditions (rather, they tend to correlate when different segments are compared) and that the determination of wages does not depend only on skills (although skills are a crucial driver for segmentation in this theory); therefore, that job quality can vary substantially in non-optimal ways. The segmentation approach also 
emphasizes the importance of the contractual relation and the conditions of employment for job quality.

\section{c) Health and safety literature}

A third strand of sociological analysis (with key contributions from occupational medicine and epidemiology) which is also relevant for the study of job quality is the health and safety literature. This tradition is strongly empirical in character (there is no unified theoretical approach behind), and it focuses on the analysis of the direct effect that the conditions of work have on workers' health. The range of conditions and outcomes studied in this tradition has varied according to the changing nature of work in the last century. Up to the 1970s, most research on this tradition focused on physical risks and hazards typically associated with industrial work; after the 1970s, the focus shifted towards psychosocial risks and their outcomes, reflecting the increasing importance of service employment (see Wilkinson 2001). This tradition has always had very strong links to social policy, in particular since the development of the Welfare State and modern labour regulation in the first half of the $20^{\text {th }}$ Century: there is an implicit assumption that, notwithstanding the monetary compensation received and whether it is willingly accepted by workers or not, there are some conditions of work which the society deems to be unacceptable (because of their impact on workers' wellbeing, hence the need to study job quality from a health and safety perspective) and which therefore must be forbidden or regulated.

\section{d) Work-life balance studies}

Finally, we will just mention a fourth strand of sociological research relevant for job quality which is relatively new but has become quite important in recent years: the studies on work-life balance. Although this issue has been in one way or another present in sociological research on work and employment from the very beginning, it is only in the last 25 years that this subject has really become a central issue. Behind this increasing centrality of work-life balance is the incorporation of married women and women with children to the workforce after the seventies ${ }^{10}$ : this incorporation brought to the surface a conflict between work and family demands which had before been precariously resolved by the gender division of labour inside and outside home (men

\footnotetext{
${ }^{10}$ Unmarried women were naturally part of the workforce of advanced capitalist economies well before the seventies.
} 
worked for pay, women carried out reproductive duties at home; see Drew, Emerek, and Mahon 1998; also Whitehead 2008).

The work-life balance literature emphasizes that work does not take place in a vacuum, but within the context of life: therefore, job quality should not only refer what happens within the workplace and its implications for workers as monads, but also its wider implications for the possibilities of having an integrated and satisfactory life outside work. This approach is specially important from a gender perspective, because the persistence of unequal gender roles implies that women are much more likely to suffer from conflicting work and family demands. The aspects of work which have a larger impact on the work-life balance are those related to the duration and scheduling of working time (working time marks the boundary between work and life), most importantly working time flexibility, predictability of schedules and the extension of working time into hours usually reserved for family duties or socializing (for a review, see Guest 2002).

Summarizing the different contributions: a list of job quality dimensions

From a sociological perspective job quality is a multidimensional phenomenon and therefore requires a multidimensional approach for its study and measurement. Although there is no encompassing theory of job quality that attempts to link all its components and their interactions within a single model, we can derive a tentative list of dimensions based on the elements considered important by the different sociological traditions discussed in this section (see table 2.I). We will use this list of dimensions to construct an alternative job quality indicator that will be compared to the two indicators that will be used in this thesis (based on wages and educational levels). This multidimensional job quality indicator will not include wages as an element (even though it is an obvious component of job quality, included in table 2.I) because we want to compare its results from those of the indicator based solely on wages. Therefore, we can consider this third indicator as a proxy of disamenities, because it will be based on different measures of aspects and conditions of work and employment that have an impact on the well-being of workers (following our earlier definition), but leaving out the monetary compensation. 


\begin{tabular}{|c|c|c|c|c|}
\hline $\begin{array}{c}\text { The orthodox } \\
\text { economic } \\
\text { approach: } \\
\text { compensating } \\
\text { wage differentials }\end{array}$ & $\begin{array}{c}\text { Traditional } \\
\text { sociological } \\
\text { approach: alienation } \\
\text { and intrinsic quality } \\
\text { of work }\end{array}$ & $\begin{array}{l}\text { The institutional } \\
\text { approach: } \\
\text { segmentation and } \\
\text { employment } \\
\text { quality }\end{array}$ & $\begin{array}{c}\text { Occupational } \\
\text { medicine and health } \\
\text { and safety literature: } \\
\text { risks and impact of } \\
\text { work on health }\end{array}$ & $\begin{array}{c}\text { Work-life balance } \\
\text { studies }\end{array}$ \\
\hline $\begin{array}{l}\text { Labour } \\
\text { compensation: } \\
\text { (1) wages; } \\
\text { (1bis) disamenities } \\
\text { (unmeasurable); }\end{array}$ & $\begin{array}{l}\text { Objective strand: } \\
\text { (2) skills; } \\
\text { (3) autonomy } \\
\text { Subjective strand: } \\
\text { (4) powerlessness } \\
\text { (5) meaninglessness; } \\
\text { (6) social isolation; } \\
\text { (7) self-estrangement }\end{array}$ & $\begin{array}{l}\text { (8) Contractual } \\
\text { status and stability } \\
\text { of employment; } \\
\text { (9) Opportunities for } \\
\text { skills development } \\
\text { and career } \\
\text { progression }\end{array}$ & $\begin{array}{l}\text { Conditions: } \\
\text { (10) Physical risks; } \\
\text { (11) Psychosocial } \\
\text { risks; } \\
\text { Outcomes: } \\
\text { (12) Perceived impact } \\
\text { of work on health; } \\
\text { (13) Absenteeism }\end{array}$ & $\begin{array}{l}\text { Working time: } \\
\text { (14) Duration } \\
\text { (15) Scheduling } \\
\text { (16) Flexibility } \\
\text { (17) Regularity } \\
\text { (18) Clear boundaries } \\
\text { Intensity: } \\
\text { (19) Pace of work } \\
\text { and workload } \\
\text { (20) Stress and } \\
\text { exhaustion }\end{array}$ \\
\hline
\end{tabular}

Table 2.I: Dimensions of job quality suggested by the different traditions

(Source: Muñoz de Bustillo, Fernández-Macías, Antón and Esteve 2009)

Therefore, we will have indicators for the three main elements of job quality in the compensating differentials approach: skills (proxied by education), pay (measured by wages) and disamenities (based on the multidimensional job quality index based on the dimensions 2 to 20 of table 2.I). In terms of the sociological approach to job quality, we will have both a multidimensional job quality index, an indicator of wages ${ }^{11}$ and an indicator of the educational level of job-holders ${ }^{12}$ : with these three indicators, we should be able to get a reasonably comprehensive view of job quality, assuming its fundamental complexity.

\section{The structural evolution of the division of labour and job quality}

But the core of this thesis (and most previous research carried out with the jobs approach) is not about the division of labour or job quality on their own, but about what are the implications of the evolution of the division of labour on the quality of jobs in advanced capitalist economies. In this section, we will discuss in some detail the most important contributions of the social sciences literature (mainly economics and sociology) on this issue, grouping them three categories: those that argue that, as a result of the structural evolution of the division of labour, job quality is degrading (the

\footnotetext{
${ }^{11}$ In sociology of work, wages and working conditions are often assumed to correlate, rather than compensate each other as in neoclassical economics. Hence the paradox that although sociological theories have often argued against a purely instrumental perspective of work, there is nothing in these theories against using wages as a proxy of job quality.

${ }^{12}$ The interest of the educational indicator from a sociological perspective can be justified on two grounds. On the one hand, because higher education leads to better jobs (through mechanisms of social reproduction, another important tradition of sociological analysis). On the other hand, because educational levels tend to be correlated with the skill requirement of jobs, which as we have discussed earlier, has been considered one of the defining elements of job quality from an intrinsic perspective.
} 
pessimists), those that argue it is upgrading (the optimists), and those that argue that both things are happening at the same time, through a polarization process that is emptying the middle. But before we review these different theories, it is necessary to make some important clarifications.

The division of labour within a society is continuously changing, transforming both the nature of existing jobs and the structure of employment. We can differentiate two types of change in the division of labour:

a) Within-jobs changes in the division of labour: these would be the changes that transform the types of tasks involved in specific jobs, the skills required or the associated hierarchical position.

b) Across-jobs changes in the division of labour: these would be the changes that affect the quantity of labour which is allocated to the different jobs in an economy. This second category would also include the creation of new jobs (which can be the result of the subdivision of a previously existing job, or of the creation of new activities for satisfying new needs, or of the creation of activities involving new forms of satisfying old needs), as well as the disappearance of pre-existing jobs (which may become unnecessary, may be substituted by machines or by jobs of a different nature). In other words, this second type refers to structural change in the division of labour.

These two types of change in the division of labour are closely connected, interacting between them and taking place simultaneously in a continuous process of shifts and transformations in the employment structure. The jobs approach which we are discussing here, and this thesis, is exclusively concerned with the second type of change in the division of labour (or employment structure). We can call this second type of change the compositional evolution of the division of labour, or more simply the transformation of the jobs structure. What we will do here is to study such transformation from the perspective of job quality: assigning a relative measure of job quality to each job, we will allocate the net change in the stocks of employment by jobs within a job quality metric.

This means, and we have to be very clear this, that this approach ignores all changes that take place within jobs, as well as changes in job quality of an absolute rather than relative nature. For instance, with this approach we would not detect an increase in the 
level of wage inequality unless it changes the relative position of jobs against each other, nor would we detect a process of generalized degradation of job quality (as the approach is relative, it is not affected by multiplying all values by a constant). This is certainly a limitation of the approach, which has to be assumed as such—but it would be wrong to overstate its implications. Evidence suggests that, in the short and medium run, the within-job changes do not affect significantly the relative positions of each job against the rest (see Krueger and Lawrence 1986; Allen 1994; for a theoretical discussion, Weiss and Fershtman 1994), so that using a fixed wage hierarchy should not generate a significant bias (for more details, see the methodological section). With respect to the potential problem of a generalized change in job quality levels, it seems implausible within a short period as the one we will study here, but even if something like that could have happened, it would not invalidate this approach. Our focus is on structural change, which is best studied with a relative approach (rather than putting the whole structure within an externally defined absolute metric, a relative approach implies studying structural change on its own terms). These are not really problems of this approach as such, but potential misunderstandings. As long as we are aware that this thesis is about the job quality implications of the evolution of the division of labour from a compositional perspective, not about changes in wage inequalities or changes in job quality in general, there should be no problem.

A further important qualification concerns the fact our approach will be totally demandoriented: that is, we will ignore the supply-side of the labour market both in our review of the existing literature and in our own empirical analysis. This necessarily implies a misrepresentation of reality to some extent, because the distinction between supply and demand on the labour market is useful but artificial: in practice, the functioning of the labour market depends on the interaction between the people that want to work (supply) and employers that want to hire them (demand). The changing division of labour and its impact on job quality is affected from factors coming from both sides, but we will focus on only one of them. Why?

First, because we need to put some limits to the range of issues that we will cover: pretending to do an evaluation of all supply and demand-side factors affecting the changing division of labour and job quality would simply be too ambitious (this thesis is already ambitious enough, despite being circumscribed to one side only). Second, because the basic definitions and assumptions explained in the previous two sections are 
most directly linked to the demand side of the labour market. Our concept of the division of labour mainly concerns the labour process from a material perspective, and our concept of job quality on the impact of work (within the production boundaries) on the well-being of workers: in both cases, our focus is on what happens at work and how work is organized, which in a capitalist economy is ultimately the prerogative of employers. Third, because the same orientation is found in most of the literature on this issue.

We believe that each of these three reasons is solid and meaningful, but they can be contested, and we have to be honest about it. With respect to the first point, the danger is to oversimplify rather than simplify: that is, to arrive to wrong conclusions because we are ignoring important factors. In particular, there are three supply-side factors whose neglect can be quite harmful, because they are likely to have played a significant role in the story we will tell in this thesis: the change in the educational level of workers, the increasing incorporation of women in employment, and the recent wave of migration in some European countries. With respect to the second point, it is true that the effect of these three factors is more indirect than the demand-side factors we will discuss in the following pages, but indirect does not mean unimportant: the increasing levels of education of the European labour force has been explicitly linked to the expansion of high-skilled jobs (Acemoglu 1999); the destandardization of employment conditions is strongly associated with the incorporation of women in the labour market (Rubery, Smith, Fagan and Grimshaw 2005); and the recent availability of a large pool of migrants has probably facilitated the expansion of low-paid employment in many European countries (Fernández-Macías and Hurley 2008). With respect to the third point, as shown by the references of the previous lines, there is a long tradition of studying these issues, even though often not explicitly from a division of labour perspective as the one taken in this thesis.

So we do not pretend that supply-side issues are unimportant, and we have to be aware that we will be leaving important issues out of our discussion. ${ }^{13}$ But we still believe that in order to keep this thesis within a manageable range of complexity, it is necessary to restrict our focus to the demand-side, following most of the literature on this field.

\footnotetext{
${ }^{13}$ In fact, in previous research, I have looked specifically at these issues (Muñoz de Bustillo, FernándezMacías and Antón 2007; Parent-Thirion, Fernández-Macías, Hurley and Vermeylen 2007), even from the same "jobs" perspective (Fernández-Macías and Hurley 2008; Stehrer, Ward and Fernández-Macías 2008).
} 


\subsection{The pessimists: the evolution of the division of labour and the degradation of work}

The discussion of the effects of the changing division of labour on job quality has been part of the general debate on the division of labour since its modern formulation by Adam Smith in "The Wealth of Nations". Most of the classical perspectives on this issue (starting with Smith himself) were deeply pessimistic. Until well advanced the $20^{\text {th }}$ century, most social thinkers assumed that the division of labour, the same principle that had precipitated the largest expansion of material wealth in human history, had the most atrocious effects on the human condition. For Adam Smith, it "mentally mutilated" workers until they became "as stupid as human beings can be". For Marx, it deskilled, alienated and dehumanized workers, until they became a "crippled monstruosity". For Frederick Winslow Taylor, who spoke as an engineer, without any moral intention, industrial workers had to become "trained gorillas".

As is well-known, Smith was the most enthusiastic supporter of the advantages of the division of labour: for him, this principle was nothing less than the ultimate source of all economic progress (Smith 1776: I.1.1). But at the same time, he was surprisingly pessimistic about the effects that such principle exerted on the nature of work and the condition of workers, as explained in this often-quoted passage from "The Wealth of Nations".

In the progress of the division of labour, the employment of the far greater part of those who live by labour, that is, of the great body of the people, comes to be confined to a few very simple operations, frequently to one or two. But the understandings of the greater part of men are necessarily formed by their ordinary employments. The man whose whole life is spent in performing a few simple operations, of which the effects are perhaps always the same, or very nearly the same, has no occasion to exert his understanding or to exercise his invention in finding out expedients for removing difficulties which never occur. He naturally loses, therefore, the habit of such exertion, and generally becomes as stupid and ignorant as it is possible for a human creature to become (Smith 1776: V.1.178).

It is interesting to note that such a devastating pessimism about the effects of the division of labour on workers did not lead Adam Smith to reconsidering the other benefits of such principle. In fact, Smith did not devote much time to discuss what 
should be done against the terrible consequences of the division of labour on the human condition. His main suggestion was that the state should provide general education for the working classes to somehow alleviate this brutalizing effect of modern work principles (Smith 1776: V.1.178).

It is, of course, Marx who fully developed a theory about the effects of the division of labour on the human condition, a theory which plays a central role in his evaluation of industrial capitalism as a social form. For Marx, the very essence of human nature is productive activity, and it is through productive activity that human beings can achieve fulfilment and realization. The division of labour in manufacture ${ }^{14}$, at least as it developed in capitalist industry, separates (alienates) workers from their very nature as human beings by separating (alienating) them from the means and fruits of their work: workers become pieces of a machine which is controlled by someone else.

As argued earlier, Marx understood the production process not only from a technical perspective (as Adam Smith), but also from a political perspective, involving asymmetrical power relations, authority and control. For Marx, labour can never be a commodity as any other, because it is purposeful human activity which cannot be separated from workers (or executed without their willingness). What is bought and sold in the labour market is labour power, the capacity to work for a specific period of time. But the input needed in the production process is labour, not labour power: somehow, labour power has to be converted into effective labour within the manufacturing process. Hence the inherent conflict in capitalist labour relations: for a given wage level, capitalists will want to extract as much labour as possible from their employees, whereas it is in the interest of workers to work as little as possible (considering that they do not benefit directly from the fruits of their labour, which belong to the capitalist that hired them, but from a salary fixed beforehand). ${ }^{15}$ It is for this reason that the capitalist production process necessarily involves the exertion of authority and control within the factory. For Marx, the manufacturing division of labour is a very powerful tool in the hands of capitalists for securing their control over the labour process and ensuring a high rate of exploitation: on the one hand, because it transforms the worker into a piece of a larger mechanism which he does not control or understand; on the other hand,

\footnotetext{
14 This argument concerns the division of labour in manufacturing, not the social division of labour. See section 2 above.

${ }^{15}$ This conflict is at the core of the Marxist concept of exploitation, which Marx linked to his labour theory of value: the benefits of the capitalists depend on his appropriation of surplus labour, that is, on the difference between the salary and the full value of what the worker produces (see Mayer 1994: chapter 3).
} 
because it simplifies the work tasks and skills required for industrial work so much that workers can be easily substituted and therefore their bargaining position vis-à-vis the employer is weakened (Marx, 1867: chapter 14, section 5).

Therefore, for Marx the principle of the division of labour (especially within manufacturing) is not a neutral technique that brings about greater efficiency in production, even if unfortunately degrading working conditions (as argued by Smith). For Marx, the ever deepening division of labour is a tool used by capitalists to degrade labour (to ensure their control over the labour process and the maximum possible exploitation of workers). Although Marx did not deny the higher efficiency (in purely technical terms) of cooperative production organized rationally according to the principles of division of tasks and specialization of workers, he emphasizes its interactions with the relations of power within the production process and therefore explicitly challenges its neutrality.

There is a certain degree of ambiguity, not to say contradiction, in Marx's arguments about the division of labour and its effects. Marx simultaneously considered the principle of the division of labour as a genuine progress in the productive capacities of humankind and as an evil tool in the hands of capitalists to dismember workers' human nature in order to facilitate their exploitation. We can illustrate this ambivalence with Marx's own words, from chapter 14 of tome I of Capital: "If, therefore, on the one hand, it [the division of labour] presents itself historically as a progress and as a necessary phase in the economic development of society, on the other hand, it is a refined and civilised method of exploitation" (Marx, 1867: chapter 14, section 5). In this sense, the analysis that Marx makes of the principle of the division of labour is similar to his analysis of machinery, which he considered both a force for the progress of humankind and a powerful tool in the hands of capitalists to further exploit workers.

This ambivalence has lead to some debate between Marxist scholars on whether the division of labour has to be considered part of the material or the social relations of production (Cohen 2000: 111-113). The material relations of production refer to the technical organization of the production process, to the way societies organize the production of the use values (goods and services) they need: therefore, these relations can be abstracted from the specific social forms of authority and power linking the people involved in production. These social forms are what Marx called the social relations of production (class relations are an example of social relation of production). 
If the principle of the division of labour brings a genuine increase in the productivity of labour, as machinery does, then the division of labour is part of the material relations; if the principle of the division of labour only brings an increase in the rate of exploitation, then the division of labour is part of the social relations of production. My understanding (following Cohen 2000: 111-113) is that, notwithstanding the use that capitalists may do of the division of labour, Marx considered the first sentence to be the correct one. $^{16}$

For Marx, the principle of the division of labour was by no means the central mechanism behind the social structure of capitalism. This centrality, in Marx's theoretical framework, is assumed by the private property of the means of production by capitalists, the deprivation of workers from them, and the support given by the state to such order of things. This institutional framework generates the bipolar class conflict around which capitalism is defined as a political, social and economic system. The division of labour is certainly a powerful tool in the hands of capitalists, as much as machinery or technology in general, to advance their interests: but it is not the mechanism that shapes capitalism as a social and economic system. For Marx, the tendency which defined the character and future of capitalism was the increasing polarization of society in two contradictory classes, bourgeoisie and proletariat. The degradation of work as a result of the increasing division of labour in manufacturing is at most a side-effect of such tendency, subordinated to it.

The ambiguity in Marx's analysis of the effects of the division of labour may appear nowadays somewhat outdated, a piece of Marxist scholasticism, but it is crucial for the overall evaluation of the effects of the division of labour from this tradition of thought. It concerns whether the division of labour is a fundamentally good development that expands human productive capacities (as Adam Smith would say, despite its negative moral impact on workers; or as Marx could add, despite its evil potential under capitalists' hands) or a historically contingent form of social organization that only increases the capacity of one social group to exploit another, and which therefore should eventually disappear in a more just social system. Some followers of Marx adopted the

\footnotetext{
${ }^{16}$ Nevertheless, a certain ambiguity remains: for instance, when Marx said in "The German Ideology", that in communism there would be no division of labour (the same way as there would be no classes), this seems to imply that the division of labour is part of the social relations of production. Otherwise, if the principle of division of labour is one of the factors behind the high level of productivity which is a necessary precondition for socialism, how could it be abolished when socialism arrives? (see Llorente 2006 for a discussion of this specific issue; also Cohen 2000: 131-133).
} 
first view (such as Gerry Cohen; see Cohen 2000: 111-113), some others the second (such as Stephen A. Marglin; see Marglin 1976).

Braverman's degradation of work hypothesis can be framed on this second strand of Marxist analysis of the effects of the division of labour. Braverman's theory is basically an update and further elaboration of Marx's analysis of the labour process and the division of labour: but Braverman goes a step further and argues that the manufacturing division of labour that takes place within capitalist firms is basically a negative development that does not increase the productive capacity of men, just the rate of exploitation.

The gist of Braverman's argumentation lies in his detailed analysis of contemporary management literature, especially of F. W. Taylor and his Principles of Scientific Management (see Braverman 1998: chapter 4). Taylor's ideas are almost a perfect managerial correlate of Marx's theory of the labour process under capitalism. For Taylor, factory workers tended to shirk or soldier, because they assumed that working fast would eliminate jobs for other workers and because it was objectively in their interests to soldier in a system which paid by the day or by the piece, but not according to their real productivity (Taylor 1911: chapter 1). He criticised the fact that, at his time, most of the responsibility and control of the work processes remained in the hands of workers, especially in the case of craft work: allowing labourers to organize their own pace of work led to a generalized shirking, to productivity levels far below the real optimum. In order to increase the efficiency of production, managers had to (re)gain control of the work process, which involved collecting and monopolizing all the knowledge previously on the hands of workers, rearranging the work process under scientific principles, selecting the workers suitable for each task and providing them with detailed instructions. Taylors' system was basically a further step in the principle of division of labour in manufacture, according to which there would be not only separation of tasks and allocation to specialized workers, but an effective separation of conception and execution of work, the first being the absolute prerogative of managers and the second being carried out without any requirement of initiative by workers.

Braverman's core argument is that Taylorism, which became the standard system of work organization in large capitalist factories throughout the first half of the $20^{\text {th }}$ Century, is simply a more explicit formulation of the principles which were inherent in capitalist work organization from the very beginning, as argued by Marx a century 
earlier: "its role [of Taylorism] was to render conscious and systematic, the formerly unconscious tendency of capitalist production" (Braverman 2000: 83). The goal behind this refinement in the division of labour is simply the disempowering and deskilling of workers in order to increase the rate of exploitation. This system is not better in terms of efficiency than the previous craft system, under which it was the worker who kept the knowledge and control of production and the degree of task differentiation was considerably lower. "Taylorism belongs to the chain of development of management methods and the organization of labour, and not to the development of technology, in which its role was minor" (Braverman 2000: 59).

The hypothesis of Braverman, therefore, with respect to the effects of the division of labour on job quality is even stronger than that of Marx: the progressive division of labour under capitalism is a purposeful degradation of work, which does not imply progress in the productive powers of humankind, but just an increase of exploitation. As happened with Marx, this mostly concerns the manufacturing division of labour (our vertical dimension of the jobs structure). Although Braverman's discussion is mostly based on the manufacturing sector, he explicitly argued that the same tendency affected clerical work and services in general, helped by technological innovations that facilitated the deskilling of these previously unaffected sectors (Braverman 2000: chapters 15 and 16).

Braverman's theory is the culmination of the pessimistic Social Sciences tradition on the effects of the division of labour, which had dominated economics and sociology of work since the $18^{\text {th }}$ Century (we have focused on Smith and Marx, but we could have mentioned also Tocqueville and Stuart Mill, for instance). The influence of Braverman's book in the field of Sociology of Work was enormous throughout the seventies and eighties, and his hypothesis of deskilling and work degradation has been empirically tested innumerable times in the most different contexts. But in fact, most of the evidence tended to refute, rather than confirm, Braverman's predictions (for a review of empirical evidence on Braverman's hypothesis, see Edgell 2006: 53-59). The structural evolution of the division of labour had apparently given a radical turn towards a less dehumanizing labour process even before Braverman published his classical study. 


\subsection{The optimists: more and better jobs in a post-industrial economy}

The most obvious difference between the previous interpretation of the implications of the evolving division of labour and this one is that they focus on different sectors of the economy. The pessimists' view was mostly based on an analysis of the conditions of work and work organization within manufacturing, which since the Industrial Revolution and up to the mid $20^{\text {th }}$ Century was clearly the dominant sector of the economy. The optimists' view (to continue with this slightly artificial dichotomy), instead, departed from the assumption that manufacturing was not the dominant sector anymore, having been substituted by services (after the 1970s, this was statistically true for many advanced capitalist economies; see Castells 2000: 224-232). This means that, even though their respective interpretation of the effects of the changing division of labour could not be more different, they are not necessarily incompatible. Many postindustrialists would agree with Braverman that the nature of work in manufacturing tends to degradation and deskilling: their point would be that manufacturing is becoming a marginal sector, and the nature of work in services (especially in knowledge intensive services, as we shall see right now) is entirely different. But Braverman and post-industralists were coetaneous, and they had direct confrontations: the Marxist American scholar argued that the same processes of deskilling and degradation was already taking place in services; and many post-industrialists argued that, even within manufacturing, the process of deskilling was counterbalanced by the increasing demand of high skilled workers to deal with new machinery and information technologies.

But why this secular trend towards services? For discussing this core element of postindustrialist theories, we will follow the argumentation of Fourastié ${ }^{17}$, which is the clearest and most carefully developed: one of the vices of post-industrialism is a certain tendency to broad generalizations and vagueness in the argumentation. The potential for productivity gains are very uneven across the different sectors: since the industrial revolution, mechanization has led to a massive productivity growth within the goodsproducing sectors (agriculture and manufacturing), but it has had very limited impact on services, whose productivity has remained at very similar levels for hundreds of years (because the very nature of services, which involve a direct and personal interaction between the producer and consumer, has remained largely unchanged). This in itself (as

\footnotetext{
${ }^{17}$ The book in which Fourastié developed this theory ("Le Grand Espoir Du Xx Siècle") was never translated into English, so in my exposition I follow the summaries of Hospers 2004 and Van Dijk 1998.
} 
argued by Baumol 1967) would imply that, even if the structure of demand would remain constant (the same proportion of goods to services would be demanded), the service sector would tend to assume an increasing proportion of overall employment. But post-industrialists go even further, arguing the structure of human wants shifts towards services as income rises. Developing Engel's law (which declared that the proportion of income spent on food tends to decrease with income), Fourastié argued that the income elasticity of agricultural and manufactured goods is lower than 1 (that is, their share in consumption shrinks with income). In fact, he argued that the consumption of goods eventually reaches a point of saturation, in which increasing income does not lead to increasing consumption of goods (Hospers 2004: 48). On the contrary, the demand of services is highly elastic with respect to income, because services contribute to well-being and to the saving of time by the consumer (and therefore, it is much more difficult or even logically impossible to reach a saturation point as in the case of material goods). The interaction of both processes (uneven productivity growth by sectors, and higher income elasticity of demand for services as income grows) leads to the rising dominance of the service sector in terms of employment. Similar reasons have led more recent writers to argue that within the service sector, it is the most knowledge-intensive activities which tend to concentrate most of employment growth (Castells 2009: chapter 4).

So the underlying argument is that in most developed capitalist societies it is the service sector rather than manufacturing which becomes the key sector in terms of employment. The next step in the post-industrialists' argumentation is that the nature of productive activity in services is completely different from the nature of manufacturing activity. Here we will draw from Daniel Bell's "The Coming of the Post-Industrial Society" (Bell 1976), which develops a more detailed account (although often imprecise) about the differences between the nature of work in services and manufacturing. Services imply working directly with people, not machines: service work is human interaction, intrinsically much less dehumanizing than industrial work (Bell 1976: 163). And within services, the fastest-growing sub-sectors are those which deal directly with information and knowledge, which require highly skilled workers with creativity and social abilities: the contrast with Braverman's degraded industrial worker is clear. In Bell's view, the production process in the service sector is directly incompatible with the hierarchical machine-like discipline of the Taylorist factory: in service activities, hierarchies need to 
be flatter, a higher level of autonomy is necessary for the work to be carried out, and the nature of the labour process is inherently more flexible. Finally, the service sector is characterized by smaller firms in which work relations, even between managers and workers, are necessarily closer (again, more "human"), flatter and less hierarchical than in the Taylorist ideal. ${ }^{18}$

Although Bell's arguments are often vague generalizations (understandable in a book which labels itself "a venture in social forecasting"), its influence has been enormous even beyond the field of academic sociology. Many of its key concepts and catchphrases have become common language in politics and mass media (starting with the label of post-industrial society itself). And it has to be said that, in terms of empirical support, the optimistic predictions of Bell fared much better than the pessimism of Braverman. Most empirical tests of the evolution of skills in the last 30-40 years find much more evidence of upskilling than deskilling (see Gallie 2007).

In his original work, Bell had prophesized that it would be the informational (or knowledge-intensive) sectors within services that would grow faster and eventually dominate the new economic order, but he had nevertheless based his argumentation on the nature of service work in general in contraposition to the industrial sector. Bell's followers have tended to focus much more on the information-producing sectors to develop their arguments about the current and future evolution of skills and job quality. The most important of Bell's followers is Manuel Castells, who developed a slightly more nuanced (and less enthusiastically optimist) view on the changes brought about by the coming of the Information Society. In his magnum opus "The Rise of the Network Society" he speaks more about polarization than upgrading (Castells 2009: 216, 266).

\section{2.bis. A not so optimist upgrading theory: skill-biased technological change}

In recent economic literature, we can find a second theoretical framework that predicts a similar upgrading in the jobs structure to the one predicted by post-industrialists. This theoretical framework is known as Skill Biased Technological Change (SBTC from now on). Under the umbrella of SBTC there are many different theories and approaches, all loosely connected by a similar overall idea but different in the details (for an review,

\footnotetext{
${ }^{18}$ Bell's arguments about the changing nature of work in Post-Industrial society are not only based on the shift to services. In general, he argued that there is an increasing premium on technical knowledge across all sectors as a result of the increasing application of technology in production processes. Bell also prophesized an expansion of public sector employment, because the market cannot provide adequately for some of the most important needs of a Post-Industrial society, such as education and health (Bell 1976: 128).
} 
see Acemoglu 2002 and Violante 2008). This family of theories are very strongly grounded in empirical analysis: in fact, their origin is the attempt to explain an empirical regularity that was difficult to explain from a standard (orthodox) economic approach. Up to the seventies, the usual assumption was that technological change is neutral with respect to skills: that is, it leads to similar increases in productivity throughout all sectors and occupations. But after the seventies, there was overwhelming evidence of a simultaneous explosion of technological innovation in production (related to the first wave of the information technology revolution) and of wage inequality by skill levels (and increasing demand for highly skilled workers). The coincidence of both phenomena suggested that, for whatever reason, technological change tended to increase the demand for skilled with respect to unskilled labour.

All formulations of the SBTC hypothesis assume some type of complementarity between technology and skills. Violante, in his review of the SBTC literature (Violante 2008), identifies three different strands (which as we will see, are not necessarily incompatible):

- The first strand links the rise in demand for skilled labour (which is behind the empirically observed wage premium) to a sharp decline in the relative prices of equipment capital, especially evident in information technologies, in the last 30 years. This assumes the already mentioned complementarity between technology and skills: a decrease in the price of capital equipment pushes up the demand for skilled labour because they are complementary factors in production.

- The second strand tries to provide an explanation for the complementarity between skills and technology, arguing that the key difference between skilled and unskilled workers is that the former are better at dealing with change. In a period of rapid changes in the workplace because of the information technologies revolution, there would be more need of skilled workers.

- Finally, a third strand focuses on the organizational shifts triggered by innovation and new technology. New technologies not only tends to be complementary with higher skill levels: in fact, they directly tend to eliminate the most routine tasks from production, directly reducing the demand for unskilled workers. Both effects take place simultaneously, which explain the significant upwards organizational shifts experienced by firms introducing technological changes. 
In contemporary economics, SBTC is widely agreed to be one of the key forces behind the substantial increase in income inequalities that have experienced most capitalist economies since the seventies. Therefore, it seems ironic to categorize it as an optimist theory. But in terms of the evolution of the jobs structure (our subject here), the corollary of this theory is as upbeat as that of the post-industrialists, although for different reasons. SBTC implies a shift upwards in the employment structure, associated with the intensity of adoption of new technologies in the different firms, sectors and countries.

\subsection{A more nuanced view: job polarization theories}

As we have seen, $19^{\text {th }}$ and early $20^{\text {th }}$ century views on the impact of the changing division of labour on job quality were predominantly pessimistic, assuming that it would lead to an increasing degradation of work. Post-Second World War Social Sciences tilted towards a post-industrial and technophile optimism (although it was in this period when the pessimistic view had its swansong, with the publication of "Labour and Monopoly Capital"). In the end of the $20^{\text {th }}$ Century, the mood shifted again, pendulating towards a more negative view, although more nuanced this time than in the earlier work degradation theories.

\section{3.a) Institutionalist and trade explanations: deindustrialization and the declining} middle

Although there had been earlier hints of job polarization in sociological theory (even Braverman spoke about polarization, although for him the most important trend was towards outright degradation), the contemporary polarization debate started in the early eighties. Probably the initiating contribution to this debate was the radical revision of the Post-Industrialist arguments about the structural transformations of the division of labour in contemporary capitalism carried out by Barry Bluestone and Bennet Harrison, two American hetherodox political economists.

The basic argument of Bluestone and Harrison's "The Deindustrialization of America" (published in 1982) unfolds as follows. In the 1970s, the capitalist economic system experienced a global crisis, a crisis which was the paradoxical result of the maturing of

the same conditions that were behind the success of the Post-War expansion. Two factors were especially important in generating this crisis. First, the increasing competition that American corporations had to face at a global scale, which put their 
profits under increasing strain (before the 1970s, American corporations benefited largely from this increase in international trade, because it meant more business opportunities: but after the 70s, many non-American corporations started to compete successfully at the global scale, threatening their privileged position). Second, the institutional system of welfare capitalism (especially the increasing levels of union activism and the expansion of social policies) had also expanded to a point in which it was directly threatening the corporate rates of profits. The explosion of these two combined factors in the seventies led big corporations, "in a desperate attempt to restore, or preserve, the rates of profit to which they had become accustomed in the halcyon days of the 1950s and 1960s" (Bluestone and Harrison 1982; 15), to resort to the key prerogative that they still maintained: their capacity to disinvest, "to shift capital as rapidly as possible, from one activity, one region, and one nation to another" (idem; 15). It is this increasing recourse to capital mobility (effective or potential, used as a threat) which is at the core of the deindustrialization hypothesis of Bluestone and Harrison. Deindustrialization, in their approach, refers to a process of disinvestment in the industrial base which not only transforms the composition of the division of labour, but also the correlation of forces between capital and labour (giving the former the upper hand). Although the triggering mechanism of this turn towards a disinvestment strategy by big corporations is primarily the shrinking rates of profits discussed earlier, technological change plays a very important role as well, as a crucial enabling factor. Without the advances in communication and information processing technologies that took place around the seventies, the possibilities of capital to move freely around the world would have been considerably more limited.

The arguments of Bluestone and Harrison, therefore, are primarily institutional (their primary variable being the correlation of forces between capital and labour), but within a multicausal explanatory framework (international trade and technology have exogenous effects on such correlation of forces that precipitate or at least facilitate its change). Bluestone and Harrison, this way, provide an alternative explanation for the shift away from manufacturing and into services within advanced capitalist economies which had been theorized in a much more upbeat manner by post-industrialists a decade earlier. Although in their 1982 book on "The Deindustrialization of America" they did not develop further the implications of such changes in the economic structure from a job quality perspective, their arguments were immediately linked to a wider debate on 
the disappearance of the middle class in America. One of the earliest and most influential formulations of the thesis of the disappearance of the middle class was an article called "The Declining Middle", published in 1983 in the Atlantic Monthly magazine and written by Bob Kuttner, an influential American economic journalist. The idea of the disappearance of the middle class is obviously analogous to the concept of polarization, although it has more resonance in the American context because the US had always styled itself as the archetypical middle class society.

Kuttner drew heavily from the ideas of Bluestone and Harrison, although he emphasizes more the importance of technological change as a direct determinant of the transformation of the division of labour from a compositional perspective. But at the same time, he argued that the distributional implications of such transformations (the disappearance of the middle and the polarization of the jobs structure) are necessarily the linked to institutional factors. For Kuttner, "middle-level jobs have been disappearing ultimately as a result of the ways in which technological gains are being distributed [...]. [But] there is nothing intrinsic in assembling cars, mining coal, or pouring molten steel that requires high wages. These jobs do pay well mainly because of the efforts of strong unions. Just as high wages are not a necessary characteristic of factory work, low wages are not a necessary characteristic of a service economy. But because unions have organized few fast-food workers, typists, bank clerks, or even computer assemblers, most of the new service jobs that are replacing factory work pay far lower wages" (Kuttner 1983: 62).

Kuttner's argument is that the forces driving the transformation of the productive structure do not determine directly the distributional implications of such transformation: institutional factors such as union power and labour regulation are a mediating factor (at the very minimum, affecting the wage structure) which can alter completely those implications. This hypothesis is especially interesting for thesis, as we will look at the transformation of the jobs structure in countries with different institutional settings, and with jobs being classified according to the specific wage distribution of each country. A possible way to test Kuttner's hypothesis would be, therefore, to see whether the structural changes themselves are very similar (that is, if the productive structures evolve in similar ways in terms of employment by sector and occupation), but the existence of different wage structures in different countries leads to diverging effects on the quality of jobs available. This way, we can test whether it is 
really the case that there is "nothing intrinsic" in fast-growing service jobs that make them low pay or in the fast-disappearing industrial jobs that put them in a middling position in the wage structure.

Harrison and Bluestone themselves, in a later book ("The Great U-Turn", published in 1988), develop the distributional implications of their earlier analysis of deindustrialization explicitly arguing that it leads to an increasingly polarized labour market. "The upper tier of the labour market includes the managers, lawyers, accountants, bankers, business consultants, and other technically trained people whose daily lives lie at the heart of the control and co-ordination of the global corporation and the corporate services that are closely linked to them [...]. At the bottom of the labour market is the other, less fortunate pool of urban residents whose collective function is to provide services to the workers in the upper tier [...]. The reality of the new service economy entails a great many low-paying jobs and a much smaller layer of high-paying ones" (Harrison and Bluestone 1988; 69-72).

\section{3.b) Technology-driven polarization: task-biased technological change (TBTC)}

The basic arguments of this approach are a derivation of the skill-biased technological change (SBTC) theory, which as we discussed earlier, predicted upgrading rather than polarization in the wage structure. In the paper "The Skill Content of Recent Technological Change", Autor, Levy and Murnane (2003) presented a task-based reformulation of SBTC (and which therefore, we will call from now on task-biased technological change, or TBTC). To conceptualize the impact that technical change would have on the demand of the different types of jobs, they categorized jobs according to their task contents, in the following four groups:

1. Routine manual: repetitive labour of a physical nature. Typically, though not necessarily, traditional industrial low- and semi-skilled jobs involve such routine manual tasks. Since the Industrial Revolution, jobs involving these type of tasks have been increasingly substituted by machines (which can easily perform repetitive physical tasks). Computerization further increases the feasibility of substitution for these type of tasks, as it allows the programming of machines to achieve much more flexibility in their functioning.

2. Routine cognitive: repetitive labour involving the processing of information. Typically, though not necessarily, traditional clerical jobs involve this type of 
routine information processing tasks. Until to the computer revolution, it was very difficult to perform these tasks with machines, which together with the increasing needs of information processing generated by managerial capitalism, led to a massive expansion of this type of employment up to the 1970s. The computerization of the last third of the $20^{\text {th }}$ Century implied for these type of tasks what mechanization had implied for routine manual tasks two hundred years ago: these jobs became as easy to "mechanize" (or more precisely, computerize) as routine manual tasks.

3. Non-routine cognitive: non-repetitive or non-codifiable labour involving the production, processing and manipulation of information. Typically, this type of tasks are carried out by managerial, scientific or professional jobs. Machines are still incapable of carry out this type of tasks, so these jobs are protected from mechanization. Autor, Levy and Murnane, following the SBTC tradition, argue that in fact these jobs are affected indirectly by mechanization in a positive way: because this type of tasks are complementary with routine tasks (whose input in production increases-not as labour input, but as machines-as the costs of mechanization go down), their demand rises with computerization (and therefore, labour demand for managerial, scientific and professional jobs rises).

4. Non-routine manual: non-repetitive tasks of a physical nature (sometimes referred to as hand-eye coordination tasks). This type of tasks are typically associated with low-skilled service jobs. Even though Autor, Levy and Murnane mention this fourth category, which is obviously implied by their model, they do not make any specific claim about how it is affected by computerization, and in most of the paper they seem to ignore it. "Because our model makes no prediction for how computerizing industries will adjust demand for non-routine manual tasks, we do not include this variable in our industry-level analysis" (Autor, Levy and Murnane 2003; 1302). This is somewhat surprising, because it does seem that the model would imply some prediction for this group as well. The simple fact that these tasks are non-routine implies that computerization has no direct negative effect on the demand of human labour of these jobs, and therefore there should be no decline in employment for this category. This would imply some type of polarization for the overall structure of employment, if we consider that these jobs tend to go in the bottom of such structure. 
But in their 2003 paper, Autor, Levy and Murnane did not pursue this line of reasoning, and therefore, they did not argue for polarization, but for outright upgrading in employment growth: this paper is still squarely within the SBTC tradition. It will be Goos and Manning (2007), in their paper on the evolution of the employment structure in the UK since the seventies, who take the TBTC model and develop it along the lines we have just discussed, to provide a theoretical framework that explains job polarization. Goos and Manning adopt the TBTC model proposed by Autor, Levy and Murnane in all respects except in its analysis of non-routine manual tasks: "The nonroutine manual tasks that make up many of the most unskilled jobs such as cleaning are not directly affected by technology, but the impact of technology in other parts of the economy is likely to lead to a rise in employment in these unskilled jobs [...].The reason is the general equilibrium effect first identified by Baumol—employment will shift toward jobs in which productivity growth is low (because technology is not applied there) in order to keep the balance of output in different products." (Goos and Manning 2007: 118-120).

Therefore, the hypothesis of Goos and Manning is that the recent process of computerization which has affected most of advanced capitalist economies tends to polarize the jobs structure. To test such hypothesis, they use a very similar methodology to the one we use in this thesis (their work can be classified within the "jobs approach" as well), applying it to the changes in the composition of the UK employment structure between 1975 and 1999. They provide abundant evidence of the polarization effect they hypothesize, with stronger job growth in the bottom and top of the wage distribution (lousy and lovely jobs) and net job destruction in the middle. Although their evidence on polarization is rather convincing, they tend to emphasize so much the polarization story that they seem to fail to recognise that (according to their own results) this polarization is strongly skewed to the top: there is at least as much upgrading as polarization. For instance, the chart representing job growth by wage deciles (Goos and Manning 2007: 121) shows that the growth of the top two deciles was five times larger than the growth of the bottom two deciles. Of course, this is not to say that there was no polarization in the UK labour market between 1975 and 1999 (undeniably, there was), but by ignoring the simultaneous strong upgrading their overall assessment of the patterns of job growth seems somewhat incomplete or even slightly misleading. 
The explanatory framework of Goos and Manning is, following Autor, Levy and Murnane, primarily technological. They briefly discuss alternative possible explanations, which they do not reject outright, but clearly consider them to be secondary to technology. For instance, they consider it plausible that international trade would have a similar effect to TBTC, but following the specialized literature, they argue that this effect is probably very minor (Goos and Manning 2007: 126). Most importantly, they only mention very briefly the potential impact of institutional changes (they speak of institutional changes rather than institutional settings), arguing that they may have had some influence (again, they imply that a secondary one) on the evolution of the lower half of the wage distribution (Goos and Manning 2007: 132).

In a later paper (Goos, Manning and Solomons, 2009), they make their point much stronger by arguing that the same technology-induced polarization that they identified in Britain in their earlier paper is taking place in all European countries (15), except in Portugal. They back their arguments with evidence which we will discuss in a later chapter, because their results are inconsistent with ours. The main point here is that, from a purely theoretical perspective, their claim that the same pattern of job growth is taking place all around Europe is quite striking, because it implies that the large country differences in institutional settings, economic structures, positions in the international division of labour, and any other factors do not have any significant effect on the types of jobs that the different European countries created in the last 15 years. According to Goos, Manning and Solomons (2007), all European employment structures experienced a very similar type of transformation (a polarization) in the nineties, as a result of the effect of a single explanatory factor: task-biased technological change.

In a new 2006 paper (“The Polarization of the US Labour Market”), David Autor, with two new co-authors (Katz and Krueger) revisited his original model along the lines suggested by the Goos and Manning paper, transforming it into an outright polarization model and replicating the type of analysis carried out by Goos and Manning with US data. They find very similar results, presenting more evidence of technology-driven job polarization. It is surprising that in this paper they do not mention the work carried out years earlier by Wright and Dwyer (Wright and Dwyer 2003), in which they find basically the same results following a very similar methodology (but different theoretical frameworks). 


\section{A model and five hypothesis}

In the previous pages, we have discussed the main elements of the jobs approach from a theoretical perspective. Can we now put all these elements within a synthetic analytical model to serve as theoretical foundation for this thesis? Figure 2.3 attempts to draw such a model.

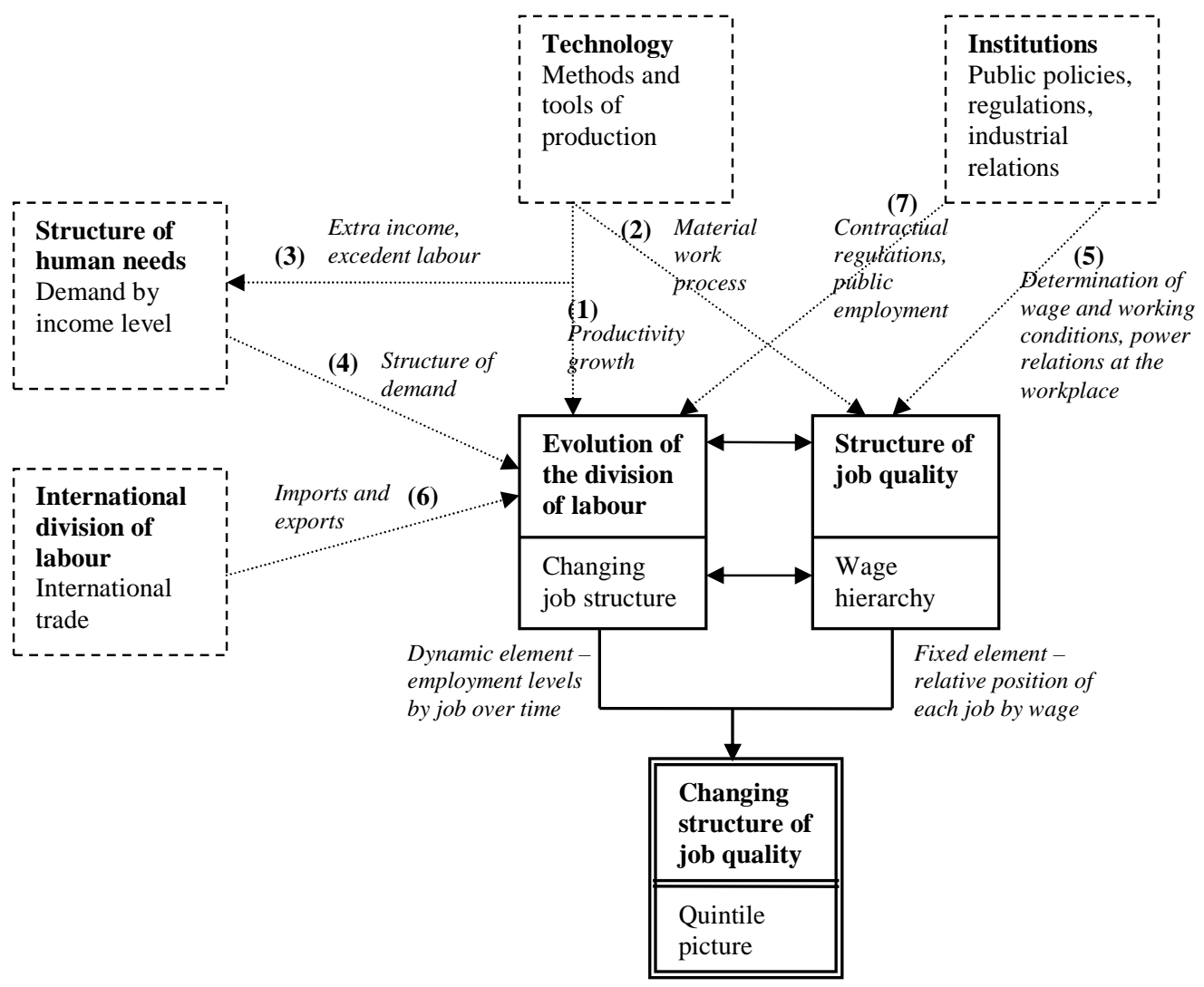

Figure 2.3: A synthetic model of the determinants of the changing structure of job quality

The box at the bottom of the picture (with a double-line frame) represents the main dependent variable of this thesis, which refers to the changing structure of jobs according to their quality and which is graphically represented by the quintile picture (growth of the jobs ranked by their job quality and grouped in five quintiles). Such dependent variable derives from the combination of two primary variables (represented by the two boxes with a single-line frame, in the middle of the picture): one which refers to the evolution of the division of labour from a compositional perspective (represented by the change in the levels of employment of each cell of the jobs matrix) 
and one which refers to the structure of job quality (represented by the hierarchy of cells in the jobs matrix according to their median wages, educational level or amenities).

As we argued in section 2 of this chapter, the changing jobs structure refers to the change in the amount of labour allocated to the production of goods and services (within the production boundaries) in a particular economy, classifying such labour by sector (horizontal, or social division of labour) and occupation (vertical, or detail division of labour). The changing jobs structure provides the link between this project and the tradition in the social sciences studying the evolution of the division of labour from a compositional perspective. As we argued in section 3 of this chapter, the structure of job quality refers to the relative impact that each job has on the well-being of workers (that is, compared to the other jobs), which in this project is proxied by the wage hierarchy (and secondarily, by the educational hierarchy and by a multidimensional job quality index). The wage hierarchy is the key element for evaluating the implications of changes in the jobs structure in terms of job quality.

Around these three central boxes representing the core dependent variable(s) of this thesis, there are four dashed-line boxes, which represent the main independent variables (forces driving compositional changes in the division of labour with an impact on job quality) identified in the literature, as discussed in section 4 of this chapter. The dashed lines represent the main mechanisms linking each factor with the changing division of labour or the structure of job quality according to the literature: these lines have been numbered in order to facilitate positioning each theory in the model. After explaining the main arguments of each theory within the context of the model shown in figure 2.3, we will operationalize it in a set of hypothesis that can be tested with our methodology.

1. The degradation of work theory concerns primarily mechanism (2), although it also refers to mechanisms (1) and (5). For the classics, mechanization and the increasingly detailed division of labour in manufacturing were boosting the productive capacities of humankind while at the same time degrading the nature of work to the point of transforming workers into mechanical beasts (mechanism 2 ). The increasing returns of such types of work organization and production technologies, furthermore, tended to shift the structure of the division of labour towards the most degrading sectors and occupations (mechanism 1). Braverman, following Marx, expanded the argument of classical economists arguing that such degradation was not only the result of a purely technical process of 
innovation in production techniques: rather, it was the result of an intentional move by capitalist employers to tighten their control of the production process, reduce the power of the proletariat both at the workshop and at the labour market, and decrease the remuneration of labour. Such mechanism would imply not only the dashed line indicated with (5), but also line (7) linking directly the institutional framework (which includes property relations over the means of production, the key independent variable in the Marxist tradition) and the changing division of labour.

- Degradation of work hypothesis: the patterns of change in the jobs structure will be downgrading, either totally downgrading or polarizing with a very strong downgrading bias. This downgrading should be taking place across sectors, in very similar ways. By occupations, we should observe a strong destruction of skilled and semi-skilled occupations, a simultaneous strong creation of unskilled occupations and some creation of technical and managerial jobs at the top of the wage structure.

We have to acknowledge, nevertheless, that although Braverman himself tried to prove his hypotheses through a compositional analysis of occupational trends similar to what we will do here (Braverman 1998: especially chapters 10 and 17), the core of his theory concerns within-job rather than across-job changes, and therefore our empirical test only concerns a secondary part of Braverman's hypothesis.

2. Job upgrading (1): Post-Industrial theories. The post-industrial hypothesis of Bell and Fourastié refers mainly to the upper left-hand side of figure 2.3. Technology leads to labour-saving productivity improvements in the goodsproducing sectors (mechanism 1). This means that there is extra income and excess labour which can be put to other uses: to which uses they are put ultimately depends on the structure of human needs (2). According to Fourastié (following Engel), the structure of human needs shifts towards an increasing demand for services (and within services, for those which are most knowledgeintensive) as income raises (3); as argued by Baumol, even if such a change in the structure of human needs would not happen, the sector-biased productivity growth leads to a shift towards the service sector. Service jobs, especially knowledge-intensive ones, are generally of a higher quality than the unskilled 
and semi-skilled industrial jobs which constituted the bulk of employment in the industrial era: hence the prediction that the pattern of change in the employment structure will be one of outright upgrading.

- Post-industrialist hypothesis: the patterns of change in the jobs structure will be unambiguously upgrading. By sector, we should see a decrease in traditional industrial (and goods-producing) jobs of a low or mid-low quality, simultaneous with an increase in middling to high quality jobs in services and a strong increase in knowledge-intensive services of high quality. By occupations, we should see a decrease in unskilled and semiskilled occupations, and a rapid growth of managerial, technical and professional occupations.

3. Job upgrading (2): Skill-Biased Technological Change. This theory concerns mostly mechanism (1), and indirectly mechanism (2) as well. According to this theory, technological change has different impacts on the demand for unskilled and skilled jobs. Machines are a substitute for unskilled workers, and therefore tend to depress their demand; whereas they are complementary to skilled work (especially work of a technical and professional nature), and therefore they tend to increase its demand. This biased effect of technological change on the demand of the different types of jobs will eventually alter the salaries (and the quality) of the different types of jobs as well, increasing wage inequality.

- SBTC hypothesis: the patterns of change in the jobs structure will be unambiguously upgrading. Across all sectors, we should see fast growth of highly-skilled (and highly paid) jobs, and stagnation or even destruction of middling and low-skilled jobs.

4. Polarization (1): trade-institutionalist explanations. These theories emphasize mechanisms (6), (5) and (1). The increasing openness of international trade alters the correlation of forces between capital and labour, because it allows capital to threat with disinvestment (this mechanism, not shown in figure 2.3, would imply an arrow between international trade and institutions: but the direct effect on the changing division of labour and job quality takes place through institutions, mainly mechanisms 5 and 7). This increased international trade also leads to an increasing international specialization or international division of labour, which implies that those jobs whose cost/productivity combination is 
less favorable tend to disappear in advanced capitalist economies (shifting to developing economies, mechanism 6). These mechanisms tend to destroy employment in the middle of the wage distribution, and generate jobs at the two extremes. This approach also acknowledges that technological change tends to transform directly the employment structure (mechanism 1), but it argues that the implications of this transformation will be mediated by the structure of job quality, which ultimately depends on the institutional framework. This approach is the only one which explicitly argues that even if the underlying transformation of the division of labour is very similar across advanced capitalist economies, their implications for job quality can vary across nations with different institutional frameworks.

- Trade/institutionalist hypothesis: the patterns of change in the division of labour of advanced capitalist economies will tend towards polarization, but the institutional framework of each country can alter substantially the implications that this underlying trend will have for job quality. Therefore, the quintile picture can vary considerably across countries, even if the underlying changes in the employment structure are broadly the same. The patterns of transformation of the jobs structure should have a strong sector bias: the jobs destroyed in the middle by international trade should mostly be in manufacturing, expanding jobs in the top are mostly knowedgeintensive services and the jobs expanding at the bottom are mostly in the personal services sector. Because of its high degree of economic integration, we should expect evidence of some degree of international specialization within Europe, with the same types of jobs appearing in developing European countries and disappearing in the most developed economies.

5. Polarization 2: technological explanation (TBTC). This theory concerns mostly mechanism 1. The basic argument is that the information technology revolution that took place after the seventies has allowed firms to substitute workers by machines (or computers) to carry out routine (manual and cognitive) tasks. At the same time, these developments have increased demand for non-routine cognitive tasks, which (as argued earlier by standard SBTC theory) are complementary with technology but cannot be carried out by machines: this has led to a boost in the demand of highly skilled labour. Productivity is increasing, 
therefore, in routine-manual, routine-cognitive and non-routine-cognitive tasks as a result of computerization: but the productivity of non-routine-manual tasks is unaffected. Hence, according to Baumol's insight, keeping constant the structure of demand (and therefore without recurring to mechanism 4), the demand for non-routine-manual tasks has to increase (and as machines cannot do these tasks, this means an increase in demand for labour of this type). Jobs involving routine manual tasks tend to go in the middle of the wage distribution, jobs involving non-routine cognitive tasks tend to go in the top and jobs involving non-routine manual tasks tend to go in the bottom: hence the prediction of polarization of the structure of job quality.

- TBTC hypothesis: the patterns of change in the jobs structure will tend towards outright polarization across most developed economies (as all are experiencing the same type of technological change). There should be some sector bias (the different types of tasks are differently distributed by sector), but the main driver of change should be occupational shifts in employment (the routine and cognitive content of tasks is mostly correlated with the vertical division of labour).

These hypotheses, which can be tested with our jobs approach, will guide the empirical analysis of this thesis. What about our own expectations? Our hypothesis would be that the current wave of technological change tends to transform the division of labour in the way argued by the TBTC (there would be, therefore, an underlying trend towards polarization induced by technological change and helped by the changing structure of demand as income raises, mechanisms 1 and 2). The direct effect of international trade in this transformation is likely to be small, as most of the specialized literature tends to emphasize (see Freeman 2003 for a review; also Krugman and Lawrence 1993). But as argued by institutionalists, we believe that this underlying technology-induced trends interact with the institutional settings of the different countries (not only through mechanism 5, as argued by Kuttner, but also through the direct regulation of employment contracts represented by mechanism 7): therefore, we would expect to see a big deal of variability in the actual patterns of change in the job quality structure, reflected in the different pictures of employment expansion by job quality quintiles. 


\subsection{A final note on two potential confounding factors}

Before moving on to the empirical analysis of the hypotheses presented above, we have to discuss briefly two potential confounding factors that could (if we did not take them into account) compromise our capacity to test these hypothesis with our approach.

The first problem concerns the fact that the countries covered in this study are in different stages of economic development. Any process of structural change is necessarily path-dependent. The initial structure will condition the changes that can take place afterwards. This affects all the mechanisms and factors driving the evolution of job structures depicted in figure 2.3. But this project is focused on the patterns of change in employment structures across Europe for a specific period of time (19952007). All the hypotheses discussed above refer to change, not to (initial) structural differences: but it is important to note that we will not be able to interpret correctly those changes if we do not take into as well these differences in the initial structures. For this reason, we will devote a whole chapter (4) to the analysis of these initial structural differences, and we will take these differences into account when interpreting the patterns of change in the rest of the thesis.

In principle, this problem should not be too important for most of the countries covered in this study. The 15 countries that were part of the EU before 2004, which will be the centre of most of the discussion in this thesis, are all similar enough in terms of their stage of development as to safely assume that most of their differences in changing patterns can be explained by the model detailed above. To a large extent, the initial differences between these countries can be assumed to be the result of different institutional settings (call them varieties of capitalism, welfare regimes or anything else) or positions in the international division of labour (Germany being more oriented towards the export of high value-added industrial goods and the UK towards financial services, for instance), rather than the result of different stages in economic development, which would be more difficult to incorporate into our model and hypotheses. In the case of the 8 New Member States that are also covered in this study, there are probably initial structural differences which cannot be explained by the factors included in the model shown in figure 2.3. These countries have experienced massive processes of structural transformation in the last two decades which result from their transition from a socialist to a capitalist economic system. Part of those transformations, without any doubt, were still taking place during the period covered in this study: this 
implies that their patterns of change in employment quality are biased by factors completely alien to those shown in figure 2.3. Partly for this reason, we will mostly include these countries in the analysis for illustrative and comparative purposes: they will always be secondary to the main line of argumentation.

The second problem concerns the length of the period covered in this study. The structure of contemporary labour markets is continuously changing, with large numbers of workers being continuously reallocated to and from different sectors and occupations, with massive numbers of jobs being created and destroyed even when the aggregate employment levels remain more or less stable. Some of these changes reflect long-term shifts in the structure of the division of labour which result from the mechanisms discussed in the previous section; but some changes are the result of short-term shifts in employment derived from cyclical factors such as boom-and-bust cycles. The problem is that, although we are mainly interested in the first type of change (structural transformations of the labour market), there is no way to differentiate it from the second type of change (cyclical or one-shot transformations of the jobs structure).

The importance of this problem is proportional to how short is the period being covered. If the time period is long enough, the short-term factors can simply be ignored because we can assume that they tend to equalize over time. But certainly, a period of 13 years as will be covered in this study is not long enough as to be able to ignore the bias introduced by short-term factors (we would probably need 20 to 30 years for that, if not more). This is not to say that a period of 13 years is short enough as to be completely biased by short-term factors: certainly, our results will reflect long-term trends as well. The problem is how to differentiate both types of change.

There is no technical solution to this problem, no way to correct the data so that it reflects only long-term trends. We have to assume the problem and rely on our good judgment to be able to interpret correctly the results. The period 1995-2007 which will be covered in this study is certainly an interesting period to study it in terms of employment creation, but recent developments have shown that this period reflected exceptional (non-sustainable in the long term) economic circumstances. Most European countries experienced high rates of economic growth compared to the previous decade (especially in the period 1995 to 2000), although there was widespread dissatisfaction because it was still lower than that of the US. Now that the bubble has busted, we can say that this long period of economic growth was a long upward move in the economic 
cycle, a cycle which abruptly changed slope around 2007 moving towards a deep recession which is still affecting most of the EU (and the rest of the world). The rates of growth of the period 1995 to 2007 were, therefore, unsustainable. But the key problem for us is whether the factors driving this unsustainable growth somehow affected the mechanisms drawn in figure 2.3. For instance, the financial sector grew enormously during this period in many countries: did this growth generate an unsustainable amount of highly paid jobs for financial professionals? Will the current recession destroy many of those jobs which were created earlier?

Furthermore: what we have just said refers to trends that were more or less pervasive across Europe. But there were also clear cases of unsustainable cyclical growth which affected only some countries: the best example is the construction sector, which grew almost insanely in countries such as Ireland and Spain while keeping a very low profile in Germany. That this growth was unsustainable was obvious even while it was taking place: we are right now suffering its terrible implications. The problem with these nonpervasive cyclical expansions of employment in specific sectors or types of jobs is that they are very likely to affect the patterns of employment growth of some countries but not others, thus confounding the overall picture of change across the different nations and making it more difficult to test the validity of the hypotheses presented in this chapter.

So again, what to do about it? As there is no way to correct the data in some way that allows the differentiation of short-term and long-term trends (they cannot be differentiated until enough time has passed), the only solution is to be aware of this problem and therefore be more careful in our interpretation. 



\section{Methodology}

\section{Introduction}

The jobs approach is, above all, a methodology for studying the evolution of labour market structures of advanced capitalist economies from a job quality perspective. The main innovation and the methodological foundation of this approach is the use of jobs (defined as specific occupations within specific sectors) as the unit of analysis, rather than individuals. This implies a structural view of the labour market, which (as we argued in the previous chapter) derives from the classical social sciences concept of division of labor (along the horizontal and vertical axes). The other methodological pillar of this approach (which is not so innovative in itself) is the use of a relative (or positional) measure of job quality to characterize the jobs of each economy and analyse the evolution of employment structures through its lens.

One of the main advantages of this approach is that the methodological ideas behind it are relatively simple and clear, which makes its results much easier to understand and communicate. But the operationalization of these ideas for the comparative study of the evolution of job quality structures across 23 different countries during a period of 13 years is understandably complex, as it involves dealing with datasets which were not designed for this purpose (and therefore require many adjustments which involve some difficult decisions), merging datasets whose methodology and definitions may not be entirely compatible, and adjusting for data inconsistencies for which there is very limited background information. In this chapter, we will provide a detailed explanation of this process step by step. Our main goal is to be as transparent and honest as possible about the methodological decisions taken: as we will see, some of them are entirely

justified by our research objectives and the theoretical approach discussed in the previous chapter; some others are purely pragmatic solutions to deficiencies of the 
datasets used which may qualify the results obtained, and therefore we need to be fully aware of them before embarking in the analysis.

The structure of this chapter will be the following. First, we will present a brief summary of the whole process that can serve as a guide for the rest of the chapter. Then, we will discuss the construction of what we call the jobs matrix, which is a very large dataset containing information about employment by occupation and sector for each country and year covered in this study, as well as information on some demographic and employment characteristics of the workers within each job. Then, we will discuss with some detail the procedure followed for the construction of the three job quality rankings which are used in this thesis (the primary rankings are by wage and educational levels, and we will explain as well the construction of a secondary multidimensional job quality ranking which will be used for testing the validity of the other two). Finally, we will discuss how the two previous elements are combined and processed for carrying out the analysis which is the main objective of this thesis.

\section{A brief summary of the methodological approach}

The method followed in this thesis was directly inspired by the work of Wright and Dwyer (2003), although the fact that our analysis concerns many instead of a single country involves some important differences that we will discuss in detail in this document. This thesis draws directly from a project that was carried out within the European Foundation for the Improvement of Living and Working Conditions between 2006 and $2008^{19}$, by an international research group that I coordinated. ${ }^{20}$

As mentioned earlier, this methodology is based on two simple, but powerful ideas: to use as the unit of analysis "jobs" rather than individuals, and to use relative measures of the wages, educational levels and non-pecuniary work characteristics of job-holders as proxies for the quality of each job. The main steps of this methodology are the following:

\footnotetext{
${ }^{19}$ The main publications from this project are Fernández-Macías 2008, Fernández-Macías and Hurley 2008, and Stehrer, Ward and Fernández-Macías 2008. More information on this project can be found in http://www.eurofound.europa.eu/research/0298.htm.

${ }^{20}$ This research group was composed by Terry Ward from Applica, a research consultancy based in Brussels; Robert Stehrer and Hermine Vidovic, from the Vienna Institute for International Economic Studies; and John Hurley, Branislav Miculic, Donald Storrie and myself from the European Foundation for the Improvement of Living and Working Conditions.
} 
1. Classifying employment into "jobs": the starting point of this study is that the whole structure of employment of any country can be classified into a matrix of "jobs", which is basically a big table in which the columns are economic sectors and the rows are occupations. The cells in the table are these so-called "jobs". Sectors are defined by the International Classification of Economic Activities (NACE) at the 2-digit level, and occupation by the International Standard Classification of Occupations (ISCO), also at the two-digit level. Examples of these "jobs" would be office clerks in the construction industry or engineers in wood manufacturing. Besides the numeric information on employment by sector and occupation per year and country, this jobs matrix includes other variables holding demographic and employment information of the people within each job.

2. Ranking the jobs by their quality: the second step consists in generating three different rankings of the jobs by their quality, specific for each country covered (except in the case of the multidimensional job quality index). These three rankings are based on: 1) the median hourly wages of workers within each job; 2) the average educational level of workers within each job, and 3) a composite index of non-pecuniary job quality constructed by the aggregation of several different variables. The wage ranking is based on information from different surveys on European wage structures; the educational ranking is based on information from the European Labour Force Surveys; the multidimensional job quality ranking is based on the 2005 European Working Conditions Survey.

3. Grouping the jobs in quintiles: using the quality rankings, the jobs are assigned to five groups, from low to high quality. These groups are constructed in such a way that, in the year 2000, they divide the whole working population of each country in five equal-sized groups (the lowest holding the $1 / 5$ of the working population in the lowest-paid jobs, and so on). The year 2000 is used for constructing the quintiles because it is more or less in the middle of the period covered, and because there are some countries with missing information for the years before 1999. This classification of jobs into quintiles is done separately for each of the job quality rankings.

4. Representing change in employment in each quintile: then, starting in 1995 and finishing in 2007 , the change in the stock of workers in each of the five job 
quality quintiles is analysed, in most cases (but not always) graphically. This way, the patterns of employment growth by job quality can be identified and the countries classified accordingly.

\section{Breaking up job creation patterns by sector and other characteristics:} finally, we look at the specific patterns of employment expansion by job quality for different sub-populations, defined by characteristics of the jobs (such as sector and occupation) or characteristics of the workers within the jobs (as gender and nationality). This helps us to understand the economic trends behind the observed patterns of employment growth, as well as their distributional implications.

\section{The first pillar of the jobs approach: the jobs matrix}

What we call the jobs matrix is a large dataset which classifies all employment within each country and year covered in this study by occupation and sector, including also secondary information on other demographic and employment variables for the workers in each job. This dataset is entirely drawn from the European Labour Force Surveys (ELFS from now on), through a special extraction requested by the European Foundation for the Improvement of Living and Working Conditions, and provided by Eurostat. All the analytical work of this thesis is based on this jobs matrix: there is no direct analysis of the ELFS microdata. In this section, we will describe the methodological characteristics of the ELFS and the variables of occupation and sector (ISCO and NACE) used for the creation of the jobs matrix. We will finish with a brief statistical description of this matrix.

\subsection{The data source of the jobs matrix: the European Labour Force Surveys}

The European Labour Force Survey has important strengths that make it without any doubt the best data source for our purposes, but it also has some drawbacks that we must have in mind when interpreting the results.

In terms of strengths, we can say that the European Labour Force Survey is simply the most important comparative data source on work-related issues at the European level. In most countries, it has a very large sample that allows to carry out quite detailed analysis, and the main concepts and definitions have been defined at the European level so that it is possible to do comparative analysis. It has been existing for a number of years, which 
means that it is possible to use it to study the evolution of many key labour-related variables at least since the mid-eighties (when the concepts and definitions were truly harmonized). Also, after years of frustrating reticence to allow external use of the data for research (which, as so often happens with the EU, was actually the result of the reticence of national member states to allow Eurostat to give away what they considered to be their data), it has been recently made available for outside research institutions ${ }^{21}$.

But it has important drawbacks too. The most important one is that there is no European Labour Force Survey as such (a proper EU-wide survey), but rather a series of national surveys carried out by each national statistical institute under EU-level coordination. Each (national) LFS is essentially designed and carried out nationally, and although the definitions and classifications, the broad methodological principles and to a relatively large extent even the questionnaire itself are basically the same, national statistical institutes retain a considerable leeway with respect to the final design of the survey. So the actual methodological details and the implementation can vary considerably among countries. Furthermore, there is very little documentation available in English on these small but sometimes crucial variations in the details of the LFS design, which makes it quite difficult to control or assess the implications of this problem. In terms of data availability, apart from the already mentioned procedural complications, the microdata made available for external researchers are full of restrictions: some very important countries are not available at all (notably, the UK and Sweden; and also Germany before 2002), and the data has been subject to a radical "anonymization process" that limits considerably its use. For instance, the microdata made available to external researchers only provides sector and occupation coded at the one-digit level, which is extremely limited for our purposes: our idea of jobs as the smallest units in the division of labour in a society along the horizontal and vertical axes clearly requires a more detailed dissaggregation of occupation and sector. For this reason, in this project we decided not to use the ELFS microdata and instead ask Eurostat officials to provide us with the necessary breakdowns of total employment for each country and year by occupation (ISCO) and sector (NACE) at the two-digit level, including also a breakdown by third variables such as sex and type of contract. With this breakdown

\footnotetext{
${ }^{21}$ The accessibility has improved, but it is still full of frustrating restrictions and problems. First, it is only accessible for a fee that puts it out of the reach of individual researchers (which should not be the case for publicly funded research); second, the process of getting access to the data is lengthy and cumbersone (each national member state has to give consent to the use of the data for every single project); and finally, as we will see later, there are considerable restrictions embedded in the microdata itself.
} 
(basically a very large table containing the number of people employed for each possible combination of sector, occupation and the other variables), we could construct the jobs matrix exactly as we wanted. The only disadvantage of this method is that it is less flexible than drawing directly from the ELFS microdata: if we wanted to add some extra information on the jobs in the matrix, we would have to make another request directly to Eurostat.

Because of the sui generis nature of the European Labour Force Surveys, it is difficult to summarize its methodology in the usual way (which would require to provide details for each of the national surveys used), so we will only briefly outline its broad characteristics $^{22}$ :

1) Coverage: The EU-LFS covers the 27 Member States of the EU, as well as member states of the EEA, Iceland and Norway, Switzerland by bilateral agreement, and the Candidate Countries Croatia and Turkey. It covers all persons living in private households. Although the first attempts to carry out a Labour Force Survey covering the whole EU (at that time much smaller than today) go back to the $60 \mathrm{~s}$, really comparable results are only available since the eighties (the microdata provided by Eurostat starts in 1983 for the earlier countries).

2) Reference period: The ELFS is a quarterly survey. In all countries but Germany, Hungary (3 weeks/month), Bulgaria, Croatia and Turkey the sample is spread over the 13 weeks of the quarter. The sample is uniformly spread over the weeks, except in Luxembourg, Slovenia, Romania and Switzerland (Eurostat 2006: 3). In this project, we use the annual ELFS results, which are generated by EUROSTAT by aggregating information of the four quarters.

3) Sampling design: according to Eurostat itself, "the sampling designs in the EULFS are extremely varied. Most National statistical institutes employ some kind of multi-staged stratified random sample design, especially those that do not have central population registers available." (Eurostat 2006: 4). But of course, this does not mean that the samples are not comparable: the design of the sample

\footnotetext{
${ }^{22}$ A detailed account of the main characteristics of each of the national surveys is available in the Eurostat publication "The European Union labour force survey: main characteristics of the national surveys" (Eurostat 2009a).
} 
is subject to constraints specified in the EU Regulation regarding the required level of statistical reliability and representativity (Charlier and Franco 2001: 2).

4) Data collection method: it also varies between countries. Most countries use a mix of data collection by personal visits and phone interviews, but in some countries the questionnaires are at least partly administered by post and in others all questionnaires are administered face to face. An increasing number of countries (at least 19) use a computerised questionnaire.

5) Weighting procedure: the EU LFS Regulation stipulates that the weighting must be based in age (using five-year bands), sex and NUTS-2 region, but in practice this also varies considerably from country to country, because of the different quality and coverage of the population registers available.

6) Non-response: Countries where the participation to the survey is compulsory tend to have a better response rate than in the countries where the survey is facultative. The response rate varies from $98 \%$ in Germany to $55-60 \%$ in the Netherlands. For some specific variables item non-response can also be quite high. Some countries do imputations for the item non-response either using auxiliary information or coping the information given by the same person in a previous interview (Charlier and Franco 2001: 6).

7) Sampling error: because of the large sample size, the standard errors of ELFS estimates are quite small. In table $\mathrm{e}^{23}$ 3.I we provide with the estimation of standard errors for 5 key variables for the year 2005, taken from the quality report published by Eurostat in 2005 (Eurostat 2006: 13).

\footnotetext{
${ }^{23}$ This table uses the two-letter country codes that we will very often use in this thesis, to be able to fit the country names in the tables and charts. The list of these codes is provided after the list of tables When possible, we will use the full country names.
} 


\begin{tabular}{|c|c|c|c|c|c|}
\hline & Number of employed & $\begin{array}{c}\text { Number of part-time } \\
\text { employed }\end{array}$ & $\begin{array}{c}\text { Number of } \\
\text { unemployed }\end{array}$ & $\begin{array}{c}\text { Rate of } \\
\text { unemployment }\end{array}$ & $\begin{array}{c}\text { Average number of } \\
\text { hours actually worked }\end{array}$ \\
\hline Country & $\times 1000$ & $\times 1000$ & $\times 1000$ & $\%$ & hrs \\
\hline EU-25 & $197467 \pm 353$ & $35983 \pm 187$ & $19514 \pm 157$ & $9.0 \pm 0.07$ & $37.8 \pm 0.04$ \\
\hline EU-15 & $167909 \pm 268$ & $33644 \pm 175$ & $14943 \pm 130$ & $8.2 \pm 0.07$ & $37.2 \pm 0.05$ \\
\hline EA-12 & $132633 \pm 242$ & $24839 \pm 146$ & $13053 \pm 121$ & $9.0 \pm 0.08$ & $37.5 \pm 0.05$ \\
\hline EEA & $199909 \pm 354$ & $36662 \pm 188$ & $19623 \pm 157$ & $8.9 \pm 0.07$ & $37.8 \pm 0.04$ \\
\hline $\mathrm{BE}$ & $4235 \pm 30$ & $932 \pm 20$ & $390 \pm 16$ & $8.4 \pm 0.3$ & $37.0 \pm 0.3$ \\
\hline BG & $2982 \pm 56$ & $60 \pm$ & $334 \pm 19$ & $10.1 \pm 0.5$ & $40.7 \pm 0.1$ \\
\hline $\mathrm{CZ}$ & $4764 \pm 33$ & $233 \pm 10$ & $410 \pm 15$ & $7.9 \pm 0.3$ & $42.0 \pm 0.1$ \\
\hline DK & $2752 \pm 16$ & $608 \pm 14$ & $140 \pm$ & $4.8 \pm 0.2$ & $35.8 \pm 0.2$ \\
\hline$D E$ & $36353 \pm 104$ & $8732 \pm 62$ & $4577 \pm 55$ & $11.2 \pm 0.1$ & $37.1 \pm 0.1$ \\
\hline $\mathrm{EE}$ & $607 \pm 18$ & $47 \pm$ & $52 \pm 6$ & $7.9 \pm 0.9$ & $40.3 \pm 0.3$ \\
\hline IE & $1952 \pm 14$ & $0 \pm$ & $89 \pm$ & $4.3 \pm 0.2$ & $37.7 \pm 0.2$ \\
\hline EL & $4369 \pm 43$ & $217 \pm \quad 13$ & $477 \pm 19$ & $9.9 \pm 0.4$ & $42.4 \pm 0.2$ \\
\hline ES & $18973 \pm 63$ & $2347 \pm 33$ & $1913 \pm 34$ & $9.2 \pm 0.1$ & $39.0 \pm 0.1$ \\
\hline FR & $24536 \pm 169$ & $4227 \pm 106$ & $2458 \pm 88$ & $9.1 \pm 0.3$ & $37.0 \pm 0.2$ \\
\hline IT & $22563 \pm 79$ & $2897 \pm 49$ & $1889 \pm 36$ & $7.7 \pm 0.1$ & $38.2 \pm 0.1$ \\
\hline CY & $348 \pm 9$ & $31 \pm$ & $19 \pm$ & $5.3 \pm 0.7$ & $39.7 \pm 0.4$ \\
\hline LV & $1034 \pm 14$ & $86 \pm$ & $101 \pm$ & $8.9 \pm 0.7$ & $42.3 \pm 1.2$ \\
\hline LT & $1474 \pm 46$ & $104 \pm 14$ & $133 \pm 15$ & $8.3 \pm 0.9$ & $38.9 \pm 0.3$ \\
\hline LU & $194 \pm 2$ & $34 \pm$ & $9 \pm$ & $4.5 \pm 0.3$ & $38.1 \pm 0.2$ \\
\hline $\mathrm{HU}$ & $3901 \pm 59$ & $160 \pm 10$ & $302 \pm 15$ & $7.2 \pm 0.3$ & $40.5 \pm 0.1$ \\
\hline MT & $149 \pm 2$ & $14 \pm$ & $12 \pm \quad 1$ & $7.3 \pm 0.6$ & $39.3 \pm 0.4$ \\
\hline NL & $8111 \pm 42$ & $3741 \pm 32$ & $402 \pm 10$ & $4.7 \pm 0.1$ & $32.0 \pm 0.2$ \\
\hline AT & $3824 \pm 36$ & $808 \pm 19$ & $208 \pm 10$ & $5.2 \pm 0.2$ & $39.2 \pm 0.3$ \\
\hline PL & $14116 \pm 213$ & $1521 \pm 62$ & $3045 \pm 82$ & $17.8 \pm 0.4$ & $41.2 \pm 0.2$ \\
\hline PT & $5123 \pm 50$ & $576 \pm 32$ & $422 \pm 22$ & $7.6 \pm 0.4$ & $39.1 \pm 0.2$ \\
\hline RO & $9115 \pm 113$ & $932 \pm 68$ & $704 \pm 37$ & $7.2 \pm 0.4$ & $40.5 \pm 0.2$ \\
\hline SI & $949 \pm \quad 9$ & $86 \pm$ & $66 \pm$ & $6.5 \pm 0.3$ & $40.7 \pm 0.2$ \\
\hline SK & $2215 \pm 16$ & $56 \pm$ & $430 \pm 13$ & $16.3 \pm 0.6$ & $41.1 \pm 0.2$ \\
\hline $\mathrm{FI}$ & $2401 \pm 16$ & $330 \pm$ & $220 \pm$ & $8.4 \pm 0.3$ & $37.4 \pm 0.1$ \\
\hline SE & $4336 \pm 19$ & $1049 \pm 29$ & $351 \pm$ & $7.5 \pm 0.2$ & $36.2 \pm 0.2$ \\
\hline UK & $28187 \pm 114$ & $7149 \pm 90$ & $1399 \pm 46$ & $4.7 \pm 0.2$ & $36.0 \pm 0.1$ \\
\hline $\mathrm{HR}$ & $1573 \pm 44$ & $158 \pm 15$ & $227 \pm 13$ & $12.6 \pm 0.7$ & $40.1 \pm 0.3$ \\
\hline IS & $160 \pm$ & $35 \pm$ & $4 \pm$ & $2.6 \pm 0.3$ & $42.3 \pm 0.3$ \\
\hline NO & $2283 \pm 13$ & $643 \pm 15$ & $105 \pm$ & $4.4 \pm 0.3$ & $35.0 \pm 0.3$ \\
\hline $\mathrm{CH}$ & $3974 \pm 28$ & $1316 \pm 27$ & $185 \pm 10$ & $4.5 \pm 0.2$ & $37.7 \pm 0.2$ \\
\hline
\end{tabular}

Table 3.I: Confidence limits for ELFS annual results (taken from EUROSTAT 2006)

As mentioned earlier, the basis we used for constructing the jobs matrix is not directly the microdata of the European Labour Force Surveys, but a dataset containing a detailed breakdown of employment for each country and year by 11 variables: occupation (ISCO-2), sector (NACE-2), employment status, type of contract, part-time status, hours worked, sex, age, education, country of birth and nationality. This breakdown was provided by Eurostat officials, following a request by the European Foundation for the Improvement of Living and Working Conditions. In practice, what this very large breakdown contains is the number of workers for each country and year which correspond to each of the possible combinations of the 11 variables mentioned earlier. This data was provided already weighted and grossed up to reflect employment in absolute numbers.

\subsection{The core variables of the jobs matrix: NACE and ISCO}

The cornerstone of the jobs matrix and of this whole project is the simultaneous classification of employment within each European country by occupation and sector 
(the units of such bidimensional classifications is what we call "jobs"). The occupational and sectorial classifications of employment in the ELFS provide the basis for the empirical operationalization of the theoretical concept of division of labour along the horizontal and vertical axes (social and detail) that we discussed in the previous chapter. These classifications also provide the operative link that allows to merge information from different sources and therefore to evaluate the implications that the evolution of the employment structure has on the quality of employment. And the fact that these classifications are internationally harmonized to a large extent is what provides the opportunity for generating comparable results across the EU. Therefore, we must discuss in some detail the rationale behind these two classifications, the level of detail which we are going to use for our project, and their consistency and comparability across the EU.

\section{2.a) The Statistical Classification of Economic Activities (NACE)}

The classification of economic activities used in the European Labour Force Surveys between 1995 and 2007, and therefore used in this project for the construction of the jobs matrix, is NACE rev. 1. This classification scheme is the European counterpart to the United Nations International Standard Industry Classification (ISIC) rev. 3. At the level of detail that we will use in this project, ISIC rev. 3 and NACE rev. 1 are absolutely identical, and therefore we can refer to them indistinctively (UN Statistics Division 2002; EUROSTAT 1996, 2002).

NACE/ISIC is a standard classification of productive economic activities. Although it is closely linked to the international classification of goods and services (CPC), it is conceptually and operationally distinct: there is no one-to-one correspondence between the two. NACE/ISIC is only concerned with types of productive activities, not with kind of ownership, type of legal organization or mode of operation (UN 2002: 11). According to the ISIC documentation, "the expression 'activity' is to be understood as a process, that is to say, the combination of actions that result in a certain type of products. In other words, an activity can be said to take place when resources such as equipment, labour, manufacturing techniques or products are combined to produce specific goods or services" (UN 2002: 12). The concept of activity behind NACE/ISIC, therefore, refers to economic activity as a process of production of use values in the sense discussed in the previous chapter (it is not concerned with ownership or legal issues, which belong to the realm of exchange values). 
In practice, NACE/ISIC is basically a classification of producing units or economic entities (entities which carry out "economic activities"). Economic entities often carry out more than one economic activity in the terms defined in the previous paragraph: in that case, they will be classified according to the activity which contributes most to value added (UN 2002: 12). Although NACE is primarily aimed at the classification of producing units, it is also routinely used for classifying workers, for instance in the European Labour Force Surveys. The basis for such classification, of course, is the main economic activity of the economic entity where the respondent works.

There are three criteria behind the classification of activities in NACE/ISIC: a) the character of the goods and services produced; b) the uses to which they are put; and c) the inputs, the process and the technology of production (UN 2002: 14). The first criterium (the character) takes into account the differences and similarities between economic activities in terms of the physical composition, stage of fabrication of the items and the needs served by them. The second criterium (the uses) takes into account the types of economic transactions in which the goods and services produced are involved, and therefore distinguishes producing units according to sources of demand and markets for their output. The third criterium is based on the material/technical characteristics of the production process.

Although NACE/ISIC was not designed with the concept of social (horizontal) division of labour in mind (at least, not explicitly), its underlying principles are so similar that it can be understood as a classification of producing units according to their position in the social division of labour. As we have just seen, it understands economic activity as a process of production of use values, and classifies producing units according to the types of goods and services they produce, how they are used and the technologies involved: all this is entirely fitting the concept of social division of labour that we discussed in the previous chapter. Of course, this is no coincidence: the enormous variety of interlinked but distinct economic activities that exists in any advanced capitalist economy is the result of the historic development of the principle of the social division of labour. The mechanisms behind the variety of economic activities that NACE/ISIC tries to classify are the principles of the horizontal or social division of labour: the specialization of labour in the production of different goods, organized by firms and externally coordinated by market mechanisms. 
The NACE/ISIC classification of economic activities has a hierarchical structure, with four nested levels of aggregation, from classes (lowest level, coded with four digit numbers) to sections (highest level of aggregation, coded as one-digit letters). There are 17 sections, 60 divisions, 159 groups and 292 classes. What level of aggregation would be adequate for our purposes? In principle, we want to identify categories which are internally more or less homogeneous in terms of job quality (ignoring for the moment the internal variability resulting from occupations, which we will discuss in the next section). In this sense, the appropriate levels of aggregation would probably be either divisions or groups: the 17 sections are likely to retain a big deal of intra-class variation, whereas the 292 classes are so detailed that they probably separate activities which are almost identical. Taking a very detailed level of disaggregation could also lead to comparability problems, as we will discuss with more detail in the next section (for the case of occupational classifications).

In any case, the decision of the level of aggregation is limited by what is available in the ELFS data provided by Eurostat: it only provides 1 and 2 digits, so we used the 2-digit disaggregation for this project. But in our view, this level of disaggregation would be a perfectly reasonable choice even if there would be no external restrictions. The 60 sectors, further broken down by 28 occupational categories as we will see in the next section, should give us an adequate and comparable picture of the composition of the division of labour in each European countries along the horizontal and vertical axes.

With respect to issues of reliability, consistency and comparability of NACE in the ELFS data, we could not find any scientific assessment in the published literature or reports from statistical offices (contrary to the case of ISCO, whose comparability has been studied in detail, as we will see in the next section). Our impression, nevertheless, is that this classification is reasonably comparable internationally, and reasonably consistent over time-much more than ISCO-. Whereas our own data shows obvious breaks in the series of employment by ISCO categories and some suspicious differences across the EU, we did not find any (apparent) problem in the NACE classification. This classification has been used for many years in a number of different statistical sources, surveys and registers, and is crucially important for the planning and evaluation of economic policy: for these reasons, it is likely to be one of the most robust statistical classifications available. 
The NACE classification has undergone a few revisions over time. Some of these revisions were minor and implied no change at the level of aggregation which we use in this project, so we can safely ignore them. One of those minor revision, from NACE rev. 1 to NACE rev. 1.1, was implemented in the European Labour Force Surveys in 2005, within the period we will study. Other revisions implied a substantial change in the structure of the classification or even in the criteria used for delineating divisions: in these cases, there is an inescapable break that does not allow the comparison of the structure of employment by sector before and after the year in which the revision is applied. The period which we will cover in this thesis is directly determined by two such major revisions, one taking place in 1993-1994 (rev. 1) and another one in 2008 (rev. 2). It would be possible to do another study covering periods before 1994 and after 2008, but it would be impossible to extend our study beyond those years.

\section{2.b) The International Standard Classification of Occupations (ISCO)}

The classification of occupations that was used in the European Labour Force Surveys between 1995 and 2008 is the 1988 version of the International Standard Classification of Occupations adapted to the European Union, called ISCO-88(COM). As in the case of NACE, this classification is the direct transposition of an international standard, in this case coordinated by the International Labour Office, which is the original ISCO-88. Again, at the level of disaggregation we will use in this project, the international and European standards are identical, so we will draw from the documentation of any of them indistinctively (ILO 1990; Elias and Birch 1994).

ISCO classifies workers according to the nature of the work they perform. In fact, what ISCO tries to classify is jobs rather than workers: but we have to be very careful here, because the concept of job in the ISCO documentation is not the same as the concept of $j o b$ which we use in this project. For ISCO, a job is a set of tasks or duties designed to be performed by a person: therefore, it concerns a single worker at a time (there would be as many jobs as workers), even if it does not concern the characteristics of the worker but the nature of the tasks and duties she is supposed to perform in the context of her labour relation. For us, a job would be a set of these ISCO-jobs with similar characteristics in terms of skills and hierarchical position, as well as in terms of the economic activity of the productive units to which they belong (in short, a job for us would be a set of ISCO-jobs classified within the same occupation and sector). To avoid 
any confusions, in this section we will use the term $I$-job for the concept of job used in the ISCO classification.

ISCO-88 classifies I-jobs according to the skills required for the competent performance of the job. It differentiates two dimensions: a) the level of skill, measuring the complexity and range of the tasks involved, and b) the specialization of skills, which refers to the field of knowledge required, the tools and machinery used, the materials worked on and the kind of goods and services produced (Elias 1997: 6). The first dimension (level of skill) derives from the ISCED classification of formal educational levels, even though the ISCO documentation argues that the skills necessary to carry out a given job can be also acquired through informal training and experience (ILO 1990: 2) —which seems somewhat contradictory-. It is this dimension which provides ISCO with a semi-hierarchical structure, with four broad skill-levels associated with specific ISCED levels of formal education.

The first dimension (skill level) assumes that the skills required for each I-job can be characterized by a single (ordinal) metric: but there are also specific skills which cannot be reduced to such single metric because they result from qualitative differences in the types of productive activity. These qualitative differences in the skills required are captured by the second dimension of ISCO (skill specialization). In many ways, this second dimension is analogous to the criteria used for the delineation of the sectorial divisions in NACE (they both refer to the goods and services produced, the materials used and the technologies involved). But this does not mean that this second dimension of ISCO is redundant with NACE. First, because despite their analogy, the second dimension of ISCO is constructed from the perspective of the skills required, whereas the NACE classification takes the perspective of the production process. Second, because NACE classifies the main activity of the unit where the I-job belongs, whereas ISCO classifies the activity of each particular I-job. An I-job may be part of the manufacturing sector, yet carry out services activities (for instance, clerical work): such I-job would be classified within manufacturing in NACE (rather than within services or administration) but as clerical in ISCO. NACE classifies producing units and ISCO classifies I-jobs: because a single producing unit can incorporate different types of economic activities (besides the main activity, used for the NACE classification, there are often secondary or ancillary activities), we need both ISCO and NACE to classify Ijobs properly in terms of the horizontal and vertical division of labour. 
This raises the question of whether ISCO is really adequate as an operationalization of our theoretical concept of the vertical division of labour (what Marx called the manufacturing division of labour). The fact that ISCO classifies I-jobs rather than economic activities or producing units fits our idea that the vertical dimension concerns the division of labour within producing units. Its emphasis on skills is also adequate for our purposes: since the classic formulation of Adam Smith, the core idea of the division of labour is the increasing returns to skill specialization. What would seem to be missing, according to the criteria behind ISCO in the ILO documentation (ILO 1990), is the dimension of authority and hierarchy, which is central to the Marxian concept of manufacturing division of labour in which our own concept is based.

As argued by Marx (for a detailed discussion, see the previous chapter), the main difference between the vertical and horizontal division of labour is the mechanism coordinating the allocation of labour to the different specialized tasks: in the horizontal dimension, the main mechanism is the market (and its units are firms); in the vertical dimension the main mechanism (in capitalism) is the authority of capitalists/managers within productive organizations (and its units are workers within firms). Authority is a central concept for distinguishing both forms of division of labour, and authority structures tend to be associated to differences in pay and job quality in general: hence, our vertical dimension of the division of labour should also classify according to the authority position of jobs within firms, which is in principle missing according to the ISCO documentation.

But is it really missing? We would argue that even if it is not explicit (to our knowledge), ISCO does take into account the authority structures within firms, as can easily be seen by a quick inspection of this classification at the one-digit level. First, this classification includes a specific category (with many sub-categories at the two-digit level) for those at the top of the firms' authority structures (managers, legislators and administrators). This category does not reflect any specific skill level (the first dimension of ISCO), so the only reason for its separate inclusion must be its position in terms of authority within firms. Second, the four skill levels of ISCO are strongly associated with the remaining authority levels within productive organizations, which may simply reflect the fact that skill levels and authority positions tend to be correlated, but it nevertheless implies that by no means is ISCO blind to within-firms authority structures. 
As NACE, ISCO has a hierarchical structure with four nested levels: 10 major groups, 28 sub-major groups, 116 minor groups and 390 unit groups. As in the case of NACE, the most detailed level of disaggregation available in the ELFS is the two-digit level, consisting of 28 categories. In principle, this seems detailed enough for our purposes, especially considering that these 28 occupations are crossed with the 60 sectors identified in NACE at the two-digit level. Furthermore, in this case we have empirical evidence supporting that this is the most detailed level at which it is possible to make reasonably valid comparisons at the EU level. Peter Elias (Elias 1997: 8-11) reviewed seven different studies evaluating the degree of agreement in the classification of the same occupations in different countries, and although he concluded that "occupational coding is an inexact process" (the same occupations are sometimes coded differently in different countries), these papers clearly show that the optimal level for international comparisons is the two-digit level. "There is a sharp improvement in agreement rates between coding frames which are compared at the 2-digit level as opposed to the 3-digit level. The further improvement at the 1-digit level is not so marked" (Elias 1997: 11). Hence, "data comparisons made at the sub-major group level (essentially the 2-digit level) are likely to be reasonably reliable in terms of the application of the underlying classification process" (Elias 1997:11).

But the ISCO classification is not only affected from potential comparability problems across countries: it is also problematic in terms of consistency over time, even within the same country. As we will see later, there are large shifts in the levels of employment of specific occupations in some countries over a single year, shifts which are completely implausible as real shifts in the employment structure and for which there is very limited or no documentation in the ELFS publications. Even though we can more or less solve these problems through some data adjustments, they suggest that (as concluded by Elias in 1997) occupational coding is an inexact process, and therefore we should be specially careful when analysing this dimension of the jobs matrix.

A final note on the implications of these ISCO comparability problems. Because all our analysis of the patterns of employment change by job quality is done using nationallyspecific job rankings and aggregated job quality quintiles, the potential impact of these problems is greatly reduced. It is important to remember that even though the jobs are equally defined by ISCO and NACE in all countries, the comparison of the patterns of job growth is made through the (national) job quality rankings and quintiles. These 
comparability issues become a real problem only when we decompose these patterns by ISCO categories, in order to understand them better. For this kind of decompositions, NACE is a more reliable basis than ISCO, and for this reason we will give more emphasis to the former than the latter. This should keep the specific comparability problems of ISCO reasonably under control.

\subsection{A brief description of the jobs matrix}

Table 3.II shows the number of jobs identified in the jobs matrix in each country covered, for 2000. According to our definition, there are 1680 potential jobs (28 occupations across 60 sectors). In practice, many of these potential jobs do not exist in reality, or they account for such a small proportion of the population that the sample of the European Labour Force Survey does not have any respondent in those categories. So the number of jobs that are actually captured in the jobs matrix varies from 401 in Estonia to 1192 in Germany (in 2000). Only 4 countries have less than 600 identified jobs (Lithuania, Luxemburg, Cyprus and Estonia).

\begin{tabular}{lcccccccccccc}
\hline & DE & UK & FR & ES & CZ & IE & HU & IT & NL & SK & FI & PT \\
\hline Freq. & 1,192 & 1,079 & 1,067 & 988 & 986 & 980 & 977 & 939 & 891 & 821 & 790 & 781 \\
\hline & AT & SI & GR & SE & BE & LV & DK & LT & LU & CY & EE & \\
\hline Freq. & 759 & 730 & 698 & 698 & 686 & 638 & 610 & 539 & 454 & 433 & 401 & \\
\hline
\end{tabular}

Table 3.II: Number of jobs identified in each country

But of course, the actual number of workers within each job varies considerably. There are some jobs which have a rather high proportion of employment and some others which are very marginal. This is illustrated in table 3.III, which shows the proportion of employment accumulated in the 5 biggest jobs of each country, then the proportion accumulated in the first 10 jobs and so on, until the proportion of employment accumulated in the first 500 jobs. The table shows that, indeed, there is quite an important accumulation of employment in relatively few jobs: in all countries, more than $50 \%$ of total employment is accumulated in the biggest 50 jobs, and around three quarters in the top 100 jobs. The biggest 5 jobs account for a relatively high share of total employment as well, from $16.3 \%$ in Luxemburg to $36.4 \%$ in Greece.

A big table showing the description of the top 5 jobs in terms of employment in each country can be found in annex 1. There are only four cases across all EU countries in which a single job accounts for more than $10 \%$ of total employment: and in all cases, these jobs are "market-oriented skilled agricultural workers" (ISCO 61) in "Agriculture" (NACE 1) - this single job account for more than $10 \%$ in Greece, Lithuania and 
Portugal- Our impression is that the ISCO classification is not very well suited for this particular sector, so that all workers tend to get aggregated into a single, large and maybe heterogeneous, category. But for the rest of the economy, the approach seems to work reasonably well, even though there are some other large jobs. The table in annex 1 shows that there are 26 jobs that account for more than $5 \%$ of total national employment in their particular countries, and the rest are below 5\%. Although these jobs are very large in terms of employment numbers, they are likely to be reasonably homogeneous in terms of work and employment conditions: the largest jobs in most countries are teaching professionals in education, building trades workers in construction, health professionals in health and social work and salespersons in retail trade. The structure of the jobs matrix seems adequate with respect to our research objectives: although there is a certain degree of concentration of employment in some specific types of jobs, this concentration reflects the structure of the labour market itself and therefore does not invalidate the approach (with the only possible exception of agriculture, where ISCO lumps all workers within a single category).

\begin{tabular}{lrrrrrr}
\hline & $\mathbf{5}$ & $\mathbf{1 0}$ & $\mathbf{5 0}$ & $\mathbf{1 0 0}$ & $\mathbf{2 5 0}$ & $\mathbf{5 0 0}$ \\
\hline Austria & $23.2 \%$ & $33.0 \%$ & $63.0 \%$ & $76.7 \%$ & $92.2 \%$ & $98.6 \%$ \\
Belgium & $19.6 \%$ & $29.0 \%$ & $59.1 \%$ & $73.3 \%$ & $90.2 \%$ & $98.2 \%$ \\
Cyprus & $24.6 \%$ & $34.0 \%$ & $66.5 \%$ & $80.3 \%$ & $95.0 \%$ & \\
Czech Republic & $18.6 \%$ & $26.6 \%$ & $51.3 \%$ & $66.1 \%$ & $84.9 \%$ & $95.4 \%$ \\
Germany & $16.4 \%$ & $25.2 \%$ & $54.3 \%$ & $67.8 \%$ & $86.3 \%$ & $96.3 \%$ \\
Denmark & $22.0 \%$ & $31.0 \%$ & $60.4 \%$ & $74.1 \%$ & $91.2 \%$ & $99.0 \%$ \\
Estonia & $18.4 \%$ & $25.5 \%$ & $54.1 \%$ & $71.0 \%$ & $92.3 \%$ & \\
Spain & $22.8 \%$ & $33.9 \%$ & $62.9 \%$ & $76.0 \%$ & $90.5 \%$ & $97.6 \%$ \\
Finland & $19.7 \%$ & $30.9 \%$ & $57.3 \%$ & $71.5 \%$ & $88.7 \%$ & $97.5 \%$ \\
France & $16.7 \%$ & $26.5 \%$ & $56.6 \%$ & $70.7 \%$ & $87.7 \%$ & $96.8 \%$ \\
Greece & $36.4 \%$ & $47.7 \%$ & $73.9 \%$ & $84.7 \%$ & $95.1 \%$ & $99.1 \%$ \\
Hungary & $20.6 \%$ & $29.4 \%$ & $54.8 \%$ & $68.9 \%$ & $86.2 \%$ & $95.9 \%$ \\
Ireland & $24.6 \%$ & $34.5 \%$ & $60.9 \%$ & $73.5 \%$ & $89.1 \%$ & $97.0 \%$ \\
Italy & $21.2 \%$ & $30.4 \%$ & $61.1 \%$ & $76.3 \%$ & $91.4 \%$ & $98.1 \%$ \\
Lithuania & $30.2 \%$ & $38.4 \%$ & $61.9 \%$ & $74.1 \%$ & $90.5 \%$ & $99.5 \%$ \\
Luxemburg & $16.3 \%$ & $26.7 \%$ & $65.0 \%$ & $80.4 \%$ & $95.1 \%$ & \\
Latvia & $20.7 \%$ & $28.9 \%$ & $57.6 \%$ & $71.5 \%$ & $90.0 \%$ & $98.6 \%$ \\
Netherlands & $18.4 \%$ & $26.6 \%$ & $59.2 \%$ & $72.4 \%$ & $88.6 \%$ & $97.1 \%$ \\
Portugal & $27.4 \%$ & $37.4 \%$ & $67.7 \%$ & $79.5 \%$ & $92.6 \%$ & $98.4 \%$ \\
Sweden & $22.5 \%$ & $31.4 \%$ & $59.6 \%$ & $72.7 \%$ & $89.7 \%$ & $98.0 \%$ \\
Slovenia & $21.2 \%$ & $29.7 \%$ & $55.1 \%$ & $69.0 \%$ & $86.8 \%$ & $97.3 \%$ \\
Slovakia & $18.5 \%$ & $26.9 \%$ & $52.2 \%$ & $65.6 \%$ & $84.5 \%$ & $95.8 \%$ \\
United Kingdom & $18.0 \%$ & $26.6 \%$ & $54.7 \%$ & $69.7 \%$ & $87.7 \%$ & $96.6 \%$ \\
\hline
\end{tabular}

Table 3.III: Share of employment in the biggest $\mathrm{X}$ jobs

But how much do the jobs matrices of different countries differ? Are they really comparable? We know that the number of jobs in the matrix varies considerably across countries, which means that not in all countries the same jobs are available. If this 
means that the actual jobs identified in the different countries are very different, then the jobs matrices may not be really comparable. The following table shows the number of jobs which are missing in any number of countries. There are 129 jobs that are available in all countries (ie., there are 0 countries with missing values in these jobs), 104 jobs which are missing only in one country, etc. Around $50 \%$ of the jobs identified in the matrix are available in at least 19 countries.

\begin{tabular}{|c|c|c|c|c|c|c|}
\hline \multirow{2}{*}{$\begin{array}{l}\text { Unavailable in } \\
\text { (n countires) }\end{array}$} & \multicolumn{3}{|c|}{ Number of jobs } & \multicolumn{3}{|c|}{ Employment } \\
\hline & $\mathbf{N}$. & Percent & Cum. & Empl. & Percent & Cum. \\
\hline 0 & 129 & 8.79 & 8.79 & 108532.7 & 62.59 & 62.59 \\
\hline 1 & 104 & 7.09 & 15.88 & 29605.3 & 17.07 & 79.66 \\
\hline 2 & 76 & 5.18 & 21.06 & 10468.8 & 6.04 & 85.70 \\
\hline 3 & 71 & 4.84 & 25.9 & 6681.8 & 3.85 & 89.55 \\
\hline 4 & 63 & 4.29 & 30.2 & 5411.7 & 3.12 & 92.67 \\
\hline 5 & 55 & 3.75 & 33.95 & 2706.3 & 1.56 & 94.23 \\
\hline 6 & 46 & 3.14 & 37.08 & 1875.8 & 1.08 & 95.31 \\
\hline 7 & 52 & 3.54 & 40.63 & 1576.0 & 0.91 & 96.22 \\
\hline 8 & 48 & 3.27 & 43.9 & 1442.4 & 0.83 & 97.05 \\
\hline 9 & 42 & 2.86 & 46.76 & 967.3 & 0.56 & 97.61 \\
\hline 10 & 53 & 3.61 & 50.37 & 869.6 & 0.50 & 98.11 \\
\hline 11 & 51 & 3.48 & 53.85 & 914.6 & 0.53 & 98.64 \\
\hline 12 & 45 & 3.07 & 56.92 & 458.3 & 0.26 & 98.90 \\
\hline 13 & 54 & 3.68 & 60.6 & 498.2 & 0.29 & 99.19 \\
\hline 14 & 38 & 2.59 & 63.19 & 233.3 & 0.13 & 99.33 \\
\hline 15 & 40 & 2.73 & 65.92 & 242.8 & 0.14 & 99.47 \\
\hline 16 & 50 & 3.41 & 69.33 & 220.4 & 0.13 & 99.59 \\
\hline 17 & 53 & 3.61 & 72.94 & 199.3 & 0.11 & 99.71 \\
\hline 18 & 48 & 3.27 & 76.21 & 125.3 & 0.07 & 99.78 \\
\hline 19 & 75 & 5.11 & 81.32 & 146.7 & 0.08 & 99.86 \\
\hline 20 & 94 & 6.41 & 87.73 & 130.8 & 0.08 & 99.94 \\
\hline 21 & 89 & 6.07 & 93.8 & 73.0 & 0.04 & 99.98 \\
\hline 22 & 91 & 6.2 & 100 & 31.6 & 0.02 & 100 \\
\hline Total & 1,467 & 100 & & 173412.0 & 100 & \\
\hline
\end{tabular}

Table 3.IV: Distribution of jobs not available in all countries

But the issue here is not so much about the number of jobs, but about the total employment that these jobs account for. And as shown also in the table above, the problem of the mismatch of the identified jobs in different countries is very small in terms of employment: the jobs which have values in all the countries account for almost $63 \%$ of total employment, and the jobs which have values at least in 20 out of the 23 countries in the dataset account for $90 \%$ of total employment. Therefore, the structures of employment as captured by the jobs approach seem reasonably comparable across different countries. In chapter 4 we will compare the jobs matrices from a more substantive perspective (the different structures of employment by occupation and sector). 


\section{The second pillar of the jobs approach: the job quality rankings}

The second step in the jobs methodology involves the ranking of jobs by their quality. Following the theoretical discussion of the previous chapter, we have constructed three different measures of quality to rank the jobs in the matrix: one based on median hourly wages, another on the average educational attainment of workers, and a third one based on the aggregation of different variables measuring non-pecuniary conditions of work and employment (what we will call the amenities index, following Adam Smith's terminology ${ }^{24}$ ). In this section, we will explain the methodology followed for the construction of those three indices, one by one. But first, we need to briefly discuss some general issues on our approach to job quality.

In this project, job quality plays a crucial but secondary role. Our intention is not to study job quality in itself, or its evolution: rather, we want to study the implications of the transformation in the jobs structure (or the changing division of labour) on the quality of existing jobs. For this reason, we will use a static and relative approach to job quality:

1. Static: the characterization of jobs by their quality will be fixed for the whole period. As mentioned earlier, the analytical strategy of the jobs approach is based on two main parameters: on the one hand, the evolution of employment across the jobs matrix, on the other hand the hierarchy of jobs by their quality. By fixing the second element (job quality), we focus our analysis on the transformation of the jobs matrix over time. Simplifying, we could say that we need is some method to identify which jobs are good and bad, and then study whether the evolution of employment over time has meant an increase of the good or the bad jobs. This requires keeping the definition of good and bad jobs (in our case, provided by the job quality rankings) constant over the period being studied.

2. Relative: our measures of job quality will be relative rather than absolute. In fact, our analysis will be based on a broad classification of jobs by their quality in five groups, from low to high (with respect to each of the three job quality measures used). A relative measure is more adequate for our purposes for the

\footnotetext{
${ }^{24}$ In fact, the term of (dis)amenities is not really used in The Wealth of Nations (instead, Smith speaks about "agreeableness or disagreeableness"; Smith 1776: I.10.4), but it has become the standard term in the debate on compensating wage differentials, which is why we use it here.
} 
following reasons: first, even though it is less precise in its characterization of the quality of the different jobs, it is detailed enough for our purposes of studying the broad implications of the changing division of labour; second, absolute measures are considerably more difficult to compare across different countries (and less reliable) than relative measures; third, relative positions and differences, especially if they are as aggregated as in this case (in quintiles), are less affected by the breaks in the time series of employment described in the previous section.

This static and relative approach provides a good basis for a compared structural analysis of job quality, but we must be aware that it has its problems too. First, the fact that it is relative and highly aggregated means that it will miss many important details in terms of job quality. Most importantly, our data will fail completely to reflect the scale of inequalities which exist in terms of job quality across jobs in the different countries (in any of the three measures, but specially in terms of wage levels; see for instance Mittag 2005). Our figures will show employment growth in all countries divided in five more or less evenly sized groups of jobs, from low to high quality: this may give a wrong impression of homogeneity in terms of labour market structures across Europe. Our figures, therefore, have to be interpreted with care, having this potential problem in mind. The second problem is related to the fact that we will use fixed job quality rankings. As we already mentioned in the theoretical chapter, the absolute and relative levels of job quality do change over time, so using a fixed ranking always implies some degree of oversimplification and misrepresentation. But as already mentioned, this simplification is necessary in order to be able to focus our attention on the implications of the transformation of the jobs structure; and as long as we are aware of its implications, this should not be a major problem either.

In fact, although of course we believe that it is fundamentally true that the relative levels of job quality are continuously changing, over a period of 12 years such as the one we are covering here they should not imply a significant realignment of jobs in the matrix. As mentioned earlier, this is one of the advantages of using a relative rather than an absolute approach. Probably between 1995 and 2007 (the period covered here) there have been important changes in the wages, educational levels or amenities of the different jobs, maybe even a few realignments in the overall ranking: but it seems quite 
implausible that a significant number of jobs would have shifted their position vis-à-vis the others, or between the five groups of job quality which are the basis of our analysis.

Unfortunately, we cannot test such idea for the wages and amenities rankings, because we only have measures of them for a single point in time (2002 for the wages ranking, and 2005 for the amenities ranking, as we shall see later). But in the case of the educational ranking, we can (re)construct the ranking year by year for each country, and see how much change there is in terms of job quality at the job level (using correlation matrices). The results of such analysis, which are shown in annex 2, corroborate our assumptions, with a few minor qualifications. Most importantly, the correlations between the yearly rankings are very high for most countries (above .85), and in most cases they are not larger for adjacent than for distant years (if they were, it would mean that the rankings changed between 1995 and 2007). In a few countries (most clearly in Finland, Netherlands, Sweden and the UK), the correlations do decrease as the period separating two rankings becomes longer: but even in those cases, the correlations between the most distant years are high enough as to ensure comparability (they are always above .75). There are, though, a few countries (most importantly, the three Baltic States; to a much lesser extent also Slovenia and Slovakia), where the correlations seem to be somewhat erratic (going up and down throughout the period): but what this suggests is that the national data is of poor quality in those cases, not that the job quality hierarchies change over time (unless we believe those changes are to some extent random). Therefore, this quick check supports the idea that using a fixed relative measure of job quality over the whole period should not be a major problem. ${ }^{25}$

\subsection{The wage ranking ${ }^{26}$}

Because there is no single European data source measuring wages for the whole of the economy and which can be broken down by occupation and sector at the two-digit level, this ranking was constructed through a complicated process of aggregation of different sources. Even though this ranking has a number of problems which we must recognise and take into account in our analysis, it is arguably as good as it can get with the currently available data at the European level; and considering that we only need a relative measure which can be used for aggregating the jobs in five ordered groups, it

\footnotetext{
${ }^{25}$ In fact, as we will see later, the educational measure used for our second job quality ranking is based on pooled data from all available years.

${ }^{26}$ The wage ranking we will use here was mainly prepared by Terry Ward and Robert Stehrer for the JOBS project of the European Foundation in 2007 (see Stehrer and Ward 2008).
} 
should serve our purposes well enough. In any case, we will contrast the results obtained with this ranking with the other two indices of job quality, based on the educational level and a multidimensional measure of amenities.

\section{1.a) A brief description of the sources}

The main sources for the wage rankings are the 2002 European Structure of Earnings Survey (ESES) and the 2005 European Survey on Income and Living Conditions (EUSILC), complemented secondarily with the European Community Household Panel (ECHP) of 2001 and the Structure of Business Statistics (SBS) of 2003. Therefore, we will provide a more detailed account of the methodology of the two main surveys used (ESES and SILC) and only a brief summary for the two complementary ones (ECHP and SBS).

The European Structure of Earnings Survey (ESES) is a relatively new European survey that aims at collecting harmonised data on gross earnings, weekly working hours and annual days of leave at establishment level (Eurostat 2005). It has only been carried out twice so far (in 2002 and 2006), but the aim is to keep on doing it every four years. The data for the 2006 edition of the SES has not been yet published (as of January 2010), so for this project we had to use the 2002 edition. As the ELFS, it is carried out jointly by National Statistical Offices (NSOs) and Eurostat. Although the concepts and principles are harmonised, the actual implementation of the questionnaire and fieldwork is done nationally. The 2002 survey covered the 25 members of the EU (including the ten NMS which had still not formally joined).

So far, Eurostat has made available only a very limited amount of documentation on this survey. Only the background Council Regulation and some brief methodological documents are available through the Eurostat website (see European Council 1999 and Eurostat 2005). In some individual Member States, there is more documentation on the national surveys that feed the ESES.

Although the unit of analysis of the ESES is the employee, the information is gathered through enterprises. Not all the employed population is covered: the survey is only representative of employees working in companies of at least 10 employees in the sectors $\mathrm{C}$ to $\mathrm{O}$ (excluding $\mathrm{L}$ ) of the NACE classification of economic activities ${ }^{27}$. ESES

\footnotetext{
${ }^{27}$ The economic activities excluded are: A - Agriculture, forestry and fishing; B - Mining and quarrying; L - Real estate activities; P - Education; Q - Human health and social work activities; R - Arts,
} 
excludes self-employed persons, those working in companies with less than 10 employees and those working in public administration and agriculture ${ }^{28}$.

Nevertheless, the ESES covers labour earnings and has a very large sample (it varies from country to country, but it is considerably larger than the EU-SILC and ECHP). For this reason, it can be used to compile information on labour earnings for very specific groups of workers. It also includes information about the number of hours worked (necessary to calculate hourly wages, the basis for our ranking) and information on the occupation and sector of each respondent (coded as ISCO and NACE). ISCO is coded at the two-digit level, but NACE is only available at one digit, which implies the need for further tweaking as we will see later.

The European Survey of Income and Living Conditions (EU-SILC) is the EU reference source for comparative statistics on income, poverty and social exclusion. The organisation of the EU-SILC is quite similar to that of the ELFS and ESES. The concepts, definitions and classifications for the questionnaire, and the principles of data collection, are decided jointly by Eurostat and National Statistical Offices (NSOs). These decisions are constrained by the need to feed the process of Open Method of Coordination (OMC) within the EU on the areas of social protection and social inclusion (Eurostat 2009b: 48). The questionnaire and fieldwork design are finalised, and the fieldwork itself carried out, by the NSOs within each member state. Eurostat coordinates the whole process and centralises the resulting data at the EU level. Therefore, the objectives, concepts and principles are the same, but not the actual formulations or methods used for collecting the information. As mentioned earlier when discussing the ELFS, this is not necessarily a problem, but must be acknowledged and taken into account when dealing with the data.

The sample size of the EU-SILC is quite large, although not as big as the ELFS or the ESES: the number of persons included in the sample ranges from 7.000 to 47.000 in the different member states (EUROSTAT 2008). But only a fraction of them are actually employed persons, which means that if the goal is to do very detailed analysis, the sample size might be somewhat limited. The information is provided in two different ways: cross-sectionally (with the reference period covering a single year, used to

entertainment and recreation; S - Other services activities; T - Activities of households as employers; undifferentiated goods and services producing activities of households for own use.

${ }^{28}$ The 2006 wave of some countries includes all establishment sizes. 
estimate the incidence on that specific year of the phenomenon of interest) and longitudinally (the information is repeatedly measured for the same individuals over a period of four years, so that the change in the incidence of the phenomena studied can be studied at the individual level). The EU-SILC is a relatively new survey, with comparable EU data available only from 2005 onwards. For this project, we used the 2005 edition of the survey, which covered the whole EU-25.

The EU-SILC includes, as already mentioned, good information on labour earnings. It collects information on net labour income for all workers living in private households, including categories of workers that are not included in the ESES, such as selfemployed workers, workers in companies with less than 10 employees and workers in the public sector or agriculture. This survey collects some basic information about the employment status of workers, including occupation and sector, but as the ESES, NACE is only coded at the one-digit level. It also includes some basic working time information (most importantly, working hours), necessary for building an indicator of hourly wages.

The European Community Household Panel (ECHP) is the precedent of the EUSILC. It has similar characteristics: the data was collected longitudinally and it covered mostly issues of income and living conditions (see EPUNET 2004). Its sample was representative of private households in each country, although it was considerably more limited than the sample of the EU-SILC (the total number of adults aged 16 years and over was approximately 130,000). It was carried out between 1994 and 2001, and it only covered the 15 countries that were members of the EU before 2004. It includes detailed information on wages, working hours, and occupation and sector at the twodigit level. For this project, we used the 2001 wave.

Finally, the Structural Business Statistics of the EU (SBS) is a completely different source because it does not draw from a specific survey, but from a range of administrative registers and official statistical information (see EUROSTAT 2007b). The observational unit is the enterprise, but it collects aggregate information on hourly wages which can be broken down by sector (at the two-digit level). It covers all members of the EU, and within each member, all private sector companies except those in agriculture and fishing, financial services an non-market services such as education and health. 


\section{1.b) The process of generating the wage rankings}

The wage ranking was constructed by aggregating information from the four sources discussed earlier, in a three-step process. The estimation of hourly wages, in all cases, concerns only employees: the earnings of self-employed are much less reliable and in any case there are no adequate sources for this particular group available in the EU. ${ }^{29}$

The first step is the construction of the backbone of the wage ranking, which is entirely drawn from the ESES. This backbone basically consists in a large matrix of data including information on the hourly earnings of each occupation (ISCO at the two-digit level) within each sector (NACE at the one-digit level). ${ }^{30}$ The assumption is that this survey is the best source for estimating median hourly earnings for the whole of the EU at the required level of detail. But it has important problems which makes it necessary to supplement it with information drawn from the other three sources. The first problem is that some sectors are completely excluded from ESES: agriculture and fishing, public administration, education, health care and personal and community services. The second problem is that even for the sectors which are included, ESES only provides a 1-digit NACE breakdown, which is not enough detailed for our purposes, especially in the case of the manufacturing sector. Whereas the 1-digit NACE is only further divided in three or four sub-sectors at the two-digit level for most of the service sectors (and therefore, the loss of information is quite small), in the case of manufacturing there is only a single one-digit sector for 23 two-digit sectors (the loss of information in this case is substantial, especially considering that there are quite important differences in these subsectors, from textile manufacturing to aeronautical industries). Therefore, it is necessary to use information from the other three sources to further break down the wage levels within the manufacturing sector in particular (for the services sectors, the potential errors introduced by the aggregation of different sources weights more than the potential gains from a more detailed breakdown, so it was decided to leave them at the one-digit level). These two problems of ESES can be more or less corrected by the estimation process explained below, but there is a third problem for which there is no solution: the ESES does not cover those working in companies with less than 10

\footnotetext{
${ }^{29}$ Nevertheless, these group of workers are included in the subsequent analysis. In the following section, we will discuss this issue specifically.

${ }^{30}$ These estimations were provided by Eurostat officials. To preserve the confidentiality of respondents, Eurostat multiplied each estimation by a constant arbitrary factor: therefore, this data cannot be used for analysing absolute wage levels, but is perfectly adequate for analysing relative wage levels. This data will also be used to construct relative wage intervals, for illustrative purposes, in the following chapter.
} 
employees. Because there is no solution, we are forced to assume (or hope) that the relative wage levels of these workers are not very significantly different from those of the rest (more on this later).

The second step in the construction of the wage ranking consists, therefore, in adding to this backbone the hourly wages for the sectors not covered in the ESES. The source used in this second step is the EU-SILC survey of 2005, which covers all sectors but, unfortunately, only provides a 1-digit NACE breakdown. The hourly earnings of each occupation (2-digit ISCO) and sector (1-digit NACE) are estimated with this survey (for the whole economy). The resulting matrix is then aligned to the matrix calculated with the ESES (what we have called the backbone of the wage rankings). For the jobs which are available in both sources, the ratio between both estimations is calculated. This ratio is then used as an adjustment factor for the jobs available in EU-SILC but not in ESES, to homogenize both matrices. This adjustment factor is the same for all EU-SILC jobs not covered in ESES (in each country), so the relative distances between their wage levels are preserved intact. Once this adjustment has been applied, the jobs only available in EU-SILC are added to the ESES backbone (for the jobs available on both sources, only the ESES estimation is used).

The third step consists in breaking down the manufacturing sector. The sources used are ECHP and SBS. ECHP has a more detailed breakdown of NACE (although still not two-digit), but unfortunately it only covers EU-15 countries. There is no survey covering hourly wages with NACE at a more detailed level of disaggregation than the 1digit in the NMS. For these countries, it was necessary to apply a correcting factor based on SBS.

In EU-15 countries, hourly wages for all 2-digit ISCO occupations within 6 broad manufacturing sub-sectors were estimated, and aligned to the previous estimations using the same procedure applied earlier for the EU-SILC. For the NMS, the average wages for two-digit sectors within manufacturing were calculated using SBS, without any occupational breakdown (ISCO is not covered in SBS). An adjustment factor based on these averages was then calculated and applied to the hourly wages within each manufacturing sub-sector, constant across all occupations. This implies the (necessary) assumption that the structure of wages by occupation within each manufacturing subsectors is broadly the same: that is, that there is no interaction between occupation and sector in the wage structure within manufacturing. 
With the resulting set of estimations of hourly earnings by occupation and sector, the jobs were finally ranked from low to high: hence the wage rankings.

\section{1.c) The validity of the wage rankings}

The process of generating the wage rankings was complex, almost tortuous: it involved the aggregation of different data sources, in some cases making (necessary) bold assumptions, sometimes pushing the data almost to the limits of what is scientifically acceptable. This tortuous process was the only way we could find to have a wage-based relative measure of quality at the job level in each European country, which was critical for this project. And notwithstanding all these problems, we believe it was worth the effort: the final result is a reasonable proxy to job quality in each of the $23 \mathrm{EU}$ countries covered in this study, which shall allow us to test the hypotheses presented in the previous section.

Nevertheless, there are some problems that must be acknowledged, and its potential implications explicitly evaluated:

1. The measure of hourly wages which served as the backbone for the wage rankings did not include those working in companies with less than 10 workers. This is a problem to the extent that there are systematic differences in wage levels between those working in very small companies compared to the rest, and to the extent that these systematic differences do not affect equally all the jobs in the matrix (if all jobs would be equally affected, considering that the approach is relative and not absolute, this would mean no problem). This problem probably has some impact on the estimated wage levels (especially in absolute terms): but in this thesis, we never analyse wage levels directly. We only use wage levels to rank the jobs and assign them to five groups (quintiles): all the analysis is based on that five-point classification. Although there may be some minor errors in the assignment of jobs to quintiles as a result of this problem, it is totally improbable that such mis-assignment is significant enough as to bias the overall results. This problem is, therefore, practically neutralized.

2. The NACE classification used for constructing the wage ranking is less detailed than the classification used for the jobs matrix. As we have seen, rather than the target 2-digit NACE, we had to use an intermediate classification (with around 30 categories, beyond the 17 categories of NACE-1 but definitely below the 60 
categories of NACE-2). The main potential implication of this compromise is some loss of precision in the analysis, which should be minimal. After all, each of these 30 NACE categories are still broken down by the 28 occupations of ISCO at the two-digit level (in total, a matrix of almost 900 potential jobs), which should provide with a fair degree of detail. The choice of 2-digit NACE classification for the jobs matrix is not the only good one: in fact, someone could have argued that a slightly more aggregated version is even better.

3. The aggregation of data from different surveys can lead to biased results if the classifications and measures are not entirely consistent. As we discussed earlier, the classification of occupations, in particular, is a complicated process which often incorporates some degree of error: the combination of different sources can compound and intensify this error. Measuring wages is also a complicated business, and for the construction of the wage rankings, sources with different methodologies for the measurement of wages were combined. As in previous cases, this problem should be minimized by the fact that our approach is relative rather than absolute, that we are only looking at the rankings, and that in the analysis stage, we even aggregate further the jobs by broad quintiles (as we will see in the next section).

4. Finally, the different sources which contribute to the wage rankings have different base years, which can add some degree of imprecision or bias. Again, the fact that the approach is relative, and quite aggregate in the final analysis, should minimize this problem. And as we argued in previous pages, the absolute levels of job quality are continuously changing, but the relative positions of each job against the other remain relatively stable for a period as short as the one we are talking about.

So we take the wage ranking as a necessarily imperfect but useful approximation to job quality. We have to assume that it includes some degree of error and imprecision, there is no point in denying that. But, on the one hand, it is only used as a relative (positional) way to classify the jobs in the matrix into a five-point scale going from lowest to highest quality (such a relative and aggregate approach is likely to minimize the potential bias); on the other hand, the potential gains of having a country-specific ranking of jobs by their median wages for all the 23 EU countries covered in this study more than compensate for the potential imprecisions. As long as we maintain this relative and 
broad approach, as long as we do not pretend the wage ranking to be a precise measure that can be used to compare jobs at a detailed level, we can use this measure safely enough. And in any case, we will always contrast the results we get from using this ranking with the results we get from the other two rankings explained below. ${ }^{31}$

\subsection{The educational ranking}

The second variable used in this thesis for ranking jobs in each country is based on the average educational level of job-holders (within each job) for the whole period covered (which varies depending on the countries). It is based on a single source, which is the same one used for the construction of the jobs matrix: the European Labour Force Survey. Because of the logic of its construction and the fact that it uses the same source as the jobs matrix, this ranking is considerably less problematic from a technical perspective than the other two.

The basis for this ranking is the variable ISCED included the European Labour Force Survey datasets. ISCED is an internationally comparable codification of national educational systems, which was originally designed by UNESCO in the 1970s and updated several times $^{32}$ (see UNESCO 2006). We used a highly aggregated version of ISCED, which only distinguishes three broad levels of education: low (basic schooling or less, below ISCED 2), medium (upper secondary and post-secondary pre-tertiary, ISCED 3-4) and high (tertiary, ISCED 5 and above). For each country, the average educational level of all workers within each job was calculated (assigning an arbitrary value of 0 to low, 0.5 to medium and 1 to high educational level), and these values were used for generating the educational ranking.

The justification for using education as a proxy for job quality was given in the theoretical chapter: but it may be good to do a quick recapitulation here. On the one hand, from a traditional economic perspective (developed in human capital theory), education is a determinant or a correlate of productivity, which is the ultimate source of job quality; on the other hand, higher education implies more chances of choosing a better job, and therefore educational attainment may be a good indirect proxy for job

\footnotetext{
${ }^{31}$ Besides the 23 national wage rankings, and in order to be able to do analysis at the EU level as well, an aggregated EU wage rankings was generated. This EU ranking is based on an average of the national wage rankings, normalized and weighted by the share of employment in each country, sector and occupation.

${ }^{32}$ The latest revision of ISCED took place in 1997, and was implemented in the ELFS from 1998 onwards. Because we use a highly aggregated ISCED (with only three categories), our educational ranking should not be affected significantly by this change.
} 
quality. A more sociological perspective would also argue that the skill content of a job is an element which contributes to its intrinsic quality: and although imperfect, the educational level of job-holders can be understood as a proxy of the skill content of jobs. $^{33}$

The main potential problem of using the average educational attainment within each job as a relative proxy for job quality comes from the existence of overqualification or underqualification. We know that, in all advanced capitalist economies, there is a significant amount of workers whose level of formal qualification is above or below the level required by their jobs. In a meta-analysis of more than 20 years of applied research on this issue, Groot and Brink estimated that on average, about one in every four workers of most advanced economies can be considered as overqualified (Groot and Brink 1999: 156) - according to these authors, the estimation of an average level of underqualification is more difficult because of the use of different definitions -. Women are more likely to be overqualified than men, and younger more than older workers (Groot and Brink 1999: 153; Sicherman 1991).

If the degree of overqualification varies systematically across jobs (that is, if more jobs tend to have more overqualified workers than others), the degree of formal education would be a biased proxy of job quality, following the arguments explained in the previous paragraph. In particular, the (proven) fact that women are more likely to be overqualified than men, and younger than older workers, means that those jobs which tend to be occupied by women and young people will probably receive a higher relative position than they should in the educational ranking. This is, in fact, something that we have already tested ourselves (see Stehrer, Ward and Fernández-Macías 2008: 21-23): female-dominated jobs have a higher average position in the education ranking than in the wage ranking, which is a direct way to prove the existence of a gender-biased overqualification by jobs. This would be a big problem if we only had the educationbased ranking to proxy job quality: but the fact that we have two other measures means that rather than a problem, it is a way to enrich the analysis (as shows the results published in Stehrer, Ward and Fernández-Macías 2009). The existence of discrepancies between the education, wage and amenities ranking can be interpreted as evidence of overequalification or other similarly interesting phenomena.

\footnotetext{
${ }^{33}$ As in the case of the wage ranking, an EU ranking of jobs by educational level was constructed by averaging the national rankings, after normalizing and weighting them according to their employment shares.
} 


\subsection{The amenities (multidimensional) ranking}

Finally, the third measure of job quality we will use in this thesis is based on a multidimensional index of amenities (understanding amenities as non-pecuniary characteristics of jobs which have an impact on the well-being of workers), constructed with data from the 2005 European Working Conditions Survey. Although this third measure of job quality is necessary to get a more complete picture of the issue at stake, it has some inescapable limitations which mean that we shall mostly consider it a supplementary measure to the other two. As usual, these limitations derive directly from limitations of the data from which the index is drawn. The only existing European survey which covers the range of issues necessary to construct a multidimensional job quality indicator is the European Working Conditions Survey: but although this survey is wide and deep in its content, it is very shallow in its sampling, not allowing for the detailed level of dissagregation that would be really adequate for our purposes. The core requirement of our approach is that the information can be broken down by sector and occupation, ideally at the two-digit level. We can do this with the EWCS, but only to some extent: on the one hand, we can only do this detailed breakdown if we aggregate all EU countries together in one large sample (and therefore, it is impossible to construct a country-specific amenities index); on the other hand, not all jobs in the matrix can be covered (for some small jobs, the number of cases is too limited for the estimation to be reliable, and therefore they have to be eliminated).

These two problems are important enough to make this index secondary to the other two, but certainly not to make it useless. The wage and educational rankings are wider in their coverage of jobs, and country-specific; but the amenities index is wider in its coverage of job quality and as we will see, can be decomposed by dimensions and indicators. And although it would be desirable to have country-specific amenities rankings, using a single European ranking should be less of a problem in this particular case: the wage and educational structures which are behind the other two rankings are likely to be more affected by institutional differences (and therefore, more countryspecific) than the amenities associated with each job, which depend much more directly on the material and technical characteristics of the labour process in each particular case, and should therefore be more similar across countries (although, of course, the levels of amenities associated with each particular labour process can also be affected by institutional factors, such as Health and Safety regulations). The fact that not all jobs 
can be covered by this index should not have major implications either, because the jobs which are not covered account for a small amount of actual employment (as we will see, less than $10 \%$ in most countries).

We should, nevertheless, keep in mind that this ranking will play mainly a complementary role to the other two: on the one hand, as a way to check their (external) validity and consistency; on the other hand, as an alternative perspective on job quality. Even with the limitations mentioned, even if it was just a crude approximation (we believe it is more than that), there are very important reasons to try to include such an amenities index in this project. We discussed these reasons with some detail in the theoretical chapter. From a standard economic approach, wages are a compensation for the (dis)amenities of work, and therefore we can only have the full picture of job quality if we take both perspectives into account. From a standard sociological perspective, job quality is much more than just pay, so in order to measure it properly we should use a multidimensional index as this one. ${ }^{34}$

\section{3.a) The source: the European Working Conditions Survey}

The EWCS is funded, designed and coordinated by the European Foundation for the Improvement of Living and Working Conditions, an EU agency based in Dublin whose mandate is to gather knowledge that can contribute to the planning and design of policies to improve the conditions of life and work of Europeans. The questionnaire is designed by a group of experts and policy makers on the area of work and employment, together with the Foundation research $\operatorname{staff}^{35}$. The Foundation also prepares the principles for the sampling and fieldwork methodology, which are then part of the technical conditions of a tender. The successful tenderer, a large EU-wide fieldwork company (Gallup in the 2005 EWCS), then finalises the design together with the Foundation and carries out the fieldwork under the supervision of the Foundation staff (see Fernández-Macías and Petrakos, 2005).

\footnotetext{
${ }^{34}$ We could, in fact, just aggregate the wage and the amenities ranking into a single ranking, which would be more fitting to the theoretical concept of job quality. But we opted for not doing such aggregation, for two main reasons: first, because the resulting index would be constructed through so many intermediate steps, assumptions and different data sources that its reliability would be compromised; second, because having separate indices for pecuniary (wage) and non-pecuniary (amenities) job quality is a strength and not a weakness: it allows for a richer analysis, from two different perspectives.

${ }^{35}$ I was part of this team, mainly involved in the coordination of the methodological aspects of the 2005 European Working Conditions Survey.
} 
One of the key advantages of the EWCS with respect to other European surveys (especially, to Eurostat's) is the fact that the whole enterprise is funded, designed and coordinated centrally, by dedicated Foundation staff and the fieldwork contractor. This ensures a level of comparability which is higher than the ELFS, at least in principle: not only the concepts, definitions and classifications are strictly the same across Europe, but also all the questionnaire items. Of course, there is some flexibility for national adaptations (the translation process itself implies an adaptation of the questionnaire to national specificities; see Hurley 2006). Another very important advantage of the EWCS with respect to the other European surveys is the high degree of transparency and documentation of the whole research process.

The sample of the EWCS is representative of all persons in employment in private households of all EU member states (and some European non-Member States, such as Switzerland or Turkey, see Parent-Thirion et al 2007). The fieldwork procedures also follow strictly the same principles across Europe: in all countries, the sample is stratified by region and size of settlement, and the interviews were clustered by geographic proximity. The actual selection of households was done by the random walk method, and within the selected household one employed individual was randomly selected. The sample size of the survey was selected with the main aim of making adequate estimations at the national and EU levels, keeping the budget within reasonable limits. The European Foundation provided all the funding for the survey from beginning to end (which is also exceptional: in all other surveys, the funding comes partly from the coordinating centre and partly from the national parties). The size of the sample for the latest EWCS was 1000 cases per country (except in six smaller countries, where the sample size was kept at 600 cases), which is the main limitation of the EWCS. This sample size allows for the production of good estimates of the overall incidence at the national level of the phenomena captured in the survey, but if we want to go deeper and break down the results within countries by gender, sectors, occupations or whatever other variables, the number of cases used for specific estimations very quickly becomes too small and therefore the estimation becomes unreliable.

\section{3.b) The construction of the amenities index}

The idea itself of amenities derives from Adam Smith's theory of compensating differentials (he argued that the utility of a job depends on the combination of wage and disamenities that it entails), but the construction of the index derives from the four main 
traditions of study of the conditions of work and employment within contemporary social sciences reviewed in section 3 of the theoretical chapter. The structure of this model and the way we operationalized it into a job quality index based on the EWCS is summarized in fig. 3.1.

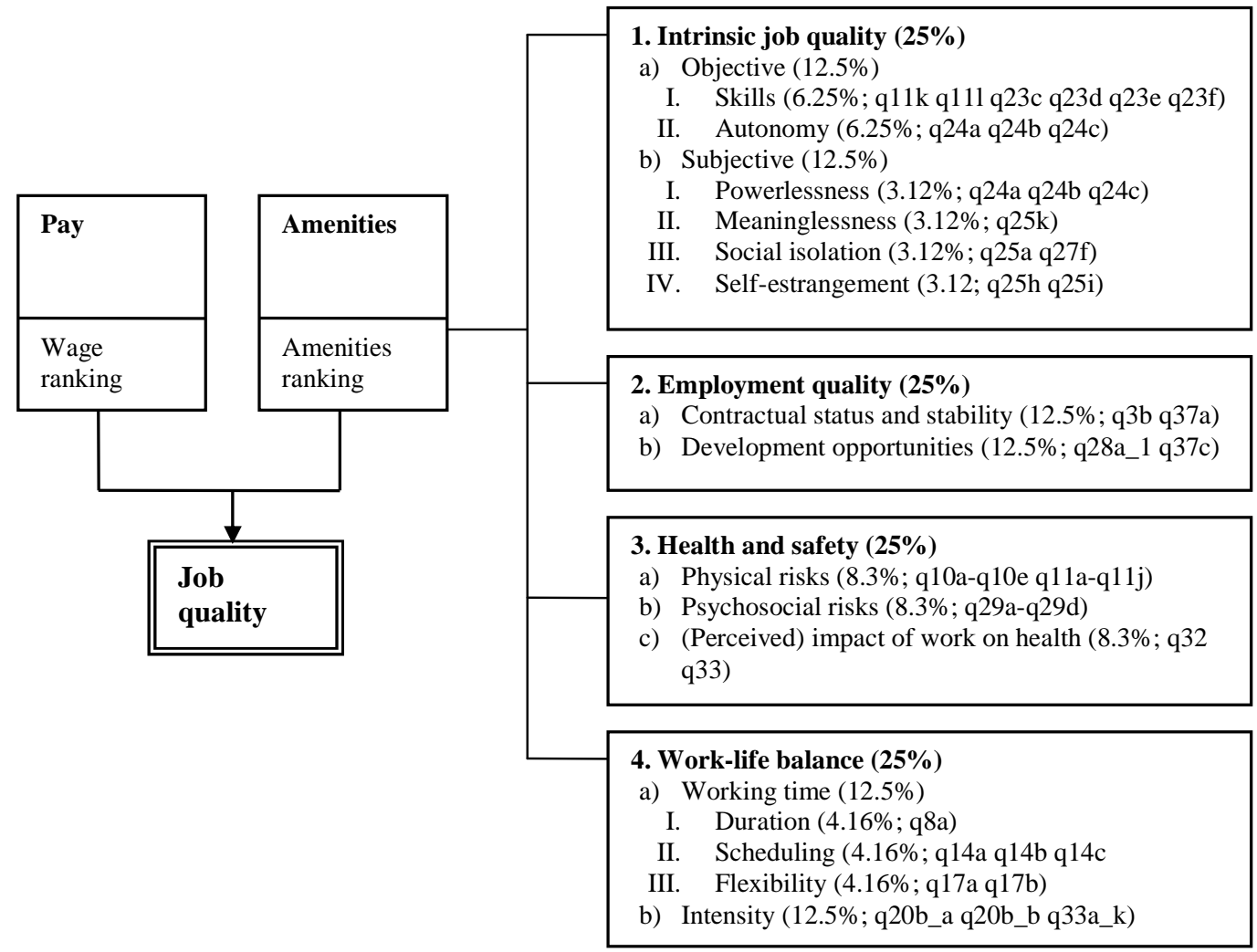

Figure 3.1: The amenities index

The left-hand side of the figure represents the link between the amenities and the wage rankings, according to the theory of compensating differentials. A more sociological approach would consider pay as another dimension of job quality, at the same level as each of the four dimensions shown at the right-hand side of the figure. In any case, even though the overall structure of figure 3.1 draws from the theory of compensating differentials (which fits our purposes, because it implies a contraposition between the wage and amenities rankings), the actual construction of the amenities index (shown at the right-hand side of the figure) draws directly from the four main sociological traditions studying the impact of the conditions of work and employment on the wellbeing of workers, as discussed in the theoretical chapter.

As can be seen in the figure, our model of amenities has a nested structure, with four levels: the overall amenities index (level 1) is composed by four dimensions (level 2, 
with Arabic numbers), which are composed by nine indicators (level 3, with small-cap letters), some of which are composed by nine sub-indicators (level 3bis, with Roman numbers), which are composed by 41 variables (level 4, indicated within brackets). The questionnaire items of the 2005 European Working Conditions Survey are those 41 variables at the lowest level of disaggregation. The process of aggregating the information contained in the 41original EWCS variables followed, step by step, the structure of the model shown in figure 3.1.

First, each variable was normalized to a single metric so that they could be aggregated. For normalizing the original variables, we opted for a method of re-scaling them to a 0 1 metric. This method is adequate for the normalizing of so many and so diverse variables as the 41 items used for the construction of the amenities index: some of these variables were continuous variables, such as working hours, some others arbitrary Likert scales and others dichotomous scales. Other normalization methods such as standardization (expressing the original variables in standard deviation units) would have led to different ranges in the indicators, and therefore the real weight of each variable in the final aggregate index would have varied. The method for re-scaling each variable depended on its original measurement. For dichotomous variables, they were simply adjusted when necessary so that 1 expressed the desirable outcome in terms of job quality, and 0 the opposite ${ }^{36}$. For ordinal variables expressing time scales or agreement scales to an item, they were re-scaled to a 0-1 metric assigning arbitrary but reasonable values to each possible outcome ${ }^{37}$. For multinomial variables of a qualitative nature, each category was assigned a value of 0 to 1 following again an arbitrary yet reasonable $\operatorname{logic}{ }^{38}$. Finally, for the only truly continuous variable used in the amenities index (working hours), the original values were recoded to discreet categories and assigned (again, arbitrary but reasonable) values within the 0-1 range. ${ }^{39}$

\footnotetext{
${ }^{36}$ For instance, variable P33, "Does your job affect your health?", was coded as 1 if the respondent said yes and 0 if respondent said no. These values were inverted, because the desirable outcome is no.

${ }^{37}$ For instance, variables q11 (physical risks exposure) were coded with a Nordic scale, from "never" to "all the time". "Never" was assigned a value of 1 (no exposure, the desirable outcome), "all the time" was assigned with a 0 (total exposure to a risk, undesirable outcome), "half of the time" was assigned a 0.5 value, "one quarter of the time" a 0.75 value, etc.

${ }^{38}$ For instance, variable q17, measured time flexibility, was coded in the following way: a value of 1 if the respondent had complete flexibility to arrange working time (desirable outcome), 0.75 if she could adapt working time within certain limits, 0.5 if she could choose between several fixed schedules, 0.25 if she had no choice but there were no regular changes to her work schedule, and 0 (most undesirable outcomes) if she had no choice and suffered regular changes in her work schedule.

${ }^{39}$ Even though working hours is strictly a continuous variable, in practice it behaves as a discrete (categorical) variable, because of the existence of norms about and restrictions to the hours people work. Our recoding reflected this assumption, as well as the assumption that, all else being equal (especially
} 
Once the original items had been re-scaled to a homogeneous metric (with equal boundaries, and with 1 always meaning best for job quality and 0 worst), we proceeded to the aggregation, following the nested structure of figure 3.1. Within each component of the index, the aggregation was carried out by simple averaging of the values of each item included, for each individual worker. For instance, the sub-indicator "skills" (1.a.I, at the fourth level of the index) contains the arithmetic average for each individual worker of the (re-scaled) values of variables q11k, q111, q23c, q23d, q23e and q23f of the 2005 EWCS. The overall principle behind the weighting of items was one of equal weighting within each level and component. That means that, in fact, the weights assigned to each variable are by no means equal: they depend on the position of each variable within the structure of the model, whether it is the main basis for an indicator or one of several variables to be aggregated into a single sub-indicator. Therefore, the weighting of each variable is directly determined by the structure of the model shown in figure 3.1, which draws from the theoretical discussion of the previous chapter ${ }^{40}$.

The aggregation process follows step by step and from bottom to top the structure of figure 3.1: from variables to sub-indicators, from sub-indicators to indicators and so on, until the final aggregation from the four dimensions to the overall composite index. This way of constructing the index has the advantage that the final result is easily decomposable: each component can be broken down into its subcomponents in a very transparent manner. In this thesis, we will mostly use the highest level of aggregation (the overall index, which is the basis of the amenities ranking): but to understand better some of the results, we will occasionally use also the four dimensions and nine indicators.

\section{3.c) The amenities jobs matrix}

Once the index had been computed at the individual level of the EWCS (that is, for each respondent), we proceeded to the construction of what we can call an "amenities jobs matrix": a large table including the average value of the index, as well as of the four dimensions and nine indicators, for each job (as jobs are defined in this project: specific

from a work-life balance perspective), working less hours is preferable to working more hours. Therefore, less than 20 hours a week was coded as 1 , between 20 and 38 as $0.75,38$ to 42 (the norm for most European countries) as $0.5,42$ to 48 as 0.25 and more than 48 as 0 (both the EU and the ILO consider 48 hours the threshold of unambiguously excessive working hours, so it makes sense to make it the lower boundary for our indicator).

${ }^{40}$ This does not mean that the weights are determined by theory: any aggregate index as this one is inescapably arbitrary in its weighting method. Still, deriving the weights from the structure of a theoretical model seems better than just assigning totally arbitrary weights. 
occupations within specific sectors). It is this structure which allows us to link the information on amenities to the evolution of employment by jobs (the jobs matrix, drawn from the ELFS 1995-2007) and the wage and educational rankings explained earlier.

For generating this amenities jobs matrix, we used the full EWCS-27 dataset, without differentiating by country. As explained earlier, the sample of the EWCS-27 (more than 25.000 cases overall, around 1,000 cases by country) makes it impossible to disaggregate by sector and occupation within each country: simply, there would not be enough people in each job (there would be, in fact, more jobs than respondents). We are thus forced to construct a single jobs matrix for the whole of Europe. How much of a problem this is depends on whether there are significant differences in the amenities of jobs depending on the country in which they are located. Ideally, we would like to test this empirically, by checking the variability of amenities within jobs across countries: but unfortunately, the same reasons that do not allow us to compute country-specific amenities indices impede the testing of such cross-country variations of amenities within the same jobs. ${ }^{41}$ In any case, our impression is that the use of a single index for the whole of Europe (and therefore, a single ranking) is less of a problem in the case of amenities than in the case of wages and educational levels (the basis of the other two rankings, which are country-specific). Whereas pay and educational structures are very strongly affected by institutional variables which are largely country-specific (even within Europe), the amenities associated with each job are much more dependent on the material, technical and organizational characteristics of each type of activity and labour process, and therefore probably less variable across countries. This is not to say that there would be no differences in amenities across Europe, or that amenities are not affected by institutional factors (for instance, differences in Health and Safety regulations or in Industrial Relations systems can be associated with systematic differences in some components): ideally, we would prefer to compute the amenities ranking also at the individual country level. But unfortunately, it is impossible to do so: therefore, we have to assess whether it is worth using a single EU amenities index. I categorically think it is: these potential problems are minor compared to the advantages of having an alternative index of job quality which is multidimensional and decomposable such as this one.

\footnotetext{
${ }^{41}$ Although in the following section we will attempt an approximate test of this assumption, which is in fact corroborated.
} 
It is debatable whether we should have used a sub-sample including only the workers of the 23 countries covered in the jobs matrix (for reasons of consistency) or the full EWCS-27 covering 5 more European countries (for keeping a sample as large as possible for the estimations). We opted for the second option (the full EWCS-27 sample) because after all, as we have just argued, the amenities index is constructed disregarding the country variable in any case. This way, we keep a larger sample, which means better estimations and a wider coverage of jobs. ${ }^{42}$

The sample used for estimating the amenities index includes all workers (that is, also the self-employed), because on the one hand, the point here is the position in the division of labour and not the type of labour relation (material and not social relations of production, in Marxian terminology); on the other hand, because in the analysis of the evolution of employment levels by job (based on ELFS) we will include both employees and self-employed workers. For the construction of the amenities index, this implies a small logistic problem: the dimension of employment quality is half missing for the self-employed, for obvious reasons (they have no labour contract as such). Our solution was simply to ignore that indicator for the self-employed, aggregating the rest of the indicators into the dimensions and overall index. ${ }^{43}$

For estimating the index of amenities and its components at the job level, the individual data has been weighted using the post-stratification factor included in the EWCS dataset (w4). This weighting variable adjusts for unequal probability of selection of individuals within households, for disproportionalities in the sampling frame and for potential biases resulting from different response rates of different groups of workers (see ParentThirion et al 2007 for a detailed explanation of this weighting factor): but it does not adjust for the differences in the working population of the different countries. The EWCS sample includes the same number of respondent in all countries ${ }^{44}$ (1000), despite the large differences in the size of employed population (for instance, the number of workers in Germany is almost 20 times larger than in Ireland, but both countries have the same number of respondents in the EWCS sample). This means that for making estimations at the EU level, a special weighting factor that corrects the

\footnotetext{
${ }^{42}$ We did not include, however, the 4 non-EU countries included in the 2005 EWCS as well (Turkey, Croatia, Switzerland and Norway). The size of Turkey is so large, that when applying the weights it has a very large influence in the results (only second to Germany). The institutional and structural differences between Turkey and the rest of Europe are so large that they could even bias this amenities index.

${ }^{43}$ In fact, whenever some item was missing for any individual respondent, such item was disregarded in the aggregation process (the aggregation was carried out ignoring the missing values).

${ }^{44}$ Except for the five smaller countries, where the sample is 600 cases.
} 
disproportionality between the sample and the size of employed population in each country has to be applied. But the problem is that applying this weighing factor implies giving much more weight to some cases (the cases of larger countries) than others (for instance, German workers are 20 times larger in the weighted sample than Irish workers): in our case, this means that the estimation of the amenities index for some small cells can be almost completely based in just a few cases from the larger countries, with many other cases from smaller countries being almost ignored. Effectively, this implies reducing the quality of the estimation. For that reason, we decided not to apply this weight. We must be aware that therefore, the estimations are not strictly representative of the European workforce as a whole, but rather an average of countryrepresentative estimations of amenities. This seems, after all, coherent with the fact that in this case we are by principle ignoring country effects, assuming that what determines amenities is primarily the position in the division of labour along the horizontal and vertical axes (occupation and sector).

Even using the whole EWCS-27 sample, crossing occupation and sector at the two-digit level generates many cells which are unacceptably small. It is necessary to set a threshold of cases below which the estimation of the amenities index is deemed to be too unreliable: we opted for 15 cases as the threshold. Taking ISCO and NACE at the two-digit level, there are 298 jobs in EWCS with 15 or more cases, accounting for roughly $84 \%$ of the sample. In order to increase slightly the coverage in terms of jobs and employment, we applied an adjustment procedure that combined cells with very similar NACE two-digit codes. The logic was the following: whenever a cell was smaller than 20 cases, its NACE classification was recoded to an intermediate level which is between 1 and 2 digits (the subsection level). Then, the indices were estimated again with this slightly more aggregated jobs matrix. Once this was done, the standard two-digit NACE codes were used for the final extraction of the amenities jobs matrix ${ }^{45}$. The result of this procedure is that 584 jobs are covered in the matrix, and the estimations use more than $91 \%$ of the sample. This way, we get a jobs matrix which is perfectly compatible with the other job matrices, covering an expanded set of jobs and workers and holding more robust estimations of the indices for small cells.

\footnotetext{
${ }^{45}$ So that there are a few jobs that have exactly the same values, which means that in the ranking they occupy adjacent positions, which is perfectly reasonable because they are very similar jobs by definition
} 


\section{3.d) The results}

The primary role of the amenities index in this thesis is to serve as the basis for a ranking of jobs in the jobs matrix, complementary to the two other rankings explained earlier. Therefore, it is beyond our objectives to carry out a detailed analysis of the distribution of this index and its subcomponents across different categories of workers, or the factors determining its variation: as we have repeatedly said, we are not interested in studying job quality as such, but in having some proxy measures of job quality which we can use for studying the implications of the changing division of labour in contemporary European economies. But nevertheless, we seems necessary to inspect the results of the amenities index to ensure that it works more or less as expected: even for a theory-driven index as this one (whose validity should not only be sustained on its empirical behaviour, but on the validity of the underlying model), it is always sensible to check the results.

Table 3.V shows a matrix of correlations (constructed at the individual level, with the full EWCS sample) between the nine indicators, four dimensions and the overall index which can give us an idea about the empirical structure of the index (to be contrasted with the theoretical structure shown in figure 3.1). To simplify the interpretation, the cells of the correlation matrix have been colour-coded with a gradient of grey tones (the darker the grey, the highest the value).

The quadrant at the lower right-hand side of the table shows the correlations between the nine indicators (remember that these indicators compose the third level of disaggregation). Generally speaking, these correlations are not very high: most of them are either moderate (between .20 and .40), or low (below .20), with a few high correlations (above .40). To some extent, these generally low correlations probably reflect some degree of heterogeneity and individual endogeneity in the variation of the different components of job quality, as well as some degree of statistical noise which is always present in this kind of international surveys. But beyond the fact that there are surely biasing factors, we have to say that our the model does not assume that the correlations between the different components of job quality should be strongly correlated. The reason why we need a multidimensional index of job quality is that we understand that each indicator is a measure of a different feature of work which may vary independently (or not) from the rest of features/indicators. Whether the correlations between the different components of job quality (or amenities) are strong or not is an 
empirical finding with interesting implications, but not an assumption of the model used here. The model does not assume either that the variability of all indicators should go in the same direction: there could be important negative correlations if, for instance, there would be compensating mechanisms between the different components of the amenities ranking. But this does not seem to be the case. There are only three negative correlation coefficients (of a total of 36): between development opportunities and psychosocial risks, between development opportunities and intensity, and between skills/autonomy and intensity. In the three cases, this result seems more than plausible: jobs involving high levels of responsibility and with good development opportunities often involve high levels of psychological strain. All the other indicators correlate positively, showing that the non-pecuniary components of job quality tend to go together rather than compensate each other. ${ }^{46}$

The grey coding of the correlations between the 9 indicators (lower right quadrant) shows that they tend to cluster in two distinct groups: the indicators that compose the first and second dimensions (intrinsic work quality and employment conditions) have moderately high correlations (around .2-.3), as well as the indicators composing dimensions three and four (health and safety and work-life balance), but these two groups have very low correlations between them (see the lighter grey tones where the indicators 1.1-2.2 are correlated with indicators 3.1-4.2). Intrinsic work quality and employment conditions, therefore, are only marginally associated with health and safety and work-life balance. The upper-left hand side quadrant of figure 3.2, showing the correlations between the 4 aggregate dimensions, confirms this pattern: dimensions 3 and 4 have a correlation between them of .59 , dimensions 1 and 2 a correlation of .35 , whereas the rest of correlations (among these two groups) are below .2.

The lower left-hand side quadrant shows the correlations between the four dimensions and the nine indicators. Because the dimensions are linear combinations of their corresponding indicators (according to the structure set out in figure 3.1), these correlations reflect the importance of each indicator in the determination of the variability of each dimension. Dimensions 1 and 2 are determined more or less equally

\footnotetext{
${ }^{46}$ It is fair to say that there are some more negative correlations at lower levels of the index, between the variables that compose the indicators. These lower-level negative correlations look perfectly plausible as well. For instance, there is an important negative correlation (-.11) between two components of the working time indicator: the duration of work and its flexibility. Overall, these low-level compensating mechanisms tend to be evened out when the sub-components are aggregated within their higher level indicator: but this is perfectly correct, because the score of the indicator should reflect such compensation (that is precisely the point of doing composite indices).
} 
by their two sub-components, but not dimensions 3 and 4 . The variability of dimension 3 is strongly dominated by indicator 3.3 (subjective perception of impact of work on health), whereas the indicator of psychosocial risk has a very limited influence on the scores of this dimension. This is because the latter indicator has a very low variance in the sample of the EWCS: most workers declare facing very little or no psychosocial risks at work, so the value of most respondents is very close to $1 .{ }^{47}$ The fourth dimension (work-life balance) is slightly dominated by the indicator or intensity (its correlation with the index is .9 , whereas the correlation of the other indicator-working time-is .6): the indicator of intensity has more variance than that of working time, partly because the latter incorporates compensations between its sub-components which tend to limit its overall variation (as explained in an earlier note).

Finally, the first column of the correlation matrix (on the left) shows the correlations between the overall index and its components (dimensions) and sub-components (indicators). Again, the index is a linear combination of its components and subcomponents, so these correlations reflect the importance of each component in the final overall score. The four dimensions have all a very similar correlation with the overall index: the latter is more or less equally determined by the former. The correlations between the index and the nine indicators are more uneven. Psychosocial risks in particular, as we have already discussed, has a small bearing on the final overall score. The highest correlation between an individual indicator and the overall amenities index is for perceived impact of work on health, the two indicators of intrinsic work quality (objective and subjective), physical risks and work intensity. ${ }^{48}$

The structure of correlations between the different components of the index, therefore, suggests that it works reasonably well, fitting our theoretical model and broad expectations. But we also need to inspect the index in terms of its actual content, for instance looking at its average score (for the overall index and its components and

\footnotetext{
${ }^{47}$ This should by no means be considered a problem of the index, but a simple reflection of the reality which it tries to measure. This is a theory-driven index in which the scales of each indicator reflect the incidence of positive or negative treats of work. If most people declare facing very low levels of psychosocial risks at work, it should be reflected in an indicator with a high average value and a very low variance, as is the case. This indicator, therefore, will have a very limited influence on the scores of its dimension and consequently on the scores in the overall index.

${ }^{48}$ Perhaps it may seem surprising that these correlations do not reflect exactly the weights assigned to each indicator in the underlying model (see figure 3.1). The empirical impact of each component on the index (reflected in these correlations) do not only depend on those weights, but very importantly also on the degree of variability of each component (the more variable, the higher the impact) and on the structure of correlations between the components themselves (a component which is highly correlated with other components will have a higher correlation with the overall index).
} 
subcomponents) for specific occupations and sectors. In order to keep the analysis manageably simple (after all, our intention here is just to check the plausibility of the scores), we will use one-digit rather than two-digit sectors and occupations. Table 3.VI shows the results for the amenities index, the four dimensions and the nine indicators for all one-digit sectors and one-digit occupations. The sectors and occupations have been ranked according to their value in the overall index (shown in the first column on the left): the absolute value of the index ranges from .752 (financial intermediation) to .598 (hotels and restaurants) in NACE, and from .725 (professionals) to .572 (machine operators) in ISCO.

The actual range of variation of the absolute scores of the index across sectors and occupations may seem small (around 15\% of the total possible variation, which would go from 0 to 1 ), but this was to be expected considering that we are comparing the average values for 1-digit sectors and occupations. The 9 broadly defined sectors and occupations shown in table 3.VI put together people with very different working conditions, which obviously reduces the variability of the means. If we use the full jobs matrix, and look at the higher and lower scores for individual jobs, the range of variability is expanded to $33 \%$ : from a value of .48 for labourers in manufacturing of non-metallic mineral products to .81 for corporate managers in computer and related activities (discounting jobs with less than 15 people).

But in any case, the absolute values of the index are secondary to us (and can always be contested, being based on ultimately arbitrary decisions with respect of the coding of the original variables). The important thing here is the relative position of each sector and occupation in terms of amenities: the rankings. And the ranking of sectors and occupations according to their "non-pecuniary job quality" seems quite reasonable according to our previous knowledge of the distribution of working conditions (see Parent-Thirion et al. 2007). As expected, the ranking of ISCO codes matches quite closely the occupational levels of this classification, with some minor but meaningful deviations: the top of the amenities ranking is occupied by professionals rather than managers (which seems plausible considering that this index leaves out remuneration) and the bottom is occupied by semi-skilled industrial workers rather than elementary occupations (as we will see, the latter have some advantages in terms of health and safety and work-life balance over the former). The extremes of the amenities ranking seem also quite plausible in terms of one-digit sectors, with financial services, education 
and real state at the top and hotels and restaurants, agriculture and fishing and construction at the bottom.

Looking at the components (dimensions) and sub-components (indicators) of the index, we can see that their scores tend to go together (which was to be expected after the pattern of correlations we discussed earlier). A semi-graphical representation has been added to table 3.VI to simplify its interpretation: superimposed on each cell of the table, a bar represents graphically each score. Each column in the table (corresponding to a specific component of the index) can be read as a bar chart: all bars within such column (vertically) share the same scale, so that the values of the different cells of the same column can be compared. The scales of the bars in different columns are different, so that they cannot be compared. This graphical representation shows clearly that, as we were saying, the values of the components tend to follow the values of the overall index (albeit with different ranges): but there are some exceptions which are very important, because they should reflect the idiosyncrasies of each sector and occupation, and therefore inspecting them is potentially a good way to check the results of the amenities index. All these exceptions look, in our view, more than plausible. Let us discuss with some detail three examples:

1. The health sector: overall, this sector occupies a middling position in terms of the amenities index, which may seem surprising considering the usually good conditions of employment that this sector enjoys in most countries. In fact, if we look at the detail (the dimensions which are behind the index), we can see that this sector has a rather good position in terms of employment conditions (where it is second in the ranking, only behind finance) and intrinsic work quality (where it is fifth), but a very low position (the $11^{\text {th }}$, the third lowest score) in terms of health and safety and a relatively low position (the $9^{\text {th }}$ ) in terms of work-life balance. We can get an even more detailed view if we go to the level of indicators: actually, the low value of health and safety in the health sector is largely the result of the lowest by far value in terms of psychosocial risks (the score in perceived impact of work on health is also relatively low, probably resulting from the high exposure to psychosocial risks as well). All this seems quite plausible and consistent with previous research on working conditions in the health sector (see Di Martino 2003). 
2. The manufacturing sector is quite low in the overall score of the amenities index (the fourth lowest). This is largely the result of having very low (the lowest of all sectors) values in intrinsic work quality (both objective and subjective, looking at the indicator level), which contrasts with its relatively high position in terms of employment conditions (especially contractual status), and even health. The indicators of health are quite interesting for this sector: although manufacturing has one of the lowest scores in terms of exposure to physical risks (that is, it suffers from high exposure of this type of risks), the overall perception of impact of health on work is not very high (which may reflect that despite the high exposure, this sector enjoys relatively high levels of protection and prevention measures compared to other sectors).

3. Elementary occupations (to use an example from ISCO as well) has the third lowest score in ISCO despite the fact that it occupies the lowest position in the hierarchy which is implicit in the ISCO classification. The dimension level shows that this derives from the compensation between quite different results in the different areas: elementary occupations have by far the lowest scores in terms of employment conditions, the second lowest value in terms of intrinsic work quality, but relatively high values in health and safety and work-life balance. In general, this seems to reflect the fact that these type of occupations often involve bad employment conditions and dead-end jobs, but low levels of commitment, intensity and even hours of work. For instance, if we compare this group with skilled and semi-skilled industrial workers we can see that the latter face considerably higher risks and are required considerably higher levels of intensity and work effort.

We can conclude, therefore, that the scores of the index (and derived rankings) and its internal structure reflect reasonably well the idea of amenities which is behind the model shown in figure 3.1. It seems like an adequate base for an alternative ranking of jobs by their quality, to complement the other measures based on wages and educational levels.

Before moving on, we would like to do a final check of the amenities index. An implicit assumption of this approach is that the position of a worker in the occupation-by-sector matrix is an important determinant of the quality of her job. Because we do not have an absolute measure of wages nor data at the individual level (necessary to test within vs. 
across jobs variance in wage or educational levels), we cannot test the validity of such assumption for the wages or educational indices. But with all the already mentioned limitations of the EWCS sample, we can do an approximate test for the amenities index. How much of the overall variation in the amenities index can be explained by differences in the average values across jobs, and how much remains within them? In table 3.VII.1, we can see the results of an Analysis of Variance (ANOVA) contrasting the total variance of the amenities index (at the individual level) with the variance that exists between the averages by job (remember that jobs holding less than 15 workers have been eliminated from the sample). The differences at the job level account for slightly less than one fourth of the total individual-level variance of the amenities index (the adjusted $\mathrm{r} 2$ has a value of .23). Although this value is obviously not exceptionally high, in our view it is high enough to justify the use of the jobs approach in this case. Considering the amount of statistical noise that exists in any cross-national survey such as the EWCS, considering the problems derived of having such a small sample, and considering the high degree of subjectivity (individual endogeneity) involved in many of the variables used for constructing the amenities index, an $\mathrm{r} 2$ of .23 is by no means disappointing. On the contrary, it empirically proves that the position of the worker in the jobs matrix has important implications for the non-pecuniary quality of her job (although we cannot test it, our hypothesis would be that the $\mathrm{r} 2$ would be even higher in a similar ANOVA for the wages variable).

Tables 3.VII.1-3.VII.7 show the results of an ANOVA for each of the four dimensions separately. The highest value (around .2) is for intrinsic work quality and the lowest (less than .08) for work-life balance. It is interesting to see that the $r 2$ for the dimensions are lower than for the overall index: this implies that the aggregation of the dimensions in a single index intensifies the explanatory power of the jobs matrix. In other words, not only the different dimensions of job quality, but the correlation between them is associated with the position one occupies within the jobs matrix.

In tables 3.VII.6 and 3.VII.7 we can finally see as well an ANOVA table for country only, and for the jobs matrix (NACExISCO) and country simultaneously. This confirms that, as we assumed earlier, the combined effect of sector and occupation is considerably more important as a determinant of job amenities than the country where each job is located. The $\mathrm{r} 2$ of the NACE and ISCO combined is two times and a half larger than that of country (.23 against .9$)$, and adding country to the model with NACE 

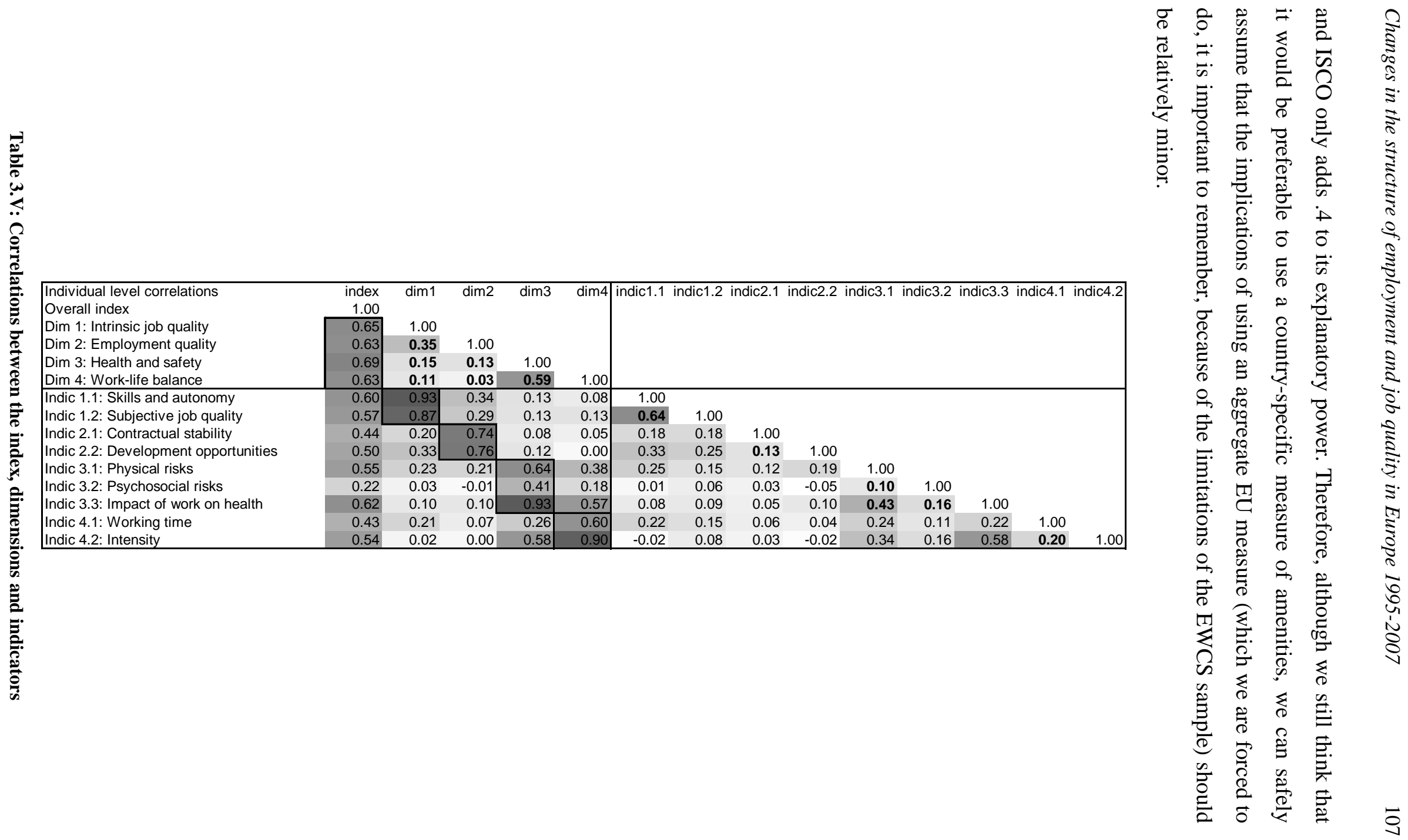


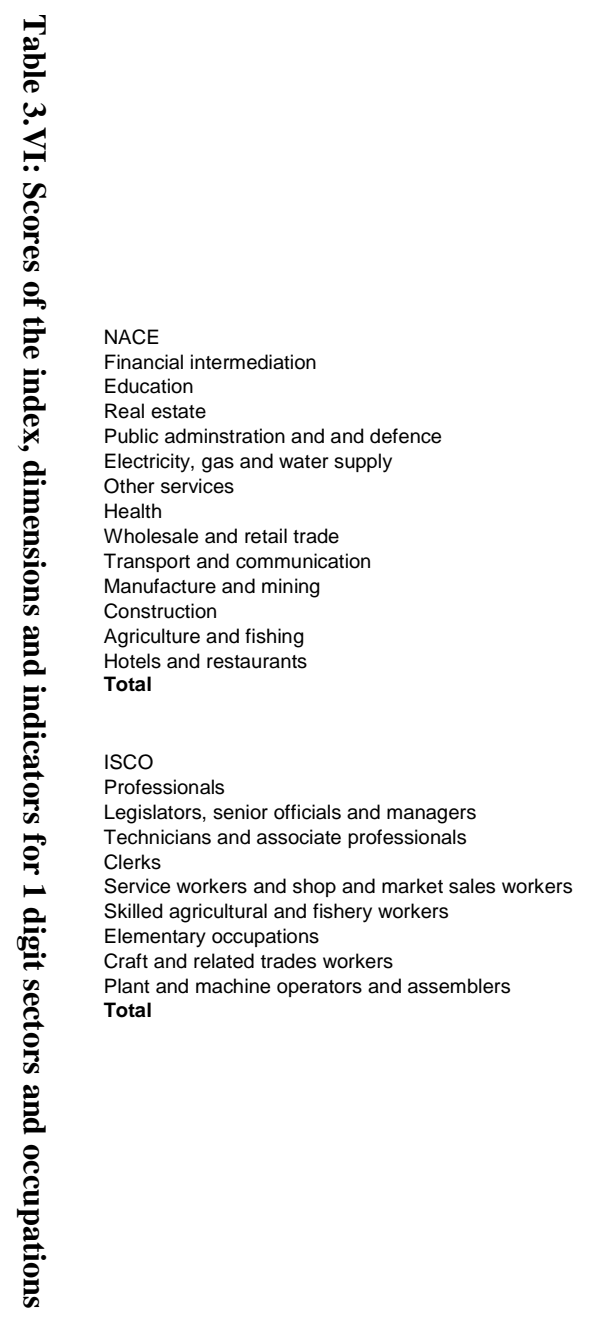

Amenities $\quad$ Dim 1: $\quad$ Dim 2: $\quad$ Dim 3: $\quad$ Dim 4: $\quad$ Indic 1.1: $\quad$ Indic 1.2: $\quad$ Indic 2.1: $\quad$ Indic 2.2: $\quad$ Indic 3.1: $\quad$ Indic 3.2: $\quad$ Indic 3.3: $\quad$ Indic 4.1: Indic 4.2: index Intrinsic Employm. Health WLB Skills Aut. Subj. Contract Dvpt. Phys. Risk Psyc Risk Perc health Worktime Intens. N

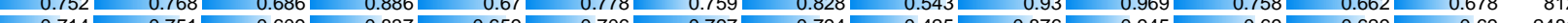

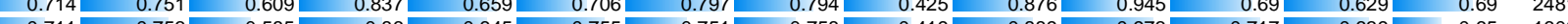

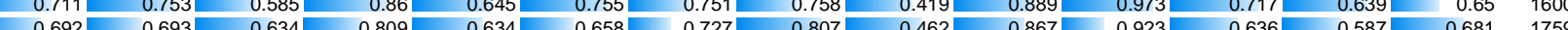

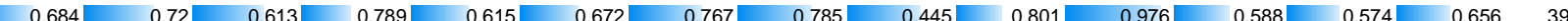

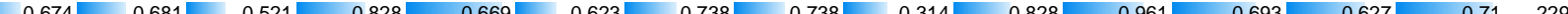

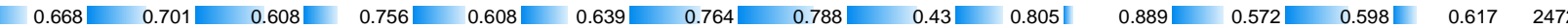

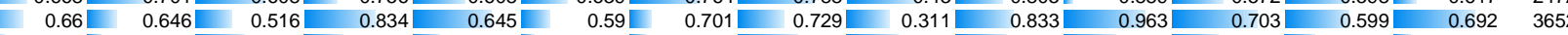

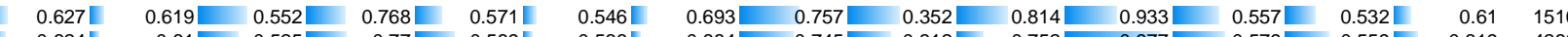
\begin{tabular}{|lllllllllllllll}
0.624 & 0.61 & 0.525 & 0.77 & $0.589 \mid$ & $0.536 \mid$ & 0.684 & 0.745 & 0.312 & 0.752 & 0.977 & 0.579 & 0.559 & 0.619 & 4282 \\
\hline
\end{tabular}

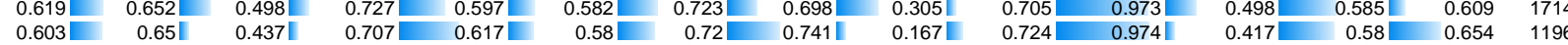
\begin{tabular}{llllllllllllll|l}
0.603 & 0.65 & 0.437 & 0.707 & 0.67 & 0.58 & 0.72 & 0.741 & 0.167 & 0.724 & 0.974 & 0.417 & 0.58 & 0.654 & 1196
\end{tabular}

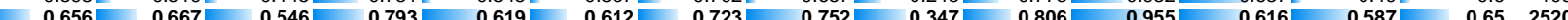

Amenities $\quad \operatorname{Dim} 1: \quad \operatorname{Dim} 2: \quad \operatorname{Dim} 3: \quad \operatorname{Dim} 4: \quad$ Indic 1.1: $\quad$ Indic 1.2: $\quad$ Indic 2.1: $\quad$ Indic 2.2: $\quad$ Indic 3.1: $\quad$ Indic 3.2: Indic 3.3: Indic 4.1: Indic 4.2: Health WLB Skills Aut. Subj. Contract Dvpt. Phys. Risk Psyc Risk Perc health Worktime Intens. N \begin{tabular}{|l|l|l|l|l|l|l|l|l|l|l|l|l|l|l|}
\hline 0.725 & 0.778 & 0.642 & 0.84 & 0.64 & 0.767 & 0.789 & 0.802 & 0.485 & 0.883 & 0.951 & 0.685 & 0.626 & 0.655 & 3485 \\
\hline
\end{tabular}

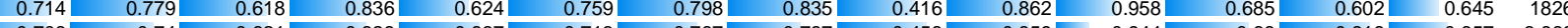

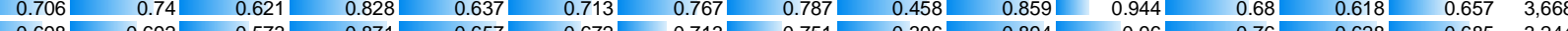

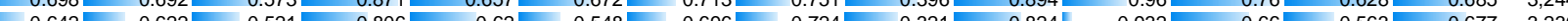

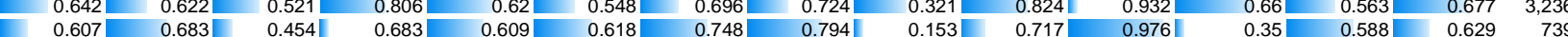

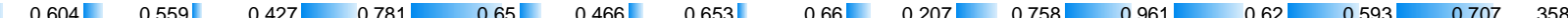
\begin{tabular}{|c|c|c|c|c|c|c|c|c|c|c|c|c|c|c|c|}
\hline 0.601 & 0.619 & 0.494 & 0.713 & 0.579 & 0.531 & 0.706 & 0.718 & $0.279 \mid$ & 0.68 & 0.976 & 0.476 & 0.563 & 0.596 & 3.543 \\
\hline
\end{tabular}

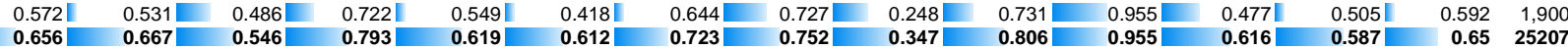




\begin{tabular}{|c|c|c|c|c|c|}
\hline \multirow[b]{2}{*}{ Source } & $\begin{array}{l}\text { Number of ob } \\
\text { Root MSE }\end{array}$ & & $s=23305$ & $\begin{array}{l}\text { R-squared } \\
\text { Adj R-squared }\end{array}$ & $\begin{array}{l}=0.2371 \\
=0.2257\end{array}$ \\
\hline & Partial SS & $d f$ & MS & $\mathrm{F}$ & Prob $>F$ \\
\hline Model & 94.4536267 & 344 & .274574496 & 20.75 & 0.0000 \\
\hline nace_rec*isco & 94.4536267 & 344 & .274574496 & 20.75 & 0.0000 \\
\hline Residual & 303.87874 & 22960 & .013235137 & & \\
\hline Total & 398.332367 & 23304 & .017092875 & & \\
\hline
\end{tabular}

Table 3.VII.1: Analysis of Variance of the overall amenities index by NACE and ISCO combined

\begin{tabular}{|c|c|c|c|c|c|}
\hline & $\begin{array}{l}\text { Number of ob } \\
\text { Root MSE }\end{array}$ & $\begin{aligned} S & = \\
& =\end{aligned}$ & $\begin{array}{l}3301 \\
9259\end{array}$ & $\begin{array}{l}\text { R-squared } \\
\text { Adj R-squared }\end{array}$ & $\begin{array}{l}=0.2062 \\
=0.1943\end{array}$ \\
\hline Source & Partial SS & $d f$ & MS & $\mathrm{F}$ & Prob $>F$ \\
\hline Model & 221.214338 & 344 & .64306493 & 17.34 & 0.0000 \\
\hline nace_rec*isco & 221.214338 & 344 & .64306493 & 17.34 & 0.0000 \\
\hline Residual & 851.458561 & 22956 & .03709089 & & \\
\hline Total & 1072.6729 & 23300 & .046037463 & & \\
\hline
\end{tabular}

Table 3.VII.2: Analysis of Variance of dimension 1 by NACE and ISCO combined

\begin{tabular}{|c|c|c|c|c|c|}
\hline \multirow[b]{2}{*}{ Source } & $\begin{array}{l}\text { Number of obs } \\
\text { Root MSE }\end{array}$ & \multicolumn{2}{|c|}{$\begin{aligned} S & =23297 \\
& =.209059\end{aligned}$} & R-squared & $\begin{array}{l}=0.1626 \\
=0.1501\end{array}$ \\
\hline & Partial SS & $d f$ & MS & $\mathrm{F}$ & Prob $>F$ \\
\hline Model & 194.816746 & 344 & .56632775 & 12.96 & 0.0000 \\
\hline nace_rec*isco & 194.816746 & 344 & .56632775 & 12.96 & 0.0000 \\
\hline Residual & 1003.13431 & 22952 & .04370574 & & \\
\hline Total & 1197.95106 & 23296 & .05142303 & 37 & \\
\hline
\end{tabular}

Table 3.VII.3: Analysis of Variance of dimension 2 by NACE and ISCO combined

\begin{tabular}{|c|c|c|c|c|c|c|}
\hline & $\begin{array}{l}\text { Number of ob } \\
\text { Root MSE }\end{array}$ & $\begin{aligned} s & = \\
& =.1\end{aligned}$ & $\begin{array}{l}3305 \\
3578\end{array}$ & $\begin{array}{l}R-s \\
\text { Adj }\end{array}$ & $\begin{array}{l}\text { quared } \\
\text { R-squared }\end{array}$ & $\begin{array}{l}=0.1583 \\
=0.1457\end{array}$ \\
\hline Source & Partial SS & $d f$ & MS & & F & Prob $>F$ \\
\hline Model & 130.08532 & 344 & .37815 & & 12.55 & 0.0000 \\
\hline nace_rec*isco & 130.08532 & 344 & .37815 & & 12.55 & 0.0000 \\
\hline Residual & 691.765675 & 22960 & .03012916 & & & \\
\hline Total & 821.850995 & 23304 & .03526652 & & & \\
\hline
\end{tabular}

Table 3.VII.4: Analysis of Variance of dimension 3 by NACE and ISCO combined

\begin{tabular}{|c|c|c|c|c|c|}
\hline & $\begin{array}{l}\text { Number of obs } \\
\text { Root MSE }\end{array}$ & $\begin{array}{l}= \\
=.\end{array}$ & $\begin{array}{l}3305 \\
1715\end{array}$ & $\begin{array}{l}\text { R-squared } \\
\text { Adj R-squared }\end{array}$ & $\begin{array}{l}=0.0888 \\
=0.0752\end{array}$ \\
\hline Source & Partial SS & $d f$ & MS & $\mathrm{F}$ & Prob $>F$ \\
\hline Model & 73.8933502 & 344 & .21480625 & 6.51 & 0.0000 \\
\hline nace_rec*isco & 73.8933502 & 344 & .21480625 & 6.51 & 0.0000 \\
\hline
\end{tabular}




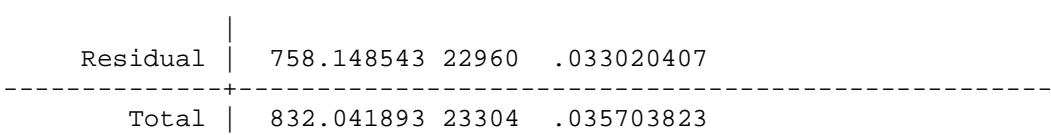

Table 3.VII.5: Analysis of Variance of dimension 4 by NACE and ISCO combined

\begin{tabular}{|c|c|c|c|c|c|}
\hline & $\begin{array}{l}\text { Number of ob } \\
\text { Root MSE }\end{array}$ & $\begin{aligned} s & = \\
& =.\end{aligned}$ & $\begin{array}{l}3305 \\
4959\end{array}$ & $\begin{array}{l}\text { R-squared } \\
\text { Adj R-squared }\end{array}$ & $\begin{array}{l}=0.0875 \\
=0.0865\end{array}$ \\
\hline Source & Partial SS & $d f$ & MS & $\mathrm{F}$ & Prob > F \\
\hline Model & 34.8516268 & 26 & 1.34044719 & 85.84 & 0.0000 \\
\hline country & 34.8516268 & 26 & 1.34044719 & 85.84 & 0.0000 \\
\hline Residual & 363.48074 & 23278 & .015614775 & & \\
\hline Total & 398.332367 & 23304 & .017092875 & & \\
\hline
\end{tabular}

Table 3.VII.6: Analysis of Variance of the overall amenities index by country

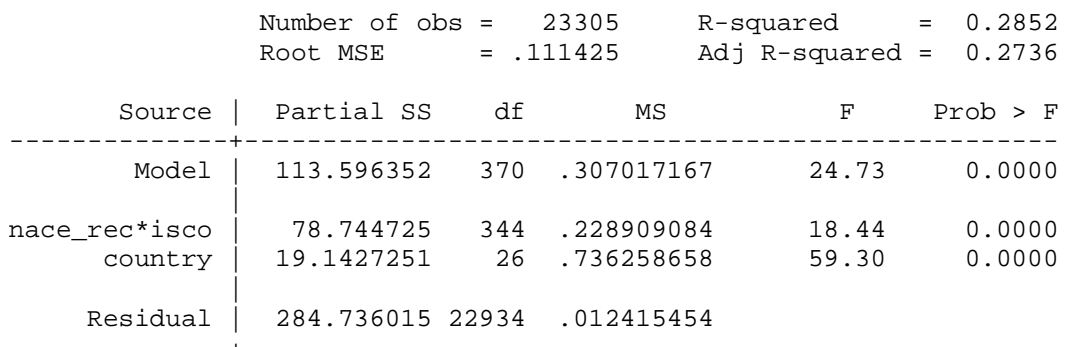

Table 3.VII.7: Analysis of Variance of the overall amenities index by NACE and ISCO combined, and country

\subsection{The correlation between the three job quality rankings}

So then we have three different measures of job quality for each of the cells (jobs) in the jobs matrix. The three are measures are of an ordinal nature: they simply identify the position of each job against the others in terms of each of the job quality dimensions. The logic behind each of these three measures is different, as well as the sources used for their construction.

The first measure, the educational ranking, is the most indirectly related to job quality: we can consider it a proxy of job quality either because it is a correlate of the relative productivity of each of the jobs in the matrix (as explained in section 3 of the theoretical chapter) or simply because workers with a higher level of education have better chances of getting better jobs. It can even be considered a correlate of the skill requirements of jobs, which is in itself an element of job quality as explained in section 4 of the theoretical chapter. This educational ranking was constructed using the same source as the (quantitative) jobs matrix itself, the European Labour Force Survey. 
The second measure is based on labour income, itself an important element of job quality for most theoretical approaches to this issue. The traditional economic approach (the theory of compensating differentials) argues that wages are a compensating mechanism for the disutility of work: job quality would be determined by the specific bundle of pecuniary (wage) and non-pecuniary (disamenities) elements that each job involves. A more sociological approach would consider wages as just one of several elements of job quality, within a more multidimensional concept. Still, in sociology of work it is often argued that wages, rather than compensate for bad working conditions, tend to correlate with good working conditions: that is, better jobs (in a non-pecuniary sense) tend to be also better paid jobs. From this perspective, wages would not only be an important element of job quality, but potentially a good proxy for overall job quality in the absence of a more encompassing measure. This wage ranking was constructed by combining the information of several different data sources, as explained earlier.

The third measure, the amenities ranking, is arguably the most direct and encompassing job quality measure of the three, even though it does not include any pecuniary element (which was purposely excluded, to avoid redundancies with the wage ranking). From a traditional economic approach, the amenities (or disamenities) of a job are the second main determinant of job quality, together with wages. From a sociological approach, this amenities ranking is (at least conceptually) the most complete of our three measures of job quality, which in this approach is assumed to be intrinsically multidimensional (although to be really complete, it should include one more dimension for wages). This amenities ranking was constructed using the Fourth European Working Conditions Survey.

Behind the construction (and subsequent use) of these three different job quality rankings there is an obvious assumption: that the three are measuring the same thing (job quality), but each of them from a different angle. Can we test empirically whether this is true? To some extent we can: even though it is impossible to test directly whether the three variables are really measuring job quality (we do not have any direct measure of job quality, otherwise there would be no need to complicate things so much), we can test whether these three measures are correlated at the job level, and in which ways. What kind of pattern of correlations would corroborate our assumptions? On the one hand, we must expect some degree of correlation between the three rankings, if they are essentially different measures of the same thing. If the theory of compensating 
differentials applies to across-jobs differentials, the correlation between wages and amenities may in fact be negative: but still, there would be correlation between them. If the theory of compensating differentials does not apply at the job level and wages and amenities tend to go together, they should correlate positively. But it would be very surprising, and would make the rankings quite suspicious, if we would find no correlation between them. The fact that the three rankings are constructed using totally different sources and with different underlying logics makes this test of correlations quite powerful, because there is no reason to expect any spurious correlation (if we do find correlation, it must be reflecting something substantial). But at the same time, we should not expect a very high degree of correlation: the three rankings should be measuring the same thing but from a different angle. Each of the rankings should capture something which the other two rankings do not: it is for this reason that using the three rankings simultaneously should give a more comprehensive picture than looking only at any one of them.

Before looking at table 3.VIII, which shows the correlations between the three rankings (including the four components of the amenities ranking), we should mention a few potential limitations of the analysis. First, it is important to keep in mind that the education and wage rankings are specific for each country (in fact, the European rankings for those two dimensions are just an aggregation of the national results), whereas the amenities ranking was constructed at the European level only (for the reasons explained in the previous section). Therefore, when we look at the correlation between the wage and educational ranking, we will be comparing two variables which are country-specific, but when we look at the correlation between education and amenities (or wage and amenities), we will be comparing, in fact, a national ranking of jobs against a European ranking. A second important thing to keep in mind is that the number of jobs covered by the wage and educational rankings is larger than the number of jobs covered by the amenities ranking. Although in terms of employment, the difference is relatively small (the number of jobs for which we do not have an amenities ranking account for less than $10 \%$ of overall employment, as explained in the previous section), it can have a significant impact in the results which is impossible to evaluate. This means that the results will only be indicative, and that they have to be taken with care. 
A further important caveat of this section refers to the statistical techniques used for carrying out the analysis. As we have repeatedly said in the previous pages, there are several problems in comparing the classification of employment by sector and occupation across different countries, so our overall strategy throughout this thesis is to try to use as much as possible non-parametric methods of analysis (which are not so sensitive to classification problems or biases in the estimations), and to keep the level of analysis relatively aggregated (not paying too much attention to the little detail, but concentrating on broad patterns). In this section, we will need to break our own rules in order to test the correlation patterns between the detailed rankings. In a previous similar analysis (see Stehrer, Ward and Fernández-Macías 2008), we used Spearman rank correlations to compare the three rankings: in principle, this non-parametric statistic seems the most adequate for our purposes. But this statistic does not allow the application of unequal weights to the jobs, and this can lead to misleading results. As we explained earlier, the jobs are very uneven in terms of their employment size: some jobs account for a rather large share of employment, whereas many others account for very little. If we just compare the rankings across jobs, a large part of the correlation score will derive from the comparison of many tiny jobs which account for a very small amount of employment: those jobs are not only unimportant for the overall results we will analyse later, but in fact they are much more likely to be wrong in one way or another - small jobs are much more likely to suffer from coding problems with respect to ISCO and NACE (the comparison is more shaky) and the estimation of education, wage and amenities is more imprecise (because it is based on smaller samples) -. To solve these problems, we want to compare jobs, but weighted by their employment size. This cannot be done with Spearman rank correlations: we will need to use the Pearson product-moment correlation instead, a parametric technique which allows weights. In practice, we checked the difference between the (unweighted) correlation matrices using Spearman and Pearson, and it is negligible - it does not change at all the overall patterns - . Therefore, we believe it should not be a big problem to use this statistic even if it is parametric (for comparison purposes, the Spearman rank correlations between the wage and educational rankings can be consulted in Stehrer, Ward and Fernández-Macías 2009).

A final caveat concerns the fact that here we will use the raw rankings (only standardized so that they range from 0 to 1 -that is, divided by their maximum value in 
each country-). The full rankings provide much more detail than the quintiles (which aggregate the full continuous rank order into just five values), and therefore provide much richer information for an analysis of the correlations between the three dimensions of job quality, which is our objective here. But we must keep in mind, again, that there is a certain degree of imprecision in the specific values of the rankings as well as in the classification of workers into jobs, which is the reason why we will use the quintiles rather than the rankings for the rest of this thesis. For this section, the gains in terms of richness and depth compensate for the higher risk of errors at the individual job level, and for this reason we will use the raw rankings rather than the quintiles. After all, this is the only section in this thesis (together with section 2 in the next chapter, which also uses the raw rankings) in which the rankings themselves are the main object of analysis, rather than a secondary element used for evaluating the nature of job creation.

\section{4.a) The correlation between the three rankings within each country}

Table 3.VIII shows the Pearson product-moment correlations between the three job quality rankings, including also the four components of the amenities ranking. Jobs have been weighted by their employment shares, as mentioned earlier (so that the importance of each job in the correlation coefficient is relative to its size with respect to total employment in each country). As in previous tables, the coefficients in the table have been shaded to facilitate their interpretation (with a grey gradient). The first 6 columns show the correlations between the educational ranking and: the wage ranking (column 1), the amenities ranking (column 2), and the four dimensions of the amenities ranking (columns 2.a-2.c). The next five columns show the correlations between the wage ranking and the amenities ranking (column 3) and its four dimensions (columns 3.a3.d). As mentioned earlier, the number of observations on which each coefficient is not the same for the different columns of the table: the correlation between education and wage uses nearly all jobs available in each country (see table 3.III), whereas all the rest only use the jobs for which the amenities ranking is available (as explained in section 4.3). 


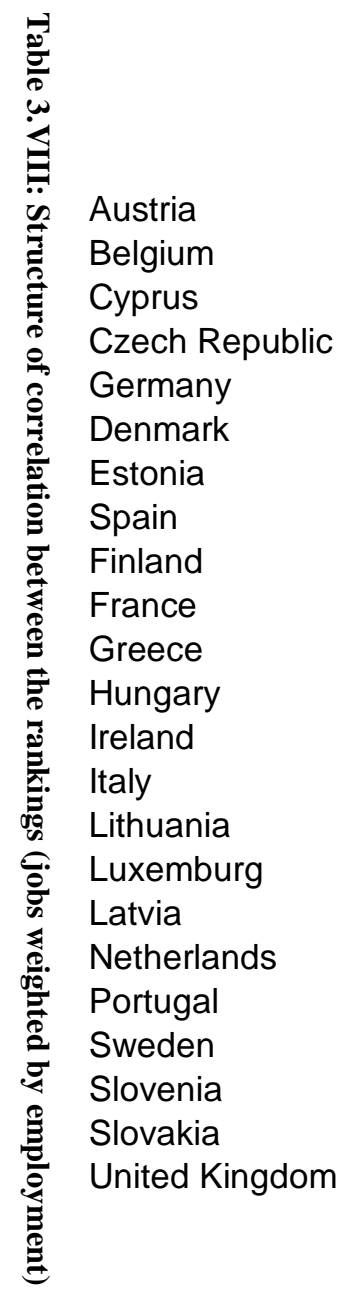

\begin{tabular}{|c|c|c|c|c|c|c|c|c|c|c|}
\hline \multicolumn{6}{|c|}{ Correlation between educational ranking and... } & \multicolumn{5}{|c|}{ Correlation between wage ranking and... } \\
\hline 1. Wages & 2. Amenities & 2.a) Dim1 & 2.b) Dim2 & 2.c) Dim3 & 2.d) Dim4 & 3. Amenities & 3.a) Dim1 & 3.b) Dim2 & 3.c) Dim3 & 3.d) Dim4 \\
\hline 0.73 & 0.72 & 0.76 & 0.82 & 0.41 & 0.18 & 0.64 & 0.76 & 0.66 & 0.26 & 0.24 \\
\hline 0.69 & 0.81 & 0.83 & 0.81 & 0.53 & 0.30 & 0.62 & 0.69 & 0.73 & 0.26 & 0.14 \\
\hline 0.58 & 0.83 & 0.77 & 0.83 & 0.62 & 0.32 & 0.50 & 0.63 & 0.64 & 0.03 & 0.02 \\
\hline 0.82 & 0.81 & 0.85 & 0.82 & 0.53 & 0.35 & 0.62 & 0.71 & 0.76 & 0.29 & 0.16 \\
\hline 0.85 & 0.72 & 0.79 & 0.78 & 0.37 & 0.23 & 0.69 & 0.72 & 0.78 & 0.38 & 0.22 \\
\hline 0.70 & 0.74 & 0.81 & 0.82 & 0.39 & 0.19 & 0.54 & 0.61 & 0.67 & 0.22 & 0.05 \\
\hline 0.63 & 0.83 & 0.79 & 0.80 & 0.66 & 0.38 & 0.56 & 0.65 & 0.70 & 0.27 & 0.01 \\
\hline 0.71 & 0.82 & 0.74 & 0.83 & 0.62 & 0.31 & 0.67 & 0.75 & 0.78 & 0.31 & 0.11 \\
\hline 0.69 & 0.76 & 0.77 & 0.82 & 0.48 & 0.26 & 0.58 & 0.66 & 0.73 & 0.32 & 0.04 \\
\hline 0.74 & 0.76 & 0.80 & 0.79 & 0.47 & 0.23 & 0.54 & 0.61 & 0.73 & 0.20 & -0.02 \\
\hline 0.51 & 0.79 & 0.55 & 0.84 & 0.72 & 0.35 & 0.43 & 0.32 & 0.68 & 0.32 & 0.00 \\
\hline 0.73 & 0.81 & 0.83 & 0.82 & 0.55 & 0.33 & 0.70 & 0.72 & 0.81 & 0.40 & 0.27 \\
\hline 0.68 & 0.73 & 0.74 & 0.80 & 0.50 & 0.16 & 0.54 & 0.65 & 0.70 & 0.12 & 0.04 \\
\hline 0.87 & 0.84 & 0.80 & 0.84 & 0.64 & 0.35 & 0.75 & 0.75 & 0.83 & 0.44 & 0.26 \\
\hline 0.77 & 0.76 & 0.59 & 0.84 & 0.66 & 0.17 & 0.67 & 0.53 & 0.82 & 0.50 & 0.11 \\
\hline 0.78 & 0.78 & 0.82 & 0.80 & 0.50 & 0.23 & 0.70 & 0.68 & 0.76 & 0.41 & 0.30 \\
\hline 0.57 & 0.84 & 0.75 & 0.84 & 0.66 & 0.26 & 0.50 & 0.47 & 0.69 & 0.24 & -0.06 \\
\hline 0.77 & 0.78 & 0.83 & 0.84 & 0.42 & 0.24 & 0.58 & 0.69 & 0.69 & 0.19 & 0.06 \\
\hline 0.80 & 0.83 & 0.66 & 0.82 & 0.68 & 0.35 & 0.76 & 0.70 & 0.83 & 0.47 & 0.26 \\
\hline 0.67 & 0.73 & 0.77 & 0.81 & 0.41 & 0.26 & 0.57 & 0.62 & 0.63 & 0.30 & 0.18 \\
\hline 0.72 & 0.81 & 0.74 & 0.86 & 0.61 & 0.37 & 0.72 & 0.79 & 0.68 & 0.36 & 0.40 \\
\hline 0.71 & 0.80 & 0.84 & 0.82 & 0.53 & 0.29 & 0.55 & 0.64 & 0.72 & 0.20 & 0.08 \\
\hline 0.83 & 0.69 & 0.79 & 0.78 & 0.28 & 0.12 & 0.54 & 0.67 & 0.73 & 0.13 & -0.07 \\
\hline
\end{tabular}


The first and most important conclusion we can derive from table 3.VIII is that the correlations are relatively high, but by no means the rankings are identical. The highest correlations for the three main rankings are those between education and amenities (which are never below .7, and in 11 cases are above .8), followed closely by the correlation between education and wages (with some rather high correlations, but some rather low as well, in 8 countries below .7). The lowest correlations are between the wage and amenities rankings, which never go above .8 and only in 5 cases above 7 . Squaring the coefficients of correlation we get the coefficient of determination, which has a more direct interpretation because it measures the extent of shared variance in the original rankings. This shared variability, therefore, would be between 50 and $75 \%$ for education and wages (except in Greece, Latvia and Lithuania, where it is below 50\%), between 50 and $75 \%$ for education and amenities (with no exceptions), and between 25 and $55 \%$ for wages and amenities.

In general terms, therefore, the correlations between the three main rankings fit our previous expectations. On the one hand, the three rankings are strongly associated despite their underlying logics and data sources: this can be taken as a proof that the three are to some extent measures of the same underlying phenomenon-of job quality - On the other hand, the correlations are low enough as to prove that each of the three measures is capturing a different aspect of that underlying phenomenon. Using the three rankings simultaneously, therefore, should provide a more comprehensive picture than using any one of them exclusively: each one of them ranks the jobs according to a different aspect of job quality, and has its own implications and interpretation.

The substantial implications of the correlations between the rankings are different for each column of table 3.VIII. Column 1, which shows the correlation between the position of each job in terms of the educational level of its workers, and the position of each job in terms of the median wage it pays, can be interpreted in terms of the match between education and wages at the job level and in positional terms. The countries with a better match are Italy, Germany and the UK, and the countries with a lowest match are Greece, Cyprus and Malta. Column 2, which includes the correlation between the position of each job by education and the position by amenities, shows that jobs fulfilled by workers with a higher levels of formal education tend to have better conditions of work and employment in non-pecuniary terms. The fact that the 
correlations are even slightly higher than between education and wages, and much more uniformly so, may suggest that higher educated workers put more emphasis in having nicer than better paid jobs, or that the educational level of jobs is in itself a proxy for an important dimension of amenities (the intrinsic quality of work; more on this later). Finally, column 3, which shows the correlation between the wages ranking and the amenities ranking, can be understood as a positional and job-based test of the theory of compensating differentials - with a negative outcome-. Even though the correlation between wages and amenities is significantly lower than the correlation between education and wages or education and amenities, it is still positive and relatively high, which implies that there is no compensation between these two aspects of job quality at the job level (we will discuss this issue more in detail in the following section).

We have also included the correlation between the educational and wage rankings with the rankings by each of the four components of amenities. The first dimension (a) measures the intrinsic quality of work (objective and subjective), the second (b) the quality of conditions of employment, the third (c) health and safety and the fourth (d) work-life balance. The educational ranking has a strikingly high correlation with the first and second dimensions of amenities (intrinsic work quality and employment conditions). Those with higher education seem to get jobs not only with better conditions of employment, but also more fulfilling and interesting. The correlation with dimension 3 is lower, but still relatively important, and rather low with the dimension measuring work-life balance. The wages ranking, on the other hand, has a rather high correlation with the dimension of employment conditions (considerably higher than with the overall amenities ranking), which seems logical considering that pay itself can be considered an element of the employment dimension (see Muñoz de Bustillo et al. 2009). The correlation between the wage ranking and intrinsic quality of work is much lower, although still of relevance (similar to its overall correlation with amenities), but very low with the third and fourth dimensions. Overall, the component of employment quality within amenities is the most linked with the other two rankings, the component of intrinsic quality of work is strongly linked to the educational ranking but not so much with the wage ranking, and the components of health and safety and (especially) worklife balance are relatively unrelated to the other two rankings. 


\section{4.b) A job-based, positional test of the theory of compensating differentials}

Earlier we argued that the interrelations between the three dimensions of job quality could be integrated within a single model using the theory of compensating differentials. Making a very rough summary, this theory argues that wages compensate for the unpleasantness of work, and that therefore the overall utility of work depends on the combination between pay and the non-pecuniary elements of work which have an impact on the well-being of the worker. The specific combination between wages and amenities depend on the preferences of the worker (and we may add, the nature of each job), but because both pay and amenities constitute costs for companies, the bundle to which a worker may aspire ultimately depends on productivity, which is a function of workers' capacities and skills.

How to translate this overall argument into a testable hypothesis regarding the correlation between the rankings at the job level is not clear. The problem is that the original argument of compensating differentials concerns the individual level, and we are dealing here with jobs. In fact, as we argued in the theoretical chapter, it could be argued that the logic of compensating differentials only applies within jobs: the original theory argues that the compensation mechanism applies to workers with a similar level of capacities and skills, because the bundle of wages and amenities is ultimately determined by productivity. Although here we speak about jobs (positions within firms involving a specific set of tasks and requiring a specific set of skills) rather than individual workers, we can consider that different jobs involve different skills and capacities, and certainly different productivities (even if there is variability in these issues within jobs as well, of course). Therefore, broadly speaking all workers within one job would have access to a similar bundle of wage and amenities: so if we compare the average values of wages and amenities across jobs, there is no reason why we should observe any compensation between the two (the compensation would take place within, not across jobs). The only possible reason why we could observe some compensation between wage and amenities across jobs would be if some jobs necessarily involve a specific type of working conditions, irrespective of each worker's preferences (for instance, a job in mining certainly involves rather tough conditions of work): in order to find people willing to do such "naturally unpleasant" jobs, employers would need to pay higher wages. Another example would be a "naturally pleasant" job, involving for instance a high level of creativity (such as arts) or public recognition: it 
seems perfectly plausible that there would be many people willing to do those jobs even for a low wage. This kind of mechanisms would involve some across-jobs compensating differentials. Whether such a thing exists or not is ultimately an empirical question that can be tested with our data. And our results show that there is no acrossjobs compensating differentials (or at least not significant enough as to lead to a negative correlation coefficient between wages and amenities). If compensating differentials do exist, they must occur within rather than across jobs. Across jobs, wages and amenities tend to go together rather than in opposite directions.

\begin{tabular}{|c|c|c|c|c|c|}
\hline & $\mathrm{n}$ & $\begin{array}{l}\text { Wage and } \\
\text { amenities }\end{array}$ & $\begin{array}{l}\text { Education and } \\
\text { wage }\end{array}$ & $\begin{array}{l}\text { Education and } \\
\text { amenities }\end{array}$ & $\begin{array}{l}\text { Education and } \\
\text { bundle }\end{array}$ \\
\hline Austria & 561 & 0.64 & 0.73 & 0.72 & 0.80 \\
\hline Belgium & 557 & 0.62 & 0.70 & 0.81 & 0.84 \\
\hline Cyprus & 440 & 0.50 & 0.59 & 0.83 & 0.81 \\
\hline Czech Republic & 567 & 0.62 & 0.83 & 0.81 & 0.91 \\
\hline Germany & 576 & 0.69 & 0.86 & 0.72 & 0.85 \\
\hline Denmark & 525 & 0.54 & 0.71 & 0.74 & 0.83 \\
\hline Estonia & 512 & 0.56 & 0.63 & 0.83 & 0.83 \\
\hline Spain & 561 & 0.67 & 0.71 & 0.82 & 0.83 \\
\hline Finland & 544 & 0.58 & 0.70 & 0.76 & 0.82 \\
\hline France & 563 & 0.54 & 0.75 & 0.76 & 0.86 \\
\hline Greece & 549 & 0.43 & 0.51 & 0.79 & 0.76 \\
\hline Hungary & 565 & 0.70 & 0.74 & 0.81 & 0.84 \\
\hline Ireland & 569 & 0.54 & 0.67 & 0.73 & 0.80 \\
\hline Italy & 566 & 0.75 & 0.87 & 0.84 & 0.92 \\
\hline Lithuania & 520 & 0.67 & 0.79 & 0.76 & 0.85 \\
\hline Luxemburg & 483 & 0.70 & 0.79 & 0.78 & 0.85 \\
\hline Latvia & 510 & 0.50 & 0.58 & 0.84 & 0.82 \\
\hline Netherlands & 559 & 0.58 & 0.78 & 0.78 & 0.88 \\
\hline Portugal & 560 & 0.76 & 0.81 & 0.83 & 0.87 \\
\hline Sweden & 545 & 0.57 & 0.67 & 0.73 & 0.79 \\
\hline Slovenia & 541 & 0.72 & 0.72 & 0.81 & 0.83 \\
\hline Slovakia & 549 & 0.55 & 0.72 & 0.80 & 0.87 \\
\hline United Kingdom & 572 & 0.54 & 0.84 & 0.69 & 0.87 \\
\hline
\end{tabular}

Table 3.IX: A job-based, positional test of the theory of compensating differentials

Table 3.IX tries to further test the within-jobs compensating differentials argument, by explicitly using the educational ranking as a proxy of the capacities and skills that each worker brings to work, which would determine the bundle of wages and amenities to which a worker can aspire. In this table, the correlations are limited to those jobs for which we have a ranking position for all three dimensions, in order to ensure that we are using the same sample. The first column shows the correlation between wages and amenities, which as we explained earlier, proves that the compensating wage differentials argument does not apply across jobs. The second and third columns show the correlation between education and wages, and education and amenities: as we 
explained in the previous section, the average educational level of job-holders seems to be the best predictor (or at least, the highest correlate) of the other two indicators of job quality (it correlates very highly with the other two, while the other two do not correlate very highly between them). This is in itself a suggestive finding, which could support the argument that the educational level of job-holders is a proxy for the main determinant of job quality (productivity) and therefore it could be a better measure than the other two, because although indirectly, it measures job quality fully, whereas the other two only measure a specific aspect of job quality.

To make an approximate test of such hypothesis, we constructed an indicator of the "wage and amenities bundle", by simply averaging the normalized ranking position of each job in these two dimensions. Making an average seems adequate in this case because it implies assuming perfect sustitubility between wages and amenities, which is precisely the compensating differentials argument ${ }^{49}$. If the educational level of workers within jobs was a good indicator for the capacities and skills of workers, and those capacities and skills were the main determinant of the bundle of wages and amenities available for workers in each job, the correlation between education and this "bundle" ranking should be higher than the correlation between education and any of the two components of the bundle, wages and amenities. And this seems to be the case. The correlation between the position of jobs in terms of the educational level of job-holders and the position of jobs in terms of the bundle of wages and amenities they enjoy is very high, and in almost all cases higher than between education and either wage or amenities separately (the values in the fourth column are higher than in the second and third column in 19 of the 23 countries). The correlation between this educational ranking and the combination between wages and amenities is, in fact, strikingly high: with the normalized educational ranking, we can predict the combination between wages and amenities with a accuracy of between 65 and $84 \%$ (with the exception of Greece, where the squared coefficient of correlation is $57 \%$ ).

\footnotetext{
${ }^{49}$ In fact, the compensating differentials argument implies sustitubility, but not necessarily perfect sustituibility. If the sustituibility is perfect, using a simple average is a good operationalization (reducing amenities and increasing wages in the same amount would imply no change in overall utility). If the sustituibility is not perfect, the components should be weighted differently. To our knowledge, there is no theory arguing for any value in the sustituibility between wages and amenities, so it seems adequate to weight them equally assuming perfect sustituibility.
} 


\section{Final data processing and analytic strategy}

In the two previous sections, we have explained how we constructed two aggregate datasets drawing from different sources: first, a dataset holding information on employment levels by job (occupations within sectors) for each country and year covered, as well as some demographic and employment variables about the people concerned (we called this dataset the jobs matrix); second, a dataset holding three different rankings of jobs (by wages, education and amenities; we can call this dataset the job quality matrix). These two matrices (one holding quantitative, the other qualitative information about the same jobs) are finally combined in a single dataset, which will allow us to study the evolution of employment numbers at the job level from a qualitative perspective. In this section, we will explain the final processing of this dataset and the broad analytical strategy that we will follow in the rest of the thesis. But first, as usual, we need to make some preliminary remarks, in this case concerning the target population of the analysis.

\subsection{The target population: should we include self-employment and part-time work?}

As we have repeatedly said, this thesis is ultimately an evaluation of the implications of the changing the division of labour from a job quality perspective. A less far-ranging formulation (but in fact a different way of saying the same thing) would be that this thesis is an evaluation of what kind of jobs were created in the employment expansion that most EU countries experienced between 1995 and 2007. In our view, both of these formulations (the theoretically- as much as the empirically-oriented) imply that the target population must be all workers, and that employment must be expressed in terms of number of workers rather than number of hours. In practical terms, this involves including not only employees (as is usual in this kind of research), but also the selfemployed and even employers (that is, anyone in employment); including not only fulltime workers (which is also usual in this type of research), but also part-timers; and finally, counting employment in terms of persons rather than in terms of working hours or full-time equivalents.

Why include self-employed (and employers) alongside employees? From a theoretical perspective, if our objective is to study the evolution of the division of labour, it is obvious that the self-employed should be also analised-they are part of the division of labour as much as employees: leaving them out is missing part of the story-. From a more practical, policy-oriented perspective, it would not make sense to exclude the self- 
employed from the analysis either: if the goal is to evaluate the kinds of jobs created in the last expansion, it is necessary to cover all employment in the analysis. For these reasons, the self-employed will be analysed together with employees, and the jobs matrix will not differentiate between them. But this does not mean that we will ignore the status of employment in the analysis: we will use this variable to break down the patterns of employment creation in a later stage, to analyse whether the status of employment made a difference in terms of the patterns of job growth.

The reasons for including part-time workers in the analysis are very similar to those just mentioned for the case of the self-employed. Part-timers participate in production, and are part of the overall division of labour, so excluding them means missing an important part of the story (especially in Europe in recent years, where there has been a substantial increase in part-time employment). A more difficult decision is whether, when analyzing the evolution of employment numbers, all jobs should count as one independently of their working hours, or they should be somehow weighted by the hours of work. From the perspective of the production process, it may be argued that what counts is the amount of labour input, and therefore, it would seem more adequate to use hours of work (or hours-weighted jobs) as the measure of employment. From the perspective of workers, the hours of work can be an important element of job quality, but they do not reflect a gradation of "degrees of employment": a job is a job (a contract involving a set of tasks and duties to be carried out for a specific number of hours in exchange for a salary). Considering that we want to evaluate the evolution of the division of labour from the perspective of job quality (that is, from the perspective of workers and their well-being rather than from the perspective of production and its efficiency), it makes much more sense to count each worker as one, independently of the number of hours they work. As in the case of the self-employed, even though parttime work will not be a defining element of the jobs matrix, we will study the specific patterns of job growth for full-time and part-time workers separately, to try to identify any systematic differences and explain them.

Summarizing: we will cover all workers; not only full-time employees, but also selfemployed and part-time workers. The figures of employment in each job for each year and country will be based on the number of people employed, without taking into account the number of hours worked. Both employment status and part-time status will be used in a second stage of the analysis as breakdown variables, to study whether they 
are related to systematic differences in the patterns of employment growth by job quality.

\subsection{Merging the quantitative and qualitative job matrices}

As we have seen in the previous pages, this project is based on the combined analysis of several different data sources. To be able to do such combination, we needed some common structure in the different datasets: this common structure was provided by the occupation-by-sector matrix. Therefore, once the two job matrices were compiled and cleaned, they could be merged, using the job as linking mechanism. The analysis could proceed thereafter, studying the evolution of employment numbers from the perspective of job quality as we will see later.

\section{2.a) The coherence of the job classifications across the different sources used}

This method of combining data from different sources can be very fruitful in terms of research, increasing substantially the richness and breadth of issues covered: but it is important to acknowledge that it implies the assumption that the classification of employment by occupation and sector at the two-digit levels is really comparable across different surveys. Is that a reasonable assumption? We believe it is, and we can defend it on two grounds: first, empirically, by trying to check the coherence of the ISCO and NACE classifications across the different surveys; second, on purely aprioristic considerations based on our knowledge of NACE and ISCO, and the surveys concerned. Unfortunately, we can only do a very limited and imperfect test of the coherence of the classification of employment by occupation and sector across the different surveys used in this project. A proper test of coherence would imply checking whether the same job (or the same worker) is equally classified across the different surveys, which is obviously impossible to do for us. The only thing we can do is to compare the employment shares within the jobs matrix across the different surveys and check whether they are more or less similar. But this has limitations so important that it is debatable whether it constitutes a proper test of coherence. At the most fundamental level, it is only a comparison at the surface level, so even if the employment structures by job would be roughly similar, the same jobs may be classified differently-and the other way around-. But the real problem is a practical one, and derives from the fact that it is impossible to differentiate the inconsistencies that derive from incorrect 
classification of workers in NACE and ISCO and the inconsistencies that derive from different non-response patterns across the different surveys.

In any case, the following table shows the correlation coefficients between the employment structures (share of employment by job) given by the ELFS, ESES, ECHP and EWCS (the first two lines of the table are taken from Stehrer and Ward 2006, the third line is new). We do not have a similar set of correlations between the ELFS and EU-SILC, which would complete the picture. The ESES is available for all 23 countries, and therefore, the correlations with the structure of employment according to the ELFS include the whole sample of countries. These correlations are the most problematic: although in most countries the correlation is above .75 , there are 8 countries where it is below 7 (highlighted in bold). The correlations between the structure of employment according to ECHP and ELFS (only available for the EU15) are above .7 in all cases, and above .8 in most. In the case of the EWCS, we have only checked the correlation at the EU-23 level, because as discussed earlier the small sample size does not allow to do meaningful comparisons at the country level. The correlation between the structure of employment according to the EWCS and ELFS at the EU-23 level is around 91\%.

$\begin{array}{lrrrrrrrrrrrrrrrrrrrrrrrr} & \text { EU-23 } & \text { AT } & \text { BE } & \text { DE } & \text { DK } & \text { ES } & \text { FI } & \text { FR } & \text { GR } & \text { IE } & \text { IT } & \text { LU } & \text { NL } & \text { PT } & \text { UK } & \text { BG } & \text { CZ } & \text { EE } & \text { CY } & \text { LV } & \text { LT } & \text { HU } & \text { SI } & \text { SK } \\ \text { ELFS/SES } & - & 0.8 & \mathbf{0 . 5 6} & 0.75 & 0.72 & \mathbf{0 . 5 3} & 0.72 & \mathbf{0 . 6 2} & 0.51 & \mathbf{0 . 6 8} & \mathbf{0 . 6} & 0.81 & 0.79 & \mathbf{0 . 6 3} & 0.82 & 0.75 & 0.8 & 0.92 & \mathbf{0 . 6 5} & 0.92 & 0.78 & 0.87 & 0.92 & 0.78 \\ \text { ELFS/ECHP } & - & 0.89 & 0.7 & 0.86 & 0.79 & 0.83 & 0.8 & 0.77 & 0.79 & 0.71 & 0.81 & 0.85 & 0.85 & 0.89 & 0.82 & - & - & - & - & - & - & - & - & - \\ \text { ELFS/EWCS } & 0.91 & - & - & - & - & - & - & - & - & - & - & - & - & - & - & - & - & - & - & - & - & - & - & -\end{array}$

Table 3.X: Correlation between job structures of the different surveys

Unfortunately, this exercise has too many problems to provide any conclusive answer to the question of whether the classification of jobs is really comparable across the different surveys. In most cases the correlation coefficients are sufficiently high as to suggest that the different sources are broadly coherent: but there are a few cases in which the correlation coefficients seem too low, especially when we compare the structures of employment by jobs given by the ESES and the ELFS. But by no means can these few cases be interpreted as conclusive evidence of a significantly different classification of employment by ISCO and NACE: as mentioned earlier, differences in non-response rates or even differences in the sampling design (remember that the ESES is atypical with respect to the other surveys, in the sense that its observational units are firms rather than workers) are probably distorting the comparison to the point of making it useless for our purposes. So we can only attempt a very weak conclusion for this exercise: first, for most countries and for most surveys the correlations are reasonably reassuring; second, that for a few (8) countries and one particular survey (the ESES) the 
correlations are below our expectations and may point to some classification problem; but (third), there are important methodological differences between ESES and ELFS that may be (more plausibly, in fact) behind the relatively lower correlations for those 8 countries.

On the other hand, there are strong aprioristic arguments supporting the assumption that the classifications are at least reasonably comparable across surveys. As we already argued in a previous section of this chapter, the classification systems of NACE and ISCO are arguably the most standardized, consolidated and tested economic statistics in Europe. They have been developed by knowledgeable statisticians backed by international agencies, which systematically follow and cross-check the results provided by the national statistical offices. At the national level, the figures of employment by NACE and ISCO are continuously published and scrutinized by the research community and even by the public in general. There is, for these reasons, a considerable pressure on national statistical agencies to ensure a consistent classification of employment by NACE and ISCO across their surveys. In fact, in most national statistical agencies there is a dedicated team of NACE and ISCO-coders, whose main task is to classify workers or economic activities in these systems across the different surveys carried out by national statistical agencies. In this sense, the fact that all but one of the data sources used in this project (all except the EWCS) were directly carried out by the same national statistical agencies is an important argument in favour of their coherence. In short, if there are economic classifications whose coherence can be trusted, they surely include NACE and ISCO among them.

Of course, there are qualifications to these aprioristic considerations. The most important one is that they apply more to NACE than to ISCO. The visibility and political importance of ISCO is considerably less than that of NACE, and as a consequence, there is also less external pressure (including from international agencies such as EUROSTAT) for coherence in the case of ISCO than in the case of NACE. This may be one of the reasons behind the fact that, even within the same data source (ELFS), we found a few clear inconsistencies in the coding of ISCO over time, but no apparent inconsistency in NACE. Also, as discussed in a previous section, there is some empirical evidence of inconsistencies in ISCO coding by national statistical agencies: although according to the literature these inconsistencies are minor as long as we stick to the two-digit level (Elias 1997: 11). 
Therefore, it seems reasonable to assume a fair amount of coherence in the classification of employment by NACE and ISCO across the different surveys used in this project, but it would be naïve to expect a perfect match. It seems more adequate, therefore, to use a fuzzy approach rather than a very precise parametric analysis at the job level. This is exactly the analytic strategy that we will use in this thesis: even though the analysis will be based on a very detailed breakdown of employment by occupation and sector, we will only analyse the data at a highly aggregated level, always using ordinal rather than cardinal measures of job quality, doing the analysis at the national level as much as possible (the coherence between sources is surely higher at the national than at the European level) and avoiding parametric analysis which is likely to be much less robust to misclassifications at the individual job level (we will attempt some pseudoparametrizations to simplify the interpretation, but in fact they retain the relative/ordinal and highly aggregated nature of the original analysis).

\section{2.b) The problem of missing cells in the amenities ranking}

A more mundane problem that emerges in the moment of linking the quantitative and qualitative job matrices is the existence of some cells for which we have no amenities ranking. As we explained in a previous sectoin, because of the limited size of the EWCS sample, we opted for eliminating all jobs for which the estimation of the amenities index was based on less than 15 cases. ${ }^{50}$ Table 3.XI shows the proportion of employment in jobs for which we do not have a ranking position in terms of amenities. Overall, these jobs account for more or less $9 \%$ of employment: only in 5 countries (all of them NMS) they account for more than 10\%. Whenever the analysis involves this ranking, these jobs will not be taken into account, although we will always try to check whether the missing jobs are biased in some way (for instance, when comparing the amenities and the wage rankings, we will check whether the missing jobs occupy a special position in terms of wages). Of course, when the amenities ranking is not applied, we will use the whole dataset, accounting for all employment.

\footnotetext{
${ }^{50}$ This was not a problem for the wages and educational rankings. In the case of the educational rankings, they are based on the ELFS, and therefore, the coverage of jobs is identical to that of the quantitative jobs matrix (also based on the ELFS). In the case of the wages ranking, the samples of the surveys used were considerably larger than that of the EWCS, and the jobs were slightly more aggregated (see section 4.1) because of the coding used in the original surveys, so the problem of small cells could be safely ignored.
} 


\begin{tabular}{rrrrrrrrrrrr}
$\mathrm{AT}$ & $\mathrm{BE}$ & $\mathrm{CY}$ & $\mathrm{CZ}$ & $\mathrm{DE}$ & $\mathrm{DK}$ & $\mathrm{EE}$ & $\mathrm{ES}$ & $\mathrm{FI}$ & $\mathrm{FR}$ & $\mathrm{GR}$ & HU \\
6.18 & 7.98 & 5.23 & 10.43 & 9.69 & 7.18 & 10.84 & 6.24 & 9.24 & 9.45 & 3.76 & 9.98 \\
\hline $\mathrm{IE}$ & $\mathrm{IT}$ & $\mathrm{LT}$ & $\mathrm{LU}$ & $\mathrm{LV}$ & $\mathrm{NL}$ & $\mathrm{PT}$ & $\mathrm{SE}$ & $\mathrm{SI}$ & $\mathrm{SK}$ & $\mathrm{UK}$ & Total \\
7.46 & 6.78 & 10.53 & 6.47 & 12.92 & 8.39 & 5.69 & 8.46 & 10.55 & 11.59 & 9.84 & 8.57
\end{tabular}

Table 3.XI: Share of employment in jobs with no amenities ranking, 2000

\subsection{The job quality quintiles}

The bulk of the analysis carried out in this thesis is based on what we call the "job quality quintiles". These quintiles are simply a reaggregation of the original jobs (within each country) into five groups, each holding $20 \%$ of employment in the year 2000, and ordered from low to high in terms of their quality according to one of the three rankings explained earlier. Even though we will sometimes use the information at the job level to decompose the patterns of job creation or do some more detailed analysis, most of our discussion will be based on the examination of the patterns of job growth within each country across those five "job quality quintiles" (simply, examining how much job creation went into each of the five quintiles).

It may seem surprising that, after taking all the trouble of constructing a very detailed dataset with information at the job level, and detailed rankings for three different job quality indices, we reaggregate all that information into five broad categories and then analyse the evolution of employment across those aggregate categories. But the fact that most of the analysis is done at the level of the quintiles does not mean that we are ignoring the more detailed information at the job level, or that such information is not used. The quintiles can be used to study the implications of the changing division of labour for job quality because they are constructed by aggregating information compiled at the job level, information on the quality character (the ranking) and on the changing numbers of employment in each job. The quintiles are just the final stage of the analytical process, a method of summarizing an amount of information that without such aggregation would be simply unmanageable. The quintile approach is simply a way of combining and studying simultaneously the quantitative and qualitative information that was earlier compiled at the job level: a way which is aggregated enough as to be able to study the evolution of 23 countries over a period of 12 years simultaneously, yet detailed enough as to answer the research questions discussed in the theoretical chapter (whether these 12 years were characterized by upgrading, degrading or polarization of the jobs structure). And in any case, doing most of the analysis at the quintile level does not prevent us from going back to the job level whenever it is useful for improve our understanding of the overall patterns. For instance, we will have to go 
back to the job level information to be able to decompose the patterns of job growth by sector, employment status or gender. In some cases, we will even inspect directly individual jobs to get a better grasp of what we see at the highest level of aggregation (for instance, by inspecting the jobs that account for a large share of job creation in each quintile).

The aggregation of jobs into quintiles does not only fit our purposes, but also the characteristics of our data. As we have discussed earlier, the combination of data from so many different sources involves a certain degree of error, something which must be taken into account when devising the analytical strategy. An approach which is relatively aggregated (looking at the broad picture rather than at the detail) and nonparametric (and therefore more robust to imprecisions in the data) is surely more adequate for this kind of data. Most previous studies in this tradition have used some type of quantile analysis: Stiglitz divided all jobs in two evenly-sized groups, the OECD used three, and Wright and Dwyer five, as we do here. Five quantiles seems a good choice because it is aggregate enough to be able to summarize the very large amount of information available (it only uses five parameters for each country and year) yet detailed enough as to reflect whether job growth was characterized by upgrading, degrading or polarization, and even how intense those patterns were.

The quintiles were constructed using the xtile instruction of the STATA software package, with some further tweaking to the allocation of jobs to each of the five groups to ensure that they end up reasonably even in the year 2000. The xtile instruction of STATA assigns jobs to quintiles in the following way: it constructs a cumulative distribution of employment by jobs, with the jobs being sorted by one of the job quality rankings; then, when the cumulative distribution reaches $20 \%$ of overall employment, it assigns all the jobs at or below that point to the first quintile, and so on. The problem is that if, for instance, the cumulative distribution up to the $77^{\text {th }}$ job (in terms of job quality) is $19.9 \%$ and the $78^{\text {th }}$ job accounts for $2.5 \%$ of overall employment, the xtile instruction of STATA will assign the $78^{\text {th }}$ job to the first quintile and therefore the first quintile will have $22.4 \%$ of employment. Because of the fact that the jobs themselves are unevenly sized, it is in most cases impossible to get a "quintile" which holds exactly $20 \%$ of employment: but nevertheless, in the previous example, it seems clear that the first quintile should hold only up to the $77^{\text {th }}$ job, and the $78^{\text {th }}$ job should go to the second quintile. This way, the first quintile would hold $19.9 \%$ of employment rather than 
$22.4 \%$. STATA cannot do that, but we prepared a correction algorithm which checks the allocation of the last job in each quintile, and if including this last job made the quintile more distant to the $20 \%$ target size than not including it, it moves it to the next quintile.

But why use 2000 as the base year to construct the quintiles? In principle, it would seem more adequate to use 1995 rather than 2000. After all, the quintiles serve as a kind of "counter" to study job creation by job quality levels: we want to set the counter to zero in the beginning of the period being studied, and then see how each of the initially equal groups of jobs grow throughout the years. As usual, the reasons behind the use of 2000 rather than 1995 to construct the quintiles are linked to limitations of the data. We only have data from 1995 for a few countries (13): for the rest of countries, the data available starts in 1997, 1998 or 1999. For homogenizing the analysis and make the national results more directly comparable, we wanted to use the same base years for all countries: therefore, the earliest possible year we could have used for constructing the quintiles was 1999 (the first year with data available for all countries). But once we moved away from the earliest available year, it does not make a big difference whether to use 1999 or 2000 , so we opted for the latter, which is more squarely in the middle of the period.

Does it make any difference which base year is used? If the base year would be the beginning of the period and the same for all countries, the interpretation would be more straightforward, because the first year would act as a tabula rasa against which the change in the number of people employed across the job quality spectrum would be compared. Using a year which is in the middle rather than at the beginning of the period means that the results are a bit less intuitive: before 2000, the change moves backward (the parameters of growth for each quintile reflect how far was each quintile from the evenly spread quintile allocation of 2000), and after 2000, forward (in the most intuitive way). ${ }^{51}$ But the results are perfectly meaningful, and reflect correctly the patterns of change in which we are interested. After all, the quintile allocation is just a way to group all jobs in five groups which mean "very bad jobs", "bad jobs", "middling jobs", "good jobs" and "very good jobs": whether this grouping is based on the number of people in the jobs in the beginning, middle or end of the period is only something to

\footnotetext{
${ }^{51}$ In fact, for simplifying further the analysis, we aggregated the "backward" change (from the earliest year to 2000) and the "forward" change (from 2000 to 2007) into a single figure of change (for each quintile) for the whole period. What this figure would actually mean is something like: "how much did employment grow between 1995 and 2007 in the $20 \%$ of jobs which had a lower quality ranking in 2000", etc.
} 
take into account when interpreting the resulting patterns (and even for this, it does not make a big difference).

\section{3.a The relative intervals behind the wage quintiles}

So we will have three sets of quintiles to characterize the quality of jobs in each European country: one based on the wage ranking, the other on the educational ranking, and a third one on the amenities ranking. Although we will use the three, the most important set of quintiles will be, without any doubt, those based on the wage rankings. In fact, the other two sets of quintiles will be there primarily to complement the findings obtained with the wage quintiles. The wage quintiles are more adequate for our purposes than the amenities quintiles because they are based on nationally-specific rankings and cover the whole economy; and they are also better than the educational quintiles because their relationship with job quality is more direct and unequivocal. Of course, this does not mean that we will ignore the other two sets of quintiles: they will provide an alternative view on the same structural developments, which will not only enrich our analysis, but contribute to the robustness of our conclusions.

The wage quintiles distribute the whole working population in five equal-sized groups, according to the median hourly wage of each job, from low to high. Therefore, the wage quintiles can, in principle, be expressed also as wage intervals that classify jobs by their median values. As we mentioned earlier, for anonymity reasons, the ESES information provided by Eurostat (the backbone of the wage ranking: see section 4.1 above) expresses the median wages at the job level multiplied by a random factor: so it is impossible to know what are the absolute wage levels associated with the wage quintiles in each country. But the relative distances between such median wages are correct, so it is possible to do a trick to get an idea about the relative wage intervals which are behind the wage quintiles. We have first calculated a central measure of the median wages by job. This central measure is, in fact, a median as well: so we basically calculated the median of the wage medians, or in other words, the median wage of the job which occupies exactly the middle position of the wage ranking in each country (the jobs were, again, weighted by employment). Then, we divided each of the wage medians by such central value, thus expressing the wage level of each job in terms of the percentage it represents with respect to the overall median. With these relative wage levels, we could construct the chart shown in figure 3.2, which expresses the relative wage intervals associated with the wage quintiles. 


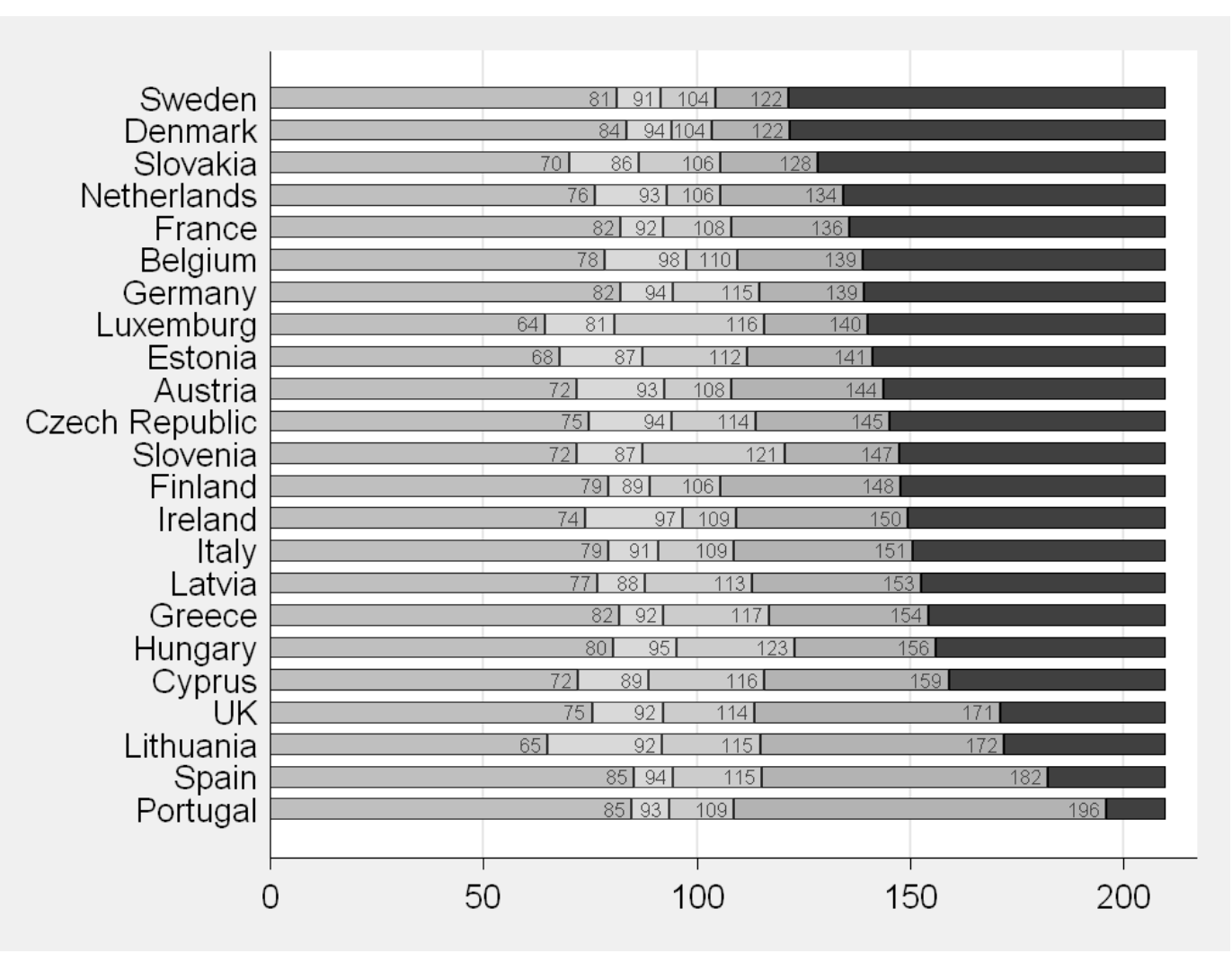

Figure 3.2: Relative wage intervals behind the quintiles

Because we are dealing with jobs rather than individuals, and with relative medians rather than absolute wage levels, it is somewhat difficult to understand what figure 3.2 shows. For each country, there is a stacked bar which represents graphically the relative wage intervals associated with each quintile. For instance, if we look at Portugal, we can see that the lowest quintile in Portugal holds jobs whose median wage is between zero (the natural lower bound of the lowest quintile) and $85 \%$ of the overall median wage. The second lowest quintile holds jobs whose median wage is between 85 and $93 \%$ of the overall median; the third quintile holds jobs between 93 and $109 \%$ of the overall Portuguese median; the fourth quintile holds jobs whose median wage is between 109 and 196\%, and the fifth quintile holds jobs whose median wage is higher than $196 \%$ of the overall median (we have not represented the upper limit for the fifth quintile, because it coincides with the value of the highest paid job in the economy, which is difficult to capture with our methodology and which in any case is irrelevant here). The countries have been ranked according to the upper boundary of the fourth quintile: that is, according to how much higher is the median wage of the top $20 \%$ of jobs than the overall median in each economy. This is, in fact, a kind of measure of the extent of between-job inequality in each European country: as we can see in the way the 
countries are ranked in figure 3.2, the European countries where the top $20 \%$ of jobs is more distant from the median are the countries where income inequality in general tends to be higher. ${ }^{52}$

An important conclusion that we can draw from figure 3.2 is that, as mentioned earlier, the wage quintiles make appear as homogeneous something which is, in fact, quite heterogeneous across Europe. In some European countries, the distance between the quintiles is much larger than in others: in Portugal, the boundary between the fourth and the fifth quintile is more than two times larger than the boundary between the first and second quintile, whereas in Sweden the same ratio is below 1.5. The countries at the bottom of figure 3.2, therefore, are characterized by a much larger inequality between the quintiles that the countries at the top. Similar developments in Sweden and Portugal, therefore, can have different implications in terms of social inequalities, because the distances between wage levels are much larger in the latter.

In any case, the picture given in figure 3.2 is important to contextualize and understand better the patterns of employment expansion by job quality quintiles which we will study in the rest of this thesis.

\subsection{Adjusting for breaks in the employment series of ELFS}

In principle, the period covered in this study is 1995-2007, although there are a few exceptions depending on two factors: first, the availability of data in the ELFS (Eurostat does not provide data for the whole period for some countries) and second, the existence of some breaks in the ELFS time series and the need to make some adjustments, whose logic we will explain in this section. Table 3.XII shows the time period covered in each country, the breaks found in the data (which, as mentioned earlier, always concern large unexplained shifts in the employment shares of ISCO categories) and the adjustments made to deal with them.

\footnotetext{
52 The ranking of countries by the Gini index is nearly identical (Portugal also has the highest value, and Sweden and Denmark the lowest), see: http://epp.eurostat.ec.europa.eu/portal/page/portal/product_details/dataset?p_product_code=ILC_SIC2
} 


\begin{tabular}{|c|c|c|c|}
\hline Country & Period covered & Breaks identified & Adjustments \\
\hline Austria & 1995-2007 & $\begin{array}{l}\text { A reclassification of occupations in } \\
\text { retail trade in } 2004 \text { (from } \\
\text { salespersons to associate } \\
\text { professionals) led to a } \\
\text { displacement of } 100,000 \text { workers } \\
\text { from quintile } 2 \text { to quintile } 5\end{array}$ & $\begin{array}{l}\text { Eliminate structural change } \\
\text { between } 2003 \text { and } 2004 \text { from the } \\
\text { series }\end{array}$ \\
\hline Belgium & $1995-2007$ & None & None \\
\hline Cyprus & $1999-2007$ & None & None \\
\hline Czech Republic & $1998-2007$ & None & None \\
\hline Denmark & $1995-2007$ & None & None \\
\hline Estonia & $1997-2007$ & None & None \\
\hline Finland & $1997-2007$ & $\begin{array}{l}\text { A reclassification of occupations in } \\
\text { the health sector in } 2002 \text { (from } \\
\text { professionals to associate } \\
\text { professionals and service workers) } \\
\text { led to a displacement of } 100,000 \\
\text { workers from quintile } 5 \text { to quintiles } \\
2 \text { and } 4\end{array}$ & $\begin{array}{l}\text { Eliminate structural change } \\
\text { between } 2001 \text { and } 2002 \text { from the } \\
\text { series }\end{array}$ \\
\hline France & $1995-2007$ & None & None \\
\hline Germany & $1995-2007$ & None & None \\
\hline Greece & $1995-2007$ & $\begin{array}{l}\text { In } 2004 \text {, there was a big sudden } \\
\text { drop in the number of skilled } \\
\text { agricultural workers. Still, there is } \\
\text { no simultaneous increase in any } \\
\text { other occupation, and the drop is } \\
\text { suspicious but does not affect much } \\
\text { the trends }\end{array}$ & None \\
\hline Hungary & $1997-2007$ & None & None \\
\hline Ireland & 1995-2007 & $\begin{array}{l}\text { In } 1998 \text {, there was a big drop in the } \\
\text { number of skilled agricultural } \\
\text { workers, simultaneous with an } \\
\text { increase in managers in agriculture } \\
\text { (looks like a reclassification) }\end{array}$ & $\begin{array}{l}\text { Eliminate structural change } \\
\text { between } 1997 \text { and } 1998 \text { from the } \\
\text { series }\end{array}$ \\
\hline Italy & $1995-2007$ & $\begin{array}{l}\text { In } 2004 \text {, there was a drop in more } \\
\text { than } 700,000 \text { salespersons in retail } \\
\text { trade, with no simultaneous } \\
\text { increase compensating it. There is } \\
\text { also a decrease of near } 400,000 \\
\text { teaching professionals and a } \\
\text { similar increase in teaching assoc. } \\
\text { professionals }\end{array}$ & $\begin{array}{l}\text { Eliminate structural change } \\
\text { between } 2003 \text { and } 2004 \text { from the } \\
\text { series }\end{array}$ \\
\hline Latvia & $1998-2007$ & None & None \\
\hline Lithuania & $1998-2007$ & None & None \\
\hline Luxemburg & $1995-2007$ & None & None \\
\hline Netherlands & $1996-2007$ & None & None \\
\hline Portugal & 1995-2007 & $\begin{array}{l}\text { In } 1998 \text {, there was a big decrease } \\
\text { in elementary occupations in } \\
\text { private households simultaneous } \\
\text { with an increase in housekeeping } \\
\text { service workers (looks like a } \\
\text { reclassification) }\end{array}$ & $\begin{array}{l}\text { Eliminate structural change } \\
\text { between } 1997 \text { and } 1998 \text { from the } \\
\text { series }\end{array}$ \\
\hline Slovakia & $1998-2007$ & None & None \\
\hline Slovenia & $1996-2007$ & None & None \\
\hline Spain & $1995-2007$ & None & None \\
\hline Sweden & $1997-2007$ & None & None \\
\hline $\mathrm{UK}$ & 1995-2007 & $\begin{array}{l}\text { A reclassification of occupations in } \\
\text { the health sector in } 2001 \text { (from } \\
\text { professionals to associate } \\
\text { professionals and service workers) } \\
\text { led to a displacement of } 500,000 \\
\text { workers from quintile } 5 \text { to quintiles } \\
2 \text { and } 4\end{array}$ & $\begin{array}{l}\text { Eliminate structural change } \\
\text { between } 2000 \text { and } 2001 \text { from the } \\
\text { series }\end{array}$ \\
\hline
\end{tabular}

Table 3.XII: Time periods covered, breaks and adjustments

In some countries, the ELFS figures of employment growth by occupation and sector are affected by strange and large year-on-year shifts that seem impossible to explain by real job growth or job destruction or by shifts in employment between jobs. We can see this clearly with a concrete example using the quintile approach. Figure 3.3 shows, for 
the UK, a graphical representation of the year-on-year changes in employment levels per quintile which illustrates these strange shifts in employment (annex 3 includes the same figure for each of the 23 countries covered in this study). We can see in this figure that the magnitude of change from one year to the following is relatively small for all years except for the year between 2000 and 2001, in which this change is completely disproportionate with the rest. This change cannot possibly reflect a real change in the level of employment across the quintiles. Although Eurostat provides no documentation for these abrupt shifts in employment, they are clearly the result of a reclassification, which can often be identified by looking at the specific jobs that are responsible for them. If we do such detailed analysis of the suspicious shifts (see annex 4), we can see that in most cases these shifts can be explained by large shifts in the number of people employed in a small number of jobs (often two or three), and that these shifts are normally related to ISCO rather than NACE code.
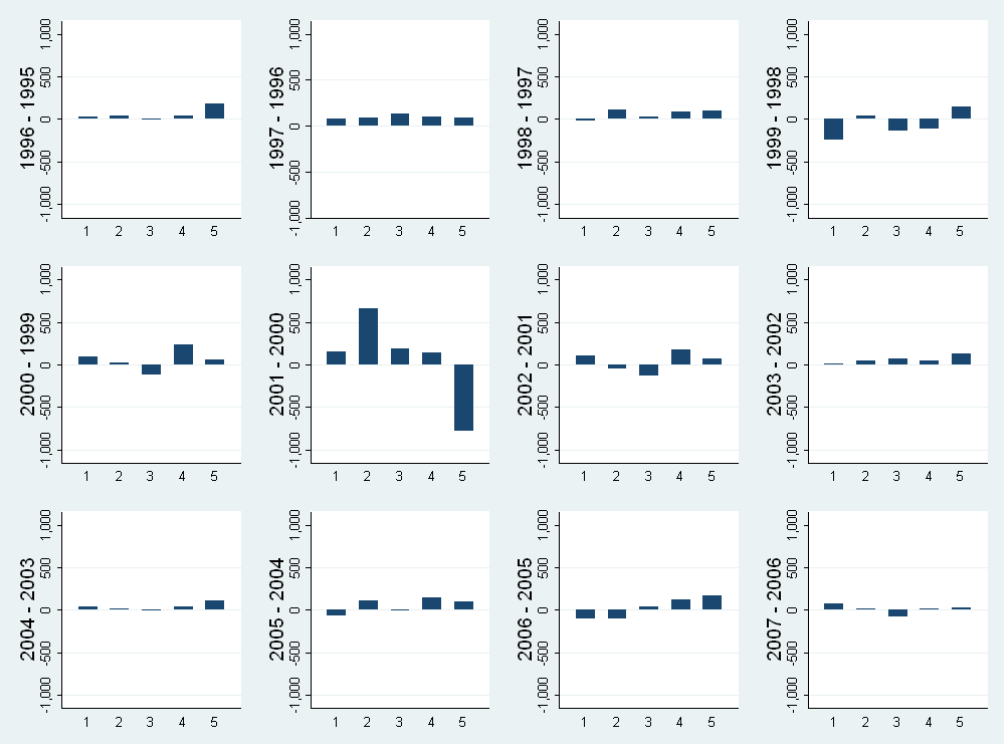

Figure 3.3: Year-on-year change in employment by quintile, UK

There are six countries with such clear shifts in employment (Austria, Finland, Ireland, Italy, Portugal and UK; see annex 3). These abrupt and discontinuous breaks point to some fundamental data problem that can compromise the validity of the trend analysis, if not dealt with in one way or another. For instance, if we look at the UK figures for year-on-year change in employment by quintile (figure 3.3), it is obvious that eliminating the change between, say, 1998 and 1999 would not significantly alter the overall pattern (for a normal, non-problematic year, the change is so small that on its 
own it does not significantly affect the overall pattern). But eliminating the problematic year (between 2000 and 2001) would alter completely the overall pattern, because of the enormous amount of (apparent, but completely implausible) change within that single year.

In fact, the comparison between the problematic and non-problematic years suggests a simple but effective way to deal with this problem. The basic idea is to eliminate the problematic year from the overall figure of change throughout the period. The logic of this adjustment is the following: as we have just seen, the change in employment numbers between two adjacent years is, in all but the problematic cases, relatively unimportant for the overall pattern of employment growth for the whole 1995-2007 period. Subtracting the structural change ${ }^{53}$ between any two (normal) adjacent years would make no difference for the overall pattern. If there is a clear disproportionate change in the size of the quintiles between any two adjacent years (see annex 1), and if this change looks like a break in the series of employment by occupation, sector, or both, this change can be subtracted from the overall change between 1995 and 2007 to keep the trend change and ignore the change resulting from the break in the series. What this adjustment means is that for these six problematic countries, the parameter of structural change for each of the corresponding five quintiles will not reflect the whole 12-year period, but just 11 (all but the problematic one). The implications of such an adjustment, for the reasons explained, should be minor in terms of substance.

There is, nevertheless, a potential problem with this adjustment, or rather a potential problem which is brought to the surface by these strange breaks in the ELFS employment series. The existence of a clear break in the series of employment by occupation and sector suggests that, for these six countries, some of the codes of occupation and/or sector may not be consistent before and after such break. In the case of the UK that we used as an example earlier, it seems that all of a sudden a whole category of nurses were shifted from one to another occupational category (see annex 4). But the job quality rankings are fixed for the whole period: therefore, either before or after the break the rankings are probably assigning an incorrect position to these

\footnotetext{
${ }^{53}$ Only structural change is eliminated, but not absolute employment growth. Basically, what we did is to substitute the original parameter of change in employment by job for the problematic year by a theoretical parameter derived by multiplying employment in all jobs by the same factor (a factor based on overall employment growth for that year). Therefore, we only eliminated structural change for that single year.
} 
suddenly recoded jobs. This may compromise the validity of the overall patterns of employment growth by job quality quintiles.

This is a very difficult problem for which a totally satisfactory solution simply does not exist. To solve this problem properly, we would need to have for these problematic countries at least two different sets of job quality rankings, one to be used for characterizing the jobs before the break, and another for the jobs after. These two figures could be added together (to give a picture of overall change in the levels of employment by quintile for the whole period), but they should be generated separately. Unfortunately, this is impossible for us because we only have a single set of job quality rankings.

But for one of the six problematic countries, we can test empirically the potential damage of this problem and the adequacy of the solution proposed earlier. In the UK (unlike most other European countries), the Labour Force Survey includes a yearly measure of wages, so that we can construct two wage rankings, one before and one after the break. This is what we have done, using 2000 as the basis for the before-the-break period and 2002 for the after-the-break period: then, we constructed job quality quintiles for the periods 1995-2000 and 2002-2007 (leaving out the problematic year), and calculated the growth in employment levels by quintile for each period. The two series were added in a single figure, and the result is shown in figure 3.4 (the bars coloured in a lighter grey). To compare these corrected results with our standard results, we have added (in darker grey) the UK figures of employment expansion by job quality quintiles using the 2002 ranking for the whole period, with the adjustment explained earlier (which simply eliminates the problematic year). 


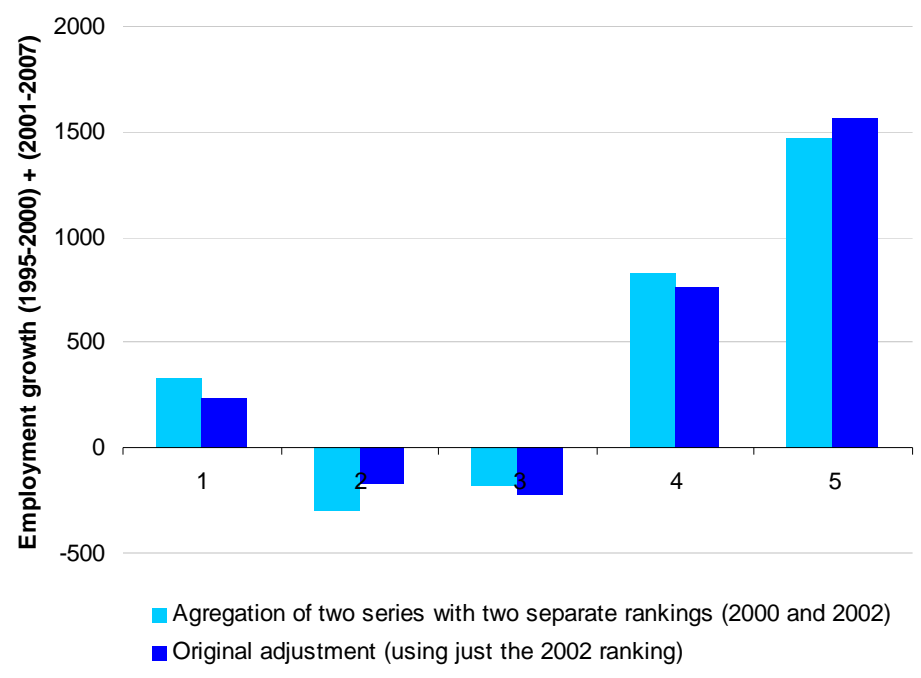

Figure 3.4: One versus two rankings, UK

This figure shows that, although there are some minor differences between using a single ranking or two separate rankings before and after the break, using one or two rankings makes very little difference. Although we cannot reproduce this test for the other 5 countries in which there are breaks in the ELFS series of employment by occupation and sector, it seems reasonable to conclude that the proposed adjustment (which assumes that a single ranking can be applied to the whole period) generates results which are broadly correct.

\subsection{General strategy of analysis for the following chapters}

First (chapter 3), we will carry out a detailed analysis of the structures of employment and the job quality rankings across Europe from a static perspective, which will serve as background for the dynamic analysis of the patterns of job creation of later chapters. This analysis will be divided in three parts: a comparison of the structures of employment by occupation and sector, a comparison of the job quality rankings and a comparison of the quintile structures (which can be understood as a combination of the two previous analysis). As the rest of the thesis, most of the analysis will be graphical, but we will make use of detailed tables and some pseudo-parametrizations when necessary to make sense of the data.

Second (chapter 4), we will inspect the overall patterns of employment expansion by job quality quintiles across Europe and try to classify them according to the principles discussed in the theoretical chapter (in particular, we will look for patterns of upgrading, degrading and polarization, which are the most pervasive images of change in the 
division of labour available in the social sciences literature). Again, the analysis will be mostly graphical (based on the quintile charts to be explained below), complemented with indices when necessary.

Third (chapter 5), we will decompose these overall patterns, inspecting the differences for specific subgroups (such as specific sectors, genders or employment status) in order to test some of the explanatory hypotheses discussed in the previous chapter.

Finally (chapter 6), we will aggregate all the national information into a single pool of data on the European labour market (although it will be an incomplete European labour market: only 23 countries are covered), to study the broad patterns of the EU, to compare these patterns with the patterns for the United States, and to attempt an evaluation of the implications of the integration of European labour markets in terms of the division of labour and job quality. 


\section{Division of labour and job quality across the EU - a static approach}

\section{Introduction}

This thesis is an attempt to evaluate, from a job quality perspective, the structural changes experienced by European labour markets in the last 12 years. It is totally focused on change, and therefore, most of the analysis is dynamic. But any analysis of structural change needs to take into account the starting position, the initial structure whose transformation will be studied. Specially when we are comparing different patterns of structural transformation (and trying to explain them), ignoring the fact that the different countries can start from different positions can lead to misinterpretations. This is so because structural change is necessarily path-dependent to some extent. Whatever our explanatory model and our hypotheses, the differences in the initial structures are also a factor to take into account when studying the different evolutions. This is exactly what we will do in this chapter: to study the division of labour, job quality hierarchies and job quality structures from a static perspective.

This chapter, therefore, will be secondary with respect to the rest of the thesis yet at the same time absolutely crucial. Its main goal will be to provide a picture of the starting position of the employment structures against which to compare the patterns of change studied in the rest of the thesis. For this reason, this chapter will be based on a detailed analysis of the main parameters of our methodological approach (the jobs matrix and the job quality hierarchies), but using different techniques to the rest of the thesis, in order to adapt our tools to a static approach.

As we have repeatedly said, the jobs approach used in this thesis is a method for studying the evolution of employment based on the combined analysis of two different parameters: first, the change in the composition of the division of labour (measured by a 
matrix of employment by sector and occupation, on a country and yearly basis) and second, the hierarchy of jobs by their quality (measured by three job quality rankings: by education, wage and amenities). The combination of these two parameters into the quintile picture is the basis for an evaluation of the structural evolution of employment from a job quality perspective. In this chapter, we will do a detailed analysis of these two parameters of the jobs approach, and their combination, from a static perspective. In the next section, we will look at the broad differences in the composition of the division of labour across Europe: for this purpose, we will use a highly aggregated categorization of overall employment in each country by sector and occupation. In the third section, we will analyse the differences in the job quality rankings across Europe (for the two job quality rankings for which we have national-specific results: education and wages), using correlation matrices and principal component analysis. In the fourth section, we will combine the two basic parameters to provide an adaptation of the quintile picture to a static analysis, to evaluate the differences across Europe in their initial job quality structures (the same structures that, from a dynamic perspective, will be studied in the rest of the thesis).

An important issue that must be clarified before embarking in this static analysis is the base year used. Because the main idea of this chapter is to set out the starting position of the changes in the employment structures since the mid-nineties, it seems logical that the base year for the analysis should be the first available year. The problem is that such first available year is not the same for all countries: some countries start in 1995 and others in 1999. The problem is exactly the same as the one discussed earlier when we had to choose a base year for constructing the quintiles. Because all countries are subject to external factors of change and because they are themselves affecting one another, it is not an option to use different base years for the different countries: we would be comparing apples and pears (the economic situation in 1995 was not at all the same as in 1999: using one or another year as the basis can make a very big difference both in terms of structural differences and differential evolutions). Therefore, we need to use the same year for all countries. As in the case of the base year for the quintiles, we opted for 2000, which is more in the middle of the period and in fact the second year which is available for all countries.

It is important to note that, although the idea is to set out the starting position, that does not mean that it is crucial to compare the first year of the period studied. First, because 
two to five years is not a period big enough as to make a big difference in structural terms; and second because the point is to anchor the dynamic analysis in some static comparison-it does not matter that much whether we use the start, the end or the middle of the period as such anchor-. Some minor details may change slightly depending on whether we are using the starting point, the finishing point or the middle: but all of these options are equally valid. Therefore, although using 1995 would be easier in terms of interpretation and presentation of results if all countries were available for that initial year, we opt for using 2000 as the base year for the static comparisons as well as for the construction of the quintiles.

\section{The division of labour across Europe: sector and occupational structures}

In this section, we will do a comparative overview of the composition of the division of labour across Europe without any reference to the job quality rankings used in the rest of the chapter, just using the information contained in the sector and occupational classifications which form the basis of the jobs matrix. Such simple distribution of employment by sector and occupation has the advantage of being immediately interpretable in terms of the division of labour (the categories of NACE and ISCO directly refer to the different economic activities and occupational structures), but it lacks the necessary elements for carrying out a qualitative evaluation of those differences (we can only describe and classify them), and it can easily become too detailed for any meaningful interpretation. In order to carry out the analysis, we will therefore have to reduce dramatically the complexity of the original jobs matrix, looking only at the univariate distribution of NACE and ISCO at a highly aggregated level (although as we will see, even at this very aggregate level, the amount of information is enormous when we are looking at 23 countries simultaneously). The main purpose of this chapter will be, for these reasons, just to provide a first approach to the broad differences in the employment structures of the different countries.

This section will be based entirely on the detailed analysis of one big table (table 4.I). This table shows the distribution of overall employment in each country (in 2000) for a reaggregation of NACE and ISCO into 9 broad categories each. The ISCO reaggregation shown in table 4.1 is simply the 1-digit level of this classification (excluding armed forces). The NACE reaggregation is partly based on its 1-digit level as well, but it has been further aggregated to simplify the analysis (keeping only 9 
categories, as in ISCO). The categories of this NACE reaggregation are the following. The primary sector contains agriculture, fishing and mining; construction corresponds exactly to the 1-digit NACE category of construction; industry has been divided in two sub-categories, high and low technology, following an OECD proposal ${ }^{54}$ (more details later); for those services which are typically private, the Eurostat differentiation of knowledge-intensive services (KIS) and low knowledge-intensive services (LKIS) has been applied (see Felix, 2006; more details later); and finally, the three large public service categories (public administration, education and health) each occupy a category on their own. This reaggregation of NACE into nine broad categories will be used in other chapters as well.

A couple of further modifications to the table have been applied in order to facilitate its interpretation. First, a semi-graphical representation has been added to the table, superimposing a bar chart to each of the columns (sectors or occupations) to facilitate the visual comparison (vertically) of each percentage. Second, for both NACE and ISCO, the countries have been sorted according to the level of employment in the most advanced sectors and occupations. This idea of "most advanced sectors and occupations" may sound strange: although we mainly use this concept to facilitate the interpretation of the table (it leads to a meaningful arrangement of countries), there is some logic behind such categorization.

In the case of ISCO, the logic is undisputable: as we discussed in the previous chapter, the ISCO classification has an implicit hierarchy, related to skill levels (we have simply taken the three top categories of ISCO at the one-digit level). But in the case of NACE, why should some sectors be more advanced than others? The argument derives from the post-industrialist theories of Bell and Fourastié (which we discussed with some detail earlier, in the theoretical chapter), as well as from the traditional economic theory of aggregate growth and large-scale structural change (the classics are Clark 1944 and Kuznets 1971). According to these theories, economic development usually implies a particular sequence of predominant sectors, from agriculture through manufacturing towards services; and within services, towards knowledge intensive and publicly provided services. In this sense, we can also understand that there is some kind of

\footnotetext{
${ }^{54}$ This categorization of manufacturing is made at the NACE two-digit sector classification, based on the intensity of research and development (ratio of R\&D expenditure to value added) and the technology embodied in the purchases of intermediate and capital goods (Hatzichronoglou, 1997, p. 6).
} 
hierarchy in the sector classification shown in table 4.I: from the primary sector (which implies the raw extraction of resources from nature) to the health sector (which implies a certain degree of economic development and is directly aimed at providing wellbeing). If we are prepared to accept that both the ISCO and NACE classifications involve some broad hierarchy in their categories, then it makes perfect sense to add up the top three or four categories and use such number to sort the countries in Europe. In any case, this is just a heuristic tool, so we can at least accept it for the time being.

In the case of our NACE reaggregation, the categories considered as most advanced are Knowedge Intensive (Private) Services, Public Administration, Education and Health: the sum of these four categories is shown in the last column, which is the basis for the sorting of countries in terms of sector. Sweden has the largest share of employment in these four categories (more than 50\% of overall employment) and Portugal the lowest ( $25 \%$ of overall employment). The ranking of countries by this ad-hoc category of NACE seems quite familiar: a similar ranking recurrently appears in many different areas of social and economic development, from working conditions (see Parent-Thirion et al 2007), to competitiveness (see Schwab and Sala-i-Martín 2009) or even happiness (see Inglehart 2004). This ranking puts the Nordics are at the top, Southern and Eastern Europe in the bottom, Continental Europe around the middle and the UK in a mid-high position.

Furthermore, this ranking is also remarkably consistent with that shown at the lower half of table 4.I, based on the three top occupations of ISCO at the one digit level (managers, professionals and technicians). Netherlands is at the top in this case (with almost $50 \%$ of overall employment in these three categories), and Portugal is again at the bottom (around 21\%). The similarity between the two rankings is so remarkable that it merits a visual exploration with a scatterplot, which is shown in figure 4.1. The percentages of employment in the "most advanced" categories of both classifications clearly tend to vary together: the coefficient of determination of the simple countrylevel association is almost .7 (which implies that knowing the percentage of employment in the "most advanced" categories of ISCO we can predict the percentage of employment in the "most advanced" categories of NACE with an accuracy of $70 \%$, and vice versa). One of the underlying mechanisms behind this association is economic development, as illustrates figure 4.2, which plots the relationship between both variables and GDP per capita in PPP: but it is not the only one, because the association 
between economic development as measured in GDP and our two structural variables is far from perfect.

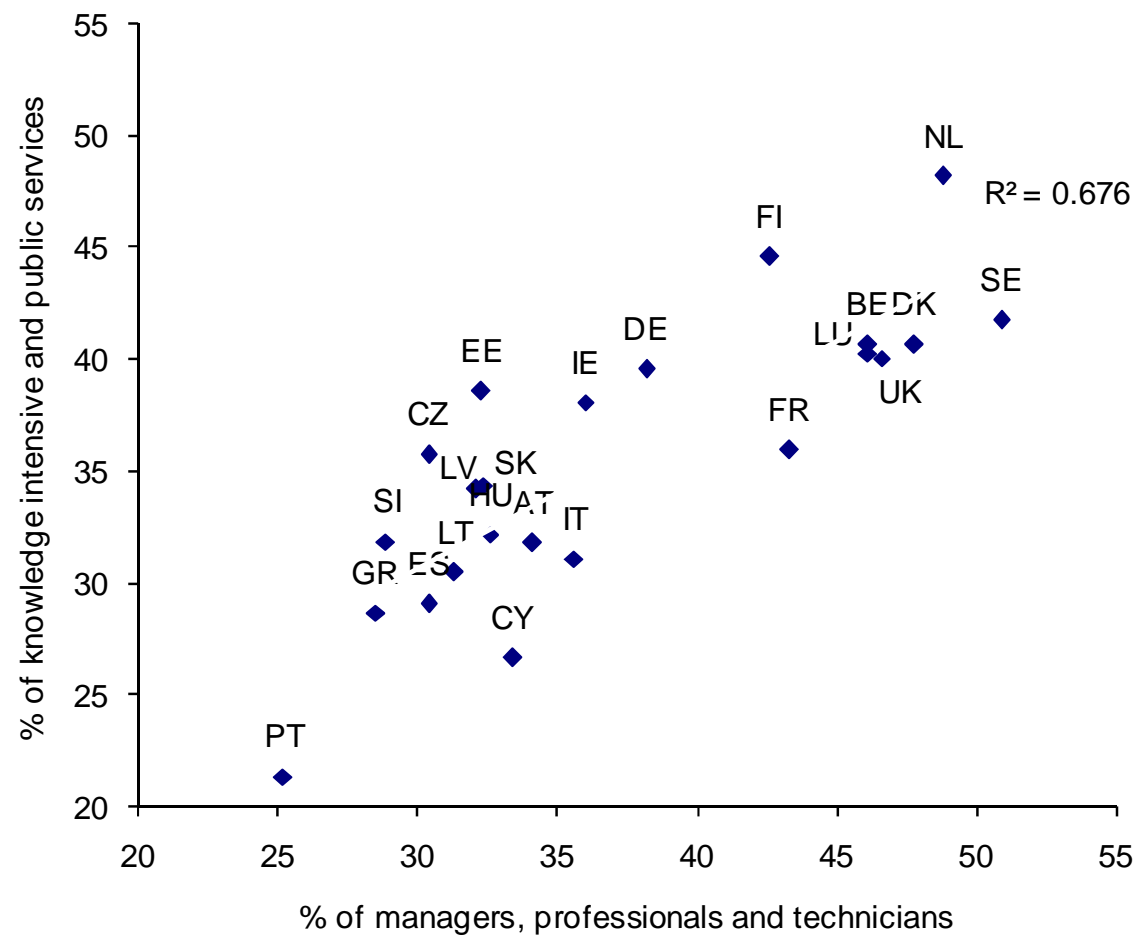

Figure 4.1: Share of employment in the "most advanced" sectors and occupations
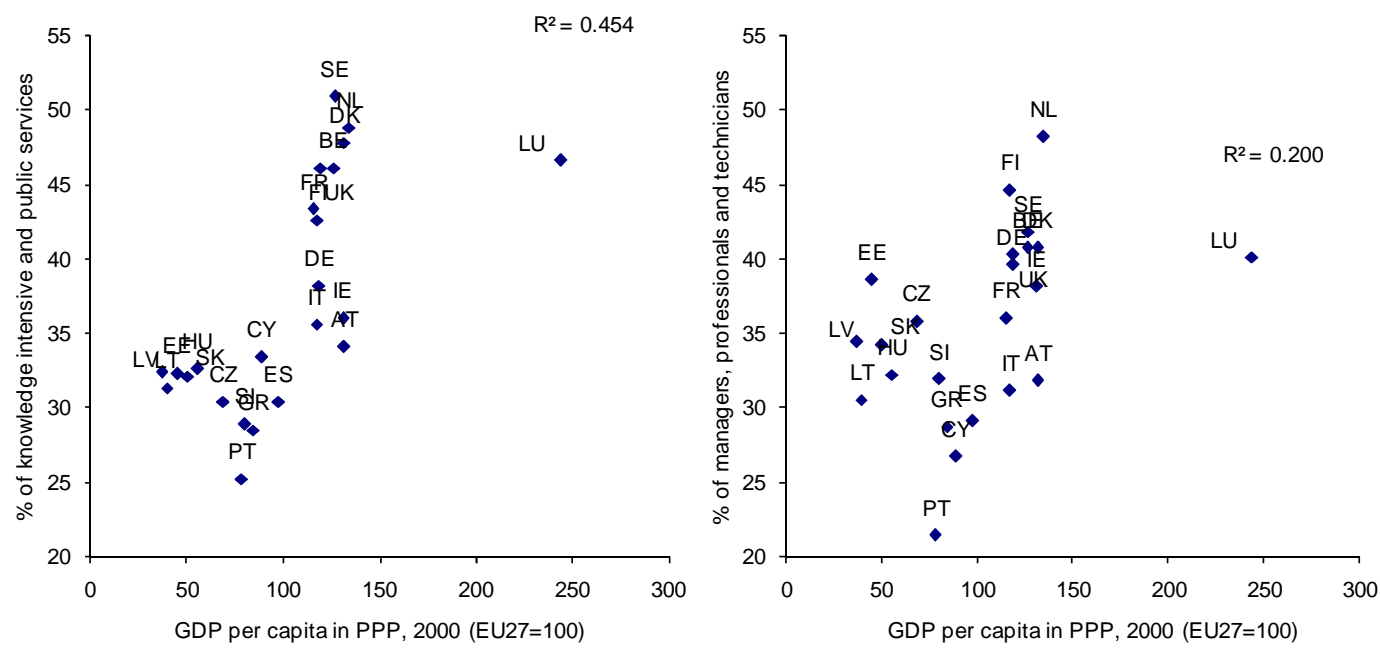

Figure 4.2: Share of employment in the "most advanced" sectors and occupations vs. GDP pc

In any case, the point we want to stress here is that the sorting of countries for the two dimensions shown in table I (sector and occupation) should help the interpretation of the table: clearly, it is not an arbitrary way of ranking countries, but a meaningful one, which tells us something about the degree of development of the division of labour in 


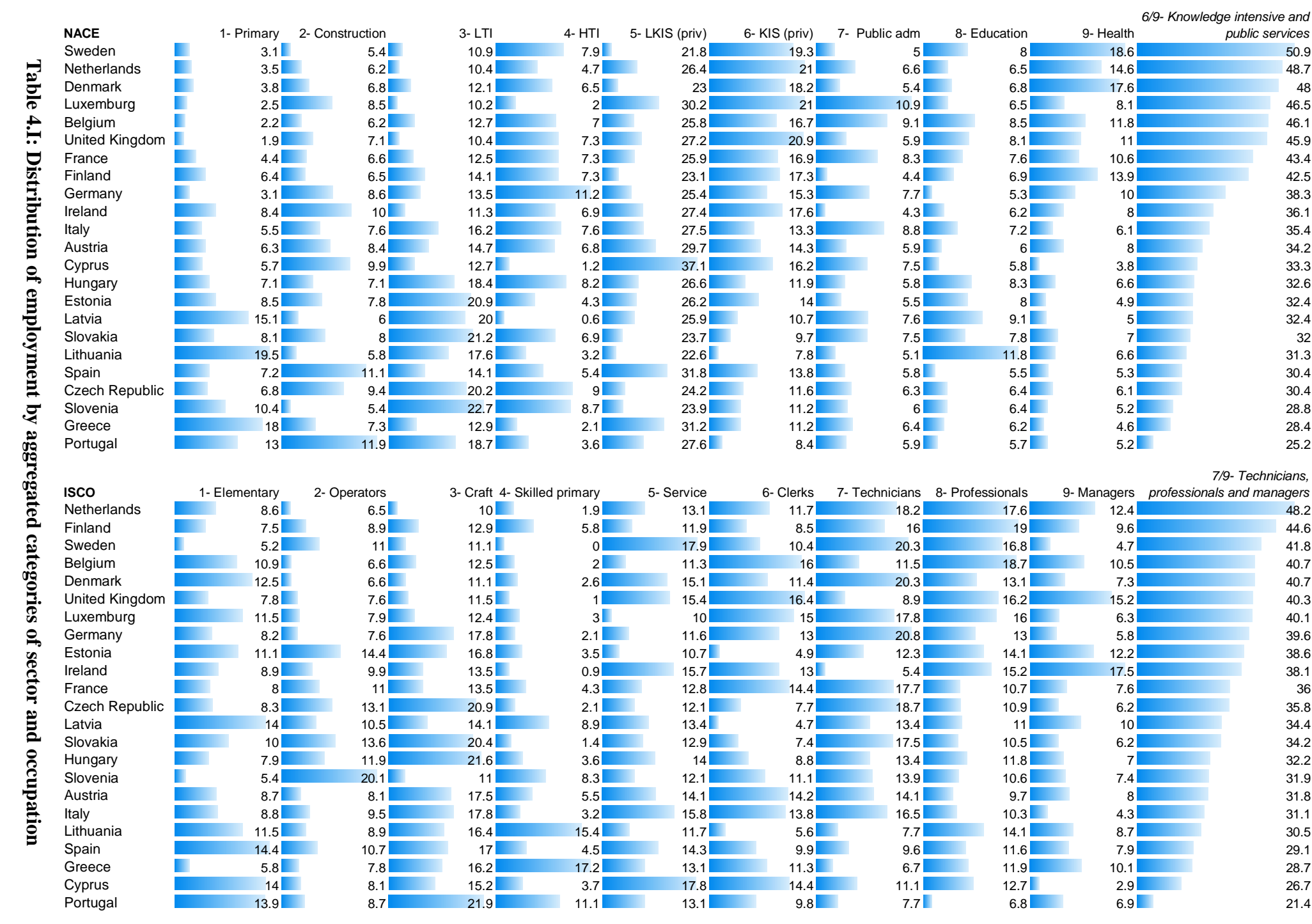


We will present the contents of table 4.I by discussing one by one each aggregated sector and occupation.

\section{1. Employment by broad categories of economic activity}

The top half of table 4.I shows how employment was distributed in the year 2000 in each of the 23 countries, in terms of the 9 broad categories of economic activity explained earlier. This table allows us to compare the composition of the division of labour in its horizontal dimension.

1. The primary sector: this sector, which incorporates agriculture, fishing and mining (NACE codes 1 to 14), is decidedly marginal in terms of employment in most of Northern and Continental Europe, and the Atlantic Isles (the UK and Ireland). In all these countries except Ireland (8.4\%) and Finland (6.4\%), the primary sector accounts for less than $5 \%$ of overall employment. On the other extreme we find that most Southern and Eastern European countries still have a relatively high share of employment in the primary sector, in all cases above 5\%, reaching almost $20 \%$ in Lithuania and Greece, and more than $10 \%$ in Latvia, Portugal and Slovenia. The overall picture of the bars superimposed to the cells of the table shows that the ranking of countries by the proportion of employment in the primary sector is the mirror image to the ranking of countries by employment in the "most advanced sectors" shown in the rightmost column of the table. These rankings, furthermore, tend to show a kind of NorthwestSoutheast axis which we will find repeatedly in many other figures. An important thing to note, nevertheless, is that the share of employment in the primary sector is so marginal in Northwestern Europe that it is very unlikely that it will play any important role in the overall patterns of change in the composition of the division of labour: but this is certainly not the case in Southeastern Europe. This sector has been in secular decline for the last 200 years across most of the developed world: therefore, where it is still sizeable, we may expect it to have fallen significantly in the period studied in this thesis.

2. Construction: as is well-known, this sector is one of the most affected by cyclical fluctuations of the whole economy (see for instance Tschetter and Lukasiewicz 1983). The year on which the data in table 4.I is based (2000) was right in the middle of a long upwards cycle in many European economies, which 
started around 1995 and finished around $2007^{55}$. In some countries, this upwards economic cycle was in fact partly based on an irrationally exuberant rise in housing prices and demand: even though the cycle still had a long way to go, in 2000 there were already signs of abnormally high employment rates in construction in some countries. Portugal, Spain, Ireland and Cyprus all had in 2000 more than $10 \%$ of overall employment in construction, a high rate both comparatively and historically. Especially in Spain and Ireland, this rate would keep on rising up to more than $13 \%$ in 2007, to plummet immediately afterwards (other countries experiencing a very sharp increase in construction employment were the three Baltics, which was still not very evident in 2000 but will appear in our dynamic analysis afterwards). It may seem surprising that Germany had a relatively high share of employment in construction in 2000, but in fact Germany experienced its own construction bubble earlier, as a result of reunification: in fact, for most of the period covered here this bubble was slowly but consistently deflating (going from almost 10 to $6.5 \%$ of overall employment). Because of its high volatility and sensitivity to the business cycle, construction is a special case that must be analysed with care when looking at the evolution of the employment structure in the subsequent chapters.

3. Low Technology Industries (LTI): this category, based on an OECD categorization of manufacturing industries (see Hatzichronoglou 1997), incorporates those industries which are less intensive in their use of technologies (the classification is based on the levels of spending on research and development, and on the technology used in production), such as manufacture of food, textiles, furniture, etc. (see table 4.II). The share of employment in this sector is highest in most Eastern European countries (around 20\%), Portugal (19\%) and Italy as well (to a lesser extent, just 16\%). In the rest of Europe, employment in this sector is between 10 and 15\%. So this sector is also associated with the NW/SE axis, but more mildly: it seems that it is a particularity of Eastern Europe that it has such a large share of employment in LTI.

\footnotetext{
55 There was, in fact, a small dip in the business cycle in 2001, which was known at the time as the bursting of the dot.com bubble. But seen from today's perspective (2009), that dip seems rather irrelevant.
} 


\begin{tabular}{|l|l|}
\hline High and medium technology industries & Low technology industries \\
\hline \multirow{2}{*}{ pr Manufacture of chemicals and chemical } & 15 Manufacture of food products and beverages \\
products & 16 Manufacture of tobacco products \\
29 Manufacture of machinery and equipment & 17 Manufacture of textiles \\
n.e.c. & 18 Manufacture of wearing apparel; dressing and dying of fur \\
30 Manufacture of office machinery and & 19 Tanning and dressing of leather; manuf. of luggage, handbags, saddlery, harness and footwear \\
computers & 20 Manuf. of wood, products of wood and cork, excl. furniture; articles of straw, plaiting materials \\
31 Manufacture of electrical machinery and & 21 Manufacture of pulp and paper \\
apparatus n.e.c. & 22 Publishing, printing and reproduction of recorded media \\
32 Manufacture of radio, television and & 23 Manufacture of coke, refined petroleum products and nuclear fuel \\
communication equipment and apparatus & 25 Manufacture of rubber and plastic \\
33 Manufacture of medical, precision and & 26 Manufacture of other non-metallic mineral products \\
optical instruments, watches and clocks & 27 Manufacture of basic metals \\
34 Manufacture of motor vehicles, trailers and & 28 Manufacture of fabricated metal products, except machinery and equipment \\
semi-trailers & 36 Manufacture of furniture; manuf. n.e.c. \\
35 Manufacture of other transport equipment & 37 Recycling \\
& 40 Electricity, gas, steam and hot water supply \\
& 41 Collection, purification and distribution of water \\
\hline
\end{tabular}

Table 4.II: Breakdown of manufacturing industries (2-digit NACE codes) into high- and low-tech (based on OECD classification; see Hatzichronoglou 1997).

4. High Technology Industries (HTI): this sector, which contains manufacturing activities which make intensive use of high technologies (such as production of chemicals, cars, electric equipment or computers: see table 4.II for a detailed list), shows almost a mirror image to the distribution of employment in LTI. HTI is very large in Germany (11\%), important in the rest of Northern and Continental Europe (around 7\% in most cases, with the exception of Luxemburg and the Netherland), and lowest in the South and the Baltics (below 5\% except in Italy). The European region that does not conform to this HTI-LTI contraposition is the East (without the Baltics), where both manufacturing sectors employ a relatively large share of the working population. Even though Eastern European countries have by far the largest shares in LTI, they tend to have rather large shares of employment in HTI as well. This means that Slovenia, the Czech Republic, Slovakia, Hungary and Estonia (even though Estonia is low in HTI, the very large share of LTI more than compensates) have a larger share of employment in manufacturing in general (including both HTI and LTI) than the traditional European manufacturing champion, Germany. In 2000, Germany had less than one in four workers in the manufacturing sector, whereas in Slovenia and the Czech Republic they were close to one in three workers. Except for Germany, all the rest of Northwestern Europe had less than one in five workers in manufacturing.

5. (Private) Less Knowledge Intensive Services (LKIS): this first breakdown of the very large services sector includes activities such as retail, hotels and restaurants 
and land transport, and is the largest category in terms of employment in all countries (see table 4.III for a list of NACE categories which are classified within this sector). In fact, this is one of the categories with a lowest variation across countries, and it is more difficult to fit into any pattern such as the NW/SE axis that we have been discussing earlier. The largest shares of employment in LKIS are those of Southern Europe (around 30\% or more in Cyprus, Spain and Greece), while this sector is below 25\% in Nordic countries and in many (not all) Eastern European countries, and between $25-30 \%$ in the rest. The distribution of employment in this sector, therefore, does not fit clearly into the usual regional/institutional European categories, probably because it occupies a somewhat ambiguous position with respect to the other sectors: on the one hand, for the simple reason of being services, it is a "progressive" sector in the sense that it tends to expand with economic growth (according to the PostIndustrialist argument explained earlier); on the other hand, it is clearly the less "progressive" segment of the services sector, and in fact it includes activities that by no means can be called new or innovative (these kind of services have occupied an important share in employment at least since the industrial revolution). Baumol's explanation of the expansion of the service sector (which concerns especially LKIS) captures this ambivalence: it would be precisely the lack of productivity growth of this category, compared with the others, that would explain its expansion (Baumol 1967).

\begin{tabular}{|l|l|}
\hline Knowledge Intensive Services (K.I.S.) & Less Knowledge Intensive Services $($ L.K.I.S.) \\
61 Water transport & 50 Sale, maintenance and repair of motor vehicles and motorcycles; \\
62 Air transport & retail sale of automotive fuel \\
64 Post and telecommunications & 51 Wholesale trade and commission trade, except of motor vehicles and \\
65 Financial intermediation, except insurance and pension funding & motorcles \\
66 Insurance and pension funding, except compulsory social security & 52 Retail trade, except of motor vehicles and motorcycles; repair of \\
67 Activities auxiliary to financial intermediation & personal and household goods \\
70 Real estate activities & 55 Hotels and restaurants \\
71 Renting of machinery and equipment without operator and of & 60 Land transport; transport via pipelines \\
personal and household goods & 63 Supporting and auxiliary transport activities; activities of travel \\
72 Computer and related activities & agencies \\
73 Research and development & 90 Sewage and refuse disposal, sanitation and similar activities \\
74 Other business activities & 91 Activities of membership organization n.e.c. \\
92 Recreational, cultural and sporting activities & 95 Private households with employed persons \\
& \\
\hline
\end{tabular}

Table 4.III: Breakdown of private services into Knowledge Intensive (K.I.S.) and the rest (based on Eurostat classification; see Felix 2006).

6. (Private) Knowledge Intensive Services (KIS): this sector includes financial and business services, communications and non-land transport, research and 
development and recreational services ${ }^{56}$ (see table 4.III). Contrary to LKIS, this sector seems to discriminate very clearly in terms of economic development, along the NorthWest/SouthEast axis. In Northern Europe (including the UK) this sector accounts for around $20 \%$ of overall employment, in Continental Europe it ranges between 15 and $17 \%$, it is around 14\% in Italy, Spain, Austria and Estonia and around or below $10 \%$ in the rest of Southern and Eastern Europe. This sector, together with health (which has similar characteristics, as we will see later) is usually referred to as the most progressive sector in the literature (see Grimshaw and Miozzo 2006; also, section 4.2.a of the theoretical chapter). Not only this sector clearly discriminates countries according to their level of economic development: as we will see later, it is one of the sectors experiencing fastest employment growth between 1995 and 2007. This will be, therefore, one of the key sectors for the following chapters, a sector whose growth reflects long-term trends in the evolution of the division of labour.

7. Public administration: the first of the public services sub-sectors does not show any clear pattern along the lines we have been discussing here. In fact, the Eurostat classification of service activities which we used for breaking up private services, and which originally referred to both private and public services, includes this sector in Less Knowledge Intensive Services (the other two typically public services, Education and Health, were classified with Knowledge Intensive Services; see Felix 2006). The proportion of employment in public administration is more likely to reflect institutional and cultural traditions (such as statism) than level of development. The highest share of employment in public administration is found in Luxemburg, Belgium, Italy and France (all of them above 8\%), and the lowest in Ireland and Finland (below 5\%). In most other countries it is between 5 and 7\%. This sector is strongly linked to national specificities and idyosincrasies, and in most countries it did not experience any important transformation over the period covered in this study: for these reasons, it is likely to be of only a minor importance in this thesis.

\footnotetext{
${ }^{56}$ We follow the relatively wide definition of this sector used by EUROSTAT (see Felix 2006), just leaving out the typically public services of education and health. Other similar concepts (such as Knowledge Intensive Business Services) are more restrictive: but the broad story is very similar (see Grimshaw and Miozzo 2006).
} 
8. Education: although it may seem surprising, this sector is to some extent similar to public administration in the sense that it does not discriminate countries in any recognisable way; instead, it seems to be determined by strictly national factors. The share of employment in education is above $8 \%$ in Lithuania, Latvia, Belgium, Hungary and Sweden (a rather disparate group of countries), and around 6-7\% elsewhere (slightly lower in Germany, Spain and Portugal). As in the case of public administration, this sector was very stable in its employment share over the period 1995-2007 in most European countries, so it will not play a very important role in the story that will be told in the next chapters.

9. Health: this third traditionally public sector (in Europe) contrasts strongly with the previous two. We can immediately see in table 4.I that this sector is strongly associated with the NW/SE axis discussed earlier, and it was one of the most dynamic sectors in terms of employment growth across most of Europe between 1995 and 2007 (as we shall see in later chapters). The share of employment in health services was in 2000 above 14\% in the North of Europe, around 10\% in Continental Europe, and around 5-6\% in the South and East.

This quick overview of the structure of employment by NACE across Europe has shown that there is a consistent differentiation of countries which seems linked to the stage of economic development as well as to a North-West vs. South-East axis. But the important thing for us, which we will be crucial for the rest of this thesis, is that this differentiation is also strongly linked to the patterns of employment expansion between 1995 and 2007 that we will discuss later. The sectors that predominate in the most developed countries (Private Knowledge Intensive Services and Health), which are considered as the most progressive in the specialized literature (for Fourastié, they were nothing less than "the hope of the $20^{\text {th }}$ Century"), happen to be also the sectors that grew faster between 1995 and 2007 in most European countries, and also the sectors that account for most of the growth of the top of the job quality structure. On the contrary, we will see that the less progressive sectors (especially, the primary and industrial sector), which predominate in the less advanced countries of the South-Eastern quadrant, were also quite dynamic but in a negative sense, destroying employment everywhere. In other words, there is an important consistency between the pictures of sectorial differentiation and economic development given by a static and a dynamic analysis. 


\subsection{Employment by broad occupational categories}

In table 4.I above, we could see that the ranking of countries in terms of the share of employment in the top three occupations of ISCO at the one digit level is remarkably similar to the ranking of countries in terms of employment share in the most progressive sectors of activity. This suggested that the two classifications partially reflect the same underlying reality, a reality linked to the level of economic development and somehow as well to a geographic axis going from the NorthWest to the SouthEast of Europe. We will see in this section that, notwithstanding the results obtained when looking at the employment share in the top three categories of ISCO (which is very consistent with the results of the previous section), when we look at the nine specific categories of ISCO at the one digit level we find many more specificities and idyosincrasies which are very difficult to fit into such overall scheme. The problem is whether those idyosincrasies, or differences which do not seem to conform to any known (to us) pattern, reflect real differences in the division of labour within firms (which is what ISCO is supposed to measure) across Europe, or whether it is somehow an artificial outcome of inconsistencies in the measurement and coding of the division of labour within firms across European countries.

It is important, in any case, to remember what ISCO is about, and what implications it may have for a summary table such as that shown in the bottom half of table 4.I. If we compare it to NACE, we can immediately see that an international comparison of employment by ISCO is necessarily more artificial, because of its very nature. The divisions of ISCO are considerably more artificial with respect to their object than the categories of NACE: they are abstract categorizations of positions, tasks and responsibilities within organizations, positions which are coordinated by the internal and subjective authority of each organization's management, rather than by an external and objective mechanism such as the market in the case of NACE. The figures for ISCO shown in table 4.I for each country are just averages (transversal summaries across firms) of these abstract categorizations of the real positions existing within firms. It seems a priori quite likely that such figures incorporate a considerably higher amount of imprecision than the figures of NACE discussed earlier.

1. Elementary occupations: as we will see in most individual categories of ISCO-1, the shares of employment in elementary positions (lowest level of skill, and lowest degree of specialization) across Europe do not display any apparent 
pattern similar to the ones discussed earlier for NACE. The different groups of countries appear scattered, without following an order related to economic development or institutional family. The share of employment in this category is higher in Spain, Latvia, Cyprus, Portugal, Denmark, Luxemburg, Estonia and Belgium (above $11 \%$ in all cases). On the other hand, it is lowest in Sweden, Slovenia and Greece (below 6\%). In both cases, we have a mix of countries from all regions, with no apparent link to their economic development.

2. Operators and assemblers: the ranking of countries by this category seems more coherent with our previous discussion. This is the typical category for semiskilled industrial workers, and as could be expected it is higher where the industrial base is stronger. Except in Latvia and Lithuania, this ISCO category is above $12 \%$ in most of Eastern Europe (reaching more than $20 \%$ in Slovenia). In most of the other countries, this category accounts for less than $10 \%$ of overall employment (with the partial exceptions of France, Spain and Sweden).

3. Craft and craft-related workers: this category includes the traditional skilled industrial worker but also craft workers in general (a category that also exists in services, and most importantly, in construction). It discriminates countries along the NW/SE axis even better than operators and assemblers: Eastern and Southern Europe is at the top (close to 20\%) and Northern and Continental Europe at the bottom (between 10 and 15\%). The exceptions are Germany and Austria, closer to the SouthEast pole in this case as corresponds to their strong industrial base and their tradition of strong institutional support for the training and learning of craft skills.

4. Skilled agricultural and fishery workers: this category (even more than the two previous ones) is more about sector of activity than about occupation (more about the horizontal than about the vertical division of labour). It basically shows the same patterns as the primary sector discussed earlier for NACE, so there is no point in repeating our earlier comments here.

5. Service occupations: this category refers mainly to occupations in personal services (such as retail) which involve direct contact with costumers and which do not require much specialization (for instance, travel attendants, waiters, protective agents, demonstrators). This category, as happened with elementary occupations, shows no clear pattern in its distribution across countries. It is 
highest in Sweden, Cyprus, Italy, Ireland and UK (above 15\%), and lowest in Luxemburg, Germany, Estonia, Belgium, Lithuania and Finland (below 12\%). The actual range of variability of the share of employment in this category across countries is the lowest of all categories (most countries are within a narrow range, between 12 and 15\%).

6. Clerks: this is a clearly transversal category with respect to sectors (there are clerks in all sectors), but a category which again shows no clear pattern. If anything, it seems that Eastern European countries have a lower proportion of workers in clerical occupations (below $8 \%$ in most cases). In the UK and Belgium this category accounts for more than $15 \%$ of overall employment.

7. Technicians: the lowest category at the top of ISCO, it shows a very mild pattern along the lines that we discussed earlier for sectors. It is relatively high in continental and northern Europe (above 15\%) and low in the south (below 10\% except in Italy), but also in the Atlantic Isles (below 10\%). The proportion of technicians in overall employment is rather diverse in Eastern European countries.

8. Professionals: this category, which as elementary occupations, clerks and technicians is really transversal to NACE, is the one that seems to discriminate countries more clearly in the NW/SE axis explained earlier. There are, though, some important exceptions that seem to be related to some porosity in the boundaries between technicians and professionals in the different countries (for instance, Ireland and UK are simultaneously very high in professionals and very low in technicians, while the opposite happens in Germany and France). The highest percentages of professionals appear in Northern Europe and the Atlantic Isles (around 15\% or higher), and the lowest in the South and East of Europe (around $10 \%$ or less), with Continental countries scattered around (relatively low in France and high in Belgium and Germany).

9. Managers: the category which occupies the top of the hierarchy which is implicit in ISCO, contrary to the previous two categories, does not show any clear pattern in terms of country differences. The most noteworthy aspect of this column is the very high values for UK and Ireland, which either have almost twice as many bosses (for the same number of workers) as the rest of Europe (the proportion of bosses is above $15 \%$ in these countries, whereas in most 
European countries it is below $8 \%$ ) or a more lax understanding of what is a managerial position.

We can therefore conclude that although the distribution of employment across the broad skill levels which are implicit in ISCO also reflect somehow the degree of economic development (as shown in figure 4.2), this effect is much less intense than in the case of NACE. Most of the categories of ISCO at the one digit level vary in their distribution across countries in ways which cannot be related to a NW/SE axis of economic/institutional development that is so powerful for explaining many other issues (including the NACE distribution). Beyond the categories of technicians and professionals, most of the national differences in the ISCO-1 distribution seem idiosyncratic: they seem to depend much more on country-specific factors than on exogenous factors which affect all European countries such as the degree of economic development or the institutional family.

Now the big question is whether these idiosyncratic differences in the percentages of employment by occupation reflect real differences in the organization of work across Europe (for instance, that in the UK and Ireland they really have twice as many bosses as other European countires) or whether they are an artefact created by inconsistent ISCO codings across Europe. We have already discussed this problem in the previous chapter: now we have a concrete example of the kinds of problems we were talking about. The most likely answer to this difficult question (as we already argued in the previous chapter) is that there is probably a bit of both: idiosyncratic occupational structures and idiosyncratic codings.

What are the reasons to think that the European occupational structures are not so easily reduced to the same axis of economic/institutional development as the sector structures? As we emphasized earlier, we must remember that we are speaking here about a kind of national average of the structures of work organization within firms (the vertical division of labour). The usual assumption is that economic development increases the demand for high-skilled occupations; and broadly speaking, figure 4.1 provides strong support for such an argument from a static perspective (the rest of the thesis will provide more support for this argument from a dynamic perspective). But beyond such a broad effect, companies still have a considerable leeway in how they actually organize their internal structure. The distribution of employment by occupations has far less external constraints than the distribution of employment by sectors, it is much more 
directly constrained by subjective decisions made by managers: this surely means that the space for national idiosyncrasies (for instance, determined by cultural differences) is larger. For instance, some societies which place more emphasis on conformism may have more developed hierarchical structures and therefore more middle management positions, for instance. Some societies may consider as specialists workers which other societies deem as elementary workers. Some societies may think that anyone with some responsibility and decision capacity is a manager, whereas others may fix a much higher boundary for such position. Probably part of the idiosyncratic differences reflected in the distribution of ISCO in table 4.I are the result of this kind of differences in national organizational cultures, if we may call them that way: these would be, therefore, perfectly valid differences even if they cannot be explained by the same factors as the differences in the distribution of NACE.

But there are also important arguments for considering that at least part of the national differences shown in the bottom half of table 4.I are a statistical artefact, even if there is no wrong coding from a national perspective. Particularly problematic is the (known) fact that there are important differences in the social status attached to the same occupations in the different countries. Even if the distribution of ISCO reflects adequately the social consideration attached to each occupation in the different countries, this can be completely wrong from the perspective of the division of labour in material terms, which is our perspective here. For instance, whether a particular occupation is considered as technical or professional often depends more on its social status than on its actual skill requirements: the boundaries between both categories are therefore porous, and in fact some of the percentages of both categories looked suspiciously contradictory in some countries (some countries seemed to have simultaneously abnormally high percentages of professionals and abnormally low percentages of technicians, and vice versa). Similar examples of porous boundaries that can shift according to the social status attached to each job would be the differences between operators and craft occupations, or between elementary occupations and service jobs. It seems very difficult to explain some of the differences in the distribution of employment by occupation across countries without mentioning this kind of social status bias in the coding of ISCO. Such a bias does not imply that the ISCO coding is wrongly done: from the perspective of each country, it could be perfectly correct, and even from an international perspective if we are interested in the social status attached 
to each particular job. But if our perspective tries to focus on the labour process as a process of production of use values, if our analysis tries to study the division of labour from a material perspective, which is the case, this would be a problem. ${ }^{57}$

The analysis of table 4.I, therefore, confirms what we already said in the previous chapter: that we must be very prudent when doing international comparisons based on the ISCO distribution. For most of the analysis we will do in this thesis, this will not be a problem, because the comparison will be based on quintiles, which are constructed according to job quality rankings that were separately generated for each country. But unfortunately, some of our analysis will need to be done under the assumption that the ISCO classification is comparable across countries: in particular, the decompositions of the patterns of job growth by ISCO (even though they will play only a secondary role), the analysis using the amenities ranking (which was constructed for the whole of Europe, and therefore assuming that their job structures are really comparable), and the analysis at the European level (which aggregates the jobs matrices of all individual countries, treating the whole of Europe as if it was a single labour market). In those cases, we will have to be careful with our analysis and interpretations, and always try to remain at a highly aggregated level (at such level, most of these problems tend to balance out and be largely inconsequential).

\section{The correlations of the educational and wage rankings across countries}

The job quality rankings provide a uniform metric to the national job matrices, and has two important functions in our analysis. First, it provides the basis for a qualitative evaluation of the nature of changes experimented by each national job matrix over time: without the rankings we would not be able to assess whether the different countries created good or bad jobs. Second, it provides a consistent and homogeneous basis for comparing meaningfully something which is potentially quite different across countries: the quality of jobs. We saw in the previous section that the structural composition of the division of labour (as captured by occupation and sector) varies considerably across countries: besides this compositional variability, there may be intrinsic variability across the national job matrices as well. The same job, involving the same tasks and

\footnotetext{
${ }^{57}$ Such a social status bias in ISCO coding would imply that the ISCO classification would already incorporate some of the functions that, in our methodology, are assigned to the job quality rankings: to provide a qualitative, evaluative element for each job in the matrix.
} 
skills, may occupy different positions in different countries with respect to any of the three aspects of job quality which we are studying here. It is because we assume that this intrinsic source of variability is potentially of importance that we have tried to construct nationally specific job quality rankings. For lack of suitable data, it was impossible to construct a nationally specific ranking of jobs in terms of amenities; but we do have nationally specific rankings of jobs in terms of relative wages and educational levels. In this section, we will explore empirically the degree of variability in the job rankings by education and wages across countries. We shall try and see whether our initial hunch that there are qualitative differences for the same jobs across different countries was correct. This has important implications for the rest of the thesis: if the same jobs have different qualities in different countries, exactly the same type of transformation in the composition of the division of labour can have a very different impact on the different countries.

Besides section 4.4 of the previous chapter, this will be the only section of the whole thesis that will look at the rankings as such, in their full detail. The rest of the analysis will be based on the quintiles, that is, on an aggregation of the rankings into five groups of equal size in terms of employment in the year 2000. As we have repeatedly said, the analysis in terms of quintiles rather than rankings not only allows to simplify and make more manageable a very large amount of information, but also minimizes some potential errors in the coding of occupations or sectors, or in the job quality estimations at the job level. Nevertheless, in a section totally devoted to a detailed analysis of the differences in the job quality rankings across the different countries, we believe it makes sense to try to use the full information contained in the original rankings, of course being careful in not paying too much attention to little details and trying to use some type of weight to make sure that the our analysis is not biased by differences in very small jobs (which are irrelevant in terms of employment and also much more likely to be affected by methodological problems such as coding errors or job quality estimations).

\subsection{Exploring the correlations of the wage and educational hierarchies across countries}

First, we will compare the educational and wage hierarchies for each possible pair of countries, using correlation matrices. Such matrices show, for each pair of countries, how similar are their rankings of jobs by education or wages. As a measure of similarity 
between national job quality rankings, we use Pearson's product moment correlation coefficient. In principle, it may seem surprising that we use such measure of correlation, which is strictly parametric, rather than a non-parametric alternative such as Spearman coefficient (which is in fact a rank comparison). But in practice, after the rankings have been normalized (divided by their maximum value), the differences between the Spearman statistic and the Pearson product-moment correlation coefficient (the standard correlation coefficient) is in this case negligible, and the latter is allows the use of weights, which is crucial for our purposes. Without weighting by employment, the correlation coefficient can give a distorted picture because there are very many small jobs, which account for a very small amount of overall employment and for which the rankings are not so reliable (being small jobs, the educational level and wage estimations are less robust). Therefore, we will show both the unweighted and weighted correlation matrices: and for reasons of consistency, we will use Pearson's correlation coefficient in both cases (in annex 5, the Spearman rank-correlation matrix for the unweighted rankings is shown as well: the results are broadly consistent with what is shown in table 4.IV).

Table 4.IV shows the matrix of correlations between the educational rankings of all possible pairs of countries. The number of observations (jobs) behind each correlation coefficient varies, because the number of jobs identified in each country varies (as discussed in section 3.3 of the previous chapter). In practice, the number of jobs behind each correlation coefficient is given by the smaller number of jobs identified in any of the two countries on which the coefficient is based (for instance, the number of jobs for which we have educational ranking in Austria and Germany are respectively 921 and 1093, and therefore, the number of observations on which their correlation coefficient is based is 921). The correlations shown in table 4.IV have no weight whatsoever: each job contributes equally to the overall correlation coefficient, without taking into account the fact that the different jobs account for very different employment levels (in other words, each job receives the same weight).

The correlation matrix shown in table 4.IV is symmetric: although the coefficients shown above the diagonal are redundant, it makes the analysis easier because the correlations between each country ranking and all the rest are all shown in a single line, horizontally and vertically (we will look at it vertically). To help the interpretation of such an enormous amount of information, we have colour-coded the cells in the table 
according to their values, with a grey gradient (darker as the values for high values, lighter for low).

Table 4.IV shows that the national educational rankings of jobs are rather similar across Europe. All correlation coefficients are relatively high: the lowest value is .56 (the correlation between the Danish and Lithuanian education hierarchies), and the vast majority ranges between .6 and .8 (for the moment we are not looking at the last row and column, showing the EU ranking, which is a linear combination of all the national rankings and therefore necessarily has very high correlation with all the rest). The lowest values, with lighter grey tones, are for the three Baltic States (whose correlation coefficients with the other countries are never above .7-with the single exception of Latvia's correlation with Hungary and Slovakia-). Denmark, Austria, Portugal and Sweden also have relatively low correlations with other countries, although never as low as the Baltics and with some significantly high correlations as well. Almost all the other correlations are between .7 and .8. If we look at the highest correlation coefficients of table 4.IV, most of them seem to make perfect sense, linking countries which are known to be institutionally or culturally similar. In particular, the correlations between Eastern Member States (except for the Baltics) are quite high, as well as the correlations between Southern European countries. The correlations among the Northern, Continental and Isles member states, on the other hand, are not particularly high. 


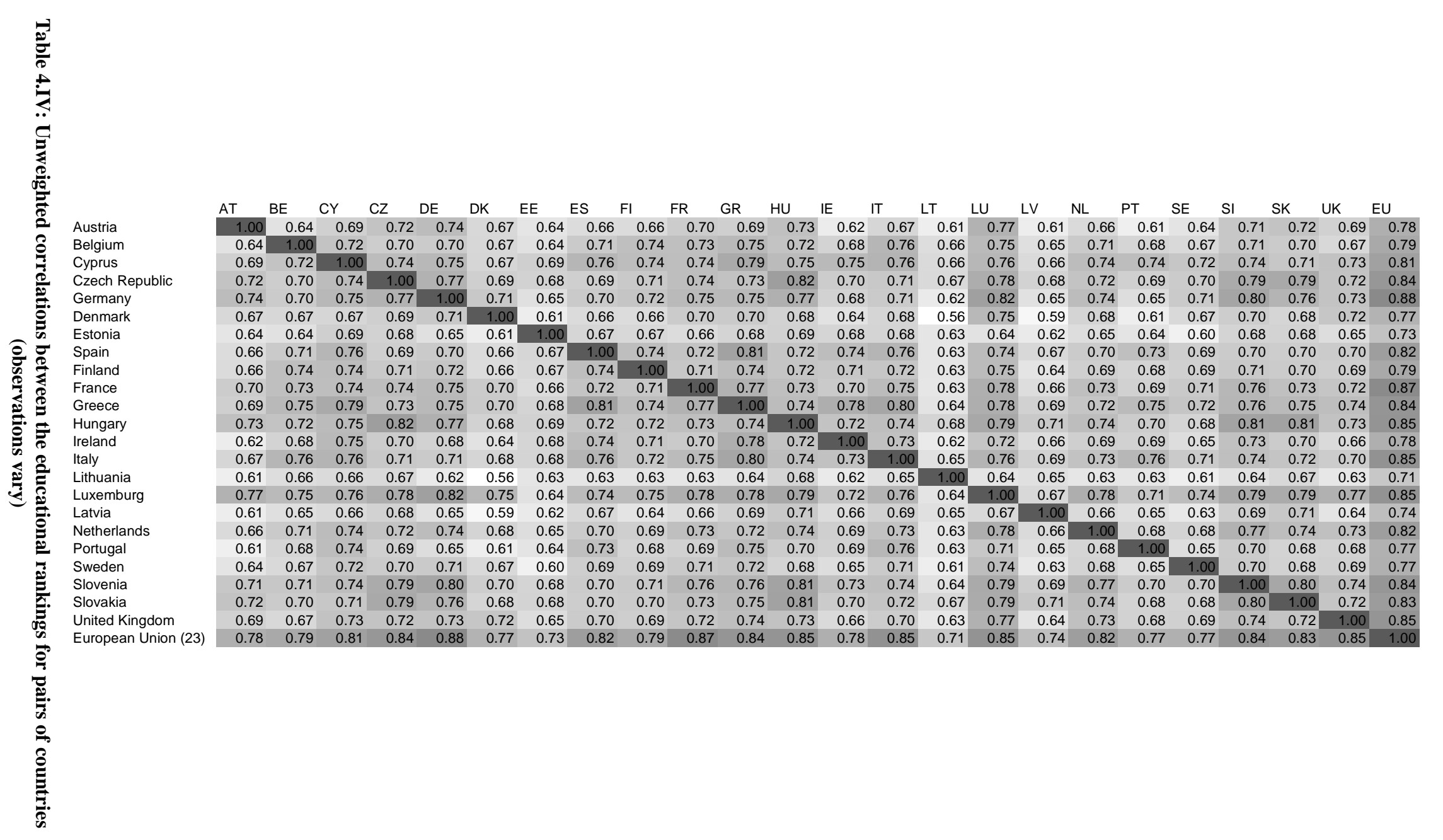




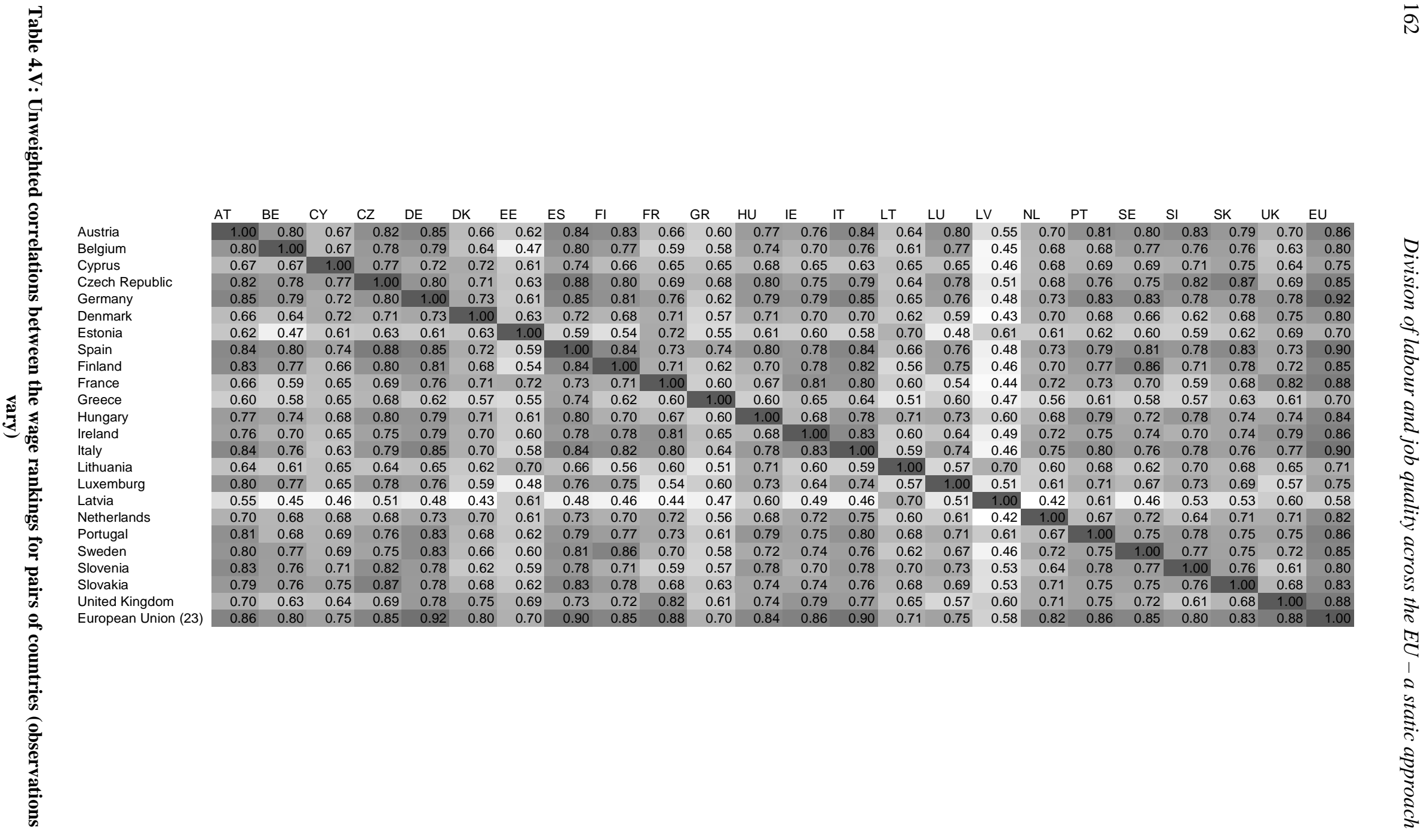




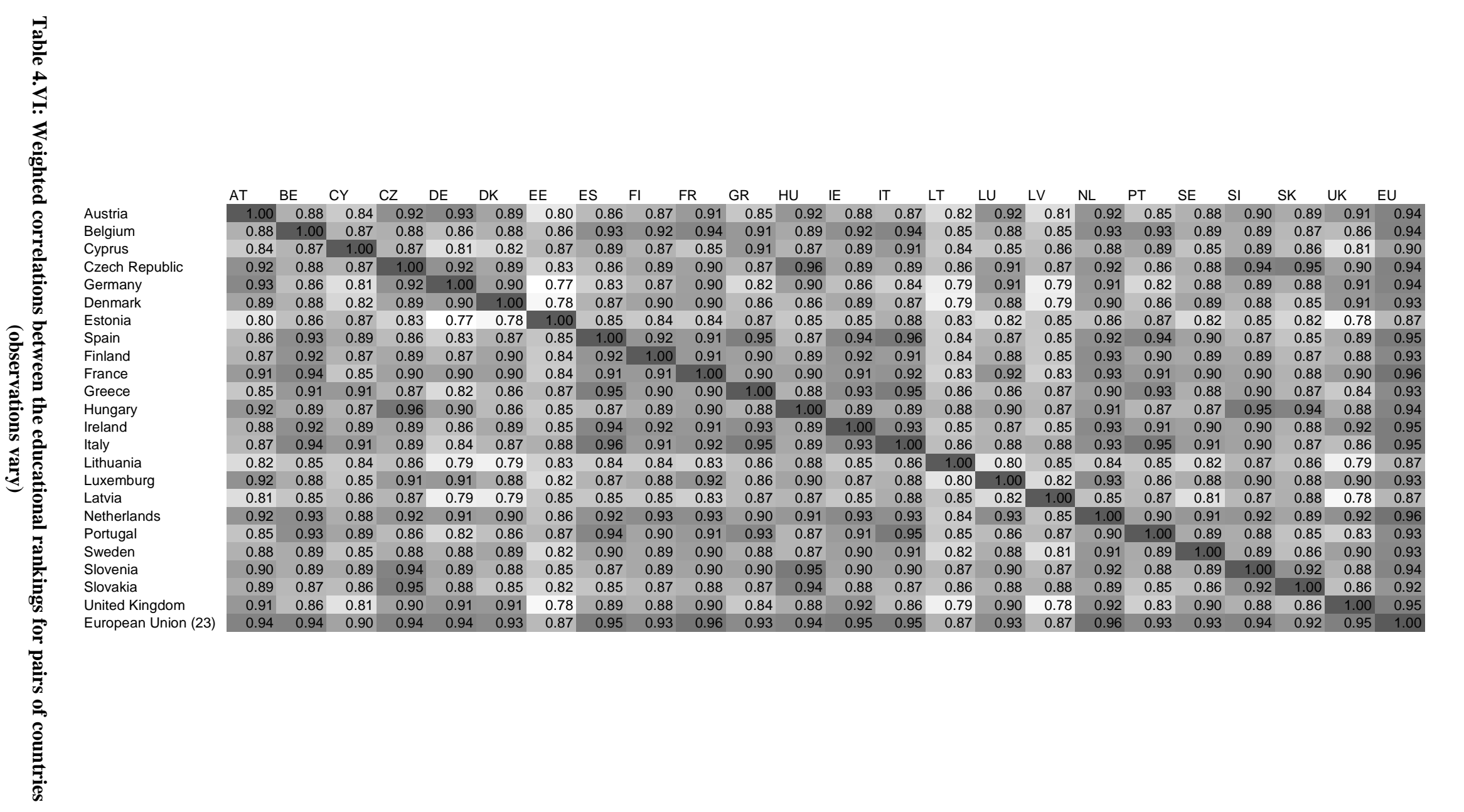




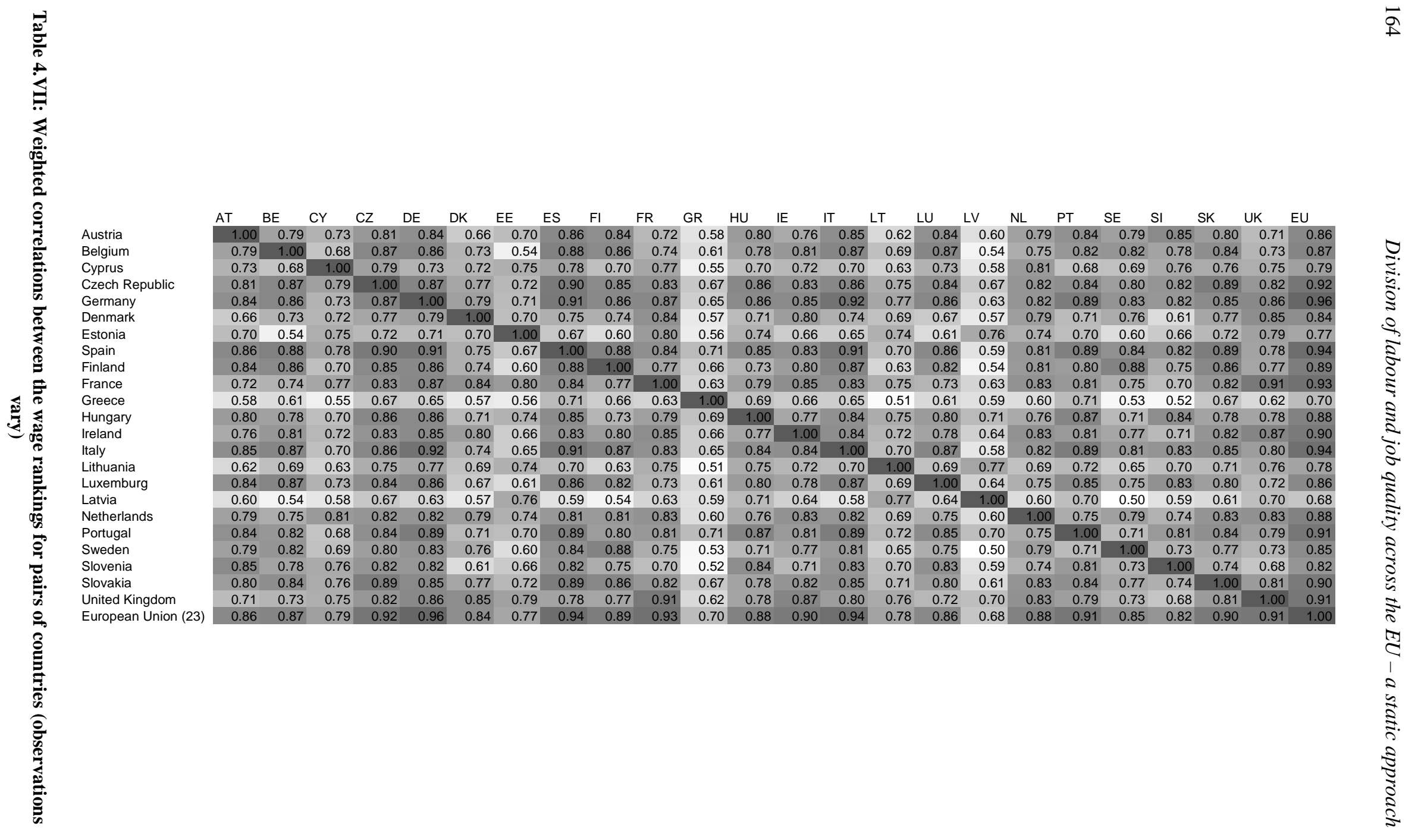


The last row and last column of table 4.IV show the correlation between the average educational ranking for the whole of the EU and each national educational ranking. Because the EU ranking is a linear combination of all the original national rankings, the correlations of the last row and column are obviously higher than the rest; yet the correlation coefficients in this last column can be used to gauge the degree of correlation between each national ranking and all the rest simultaneously (represented here by the EU ranking). This way, we can confirm that the three Baltic States have the lowest similarity in their educational rankings with the other countries (correlations with the EU: .71, .73 and .74), followed by Denmark, Portugal and Sweden. In the following section we will do a more detailed analysis of the commonality and uniqueness of each national ranking using principal components factor analysis.

So what do we make of these results? The square of the correlation coefficient, the coefficient of determination $\mathrm{r}^{2}$, has a more immediate interpretation: it measures directly the proportion of shared variance between each pair of rankings. The fact that the vast majority of the coefficients in table 4.IV are between .6 and .8 means that knowing the ranking position of a job in country $\mathrm{x}$ we can predict its ranking position in country $\mathrm{y}$ with an accuracy between $36 \%$ and $64 \%$. So we can conclude that the educational rankings are broadly similar across Europe, but that we still need to use nationallyspecific rankings in order to properly account for the specificities of each country's educational hierarchies at the job level. In other words, the rankings are similar enough as to prove that they are measuring the same thing in the different countries, yet low enough as to demonstrate the existence of significant and systematic country differences.

Table 4.V shows the (unweighted) correlation matrix for the wage rankings, constructed and represented in the same ways already explained for the educational ranking. We can immediately see that the correlations are in this case lower, although it would be more precise to say that their range is significantly higher. In this case, the lowest correlation (between Netherlands and Latvia) is .42, and the highest .88 (Spain and the Czech Republic). Therefore, many pairs of countries are more dissimilar in terms of wage hierarchies than in terms of educational hierarchies, but there are also some pairs of countries which are more similar. 
Again, the Baltics have the lowest values (their correlation with most countries are below .7), but in this case there is more similarity between the three of them. Neither Austria, nor Sweden or Portugal are atypical countries in terms of their wage hierarchies, in contrast with their educational hierarchies. Denmark is still a relatively atypical country, although much less than in terms of the educational ranking. But the country that is clearly very atypical in this case, almost as much as the Baltics, is Greece, which only has a correlation coefficient above .7 with one country (Spain), and its correlation coefficient with the EU wage ranking is .7, the third lowest of all countries. The correlation coefficients of Luxemburg and Cyprus are also relatively low (.75 with the EU ranking).

The conclusions for the correlations of the wage rankings are very similar to those for the educational hierarchies. The main difference is the range of variability across countries of the two rankings: the correlations of the wage hierarchies are slightly more variable than the educational hierarchies. A possible explanation for such difference is that the link between education and the division of labour is stronger and more universal (insofar as there is a link between education and the skills required for each job) than the link between wages and the division of labour. The relative position of each job in terms of wage seems, all else being equal, more likely to be sensitive to institutional (and other) differences across countries.

In any case, as we mentioned earlier, the analysis so far has the problem that each job weighted equally in the computation of the correlation coefficient. This can lead to some important distortions because the actual share of employment in each job varies considerably: there are some jobs that account for quite a large share of employment, and many other jobs that account for a negligible share of employment. It is in the (many) small jobs that most of the methodological problems tend to concentrate: on the one hand, they are more likely to have been miscoded in terms of NACE or ISCO (which would distort the international comparison); on the other hand, the estimation of average education or median wage in those cases is based on a smaller set of observations and therefore less reliable. For this reason, it is necessary to complement what we have said so far with an analysis in which each job is assigned a weight according to its employment size.

The problem is, to the employment size of what? We must remember that what we are doing here is comparing the rankings of pairs of countries. The share of employment in 
each job varies across countries, so it is simply not possible to weight the country rankings by their own employment levels (it would mean that we would use two weights simultaneously, which is obviously impossible). Therefore, we have opted for using a weight which is proportional to the level of employment in each job at the overall EU level, which seems like a second best option considering that using the national weights is not possible. After all, the function of this weight is simply to make the contribution of each job proportional to its importance in terms of employment: it seems like a reasonable compromise to make the importance of each job in the correlation matrix proportional to its importance in overall EU employment.

Table 4.VI, like table 4.IV earlier, shows a correlation matrix between the educational rankings across the EU, but in this case the jobs have been weighted by their share in overall EU employment. Clearly, when the contribution of each job is proportional to its employment share, the correlations increase considerably: most of the correlations in the educational rankings are above .8 , none of them below .7. The contrast between tables 4.IV and 4.VI proves that an important part of the variability in the educational rankings takes place in very small jobs, jobs that account for a small share in employment. A correlation between .8 and .9 between two national rankings means that they share between $65 \%$ and $80 \%$ of their structure. This finding reinforces our previous conclusion that the rankings are similar enough as to prove that the rankings are certainly measuring the same thing across different countries. In fact, the level of correlation is in this case quite striking: it seems to suggest that, notwithstanding the important economic and social differences across Europe, there is an underlying hierarchy of jobs (at least in terms of relative educational levels) behind most European employment structures. This is an interesting and important finding, but it should not be taken too far: even when using weights, there is between 20 and $40 \%$ of variability in the educational rankings across Europe, which (assuming that the educational ranking is a proxy of the quality of each job relative to the other jobs within each country) is a considerable amount of difference. And the weighted results should not be taken as the true results, but as another approximation to complement the unweighted results shown in tables 4.IV and 4.V.

Table 4.VII shows the same weighed correlation matrix, but in this case for the wage rankings. As with the educational rankings, most coefficients are higher when the jobs are weighted (most of them are above .7): but the increase is smaller in this case than in 
the case of the educational rankings, which means that some of the large jobs occupy significantly different positions in terms of relative wages across Europe. The patterns identified for the unweighted data remain quite similar when the data is weighted.

\subsection{Exploring the correlation patterns by sector using principal component analysis}

The analysis of the correlation matrices of the previous section allowed us to study in detail the similarities between the wage and educational rankings of all possible pairs of countries. To get a better understanding of such patterns of similarity in the national rankings, it would be useful to study separately each of the broad sectors of the economy that we constructed in section 1 of this chapter. But using the correlation matrices for exploring the sector differences is totally impractical: we would be confronted with too much information, so much that it would be impossible to make any sense of it (we would need to inspect nine different correlation matrices-one for each sector-for each of the two rankings). We need some statistical tool to summarize the information contained in the correlation matrices so that it becomes more manageable. The obvious choice is factor analysis, a method specifically designed for reducing the information contained in many associated variables, and which is in fact basically a transformation of the correlation matrix (see Kim and Mueller 1978a; Kim and Mueller 1978b). We will use principal components analysis, a variant of factor analysis which decomposes the correlation matrix in its main components ${ }^{58}$ (factors which account for the maximum variance of the original variables, and which are linear combinations of the latter). We will use this technique with the sole purpose of summarizing and making more manageable the information contained in the correlation matrices, not to study the extracted common factor, which is the usual purpose of factor analysis.

The logic of the following pages will be the following: for the whole dataset, and for each sector separately, we will carry out a principal component analysis of the correlations between the country rankings, extracting a single factor; the variance explained by the extracted factor will be used as an indicator of the similarity in the rankings in each particular case, and the uniqueness of each national ranking (percentage of the variance not explained by the extracted factor) will be used as an

\footnotetext{
${ }^{58}$ The differences between using principal components and factor analysis in this case are very small and inconsequential, because the rankings are very highly correlated. Nevertheless, we opted for principal components because it is more directly a transformation of the raw correlation matrix, which is what we want to do. Factor analysis uses as the basis an adjusted correlation matrix and only tries to summarize the common variance, not all: it is more adequate for causal or explanatory analysis, whereas here we simply want to summarize the information contained in the original correlation matrices (see Suhr 2005).
} 


\begin{tabular}{|c|c|c|c|c|c|c|c|c|c|c|c|}
\hline \multirow[b]{2}{*}{$\mathrm{n}$} & Overall & Primary & \multicolumn{2}{|c|}{ Construction LTI } & HTI & LKIS & KIS & \multicolumn{2}{|c|}{ Publicadm } & \multicolumn{2}{|l|}{ Health } \\
\hline & & 523 & 21 & 17 & 141 & 40 & 127 & 117 & 21 & 18 & 21 \\
\hline Var expl. & & $89.29 \%$ & $79.17 \%$ & $89.89 \%$ & $82.77 \%$ & $86.79 \%$ & $82.16 \%$ & $88.95 \%$ & $90.19 \%$ & $95.32 \%$ & $94.78 \%$ \\
\hline Austria & 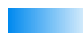 & $10.52 \%$ & $55.74 \%$ & $6.52 \%$ & $15.27 \%$ & $8.88 \%$ & $15.21 \%$ & $10.18 \%$ & $21.09 \%$ & $2.77 \%$ & $1.38 \%$ \\
\hline Belgium & 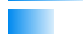 & $7.89 \%$ & $22.35 \%$ & $9.53 \%$ & $15.08 \%$ & $12.31 \%$ & $11.03 \%$ & $7.42 \%$ & $9.79 \%$ & $1.61 \%$ & $6.49 \%$ \\
\hline Cyprus & & $13.77 \%$ & $19.61 \%$ & $10.99 \%$ & $25.16 \%$ & $30.65 \%$ & $18.66 \%$ & $13.92 \%$ & $19.84 \%$ & $3.14 \%$ & $7.20 \%$ \\
\hline Czech Republic & 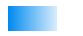 & $8.09 \%$ & $15.40 \%$ & $8.91 \%$ & $8.18 \%$ & $4.14 \%$ & $15.58 \%$ & $5.33 \%$ & $6.42 \%$ & $3.15 \%$ & $5.52 \%$ \\
\hline Germany & 口 & $14.55 \%$ & $14.53 \%$ & $11.89 \%$ & $20.73 \%$ & $4.84 \%$ & $27.39 \%$ & $5.74 \%$ & $12.73 \%$ & $2.76 \%$ & $3.39 \%$ \\
\hline Denmark & - & $12.73 \%$ & $45.27 \%$ & $19.53 \%$ & $23.23 \%$ & $9.33 \%$ & $13.17 \%$ & $11.27 \%$ & $17.50 \%$ & $4.06 \%$ & $5.04 \%$ \\
\hline Estonia & & $17.76 \%$ & $20.27 \%$ & $9.56 \%$ & $26.84 \%$ & $34.32 \%$ & $28.74 \%$ & $37.14 \%$ & $6.41 \%$ & $3.71 \%$ & $7.60 \%$ \\
\hline Spain & 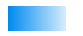 & $8.89 \%$ & $5.02 \%$ & $11.09 \%$ & $13.49 \%$ & $4.67 \%$ & $19.65 \%$ & $6.19 \%$ & $8.33 \%$ & $4.99 \%$ & $3.77 \%$ \\
\hline Finland & E & $8.52 \%$ & $17.07 \%$ & $4.69 \%$ & $16.95 \%$ & $6.48 \%$ & $13.28 \%$ & $9.04 \%$ & $5.29 \%$ & $13.90 \%$ & $4.51 \%$ \\
\hline France & 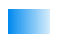 & $7.53 \%$ & $24.58 \%$ & $3.30 \%$ & $11.30 \%$ & $7.22 \%$ & $10.30 \%$ & $5.04 \%$ & $9.22 \%$ & $5.52 \%$ & $5.34 \%$ \\
\hline Greece & - & $9.48 \%$ & $16.34 \%$ & $12.00 \%$ & $11.76 \%$ & $6.87 \%$ & $20.18 \%$ & $12.50 \%$ & $8.22 \%$ & $2.75 \%$ & $3.15 \%$ \\
\hline Hungary & 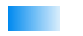 & $8.33 \%$ & $12.63 \%$ & $10.68 \%$ & $7.82 \%$ & $5.63 \%$ & $14.33 \%$ & $4.75 \%$ & $3.25 \%$ & $2.23 \%$ & $3.71 \%$ \\
\hline Ireland & E. & $6.98 \%$ & $31.93 \%$ & $5.39 \%$ & $10.79 \%$ & $10.61 \%$ & $9.80 \%$ & $5.03 \%$ & $14.31 \%$ & $10.09 \%$ & $6.36 \%$ \\
\hline Italy & E. & $7.22 \%$ & $10.25 \%$ & $13.09 \%$ & $9.12 \%$ & $3.63 \%$ & $16.86 \%$ & $8.53 \%$ & $4.23 \%$ & $4.11 \%$ & $3.91 \%$ \\
\hline Lithuania & & $17.71 \%$ & $21.67 \%$ & $19.08 \%$ & $33.66 \%$ & $31.77 \%$ & $26.17 \%$ & $25.24 \%$ & $11.50 \%$ & $2.72 \%$ & $8.18 \%$ \\
\hline Luxemburg & E & $11.24 \%$ & $40.37 \%$ & $4.11 \%$ & $23.56 \%$ & $17.54 \%$ & $13.81 \%$ & $9.79 \%$ & $15.92 \%$ & $3.61 \%$ & $3.37 \%$ \\
\hline Latvia & & $17.48 \%$ & $7.33 \%$ & $9.54 \%$ & $33.18 \%$ & $47.23 \%$ & $23.51 \%$ & $27.00 \%$ & $4.97 \%$ & $4.70 \%$ & $11.76 \%$ \\
\hline Netherlands & I & $5.32 \%$ & $13.09 \%$ & $1.89 \%$ & $14.23 \%$ & $5.67 \%$ & $7.25 \%$ & $4.39 \%$ & $7.13 \%$ & $7.71 \%$ & $3.27 \%$ \\
\hline Portugal & 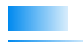 & $9.02 \%$ & $6.80 \%$ & $13.35 \%$ & $15.69 \%$ & $13.01 \%$ & $19.41 \%$ & $9.17 \%$ & $7.45 \%$ & $7.81 \%$ & $4.59 \%$ \\
\hline Sweden & E & $10.69 \%$ & $26.83 \%$ & $12.77 \%$ & $19.89 \%$ & $7.32 \%$ & $23.26 \%$ & $8.40 \%$ & $10.00 \%$ & $6.85 \%$ & $6.63 \%$ \\
\hline Slovenia & 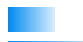 & $7.92 \%$ & $6.70 \%$ & $7.44 \%$ & $10.55 \%$ & $5.76 \%$ & $16.82 \%$ & $7.65 \%$ & $8.91 \%$ & $1.23 \%$ & $1.96 \%$ \\
\hline Slovakia & - & $11.74 \%$ & $16.23 \%$ & $8.69 \%$ & $11.52 \%$ & $7.74 \%$ & $25.33 \%$ & $11.68 \%$ & $4.94 \%$ & $2.13 \%$ & $5.85 \%$ \\
\hline United Kingdom & & $12.90 \%$ & $29.09 \%$ & $18.48 \%$ & $18.28 \%$ & $18.14 \%$ & $20.55 \%$ & $8.78 \%$ & $8.21 \%$ & $6.06 \%$ & $7.13 \%$ \\
\hline
\end{tabular}


That is what is shown in tables 4.VIII (for the educational ranking) and 4.IX (for the wage ranking). There are ten columns in the table: the first one shows the results of a principal component analysis for the whole economy, and the nine subsequent columns the same analysis for each of the broad sectors explained in section 2.1 of this chapter. The first row shows the number of observations (jobs) used for each principal components analysis. It may seem surprising that the numbers are relatively low: even for the whole economy, the analysis is based on only 452 jobs. The reason is that any job which is missing in at least one country cannot be included in the analysis, which means that we can only use those jobs for which we have a value in the educational ranking in all countries. Although it would obviously be better to cover all jobs identified in any country, this should not be a major problem because the missing jobs (those missing in at least one country) account for less than $8 \%$ of overall employment in the EU (the country with a highest proportion of missing employment is Estonia, where it is around 13\%; in most other countries it is below 8\%). For carrying out the analysis, the data has been weighted by overall EU employment, as explained earlier.

The number of observations becomes really small, though, for some of the sector breakdowns: for instance, the principal components analysis in construction is based only on 17 jobs. This may seem problematic in terms of the reliability of the results of the analysis, but in fact it is not necessarily the case, because we are not doing statistical inference here. We are simply using principal components analysis as a data reduction tool, to summarize the information contained in the correlations between the 23 national rankings so that it becomes easier to analyse. In the background, what we have is a data table with the relative position of each construction job within each national labour market in terms of the education of its workers: the correlation matrix is a way to summarize those relative positions (into a set of coefficients reflecting the association between the rankings of each pair of countries), and what table 4.VIII shows is only an even more summarized version of that correlation matrix.

The second row contains the variance explained by the first factor extracted (this factor is simply a linear combination of the original rankings, constructed in such a way that it explains as much of the total original variance as possible): for the whole of the economy (all sectors), the variance explained by the first factor extracted by the principal components method is $89 \%$. What this means is that almost $90 \%$ of the total variance contained in the original 23 rankings can be explained by a single common 
variable which is a linear combination of them. Although this percentage provides an indication of the existence of a very significant correlation between the national rankings (which we already identified in the previous chapter), we should be aware that this percentage is artificially inflated to some extent: first, because we are leaving out those jobs which are missing in at least one country, which account for less than $8 \%$ of total EU employment but which are likely to contain more variation than the jobs available everywhere; second, because as we saw earlier the fact that the data is weighted by employment also tends to inflate the correlation between the rankings. The extent of overall correlation was already discussed in the previous section, so we will not go back to it. The percentage of variance explained in the second row of table 4.VIII is there mostly to be able to compare the variability of the different sectors, not to evaluate, once again, the extent of total variability.

The 23 rows after the third contain the uniqueness scores for each of the national educational rankings. This uniqueness coefficient expresses how much of the variance of each variable (each national ranking in this case) is not accounted for by the factor extracted: therefore, as its name suggests, it is an indicator of how much does each country differ from the pattern shared by all countries. For the whole economy (first column), these coefficients show (in most cases confirming what we said in the previous section) that the most unique rankings are those of the three Baltic States (almost 18\% of unique variance in the three cases), followed by Germany (which we did not pick out as a atypical ranking in the previous section), Cyprus, the UK and Denmark.

But as we said earlier, we already discussed the patterns for the overall economy and we do not wish to go back to our previous conclusions based on a detailed analysis of the correlation matrix. The main objective of table 4.VIII is to compare the similarities in the rankings for each of the broad sectors of the economy (the first column, showing the whole economy, is there mainly for comparative purposes). We can see in the values of the second row that the most similar sectors across all countries in terms of the educational rankings are the public sectors: education, health and public administration. For these three sectors, the common factor explains more than $90 \%$ of overall variance. Construction and private knowledge intensive services are also relatively similar across Europe in their position within the national educational rankings, with almost $90 \%$ of variance explained by the first factor. The most dissimilar sectors are low technology 
industries, (private) less knowledge intensive services and the primary sector (around or below $80 \%)$.

In table 4.VIII, we can also inspect the uniqueness of each national ranking within each sector: in other words, how dissimilar are each countries in the educational positions they assign to the jobs of the different sectors. In many cases, the highest uniqueness is again that of the Baltic countries (in both industries, knowledge intensive services and health). But there are other countries that have high values of uniqueness in some specific sectors even if they are very similar to the rest in others. For instance, we can see in table 4.VIII that the relatively high uniqueness of Germany comes mostly from jobs in less knowledge intensive services, low technology industries and construction. Austria differs from the rest mostly in the primary sector and public administration. Finland and Netherlands only stand out in the education sector.

Table 4.IX shows the same type of analysis for the wage rankings. As could be expected (we had already identified this in the analysis of the correlation matrices), the variance explained by the first factor (which summarizes the overall similarity of all rankings) is considerably lower than in the case of education: around $77 \%$. The most atypical country (again, we had identified this earlier) is Greece, followed closely by the three Baltics, Cyprus and Denmark. By sector, the patterns are also rather different from those shown in table 4.VIII: the lowest variability appears in knowledge intensive services and the two industries, while the highest variability in the relative positions of jobs by wage appear in the primary sector, public administration and construction. 


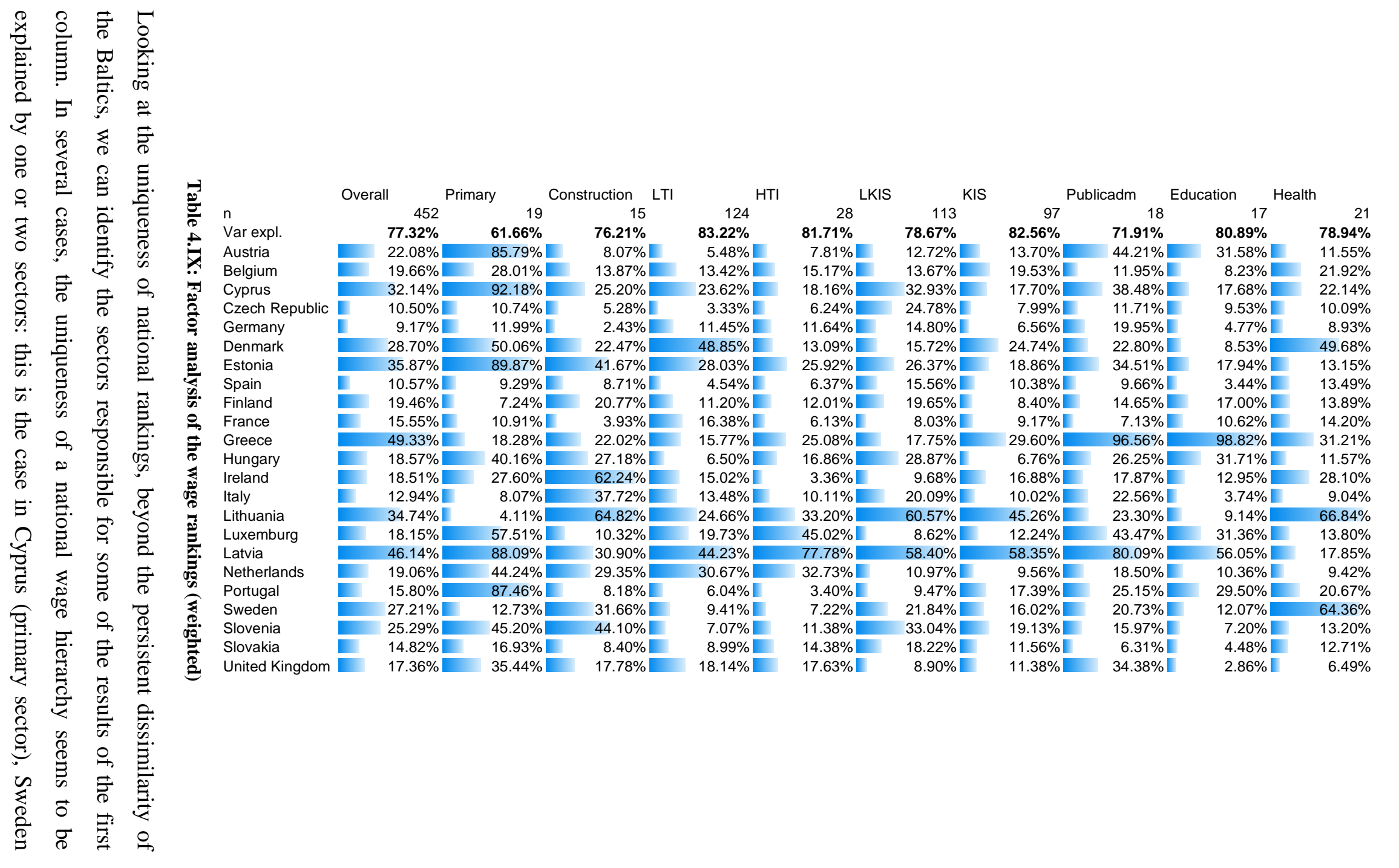


(health), Ireland (construction and health) or Denmark (health, low technology industries and the primary sector). But the most spectacular case is Greece, which is the most dissimilar country overall, and this dissimilarity seems to be totally concentrated in two public sectors: public administration and education. The uniqueness of these two sectors is so large (close to $100 \%$, meaning that the relative wages within those sectors in Greece have no relation whatsoever with those of the rest of Europe) that it seems suspicious. The very high uniqueness of Greece in the education sector is mostly due to a single job (teachers, ISCO code 23), which is by far the largest in this sector and which in Greece has a position in the wage hierarchy far below any other country. The wages of this job in Greece (teachers in the education sector) puts it in the second lowest quintile, whereas in all the other countries this job is either on the fourth quintile or even in some cases in the fifth. It is difficult to tell whether this large discrepancy between the Greek wage hierarchy for teaching professionals and the rest of Europe is real or the result of some error in the wage estimations: but the latter is not at all implausible considering recent news of very large-scale mobilizations of Greek teachers (see EIRO 2006; Vassilopoulos 2006). Wages in public administration in Greece are also highly unique (lower than elsewhere) according to our analysis.

\subsection{Some final remarks}

In this section, we have compared the job hierarchies for the two dimensions of job quality for which we have nationally-specific rankings. We have been able to provide some rough estimates of the degree to which the rankings vary across countries: depending on whether the data is weighted by employment or not, between $50 \%$ and $75 \%$ of the education and wage hierarchies are shared across Europe (only the Baltic states, and Greece in the case of wages, go significantly below 50\%). We could identify which countries and sectors differ more from the rest in terms of educational and wage hierarchies, and we also found that there is more variability in terms of wage than educational hierarchies. What is the significance of these findings? How much difference in the education and wage hierarchies across Europe could we expect? Do these results have substantial implications for the questions we made in the theoretical chapter?

A certain degree of correlation in the educational and wage hierarchies was certainly to be expected. After all, what we are doing is comparing the relative positions in two different quality dimensions for jobs which are defined homogeneously across 
countries. Going back to our justification of the jobs approach, the jobs in our jobs matrix correspond to relatively stable positions in the division of labour across the horizontal and vertical axes: each of these positions involve specific sets of tasks, requires specific skills, etc. From a material perspective, which is the way the jobs matrix is defined, the jobs in the matrix should be roughly the same irrespective to where they are located. To the extent that the formal educational level of job holders and the remuneration associated to each job is associated with its material characteristics (to its position within the division of labour), the hierarchies of jobs by those two dimensions should be similar across countries.

The main empirical finding of this section is that the rankings are similar, but not identical. On the one hand, this confirms the core assumption that the job structures are equivalent classifications of employment in terms of positions in the division of labour. The existence of a significant degree of cross-country correlation in the ranking of jobs by their educational level and wages (two rankings constructed with different data sources and through different processes) would be difficult to explain if the jobs approach was not capturing something real about how employment is structured in the different European countries. But at the same time, the fact that the correlation is by no means total, but leaves an important degree of national variability (as we said earlier, between $25 \%$ and $50 \%$ of the variability in the rankings is purely national) proves that it is necessary to use national rankings, and that similar evolutions in the structure of employment can have very different implications depending on how the different societies valuate the different jobs.

\subsection{A small illustration of the ranking variability at the job level across countries}

Before finishing this section, and in order to put some flesh into the very abstract analysis that we have been carrying out so far (such a high level of abstraction makes it very difficult to really grasp what we are talking about), we will present a brief illustration of the variability in the rankings at the job level. Table 4.X shows the quintile location in each country of the 10 jobs which account for a largest share of overall employment at the EU level (the share of EU employment accounted for each of these 10 jobs is shown in the third column of the table). For instance, the job which accounts for a largest share of overall employment in the EU is "extraction and building trades workers" in "construction" ( $4.48 \%$ of all employment). For most of the countries, the educational quintile of this job is the second one, but there are some exceptions: in 
Belgium, Estonia, Italy, Luxemburg and Netherlands, they are located in the first educational quintile, whereas in the Czech Republic, Germany and the UK, they are in the third quintile. In terms of wage, the position of this job is a bit better, because in most countries it falls in the third quintile, whereas in terms of amenities, this job is located in the first and second quintiles in more or less the same number of countries.

For this illustrative exercise, therefore, we have opted to look at the quintiles directly rather than at the raw rankings, to simplify the presentation and also to link this section with the following one, which is based on a static analysis of the quintiles. We have also included the position of these top ten jobs in the amenities quintiles, which we did not analyse before because in this case, the ranking is invariant across countries. We can see in the table that, although the ranking is invariant, the quintile allocation is not: the reason is that even if job $\mathrm{X}$ occupies the same ranking position in each country, the specific quintile position depends also on the employment levels by job. For instance, in the case of building trades workers in construction (the first job shown in table 4.X), the value of its normalized amenities ranking is .18: depending on whether the jobs which have a value below .18 account for less or more than $20 \%$ of employment in each country, this job is positioned in the first or second quintile (as shown in table 4.X, in more or less half of the countries this job is positioned in the first and the other half it is positioned in the second). This illustrates the relationship between the ranking and the quintiles: the quintiles are not only a reclassification of the rankings in five groups, but a reclassification that takes into account the employment size of each job when defining the boundaries between quintiles. The quintiles are, in fact, a kind of aggregated ranking weighted by employment in 2000 .

In any case, by looking at the quintile position of these ten most important jobs (numerically), we can get an idea of the implications of the variability in the rankings that we discussed in the previous pages. The two large professional occupations in the public sector (teachers in education and doctors in health), are extremely similar in terms of education (they are in the fifth quintile everywhere) and to a lesser extent in terms of wage (also in the fifth quintile in most cases), but not in terms of amenities (teachers are between the fourth and fifth quintile of amenities, and doctors between the second and fourth, considerably lower than in the other two rankings). The strange position of teachers in Greece is clearly illustrated in table 4.X: although in terms of education and amenities they are in a very similar position to the other countries, in 
terms of wages they are three quintiles below most of Europe, with no other country getting even close. In fact, with this single exception, all other jobs are concentrated in one, two or a maximum of three quintiles in table 4.X. This illustrates the extent of correlation in the rankings across countries, discussed in the previous pages: the rankings are very similar, and the resulting quintile positions of jobs across countries are rather similar as well; but this similarity (no job is spread across the table in its quintile allocation) does not mean that there is no variability. In most cases, the same jobs occupy different quintiles in different countries, even if they are adjacent: this variability implies that the differences in the job quality structures that we will study in the following section do not only depend on different employment structures, but also on different job quality rankings across countries (for the education and wages dimensions). 


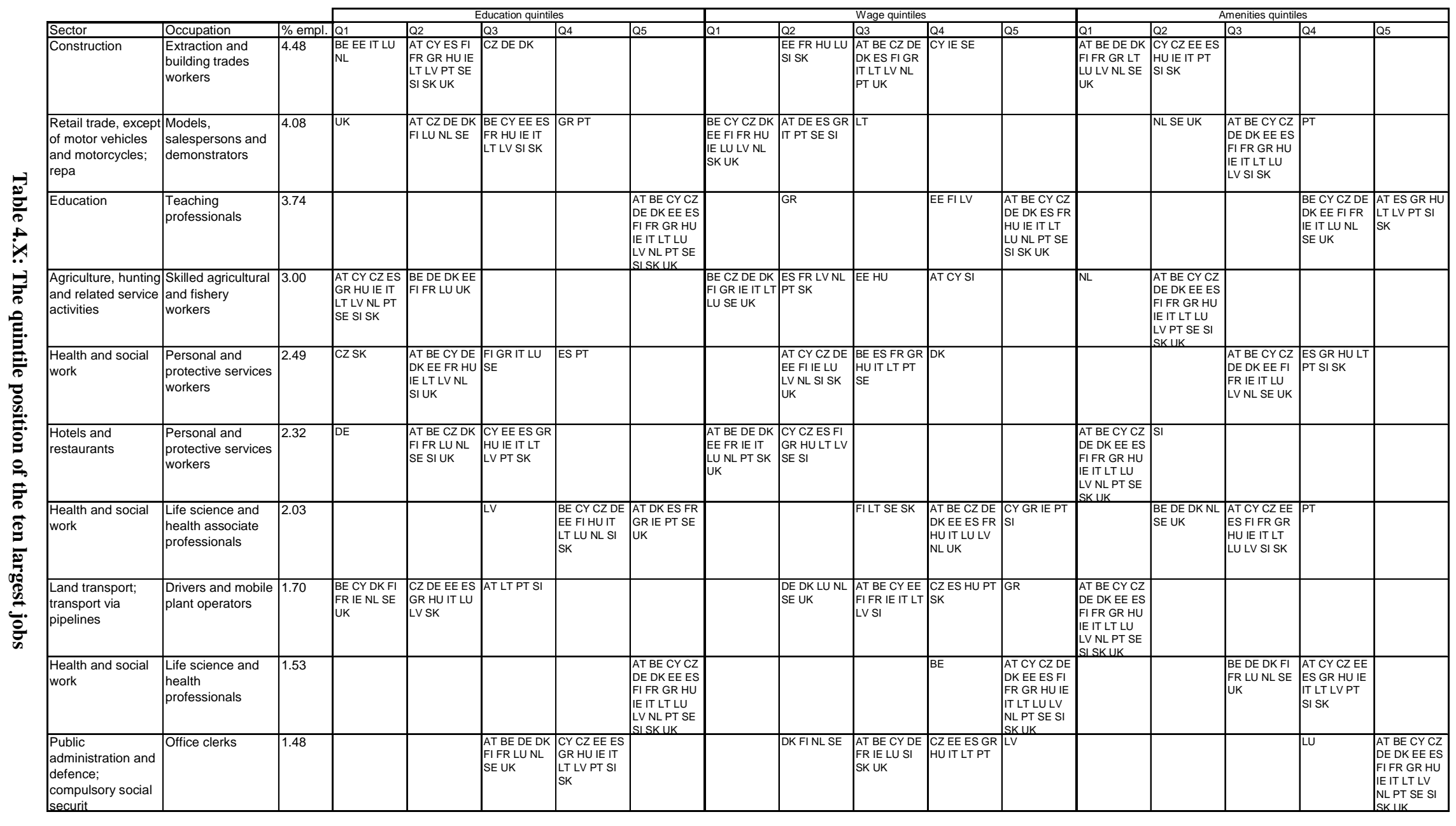




\section{A static comparison of job quality structures across Europe}

In the two previous sections, we compared from a static perspective the two main parameters behind the jobs approach: the composition of the division of labour (as captured by the occupation by sector matrix) and the job quality hierarchies (as captured in the wage and educational rankings). To finish this chapter, we will combine those two parameters into one and compare the employment structures from a job quality perspective, adapting the quintile method explained in the previous chapter to a static comparative analysis.

For the most part, the rest of this thesis will be based upon a detailed analysis of the patterns of job growth by job quality quintiles. Those quintiles are five groups of jobs aggregated in such a way that, for each country, they contain each the same number of workers in the year 2000, sorted from low to high in terms of one of the three job quality rankings. By looking at how the size of each of those quintiles changed between 1995 and 2007, we can evaluate the implications for job quality of the changes in the composition of the division of labour.

In this sense, the idea of comparing the quintiles from a static perspective seems, in principle, completely absurd. By definition, for the year 2000 (the year we are using for the static comparisons), each country will have exactly the same quintile structure, with around $20 \%$ of employment in each of the quintiles. If we would compare the quintile structure for any other year, what we would in fact be comparing is the different patterns of job growth, because the quintiles can only differ insofar as there is differential job growth across them in the different countries. If we would compare the allocation of jobs to the different quintiles, we would be doing essentially the same exercise as that of the previous section, only using the quintiles instead of the rankings. ${ }^{59}$ So what do we mean when we say we will compare the quintile structures in $2000 ?$

What we will do is to apply the allocation of quintiles of one particular country to all the others, generating a new set of counterfactual quintile charts that will reflect the difference between the countries, using Germany as reference. Each of those counterfactual quintile charts can be understood in the following way: how would the

\footnotetext{
${ }^{59}$ In fact, this is exactly what is done in the small illustrative appendix of the previous section, for the top ten jobs in terms of employment.
} 
German job quality structure look if its employment structure was transformed into the employment structure of Spain, for instance? What these counterfactual charts represent is the distance between the job quality structures of each country and that of Germany in 2000: and therefore, they allow us to evaluate the (differences in the) starting point of the dynamic quintile charts that we will analyse in the next chapter.

Why use Germany as the reference country? We could have used any other country as a point of reference instead of Germany, or even the EU as a whole. But Germany seems to be more or less in the middle of Europe, both geographically and in terms of its economic structure, so it seems like a good choice for a reference point. Using the EU aggregated figure, on the other hand, would be more difficult to interpret, because we would be effectively comparing each country to a chimera.

Figure 4.3 shows the set of counterfactual quintile charts for 2000, based on the German educational ranking. We have included also the German quintile chart (even if it is unnecessary) to help the understanding of the figure (Germany is shown at the top righthand side of figure 4.3). The German chart of figure 4.3 is, in itself, tautological: it only reflects that, by definition, each of the quintiles holds around $20 \%$ of overall German employment in 2000 , with jobs being sorted by their educational ranking. But this figure is interesting as a reference point, because the same quintile allocation which is behind this tautological German figure has been used in all other countries, and therefore the difference between each other country and the German pattern reflects the difference in their job quality structures. For instance, if we look at the Spanish picture, we can see that the Spanish employment structure is considerably biased downwards with respect to the German employment structure in terms of the educational level of job-holders. In particular, the same jobs that occupy the lowest $20 \%$ of the employment structure in terms of education in Germany, account for almost 30\% of employment in Spain; at the same time, the jobs that occupy the top $20 \%$ in terms of education in Germany account for $15 \%$ of employment in Spain (and roughly the same for the fourth quintile).

What we are doing here is taking advantage of the fact that the jobs structure is identically defined in all countries (each country has the same classification of sectors and occupations): what changes is the actual proportion of people in each job. Therefore, we can apply the quintile allocation of one country to any other country, and the resulting picture will tell us how do their employment structures differ in terms of 
one of the three job quality dimensions. In other words, we keep fixed the quintile allocation to focus our attention on the differences in the structure of employment.

This counterfactual quintile picture can be easily compared with the dynamic quintile picture which we will discuss in the following chapter, and which constitutes the main finding of this thesis. In the following chapter, we will see how the different quintiles grew between 1995 and 2007 across Europe, whereas in figure 4.3 we see how much did quintiles differ originally (in 2000).

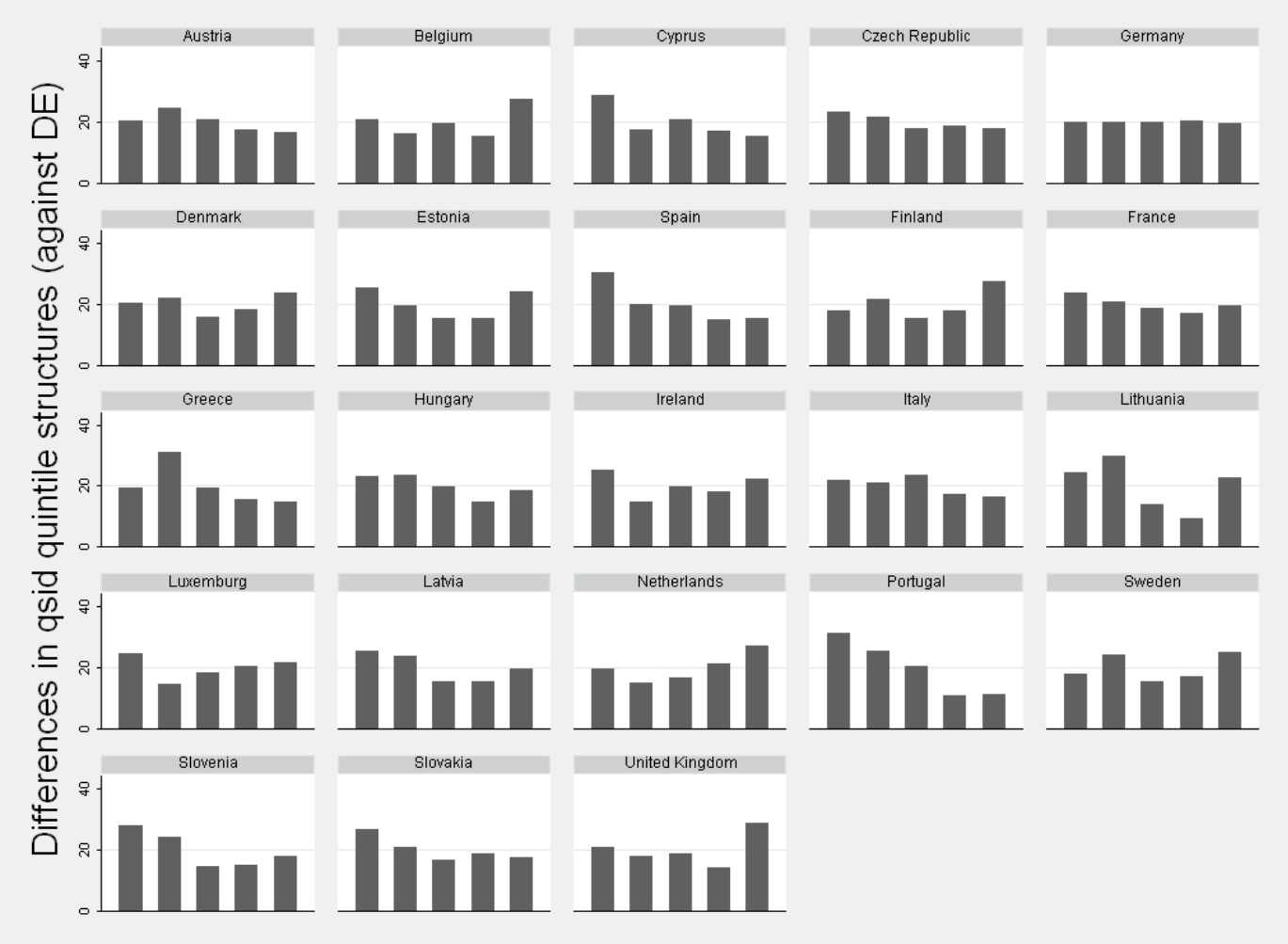

Figure 4.3: Counterfactual distribution of educational quintiles, using Germany as reference 


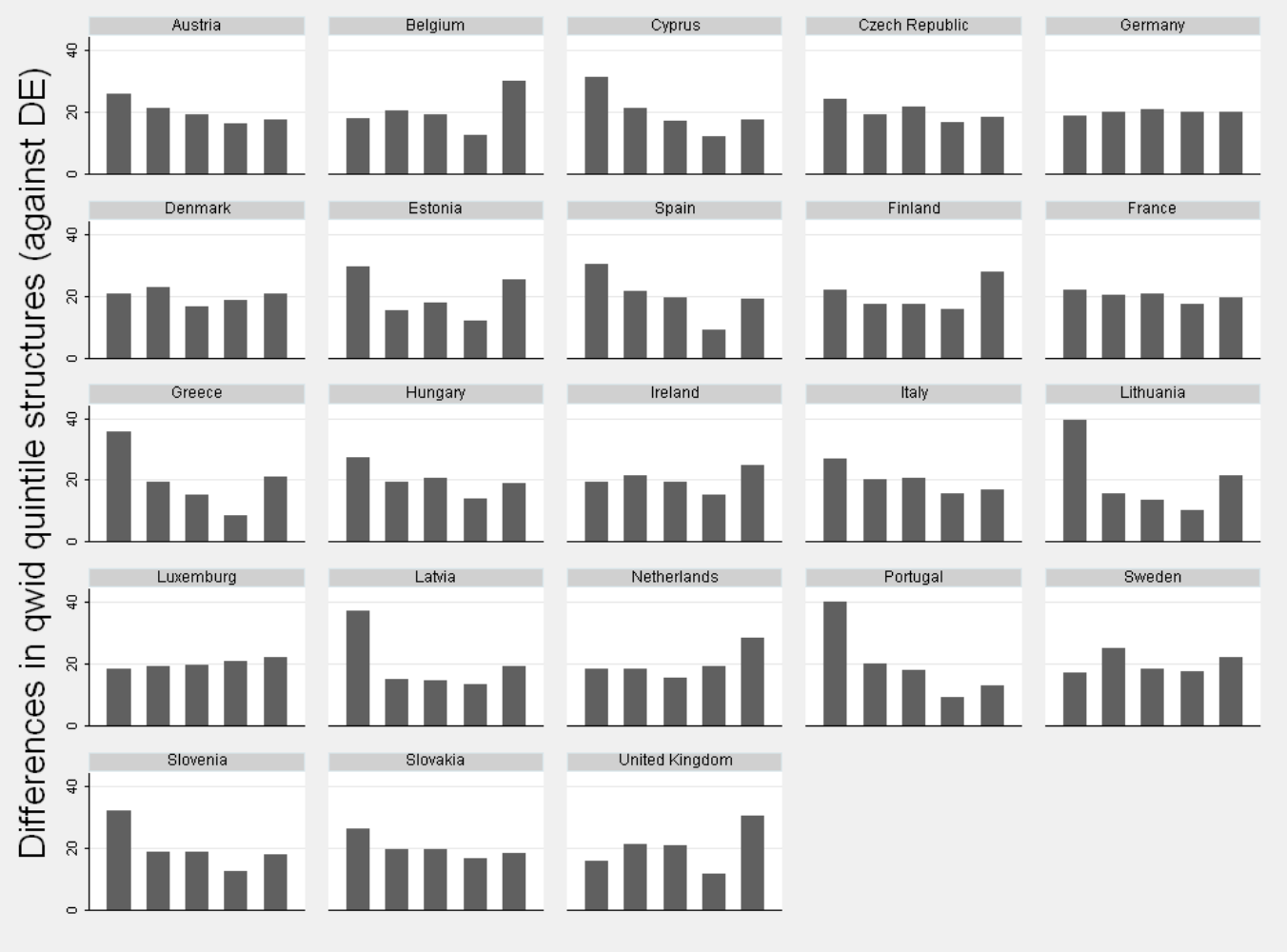

Figure 4.4:Counterfactual distribution of wage quintiles, using Germany as reference

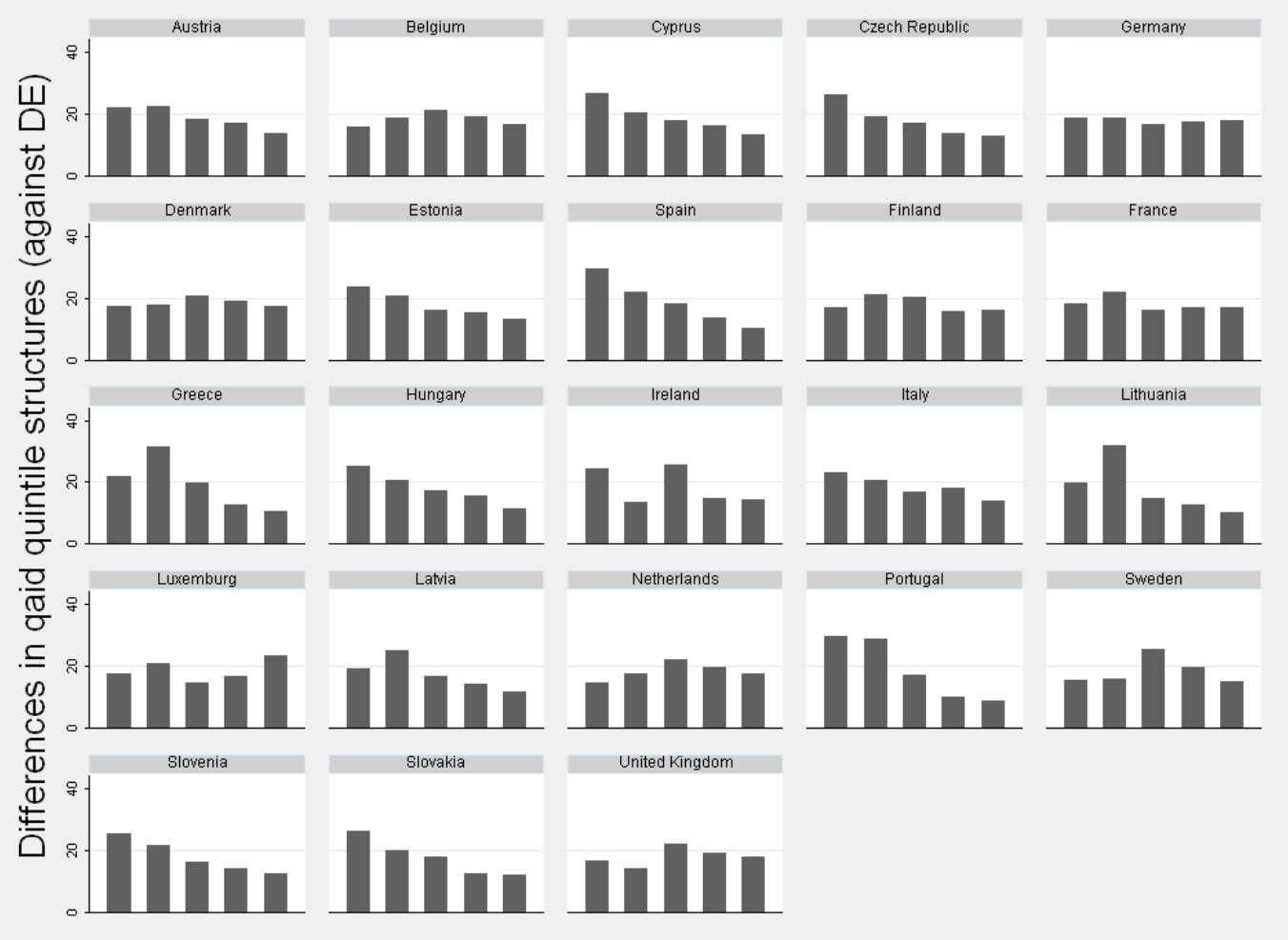

Figure 4.4: Counterfactual distribution of amenities quintiles, using Germany as reference 
In any case, figures $4.3,4.4$ and 4.5 show these counterfactual quintile pictures (with Germany as reference point) for the educational, wage and amenities job quality measures. The first thing to be noticed is how remarkably consistent the picture given by the three job quality measures is. Of course, this was to be expected after the results of the correlation analysis discussed in section 4.4 of the previous chapter: but although the correlations were quite high, they were not perfect, and it is always difficult to evaluate what is "high" or "low" in a statistical coefficient unless we look at how it impacts on the actual results. What the consistence between figures $4.2,4.3$ and 4.4 proves is that the three different rankings do imply rather similar pictures of the structure of employment by job quality. They are so similar, in fact, that with very few exceptions the interpretation is more or less the same in the three cases. Therefore, we will discuss the country differences in terms of job quality in general, without making much reference to the differences between the three job quality measures, except when we find it necessary because of some significant exception.

A second important conclusion that we can draw from figures $4.3,4.4$ and 4.5 is that the countries tend to group together in a similar way as we discussed in the first section of this chapter. This seems perfectly logical: it shows that the differences in the composition of the division of labour by sector and occupation are linked to differences in job quality structures. The same European regions that-as we argued in section 2 of this chapter-have a less developed structure of division of labour (higher share of employment in agriculture and industries, lower share in knowledge intensive and public services, as well as in professional/technical occupations) are characterized by a bigger share of low quality jobs, defining job quality with the German standard. This result is consistent across the three job quality quintiles, with few exceptions. The clearest case is Southern Europe: the counterfactual German-based quintile picture is in these countries clearly biased downwards. The jobs that occupy the lowest quintile in Germany occupy a much larger share of employment in Europe (between 30 and $40 \%$ in Greece, Portugal, Spain and Cyprus), while the jobs that make the top two quintiles in Germany (40\% of employment in better jobs) account for less than $30 \%$ in Southern European countries. The only Southern European country that departs slightly form this pattern is Italy, although even in Italy the quintile structure is slightly downwards biased. Most of Eastern Europe is also downwards biased with respect to Germany in their job quality structures, although in most cases the bias is milder than in the 
Southern region. The exceptions are Estonia and Lithuania (and Latvia to a lesser extent, depending on the job quality dimension), which seem to have a structure of employment which is polarized in terms of job quality in comparison with the German structure.

On the other hand, most Continental and Northern European countries look rather similar to Germany in their job quality structures, with some exceptions. In particular, the Netherlands, Belgium and the UK seem to have a higher share of good jobs than Germany: that is, the jobs which occupy the top $20 \%$ of the distribution in Germany account for a significantly larger share of employment there (near 30\% of employment in these three countries). This only happens, though, for the educational and wage rankings: in terms of amenities, in fact, Germany seems to be one the best countries in Europe. Only Luxemburg has a slightly better job quality structure in terms of amenities than Germany: all the other countries (including those that have an upwards biased structure in the other two dimensions) have either a similar picture (such as France) or clearly worse (most of the rest).

\section{Conclusions}

In principle, this chapter had a secondary role to play in this thesis. Its only objective was to set the background for the dynamic analysis of the following three chapters. Yet, the results of this static analysis of the two parameters behind the jobs approach have substantial interest on their own, as a comparison of the employment structures across the EU from a job quality perspective.

The first section of this chapter presented a broad overview of employment structures across Europe, from the perspective of the composition of the division of labour which is at the core of this approach, and using the univariate distributions of employment by broad sectors and occupations as main tool. The employment structures of NorthWestern Europe seem more advanced in terms of a hypothetical transition towards a Post-Industrial/Informationalist division of labour, whereas the SouthEast has still a

long way to go. This way, the static analysis seems to provide some hints with respect to what kind of results may be expected in the rest of this thesis: the existence of such an axis suggests a specific pattern of job growth for SouthEastern Europe (assuming that they would take a similar development path as the most developed European 
regions), and begs the question of whether the changes between 1995 and 2007 led to some type of convergence in employment structures.

In the second section, we analysed in detail the qualitative element of our methodology, the three job quality rankings, in terms of their differences across countries. Whether there are significant differences in the job quality rankings has important implications for this thesis, both methodological (is it necessary to use different national rankings?) and substantial (are identically defined jobs in terms of their content different in their quality across Europe?). One of the hypotheses of this thesis, in fact, is explicitly predicated on the existence of different rankings (the idea that a similar development in terms of structural change can lead to different human implications depending on the way societies value the different jobs). The empirical findings of this section were rather positive for our objectives, precisely because they were not very decisive in any direction. We could estimate that the educational and wage rankings of jobs are shared to an extent ranging between 50 and $70 \%$ (the results differ depending on whether the jobs are weighted by employment or not). We believe that this result supports the robustness of the whole approach, because it shows that the job matrices are comparable and that the rankings are measuring the same thing in different countries (otherwise, such a high correlation would be impossible to obtain); yet, at the same time, the fact that between 30 and $50 \%$ of the rankings are specific for each country implies that the national differences are important, that we should use the national rankings where possible and that indeed, the same structural evolutions can have different implications for job quality.

The final section put together the two parameters discussed previously and used the quintile picture to compare statically the initial job quality structures across Europe. On the one hand, this section corroborated the findings of the previous two sections in a simpler and more compact manner. On the other hand, it provides a direct link with the rest of this thesis. In the following three chapters, we will use the quintile pictures to analyse the nature of employment creation in the period between 1995 and 2007: the charts shown in figures $4.3,4.4$ and 4.5 of this chapter provide a first approximation too the job quality structures whose transformation we will study later.

The coherence between the quintile pictures of section 4 and the broad differentiation of employment by sector and occupation of table 4.I is not a trivial finding. It proves that the development of the division of labour is strongly associated with the quality of jobs 
existing in the different economies, and it suggests that at least in broad terms, the arguments of Post-Industrialists are corroborated by the facts. The same countries which are characterized by a less advanced division of labour are those with more abundance of relatively low quality jobs (we used here the German quintiles as reference, but any other reference would yield very similar results), and vice versa. Still, because in this section the analysis is only static, we can only provide suggestive, but not conclusive, evidence for any of the hypotheses that we are trying to validate. In the following three chapters, we will look at the same issues but from a properly dynamic perspective: then we shall be able to answer our hypotheses more conclusively. 


\section{Overall patterns of job growth in Europe 1995-2007}

\section{Introduction}

This chapter constitutes the core of the whole thesis. Although the analytical tools we will use are relatively simple (basically a graphical presentation of job growth by job quality quintiles), they provide a direct test of the validity of the three main hypotheses extracted from the literature on the impact of the changing division of labour (from a compositional perspective) on job quality.

As we argued in the theoretical chapter, the economic and sociological literature on the impact of the changing composition of the division of labour on job quality has been dominated by three broad images of change. The first one, which is as old as the modern concept of division of labour (starting with Adam Smith and finishing with Harry Braverman), is the most pessimistic: the nature of technological change and the very principle of the division of labour inevitably tends to degrade human labour, continuously shifting employment from skilled and semiskilled to deskilled routine occupations. The second one, which gained ground with the Post-War shift to services which is in fact its stronger argument, is the most upbeat: people like Fourastié and Daniel Bell argued that the huge increases in productivity brought about by industrialization tended to shift large amounts of labour to new activities in the service sector, characterized by much more human conditions of work and employment, and higher rather than lower skill requirements. The third and most recent hypothesis, a kind of reconciliation between the pessimists and optimists, argues that technological change and globalization tend to expand simultaneously both extremes of employment structures, while depressing the activities that in the Post-War period formed the middle of the job quality distribution: all in all, leading to a polarized pattern of job growth. Each of these three hypotheses predicts a very specific pattern of change of job quality 
structures, and therefore, should be relatively easy to test with our jobs approach: we will simply evaluate whether the transformation of the jobs structure across 23 European countries between 1995 and 2007 fits a downgrading, upgrading or polarizing image of change.

Of course, testing these three broad hypotheses of change is not going to be so simple. Although this chapter (and more specifically, its second section) arguably constitutes the core of the thesis, the simple inspection of the empirical patterns of employment growth by job quality leaves many things still unclear or difficult to interpret: the third and fourth sections of this chapter, and the two following chapters, are basically an attempt at clarifying those dark spots to be able to evaluate adequately what has been the dominant pattern (or patterns) of job growth in Europe between 1995 and 2007, and why. The structure of this chapter, therefore, will be the following. Its second section will be a direct analysis and evaluation of the overall implications of the changing composition of the division of labour for job quality (in 23 European countries, between 1995 and 2007). Sections three and four play around with the two main parameters of the jobs methodology (the change in the distribution of employment across jobs, and the job quality rankings) in order to shed some light on two particular confounding factors, without leaving the simple analysis of the overall patterns of job growth. Section 3 will try to evaluate whether the main determinant for the observed patterns of job growth across Europe are the changes in the distribution of employment across jobs, or differences in the job quality structures in the different countries. In section 4, we will try to evaluate whether (and to what extent) do the observed patterns of job growth between 1995 and 2007 simply reflect the fact that the initial positions of the 23 European countries were very different in terms of economic development and composition of the division of labour. ${ }^{60}$

\section{The transformation of job quality structures across Europe 1995-2007: identifying and classifying broad patterns of change}

In this section, we will discuss in detail the patterns of change in the job structures of the 23 European countries covered here, between 1995 and 2007. We will discuss these

\footnotetext{
${ }^{60}$ A small terminological clarification: in this chapter, we will use indistinctively "patterns of job growth" and "patterns of employment expansion" to refer to the shapes of the quintile pictures, which represent the transformation of national employment structures in terms of job quality between 1995 and 2007. The reason is simply to avoid too much repetition of the same (and very long) terms.
} 
patterns in the light of the theoretical arguments presented above: our main goal will be to identify to which image of change each national pattern resembles most, classifying them accordingly. Therefore, this chapter will be mostly descriptive and classificatory: but because the hypotheses which derive from the literature make very explicit predictions about how the patterns of change in the employment structure should look like, such description/classification will have a very important role in the refutation or validation of our primary hypotheses.

Our interests in this section are totally focused on change: more specifically, on how the job quality quintiles changed quantitatively between 1995 and 2007 in each European country. For this reason, the core of this section (and in many ways, the core of this thesis) will be a graphical representation of the change in the absolute levels of employment for the five job quality quintiles across Europe (figures 5.1-5. 3). In this initial analysis, we will focus on the shape taken by overall employment growth when such growth is split in five segments of equal initial size and ordered by job quality. To be able to identify better and classify those shapes, we will initially ignore the actual scale of change in the different countries, which varied widely between 1995 and 2007. A second, complementary representation of the patterns of employment growth by job quality is therefore necessary, one which puts those patterns in the context of the initial structures and therefore allows an evaluation of the scale of those changes in the different countries (figures 5.4-5.6). With those two different representations of essentially the same data (one looking at change in abstraction from everything else, another one putting it in the context and scale of the initial structure), we will try to provide a first answer to the main question of this thesis, that is, what were the implications for job quality of the compositional changes in the division of labour in Europe between 1995 and 2007.

\subsection{Focusing on the patterns of change}

So what do figures 5.1, 5.2 and 5.3 tell us about the transformation of European job structures in the last decade and a half? The first and most obvious observation we can make from these figures is that there was a considerable amount of variability in the patterns of employment expansion across the different countries (in any of the three job quality dimensions). Such variability does not seem to be related to differences in the levels of economic development: countries with a similar level of development look as different as countries with a different development level. In some ways, this may seem 
like an obvious finding, but it is certainly not trivial from a theoretical perspective. None of the theoretical approaches discussed earlier, each of which proposes a specific image of change, can on its own explain what happened to European employment structures between 1995 and 2007. In fact, our findings are in sharp contradiction with the findings of another recent study using a very similar methodology and also trying to evaluate the patterns of job growth across Europe: the main conclusion from that study was that all around Europe, the expansion of employment had been of a polarized nature in terms of job quality (Goos, Manning and Solomons 2009). The contrast is so important that we will devote a sub-section (2.3) to explain the reasons for this discrepancy, and to justify why we believe that our findings are considerably more robust. Our figures 5.1-5.3, in their conspicuous pluralism, suggest the existence of multiple causal forces behind the evolution of job structures across different countries. They make it very difficult to invoke any single-factor or universalistic explanation. The variability of patterns in figures 5.1-5.3 suggests that it is necessary to look at nationalspecific factors, such as institutions, to explain the evolution of job quality structures.

There would be, nevertheless, two possible lines of defence of a deterministicuniversalistic argument in the face of the empirical variability of figures 5.1-5.3. What we are looking in these figures is the job quality structures rather than the employment structures as such. Because the rankings vary across countries, it may be that a similar evolution in terms of the compositional division of labour leads to different outcomes in terms of job quality. This is an important argument, so we will discuss it in detail in section 3. A second argument in defence of a universalistic hypothesis would be that the different countries did not start from the same position: hence, all countries may have experienced exactly the same path of change in their employment structures, but as we are studying just a slide of such path (and a different one in each case), the observed variability could only be apparent. This is another important argument, which argues for some type of convergence or parallel evolution of European employment structures, and which will be discussed in detail in section 4 .

In any case, once verified that there is a fundamental plurality of patterns of job growth across countries, we can try to identify to which of the three images of change do the empirical patterns resemble most. We can quickly conclude that the hypothesis of downgrading does not find any empirical confirmation with our jobs-based approach. The only cases that could fit this hypothesis are Estonia (where the lowest or second 
lowest quintile - depending on the ranking used - grew relative to the rest) and Slovakia (where there was a polarization strongly skewed to the bottom): no other country, using any of the three job quality rankings, experienced a downgrading in its employment structure (a shift towards the bottom of the job quality structure). This is consistent with most empirical research which, since the 1980s, and from different perspectives, has refuted the predictions of the degradation of work hypothesis (for a review, see Edgell 2006: 53-59).

Most of the patterns in figures 5.1-5.3, therefore, conform to either the upgrading or the polarization image of change, or some combination of both. There are a few instances (such as Spain, Greece and Portugal, mostly in the wage quintiles) where there seems to be a pattern of growth in the middle of the quintile structure which does not fit into any of the images of change discussed in the literature: but even in these cases, such "growth in the middle" is considerably biased upwards, so that we can say that these are more or less idiosyncratic cases of upgrading. In fact, if we have to identify one single image as the most pervasive across all countries (and rankings) - assuming the existence of a fundamental variability in these patterns, as mentioned earlier - this image would be, without any doubt, one of upgrading. With few exceptions, almost all the patterns of job growth across countries and rankings involve some type of upgrading: even in those cases in which there is a more evident polarization, such polarization is often clearly biased upwards (for instance, in France, Germany or Cyprus). The exceptions are Netherlands (which is the clearest case of polarization in the wage ranking, with only a marginal upgrading in the educational ranking and a more or less flat pattern in the amenities ranking of jobs), Slovakia (consistent polarization across the three rankings), Austria (which, as can be seen in the incoherence between the pictures given by the three rankings, is a rather problematic country in any case; see annex 6) and the three Baltic countries (which as we discussed in the previous chapter, are rather problematic as well: the quintile charts seem to be rather unstable across the quintiles, and therefore we have to be careful with their results).

So although there is a variety rather than a single pattern characterizing the transformations of the employment structures across Europe, the most pervasive image of change of the three that were postulated in the theoretical discussion is, without any doubt, one of upgrading in the employment structure. Besides, there are some countries characterized by a polarizing development, and a few unclassifiable cases. The 
individual country charts of figures 5.1-5.3 have been rearranged in columns, putting together countries with relatively similar patterns of employment growth according to the wage ranking. This arrangement of countries will be used throughout the rest of this chapter, and the following one as well. As can be easily seen, each ranking yields slightly different results, so no classification of countries can be consistent for the three rankings (for instance, France is clearly polarizing in terms of wage; marginally polarizing but mostly upgrading in terms of education; and totally upgrading in terms of amenities). We had to opt, therefore, for one of the three rankings in order to classify the patterns: the amenities ranking is not an option because, as argued earlier, it is the most problematic (it is not country-specific and it does not cover the whole sample) and should play only a complementary role; between the educational ranking and the wage ranking we opted for the wage ranking (figure 5.1) because it is the most directly linked to job quality of the two, and because it is the ranking we used for classifying countries in our earlier work (see Fernández-Macías and Hurley 2008). In any case, this categorization is just a heuristic tool, a way to describe the results more meaningfully. The departures from this classification in the educational and amenities rankings will be discussed as well.

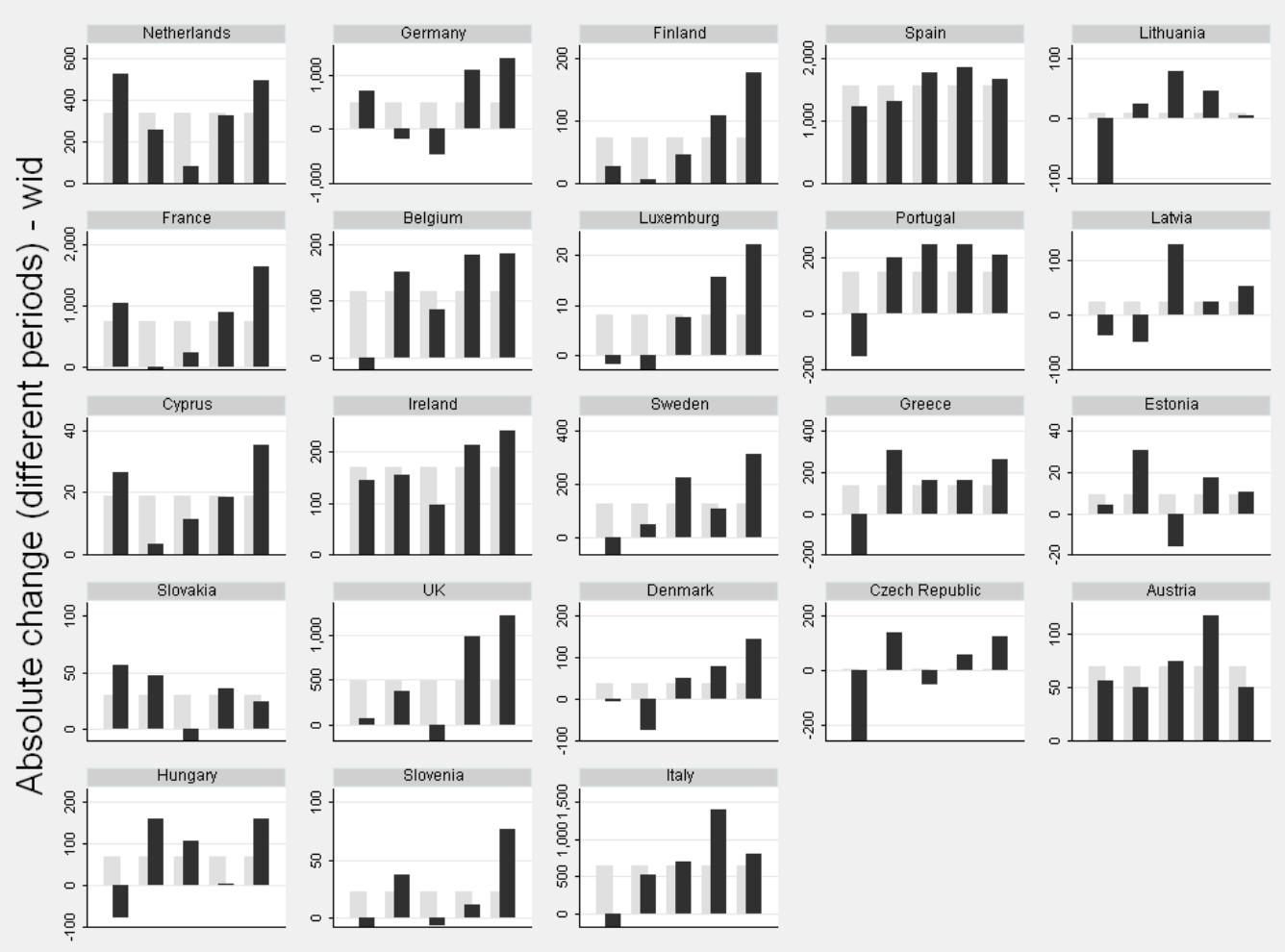

Figure 5.1: Absolute change in the wage quintiles (different periods) 


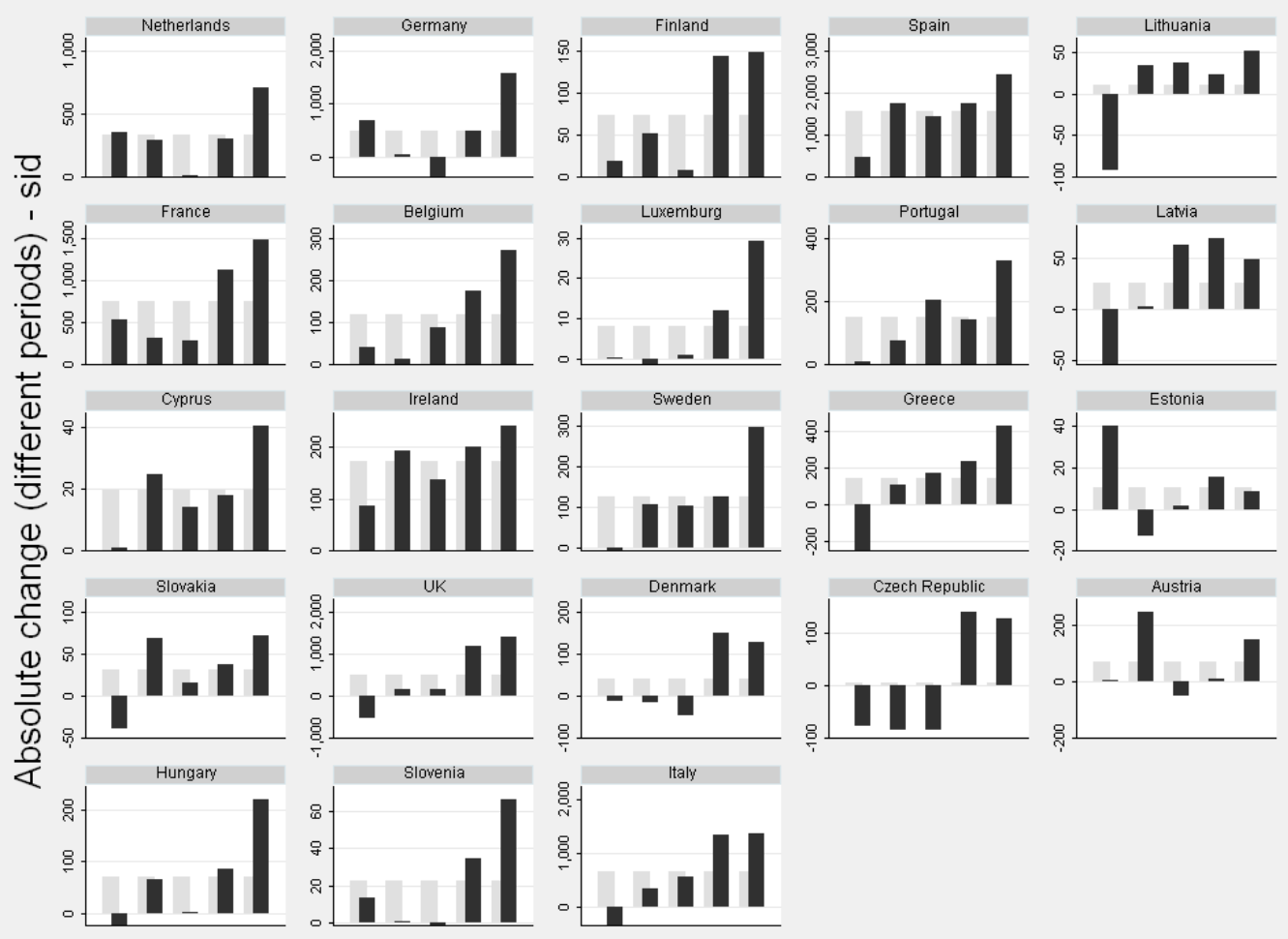

Figure 5.2: Absolute change in the educational quintiles (different periods)

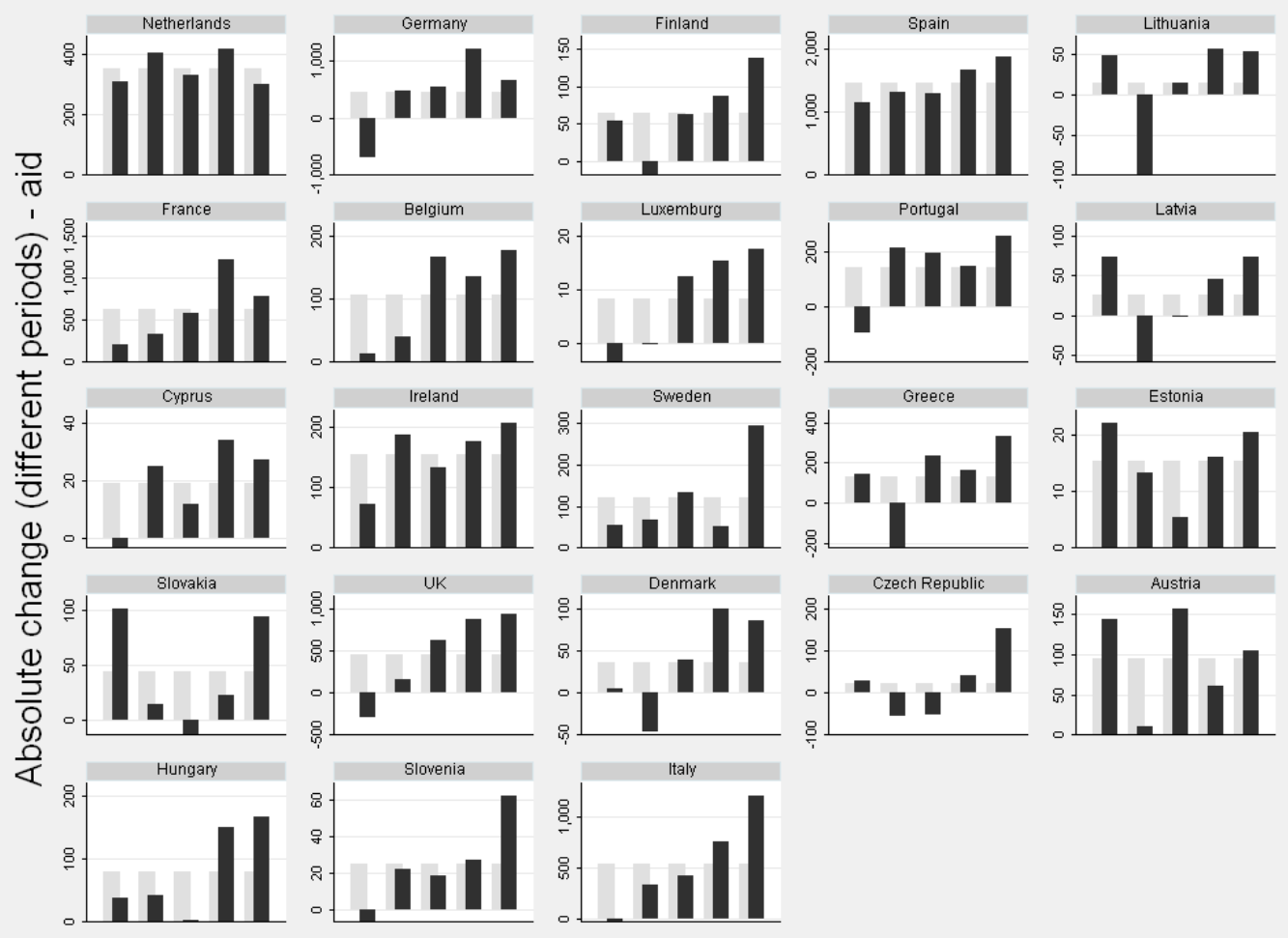

Figure 5.3: Absolute change in the amenities quintiles (different periods) 
Therefore, figure 5.1 shows the patterns of employment expansion based on a ranking of jobs by their median wage, and serves for classifying countries according to their resemblance to the theoretical images of change discussed earlier. Each of the country charts shows growth in absolute employment across the five quintiles (in thousands) in the period covered (in most cases 1995 to 2007, but with some exceptions as explained in chapter 3), represented with black bars. The grey bars behind show how each bar should be if all five quintiles had grown equally (that is, if overall employment growth was equally split across the quintiles): the comparison between the black and the grey bars gives an indication of how much did the expansion of each quintile deviate from overall job growth. The bigger the gap between the black and the grey bars, the more change in the job quality structure over the period (if the black and grey bars would be of the same size, there would have been no change in the job quality structure from a quintile perspective).

Each column of charts in figure 5.1 corresponds to a distinct category with respect to the images of change discussed in the literature. We will discuss each of these columns one by one:

- The first column (on the left) includes the five countries whose pattern is closer to the polarizing image: the Netherlands, France, Cyprus, Slovakia and Hungary. In all these countries, job growth was concentrated at the extremes of the wage distribution, with an important through in the middle: accumulated over the years, such development amounts to a polarization of the employment structure (later, we will discuss the actual impact of this effect on the overall structure of employment).

- The second column shows countries which were also polarizing, but simultaneously upgrading their employment structures. This category includes Germany (which although upgrading, is strongly polarizing as well: it could have been placed within the first column), Belgium, Ireland, the UK (the mildest case of polarization) and Slovenia. In all these cases, job growth tended to concentrate on the extremes as well (again, with some type of gap in the middle), but the growth at the top was much more important that at the bottom, so that the overall effect is more one of upgrading than one of polarization. 
- The third column includes the clearest cases of outright upgrading: these are Finland, Luxemburg, Sweden, Denmark and Italy. In these five countries, nearly all job growth was concentrated at the top - in some cases there was even net job destruction at the bottom -. Overall, this development implies a significant upgrading, which we shall evaluate later when we put it in the context of the initial structure.

- The fourth column includes four countries which experienced some degree of upgrading as well, but with strong growth in the middle of the wage distribution: the clearest case is Spain, but Greece, Portugal and the Czech Republic have also been classified in this group. In the three later cases, there was also a very significant destruction of employment in the very bottom of the wage hierarchy (quintile 1).

- Finally, the fifth column includes four countries that are difficult to classify and generally problematic: the three Baltic countries and Austria. Lithuania and Latvia could more or less fit in the fourth column, because job creation was somewhat concentrated in the middle of the wage structure: but clearly, the patterns of job growth of these countries look different from the rest. As we have already said, Estonia is a clear outlier, maybe the only case that clearly the downgrading hypothesis. And although Austria looks relatively similar to the patterns of the fourth column, it changes so much when using the other rankings (it is the country that changes most) that we preferred to put it on the category of oddities. We have reasons to suspect the reliability of the results for these four countries: not only do their patterns look weird, but they are very inconsistent when using the three rankings. ${ }^{61}$

As previously mentioned, the distance between the black and the grey bars gives us an idea of how biased in terms of job quality was employment change in each country. In Spain, Austria and Ireland, the five wage quintiles expanded rather evenly, and therefore the extent of change in the job quality structure was in fact relatively limited.

\footnotetext{
${ }^{61}$ The wage and educational rankings for the three Baltic states, as we saw earlier, are themselves rather different from those of the rest of Europe. It may be that the strangeness of the Baltics simply reflect the peculiarities of their economic evolution in recent years (which has had the pace and consistency of a roller coaster), or it may be that the ELFS data is not so good in those countries: in any case, we cannot make much sense of their results, so we will show them for reference, without paying them much attention to them. With respect to Austria, to some extent, its patterns are a statistical artifact derived from a reclassification of ISCO in the middle of the period. In annex 6, we can see an illustration of the impact of such reclassification on the Austrian patterns of job growth.
} 
In most of the other countries, the transformation of the jobs structure seemed to be considerably biased by job quality. But in any case, when we discuss figures 5.7-5.9 we will make a more adequate evaluation of the extent of change in the initial structure of employment.

How much does the picture change when we look at job creation by the educational and amenities quintiles? As we already saw in section 4.4 of chapter 3, the three rankings are considerably, but not totally correlated: therefore, we could expect broadly similar patterns of change but with some significant differences - which is exactly what we find -. The educational ranking (figure 5.2) makes the job creation patterns more upgrading in nearly all cases. If this was the only ranking we would have, we could have concluded that, in fact, the upgrading hypothesis can explain the patterns of job growth between 1995 and 2007 for nearly all of Europe. Still, that does not mean that the polarization image disappears: in most of the cases with polarization in terms of wage, there is some polarization in terms of education as well, but considerably milder and more upwards biased. Across most of Europe, employment expanded more strongly on those jobs with a higher average educational level, in many cases even moving away from the jobs with a lower educational level.

The amenities ranking makes the patterns even more upgrading in most cases (figure 5.3). In fact, some important countries which were characterized by polarization in terms of education and wages, become purely upgrading in terms of the non-pecuniary conditions of work and employment (for instance, France and Germany). The clearest case of polarization in terms of the wage and educational structures, the Netherlands, becomes nearly flat when we rank the jobs by amenities. Only Slovakia, and to a lesser extent Cyprus and Hungary could still be classified as polarizing countries in terms of the amenities ranking. Therefore, in nearly all of Europe, most job growth is concentrated on jobs with relatively good non-pecuniary attributes. ${ }^{62}$

We could summarize our conclusions so far in the following points: 1) there is considerable variation in the patterns: no single theory can explain everything; 2) the most pervasive image of change is that of upgrading; there are some cases of clear polarization, but no downgrading; 3) the three rankings give consistent results, but each

\footnotetext{
${ }^{62}$ The results derived from the use of the amenities ranking, as mentioned in previous chapters, must be taken with some caution because this ranking does not cover the whole economy (some job creation, therefore, is not included in the picture of figure 5.3), and it forces the same job quality hierarchy across all Europe.
} 
with a slightly different emphasis: the wages ranking generates more plurality of patterns (basically, more polarization); the educational ranking a very similar picture to the wage ranking but more biased upwards; and the amenities ranking generates the most similar results across all countries (pervasive upgrading). These three points constitute the basic elements of our story: the rest of this thesis will basically qualify, complement and deepen our understanding of those basic elements. The first qualification to be made concerns the scale of the transformations which we are talking about.

\subsection{Putting change in its proper scale}

Figures 5.1-5.3 expressed only the change of each quintile in absolute terms, with the vertical scales adapted to the level of employment growth in each country. This is the most adequate representation to identify and classify patterns, but not to evaluate or compare the magnitude of change in the different European countries. For that purpose, it is necessary to show change rescaled to the initial structure: that is, representing the size of each bar in the beginning and end of the period. That way, we should be able to see whether the change represented earlier in isolation implies a significant transformation of the initial structure or just a minor reallocation of some jobs in the margin.

Figures 5.4-5.6 show such rescaled quintile pictures. For each quintile, rather than a single parameter, we represent now three: first, with an empty black box with thick lines, the initial size of the quintile; second, with a very thin line (in many cases hardly visible because it is very close to the thick black box), the size of the quintile in 2000 (therefore, the moment when it was really a quintile, or close to it) ${ }^{63}$; and third, with a grey bar (on the back), the size of the quintile in the end of the period. What we represented earlier in figures 5.1-5. 3 is the difference between the thick black box and the grey bar in figures 5.4-5.6, as can be easily seen. The picture, nevertheless, looks very different: now we can see change in its proper context.

\footnotetext{
${ }^{63}$ We can see in figure 5.4 that the quintiles did not in all cases splits all employment in five strictly equal-sized groups of jobs: that is, they are not always proper quintiles. The clearest case, in the chart based on the wage ranking, is Sweden, where in 2000 the fourth "quintile" was clearly smaller than the third and fifth. The reason, as explained in chapter 3, is that the jobs which are the basis for the quintiles are rather uneven in their size, which sometimes makes it very difficult to achieve a proper distribution of jobs in five $20 \%$ employment groups. In most cases, nevertheless, the quintiles are reasonably even in terms of employment; and in any case, this has no major implications for the analysis.
} 


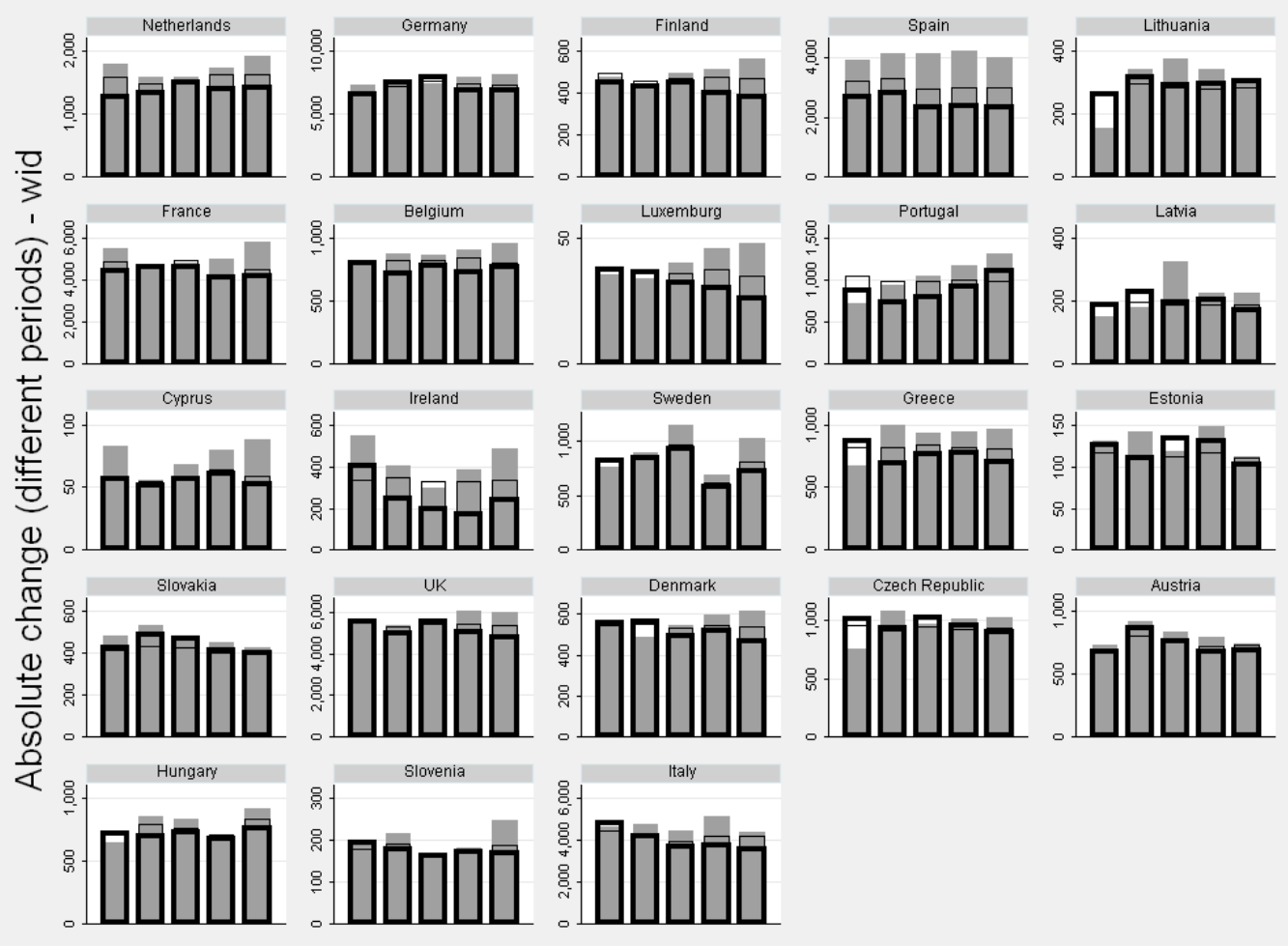

Figure 5.4: Initial, middle and final size of the wage quintiles (different periods)

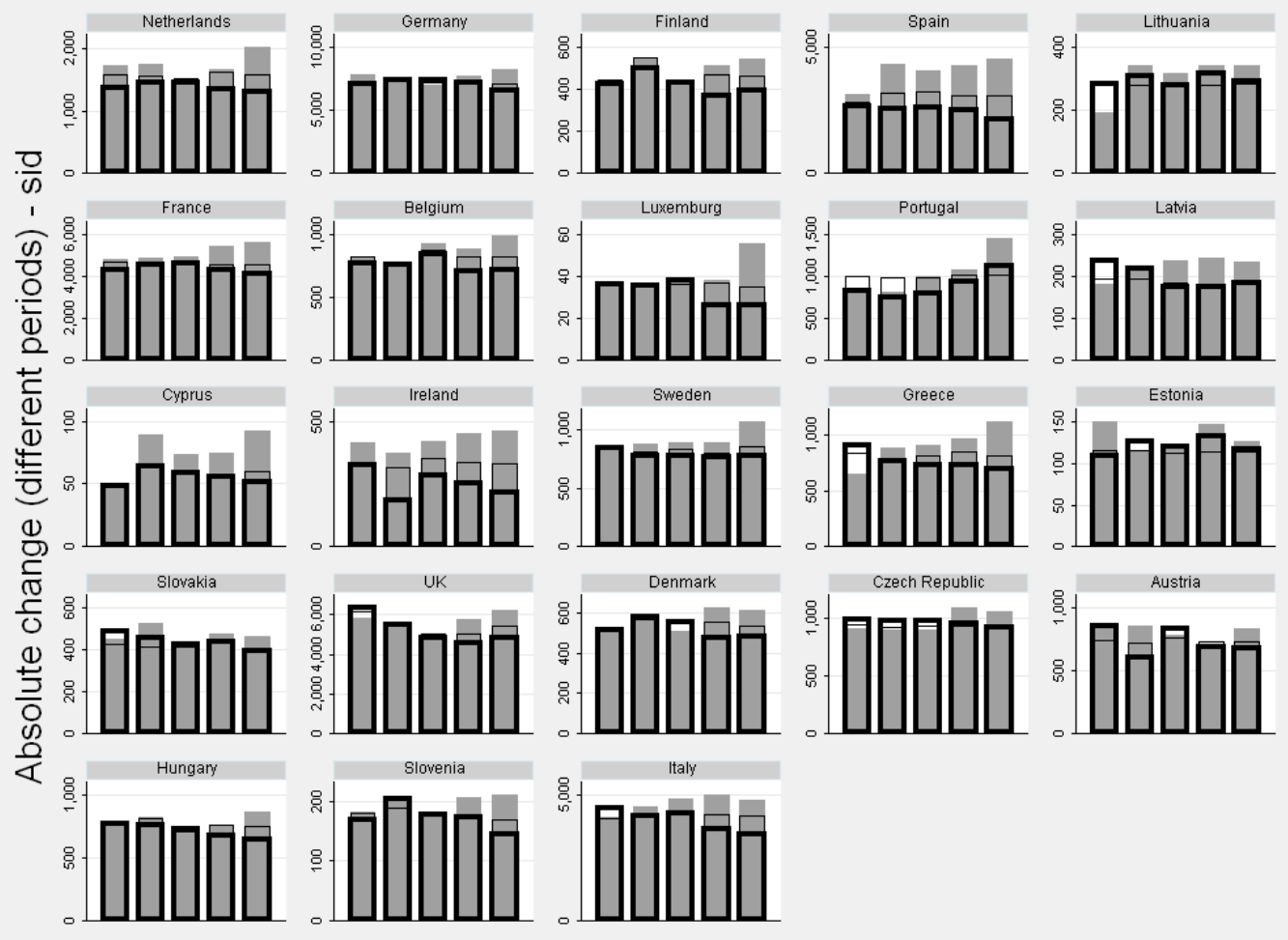

Figure 5.5: Initial, middle and final size of the educational quintiles (different periods) 


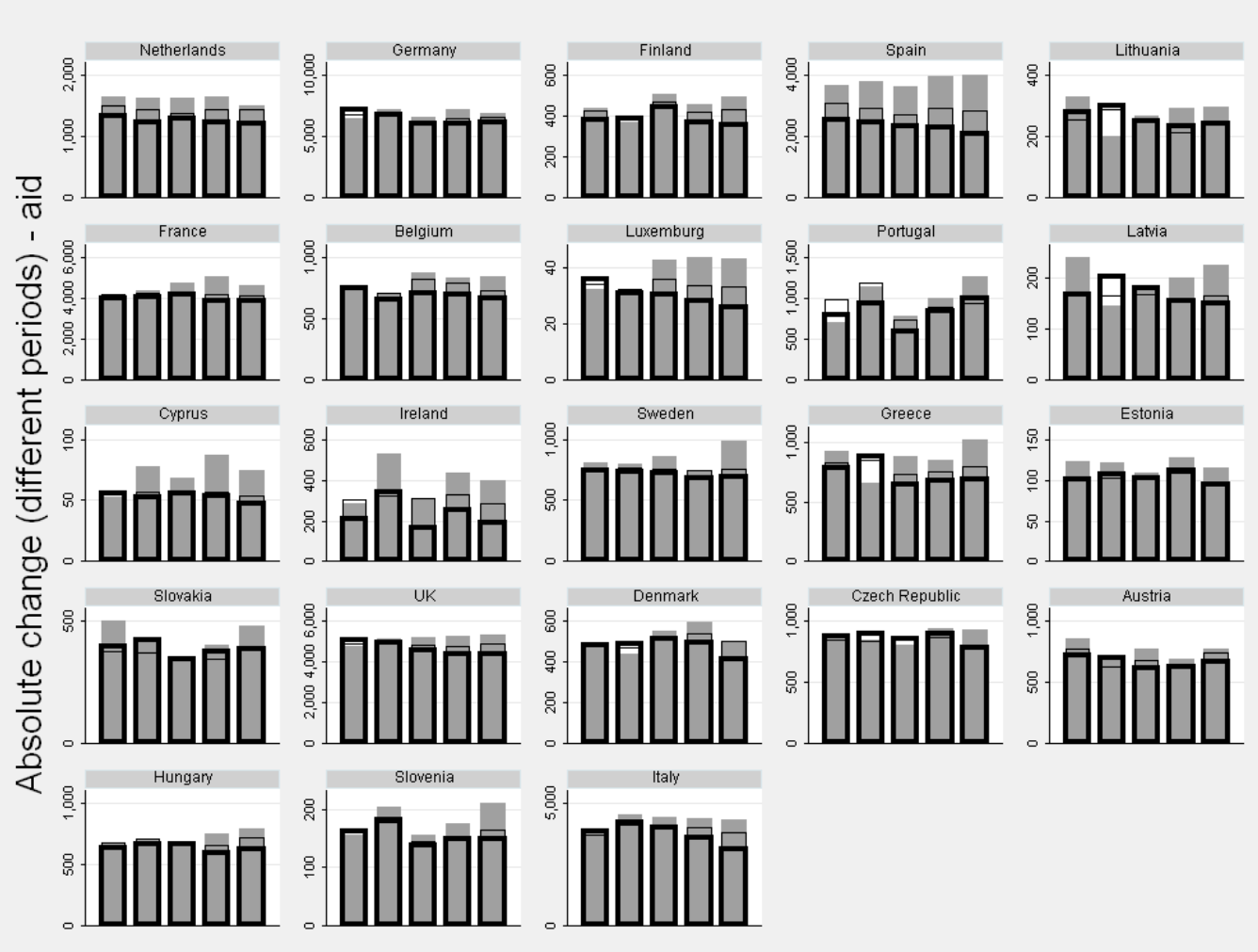

Figure 5.6: Initial, middle and final size of the amenities quintiles (different periods)

The first conclusion we can derive from inspecting figures 5.4-5.6 is that the scale of change in the initial structure varies considerably across countries. This variation was completely missing in pictures 5.1-5.3: because each country chart was rescaled to maximize the range of quintile change in each country (to make the pattern more visible and identifiable), the wide differences in the scales of change were completely out of the picture. We can see now, for instance, that the actual amount of employment expansion in Germany, the UK or Austria were much smaller than in Spain, Ireland or Cyprus.

Nevertheless, although the scale of the employment expansion is an important parameter of the transformation of the job quality structure, it is not the only one. The other parameter is the extent of employment reallocation across jobs, which can transform the structure very significantly even without any employment expansion. And the opposite: there can be very a very large expansion of employment with no transformation in the job quality structure, if the expansion is evenly spread across the quintiles. A comparison between Spain and Luxemburg, for instance, illustrates this. Spain experienced an extraordinary expansion of employment over the 1995-2007 period, as can be seen in the sheer distance between the grey bars and the black boxes of 
each quintile. But because job creation affected very similarly the five job quality quintiles, the (final) shape of the grey bars does not differ much from the (initial) shape of the black boxes. In contrast, in Luxemburg job creation was more limited between 1995 and 2007: the area of the grey bars which is above the black boxes is considerably smaller in Luxemburg than in Spain. Yet, the transformation of the wage structure in Luxemburg was much more important than in Spain over this period: the contrast between the shape of the black boxes and the shape of the grey bars in Luxemburg is quite striking (they became significantly more inclined upwards), whereas in Spain such difference is relatively minor.

We shall not discuss figures 5.4-5.6 with the same detail as figures 5.1-5.3. As mentioned earlier, the main point of figures 5.4-5.6 is to complement and qualify the picture given by figures 5.1-5. 3. We can see now that, over the period of 12 years (maximum) that we cover here, the actual scale of the transformation of European employment structures was in most cases rather limited, implying just a marginal change of the initial structures of job quality. The countries where the transformation was deepest were Luxemburg (with a very significant upgrading), Cyprus, the Netherlands, France, Finland, Slovenia, Portugal and Greece. The Irish case seems quite unique, because in the first half of the period the wage structure changed in the complete opposite way as in the second half of the period, which means that although this country experienced radical transformations in its wage structure, the initial and final shapes look relatively similar. On the other hand, Germany, Slovakia, the UK, Spain, Austria and the Czech Republic all ended the period with a wage structure very similar to the initial one (in some cases, because job creation was very limited; in others, because job creation was evenly spread across the five quintiles). There seems to be no link between the different patterns and the scale of transformation of the initial structure (cases of deep and shallow transformation appear across all types of patterns of job expansion).

\subsection{Job polarization in Europe? A response to Goos, Manning and Salomons}

A recent paper using a very similar approach to the one used in this thesis reached surprisingly different conclusions. Goos, Manning and Salomons (GMS from now on), in their 2009 paper "Job Polarization in Europe" (GMS 2009: 58-60), argued that over roughly the same period studied here, all EU15 countries except Portugal experienced a polarization in their job quality structures (a development that they link to the impact of 
task-biased technological change, as explained in chapter 2). In the previous pages, we did find polarization of job structures in some countries, but certainly not in all. Our main conclusion from the analysis, in fact, was that there was a considerable plurality in the patterns of employment growth by job quality across Europe; and that, if we would have to name a single image of change that fitted most countries, it would be one of upgrading rather than polarization. How can we explain such discrepancy in the conclusions of two very similar studies?

There are three potential sources for this discrepancy: the data used, the analytical strategy, and the interpretation.

1. The data used: although there were some differences in the data used ${ }^{64}$, we can be confident that the discrepancy of results does not come from there, because in fact we exchanged data sources and could replicate our original results with the data of the other party. ${ }^{65}$ There is just one difference in the data used which is of great significance: that GMS used a single wage ranking for all EU countries (based on UK LFS data), whereas we used country-specific wage rankings. But in fact, this difference was the result of a difference in the approach, so we will discuss it in the following point.

2. The analytic strategy: although the paper by GMS and this thesis follow a similar (jobs-based) approach, there are two apparently small differences in the analytic strategy which have important implications for the results obtained:

a. As mentioned earlier, the GMS paper used a single ranking to characterize the quality of jobs across all the EU15 (based on the 1994 UK LFS), whereas we used country-specific wage rankings. In note 3 of their paper (GMS 2009: 59), the only reason given for the use of a single ranking is the lack of EU-wide data, but in subsequent discussions they argued that it is necessary to use a single ranking to be able to compare correctly different employment structures (Goos 2009). As we will see in the next section, it is true that the main driver behind the plurality of patterns across Europe is the differential evolution of employment by occupation and sector, and not the job quality

\footnotetext{
${ }^{64}$ Most importantly, GMS only analysed EU15 countries (we analysed 23), used a single wage-based ranking (we added two secondary rankings, by education and amenities), covered the period 1993-2006 (we covered 1995-2007) and studied change in hours worked rather than number of workers.

${ }^{65}$ This exchange took place in the meeting of the JOBS project that took place in Salamanca in July of 2009 (see Fernández-Macías 2009; Goos 2009).
} 
rankings. But this does not mean that the wage ranking of a single country can be used to characterize the quality of jobs of all other countries: in fact, it can generate quite different and potentially misleading results. In FernándezMacías 2009, it is shown that using the UK wage ranking tends to generate slightly more polarized results than using the national wage rankings: so the use of the UK wage ranking is likely to have biased (polarized) the national results shown in GMS 2009. ${ }^{66}$ In our view, the use of a single ranking for all countries is justified only when there is no other alternative: for instance, in the case of the amenities ranking or for the analysis at the EU-level we ourselves use such approach. But by no means can it be argued that such an approach is more adequate than using country-specific rankings.

b. A second important difference in the analytic strategy resides in the way the job quality tiers are constructed. In this thesis, following the earlier example by Wright and Dwyer (2003), we grouped the jobs in five equal-sized groups ranked by their median hourly wages (what we called "quintiles"). GMS also rank the jobs by their median wages, but they classify them in three categories (good, middling and bad jobs) which have very uneven sizes in terms of number of occupations (8-9-4) and in terms of employment shares in the first year of the period studied (29\%-49\%-22\%). There is no explanation in the paper for the rationale behind this particular grouping of jobs. This is quite striking, because the way the jobs are grouped has very important implications for the results, especially when the relative rates of growth are the main parameter of the analysis (as is the case in the GMS paper). In table 5.I, using the EU figures given by GMS (2009: 59), we show an example of the importance of the grouping criteria. Under column 1, we can see the original grouping of jobs made by GMS, which generates the rates of growth of $8 \%$ for good jobs, $-9 \%$ for middling jobs and 1.2 for bad jobs (hence the polarization). In column 2, we have made an alternative grouping in which the initial shares of employment in good, middling and

\footnotetext{
${ }^{66}$ The idea that using a single ranking improves the comparability of the analysis is also wrong. As we discussed in chapter 3, the comparability of the classification of employment by sector and (especially) occupation is only approximate. Using country-specific rankings is a good way of minimizing the potential implications of such comparability problems (as we are sure we are comparing likes with likes, that is, jobs of similar quality), whereas using one ranking for all countries intensifies these problems (as it necessarily assumes that the jobs are identically classified in all countries)
} 
bad jobs are roughly the same (one third each): we can immediately see that, even though there is still a gap in the middle, the degree of polarization diminishes considerably (the rates of growth now are: 7.6 for good jobs, -5.4 for middling jobs and -2.2 for bad jobs). The same (uneven) grouping shown in column 1 was applied to all European countries, and the resulting patterns of job growth (table 2 of the GMS paper; see GMS 2009: 61) were the evidence provided for the pervasiveness of polarization. Although we cannot prove it directly (the occupational breakdown for each country is not provided in the paper), we suspect that the way the jobs are grouped tend to inflate the degree of polarization for each country as much as it did for the EU as a whole as shown in table 5.I below.
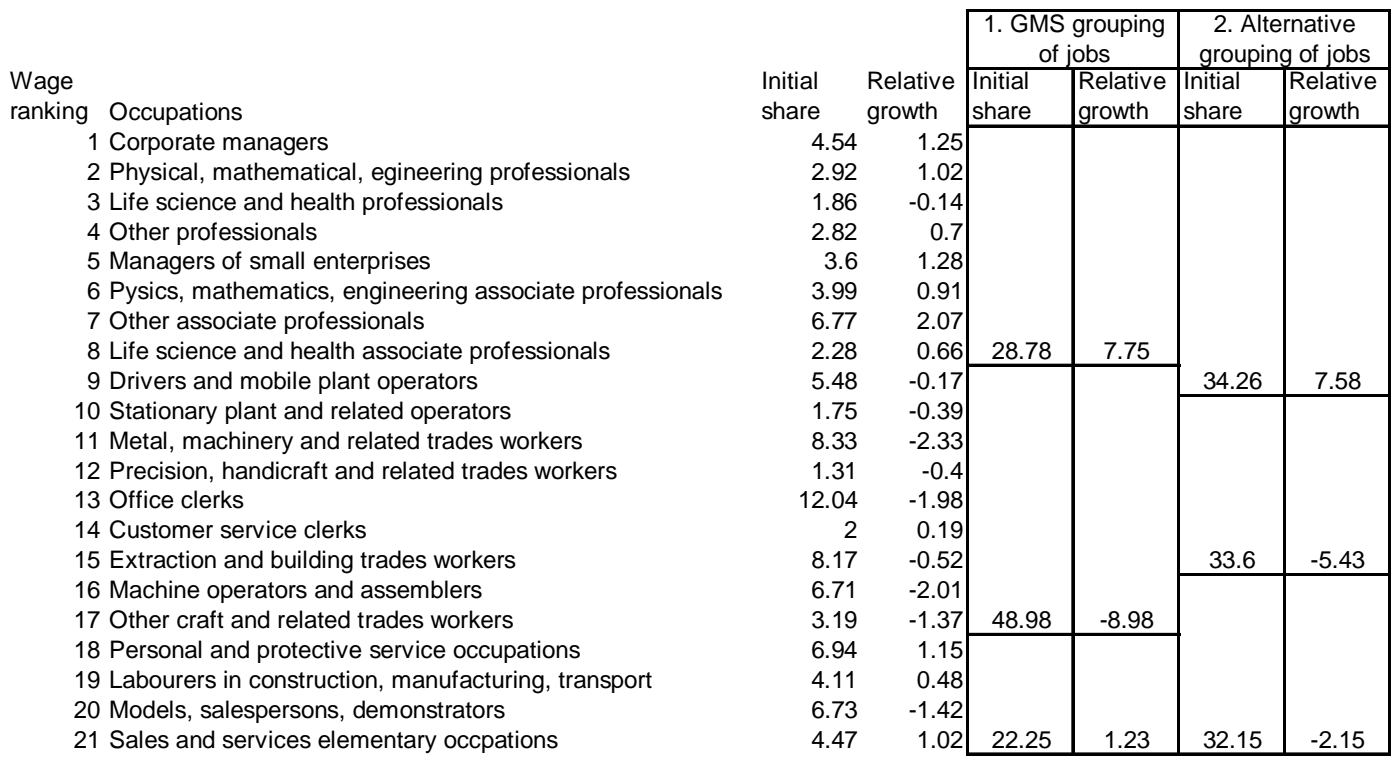

Table 5.I: Uneven vs. even groups of jobs in the GMS 2009 paper

3. The interpretation: in our view, the GMS paper places too much emphasis on the polarization story, ignoring almost completely the simultaneous (stronger) trend of upgrading which is apparent even in the figures that they provide. Let us go back to the original GMS results (shown in column 1 of table 5.I above): nobody could deny that relative growth figures of $8,-9$ and 1.2 for good, middling and bad jobs imply a polarization effect; but I guess nobody would deny either that they imply a rather significant upgrading of the jobs structure as well (good jobs grew 8 times faster than bad jobs). Even if there were not problems with the way the analysis is constructed, the polarization story seems to be over-emphasized in the GMS paper. 
But we would not like to make much emphasis on the differences in the interpretation, as they are always much more open to debate. The main source of differences between our results and the results of the 2009 GMS paper lies in the different analytic strategy: basically, in the use of the UK rankings to characterize the quality of jobs of the whole of Europe, and in the construction of uneven job quality tiers. Both elements combined generate results which are more polarized than they should be, leading to the incorrect conclusion that polarization was a pervasive development across all European countries.

\subsection{Some final remarks}

Our results show that there was no universal pattern of evolution in the employment structures of 23 European countries between 1995 and 2007. Instead, there was a considerable diversity, not only in the patterns but also in the intensity of change of the job quality structures across different European economies. This in itself suggests that neither of the three types of hypotheses which predominate in the literature (which we summarized earlier in three images of change: downgrading, upgrading and polarization) suffices to explain the empirical patterns of change according to our jobs approach. But to transform this hint into evidence, we still need to make a much more detailed analysis of what lies behind the patterns of figures 5.1 and 5.2 in this section. In the remaining of this chapter, we will try to evaluate to what extent did the differences in the observed patterns across Europe result from different evolutions in the composition of the division of labour or from different job quality structures (section 3), and also to what extent could these different evolutions be the result of different starting positions within a general process of convergence or parallel evolutions (section 4). In both cases, what we will evaluate is whether we can still maintain some type of universalistic explanation, according to which behind the superficial diversity there would still be universal underlying forces.

But although no single explanation suffices, the three main hypothesis that we distilled from the literature have varying rates of success in their applicability to the observed patterns of job growth. The degradation hypothesis, in particular, seems totally knocked out: from our compositional, job-based approach, we could find no evidence at all of downgrading in the job quality structure. The outcome is more positive for the polarization approach, but certainly not totally successful: we could see some cases that fit the image of change anticipated by this theory, but not a universal validation. The theory that finds more widespread support in our analysis is the upgrading hypothesis. 
In fact, in many ways it could be argued that almost all cases involve some type of upgrading in the job quality structure (something which certainly cannot be said of the other two hypotheses). In nearly all countries ${ }^{67}$, for the three job quality rankings, the one or two bars representing job growth at the top of the job quality structure between 1995 and 2007 were the tallest. In this sense, most of the country differences lie at the middle and bottom of the job quality structure: the top looks rather similar everywhere (the main differences at the top being with respect the scale of growth, rather than with its shape). So there may be, after all, some universal factors that, across most of Europe, explain the fact that the share of employment in jobs of a relatively high quality tended to increase. In chapter 6 , we will try to identify those universal factors and also the factors that explain the variability in the degree of job expansion in the middle and bottom of the job quality structure.

\section{The importance of the national rankings}

The picture of change in the employment structure by job quality quintiles is determined by two main parameters: on the one hand, the evolution of employment within each of the cells in the jobs matrix and, on the other hand, the job quality ranking which is used to assign each job to one of the five quintiles. It could be that all countries experienced a very similar evolution in terms of the division of labour, yet the patterns of employment growth by job quality differ because the jobs are differently ranked in different countries. It could also be that the job quality rankings did not play any role in the observed differences, because these are totally the result of differences in the structural evolution of employment. Or it could be that the differences are the result of differences in structural change and differences in job quality structures. Each of these possibilities would have different implications for the overall interpretation of the patterns of employment growth across Europe.

The methodology used in this thesis makes it relatively easy to test the relative importance of each of the two basic parameters in the determination of the differences across Europe in the evolution of job quality structures. As we have already seen previously (section 4 of chapter 4 ), the fact that the job matrices of all countries have essentially the same structure (all of them are defined by a crosstabulation of sector and

\footnotetext{
${ }^{67}$ The exceptions are Slovakia (for the three rankings), the Baltics (for the three rankings), Netherlands (for wages and amenities) and Austria (for education).
} 
occupation and the two-digit level) means that we can cross the parameters, applying the ranking of one country to another, or the quintile allocation, or the employment structure. This makes it possible to generate counterfactual results, in which we make one of the two parameters constant across the countries and therefore all the remaining variation necessarily a result of the other one. ${ }^{68}$ This is exactly what we will do in this section: we will apply the rankings of one reference country (Germany) to all the other countries, reconstruct the quintiles (making them as they would have been if all countries had the same educational and wage job hierarchies as Germany) and then compare the resulting (counterfactual) picture with the real quintile picture which we presented in the previous section. The comparison between this counterfactual and the real picture shall allow us to evaluate the importance of the national rankings in the determination of the different patterns of job growth across Europe.

As usual, it is necessary to make some qualifications before embarking in the analysis. The first one is that this section will only concern the wage and educational rankings, because the amenities ranking is the same for all countries. In fact, the pictures of job growth across the amenities quintiles discussed in the previous section (figures 5.3 and 5.6) can be understood as a first approximation to the analysis we are going to carry out here: because the amenities ranking is the same for all countries, all differences in the shape of the amenities quintiles across countries result from differential evolutions in the employment structures. A second qualification concerns the fact that this type of analysis assumes that the classification of employment by sector and occupation is strictly comparable across Europe (so that we can cross the rankings), which is only true in broad terms (for a detailed discussion of this issue, see section 3.2 of chapter 3 ). Therefore, we need to keep a broad-brush approximation, focusing on general trends and not being too finicky about small details. A third qualification relates to the overall aim of this section. This section should not be taken as an evaluation of the differences in the national job quality rankings: this was already done in chapter 4. It may be useful, in fact, to remember that we found the national rankings to be generally rather similar, but still different enough as to be necessary to use them (rather than a single EU ranking) in order to characterize the nature of employment growth across Europe. Our aim here is not to discuss the same issue again: but to start from where we left it in chapter 4 . What is the relative influence of the rankings on the different patterns of job

${ }^{68}$ This method is in fact very similar to shift-share analysis. 
growth across Europe? Do these differences derive mainly from the fact that the rankings differ, or from differential evolutions in the employment structure?

\subsection{A visual exploration}

Figures 5.7 and 5.8 show a graphical comparison of the counterfactual and real quintiles for each of the job quality rankings. There are two bars for each quintile, one black and one grey. The black bars represent the counterfactual: that is, how much job creation would have taken place in each quintile if country $\mathrm{X}$ had the same educational or wage structure as Germany. The grey bar behind represents the real growth of each quintile, using the educational or wage structure that truly corresponds to each country: therefore, these grey bars are identical to the black bars of figures 5.1 and 5. 2. There are two important things to look for in these charts: first, the difference between the black and grey bars for each country, which is an indication of how much does the quintile picture change when we use the German job quality ranking instead of the national one; second, the difference between the black bars of each country and the black bars of Germany, to see whether using the German ranking makes other countries look more similar to Germany than using their own ranking.

We are not going to do a detailed analysis of these charts country by country: as mentioned earlier, we want to keep an eagle-eye perspective. But just a quick overview of figures 5.7 and 5.8 suggests that the different job quality rankings are not obscuring a similar evolution of the division of labour in the different countries. That is, using the German job quality ranking does not make all country patterns look like those of Germany. That does not mean that applying the German ranking makes no difference: the quintile shapes do change in most cases, which proves that the differences in the national rankings have an influence on the shape of the quintile picture. But the crucial point here is that eliminating the variation which derives from using different national rankings does not seem to reduce significantly the original variability, nor does it seem to make all countries look like Germany. The rankings, therefore, would not seem to be the main source of variability in the patterns of job growth across Europe.

Of course, there are some qualifications to such eagle-eye's picture. If we pay close attention to the country charts in figures 5.7 and 5.8, we can see that in some cases the German ranking does seem to make the quintile shape more German-like, especially in the case of the educational quintiles. The black bars of figure 5.8 include noticeably more polarization than the grey bars. The amount of variability in the original 
educational quintiles, on the other hand, does not seem to diminish (the variability in the educational picture was always lower than in the wage picture). Applying the German ranking across the board makes the educational quintile pictures become slightly more polarized, not more homogeneous (which suggests that the German ranking has something to do with its asymmetrically polarized picture, but it does not prove that the variability was the result of having different rankings because most of it remains after using only one ranking). Nothing like this, on the other hand, happens with the wage quintiles: in this case, the shapes also change somewhat, but not moving in a more "German" direction; and of course, the amount of variability of the black and grey shapes is very similar.

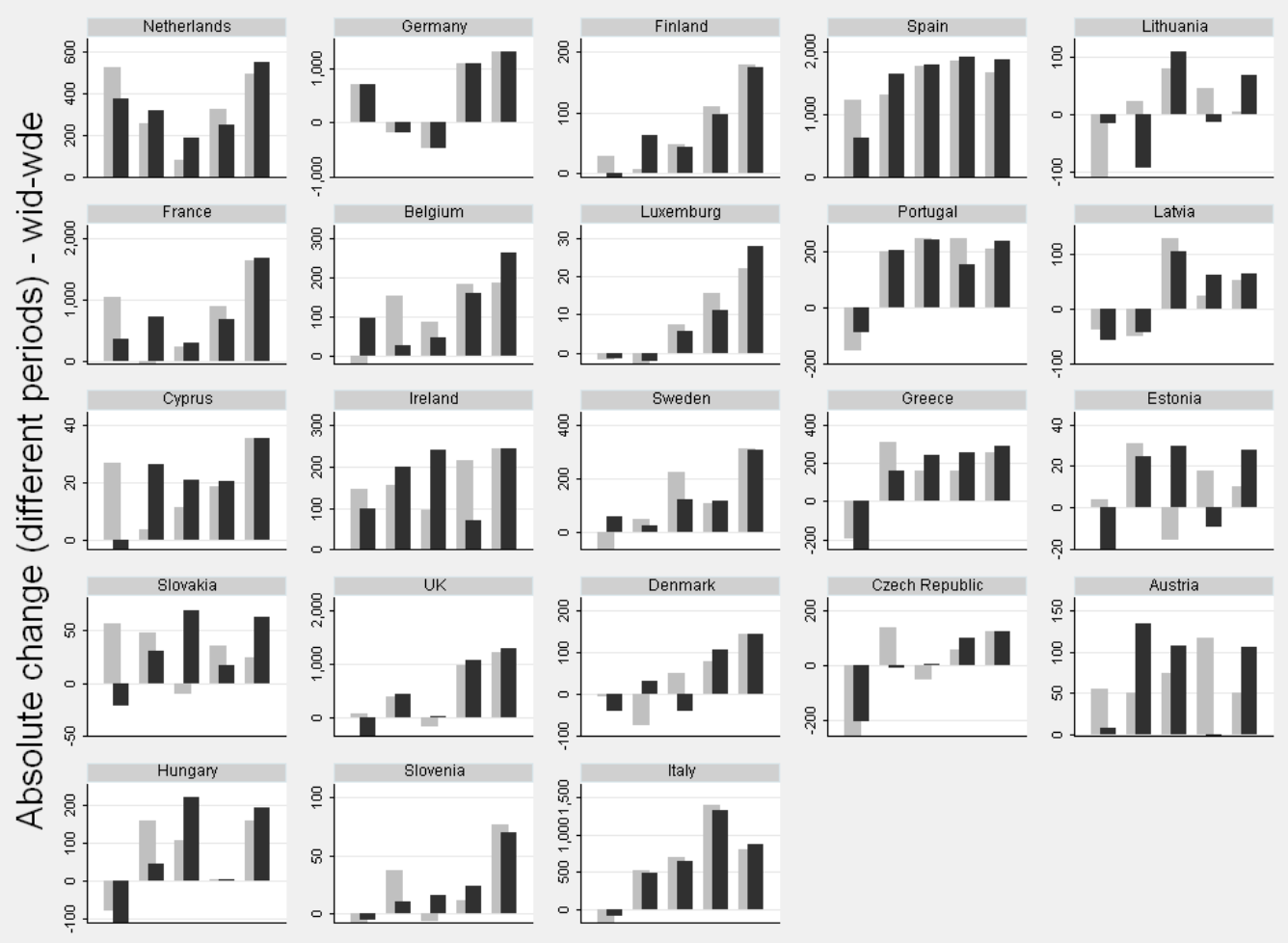

Figure 5.7: Counterfactual (Germanic) vs. real wage quintiles (different periods) 


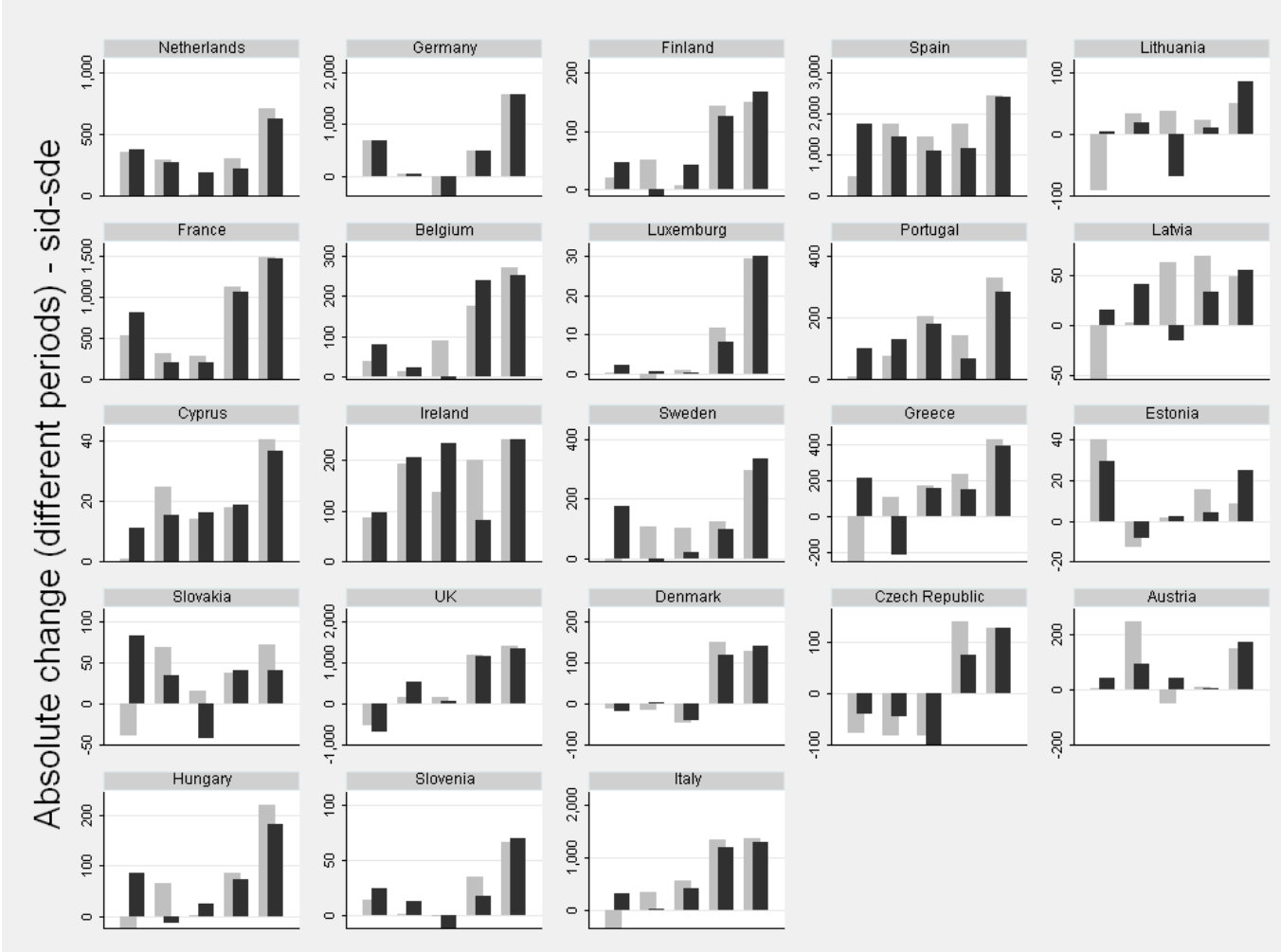

Figure 5.8: Counterfactual (Germanic) vs. real educational quintiles (different periods)

One way to think about the implications of figures 5.7 and 5.8 is to imagine how would the overall analysis of the previous section had been if, because of lack of national sources, we had been forced to use the German wage and educational hierarchies to characterize the quality of jobs of the 23 countries. In some cases, the classification of countries as polarizing or upgrading would have changed, in others it would not; the overall interpretation of the educational quintiles would have shifted slightly towards polarization. But clearly, we would still have concluded that between 1995 and 2007 there were significantly different patterns of job growth across Europe, that none of the three hypotheses of change (downgrading, upgrading and polarization) can explain on its own what happened everywhere. Therefore, the national rankings do matter: but they are not the reason why the patterns of job growth differ across Europe.

\subsection{A pseudo-parametric approach}

Although the previous section probably provides enough evidence of the fact that the national patterns do not differ only (not even mainly) because of the use of nationallyspecific rankings, the fact that such evidence is purely based on a visual exploration may make it seem confusing or inconclusive. For this reason, we are going to use a pseudo-parametric approach which puts numbers to the comparison that we carried out 
visually in the previous section. We call this approach pseudo-parametric because in fact, it is only a numerical translation of what we see in figures 5.7 and 5.8. But by expressing numerically figures 5.7 and 5.8, the comparison between the different countries is simplified, and it is easier to reach an overall conclusion.

This whole section is based on a kind of difference-in-differences analysis. Basically, we are analyzing whether the difference between, say, the Spanish quintile picture and the German quintile picture is diminished when we apply the German job quality ranking to Spain. This idea can, in principle, be expressed mathematically. What we need is a way to measure the original difference between two country patterns, and then see whether that measure diminishes if we apply the same ranking (the German one in this case) to both countries. Such pseudo-parametrization was carried out in 3 steps:

1. First, all the figures were normalized, to ensure that they were expressed in a comparable scale. Our interest here is not job growth per se, but change in the structure of employment by job quality as characterized by the quintile picture. Therefore, we have opted for a normalization that expresses the change in each quintile with respect to the average change of the five quintiles in each country (each quintile is divided by the average absolute growth of the five quintiles).

2. Then, two dissimilarity indices were calculated:

a. First, we calculated $x$, which expresses the difference between the national patterns of employment growth that result from applying the national rankings and the German patterns of employment growth. This $x$ index is the sum of five sub-parameters, each expressing the difference between the size of each quintile in the national pattern and Germany. Rather than comparing each quintile in isolation, they are compared in regions, that is, taking also into account the quintiles which are adjacent. The formula used for calculating these sub-parameters is the following ${ }^{69}$ :

\footnotetext{
${ }^{69}$ As can be seen, each $x$ sub-parameter derives from the absolute addition of two values: the first one is simply the difference between that quintile in the national figures and the same quintile in the EU figures; the second one is the difference for that quintile and its neighbor/s (depending on which quintile it is) in the national and EU figures. The second measure is added because this way the dissimilarity index is sensitive to dissimilarities not only for each particular quintile, but also for its area (its neighbors).
} 


$$
\begin{aligned}
& x_{1}=\left|q_{1} n a c-q_{1} d e\right|+\left|\left(\frac{\left(q_{1} n a c+q_{2} n a c\right)}{2}\right)-\left(\frac{\left(q_{1} d e+q_{2} d e\right)}{2}\right)\right| \\
& x_{1}=\left|q_{i} n a c-q_{i} d e\right|+\left|\left(\frac{\left(q_{(i-1)} n a c+q_{i} n a c+q_{(i+1)} n a c\right)}{3}\right)-\left(\frac{\left(q_{(i-1)} d e+q_{i} d e+q_{(i+1)} d e\right)}{2}\right)\right| ; \text { for } \mathrm{i}=2,3,4 \\
& x_{1}=\left|q_{5} n a c-q_{5} d e\right|+\left|\left(\frac{\left(q_{4} n a c+q_{5} n a c\right)}{2}\right)-\left(\frac{\left(q_{4} d e+q_{5} d e\right)}{2}\right)\right|
\end{aligned}
$$

The overall $x$ is then calculated as the summation of the absolute value of each of the $x$ sub-parameters.

$$
x=\sum\left|x_{i}\right|
$$

b. Then, we calculated $y$, which expresses the difference between the national patterns of employment growth that derive from applying the German rankings and the German patterns of employment growth shown in figure 5.7. The calculation is identical as for $x$ (and therefore, there is no need to repeat the formulas), only that in this case the national figures are those that derive from applying the German rankings.

3. With $x$ and $y$, we can finally calculate our difference-in-differences index. Two versions of the index are calculated:

a. $z 1$, which is simply $x-y$, and expresses in absolute terms how much the difference between each country and Germany changes when the German ranking (rather than the national ranking) is applied;

b. $z 2$, which is $z 1$ divided by $x$, and therefore expressed in relative terms, as a ratio (reflecting the proportion by which the difference between the national and German patterns changes when applying the German rankings to the national employment figures).

The most important index for our purposes is $z 2$, which ranges between $-\infty$ and 1 , taking a value of 0 when if the German ranking does not change the original differences between the national and the German pattern (which would imply that the national ranking makes no difference whatsoever, and therefore all the observed differences are the result of different structural changes in employment); a value approaching 1 if the German ranking makes the national pattern more similar to the German pattern (with an upper limit of 1 when applying the German ranking makes the national pattern identical to the German pattern: that is, when all the original 
differences were the result of different rankings); and a value below 0 if the German ranking makes the differences between the national and German pattern even larger (in this case, the limit is $-\infty$ because of the fact that $x$ is in the denominator: if the German and national results were originally very similar, $x$ would be close to zero, so any increase in the difference measured by $y$-which is subtracted from $x$ in the numerator-would be magnified). It may seem surprising that applying the same ranking to two countries would make their results more dissimilar, but it is theoretically possible, and it does happen in practice, as we will see. What this means is that the national ranking happened to make different structural evolutions in employment appear more similar to the German pattern than they really were, so that when the same ranking is applied to both, these underlying differences surface.

Tables 5.II.1 and 5.II.2 show the results of this exercise. The countries have been arranged in the same way as in figures 5.3.1 and 5.3.2, to facilitate the analysis of the table. For each country, the four parameters explained above are shown, including the values for each quintile (so that we can identify where the differences in the patterns come from). For instance, the upper left-hand frame of table 5.II.1, which shows the comparison between the Dutch patterns obtained from using the Dutch and German rankings would be interpreted in the following way: $x N L$ shows that the overall difference between the original Dutch (NL) patterns (as shown with black bars in figure 5.1, or grey bars in figure 5.7) and the German (DE) original patterns (shown in the second chart from of the top row in figures 5.1.1 and 5.3.1) is 5.8 according to our measure (above the overall index $x N L$ we see the contribution of each quintile: the biggest dissimilarity lies in quintiles 2,1 and 3). The following column, $y N L$, shows the difference after the German ranking has been applied to the Dutch figures of employment growth (therefore, this comparison corresponds to the difference between the black bars of figure 5.7 and the German pattern): the value of this overall dissimilarity index is 6.02. Therefore, applying the German ranking to the Dutch figures of job growth makes the Dutch pattern slightly more dissimilar to Germany. Inspecting the results by quintiles, we can see where these differences come from: whereas the first quintile becomes more German-like, the second and third quintiles become more different, because they expand considerably when we apply the German ranking to the Dutch employment figures. The indices $z 1$ and $z 2$ show this: in absolute terms, the similarity decreases by $.24(z 1)$ which in relative terms corresponds to a decrease of .04 . 


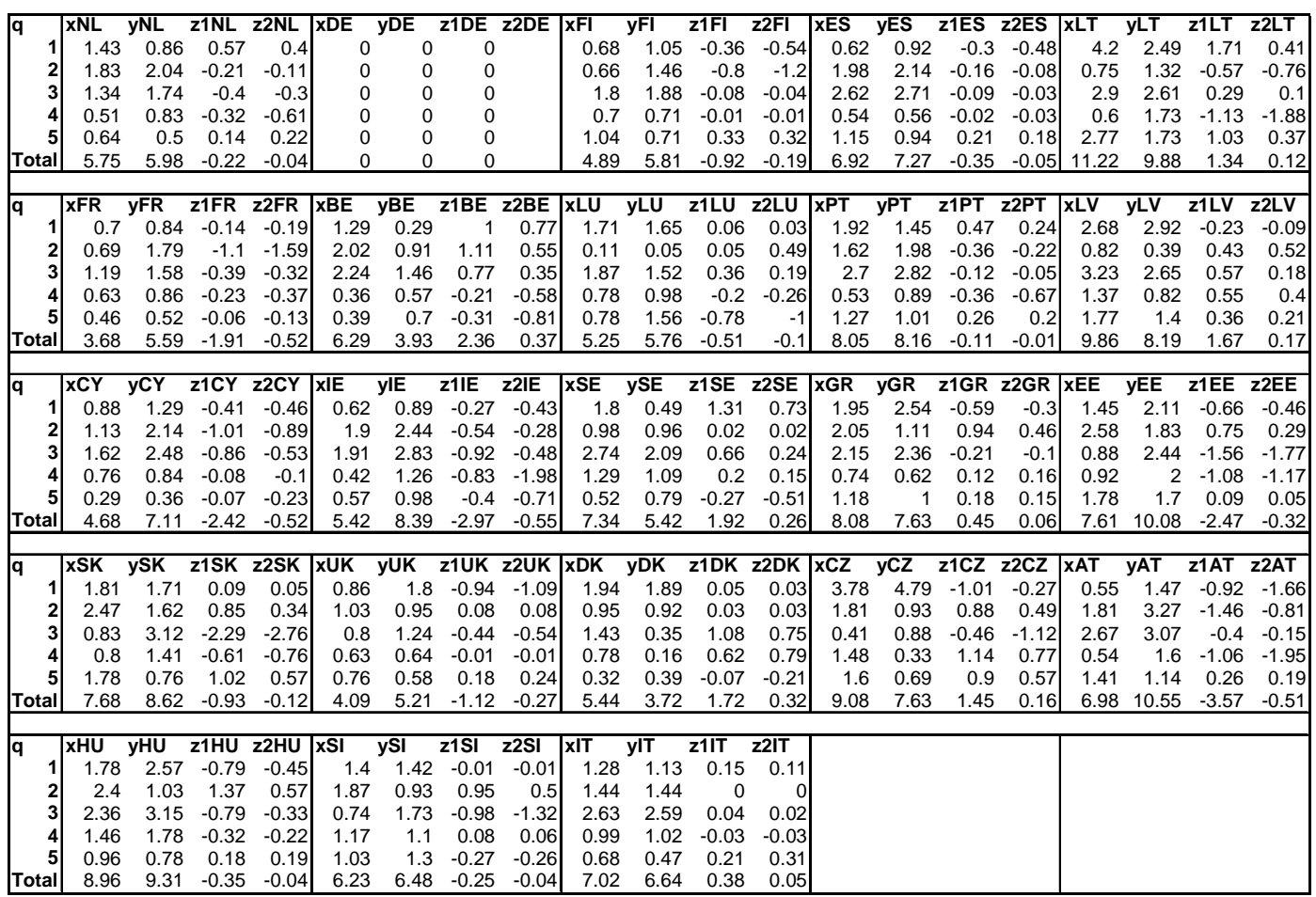

Table 5.II.1: Pseudo-parametric comparison between counterfactual and actual patterns (wage)

\begin{tabular}{|c|c|c|c|c|c|c|c|c|c|c|c|c|c|c|c|c|c|c|c|c|}
\hline q & $\mathrm{xNL}$ & yNL & Z1NL & $\mathrm{z2NL}$ & XDE & yDE & z1DE & Z2DE & XFI & yFI & z1FI & Z2FI & XES & yES & z1ES & \begin{tabular}{l|} 
Z2ES \\
\end{tabular} & xLT & yLT & Z1LT & z2LT \\
\hline 1 & 0.41 & 0.42 & -0.01 & -0.02 & 0 & 0 & 0 & & 0.92 & 0.82 & 0.1 & 0.11 & 0.91 & 0.48 & 0.44 & 0.48 & 4.22 & 1.17 & 3.04 & 0.72 \\
\hline & 1.29 & 1.37 & -0.08 & -0.06 & 0 & 0 & 0 & & 0.8 & 0.35 & 0.44 & 0.55 & 1.64 & 1.57 & 0.07 & 0.04 & 0.98 & 1.01 & -0.03 & -0.03 \\
\hline 3 & 1.19 & 1.75 & -0.56 & -0.47 & 0 & 0 & 0 & & 1.54 & 1.72 & -0.17 & -0.11 & 2.49 & 2.01 & 0.48 & 0.19 & 1.96 & 1.59 & 0.37 & 0.19 \\
\hline & 0.27 & 0.24 & 0.04 & 0.13 & 0 & 0 & 0 & & 1.64 & 1.39 & 0.25 & 0.16 & 0.68 & 0.13 & 0.55 & 0.8 & 0.36 & 1.12 & -0.76 & -2.1 \\
\hline & 0.49 & 0.98 & -0.49 & -0.98 & 0 & 0 & 0 & & 0.83 & 0.6 & 0.22 & 0.27 & 1.22 & 1.44 & -0.22 & -0.18 & 2.25 & 0.57 & 1.68 & 0.75 \\
\hline Total & 3.66 & 4.76 & -1.1 & $\begin{array}{l}-0.3 \\
\end{array}$ & 0 & 0 & 0 & & 5.73 & 4.89 & 0.84 & 0.15 & 6.94 & 5.63 & 1.31 & 0.19 & 9.77 & 5.47 & 4.3 & 0.44 \\
\hline & XFR & yFR & z1FR & 2FR & $3 E$ & yBE & 1BE & & & & & & & & & & & LV & Z1LV & z2LV \\
\hline 1 & 0.38 & 0.1 & 0.28 & 0.74 & 1.1 & 0.57 & 0.52 & 0.48 & 1.67 & 1.2 & 0.47 & 0.28 & 1.35 & 0.59 & 0.76 & 0.56 & 3.31 & 0.9 & 2.41 & 0.73 \\
\hline 2 & 0.65 & 0.55 & 0.11 & 0.17 & 0.25 & 0.2 & 0.04 & 0.18 & 0.41 & 0.08 & 0.33 & 0.81 & 0.87 & 1.48 & -0.61 & -0.7 & 0.1 & 1.46 & -1.36 & -14.2 \\
\hline & 1.67 & 1.45 & 0.22 & 0.13 & 2.06 & 1.21 & 0.86 & 0.42 & 1.05 & 0.87 & 0.18 & 0.18 & 2.8 & 2.51 & 0.29 & 0.1 & 2.8 & 0.67 & 2.14 & 0.76 \\
\hline & 1.16 & 1 & 0.17 & 0.14 & 1.38 & 1.73 & -0.36 & -0.26 & 1.33 & 0.88 & 0.44 & 0.33 & 0.78 & 0.59 & 0.18 & 0.24 & 1.1 & 0.42 & 0.68 & 0.62 \\
\hline & 0.63 & 0.59 & 0.04 & 0.06 & 0.46 & 0.83 & -0.37 & -0.81 & 1.61 & 1.85 & -0.24 & -0.15 & 0.36 & 1.07 & -0.71 & -1.97 & 1.85 & 1.04 & 0.81 & 0.44 \\
\hline Total & 4.5 & 3.68 & 0.82 & 0.18 & 5.24 & 4.54 & 0.7 & 0.13 & 6.07 & 4.87 & 1.2 & 0.2 & 6.16 & 6.24 & -0.08 & -0.01 & 9.16 & 4.48 & 4.69 & 0.51 \\
\hline $\mathbf{q}$ & $\mathrm{xCY}$ & $y C Y$ & z1CY & $\mathrm{ZZCY}$ & XIE & yIE & z1IE & z2IE & XSE & & z1SE & z2SE & XGR & & & z2GR & XEE & yEE & z1EE & z2EE \\
\hline 1 & 1.12 & 0.61 & 0.51 & 0.45 & 0.81 & 0.82 & -0.01 & -0.01 & 1.33 & 0.38 & 0.95 & $; \quad 0.72$ & 3.03 & 0.72 & 2.31 & 0.76 & 1.72 & 1.22 & 0.5 & 0.29 \\
\hline & 1.69 & 1.26 & 0.43 & 0.25 & 1.68 & 1.98 & -0.31 & -0.18 & 1.11 & 0.45 & 0.66 & 0.59 & 0.53 & 1.08 & -0.55 & -1.03 & 1.33 & 1.05 & 0.27 & 0.21 \\
\hline & 2.21 & 2.21 & 0 & 0 & 2.35 & 2.89 & -0.54 & -0.23 & 2.22 & 0.98 & 1.23 & 0.56 & 1.96 & 1.33 & 0.62 & 0.32 & 0.73 & 0.9 & -0.17 & -0.24 \\
\hline & 0.5 & 0.53 & -0.03 & -0.06 & 0.65 & 0.47 & 0.18 & 0.28 & 0.72 & 0.31 & 0.41 & 0.57 & 0.5 & 0.25 & 0.25 & 0.5 & 0.55 & 0.59 & -0.04 & -0.08 \\
\hline & 0.57 & 0.85 & -0.29 & -0.5 & 1.41 & 1.76 & -0.35 & -0.25 & 0.21 & 0.19 & 0.01 & 0.07 & 0.93 & 1.16 & -0.23 & -0.24 & 2.81 & 1.25 & 1.56 & 0.56 \\
\hline Total & 6.08 & 5.47 & 0.62 & 0.1 & 6.91 & 7.94 & -1.03 & -0.15 & 5.59 & 2.31 & 3.27 & 0.59 & 6.96 & 4.54 & 2.41 & 0.35 & 7.13 & 5.01 & 2.13 & 0.3 \\
\hline q & SK & ySK & z1SK & z2SK & $\mathrm{xUK}$ & yUK & z1UK & z2UK & xDK & yDK & z1DK & Z2DK & $\mathrm{xCZ}$ & yCz & z1CZ & $\mathrm{z2CZ}$ & xAT & yAT & Z1AT & Z2AT \\
\hline 1 & 2.18 & 1.27 & 0.91 & 0.42 & 2.65 & 2.63 & 0.01 & & 2 & 2.06 & -0.06 & -0.03 & 3.21 & 2.72 & 0.49 & 0.15 & 1.86 & 0.87 & 0.98 & 0.53 \\
\hline 2 & 1.55 & 0.97 & 0.57 & 0.37 & 0.43 & 0.85 & -0.42 & -0.97 & 0.83 & 0.56 & 0.27 & 0.33 & 1.88 & 1.63 & 0.25 & 0.13 & 3.17 & 1.93 & 1.24 & 0.39 \\
\hline 3 & 1.74 & 0.43 & 1.32 & 0.76 & 1.52 & 1.38 & 0.14 & 0.09 & 0.4 & 0.38 & 0.03 & 0.06 & 0.39 & 1.08 & -0.69 & -1.78 & 0.7 & 1.79 & -1.08 & -1.52 \\
\hline & 0.06 & 0.69 & -0.63 & -10 & 1.44 & 1.05 & 0.39 & 0.27 & 1.57 & 1.39 & 0.18 & 0.11 & 0.89 & 0.67 & 0.22 & 0.25 & 1.1 & 0.88 & 0.27 & 0.23 \\
\hline & 1.4 & 2.41 & -1 & -0.72 & 0.72 & 0.75 & -0.03 & -0.05 & 1.01 & 0.7 & 0.31 & 0.3 & 1.58 & 1.19 & 0.39 & 0.25 & 1.63 & 0.44 & 1.19 & 0.73 \\
\hline Total & 6.94 & 5.76 & 1.17 & 0.17 & 6.76 & 6.67 & 0.09 & 0.01 & 5.81 & 5.09 & 0.72 & 0.12 & 7.95 & 7.29 & 0.66 & 0.08 & 8.51 & 5.91 & 2.6 & 0.31 \\
\hline & XHU & $\mathrm{yHU}$ & z1HU & HU & xSI & ySI & z1SI & z2SI & $\mathrm{xIT}$ & yIT & & & & & & & & & & \\
\hline 1 & 1.69 & 0.14 & 155 & 0.92 & 0.76 & 0.29 & 0.47 & 0.62 & $\begin{array}{r}x+1 \\
2.09\end{array}$ & 0.91 & 1.18 & 0.56 & & & & & & & & \\
\hline 2 & 0 & & 0.28 & & & & -0.49 & -10 & 0.41 & & 0.19 & & & & & & & & & \\
\hline 3 & 1.17 & 1.21 & -0.04 & -0.04 & 0.96 & 0.36 & 0.61 & 0.63 & 2.18 & 1.99 & 0.19 & 0.09 & & & & & & & & \\
\hline 4 & 0.74 & 0.55 & 0.18 & 0.2 & 1.27 & & 1. & 0.88 & 1. & 1.71 & -0.25 & -0.17 & & & & & & & & \\
\hline 5 & 0.61 & 0.13 & 0.4 & & 0.9 & & & 0.9 & 86 & 0.79 & 0.07 & 0.08 & & & & & & & & \\
\hline Total & 4.98 & 2.53 & 2.45 & 0.49 & 3.93 & 1.43 & 2.51 & 0.64 & 7 & 5.63 & 1.37 & 0.2 & & & & & & & & \\
\hline
\end{tabular}

Table 5.II.2: Pseudo-parametric comparison between counterfactual and actual patterns (education)

The results of tables 5.II.1 and 5.II.2 largely confirm our preliminary conclusions from the previous visual inspection. In the case of the wage quintiles, we can now corroborate that the impact is limited in most cases (most values of $z 2$ are very close to zero). In 
fact, in 13 out of 22 cases $z 2$ is negative, which means that applying the German ranking generates more rather than less differences with the national rankings (of course, this effect is very marginal, so it has no implications on its own beyond the fact that it reinforces the conclusion that the German ranking does not homogenize the national patterns). The important point is that, in the case of the wage quintiles, applying the German ranking across the board does not reduce the amount of variability between countries, nor does it make the patterns of other countries more German-like.

In the case of the educational rankings, the results are not so clear-cut. In most countries, the value of $z 2$ is positive, in a few cases significantly so (in seven countries, the reduction in the original dissimilarity index produced by applying the German ranking is above 20\%). The German educational ranking does seem to make other countries more German-like, which is as saying more asymmetrically polarized ${ }^{70}$. Still, this effect is by no means strong enough as to argue that the differences in the rankings are the main parameter behind the differences in the patterns. Only in 2 of the 22 countries which are compared to Germany is the dissimilarity index reduced by more than $50 \%$ when applying the German ranking (taking $z 2$ as an index of the extent to which the difference in the national rankings explain the difference in the pattern). In five more countries the reduction in the dissimilarity is between 20 and $50 \%$. And in the remaining 15 countries, even after fixing the same ranking more than $80 \%$ of the original differences between the national and German patterns remain. So even in the case of the educational quintiles, it is clear that the differences in the patterns are mainly the result of different evolutions in the division of labour. ${ }^{71}$

\subsection{Concluding remarks}

The original aim of this section was to test whether the observed variability in the patterns of employment expansion by job quality across Europe was primarily the result

\footnotetext{
${ }^{70}$ We saw in section 4 of chapter 4 that the German educational ranking was rather unique in Europe (its uniqueness in a principal components analysis of the educational rankings was the highest except for the Baltics), whereas its wage ranking was very common. This must be surely related to the fact that the German educational ranking makes a much bigger impact on the patterns of job growth of the other countries than the German wage ranking.

${ }^{71}$ There is yet another way to prove the same point. As we did in section 4 of chapter 4 for the different rankings, we can produce correlation matrices of the change in the employment levels of each job across the different countries. The correlations are in this case much smaller (in most cases, between .1 and .3) than those of the rankings (which ranged between .6 and .9, see sct. 4, ch. 4). Because the differences in the quintile pictures can only come from those two parameters, the fact that the correlations between rankings is much higher than the correlations between employment change matrices proves that most of the variability in the quintile picture derives from the latter.
} 
of using nationally-specific job quality rankings. In other words, whether behind an apparent plurality of patterns there could be a similar evolution in the division of labour, confounded by different national job quality rankings. In light of our results, we can reject this hypothesis. The main source of the observed variability in the patterns of job growth is, in fact, a different distribution of employment growth across the jobs structure, not differences in the job quality rankings. But it would be wrong to infer from this that the national job quality rankings do not matter. They do have an impact on the observed patterns of job growth: shifting the job quality rankings leads in many cases to significantly different results, which would lead to different conclusions. What we have proved is that the job quality rankings are not the main source of variability, not that they do not matter.

\section{Convergence or parallel evolution of European job structures}

Why should we expect some process of convergence or parallel lagged evolution of job structures? We have empirical evidence suggesting that such a process could be important: in the last section of chapter 4, we observed an association between the level of economic development and the structure of employment, with less developed European countries having a downwards-biased job quality structure (using the German job quality quintiles as reference). This suggests some type of universal process of evolutionary change in employment structures, a process that would involve parallel developments but with time lags associated to the different initial positions, or a convergence if the less developed countries would evolve faster to catch up with richer countries. This kind of development would have ample support in the Social Sciences literature, which has often taken an evolutionary approach that assumes that most countries experience a roughly similar process of structural change, only varying in speed and timing. In fact, any explanation of the patterns of transformation of employment structures which is based in the effect of one or more universal factors (such as the TBTC theory discussed in section 2.3) involves the assumption that all employment structures evolve in similar ways, and therefore that all differences are just the result of different points of departure.

There are important reasons, however, to expect the opposite as well. The growing degree of integration of national economies within European or even global markets could lead to the formation of a European (or global) division of labour, and 
consequently to a growing differentiation rather than convergence of national employment structures. If different countries or regions specialize in the production of different goods or services for the international market, their employment structures could tend to diverge, and this is likely to have different implications in terms of job quality as we are studying it in this thesis. Theories of structural economic change that emphasize the importance of institutions, as well, tend to be much less unidirectional in their predictions: the transformation of employment structures is not only affected by universal factors such as technology or trade, but by the intended or unintended consequences of human behaviour, expressed or canalized through the action of institutions.

In the end, what we have is different alternative views of the factors explaining structural change in employment, which lead to different hypothesis that ultimately must be validated or invalidated empirically. In this section, we will specifically try to assess whether there is evidence of similar evolutionary paths in the transformation of European employment structures in the last 15 years, from our jobs perspective. Even though we will try to contribute to the clarification of this issue, quite obviously we will not be able to settle it. In fact, the following two chapters will also deal with this fundamental issue of the degree of universalism in the patterns of job growth in Europe between 1995 and 2007.

In order to evaluate the importance of initial differences in the patterns of job growth between 1995 and 2007, we need to use a different representation of change to the one used previously in this chapter. The quintile method used in figures 5.1-5.3 is not suitable for our purposes here, because such representation is totally focused on nationally-specific relative change. The quintiles for the year 2000 were constructed in such a way that they divide total employment in each country in five equal-sized groups. But it should be very clear by now that those five groups are equally-sized, not equally-defined: the same job can be in the first quintile in one country and in the third one in another. And this is not only because of the fact that we use country-specific rankings: the different quintile position of the same job in different countries is, in fact, largely the result of the existence of differences in the (initial) composition of the division of labour. For instance, let us take a job which occupies exactly the same ranking position (a low one) in countries A and B: if country A has a large share of employment in low-quality jobs, this job can be assigned to the second quintile, whereas 
if in country B the share of employment in low-quality jobs is very low, it may be assigned to the first quintile instead. ${ }^{72}$ If we want to compare employment structures ignoring differences in job quality hierarchies, we need to use the same quintile allocation across all countries. This is what we did in figure 4.3 of the previous chapter: using the German job quality quintiles as reference point, we created a set of "counterfactual" quintile pictures which summarized graphically the differences in the division of labour from a job quality perspective, for the year 2000. In this section, we use the same analytic strategy, but this time in a dynamic manner: using the 2000 German job quality quintiles, we will create a series of counterfactual charts that show how the quintile picture of each country differed from the German in 1995 and 2007. The comparison between those counterfactual quintile pictures shall allow us to evaluate whether there is some evidence of convergence in job quality structures or similar paths of evolution. Figures 5.9-5.11 show graphically this counterfactual dynamic analysis, while tables 5.III.1-5.III.3 present a pseudo-parametrization similar to the one utilized in the previous section.

Obviously, this analysis is constructed ignoring the differences in the national job quality rankings. This is necessary because, to test the convergence hypothesis, we need to fix a homogeneous metric, which is provided by the German job quality quintiles in this case. This does not mean that the differences in the rankings are not important here: in fact, as was the case in the previous section, the comparison between the counterfactual patterns shown in figures 5.9-5.11 and the real patterns shown in figures 5.1-5.3 is a way to evaluate the role played by the rankings and the initial differences in job quality structures in our results.

\footnotetext{
${ }^{72}$ For instance, the high share of low-quality agricultural employment in Southern European countries fills most of the lowest quintile, leaving less room for low-quality service jobs that occupy most of the lowest quintile in more advanced economies.
} 


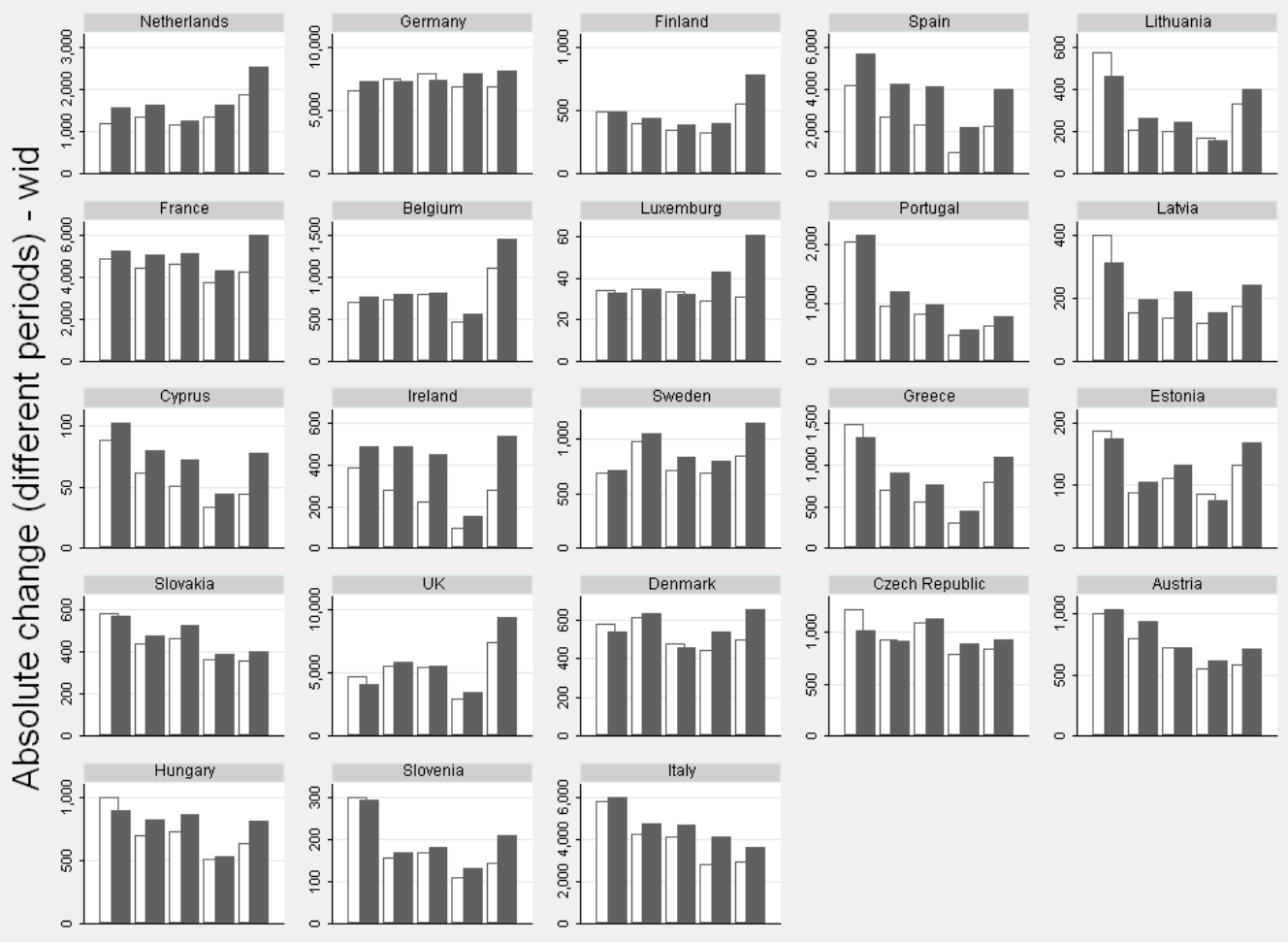

Figure 5.9: Counterfactual job quality structures (wages), 1995 (white bar) to 2007 (black bar)

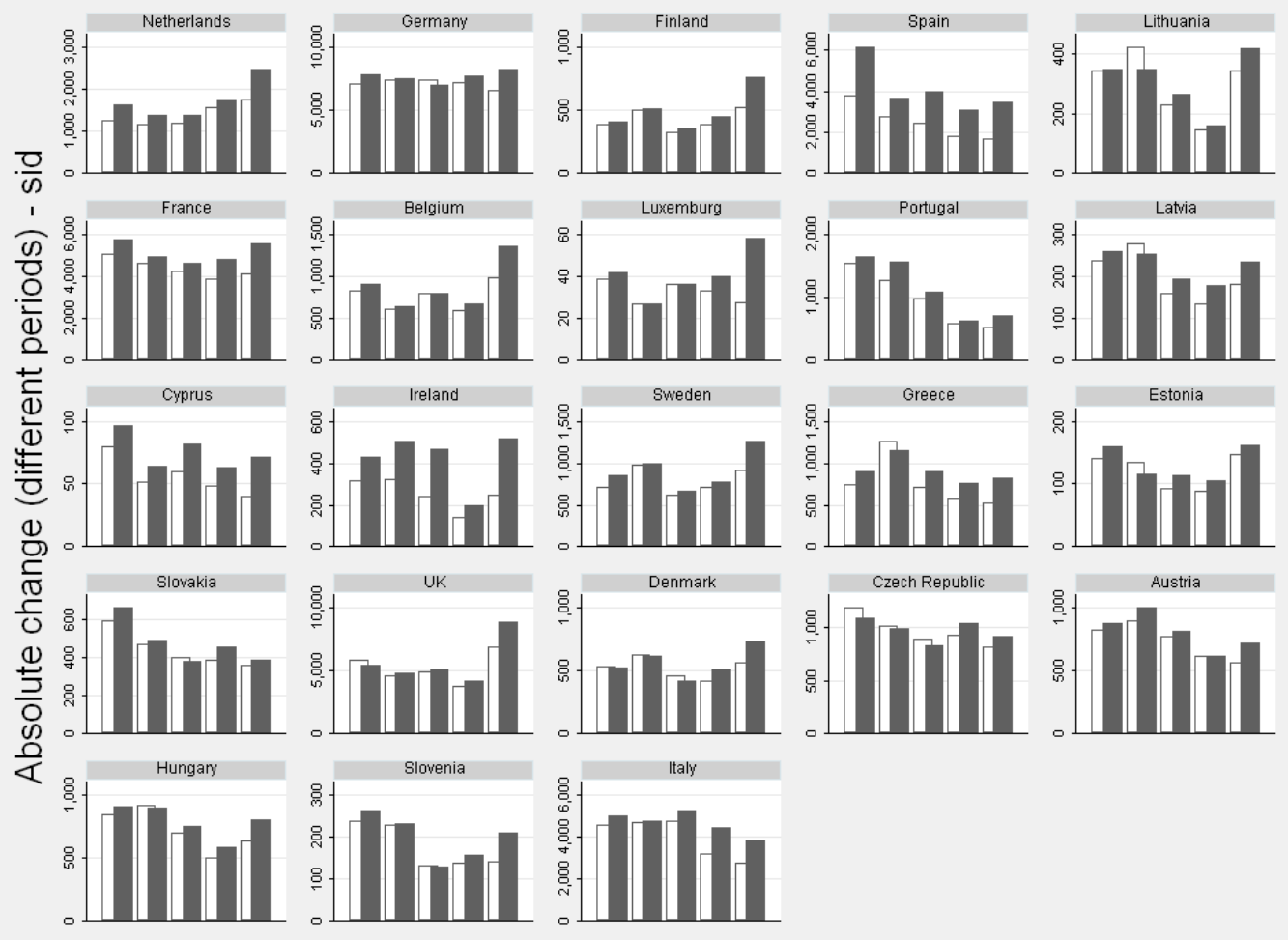

Figure 5.10: Counterfactual job quality structures (education), 1995 (white bar) to 2007 (black bar) 


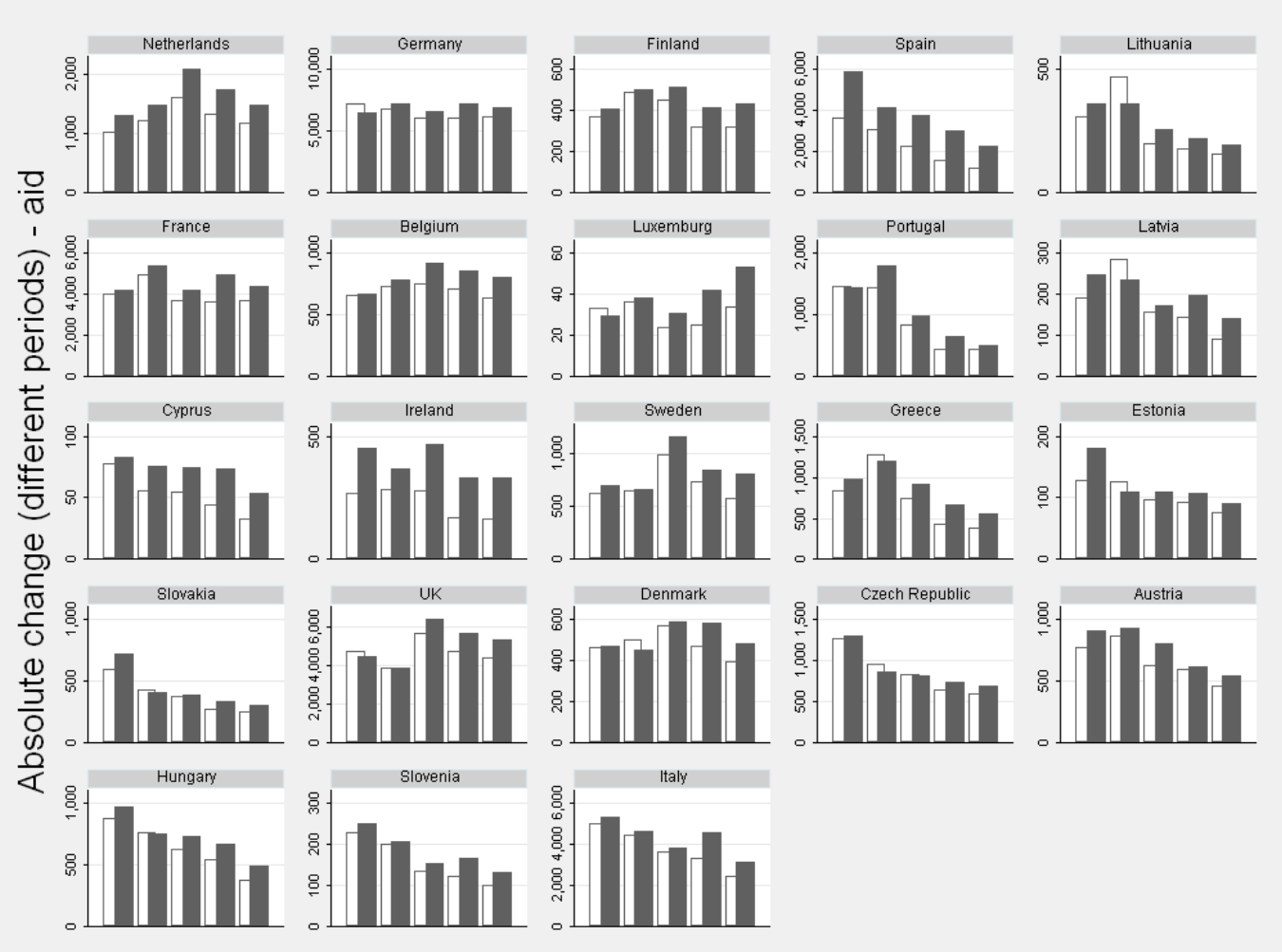

Figure 5.11: Counterfactual job quality structures (amenities), 1995 (white bar) to 2007 (black bar).

First, let us look at the country that will serve as reference for this exercise: Germany. The German structure of employment, as we already know, changed little between 1995 and 2007, destroying some employment in the middle, creating many jobs at the top and some, though not so many, at the bottom (according to the wage and educational ranking: the amenities ranking shows an unambiguous upgrading). In overall terms, the German transformation can be summarized as a mild polarization strongly skewed upwards (or alternatively, a slightly polarized upgrading). The fact that the German job quality structure changed so little over the 1995-2007 period makes it a convenient reference point: basically, a convergence of job quality structures would require the counterfactual (non-German) job quality quintiles becoming more even.

Do figures 5.9-5.11 provide evidence of such a convergence? Certainly, not overwhelmingly, nor everywhere. Some of the countries which were different initially (such as Spain, Latvia, Lithuania, Greece, Italy or Slovenia) became significantly more similar to Germany over the years (their quintile pictures reduced their initial skewness towards the bottom). But some other countries which were also initially quite different (such as Portugal, Finland, the UK or even Netherlands) seemed to become even more different, or at least their original difference from the German job quality structure was 
not reduced. Other countries which were initially very similar to Germany (such as France, Luxemburg or Denmark) became clearly more dissimilar over time.

The convergence hypothesis does not seem to fit very well these results. There are some cases where our results may suggest some type of convergence, but there are many others that do not fit such hypothesis at all, even suggesting divergence rather than the other way around. The effect is, nevertheless, very minor in all cases: even where there seems to be some convergence, most of the initial difference remains.

That is what can be said by means of a simple visual inspection. But as in the previous section, there are so many parameters being represented simultaneously in figures 5.95.11 that it may be difficult to identify clear patterns in the differences between each country and Germany. Again, we are doing here a difference-in-differences type of analysis (comparing the difference between each country and Germany at the beginning and at the end of the period), so that a similar pseudo-parametrization to the one introduced in the previous chapter may help interpreting our results. This is done in tables 5.III.1-5.III.3, which for each country shows: first, the differences between the quintiles of each country and Germany in $1995(x)$; second, the differences between the quintiles of such country and Germany in $2007(y)$; third, the absolute difference-indifferences between $x$ and $y(z 1)$; and fourth, the relative difference-in-differences between $x$ and $y(z 2)$. The latter index, a ratio expressing how much of the initial difference remained in 2007, can be understood as a kind of convergence index in this case: positive values implying convergence with Germany (increased similarity), and negative values implying divergence (decreased similarity). 


\begin{tabular}{|c|c|c|c|c|c|c|c|c|c|c|c|c|c|c|c|c|c|c|c|c|}
\hline & xNL & yNL & $211 \mathrm{vL}$ & Z2NL & |XLL & yDE & IDL & z2DE & xFI & yFI & z1FI & z2FI & xES & yES & z1ES & z2ES & $x$ LT & YLI & z1LT & z2LT \\
\hline 1 & 0.15 & 0.09 & 0.05 & 0.36 & 0 & 0 & 0 & & 0.31 & 0.05 & 0.26 & 0.83 & 1.13 & 0.7 & 0.42 & 0.38 & 1.35 & 0.79 & $\begin{array}{ll}9 & 0.56\end{array}$ & 0.41 \\
\hline 2 & 0.21 & 0.12 & 0.08 & 0.4 & 0 & 0 & 0 & & 0.16 & 0.17 & -0.01 & -0.06 & 0.23 & 0.28 & -0.06 & -0.24 & 0.43 & 0.2 & 0.24 & 0.55 \\
\hline & 0.38 & 0.36 & 0.03 & 0.07 & 0 & 0 & 0 & & 0.5 & 0.38 & 0.12 & 0.24 & 0.39 & 0.17 & 0.23 & 0.58 & 0.82 & 0.43 & 0.39 & 0.47 \\
\hline & 0.07 & 0.13 & -0.06 & -0.91 & 0 & 0 & 0 & & 0.24 & 0.26 & -0.02 & -0.08 & 0.81 & 0.67 & 0.13 & 0.17 & 0.61 & 0.68 & -0.08 & -0.13 \\
\hline 5 & 0.61 & 0.57 & 0.04 & 0.06 & 0 & 0 & 0 & & 0.44 & 0.64 & -0.2 & -0.46 & 0.36 & 0.37 & -0.01 & -0.03 & 0.27 & 0.38 & -0.11 & -0.42 \\
\hline Total & 1.41 & 1.27 & 0.14 & 0.1 & 0 & 0 & 0 & & 1.65 & 1.5 & 0.15 & 0.09 & 2.92 & 2.19 & 0.72 & 0.25 & 3.48 & 2.49 & 0.99 & 0.28 \\
\hline q & XFR & yFR & z1FR & Z2FR & $\mathrm{xBE}$ & yBE & z1BE & Z2BE & $x L U$ & yLU & Z1LU & Z2LU & XPT & $y=1$ & z1PT & Z2PT & xLV & $y \mathrm{yLV}$ & Z1LV & Z2LV \\
\hline 1 & 0.26 & 0.11 & 0.15 & 0.58 & 0.05 & 0.15 & $5 \quad-0.1$ & -2.1 & 0.19 & 0.29 & -0.1 & -0.54 & 1.74 & 1.51 & 0.23 & 0.13 & 1.52 & 0.59 & $\begin{array}{ll}9 & 0.92\end{array}$ & 0.61 \\
\hline 2 & 0.08 & 0.06 & 0.01 & 0.17 & 0.14 & 0.12 & 0.02 & 0.11 & 0.05 & 0.25 & -0.2 & -4.41 & 0.36 & 0.42 & -0.06 & -0.18 & 0.41 & 0.21 & 0.21 & 0.5 \\
\hline & 0.11 & 0.07 & 0.04 & 0.35 & 0.23 & 0.22 & 0.01 & 0.05 & 0.1 & 0.28 & -0.17 & -1.69 & 0.54 & 0.31 & 0.23 & 0.43 & 0.76 & 0.15 & 0.6 & 0.8 \\
\hline & 0.15 & 0.24 & -0.08 & -0.56 & 0.37 & 0.45 & -0.08 & -0.2 & 0.11 & 0.1 & 0.01 & 0.07 & 0.88 & 0.92 & -0.05 & -0.05 & 0.63 & 0.46 & 0.16 & 0.26 \\
\hline & 0.06 & 0.15 & -0.09 & -1.68 & 0.58 & 0.68 & -0.11 & -0.19 & 0.04 & 65 & -0.61 & -13.5 & 0.77 & 87 & -0 & -0.14 & 0.28 & 18 & 0.1 & 0.37 \\
\hline Total & 0.65 & 0.63 & 0.02 & 0.04 & 1.36 & 1.62 & -0.26 & -0.19 & 0.49 & 1.56 & -1.07 & -2.2 & 4.28 & 4.04 & 0.25 & 0.06 & 3.59 & 1.59 & 2 & 0.56 \\
\hline & & & & & & & & & & & & & & & & & EE & & 1EE & 2Cᄃ \\
\hline 1 & 1.01 & 0.65 & 0.36 & 0.35 & 0.94 & 0.38 & $\begin{array}{ll}3 & 0.56\end{array}$ & 0.59 & 0.13 & 0.18 & -0.06 & -0.43 & 1.43 & 0.77 & 0.67 & 0.46 & 0.76 & 0.47 & 0.29 & 0.39 \\
\hline 2 & 0.23 & 0.26 & -0.03 & -0.13 & 0.21 & 0.35 & -0.15 & -0.72 & 0.22 & 0.21 & 0.01 & 0.06 & 0.3 & 0.17 & 0.13 & 0.43 & 0.36 & 0.24 & 0.12 & 0.34 \\
\hline 3 & 0.36 & 0.13 & 0.23 & 0.64 & 0.48 & 0.22 & 0.26 & 0.54 & 0.21 & 0.07 & 0.15 & 0.68 & 0.73 & 0.35 & 0.38 & 0.52 & 0.43 & 0.22 & 0.21 & 0.48 \\
\hline 4 & 0.59 & 0.61 & -0.02 & -0.04 & 0.79 & 0.81 & -0.02 & -0.02 & 0.13 & 0.16 & -0.03 & -0.25 & 0.85 & 0.72 & 0.13 & 0.16 & 0.34 & 0.52 & -0.18 & -0.52 \\
\hline & 0.41 & 0.29 & 0.13 & 0.31 & 0.35 & 0.44 & -0.09 & -0.26 & 0.13 & 0.22 & -0.09 & -0.68 & 0.31 & 0.34 & -0.02 & -0.08 & 0.19 & 0.34 & -0.14 & -0.76 \\
\hline Total & 2.6 & 1.93 & 0.67 & 0.26 & 2.77 & 2.21 & 0.56 & 0.2 & 0.82 & 0.84 & -0.02 & -0.03 & 3.62 & 2.34 & 1.28 & 0.35 & 2.09 & 1.79 & 0.3 & 0.14 \\
\hline q & XSK & ySK & z1SK & z2SK & xUK & yUK & $210 \mathrm{~K}$ & z2UK & XDK & yDK & z1DK & Z2DK & $\mathbf{x C Z}$ & & $\mathrm{z1CZ}$ & & XAT & yAT & Z1AT & Z2AT \\
\hline 1 & 0.56 & 0.39 & 0.17 & 0.31 & 0.04 & 0.32 & -0.28 & -7.03 & 0.34 & 0.08 & 0.26 & 0.76 & 0.44 & 0.11 & 0.33 & 0.75 & 0.68 & 0.59 & $\begin{array}{ll}9 & 0.1\end{array}$ & 0.14 \\
\hline 2 & 0.15 & 0.19 & -0.04 & -0.29 & 0.04 & 0.12 & -0.09 & -2.45 & 0.17 & 0.16 & 0.01 & 0.04 & 0.18 & 0.1 & 0.08 & 0.45 & 0.16 & 0.35 & -0.19 & -1.2 \\
\hline 3 & 0.13 & 0.15 & -0.02 & -0.14 & 0.21 & 0.12 & 0.08 & 0.4 & 0.25 & 0.2 & 0.06 & 0.22 & 0.09 & & -0.1 & -1.05 & 0.2 & 0.13 & 0.09 & 0.42 \\
\hline 4 & 0.25 & 0.31 & -0.06 & -0.24 & 0.4 & 0.48 & -0.08 & -0.21 & 0.21 & 0.14 & 0.07 & 0.34 & 0.23 & 0.15 & 0.08 & 0.34 & 0.37 & 0.45 & -0.08 & -0.22 \\
\hline 5 & 0.3 & 0.44 & -0.14 & -0.47 & 0.51 & 0.66 & -0.15 & -0.28 & 0.07 & 0.09 & -0.03 & -0.44 & 0.22 & 0.24 & -0.02 & -0.09 & 0.34 & 0.4 & -0.06 & -0.19 \\
\hline Total & 1.38 & 1.47 & -0.09 & -0.06 & 1.19 & 1.7 & -0.51 & -0.43 & 1.04 & 0.68 & 0.36 & 0.35 & 1.16 & 0.79 & 0.37 & 0.32 & 1.76 & 1.92 & -0.15 & -0.09 \\
\hline$q$ & $\mathrm{xHU}$ & yHU & $\mathrm{z} 1 \mathrm{HU}$ & $\mathrm{z2HU}$ & & ySI & z1SI & Z2SI & XIT & & Z1IT & Z2IT & & & & & & & & \\
\hline 1 & 0.68 & 0.32 & 0.37 & 0.54 & 1.1 & 0.75 & 0.35 & 0.32 & 0.81 & 0.53 & 0.28 & & & & & & & & & \\
\hline 2 & 0.18 & 0.22 & -0.04 & -0.21 & 0.3 & & 0. & 0. & & 0.2 & -0.02 & -0.1 & & & & & & & & \\
\hline 3 & 0.22 & 0.17 & 0.05 & 0.22 & & 0. & 0. & 0.38 & & 0. & 0.12 & & & & & & & & & \\
\hline 4 & 0.38 & 0.45 & -0.07 & -0.19 & 0.55 & 0.52 & 0.03 & 0.06 & 0.44 & 0.28 & 0.16 & 0.3 & & & & & & & & \\
\hline 5 & 0.23 & 0.23 & 0 & & & 0. & & 0.4 & 0. & 0.51 & -0.04 & -0. & & & & & & & & \\
\hline Total & 69 & 1.39 & 0.3 & 0 & 2.72 & 1.93 & 0.79 & 0.29 & 2.07 & 1.57 & 0.5 & 0.24 & & & & & & & & \\
\hline
\end{tabular}

Table 5.III.1: Convergence towards Germany, wage quintiles

\begin{tabular}{|c|c|c|c|c|c|c|c|c|c|c|c|c|c|c|c|c|c|c|c|c|}
\hline & XNL & NL & & $2 \mathrm{NL}$ & xDE & yDE & & DE & XFI & yFI & Z1FI & Z2FI & XES & yES & z1ES & Z2ES & xLT & yLT & z1LT & Z2LT \\
\hline 1 & 0.24 & 0.2 & 0.04 & 0.15 & 0 & 0 & 0 & & 0.12 & 0.27 & -0.15 & -1.28 & 0.83 & 0.69 & 0.14 & 0.17 & 0.44 & 0.25 & 0.19 & 0.44 \\
\hline 2 & 0.37 & 0.31 & 0.06 & 0.16 & 0 & 0 & 0 & & 0.22 & 0.17 & 0.05 & 0.22 & 0.24 & 0.24 & 0 & 0 & 0.47 & 0.22 & 0.26 & 0.54 \\
\hline & 0.25 & 0.22 & 0.03 & 0.13 & 0 & 0 & 0 & & 0.35 & 0.29 & 0.06 & 0.18 & 0.16 & 0.15 & 0 & 0.03 & 0.4 & 0.2 & 0.2 & 0.51 \\
\hline 4 & 0.22 & 0.1 & 0.12 & 0.55 & 0 & 0 & 0 & & 0.12 & 0.16 & -0.04 & -0.35 & 0.48 & 0.38 & 0.1 & 0.21 & 0.69 & 0.57 & 0.12 & 0.17 \\
\hline & 0.58 & 0.55 & 0.02 & 0.04 & 0 & 0 & 0 & & 0.41 & 0.63 & -0.21 & -0.52 & 0.53 & 0.46 & 0.07 & 0.13 & 0.37 & 0.39 & -0.01 & -0.04 \\
\hline Total & 1.66 & 1.39 & 0.27 & 0.16 & 0 & 0 & 0 & & 1.22 & 1.52 & -0.3 & $\begin{array}{l}-0.24 \\
\end{array}$ & 2.23 & 1.92 & 0.32 & 0.14 & 2.37 & 1.62 & 0.75 & 0.32 \\
\hline & XFR & yFR & Z1FR & z2FR & $\mathrm{XBE}$ & & 1BE & $2 \mathrm{BE}$ & $x L U$ & $y$ LU & z1LU & z2LU & xPT & PT & Z1PT & & $x L V$ & yLV & $1 \mathrm{LV}$ & $22 \mathrm{LV}$ \\
\hline & 0.26 & 0.14 & 0.12 & 0.46 & 0.17 & 0.13 & 0.03 & 0.2 & 0.21 & 0.17 & 0.04 & 0.21 & 1 & 0.86 & 0.14 & 0.14 & 0.49 & 0.29 & 0.2 & 0.42 \\
\hline 2 & 0.06 & 0.04 & 0.02 & 0.34 & 0.28 & 0.34 & -0.06 & -0.2 & 0.24 & 0.43 & -0.2 & -0.83 & 0.53 & 0.71 & -0.18 & -0.33 & 0.48 & 0.23 & 0.26 & 0.53 \\
\hline & 0.13 & 0.05 & 0.08 & 0.63 & 0.17 & 0.16 & 0 & 0.03 & 0.12 & 0.14 & -0.02 & -0.18 & 0.09 & 0.05 & 0.04 & 0.43 & 0.3 & 0.09 & 0.21 & 0.7 \\
\hline & 0.2 & 0.1 & 0.09 & 0.48 & 0.29 & 0.32 & -0.03 & -0.11 & 0.01 & 0.12 & -0.11 & -12.8 & 0.69 & 0.73 & -0.04 & -0.06 & 0.52 & 0.31 & 0.21 & 0.4 \\
\hline & 0.07 & 0.04 & 0.03 & 0.47 & 0.43 & 0.6 & -0.17 & -0.4 & 0.11 & 0.52 & -0.41 & -3.68 & 0.81 & 0.9 & -0.09 & -0.11 & 0.18 & 0.14 & 0.04 & 0.21 \\
\hline Total & 0.71 & 0.37 & 0.34 & 0.48 & 1.33 & 1.55 & -0.22 & -0.17 & 0.69 & 1.38 & -0.7 & -1.01 & 3.12 & 3.24 & -0.12 & -0.04 & 1.97 & 1.05 & 0.92 & 0.47 \\
\hline & $\mathrm{xCY}$ & $\mathrm{yCY}$ & Z1CY & $\mathrm{Z2CY}$ & XIE & ylE & Z1IE & Z2IE & XSE & ySE & z1SE & Z2SE & XGR & GR & Z1GR & GR & xEE & & Z1EE & Z2EE \\
\hline & 0.59 & 0.32 & 0.27 & 0.45 & 0.49 & 0.1 & 0.39 & 0.79 & 0.15 & 0.1 & 0.05 & 0.33 & 0.32 & 0.16 & 0.16 & 0.49 & 0.29 & 0.24 & 0.05 & 0.17 \\
\hline & 0.23 & 0.23 & 0 & 0.01 & 0.35 & 0.34 & 0.02 & 0.05 & 0.25 & 0.17 & 0.08 & 0.32 & 0.78 & 0.4 & 0.38 & 0.49 & 0.08 & 0.11 & -0.03 & -0.43 \\
\hline & 0.11 & 0.21 & -0.11 & -0.98 & 0.2 & 0.23 & -0.03 & -0.16 & 0.28 & 0.27 & 0.02 & 0.06 & 0.19 & 0.15 & 0.04 & 0.21 & 0.41 & 0.16 & 0.25 & 0.62 \\
\hline & 0.24 & 0.22 & 0.03 & 0.12 & 0.61 & 0.61 & 0 & 0.01 & 0.15 & 0.16 & -0.01 & -0.08 & 0.46 & 0.26 & 0.2 & 0.43 & 0.35 & 0.24 & 0.11 & 0.32 \\
\hline & 0.39 & 0.28 & 0.11 & 0.29 & 0.26 & 0.34 & -0.09 & -0.33 & 0.3 & 0.39 & -0.1 & -0.32 & 0.48 & 0.33 & 0.15 & 0.3 & 0.3 & 0.18 & 0.12 & 0.4 \\
\hline Total & 1.56 & 1.25 & 0.3 & 0.2 & 1.91 & 1.62 & 0.29 & 0.15 & 1.13 & 1.09 & 0.04 & 0.03 & 2.22 & 1.31 & 0.92 & 0.41 & 1.43 & 0.93 & 0.5 & 0.35 \\
\hline & XSK & & $\overline{\text { SK }}$ & Z2SK & XUK & yUK & z1UK & \begin{tabular}{l|l} 
Z2UK \\
\end{tabular} & $\mathrm{xDK}$ & yDK & z1DK & Z2DK & $\mathrm{xCZ}$ & & z1CZ & $\mathrm{z2CZ}$ & XAT & yAT & Z1AT & Z2AT \\
\hline & 0.54 & 0.59 & -0.05 & -0.09 & 0.14 & 0.16 & -0.02 & -0.14 & 0.14 & 0.1 & 0.04 & 0.3 & 0.35 & 0.16 & 0.19 & 0.54 & 0.29 & 0.24 & 0.05 & 0.17 \\
\hline & 0.11 & 0.15 & -0.04 & -0.33 & 0.2 & 0.2 & 0 & 0.01 & 0.17 & 0.16 & 0.01 & 0.07 & 0.06 & 0.06 & 0 & -0.08 & 0.3 & 0.4 & -0.11 & -0.36 \\
\hline & 0.2 & 0.16 & 0.04 & 0.19 & 0.27 & 0.15 & 0.12 & 0.44 & 0.23 & 0.22 & 0.01 & 0.03 & 0.16 & 0.08 & 0.08 & 0.49 & 0.04 & 0.12 & -0.09 & -2.49 \\
\hline 4 & 0.27 & 0.19 & 0.08 & 0.3 & 0.29 & 0.34 & -0.05 & -0.17 & 0.27 & 0.1 & 0.17 & 0.64 & 0.13 & 0.11 & 0.02 & 0.16 & 0.28 & 0.36 & -0.08 & -0.27 \\
\hline & 0.24 & 0.41 & -0.16 & -0.68 & 0.47 & 0.59 & -0.12 & -0.2 & 0.19 & 0.3 & -0.12 & -0.64 & 0.15 & 0.17 & -0.02 & -0.12 & 0.3 & 0. & -0.06 & -0.18 \\
\hline Total & 1.36 & 1.49 & -0.13 & -0.09 & 1.37 & 1.44 & -0.07 & -0.05 & 0.99 & 0.88 & 0.12 & 0.12 & 0.86 & 0.59 & 0.27 & 0.31 & 1.23 & 1.51 & -0.28 & -0.23 \\
\hline & HU & HU & $\mathbf{Z}$ & $\mathrm{z} 2 \mathrm{HU}$ & |xS| & ySI & z1S| & Z2SI & T & & Z1IT & $21 \mathrm{~T}$ & & & & & & & & \\
\hline & 0.39 & 0.27 & 0.12 & 0.31 & 0.67 & 0.55 & 0.12 & 0.18 & 0.3 & 0.1 & 0.19 & 0.65 & & & & & & & & \\
\hline 2 & 0.36 & 0.27 & 0.08 & 0.23 & 0.39 & 0.27 & 0.12 & 0 & 0.28 & 0.14 & 0.14 & & & & & & & & & \\
\hline 3 & 0.11 & 0. & 0.05 & 0.49 & 0. & 0.37 & 0 & -0. & 0.18 & 0.28 & -0.1 & -0.53 & & & & & & & & \\
\hline & 0.46 & 0.37 & 0.09 & 0.19 & 0.43 & 0.38 & 0.05 & 0. & 0.3 & 0.09 & 0.22 & 0.71 & & & & & & & & \\
\hline & 0.22 & 0.22 & 0 & 0 & 0.3 & 0.12 & 0.17 & 0.58 & 0.45 & 0.41 & 0.05 & 0.1 & & & & & & & & \\
\hline Total & 54 & 1.19 & 0.35 & 0.23 & 2.15 & 1.69 & .46 & 0.2 & 1.52 & 1.02 & 0.5 & 0.33 & & & & & & & & \\
\hline
\end{tabular}

Table 5.III.2: Convergence towards Germany, educational quintiles 


\begin{tabular}{|c|c|c|c|c|c|c|c|c|c|c|c|c|c|c|c|c|c|c|c|c|}
\hline & xNL & NL & z1NL & $\mathrm{z} 2 \mathrm{NL}$ & XDE & yDE & z1DE & z2DE & xFI & $\mathrm{yFI}$ & z1FI & z2FI & xES & yES & z1ES & z2ES & xLT & yLT & z1LT & Z2LT \\
\hline & 0.51 & 0.26 & 0.24 & 0.48 & 0 & 0 & 0 & & 0.19 & 0.04 & 0.14 & 0.76 & 0.8 & 0.91 & -0.12 & -0.15 & 0.47 & 0.65 & -0.18 & -0.39 \\
\hline 2 & 0.12 & 0.16 & -0.04 & -0.37 & 0 & 0 & 0 & & 0.29 & 0.11 & 0.18 & 0.61 & 0.52 & 0.26 & 0.26 & 0.5 & 0.95 & 0.43 & 0.52 & 0.55 \\
\hline 3 & 0.45 & 0.41 & 0.04 & 0.09 & 0 & 0 & 0 & & 0.34 & 0.21 & 0.13 & 0.37 & 0.04 & 0.09 & -0.06 & -1.55 & 0.28 & 0.04 & 0.24 & 0.86 \\
\hline & 0.24 & 0.11 & 0.13 & 0.53 & 0 & 0 & 0 & & & 0.15 & -0.02 & -0.17 & 0.52 & 0.47 & 0.05 & 0.09 & 0.53 & 0.46 & 0.07 & 0.12 \\
\hline 5 & 0.08 & 0.12 & -0.05 & -0.62 & 0 & 0 & 0 & & 0.28 & 0.14 & 0.13 & 0.48 & 0.83 & 0.73 & 0.1 & 0.12 & 0.69 & 0.59 & 0.1 & 0.14 \\
\hline Total & 1.39 & 1.07 & 0.32 & 0.23 & 0 & 0 & 0 & & 1.22 & 0.66 & 0.56 & 0.46 & 2.7 & 2.47 & 0.23 & 0.08 & 2.91 & 2.17 & 0.73 & 0.25 \\
\hline $\mathbf{q}$ & XFR & yFR & z1FR & & XBE & & & & xLU & & 110 & $22 L$ & (1) & & z1PT & & $x L V$ & & z1LV & Z2LV \\
\hline 1 & 0.15 & 0.07 & 0.08 & 0.53 & 0.25 & 0.21 & 0.04 & $\quad 0.15$ & 0.08 & 0.3 & -0.22 & -2.86 & 0.97 & 0.93 & 0.03 & 0.04 & 0.3 & 0.53 & -0.22 & -0.74 \\
\hline 2 & 0.21 & 0.12 & 0.09 & 0.43 & 0.01 & 0.09 & & -7.27 & 0.15 & 0.2 & -0.05 & -0.31 & 0.82 & 0.95 & -0.13 & -0.16 & 0.78 & 0.24 & 0.54 & 0.69 \\
\hline & 0.06 & 0.07 & -0.01 & -0.21 & 0.21 & 0.22 & -0.01 & -0.05 & 0.2 & 0.23 & -0.03 & -0.15 & 0.03 & 0.08 & -0.05 & -1.88 & 0.18 & 0.09 & 0.09 & 0.48 \\
\hline 4 & 0.05 & 0.04 & 0.01 & 0.21 & 0.13 & 0.07 & 0. & 0.44 & 0.15 & 0.11 & 0.05 & 0.29 & 0.79 & 0.8 & -0.01 & -0.01 & 0.3 & 0.2 & 0.09 & 0.32 \\
\hline & 0.07 & 0.06 & 0.01 & 0.17 & 0.06 & 0.01 & & 0.87 & 0.16 & 0.58 & -0.42 & -2.64 & 0.97 & 1.03 & -0.06 & -0.06 & 0.72 & 0.46 & 0.26 & 0.36 \\
\hline Total & 0.54 & 0.36 & 0.18 & 0.34 & 0.66 & 0.6 & 06 & 0.09 & 0.74 & 1.41 & -0.67 & -0.9 & 3.58 & 3.8 & -0.22 & -0.06 & 2.28 & 1.52 & 0.75 & 0.33 \\
\hline & S & & & Z2CY & & & & Z2IE & xSE & & ISE & Z2SE & xGR & & z1GR & Z2GR & XEE & yEE & z1EE & Z2EE \\
\hline 1 & 0.53 & 0.31 & 0.22 & 0.41 & 0.13 & 0.27 & -0.13 & $3-1$ & 0.43 & 0.29 & 0.14 & 0.33 & 0.38 & 0.46 & -0.09 & -0.23 & 0.26 & 0.79 & -0.53 & -2.05 \\
\hline 2 & 0.15 & 0.1 & 0.05 & 0.34 & 0.33 & 0.22 & 0.1 & 0.32 & 0.17 & 0.28 & -0.11 & -0.66 & 0.96 & 0.55 & 0.41 & 0.43 & 0.26 & 0.26 & -0.01 & -0.02 \\
\hline 3 & 0.09 & 0.09 & 0 & 0.01 & 0.34 & 0.27 & 07 & 0.21 & 0.58 & 0.49 & 0.09 & 0.16 & 0.22 & 0.16 & 0.07 & 0.3 & 0.04 & 0.15 & -0.11 & -2.7 \\
\hline 4 & 0.22 & 0.1 & 0.13 & 0.56 & 0.27 & 0.24 & 03 & 0.1 & 0.22 & 0.16 & 0.06 & 0.26 & 0.59 & 0.46 & 0.13 & 0.22 & 0.14 & 0.31 & -0.17 & -1.22 \\
\hline & 0.56 & 0.39 & 0.17 & 0.3 & 0.5 & 0.32 & 0. & 0.36 & 0.18 & 0.07 & 0.11 & 0.61 & 0.84 & 0.67 & 0.17 & 0.2 & 0.38 & 0.44 & -0.06 & -0.17 \\
\hline Total & 1.56 & 0.99 & 0.57 & 0.36 & 1.57 & 1.32 & 0.24 & 0.16 & 1.58 & 1.29 & 0.29 & 0.18 & 2.99 & 2.3 & 0.69 & 0.23 & 1.07 & 1.96 & -0.88 & -0.82 \\
\hline & XSK & & & z2SK & & & & z2UK & & yDK & $\overline{\mathrm{DK}}$ & 2DK & & CZ & 2102 & & & yAT & Z1AT & Z2AT \\
\hline 1 & 0.68 & 1.05 & -0.37 & -0.55 & 0.26 & 0.26 & 0 & -0.01 & 0.23 & 0.13 & 0.1 & 0.44 & 0.58 & 0.77 & -0.19 & -0.32 & 0.21 & 0.47 & -0.26 & -1.25 \\
\hline 2 & 0.25 & 0.3 & -0.05 & -0.2 & 0.24 & 0.33 & -0.09 & -0.4 & 0.04 & 0.18 & -0.14 & -3.72 & 0.22 & 0.21 & 0 & 0.02 & 0.36 & 0.34 & 0.02 & 0.06 \\
\hline 3 & 0.08 & 0.2 & -0.11 & -1.36 & 0.31 & 0.3 & 0.02 & 0.05 & 0.35 & 0.22 & 0.13 & 0.36 & 0.07 & 0.14 & -0.07 & -1.14 & 0.09 & 0.11 & -0.03 & -0.28 \\
\hline 4 & 0.39 & 0.48 & -0.09 & -0.24 & 0.17 & 0.17 & 0 & 0.02 & 0.09 & 0.14 & -0.05 & -0.58 & 0.33 & 0.37 & -0.04 & -0.13 & 0.15 & 0.38 & -0.23 & -1.55 \\
\hline 5 & 0.59 & 0.59 & 0 & -0.01 & 0.05 & 0.09 & -0.04 & -0.91 & 0.19 & 0.07 & 0.12 & 0.62 & 0.5 & 0.43 & 0.08 & 0.15 & 0.44 & 0.55 & -0.11 & -0.25 \\
\hline Total & 1.99 & 2.62 & -0.64 & -0.32 & 1.03 & 1.15 & -0.12 & -0.12 & 0.89 & 0.75 & 0.14 & 0.16 & 1.7 & 1.92 & -0.22 & -0.13 & 1.24 & 1.85 & -0.61 & -0.4 \\
\hline 4 & $\mathrm{xHU}$ & $\mathrm{yHU}$ & HU & $\mathrm{z} 2 \mathrm{HU}$ & xSI & ySI & z1SI & Z2SI & & & z1IT & Z2IT & & & & & & & & \\
\hline 1 & 0.47 & 0.6 & -0.13 & -0.29 & 0.63 & 0.71 & -0.08 & $3-0.12$ & 0.4 & 0.45 & -0.05 & -0.12 & & & & & & & & \\
\hline 2 & 0.3 & 0.16 & 0.14 & 0.47 & 0.39 & 0.22 & 0.17 & 0.4 & 0.26 & & 0.15 & 0.57 & & & & & & & & \\
\hline 3 & 0.09 & 0.08 & 0.01 & 0. & 0.0 & 0. & -0.09 & -0.97 & & 0.0 & -0.01 & -0.1 & & & & & & & & \\
\hline 4 & 0.22 & & -0.04 & -0.18 & & & & & & & & & & & & & & & & \\
\hline 5 & 0.61 & 0.55 & 0.06 & 0.1 & 0.5 & 0.47 & 0. & 0.17 & 0.52 & 0. & 0.12 & 0.24 & & & & & & & & \\
\hline Total & 1.69 & 1.65 & 0.04 & 0.02 & 2.04 & 1.89 & 0.15 & 0.07 & 1.42 & 1.15 & 0.27 & 0.19 & & & & & & & & \\
\hline
\end{tabular}

Table 5.III.3: Convergence towards Germany, amenities quintiles

The $z 2$ index shows that most of the original dissimilarity persisted in 2007 (only in one country did the dissimilarity reduce by more than half, in Latvia). But of course, there was no reason to expect a complete homogeneization of European job quality structures over this particular period of 12 years. In fact, in most countries the original dissimilarity was reduced over this period (in 16 countries for the wage index, 15 for the educational index, and 16 for the amenities index, the values of $z 2$ are positive), which suggests some convergence whose importance is difficult to evaluate. A possible way to evaluate the extent of this convergence is to study the relationship between the extent of initial differences and the degree of convergence: in principle, the countries which were initially more different from Germany should have converged faster than the countries which were initially rather similar (whose dissimilarity index should not have changed that much). This is shown in figure 5.12, which plots the convergence index ( $z 2)$ against the initial dissimilarity index $(x)$.

A process of convergence in job quality structures would require a clear positive association between initial dissimilarity and convergence, and figure 5.12 does not show that. Only the wage ranking suggests a significant degree of convergence, and only very mildly (there are very clear outliers, such as Portugal, the Czech Republic or Denmark). Certainly, figure 5.12 does not allow us to conclude that convergence was an important 
driver behind the evolution of job quality structures across Europe. All our evidence (be it the visual inspection of the convergence in counterfactual job quality quintiles or the pseudo-parametric analysis) points to this same direction. Convergence was not a determinant factor, or only a very marginal one.
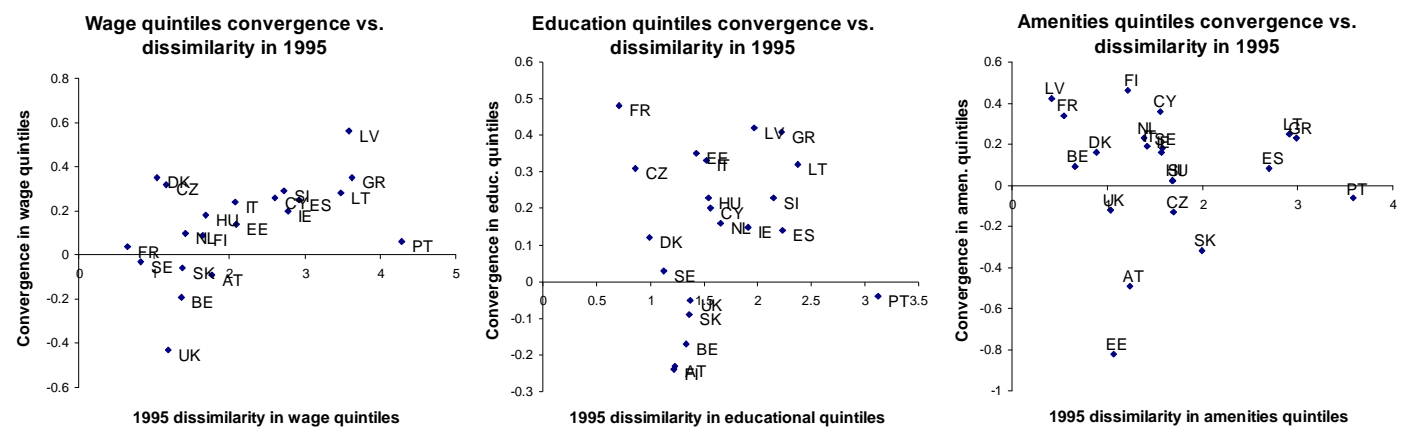

Figure 5.12: Convergence in the three job quality quintiles vs. original dissimilarity

But there is a second way in which the initial differences would affect the patterns of employment growth, even if there is no convergence. Insofar as economic development is associated with a particular sequence of transformations in the employment structure, the observed differences in the patterns of job growth may simply reflect the different stages of economic development of the European countries we are studying here. Our earlier analysis of convergence would not reflect this effect, because we used a moving target as point of reference: the fact that the other countries did not become more like Germany between 1995 and 2007 may prove that there was no convergence, but not that there was not a similar pattern of development. In order to test this second idea, we can keep Germany as reference, but rather than comparing the initial structures of each country and Germany versus the final structures of each country and Germany, we can compare both the initial and final structures of each country to the initial structure of Germany. If between 1995 and 2007 the countries which are on a lower stage of development became significantly more like Germany in 1995, whereas those on a similar or even higher stage of development became more dissimilar, we could take it as evidence of the existence of a shared sequence of transformations in the jobs structure.

This second hypothesis finds slightly more support in our data, although still the support is by no means overwhelming or even conclusive. In figure 5.13, we can see a plot of the $z 2$ index (in this case, based on the convergence towards the 1995 German job quality structure) against the initial differences measured by the $x$ index (which in this case is identical to the horizontal axis of figure 5.12, as the initial differences refer to 
Germany in 1995). The association between the degree of original dissimilarity and the degree of final dissimilarity when the point of reference is fixed to Germany in 1995 is slightly better for the three job quality measures. We can take the argument even further and plot the $z 2$ index against GDP per capita in PPP in 2000 (see figure 5.14), which can be taken as an indicator of the level of economic development of each country. The job quality structures as measured by education and wages became more similar to Germany in 1995 for most of the countries whose GDP per capita was below that of Germany, whereas they became more dissimilar for most of the countries whose GDP per capita was around the same level as Germany or even higher (of course, there are exceptions; like Ireland, which became much more similar to Germany over these years despite the fact that its PC GDP was already higher). The effect, though, remains mild.
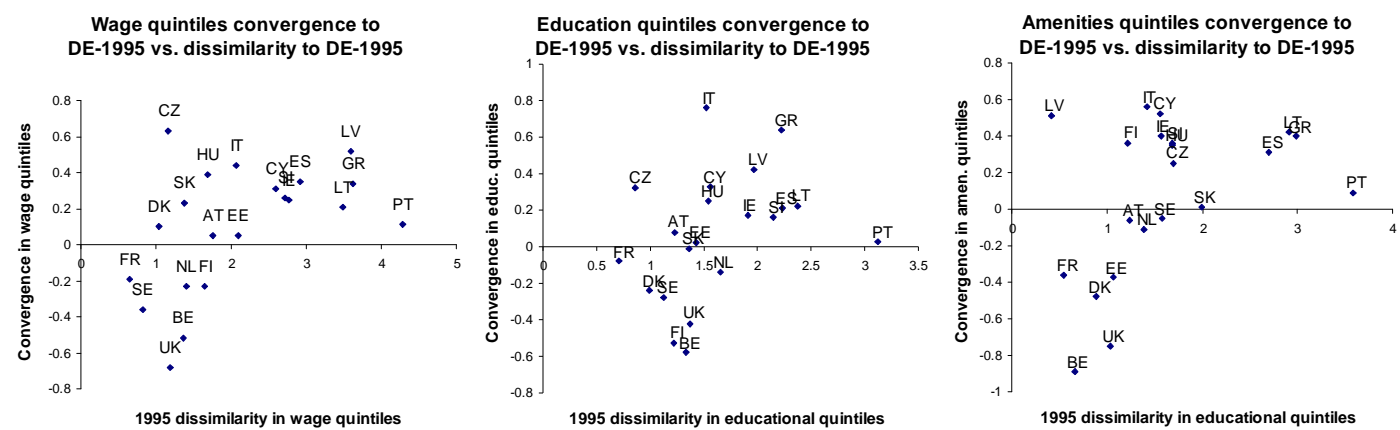

Figure 5.13: Convergence in the three job quality quintiles to Germany 1995 vs. original dissimilarity
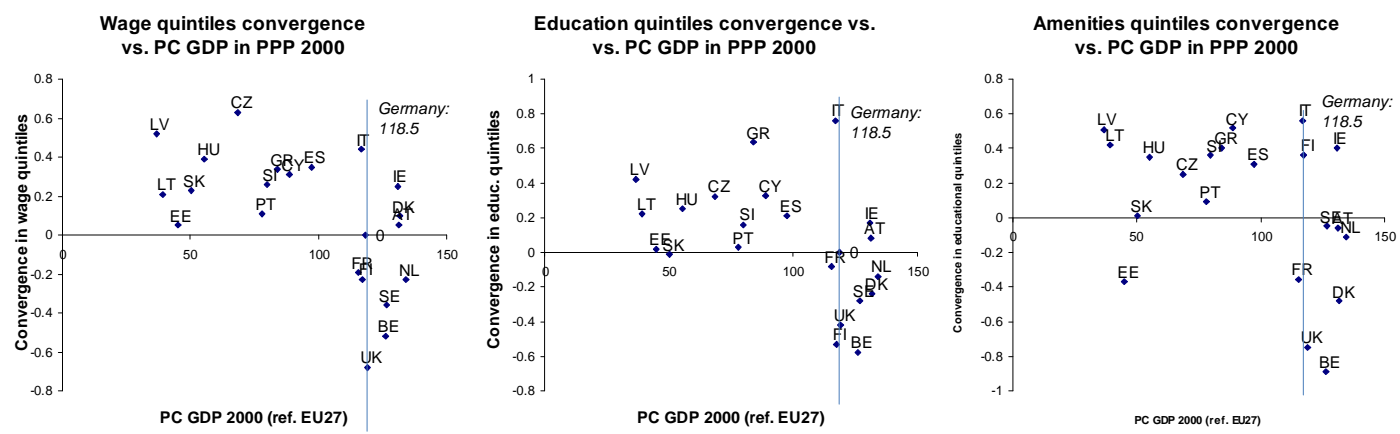

Figure 5.14: Convergence in the three job quality quintiles to Germany 1995 vs. GDP PC in 2000

The overall conclusion for this exercise, therefore, is that although there were important differences in the job quality structures at the beginning of the period, these differences are not the main factor behind the observed patterns of job growth by job quality. Our evidence suggests that such initial differences played only a minor role. Throughout the period of 13 years covered here, the 23 countries did not experience a process of 
convergence in their job quality structures, or if they did, it was very limited. Clearly, there are other more important factors behind the different patterns of employment growth by job quality, factors that we shall explore in the next section. We found slightly more convincing evidence of the possibility that the evolution of employment structures would have followed a similar path in the different countries (which rather than convergence, involves parallel paths with different starting points); but even in this case, such an effect seems rather weak and probably secondary to other most important explanatory factors.

\section{Conclusions}

The first and most obvious conclusion that can be drawn from the analysis carried out in this chapter is that there was a considerable plurality in the 1995-2007 patterns of job growth across the European Union. Such finding, which in itself would not seem very surprising (after all, we are speaking about very different countries, economic situations, social systems), contrasts with most of the literature on the implications of the changing division of labour. Such literature, as argued in chapter 2 , is dominated by three broad approaches which have in common a relatively deterministic explanation, in which organizational, technological and economic exogenous factors would affect the employment structures of advanced economies in similar and one-directional ways ${ }^{73}$. Our findings prove that this is not the case, or at least it was not in the sample of 23 European countries and the period of 12 years studied here. There was a plurality of patterns, and therefore a certain degree of indeterminacy in the effects of the structural evolution of the division of labour on job quality. This suggests that even if all countries are subject to similar exogenous mechanisms, there are endogenous mechanisms that can alter the equation significantly, leading to diverging results for job quality.

In terms of the method used here to study the patterns of job growth by job quality, this observed plurality could have two different sources: the different evolution in the employment structures and the different job quality rankings across Europe. In the second section of this chapter, we tried to evaluate the relative importance of those two main parameters behind the patterns of job growth across Europe. We concluded that

\footnotetext{
${ }^{73}$ Not all these theories have such deterministic bias: in particular, the trade/institutionalist polarization hypothesis explicitly argues that the outcome of the evolution of the division of labour depends on the interaction between such exogenous factors (technology and international trade for this hypothesis) and endogenous institutions and political action.
} 
the main determinant for the observed country differences is the differential transformation of employment structures, with the national job quality rankings playing only a secondary role. If the national job quality structures changed in different ways across Europe between 1995 and 2007, it is primarily because European countries transformed their employment structures in different ways. This reinforces the hypothesis that the different countries had some flexibility to accommodate the economic transformations brought about by technological change and globalization to the organization of labour, with rather different implications for the quality of existing jobs.

But there are still alternative possible explanations of the observed plurality of patterns which would retain a universalistic/deterministic bias. It could be that the observed plurality of patterns was simply a result of the different initial positions: even if all countries shared exactly the same sequence of development in the division of labour, if we compare different segments of that same sequence (which is the case, because not all countries were in 1995 in the same stage of economic development) we will inevitably see different patterns. In section three of this chapter, we tested the validity of this hypothesis by fixing the same quintile structure to all countries and evaluating whether their evolutions were somehow converging or parallel. All national employment structures were reconstructed as if they were pictures of the German employment structure at a different stage: the evolution of employment in less developed European countries over such counterfactual German structure did not lead to a significant level of convergence towards the current German structure, which can be taken as evidence that convergence played a very secondary role (if any) in the patterns of job growth across Europe between 1995 and 2007.

In any case, once we assume the existence of a plurality of patterns of job growth across Europe, we can try to fit each of them to the three images of change distilled from the theoretical discussion of chapter 2. The downgrading hypothesis does not fit any of the observed patterns of job growth (with the partial and not very convincing exception of Estonia), so we can safely reject it from our jobs perspective. The polarization image of change fits the patterns of job growth of many important countries (especially, some of the big countries of continental Europe, such as Germany, France and the Netherlands), though only in two of the three job quality measures (education and wages, not amenities). But in the sample of European countries studied here, the image of change 
which clearly predominates is one of upgrading in the job quality structure. When jobs are ranked by wages, around half of the countries show an upgrading pattern; when jobs are ranked by their educational level, three quarters; and when jobs are ranked by amenities, nearly all countries seem to upgrade their job quality structures.

In fact, if we look at the patterns of job growth quintile by quintile, the support for the upgrading hypothesis is even more overwhelming. In nearly all countries, for the three job quality dimensions, employment at the top grew faster than in the middle or the bottom. Even in some of the clearest cases of polarization, as Germany or France, such polarization was clearly skewed to the top. Therefore, another way to describe the patterns of job growth across Europe between 1995 and 2007 is that good jobs grew vigorously nearly everywhere, whereas bad jobs grew significantly in some countries (those in the first and second columns of figure 5.1) and middling jobs in some others (those in the fourth column of figure 5.1).

Most of the differences in the patterns of job growth, therefore, took place in the bottom and in the middle of the job quality structure: the top looked rather similar everywhere. In section 3 of this chapter, we proved that such differences are mainly the result of a different distribution of job creation and destruction across the different sectors and occupations (not of the different job quality structures). This raises many questions that we will try to answer in the following chapter. Why did most countries expand significantly their share of good jobs? What types of jobs are we talking about? Were they the same types of jobs in all countries? On the other hand, why did some countries expand significantly their share of bad jobs? What sectors and occupations were responsible for such development? In which ways were those countries different from the rest? By decomposing the patterns of job growth by broad sectors of the economy, we shall be able to get a better understanding of the mechanisms that explain the differences observed across Europe. By further decomposing those patterns by employment status, we shall link the evolution of job quality structures with recent changes in employment regulation. 



\section{Understanding the patterns: decomposing the patterns of job growth by sector, occupation and type of employment relation}

\section{Introduction}

In the previous section, we identified the broad patterns of employment growth by job quality between 1995 and 2007 in 23 European countries, and linked them to the three main images of change in the composition of the division of labour that appear in the Social Sciences literature. In this section, we will go one step forward and try to explore what lies behind those developments, by decomposing them in terms of sector, occupation and type of employment relation. In particular, we will try to identify the sources of variation and similarity in the patterns of job growth across Europe, using the theoretical framework discussed in the second chapter as the yardstick of our analysis.

It is, in fact, debatable whether in this chapter we will really do explanatory analysis or we will remain at an exploratory or even descriptive level. A proper explanatory analysis of the differences in the patterns of job growth across Europe would require studying directly the mechanisms behind technical, organizational and social innovation, which is obviously beyond our possibilities in this thesis. Our approach in this chapter is much less ambitious, yet we believe it can provide very useful information for the interpretation of the differences in the patterns of job growth across Europe, and for linking them to the theoretical hypotheses discussed earlier.

The approach used here for the analysis of the evolution of European employment structures is based on what we have called the jobs matrix, which is a detailed system of classification of employment into jobs defined simultaneously by sector and occupation at a relatively detailed level. So far, we have analysed this evolution only from the 
perspective of job quality: the information on sector and occupation has only been used to classify employment into jobs. But in fact, sector and occupation contain very useful information for the interpretation of the patterns of evolution of employment by job quality: they directly classify employment in terms of the horizontal and vertical dimensions of the division of labour, as argued in chapter 2. Because sector and occupation are the basis of the jobs matrix, the patterns of job growth shown in the previous chapter can be easily decomposed by any reaggregation of those two dimensions. In other words, we can see what was the precise contribution of each sector and occupation to the change of each of the quintiles. This shall allow us to evaluate more directly the evolution of employment by job quality from the perspective of changes in the composition of the division of labour: it is this type of analysis that can most directly answer the big questions posed at the end of chapter 2.

This detailed analysis of the contribution of each sector and occupation to the different patterns of job growth in Europe will be done in section 3. But before such analysis, in section 2 we will evaluate the relative contribution of each of the two dimensions defining the jobs matrix (sector and occupation) as a whole. The jobs matrix is defined by both sector and occupation, the assumption being that the division of labour is defined by those two dimensions: but this does not mean that both dimensions are equally important in terms of the evolution of job quality from a compositional perspective. It may be that the change in the composition of employment by sector, for instance, is irrelevant for the evolution of job quality as defined in this project, and that such evolution is totally determined by shifts in employment across occupational levels. By constructing two counterfactual pictures of employment growth by job quality, one which keeps constant the structure of occupations and the other the structure of sectors, we will be able to evaluate the relative contribution of each dimension to the transformation of the employemnt structure with respect to job quality.

Then, in section 4, we will add a further dimension to the analysis, by differentiating employment by type of contract. This differentiation will be done in terms of two different variables: the employment status of the job (with three categories: selfemployed, permanent employees and contingent employees) and part-time status ${ }^{74}$ (with

\footnotetext{
${ }^{74}$ As explained in section 4 of the theoretical chapter, we opted to leave out supply-side factors from our analysis, which means that we will not discuss the association between gender and part-time employment, nor gender in general. For the association between gender and the expansion of part-time employment in Europe, see our previous work in Muñoz de Bustillo, Fernández-Macías and Antón 2008 (especially,
} 
two categories: full-time and part-time workers). The jobs matrix is defined in terms of the division of labour from a material perspective: roughly speaking, it is a classification of all workers within an economy in terms of the types of products and services they produce (horizontal division of labour, or sectors) and in terms of the types of skills and responsibilities they deploy at work (vertical division of labour, or occupations). The contract of employment is a transversal dimension to this basic differentiation, which cuts across its categories and belongs to a different realm. Sector and occupation classify workers in terms of their contribution to production, and reflect the material relations of production; type of contract classifies workers in terms of the relation binding them to the organization where they work, and reflect the social relations of production. Therefore, the decomposition of the patterns of job growth by type of contract introduces an institutional element in the analysis, which shall allow us to evaluate whether the regulation of production relations (in a very basic sense) played some role in the patterns of employment growth by job quality.

In the previous two sections, we used three different job quality rankings to characterize the structure of employment: one based on wages, the other on the educational level of job-holders and the third one based on non-pecuniary job attributes. Having three different measures contributed to the robustness of our analysis and interpretations, because we could contrast the results obtained using the three approaches. In this section, which will be based on a graphical decomposition of the broad patterns discussed earlier, it would be totally impractical to use again the three rankings, as it would require multiplying by three all the figures, without adding much information in most cases. It seems much more sensible to use only one of the three rankings for all the decompositions of this chapter, or alternatively to merge the three rankings into one and use it for the decompositions. The latter alternative has the problem of introducing a new ranking (a merged one) that has not been used so far, and which therefore cannot be related to any earlier analysis. It is obviously preferable to use one of the three rankings which we have been using up to now, but which one? The ranking based on the multidimensional non-pecuniary index of job quality is out of the question because it does not cover the whole of the jobs matrix (it leaves out more or less $10 \%$ of employment) and it is not country-specific: this ranking is useful as a complement to the other two, but it cannot be used as a standalone job quality measure. The educational 
ranking has very good technical properties (it covers the whole sample, it is countryspecific, and draws from the same source as the employment data), but it is the most indirectly linked to job quality, which also disqualifies it as a standalone job quality measure. The best choice, therefore, is the wage ranking, which covers the whole sample, is country-specific and has a direct indisputable relationship (even if this relationship is not without problems) to the concept of job quality. Nevertheless, in annex 7 we include the same graphical decompositions used in the body of this chapter but based on the two other job quality rankings.

\section{What drives the change in the quintiles: sector or occupation?}

The jobs matrix, our picture of the division of labour within each country, is constructed as a big table of employment by sector and occupation. The main aim of this thesis is to evaluate from a job quality perspective how such jobs matrix changed between 1995 and 2007: hence the quintile approach used in the previous chapter. But because each of the two variables defining the matrix represents a dimension of the division of labour, a natural question to ask oneself is whether one of those two dimensions is driving the change in job quality represented by the quintile picture, or whether the two are equally important.

Although our general approach in this thesis does not involve any strong assumption in this respect, it may be argued that both dimensions should have some importance in terms of the evolution of the division of labour from a job quality perspective. If only one of the two dimensions would explain most of the transformations of the jobs structure from a job quality perspective, it would simply be unnecessary to use the other one. The theoretical frameworks discussed in chapter 2, on the other hand, have different assumptions with regards to the relative importance of each of these two dimensions. For instance, the downgrading hypothesis puts more emphasis in the importance of changes in the organization of work within companies: hence, it would argue that changes in the occupational structure would drive the effects of the evolving division of labour on job quality. The upgrading hypothesis puts much more emphasis in big-scale sector shifts in employment as the main driver of changes in the composition of job quality (from industries to services, and from personal and distribution services to information and knowledge services), although it also assumes an upgrading trend at the occupational level (with intense growth of technical, 
professional and managerial occupations). The different flavours of the polarization hypothesis vary in this respect: the trade/institutionalist theories seems to put somewhat more emphasis on sectorial shifts in employment, whereas the technological variety emphasizes much more shifts in the occupational structure ${ }^{75}$.

A simple but effective way of testing whether one dimension of the division of labour is driving the changes in the structure of job quality is to redo the analysis using only one dimension for constructing the jobs matrix, the rankings and the quintiles. The basic unit of analysis in our approach is the job, defined as a specific combination of occupation and sector. If one of those two dimensions was irrelevant for the changes in the structure of job quality, the results obtained using only the other dimension should be very close to the results obtained using the two dimensions. For instance, if only the sector was relevant for job quality, all occupations within each sector would tend to cluster together (with very similar values in terms of job quality, and very different from the values of the same occupations in different sectors) and only the sector would determine the allocation of each job to the quintiles. In such case, using only the 60 sectors as the basis of the analysis (ignoring occupations) would produce essentially the same results as using the full jobs matrix.

Figure 6.1 presents again the original patterns of job growth discussed in the previous chapter (shown as white bars; these patterns of job growth are based on using the full jobs matrix, defined by sector and occupation), but adding two new series of figures which represent the patterns of job growth obtained when using only ISCO and only NACE as a basis. At the left-hand side of the bar which represents the original results, we can see the patterns of job growth obtained by using only ISCO for defining the jobs matrix: this bar can be identified by its dark grey colour. At the right hand-side, in a light grey colour, we can see the pattern of job growth obtained when only sectors (NACE) are used.

\footnotetext{
${ }^{75}$ In fact, the main exponents of the technologically-induced polarization hypothesis within this jobs approach, Goos and Manning, in some cases use an alternative jobs matrix defined only by occupation, assuming that the results are broadly the same (Goos and Manning 2007).
} 


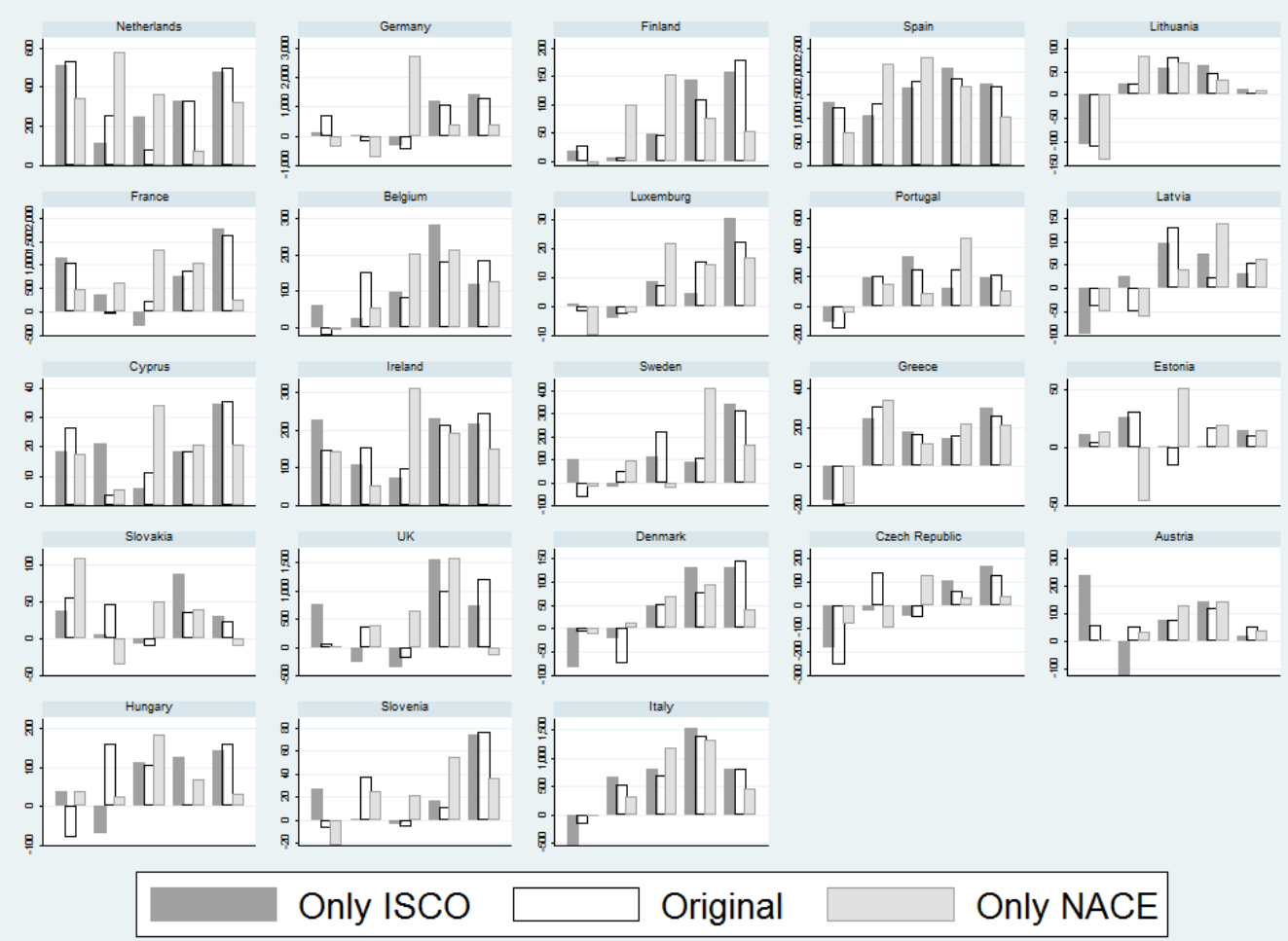

Figure 6.1: Patterns of job growth using only ISCO, ISCO and NACE (original), and only NACE

These alternative figures must be understood as just an approximation. The wage ranking we have at our disposal was originally constructed for jobs (occupations within sectors), so for redoing the quintiles based only on sectors or occupations, we had to generate a weighted average of the normalized ranking positions of all the jobs whithin each sector or occupation. Then, all employment was assigned into five groups (quintiles), according to the average ranking position of each sector (or occupation). Because the number of sectors (60) and occupations (26) is much smaller than the number of jobs (1600), these quintiles are more uneven than the original quintiles, which may generate some distortions in the patterns - but should serve as a reasonable approximation -. Finally, the change in the number of people employed in each of those five groups between 1995 and 2007 is plotted in figure 6.1, together with the original patterns as explained earlier. These new figures, therefore, are just than a simpler version of the original analysis, using only 26 occupations or 60 sectors as the basis instead of 1,600 jobs.

Neither of these two alternative figures are a very good match of the original patterns of job growth, which proves that both dimensions matter. In other words, using only one of the two dimensions for the analysis implies loosing too much information: the results 
are so distorted that they would lead to different conclusions. That said, it seems quite clear by looking at figure 6.1 that using only ISCO we get a much closer approximation to the original results than using only NACE - despite the fact that the number of categories in ISCO is three times smaller. In most cases, the patterns of job growth obtained by using only NACE are rather different from the original patterns, whereas the patterns obtained by using only ISCO look in many cases approximately similar. So if we have to point to one of the two dimensions as the main driver behind the transformation in the structure of employment by job quality, it would be ISCO. But again, even in the case of ISCO, using only one dimension would mean loosing too much information and distorting the real patterns of job growth. Neither ISCO nor NACE on their own can reflect adequately the evolution of employment from a job quality perspetive.

In fact, the results shown in figure 6.1 point to the existence of a significant interaction between the two dimensions that form the jobs matrix. If there is a different occupational composition in each sector, for instance, a change in the structure of employment by sectors will transform the overall composition of employment by occupations indirectly, even if there is no change in the occupational composition within each sector. The interpretation of the impact of occupation on the patterns of job growth would in that case be totally different: we could even argue that all the impact of occupation is spurious, because it would just be a (compositional) result of a change in the structure of employment by sectors.

Following this argumentation, we can try a second test of the impact of each individual variable: by fixing the structure of occupations within sectors at the level of 2000, we can eliminate the net impact of occupation (leaving only the compositional effect through the change in sectors explained earlier), and then see how much do the results change. The underlying question of such exercise is how much would the job quality structure have changed if only the sectorial composition had changed, and the composition of employment by occupation within each sector had remained constant. We can do the same exercise keeping constant sectors within occupations, and by comparing the results in both cases, we can see which individual dimension had a largest effect on its own. 


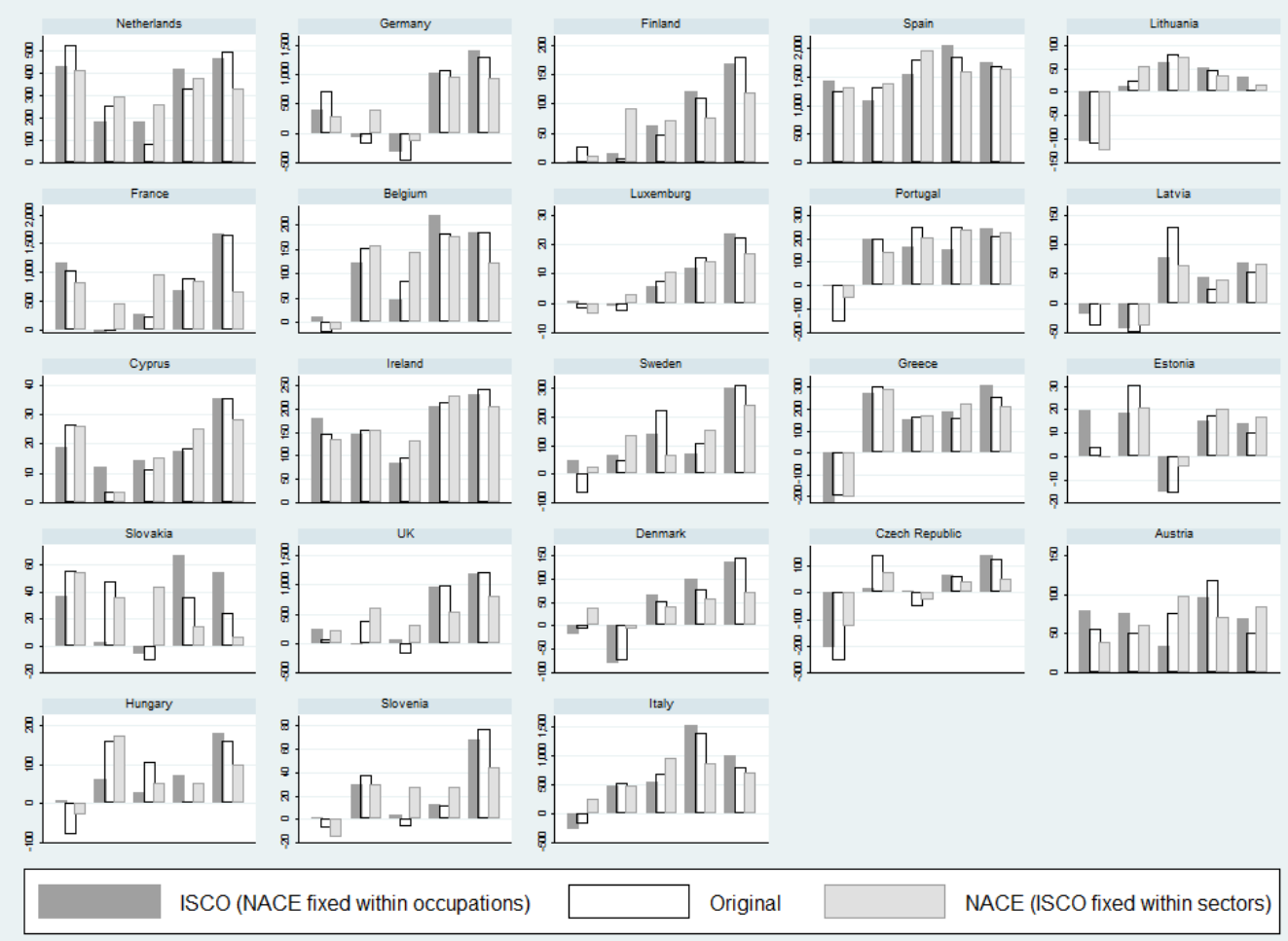

Figure 6.2: Counterfactual (keeping constant one dimension) vs. original patterns of job growth

The results of this analysis is shown in figure 6.2. It is important to notice that the logic behind this exercise is different from that behind figure 6.1. In figure 6.1, we just constructed two simpler versions of the jobs approach, using only sector or occupation as the basis. In figure 6.2, we used the full jobs matrix of the original analysis, but we manipulated it to generate two sets of counterfactual results to eliminate the net effect of each of the dimensions. These counterfactual results were generated by making the occupational structure within each sector constant (in proportional terms) at the level of $2000 .^{76}$ Therefore, only the sector structure changed; and all the change in the occupational structure was due to the change in the relative size of each sector.

\footnotetext{
${ }^{76}$ For instance, for constructing the quintile picture with ISCO fixed at the 2000 level, we did the following transformations to the each original national jobs matrix: 1) we calculated the proportion of employment that each occupation represented within each sector (for instance, the proportion of office clerks - ISCO 41 - in construction - NACE 45 - was 3.66\% in Spain in 2000); 2) we multiplied the number of people employed in each sector and each year by the vector of proportions generated in the previous step (that is, the original number of office clerks in construction in 1995 was substituted by the total number of workers in construction in 1995 multiplied by 0.0366, etc.); 3) with such counterfactual jobs matrix (in which only NACE changed throughout the years, with the ISCO structure frozen in 2000 for the whole period) we recalculated the quintile patterns in the same way as we did in the previous chapter.
} 
As in the previous case, figure 6.2 contains three different parameters for each country and quintile. The first one, on the left, and represented with dark grey bars, shows the patterns of job growth with NACE fixed at the 2000 structure: therefore, all the change represented by these bars is solely the result of shifts in employment across occupations. The second one, represented in the middle and with a white bar, represents the original results: that is, this bar is identical to the bars shown in figure 6.1 of the previous chapter. The third one, represented at the right-hand side for each quintile with a light grey bar, shows the patterns of job growth with ISCO fixed at the 2000 level: hence, all the change represented by these light grey bars is solely the result of shifts in employment across sectors.

In this case, the alternative figures are much closer to the original results, both for ISCO and NACE. This was to be expected, since the effect of each variable on the patterns of job growth also includes the indirect compositional impact through the other variable. But the important thing is that the results shown in figure 6.2 confirm our earlier conclusions based on a simpler approach. If one of the two dimensions explained all the change in the structure of job quality between 1995 and 2007, the counterfactual results keeping fixed such dimension would be flat (as we would have eliminated all change), whereas the counterfactual results keeping constant the other dimension would look identical to the original results. This does not happen in any case: which proves that both variables have a significant influence on the patterns of job growth. But as we also observed in figure 6.1, it seems quite clear that ISCO played a more significant role than NACE in the change of the structure of job quality. In most cases, the patterns of job growth by occupations (keeping sector constant) are quite similar to the original results, much more than the patterns obtained when only the sector shifts in employment (keeping occupations constant) are taken into account. The shape of the light grey bars representing change with ISCO fixed within each sector are clearly flatter than the dark grey bars representing change with NACE fixed.

Figure 6.2 suggests a systematic geographic pattern in the relative importance of both dimensions, something which was not so clear in figure 6.1. In a few countries we cannot say that ISCO drives the transformation of the job quality structure, but the evolution of both dimensions of the division of labour seem to be equally important (the shifts across NACE categories are as important as the shifts across ISCO). This is the case in most Southern and Eastern European countries, and to a lesser extent, also in 
Germany, Austria and Luxemburg. What this seems to suggest is that the vertical dimension of the division of labour is crucial for the evolution of job quality in structural terms in all stages of economic development, whereas the horizontal dimension seems to loose importance with economic growth. This seems rather plausible. The move towards a service economy, which takes place at relatively low levels of economic development (in an European context), involves large-scale shifts in employment across sectors with important implications for job quality. In countries which are already service economies, sector shifts are likely to loose relevance for the structural evolution of job quality (with occupational shifts taking an even more prominent role).

\section{Decomposing the patterns of job growth by occupation and sector}

Now that we have seen that both dimensions of the division of labour play an important role in the evolution of job quality as presented in the quintile picture (although occupation is more important than sector, especially at higher levels of economic development), we can proceed to a detailed analysis of the contribution of the different sectors and occupations to the growth of the quintiles in the different countries. Of course, we will not be able to analyse in full detail the whole list of 60 sectors and 26 occupations: instead, we will use a reaggregation of both variables into 9 broader categories. In the case of occupation, we will simply use the one-digit level of aggregation of the ISCO classification; in the case of NACE, we will use our own reaggregation based on different proposals available in the literature, and explained in section 2.1 of chapter 4 of this thesis.

\subsection{A decomposition of the patterns of job growth by 9 broad occupational levels}

The 9 one-digit occupational codes of ISCO summarize the information contained in the full list of 26 occupations used for the construction of the jobs matrix in a way which is both meaningful for our purposes (in terms of the vertical division of labour) and manageable. These 9 categories, which are defined in terms of the skill contents and skill levels of jobs, broadly correspond to the usual hierarchical levels within productive organizations, from managers (group 1), through professionals, technicians (groups 2 and 3), administrative and service workers (groups 5 and 6), to skilled, semi-skilled and unskilled workers (groups 7, 8 and 9). Of course, this does not mean that all real-life 
organizations have employees of all these different types: many organizations, depending on their type of activity (hence the interaction between occupation and sector mentioned in the previous section), would only have workers in two or three categories. Furthermore, the one-digit ISCO classification has one group that does not fit into such idealized organizational structure: group 6 refers to skilled agricultural workers, which is a category more pertinent in terms of sector than in terms of occupation (although it certainly involves distinct skills, which is why it is a category of ISCO). But nevertheless, the analysis of the contribution of these 9 broad categories to the patterns of job growth across Europe should help us interpret those patterns from the perspective of the division of work within productive organizations.

In line with the analytical strategy employed so far in this thesis, our analysis will be primarily based on a graphical representation of the contribution, in absolute terms, of each occupational category to the growth (or decline) of each quintile over the period 1995-2007, country by country. Figure 6.3 shows such representation for the three upper categories of ISCO at the one-digit level: managers, professionals and technicians. The five bars for each country should look rather familiar, because they are in fact basically the same as those discussed in the previous chapter (in fact, they are not completely identical, because the different occupational categories can have contradictory and therefore compensating contributions - the overall figure being the net sum of the contribution from all categories -). Within each bar, the contribution of each occupational group is represented with different colours: in this case, managers are represented in black, professionals in dark grey, technicians in light grey and all the other categories (together) in white. The interpretation of these charts, therefore, should be relatively easy. For instance, for the Netherlands, we can see that almost all job growth in the $5^{\text {th }}$ quintile (that is, the highest paid jobs) corresponded to professional occupations, with some small contribution from managers. The growth of the fourth quintile was the result of the expansion of technicians and professionals - both categories contributed almost equally -, whereas the contribution of managerial jobs to this quintile was in fact negative (though very small). The category of technicians also contributed marginally to the expansion of the second quintile. The bottom and the middle quintiles had almost no contribution from these three upper categories of ISCO in the Netherlands. 


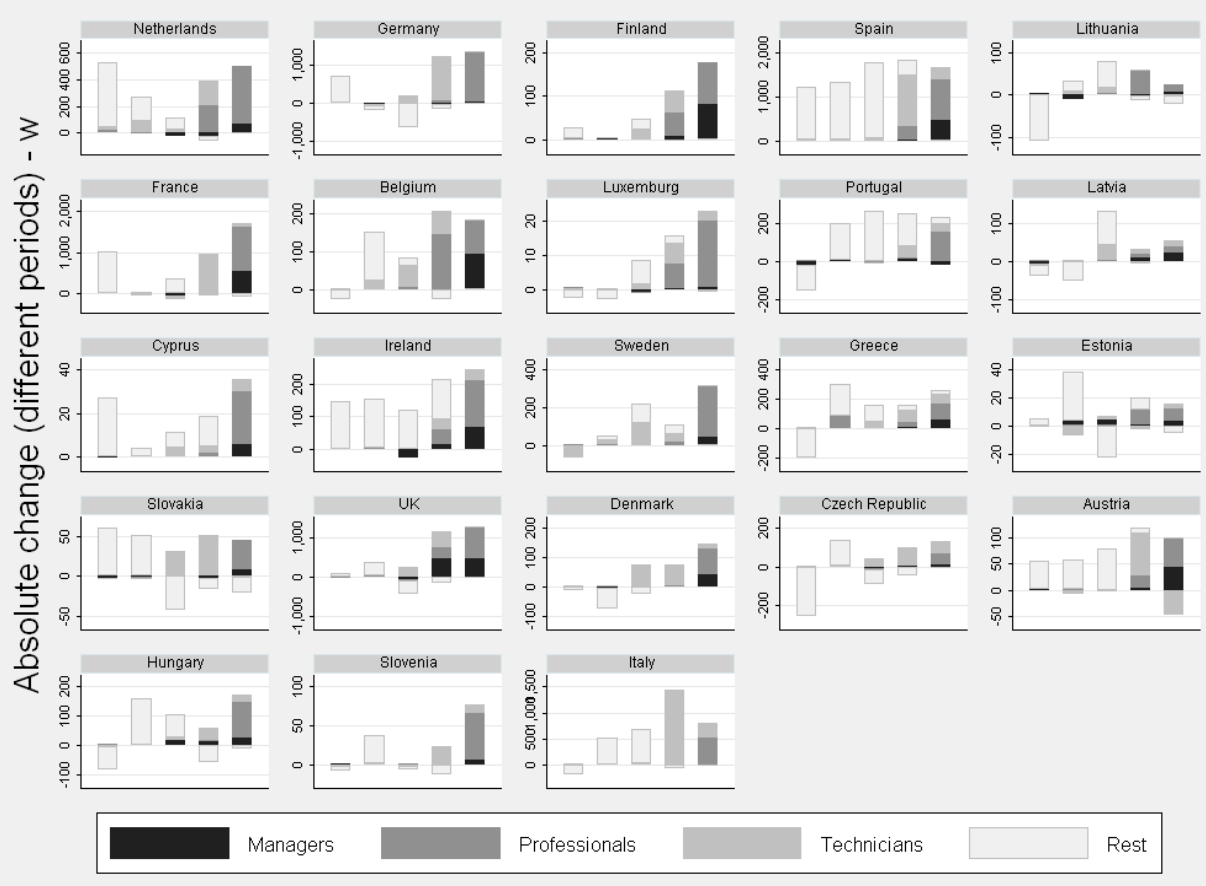

Figure 6.3: The contribution to job growth of managers, professionals and technicians

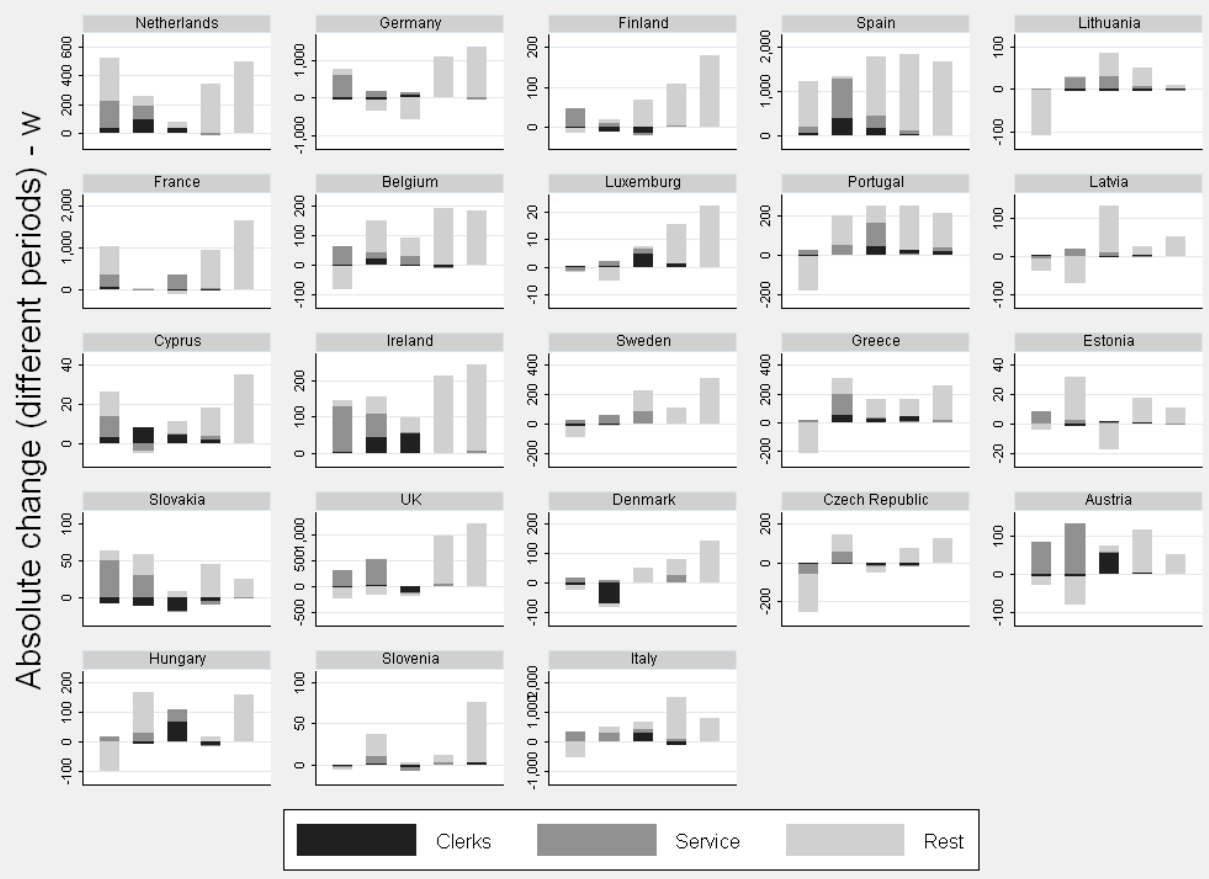

Figure 6.4: The contribution to job growth of clerks and service workers 


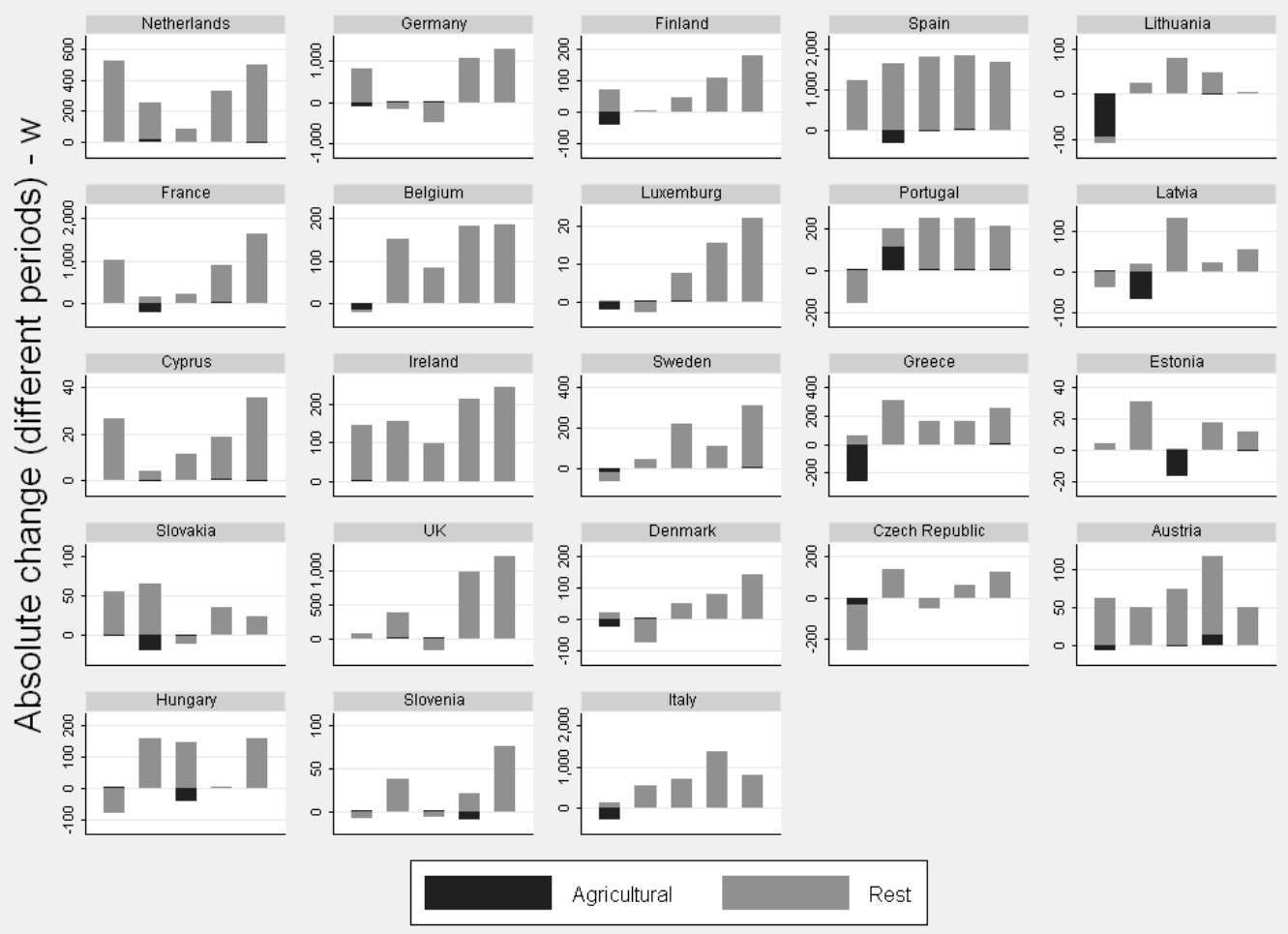

Figure 6.5: The contribution to job growth of skilled agricultural workers

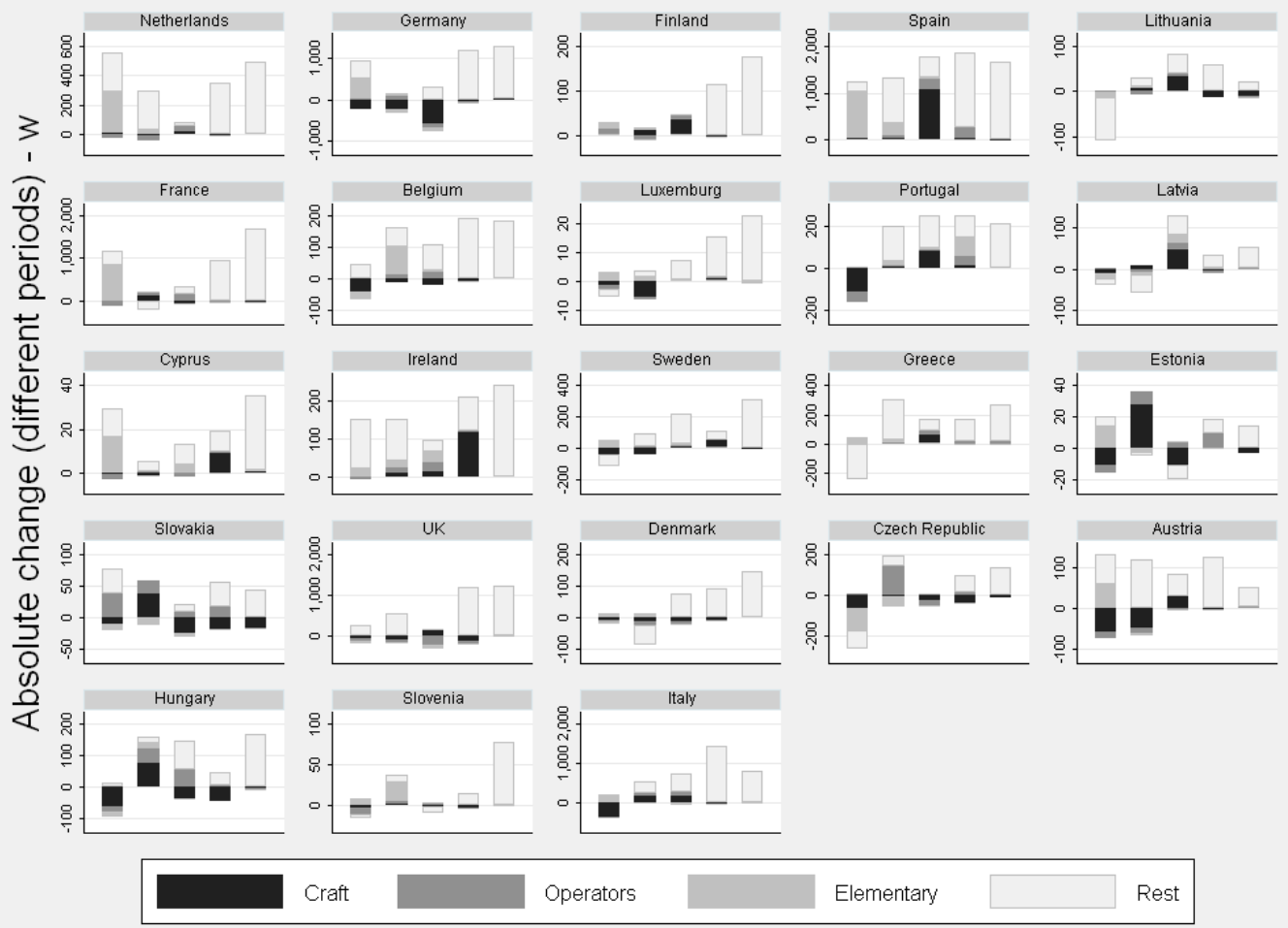

Figure 6.6: The contribution to job growth of craft workers, operators and elementary occupations 


\section{1.a) Managers, professionals and technicians}

In the 23 countries shown in figure 6.3, without exception, the growth of the top quintile was dominated by the category of professional workers. In some cases, the category of managers also added significantly to the growth of the jobs with highest median earnings: most notably, in Belgium, Finland, France, Austria, Spain, Ireland and the UK. In many other countries, the category of managerial jobs grew very marginally, although always around the top of the wage structure. Although not as universally as for professionals in the top quintile, the category of technicians tended to dominate the growth of the second highest quintile, in a few cases even extending its reach into the third quintile. The contribution of these three categories to the bottom quintiles was close to zero in all countries (and negative in Sweden).

\section{1.b) Clerical and service workers}

Figure 6.4 shows a similar breakdown for clerical and service occupations, which would occupy a middling position in our earlier idealized organizational structure. In fact, as we can see, these two categories contributed mostly to the growth of the mid-low quintiles, although there is considerably more variability here than in the case of managerial-professional occupations. The growth of service occupations explains most of the expansion of the bottom quintile in those countries where such quintile did expand: basically, in the polarizing countries. In Spain, Austria, the UK and Greece, service occupations tend to be in the second rather than the lowest quintile: in all these countries, the second quintile grew much more than the first. Portugal is the only country where service occupations account for a significant part of the growth of the third quintile. In general, the contribution of clerical occupations was considerably smaller (in some cases, most notably in Denmark, even negative), and generally concentrated on the second and third quintiles.

\section{1.c) Skilled agricultural workers}

Figure 6.5 shows the contribution to the quintile patterns of the category of skilled agricultural workers. As mentioned earlier, this category is a hybrid of sector and occupation: in fact, as we shall see later, its results are almost identical as those of the primary sector, so we will discuss them with more detail later. Here, we will only briefly mention that this occupational group contributed negatively to job growth in 
most cases ${ }^{77}$, especially where the primary sector still employs a sizeable proportion of the population. In most cases, such (negative) contribution took place in the bottom two quintiles: in fact, where agriculture contributed significantly to employment change, it tended to depress growth at the bottom of the wage structure.

\section{1.d) Skilled, semi-skilled and unskilled workers}

Much more interesting for our present purposes is figure 6.6, which shows the contribution to the patterns of job growth of the three categories of "workers" in the most traditional sense. The category of craft occupations includes not only skilled workers in the industrial sector, but also building trades workers: because the construction sector has had such a dynamic and diverse evolution across Europe in recent years, the evolution of employment in the category of craft occupations has been in many countries dominated by developments in construction. In most countries, craft occupations tend to occupy the middle quintile, and what happened in this particular category explains in many countries a large part of what happened in the middle quintile, with widely varying developments across countries. In those countries which experienced a construction boom between 1995 and 2007, such as Spain, Ireland and most Eastern Member States, the category of craft workers expanded considerably and consequently, the middle quintiles as well $^{78}$ (although in Ireland the position of this category is abnormally high, contributing mostly to the growth of the fourth quintile ${ }^{79}$ ).

On the other hand, there were some countries, notably Germany, where the construction industry collapsed over this period, and the category of craft workers destroyed large amounts of employment in middling and mid-low jobs. Yet in other countries, this category did not change much in terms of employment, so it contributed little to the patterns of job growth. In the next section we will look more in detail at the specific contribution of construction to the patterns of job growth.

\footnotetext{
77 The main exception is Portugal; see note 76.

${ }^{78}$ In the transition economies of Eastern Europe, there were simultaneously important expansions and contractions in this category across the different quintiles (for instance, in Slovakia there was an important expansion of craft workers in the second quintile, while the third, fourth and fifth quintiles shrinked for this category). Where there is a expansion, it tends to be associated to the construction sector; where there is a contraction, it tends to be associated with skilled industrial workers affected by the largescale restructuring carried out in these countries in the last couple of decades (see Kattel, Reinert and Suurna 2009: 12; also Crespo and Fontoura 2007: 615-616).

${ }^{79}$ In the early 2000 s, activity in construction in Ireland was growing at a truly phenomenal pace, which led to considerable shortages of labour (especially skilled and semi-skilled craft construction workers) and consequently to abnormally high increases of salary levels. From 1998 to 2003, hourly earnings in the construction sector in Ireland increased by $57 \%$, the highest proportional increase among all sectors of the Irish economy over that period (see Casey 2004, p. 9).
} 
The second main category of workers, operators and assemblers, is the most directly linked to traditional industrial employment, and saw very little change over this period except in three Central-Eastern Member States (Czech Republic, Slovakia and Hungary) which experienced a very important expansion in heavy industries over this period. As we can see in figure 6.5, this expansion led to the creation of many jobs of mid-low salaries in these countries.

Finally, the evolution of elementary occupations (a catch-all category which basically comprises unskilled jobs across different types of activity) was strongly linked to the growth of the bottom quintile. As in the case of service occupations, employment in this category only expanded in those countries which experienced growth in the very bottom of the employment structure (basically, the polarization countries plus Spain and Austria).

\section{1.e) Some final remarks}

In all European countries, there is a strong association between the five quintiles (which we should recall, are five equal-sized groups of jobs ranked by their median hourly wages) and the nine occupational levels of ISCO. In most countries, professionals and managers dominate the fifth quintile, technicians the fourth, clerks and skilled workers the third, service and unskilled workers the two bottom quintiles. The association is so strong, that the evolution of employment by quintiles and the evolution of employment by the nine occupational levels of ISCO are to some extent the same thing: the top expanded everywhere because there was a widespread expansion of the professional and technical groups; the behaviour of the middle depended largely on the evolution of craft workers; and the bottom expanded where low-skilled service and industrial occupations expanded.

As we argued earlier, it is debatable whether this type of analysis is really explanatory or simply exploratory. In our view, the most important thing is that, by putting the patterns of job growth in the context of the evolution of employment by occupational levels, it does expand our capacity to understand them. If we go back to the hypotheses derived from the specialized literature, we can see that our results do fit relatively well the predictions of the post-industrial theories (which not only predicted a generalized upgrading of employment structures, but specifically linked it to a large expansion of the professional and technician occupations), while contradicting strongly the predictions of the downgrading hypothesis (not only there was no generalized 
downgrading, but there was obviously not a shift towards the most unskilled occupations) and mildly the hypothesis of polarization (in those countries that did experience a polarization of their employment structure, the occupational patterns do fit the predictions of the polarizing hypothesis: but nevertheless, this only happened in a minority of countries in Europe).

\subsection{A decomposition of the patterns of job growth by 9 broad sectors}

In this section, we are going to study the sector dynamics which are behind the patterns of job growth identified in the previous chapter. For this, we will use a reclassification of the 60 sector codes used in the construction of the jobs matrix into 9 broad categories which capture the most important distinctions between the types of economic activity mentioned in the literature.

In many ways, this breakdown of the patterns of job growth is even more informative than the occupational one which we discussed in the previous section, precisely because the association between the quintiles and the sectors is not so strong as the association between the quintiles and the nine 1-digit occupational codes of ISCO (so that it adds more new information). The analysis of this section can be linked to the tradition of studying the sector dynamics of economic development of Clark (1944), Kuznets (1963) and Fourastié (1963), with the limitation of covering a much shorter time span than those seminal works but the added value of putting it in the context of our two core concepts: the division of labour and job quality. The shorter time span of our study means that the long-term dynamics which were the focus of these classic studies will be confounded by developments of a cyclical nature, which may make it difficult to evaluate the implications of our results in the longer term. Nevertheless, we will try to take this into account as much as we can while interpreting our results, trying to differentiate long-term and short-term transformations in the employment structure.

We will use the same analytical strategy as in the previous section: taking advantage of the fact that the patterns of job growth are perfectly decomposable by any classification of jobs by sector or occupation, we can represent graphically the individual contribution of each sector to the change in each quintile (and country) over the period 1995-2007. Figures 6.7 to 6.10 present such graphical decompositions of the patterns of job growth by nine broad sectors of the economy, which are the same ones used in chapter 4 of this thesis for a static analysis of the different employment structures across Europe (see that chapter for details of the construction of these 9 aggregated sectors). 
Figure 6.7 shows the contribution to the growth of each quintile over the period of the primary sector and construction. Contrary to figures $6.8,6.9$ and 6.10 , there is no particular reason for representing these two sectors together: they are very distinct on all accounts, and we only represent them together in order to limit the number of figures. Therefore, we will discuss the contribution of each of the primary sector and construction separately, as if they were represented in different figures.

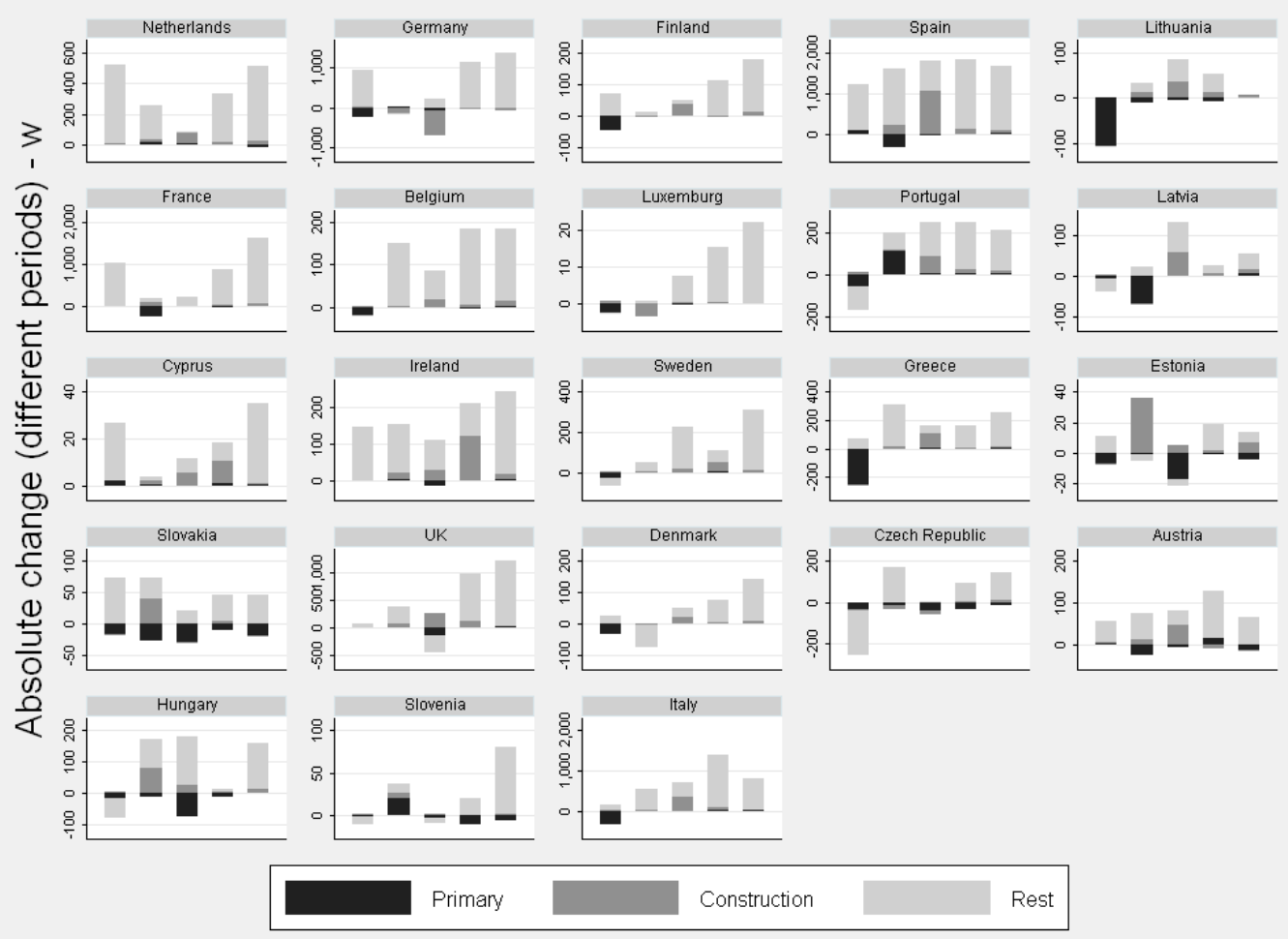

Figure 6.7: The contribution to job growth of the primary sector and construction 


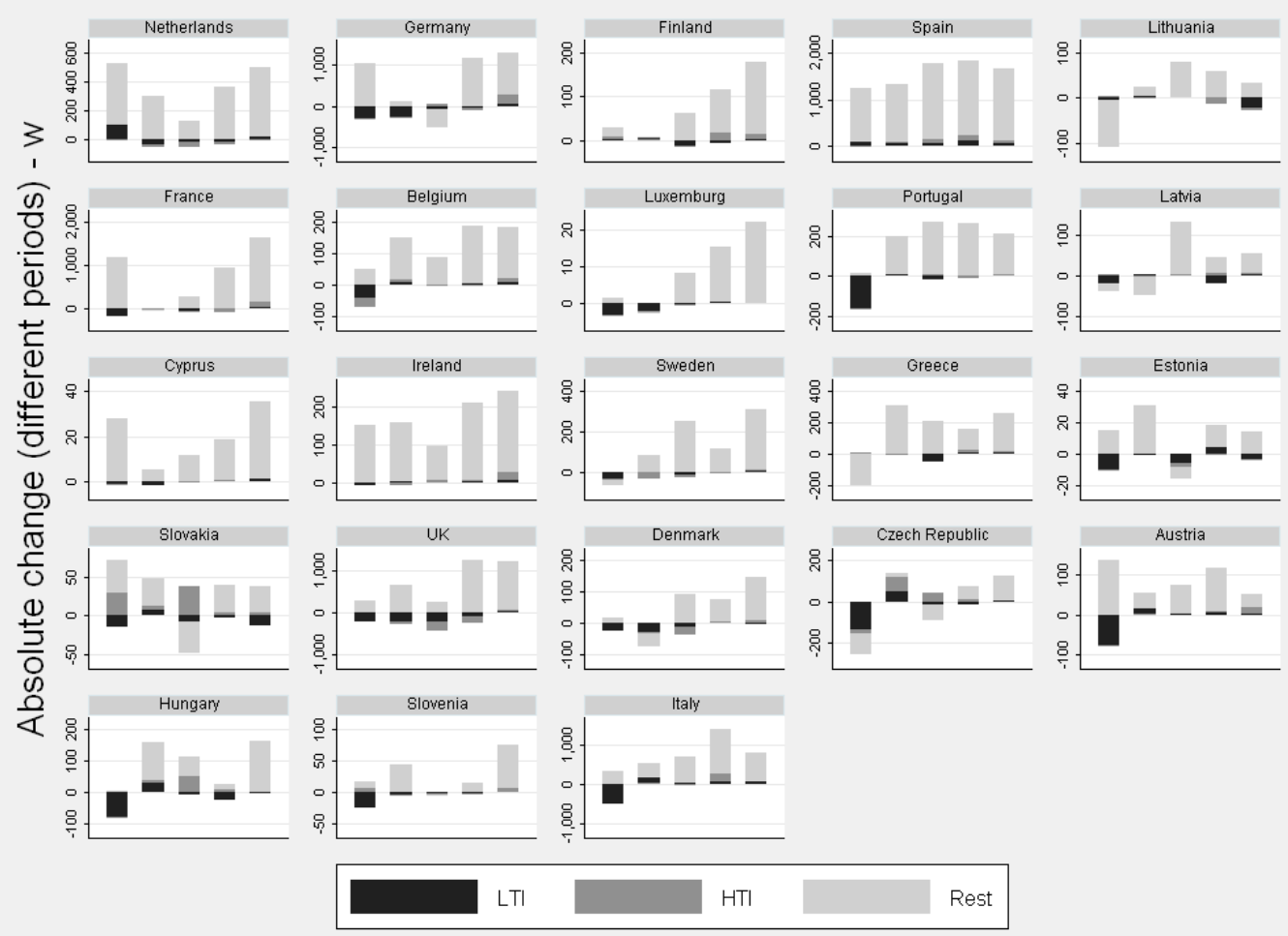

Figure 6.8: The contribution to job growth of low and high technology industries

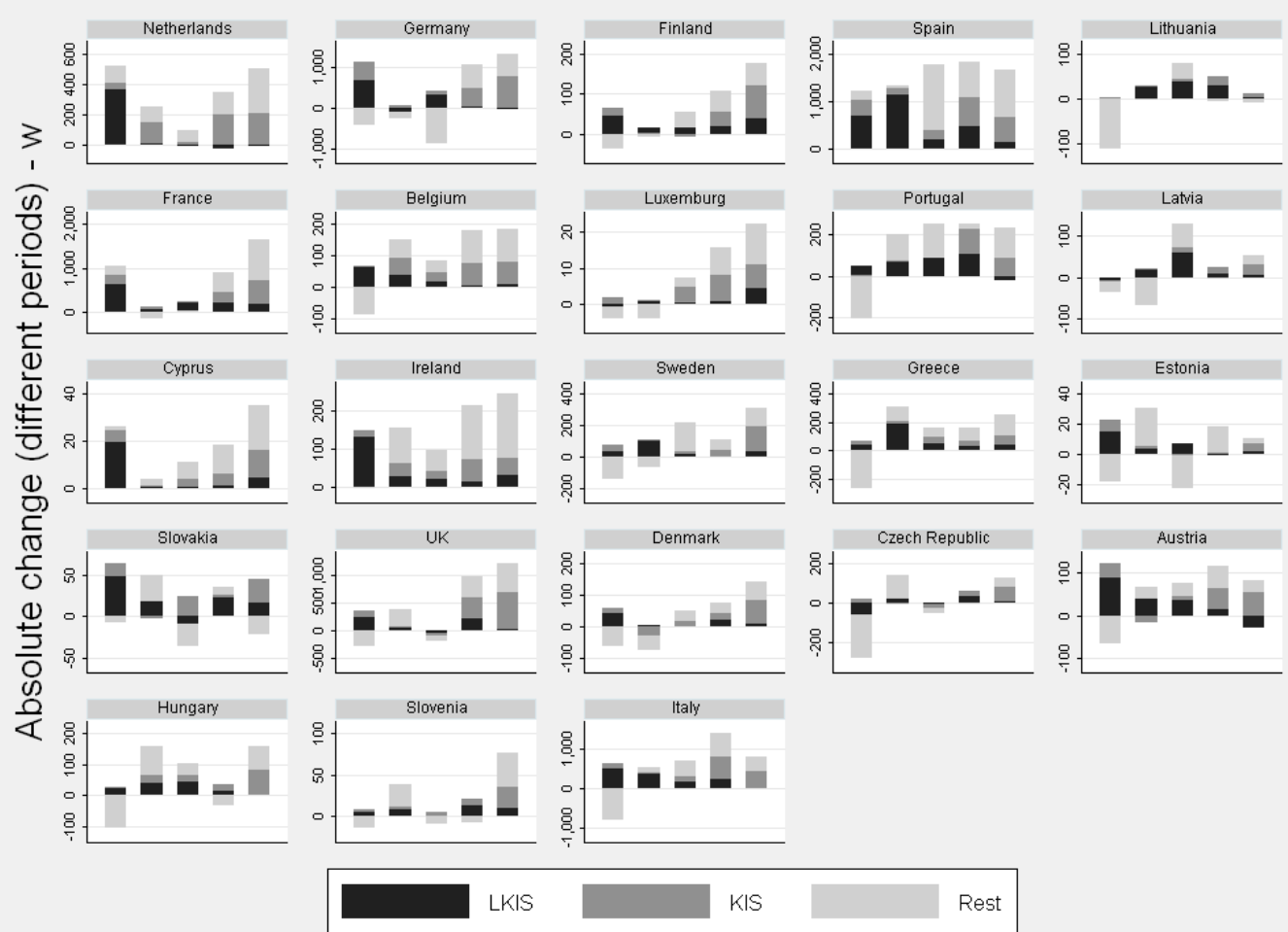

Figure 6.9: The contribution to job growth of low and high knowledge intensive services 


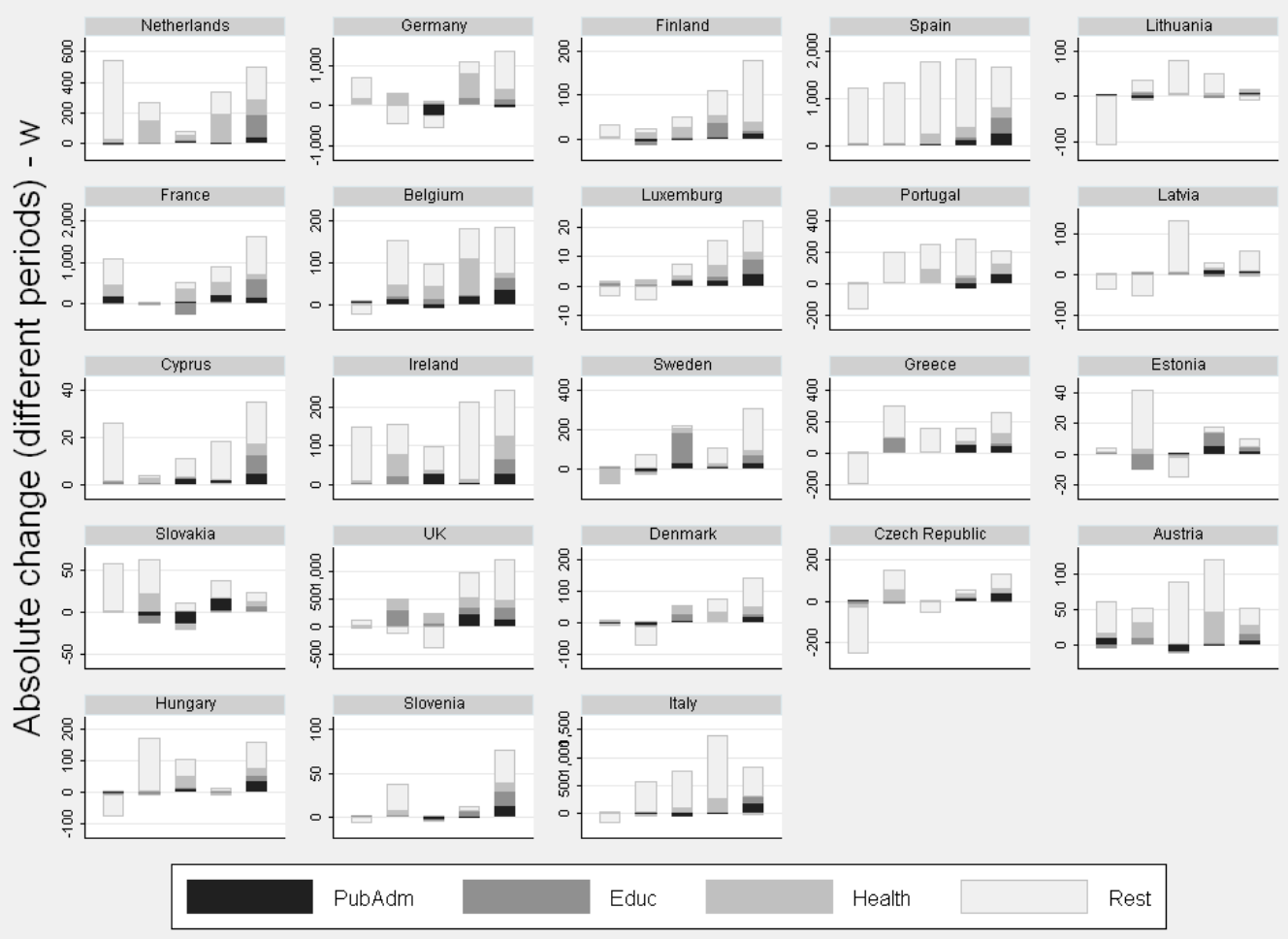

Figure 6.10: The contribution to job growth of public administration, education and health

\section{2.a) The primary sector}

The evolution of employment in the primary sector in Europe between 1995 and 2007 reflects the last 12 years of a very long process of decadence which started with the Industrial Revolution. The agricultural sector destroyed employment across Europe between 1995 and 2007, most intensively in those countries which still had a sizeable proportion of agricultural employment in the beginning of this period ${ }^{80}$ (in the South and East of Europe), but also in some of the most advanced economies (such as Germany, France, Finland or Luxemburg). As we can see in figure 6.7, this net destruction of agricultural employment affected mostly the three lowest quintiles: that is, the evolution of employment in the primary sector contributed to the destruction of relatively low paid jobs, quite significantly in the South and East of Europe.

\footnotetext{
${ }^{80}$ The only exception is Portugal, which according to the Eurostat ELFS data used in this Project, expanded its agricultural sector by more or less 100.000 workers between 1995 and 2007. Between 1995 and 1997 in Portugal, according to this data, employment in agriculture expanded rather strongly, and after that it went down and stabilized in relative terms (although in absolute terms it increased, as overall employment grew, which is what explains the growth of 100.000 workers shown in figure 6.7). These figures are not only contradictory with historical trends and the developments in similar countries, but even with other statistical sources from Eurostat, such as the agricultural statistics published in Eurostat 2007a. There must be some problem with the ELFS data of agricultural employment in Portugal, but we could not find any documentation. Slovenia also experienced a very small expansion of agricultural employment over the period 1996-2007, of around 10.000 workers.
} 


\section{2.b) Construction}

Of all sectors discussed here, construction is the most obviously affected by cyclical economic factors. Employment in construction tends to grow very rapidly in economic expansions, and to plummet in crises, so it is always difficult to evaluate the long-term implications of any development in this sector (see Tschetter and Lukasiewicz 1983). The period we are analysing here, from 1995 to 2007, is paradigmatic in this respect: in many countries, there was a long construction boom which led to an exceptional expansion of employment, only to collapse dramatically after 2007 destroying as many jobs as it had created earlier, and a few more. The two chief examples of such development are Spain and Ireland, but similar evolutions took place in many Eastern European countries (and to a lesser extent also in Italy, Greece, Portugal, the UK and Austria). On the other hand, during this same period there was one European country which actually experienced, for idiosyncratic reasons, a long and deep contraction of employment in this sector: Germany. The reunification of Germany in the first half of the nineties inflated an earlier bubble which slowly deflated between 1995 and 2007 (see Walter 2002), while many other European countries were creating their own ones. And finally, in many other countries (all except those mentioned earlier) construction did not expand nor contract very significantly in this period.

All these developments are certainly reflected in our data, as can be seen in figure 6.7. It is particularly interesting to see their implications for the overall structure of job quality in the different countries ${ }^{81}$. In general, construction tends to be located around the middle quintile (with some exceptions), and as there were such wide variations in the evolution of employment in the construction sector across Europe, this sector determined in most cases the evolution of middling jobs. Therefore, the countries which experienced a boost in construction tended to expand considerably their share of

\footnotetext{
${ }^{81}$ Nevertheless, we have to mention a limitation of our approach with respect to this specific sector. Because of the way NACE and ISCO are defined at the two-digit level, around two thirds of all workers in construction are in most countries assigned to a single cell (NACE code 45, ISCO code 71: that is, trade workers in the construction sector). This means a large number of workers: in many countries, around $4 \%$ of all employment is concentrated in this single cell. This may be stressing too much the jobs approach, which assumes that all workers within one cell carry out the same type of economic activity, are required the same type of skills and responsibilities, and enjoy the same type of job quality (without taking into account differences between workers in the same cell which are the result of career effects or working in different companies). The ISCO and NACE classifications at the two-digit level may not be detailed enough for the construction sector. This does not mean that the results shown in figure 6.7 are wrong, only that they are probably less precise than for other sectors (many jobs that should be differentiated within this sector are aggregated into a single one, and its aggregate median wage positions this job in the middle quintile in most countries).
} 
middling jobs (middle-high paying jobs in the case of Ireland, middle-low in Slovakia, Hungary and Estonia). In Germany, the long recession in construction over this period is the main factor behind the deep through in the middle of the job quality structure (more than three quarters of a million jobs were lost in construction over this period, most of them located in the third quintile). Such large shifts in the middle of the job quality structure were absent in most of the countries where construction remained more or less stagnant over this period. So the evolution of employment in the middle was mostly determined by developments in the construction sector. ${ }^{82}$

The fact that any large expansion (or contraction) in the construction sector is likely to be a cyclical development, fundamentally unsustainable in the long run, makes it interesting to consider how would the evolution of employment have looked without the construction effect. In the case of Spain and Austria, the patterns of job growth would have fitted more a polarizing than an upgrading image. In the case of Ireland, the polarization would have looked even stronger (by depressing the second highest quintile). The polarization of Germany, on the other hand, would remain, but considerably softened (something similar would have happened in Slovakia and Hungary). So overall, we would have seen a bit of more polarization, although the overall plurality of patterns would remain (the evolution of employment in construction had little impact on the overall patterns in many countries).

\section{2.c) Manufacturing industries}

Figure 6.8 shows the contribution to the patterns of job growth of the manufacturing sector, which is split in two sub-sectors according to their technological intensity following an OECD proposal, as explained in chapter 4 (Hatzichronoglou 1997). We can immediately see that the contribution of this sector to the patterns of job growth was quite small between 1995 and 2007 in most countries, although this does not mean that such development is not relevant for our purposes. The fact that manufacturing, which still accounts for around one fifth of the working population in most countries, remained more or less stagnant in absolute terms in a period of generalized employment expansion means that it shrinked in relative terms, and this certainly had implications for the structure of employment by job quality, as we shall see now.

\footnotetext{
${ }^{82}$ Construction tended to dominate the evolution of the third quintile for another important reason, as we will see later: the manufacturing sectors that traditionally pulled the expansion of mid-paid jobs were stagnant over this period (despite the fact that their share in the third quintile was still important), and services did not create mid-paid jobs.
} 
In general, Low Technology Industries tend to be located quite low in our job quality quintiles: in most countries, between one and two thirds of the lowest quintile is accounted for by this sub-sector. Low Technology Industries destroyed employment everywhere in Europe (with the single and surprising exception of the Netherlands), contributing in some cases quite significantly to the shrinking of the lowest quintiles. Such negative contribution was specially important in New Member States, as a consequence of the economic transition that these countries experienced over the period studied and which involved the intense restructuring of many old and inefficient industries of the Communist period (see Crespo and Fontoura 2007). Low Technology Industries also contributed importantly to the shrinking of the low-paid tier in Italy, Portugal, Austria and the UK.

On the other hand, High Technology Industries tend to be positioned in the middle quintile, and in some countries it also accounts for an important share of employment in the highest paid segment: in France and Germany they account for nearly $20 \%$ of all employment in the fifth quintile, and for $10 \%$ in the UK. In this sector, there were very diverging developments in EU15 and in NMS (except the Baltics). Across EU15, High Technology Industries hardly contributed at all to job creation (or destruction), except in the fifth quintile in Germany, France and Austria. In the Czech Republic, Slovakia and Hungary, this sub-sector contributed very importantly to the expansion of jobs with wages around the median (third quintile; in the Czech Republic, it also contributed to the expansion of the second quintile, and in Slovakia to the expansion of the first quintile). East-Central European countries grew in middle and mid-low paid jobs in High Technology Industries while neighbouring West-Central European countries grew in high paid jobs in the same sector: the type of development we would expect in a process of Europeanization of production systems. Our results, nevertheless, point to specialization rather than relocation: there was no large-scale destruction of employment in middle and low-paid jobs in this sector in the West to match the expansion of these jobs in the East.

So in general, Low Technology Industries contributed to the shrinking of the low-paid segments of employment, whereas High Technology Industries had very little impact on overall employment change except in the mentioned (Eastern and Western) Central European countries. Surely the weak growth of low and mid paid jobs in many European countries must be linked to the very small contribution of manufacturing to 
overall job creation. Where there was expansion of employment in the middle, it was more because of the cyclical boom in the construction industry than because of the growth of middle-paid industrial workers. Sometimes an absence can be as significant as a presence. Mid-paid industrial jobs used to be considered as the foundation of the Post-War European middle class: although such group still represents an important segment of overall employment (around 20\%), its irrelevance in the dynamic analysis of the patterns of job growth between 1995 and 2007 testifies its decadence.

\section{2.d) Services}

Most of the action, of course, took place in the service sector. For analysing the contribution to the patterns of job growth of this very large sector of the economy, which employs between 50 and $70 \%$ of overall employment in all European countries, we will split it into five different segments. First, we will differentiate between services which are typically (in Europe) provided privately and publicly. Within the private service sector, we will use a Eurostat proposal to differentiate between the knowledge intensity of each activity. Within the (typically, but not necessarily) public service sector, we will differentiate the three largest branches: public administration, education and health. For more details of this sector breakdown, see chapter 4.

Figure 6.9 shows the contribution of private services to the overall patterns of job growth. It seems quite clear that private services contributed disproportionately to the expansion of the two extremes of the job quality structure, with little impact on the evolution of middling jobs. And it seems also quite clear from a quick look at the picture that there is a sharp differentiation between the contribution of the two groups of private services: Less Knowledge Intensive Services (LKIS, which coincide more or less with the more meaningful denomination of personal and distributional services) contributed mostly to the expansion of low-paid jobs, whereas Knowledge Intensive Services contributed disproportionately to the expansion of the highest-paid jobs.

Such development results from two reinforcing effects: on the one hand, a purely compositional effect; on the other, what we can call an internal centripetal effect. Personal and distributional services (or LKIS) tend to be concentrated on the bottom of the pay structure, so any expansion of this sector will necessarily involve an expansion of the bottom. But beyond such purely compositional effect, the expansion of employment within personal and distributional services is also downwards biased: that is, the jobs within personal and distributional services that expanded most rapidly 
between 1995 and 2007 were those with lowest median wages. The expansion of LKIS, therefore, has a compounded effect on the employment structure that tends to boost the growth of its lowest paid segments. Something very similar, but at the other extreme of the employment structure, happens with knowledge intensive (private) services: not only does the shift of employment towards this sector tend to expand the top of the employment structure because of its initial high position, but it is precisely the best paid jobs in this sector which expanded most, leading to an intensified upgrading effect.

But whereas knowledge intensive services expanded considerably everywhere, personal and distributional services only expanded significantly in a few countries. In this sense, we can consider private services as the main driver behind both the upgrading and the polarizing transformation of European job quality structures. There was upgrading nearly everywhere because everywhere there was a similar (though with different intensities, of course) expansion of (private) knowledge intensive services. And there was polarization in some countries because such countries simultaneously expanded their share of private personal and distributional services - where such expansion was missing, there was no polarization ${ }^{83}$. We can even link the development of private services with the weak expansion of the middle in most countries: neither LKIS nor KIS contributed significantly to the change of the middle quintile, despite the fact that together they account for more or less one third of this quintile (as a result of the centripetal effect mentioned earlier).

Finally, figure 6.10 presents the contribution of public services to the patterns of job growth across Europe between 1995 and 2007, in three categories: public administration, education and health. After the thatcherite privatization wave of the eighties and early nineties, it may seem somewhat outdated to characterize these sectors as public. Even in the case of public administration, which is in principle public by definition, there is in some European countries a significant proportion of workers which are in fact employees of the private sector (such as the British Public-Private Partnership schemes). In the case of education and health, the proportion of private sector employees is larger, in a few cases even outnumbering those directly employed

\footnotetext{
${ }^{83}$ The main exceptions to this general argument are Spain and Austria: in both countries, personal services expanded very significantly, so we would expect a polarization pattern of job growth. In fact, such polarization pattern would have been there if there had not been a massive increase of the construction sector boosting job growth in the middle of the employment structure, generating a picture which looks like upgrading with strong growth in the middle. Without the construction boom, as argued earlier, Spain and Austria would probably have been clear cases of polarization.
} 
by the State (for instance, the health sector in Ireland and Netherlands; see Nolan and Nolan 2004; WHO 2000). These three sectors, nevertheless, remain mostly public in most European countries, and certainly account for the vast majority of public sector workers. Being mostly public, the evolution of employment in these sectors responds to a completely different logic than in the case of private sector services. For this reason, we opted to study them separately, as shown in figure 6.10 .

Across most of Europe, public sector jobs contributed positively to employment growth, especially to the expansion of the highest paid quintiles. Of the three sectors represented in figure 6.10, the largest contribution was by far that of the health sector. In almost all European countries (the exceptions being Estonia, Latvia, Slovakia and Sweden), employment in the health sector grew above trend, contributing mostly to the expansion of the two highest quintiles. In a few countries (Ireland, Netherlands, Austria and the UK) this sector also contributed significantly to the expansion of the second lowest quintile: but only in Ireland was this contribution larger than that of the top quintiles. The contribution of education to employment growth was more uneven: in some countries, it hardly contributed to employment growth at all, in others it contributed significantly to the expansion of well paid jobs; but in most cases the contribution was smaller than that of health. Something very similar can be said about employment in public administration: small or even negative contribution to employment growth in some cases, significant contribution to the expansion of high paid jobs in others. In Germany, Austria, Belgium and Slovakia there was a significant destruction of public administration employment in the middle quintile over this period as well.

The period analysed in this thesis represents a turnaround of previous trends in the evolution of public sector employment in Europe (for a review, see Rothenbacher 1997). Following the earlier example of the United Kingdom under Margaret Tatcher, many European governments reduced the share of employment in the public sector in the late eighties and early nineties; only in some Eastern European countries did public sector employment increase during this earlier period (Rothenbacher 1997). Between 1995 and 2007, in the context of a more bening economic situation, there was a more or less generalized expansion of public sector employment, which as we have seen contributed significantly to the expansion of the highest paid segments of employment. Such contribution was specially the result of a widespread and above-trend growth of 
the health sector, but in some countries education and even public administration also had significant positive contributions.

\section{2.e) Some final remarks}

Since the 1970s, the debate on the sector dynamics in employment is primarily a debate about the shift to services, as all advanced economies have become largely dominated by this broad sector. In this section, we have been able to evaluate some of the implications of this shift (within a relatively short time span, but covering a large sample of European countries) for the composition of employment in terms of job quality. As discussed in chapter 2, there are two dominant hypotheses with respect to the impact of the shift to services on the quality of employment: the relatively rosy picture of post-industrialists, which consider the shift to services as "the great hope of the 20th Century", leading to a generalized upskilling and upgrading in the employment structure; and the most nuanced arguments of polarization theories, according to which the shift to services is associated with an expansion of highly paid knowledge intensive jobs as well as low-paid personal services occupations. In both hypotheses, industrial employment tends to shrink, depressing the middle of the wage distribution.

Across Europe, we could see instances of both polarization and upgrading, so we could try to identify the sector dynamics behind each pattern, and evaluate whether they corresponded to the hypotheses described earlier. The primary sector and manufacturing shrinked across most of Europe between 1995 and 2007, depressing the expansion of the bottom and middle of the job quality structures of most countries (although with different intensities). Such development does not seem so interesting anymore, once the shift to services has been widely accepted as inevitable: but its impact on the overall structure of employment is still very important (in negative terms), depressing some specific types of jobs, so it should not be forgotten. Without this development, the net growth of the bottom and middle of the employment structure would have been much more important in many countries.

Partly because of purely cyclical reasons (the post-1995 boom which has recently collapsed), the construction sector had a suprisingly important impact on the different patterns of job growth across Europe. It tended to determine the evolution of the middle of the employment structure, partly because other jobs that should have contributed to the middle were stagnant (such as manufacturing jobs, as already mentioned). Where construction grew, mid-paid jobs tended to grow as well; where it contracted, mid-paid 
jobs were destroyed; and where nothing happened, the middle remained quite stagnant. Without this development, we may have seen a bit of more polarization in the patterns of job growth: in particular, Austria and Spain would look more polarizing. Still, most of the variability in the patterns would have remained even without this clearly cyclical development.

But of course, the most dynamic development was that of the service sector, which we divided into five different sub-categories, according to its (typical) ownership (publicprivate services) and the type of activity (knowledge intensive or not in the case of private services; public administration, health and education in the case of public services). The most important driver behind the overall patterns of job growth are clearly those of private services, which match very closely the patterns of the whole economy. Knowledge intensive services expanded everywhere, and were responsible for the pervasive expansion of the top of the employment structure, whereas less knowledge intensive services (personal and distributional services) were responsible for an expansion of the bottom of the employment structure, where the bottom did expand. In other words, the differential expansion of the bottom of the employment structure seemed to be related to the expansion of personal and distributional services, whereas the pervasive expansion of the top was partly the result of an expansion of private knowlege intensive services. This expansion of the top of the employment structure was significantly helped by an important growth of the public sector in many European countries, especially in health activities.

So again, our results seem to support the post-industrial hypothesis more than any other one. The shift to services, and within services to knowledge intensive and social services, is clearly associated with a rather pervasive expansion of relatively high paid jobs. In the few cases of polarization, the arguments of polarization theories seem to fit our results; but the simple fact that this pattern was only observable in a few countries challenges the universalist claims of this hypothesis.

We know that where there was an expansion of the bottom of the employment structure, it was associated to an expansion of distributional and personal services. But what we still do not know is why such expansion only happened in a few cases. 


\section{Patterns of job growth by employment status}

All the analysis carried out so far has been limited to the evolution of employment by sector and occupation (using the job quality rankings to characterize the resulting jobs). The decompositions carried out in the previous two sections did not add any new information to this analysis, it only organized the information differently by merging or breaking up the occupations and sectors in more meaningful ways. In this section, on the contrary, we will add information which is external to the primary jobs matrix: information which will allow us to characterize each job in terms of its status of employment, and to see to what extent such status was related to the observed patterns of job growth.

In fact, we have used this kind of information before. An important component of the multidimensional index of job quality (what we called "amenities") was in fact the status of employment: having a non-permanent job was considered as a negative attribute (see chapter 3). But we only used that information to rank the jobs according to their non-pecuniary qualities: as we have mentioned several times, such ranking was fixed over time, and it only served as the basis of a qualitative characterization of the jobs. In this section, we will use the information on the status of employment in a completely different way: to decompose the patterns of job creation, trying to test the association between the quantitative evolution of employment by its status and the quantitative evolution of employment by job quality. The source of data will also be different: whereas for the construction of the amenities index we used the 4th EWCS, for the decompositions of this section we will use the ELFS, the same source used for studying the evolution of the structure of employment between 1995 and 2007. For ranking the jobs, we will use the wage index (as in the rest of this section).

Most Western European countries experienced, during the three decades after World War II, a process of standardization and regulation of the employment relation. Such process was a core element of the development of European Welfare States and Industrial Relations systems (Esping Andersen 1999: 22). As a result of this development, the conditions of employment were considerably standardized (and improved) for a majority of workers, under what we can call a Post-War norm of employment, which was mostly defined by being full-time (around 40 hours a week) and permanent. Such norm, which was a victory for the traditional industrial working class (fulfilling some of their historical aspirations), came under crossed fire in the late 
seventies and eighties: from the right, neoliberals argued that the standardization had gone too far and was asphyxiating economic activity, especially in the context of globalization (Huber and Stephens 2001: 48); from the left, the feminist movement argued that such norm assumed and perpetuated an unfair distribution of household tasks (Wilson 1977). Such crossed fire changed the political consensus, and many European countries initiated a process of deregulation of the employment relation that explicitly tried to facilitate more flexibility in terms of working time arrangements and the stability of employment contracts (Stanford and Vosko 2004). As a result, since the 1980s in many countries there was a very significant decrease in the proportion of workers whose employment conditions fitted the Post-War norm (for a detailed discussion of the concept of destandardization of labour, see Beck 1992: chapter 6).

Such regulatory changes have been explicitly linked to a polarization or even to an outright downgrading of the employment structure (see, for instance Peck 1996: 123; or Polavieja 2005). In this sense, the status of employment can be understood not only as an indicator of job quality (our main dependent variable in this thesis), but also as an indicator of regulatory changes in employment conditions, which is one of the most important potential determinants of the evolution of the division of labour from a compositional perspective. It is in this second sense that we will look at the status of employment in this section.

In what sense could a destandardization of the employment relation be related to the structure of employment from a job quality perspective? On the one hand, it could facilitate the creation of many low-productivity (and low-quality) jobs that would otherwise be too costly (thus, directly boosting the expansion of the bottom of the job quality structure). The 90s "eurosclerosis" appraisal, which blamed strict European labour regulation for the high levels of unemployment because it impeded the creation of low-productivity jobs (OECD 1994; Siebert 1997), is based in this same idea. This first relationship would imply a direct causal link between destandardization and the growth of low quality employment. On the other hand, even if a destandardization would not affect directly job creation, it could be specifically associated with some segments of the job quality structure: most plausibly, to the bottom. A deregulation of employment would not contribute to the expansion of low-paid jobs, but would make low-paid jobs more precarious. Finally, it could be that there is no relationship between the destandardization of employment and the structure of job quality: in this case, the 
expansion of non-standard employment should have been evenly spread for jobs of different qualities.

How do we operationalize the destandardization hypothesis? It mainly concerns the contractual conditions of employment: 1) the permanency of the employment contract: we can differentiate between self-employed workers (with no employment contract as such), non-permanent employees (with an employment contract with a limited duration) and permanent employees (with an unlimited contractual bond); 2) the (time) intensity of the employment relation: we can differentiate between full-time (defined by the norm of standard working time, in most countries 40 hours a week) and part-time (whose definition is more complicated, as we will see later: in general, we can define it as working hours which are below the full-time norm). In both cases, the standard relationship implies a stronger bond between the employee and the employer than the non-standard alternative (stronger over time in the case of the duration of the contract, stronger in time in the case of the duration of the work schedule). The standard will be defined here as a permanent full-time contract, the non-standard employment relation will be anything that deviates from such norm. ${ }^{84}$

\footnotetext{
${ }^{84}$ The consideration of self-employment as a non-standard employment relation may result somewhat more problematic than the other distinctions. Self-employment is associated to some types of economic activity that have always been carried out by self-employed workers, even in the heyday of the Post-War norm of employment. Still, it is clearly a departure from such norm, and it has been argued that one of the forms of destandardization of employment in recent years has been an artificial increase in the proportion of self-employed (see Frade, Darmon and Laparra 2004: 56). In practice, as we will see, the inclusion or not of self-employed does not change much the picture in most cases: and in the few cases where it does, it does look like an idiosyncratic process of destandardization.
} 


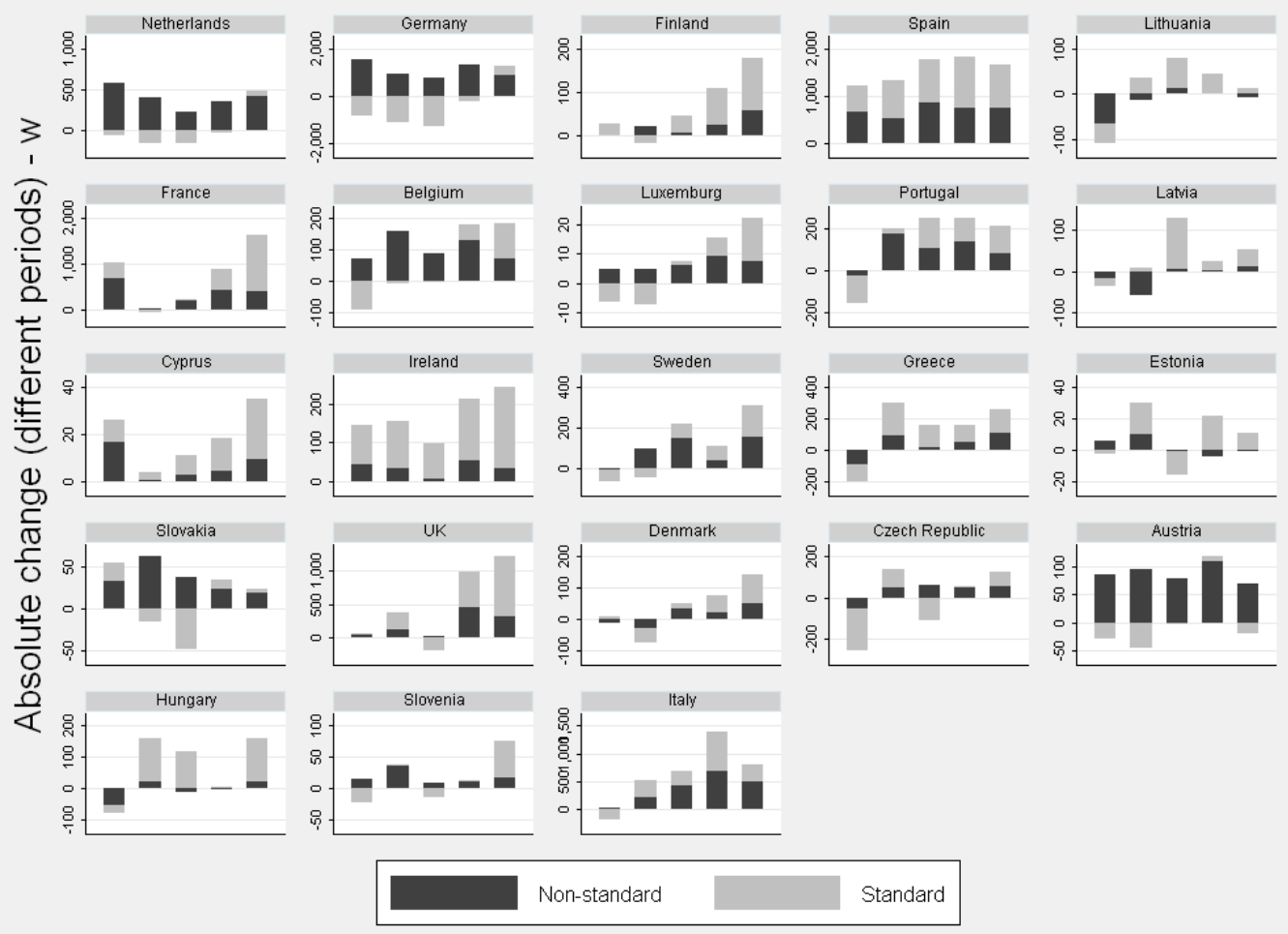

Figure 6.11: Breakdown of job growth by type of employment relation
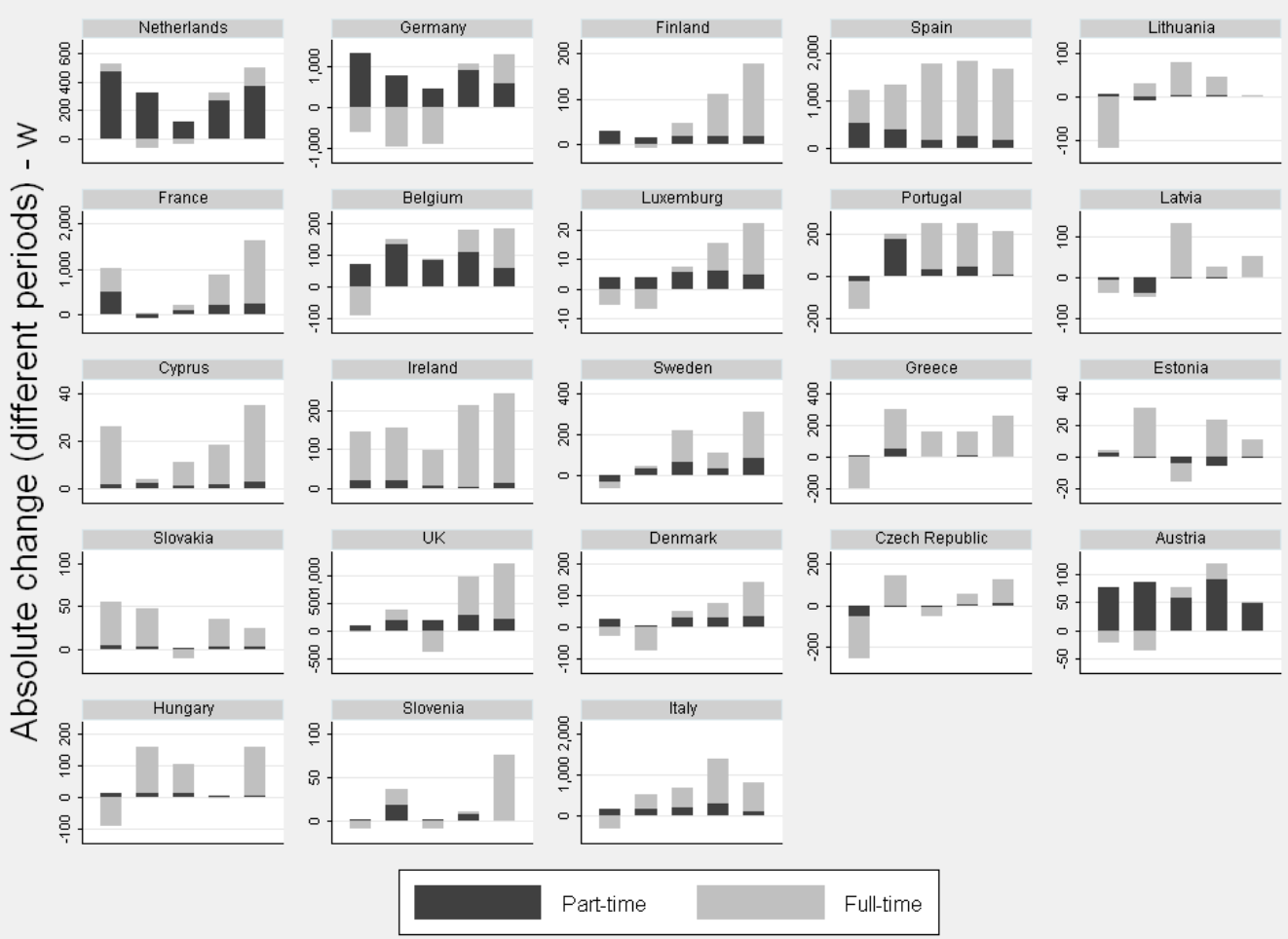

Figure 6.12: Breakdown of job growth by full-time or part-time status 


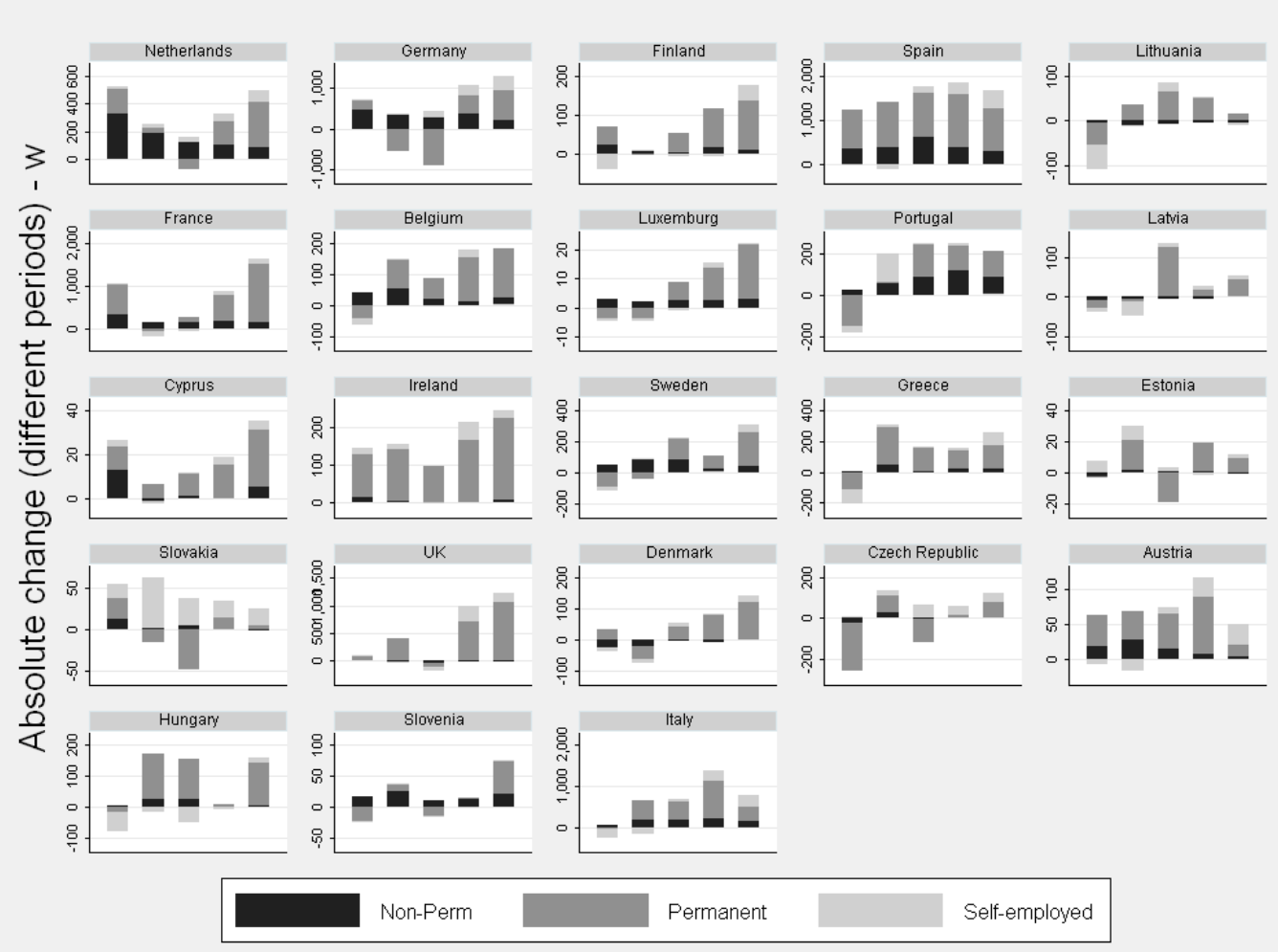

Figure 6.13: Breakdown of job growth by employment status

Figure 6.11 shows the patterns of job growth between 1995 and 2007 in Europe, broken up in two categories, one including workers with a standard employment relation (fulltime permanent employees) and the other category including all workers that depart from such norm. This figure will be used to discuss the overall relationship between the patterns of job growth and the destandardization of employment: but because the developments in terms of permanency of employment and part-time status are not the same in many countries, we will also discuss separately the specific developments for those two variables (presented in figures 6.12 and 6.13).

Before discussing these figures, it is important to recognise some difficulties of this particular exercise. The contractual and part-time status depends on the regulation of employment, which is in Europe (still?) an attribution of member states: there are very important differences across countries, which means that the same concept can mean rather different things in different contexts. For instance, in some cases part-time work it is defined with respect to what is stipulated in the contract (a worker works part-time if she has a part-time contract), in others it is defined by the hours of work (a worker works part-time if she works less than $\mathrm{X}$ hours a week). The boundaries between part- 
time and full-time are so different that they overlap across countries: for instance, in France 35 hours defines the full-time norm, whereas in Hungary it represents the upper boundary of part-time work (see Muñoz de Bustillo, Fernández-Macías and Antón 2008: 51). We are not speaking (or not only) about a measurement problem, but about a problem of different regulatory definitions of the same concept. The same happens in the case of contractual status: for instance, in the UK, Ireland and Denmark, the distinction between permanent and temporary contract does not make much sense because permanent contracts have themselves very little protection against dismissal, whereas in Spain the difference between permanent and temporary contracts in terms of employment protection is enormous. Still, these problem should not be taken too far because, after all, our interest is in the process of destandardization (an abstract concept which simply implies the degree of departure from the norm, in this case exemplified by the percentage of workers that do not fit the standard), not in comparing identically defined contractual relations.

Saying that after the eighties there was a process of destandardization of the employment relation in Europe is, of course, a very gross generalization. Many European countries did reform their labour market regulations in the eighties and nineties, but at different paces and to different degrees - and some did not deregulate at all. This is advantageous for our purposes, as we have countries that did experience a process of destandardization between 1995 and 2007 and countries which did not, so that we can compare their outcomes in terms of employment; but it also makes our task more difficult, because again we will have to deal with a very high level of complexity. Another potential problem for the analysis is how to deal with destandardization processes that took place before the period covered in this study, and which may therefore have already transformed the employment structure before our dynamic analysis kicks in. For instance, one of the most dramatic examples of destandardization (in Europe and beyond) is Spain, which after the labour market reforms of the mideighties generated an enormous amount of temporary jobs while permanent employment was being massively destroyed (in a few years, the share of temporary employment reached 35\% of all employment; see Fernández-Macías 2003). Such enormous change would obviously be of great interest for us: but most (or all) of its effects would have taken place before 1995, the first year which we cover here. Around 1995, the percentage of temporary employment stabilized, and as we shall see, it did not play a 
very significant role in the employment dynamics of the following years (the expansion of temporary employment in Spain was in fact below trend). How much of a problem can this be? It can be a problem if we want to say something about destandardization per se, and its implications on the quality of employment. But our analysis goes the other way around. We want to see whether a destandardization process had anything to do with the patterns of job growth observed in Europe between 1995 and 2007. What happened before determines the starting point: but as we are totally focused in analysing the change over a particular period, what happened before is not so important here.

The first thing we can say about figure 6.11 is that, as expected, the contribution of nonstandard employment to job growth was very diverse across Europe between 1995 and 2007. In some countries, there was an indisputable process of destandardization of employment, with nearly all growth corresponding to non-standard employment and a simultaneous net destruction of jobs with standard employment contracts (for instance, in Germany, Netherlands, and Austria); in other countries, non-standard was clearly secondary to standard employment in terms of job growth, in some cases even irrelevant (or negative, as in Estonia).

But as mentioned earlier, our real interest is not on the process of destandardization per se, but on its links to the patterns of change in the job quality structure. Figure 6.10 does suggest that they are related, but as usual, the relationship is complex and full of exceptions:

- The five clearest cases of polarization (Netherlands, Germany, France, Belgium and Cyprus) experienced some of the strongest processes of destandardization in Europe between 1995 and 2007 (the only case of very strong destandardization that did not fit a polarization pattern of job growth is Austria, which as we keep on saying, may be problematic). In Netherlands, Germany and Belgium, the expansion of non-standard employment was truly phenomenal, and affected the whole quintile structure although it was slightly skewed to the bottom; but at the same time these countries experienced a significant destruction of standard employment in the low-mid quintiles and a small expansion at the top. Together, these two developments amount to an important destandardization of the bottom of the employment structure, and contribute to the polarization of job growth. In France and Cyprus, the expansion of non-standard employment did not affect the whole structure of employment: it was polarized but strongly skewed to the 
bottom, whereas the expansion of standard employment was polarized but strongly skewed to the top. The two developments together, again, involve a process of destandardization of the bottom and a polarization of job growth.

- Ireland and the UK, which have been classified earlier as mild cases of polarization with an upwards bias, look completely different. Although there was some expansion of employment of a non-standard nature, such expansion was not biased downwards (in fact, in the UK it was biased upwards) nor did it seem to contribute significantly to the overall patterns of job growth.

- In most of the Transition countries of Eastern Europe, there is very little evidence of any ongoing process of destandardization of employment, and therefore it could not be a determining factor of the evolution of the employment structure (in fact, in Latvia, Lithuania and Hungary, there was a small but significant destruction of non-standard employment). The only exception to this general pattern is Slovakia, where there was a very significant expansion of nonstandard employment in mid-low paid jobs (especially the second lowest quintile), simultaneous with a very significant net destruction of standard employment in the middle quintiles (to a much lesser extent, something similar happened in Slovenia). As we will see, these developments have the peculiarity of being related to self-employment rather than to temporary or part-time work, contrary to the rest of Europe.

- With respect to the countries in the third and fourth column of figure 6.10, characterized by upgrading with or without growth in the middle of the job quality structure, destandardization was clearly much less of an issue between 1995 and 2007. In some of these countries (Finland, Denmark, Greece), there is no evidence of destandardization at all (the expansion of non-standard employment was negligible, and clearly below trend). In Sweden, Italy and Spain, the expansion of non-standard employment was more significant (although not nearly as much as in the polarization countries), but there was no bias of such development in terms of the job quality structure (the patterns of job growth were rather similar for non-standard and standard employment). Among the upgrading countries, only Portugal and Luxemburg seem partial exceptions in the sense that there was a significant growth of non-standard employment, and such growth was biased with respect to the job quality structure (leading to a 
mild destandardization of low-paid jobs). But even in these two cases, the scale of such effect was much smaller than in the polarization countries.

Figures 6.12 and 6.13 show the specific patterns of employment growth broken up by employment status (differentiating permanent employees, non-permanent employees and self-employed workers) and by full-time or part-time status. These two figures present a more detailed view of the issue of destandardization that we have just discussed in broader terms: we will only briefly discuss any departure from the overall picture discussed above.

In the Netherlands, Germany, Belgium and Austria, the move towards part-time employment was really important (see figure 6.12). In net terms, all job creation was part-time in these four countries, with a simultaneous important destruction of full-time employment in Germany and Austria. In many other EU15 countries there was some creation of part-time employment, but much less important than in those four countries (we can mention Spain, Portugal, Luxemburg, Sweden and France). There was no significant expansion of part-time employment in New Member States, nor in Cyprus, Ireland and Greece. In many countries, the expansion of part-time work was spread across the five quintiles, but at the same time, most new full-time employment was concentrated in mid-high paid jobs: so in relative terms, the expansion of part-time employment is much stronger on the bottom.

The expansion of non-permanent employment was much more clearly skewed towards the lowest quality jobs across all Europe, except Spain and Portugal (where the bulk of new non-permanent jobs concentrated around the middle: this development is probably linked to the expansion of middling jobs in construction, a sector which makes more use of non-permanent contracts in Southern Europe). Non-permanent employment grew most rapidly in the same countries that generated more part-time jobs: especially, in the Netherlands, Germany, Belgium and Austria. The expansion of non-permanent employment was also important in Sweden and Slovenia over this period.

In figure 6.13 we can also observe the developments in terms of self-employment. There is a clear difference in this sense between Old and New Member States. In Old Member States, the expansion of self-employment was very small, and tended to be concentrated in relatively well-paid jobs (the only exception is Portugal, as a result of the expansion of agricultural employment discussed in the previous section). In New Member States, the contribution of self-employment to the change in the employment 
structure was much more important, though not homogeneous. In Slovakia, there was a very large expansion of mid-low paid self-employment over this period: the destandardization of employment in this country that we identified in figure 6.10 is totally due to this expansion in the number of self-employed workers, which has no match in Europe. In Estonia, there was also a significant expansion of self-employment of mid-low quality, although not as important as in Slovakia. On the other hand, selfemployment contributed significantly to a net reduction of low-paid employment in Hungary, Latvia and Lithuania.

Therefore, in many continental European countries there was a destandardization process between 1995 and 2007 that involved simultaneously the expansion of part-time and non-permanent employment, and which tended to be skewed towards the lower half of the job quality structure. Although there is some evidence of destandardization in other EU15 countries between 1995 and 2007, its scale is considerably smaller in all cases. And the destandardization story does not seem to apply (at least, not yet) to the New Member States that we are studying here ${ }^{85}$ : only in Slovakia there was a significant increase in non-standard employment, and such development was the result of a sui generis process of expansion of self-employment.

Can we say that there is some relationship between the destandardization of employment and the patterns of job growth identified in the previous chapter? We believe there is, although as usual, such relationship is not unambiguous and there are some important exceptions. Broadly speaking, the countries that experienced a most intense process of destandardization happen to be the countries where the pattern of job growth was more polarized (especially, the Netherlands, Germany, France, Belgium and Cyprus; maybe also Slovakia): it is difficult not to see some relation between the two phenomena. This interpretation is strengthened by the fact that the countries that experienced a generalized upgrading in their employment experienced a considerably smaller expansion in non-standard forms of employment, and an expansion which was less downward-biased (as the countries in the third column of figure 6.11). There are, though, some exceptions that make such relationship between polarization and destandardization less pristine. Austria, for instance, should have experienced more polarization according to this interpretation, because it experienced a destandardization

\footnotetext{
${ }^{85}$ Unfortunately, our data does not cover Poland, a New Member State where there was a very significant process of destandardization in recent years.
} 
as important as that of Germany, Belgium or Netherlands. There was no destandardization in Ireland, so its polarization must have other reasons behind.

So there is a relationship between the destandardization of employment and the polarization of the employment structure, but only in broad and general terms. But doing analysis at such a high level of abstraction, comparing the developments of so many different countries, should make us suspicious of any pattern which looks too clear and unambiguous rather than the other way around. The task of social sciences is to simplify reality to be able to understand (and eventually change) it - not to pretend that reality is simpler than it really is.

\section{Conclusions}

We started this chapter with a general evaluation of the relative importance of the two dimensions behind the jobs matrix on the evolution of the employment structure in terms of job quality. We found that such evolution is primarily driven by shifts in the vertical division of labour (occupations). In section 3.1 we explored directly those shifts: the expansion of the top two quintiles in most countries is the result of a generalized growth in professional and technical occupations; in the few cases where the bottom also expanded, it was the result of growth in service and elementary occupations, whereas the change in the middle (whatever its sign and scale) was mostly related to the evolution of craft industrial and construction occupations.

Although the vertical dimension of the division of labour is the most strongly linked to the patterns of employment growth by job quality (because of the strong association between occupational levels and job quality quintiles), the horizontal division of labour is by no means irrelevant. Not only because there are systematic differences in the quality of employment across the different sectors (though smaller than across occupations), but also because the structure of occupations differs significantly across sectors (which intensifies the impact of sector shifts in employment over the job quality structure).

In section 3.2, we studied in detail the sector dynamics which are behind the patterns of employment growth by job quality. Such dynamics are even more interesting for our purposes than the occupational dynamics: on the one hand, because the link between occupational levels and quintiles was so strong that the analysis seemed almost 
tautologic, whereas - paradoxically - the weaker link between quintiles and sectors means that the sector decomposition adds more new information to the original analysis; on the other hand, because this type of analysis has more theoretical resonance: not only there are more references to sectorial dynamics in the literature summarized in chapter 2 , but this type of analysis is directly related to the traditional economic debate on structural economic change of Kuznets, Clark and Fourastié.

Although we can say that all sectors contributed to the patterns of job growth (expanding, contracting or keeping stable), the main stars of this story are private knowledge intensive services and the health sector. These two sectors expanded above trend in almost all European countries over this period, and were primarily responsible for the pervasive growth in the share of employment in relatively high paid jobs. Knowlege intensive services are routinely mentioned in the literature as a key driver of the expansion of good quality jobs; there are not so many references to the health sector in this respect, which being predominantly public is subject to a completly different logic (more political than strictly economic) in its evolution. The expansion of personal and distributive services (which we called "less knowledge intensive services") was clearly associated with the growth of low-paid jobs: where they did not expand, neither did the bottom of the employment structure. Quite surprisingly, the evolution of middling jobs depended mainly on the evolution of the construction sector: the sector which traditionally pulled the expansion of this type of jobs, manufacturing, was largely absent from the picture except in the three Central-Eastern European countries whose analysis we covered here; and although there is a significant proportion of middling jobs in private services, they tended to grow mostly on the extremes. The primary sector, unsurprisingly, contributed negatively to employment growth, depressing the lowest paid quintiles in those countries where the primary sector had still some room to fall.

In the previous chapter, we saw that the broad patterns of job growth tended to conform mostly to the (upgrading) predictions of post-industrialists, with only a few countries following a polarizing pattern of job growth and no support whatsoever for the downgrading hypothesis. The variety of patterns found, nevertheless, implied a rejection of the universalist claims which were somehow behind the three theories. The occupation and sector decompositions of this chapter confirm and qualify those earlier interpretations. The sector dynamics behind the (almost) generalized upgrading did conform to the arguments of post-industralists: increasing irrelevance of the industrial 
sector, expansion of services of high information and knowledge content as well as social services, expansion of professional and technical occupations. This type of development was nearly universal in the 23 countries for which we have data, even in those economies whose employment structures tended towards polarization between 1995 and 2007. What differentiated the latter economies was, in fact, a bigger expansion of low-paid private service occupations (mostly in personal and distributive services). Such development seems to confirm the hypotheses of polarization summarized in chapter 2: but as we mentioned earlier, the fact that it only happened in a few cases puts a question mark to the whole argument, especially in the technological version. If all countries were under the same type of technological constraints, why did the bottom only grow in a few countries?

The final section of this chapter provided suggestive answer to this question, an answer that is more anchored on institutional than technological factors. There seems to be an important (although not totally unproblematic, as discussed earlier) association between polarization and destandardization of employment. Most of the countries that expanded the bottom of their employment structures experienced a very important destandardization of employment, especially acute in the low-paid segments of the labour market. Most of the countries where there was no polarization did not experience such a destandardization, or it was not nearly as important. This suggests an institutional explanation for the expansion of low-paid employment, rather than (or complementary to) the technological explanation which is often mentioned in the literature. Only where the regulatory framework facilitates the expansion of low-paid employment does the bottom grow, most usually in personal and distributive services. The expansion of the top, on the other hand, seems more fitting for a technological explanation for its pervasiveness. The middle, as we have mentioned several times, is either absent from the picture, or (in a few cases) determined by the cyclical evolution of the construction sector. 



\section{Patterns of employment growth for the whole $\mathrm{EU}$}

\section{Introduction}

It may happen at some point; but it is indisputable that nowadays, there is not such a thing as an European Labour Market (Muñoz de Bustillo and Bonete 2009). Despite some recent efforts of "soft" harmonization (Trubek and Trubek 2005), the differences in the labour regulation of the different European countries remain very important (Dehejia and Samy 2006: 10). In fact, the impetus to harmonize employment and social regimes that started with the Lisbon strategy seems to be fading recently (as the repeated failure to update the working time directive testifies), in favor of more pressing priorities as the financial crisis or climate change. But even if there was a real harmonization of labour regimes across Europe, there would be still enormous difficulties to overcome for the construction of an European labour market. The existence of 23 different official languages is both a crucial barrier and a symbol of the difficulties for integrating so many different cultures within a single frontier.

For these and other reasons, the levels of labour mobility across EU countries (which can be understood as an indicator of the extent of labour market integration) remain strikingly low. Despite the fact that the EU actively encourages the mobility of labour within Europe and despite the enormous disparities in unemployment and wage levels, the proportion of European citizens working in a country which is not their own is less than $2.6 \%$ (less than 6 million workers of a total workforce of 222 millions). To understand the significance of this figure, we can compare it to the proportion of nonEU nationals working in the EU, which is almost double (4\%). Or we can compare it to the other large Union of States: although the figures are not totally comparable (there 
are no nationalities within the US), the proportion of US citizens living in a state which is not the one where they were born is $32 \%$ (Vandenbrande 2006: 14).

So instead of a single European Labour Market, there are 27 different labour markets with formally open borders between them which are rarely used for work-related reasons. That is the main reason why, up to this point, we have maintained the analysis strictly at the country level: the patterns and dynamics of employment growth by job quality are naturally circumscribed by the national frontiers, so only at that level are they really comprehensible. Still, there are interesting questions regarding the patterns of job growth for which using an European approach may be useful. Despite the mentioned deficit in terms of labour mobility and labour regulatory harmonization, the countries of the European Union have achieved in the last few decades a degree of economic integration that has no match in any other region of the world. There are open borders not only for labour, but also for services, capital and goods: and at least in the two later cases, the degree of institutional harmonization and effective mobility is really important. There is no European labour market nor probably an European services market, but there is probably an European capital market and certainly an European goods market (see Jones and Verdun 2005: 82-83). Even without effective labour mobility, a high level of integration of capital and goods can surely have important implications for the evolution of the division of labour within each European country, and for job quality too. If the national divisions of labour are becoming engrained within a wider European division of labour, it makes sense to carry out a specific analysis of the patterns of job growth from an European perspective, and from that perspective go back to an analysis of their implications at the regional level.

So in this chapter, we are going to take the data of the 23 European countries which have been studied separately in the previous countries, pool it into a single large dataset and analyse it as if the EU really was a single labour market. As the data is essentially the same, only arranged differently, we have to be careful not to repeat what we have already said in previous chapters. In this chapter, we will try to answer only those questions which specifically need a pan-European approach. What kinds of jobs has the EU as a whole created in the last job expansion? How does the EU pattern of employment growth by job quality quintiles compare with the US patterns for a similar period? What was the contribution of each European region (or institutional country clusters) to the overall patterns of job growth? Is there any evidence of a geographic 
division of labour within Europe, and what are their implications for job quality? This is the kind of questions that we will try to answer in this chapter.

The period that goes between 1995 and 2007 seems very adequate for our purposes. This was an incredibly eventful period for EU integration, especially from an economic perspective. During this period, under the Maastricht Treaty (which came into effect in 1993), the European Economic Community became the European Union. This was a period of intense deepening and widening of the EU, especially in economic terms. The European Central Bank was created to manage a full monetary union which came into effect for a core group of countries in 1999, and fully substituted national currencies by the Euro in 2002. Three new countries joined the EU in 1995 (Sweden, Finland and Austria); but the most crucial expansion of the EU took place in 2004-2007, when 10 Eastern European countries of the former Communist Bloc joined the EU. Although the actual accession of these 10 countries took place near the end of the period studied here, the process of adaptation to EU membership and economic integration coincided broadly with the period we are studying here. Finally, it is in this period when for the first time the EU attempts some form of harmonization of labour regulation and employment policies, with the European Employment Strategy (EES) which was kickstarted in 1997. Initially, this strategy was nearly totally focused on employment growth: but the critiques by social partners as well as the better economic conditions of the late nineties led to a shift towards job quality that made the EES adopt (at least rhetorically) the motto of "more and better jobs" - a motto that, transformed into a question, summarizes the objectives of this thesis -. The implementation of EES, though, is not based on "hard law" as many other areas of EU integration, but on a semi-experimental "Open Method of Coordination" (basically consisting on declaring objectives, benchmarking, reviewing performance and sharing good practices; for many, this simply proves the lack of will for harmonizing labour standards in Europe - see Chalmers and Lodge 2003). The EES may be little more than a declaration of intentions, lacking effective enforcement mechanisms: but at least, it justifies studies as this one. 


\section{Some prior methodological considerations}

In this chapter, the EU(23) will be analysed as if it was a single country. The two core elements of the jobs approach, the ranking and the employment matrix, are aggregated at the EU level by jobs, in the following way:

1. The rankings: the original wage and education ranking were constructed separately for each country. In order to have an EU ranking of jobs for wage and education, the national rankings were averaged, weighting by employment for occupations, sectors and countries. It is important to be aware that for wages and education the EU ranking is just an average of the national rankings, but this has its advantages too. By averaging 23 national rankings, rather than creating a new index based on absolute wage and educational levels in Europe, we solve the problem of how to put the incommensurate wage and educational levels of the different countries within the same metric. With respect to the amenities ranking, it was from the start constructed at the EU level, so we will just use it directly.

2. The employment matrix: the matrix of employment by occupation and sector for the whole of the EU was constructed by adding the absolute levels of employment of the 23 countries per year. As for most countries we only have information from 1998 onwards, the period covered for the EU analysis starts in that year instead of $1995 .^{86}$ The same adjustments applied to the national data (for the 8 countries with breaks in the ELFS employment series) were applied to this data (the adjustments were made at the national level, and then all the national data aggregated into the European totals).

3. The quintiles: finally, the quintiles were constructed for the whole of the EU for the year 2000, for the three rankings, and the evolution of employment by quintile is analysed in exactly the same way as in the previous chapters.

Before embarking on our analysis, it is important to be aware of its limitations. First, we would like to stress that the natural limits for the analysis of the patterns of employment growth are the national boundaries: the analysis in this section refers to a chimera, to a

\footnotetext{
${ }^{86}$ In fact, for Cyprus the data starts in 1999 rather than 1998. But it would not make much sense to loose a full year of data for 22 countries just to make sure that the smallest country of our sample is there. So the data for Cyprus in 1998 is in reality the same as for 1999 (as if nothing had changed in that year). The implications of this little trick are negligible.
} 
purely theoretical artifact which is constructed by forcefully merging completely different realities. It is important to be aware about the implications of this merging, in terms of our methodology. The same job will receive the same quality ranking across the whole EU, which implies assuming that, within the EU, the differences by occupation and sector are more important for job quality than the differences by country. It will be (necessarily) assumed that the ISCO and NACE classification are strictly comparable across Europe, which is only true in broad terms (see chapter 3 for more details). And the evolution of employment within the whole of the EU will be studied as if it was a coherent one, when we know that the economic cycles and trends varied considerably between countries.

A second important limitation is the fact that, because of data availability, we can only cover 23 member states. This was not such a big problem in the previous pages because the analysis was carried out at the country level: missing countries meant that the scope was narrower, but there was no misrepresentation of reality. In this case, our European results will in fact represent only a sub-sample of 23 European countries. Most importantly, we leave out Poland, which joined the EU in 2004 (as the other 8 new member states which are included in our analysis), and which has a large employed population (around $7 \%$ of the whole EU), so that its inclusion may have slightly changed the results. Poland is the only country of the whole EU that did not experience employment growth between 1998 and 2007, the period covered in our analysis here: but this was surely a period of deep changes in the Polish division of labour because of the intense restructuring of its economy. But unfortunately, the Polish LFS data does not include information on economic sector before 2004, so it was simply impossible to include Poland in the jobs matrix. The question is: then, why include at all the New Member States in the analysis? Leaving out Poland means leaving out almost half of the story, and after all, these countries joined the EU in 2004 (only three years before the end of the period covered here). But after all, this is just a theoretical exercise, and it is interesting to see what are the implications of the inclusion of the NMS for the evolution of the division of labour within the EU. Even though the NMS formally joined the EU in 2004, the impact of their inclusion was being felt much earlier (the process of integration started in the early nineties). So we decided to include the NMS (minus Poland and Malta) in the analysis, and simply be aware of this limitation. 


\section{Overall EU patterns of employment growth}

Figures 7.1-7.3 show the overall EU(23) patterns of job growth, ranking the jobs by wages, educational level and amenities. The first thing that we can say by looking at these pictures is that the overall EU figures provide a good summary of the national patterns for each ranking. The patterns of job growth ranking the jobs by wages are the most polarized of the three, yet strongly biased upwards. The jobs occupying the $20 \%$ of employment with higher wages grew more than twice as fast as the jobs occupying the lowest 20\%; and the fourth highest quintile grew also quite vigorously (expanding by nearly 6 million workers), whereas the second lowest and middle quintile grew much less than the rest. If we put these patterns in the context of the national results discussed in chapter 5, the EU would fit in the second column, together with Germany and the UK, as a case of mild polarization with strong upgrading of the employment structure. When the jobs are ranked in terms of their average educational level, we get a very similar pattern, but the degree of polarization is milder because rather than the lowest quintile, it is the second lowest which grows slightly above average (with the middle and lowest quintiles growing below trend). Finally, the results based on the amenities ranking show much less polarization, with the top and middle of the structure growing relatively fast and the bottom growing below trend.
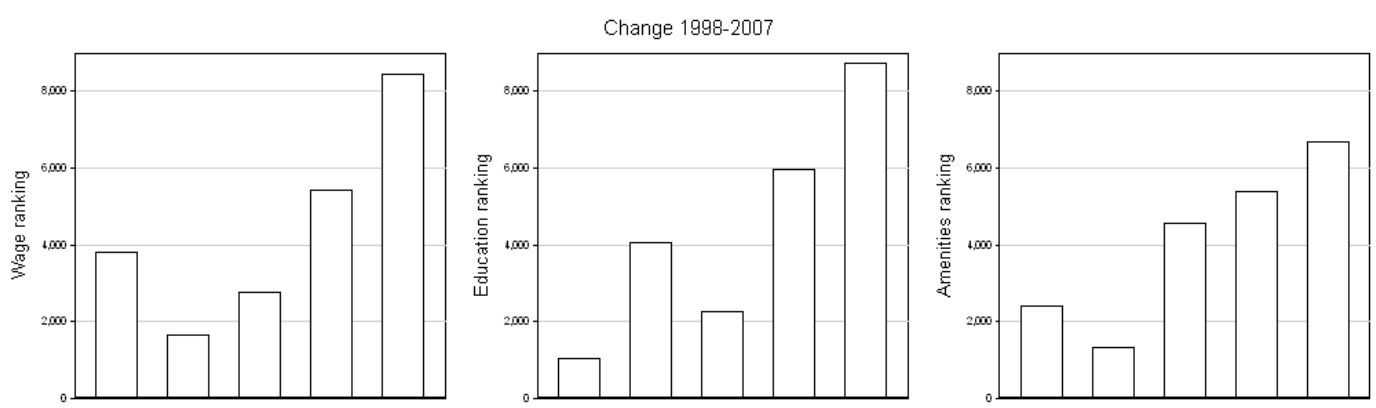

Figures 7.1-7.3: Patterns of job growth by job quality, EU(23) 1998-2007

These overall EU patterns, constructed as if the whole of the EU was a single labour market, provide a good summary of the 23 national patterns discussed in chapter 5: the main pattern was one of upgrading, with some degree of polarization (most clear when the jobs were ranked by wages, but apparent in the three rankings) and rather limited expansion of those jobs occupying middle positions overall (except in the amenities ranking). But although these EU patterns may be a good summary of the national patterns, we should not forget that they conceal what may be the most interesting finding of all this study: the plurality of patterns of job growth that existed in Europe 
between 1995 and 2007. That is one of the reasons why the country results are always the main result, and these aggregated EU figures are just a useful analytic tool to answer some specifically transnational questions.

\section{A comparison between Europe and the US}

One important question that the EU-level analysis can answer is: how did the patterns of job growth in Europe as a whole compare with the patterns of job growth in the United States over a similar period? Figure 7.4 compares the EU wage-based pattern shown in figure 7.1 above with the pattern of employment growth for the United States between 1992 and 2000. This figure has been directly taken from the paper that Wright and Dwyer published in 2003 in the Socio-Economic Review, which was one of the main inspirations for this thesis (see Wright and Dwyer 2003). Although there are some differences in the details ${ }^{87}$, the methodological principles behind the US chart shown on the right-hand side of figure 7.4 and the EU chart shown in the left-hand side (both are based on a ranking of jobs by their median wages) are exactly the same, so the comparison is perfectly legitimate. And even though the periods covered are not the same, they are comparable in a very important way: whereas in the US, the strong employment expansion took place precisely between 1992 and 2000 (the years of the "American Jobs Machine"), in the EU the expansion took off a bit later, covering the period between 1995-6 and 2007. After the year 2000, US employment growth has been much milder, whereas in the EU the expansion of employment remained quite strong.

\footnotetext{
${ }^{87}$ The most important difference is that the American analysis referred only to full-time employees, whereas our own analysis is based on all workers. In the EU analysis, leaving out part-time workers is not an option because of the enormous importance of this type of employment for the patterns of job growth between 1995 and 2007 in many countries (see section 4 of chapter 6 for a discussion of the patterns of job growth for part-time and full-time employees).
} 

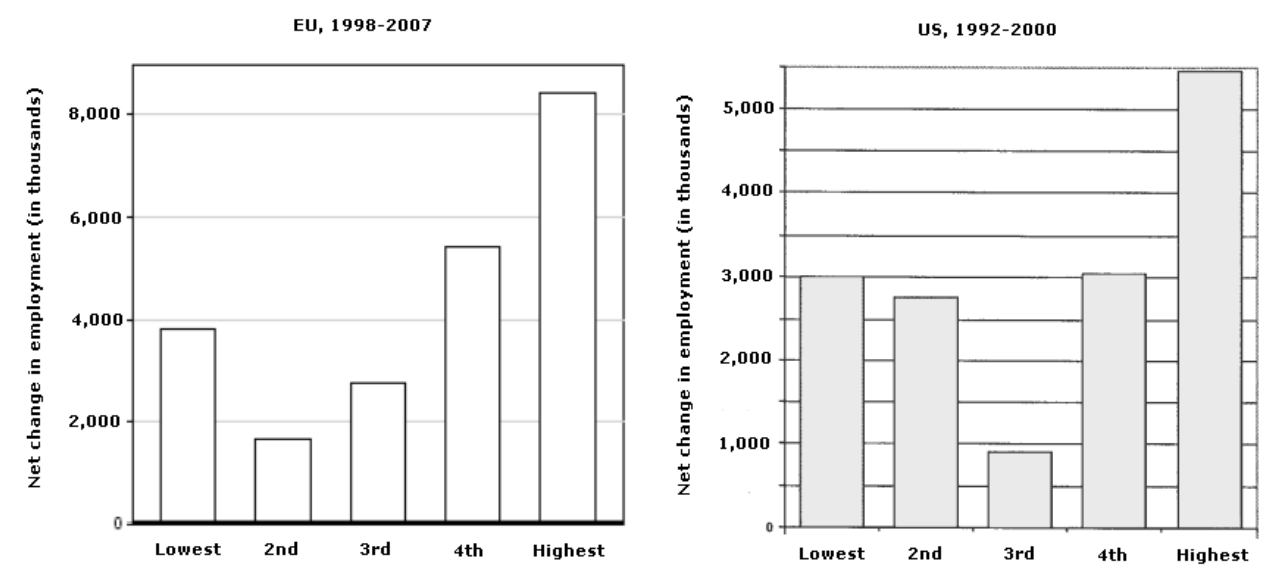

Figure 7.4: EU vs. US patterns of job growth (wage quintiles)

In any case, the comparison shown in figure 7.4 is very suggestive, because of their similarities as well as their differences. In both cases, the pattern of job growth is one of asymmetric (upwards) polarization; but there is much more polarization in the US than in Europe, and much more upgrading in Europe than in the US. In fact, the quintile picture in the US and Europe is remarkably similar for the three upper quintiles, and quite different for the bottom two. The ratio of better-to-worse jobs in the US expansion of 1992-2000 (taking the first and fifth quintiles) is 1.8/1; in the $\mathrm{EU}(23)$ expansion between 1998 and 2007 it is 2.1/1. But if we take the two lowest versus the two highest quintiles, the ratio in the US is $1.5 / 1$ while in the EU it is 2.5/1. Another interesting comparison is between the US patterns and the national patterns discussed earlier in chapter 5: the US figures would be among the most polarizing of our sample of 23 countries, only matched by the countries of the first column of figure 5.1 .1 of chapter 5 (Netherlands, France, Cyprus and Slovakia).

A similar pattern of job growth, but with more polarization: that is how the US pattern looks in the light of our European results. A similar picture suggests that there could be similar dynamics behind. Is that the case? In the same paper where figure 7.4 is taken from, Wright and Dwyer make a decomposition analysis very similar to the one we carried out in the previous chapter, and their results are strikingly coincidental with ours. According to their analysis, the gap in the middle was the result of "the collapse of durable manufacturing as a source of employment growth" and of "the absence of a countervailing expansion of middle quintile jobs in high-end services" (Wright and Dwyer 2003: 321). The expansion of the bottom quintiles was largely the result of a strong expansion of retail trade and personal services, and the very strong expansion of 
the top of the employment structure came mostly from an expansion of business services and high-tech activities (Wright and Dwyer 2003: 318). In broad terms, these were also the main sector dynamics behind the European patterns of employment growth that we discussed in the previous chapter: probably the biggest difference would concern the role of the public sector, which was very important in Europe for the expansion of high paid jobs, whereas Wright and Dwyer do not even mention them; also, the role played by high technology industries in the expansion of good jobs seemed to be more important in the US than in most European countries (except in Germany, Austria and Ireland).

For explaining why there was such a large expansion of low-paid jobs in distributive and personal services, Wright and Dwyer refer mostly to two factors: immigration and the historically low minimum wage of the 90s. Although we did not analyse such factors in the previous chapter, it is unlikely that they would be the main determinants of the expansion of low-paid jobs in the EU, although they may have played a role. There were some European countries that experienced a surge in migration over the period covered here (such as Spain, Ireland or Greece), and countries with traditionally high shares of migrant workers (such as Germany, the UK or Austria): but although migrant employment tends to be located at the bottom, neither the number of new migrants nor the number of existing migrants seem to be related to whether the bottom of the employment structure grew in Europe (for a detailed analysis, see FernándezMacías and Hurley 2008: 45-46). With respect to whether the level of minimum wages played a significant role, answering such question would require a detailed analysis that we cannot carry out here, although the countries which expanded most their shares of low quality jobs do not seem to be those with lowest minimum wages, at least at first sight. ${ }^{88}$ Our prime explanation for the expansion of the bottom in some European countries and not others was the destandardization of employment, something that is completely missing in the US picture according to Wright and Dwyer. So the expansion of the top and the stagnation of the middle seem to have very similar explanations in Europe and the US, but the expansion of the bottom does not, despite their similarity in terms of the sectors affected (in both cases distributive and personal services).

\footnotetext{
${ }^{88}$ Some of the countries which expanded most the bottom two quintiles in relative terms are among the highest as well in terms of their minimum wages, in absolute and relative terms (such as Netherlands or France; see Hurley 2007: 1-2).
} 


\section{Decomposing the EU patterns of job growth by regions}

But the real benefits of using an EU approach do not lie in looking at the overall patterns, but in the possibility of breaking up those broad patterns by regions and industries. We must remember that the analysis of this chapter is constructed as if the whole of the EU was a single labour market. That is, all European workers within the same occupation and sector are classified together, irrespective of where do they work, and are located in one of the five job quality tiers according to the average wage ranking of their jobs for the whole of the EU. But even though we ignore the nationality of each worker when we generate the quintiles and represent their change between 1998 and 2007, we do have such information, and therefore we can break up the overall patterns and examine exactly how much of all EU employment creation of each job quality tier goes to (or more precisely, comes from) the different European regions.

In this section, and in the rest of this chapter, we will classify the 23 European countries of our sample in five groups. On the one hand, we do this to reduce the complexity of the analysis; but an even more important reason behind the use of country groups is the fact that such grouping is meaningful on its own, because it puts together countries with similar institutional frameworks. The idea of clustering European countries in a few (ideal) types according to their similarities and differences in institutional terms goes back to the analysis of welfare regimes that Esping-Andersen published in $1990 .{ }^{89}$ Although Esping-Andersen was primarily concerned with the classification of welfare systems, and although there were many critiques and amendments to his original scheme, this approach proved to be enormously fruitful for many areas of comparative social analysis (for a review of the field, see Arts and Gelessen 2002). In the last two decades, there have been countless studies which have used some type of EspingAndersen-inspired scheme to explore or explain the European distribution of many different social issues.

Esping-Andersen (1990: 26-29) originally spoke about three types of welfare regimes: the Conservative (corporatist, which puts the family at the centre of social provision), the Liberal (which relies primarily on the market) and the Social-Democratic (universalist, which puts more emphasis on the State as a provider). This typology broadly coincided with the geographic regions of Continental Europe (the Conservative

\footnotetext{
${ }^{89}$ In fact, this idea goes further back, to Titmuss 1958; but it was the publication of Esping-Andersen's book in 1990 which really initiated this field.
} 
type), the UK and Ireland (as well as the US, within the Liberal type) and Scandinavian countries (the Social-democratic type). Most later researchers added a fourth group, following the arguments of Ferrara (1996), specific for Mediterranean countries (Esping-Andersen considered Mediterranean countries as underdeveloped examples of the Conservative type), characterized by clientelism, low social expenditure and even more reliance on the family as a welfare provider than the continental Conservative group. The collapse of the Eastern European Communist bloc and its increasing integration into Europe has stretched even further this type of typology: it is widely debated whether Eastern European countries should be considered as a new type (or types) or whether they can be fitted within the existing typologies (see, for instance, Fenger 2007).

Our classification of countries will be based, therefore, on the original Esping-Andersen typology, with the addition of a Southern European group and a group for Eastern European Transition countries (hence, five types). Rather than labelling these types in terms of their institutional settings (as in the original scheme by Esping-Andersen), we will use the more neutral geographic labels of Northern, Continental, Southern, Eastern and Isles (table 7.II shows the classification of each country), which correspond to the Social-democratic, Conservative, Mediterranean, Transition (or Post-Communist) and Liberal types. Although the institutional labels are more informative, they involve a stronger commitment to a specific analytic framework than is necessary here. Of course, we use these groupings because we believe that the institutional framework that they refer to is relevant for our core subject (the patterns of employment growth by job quality): but it remains secondary to our general approach. This is not a study about welfare regimes and the transformation of employment structures: we will just use this institutional typology in order to understand better the regional dynamics which are behind the European patterns of job growth shown earlier.

Figure 7.5 shows the patterns of employment expansion in the EU by job quality quintiles (from now on, we will only use the wage-based quintiles), broken up by the mentioned five European regions. This figure represents employment growth in absolute numbers (in thousands): below, table 7.I shows the relative contribution of each region, in percentages. Just by giving a quick glance to these results, we see that the different European regions had different contributions to the overall European patterns of job growth. We will discuss each region separately: 
1. Continental Europe. Figure 7.5 makes quite clear that job polarization was primarily a Continental development: the white segment of the bars is what gives the EU quintiles picture its polarized shape. By comparing the percentages to the row marginals of table 7.I, we can get an idea about how skewed was the contribution of each country cluster to the expansion of the EU quintiles: the more distant the percentages to the marginals, the more biased was the contribution of each cluster to the overall expansion of employment. In the case of Continental Europe, its contribution to the expansion of the lowest and second lowest quintiles was far above its overall contribution to employment (48\% and $45 \%$, compared to $36 \%$ overall), whereas for the quintile holding middling jobs the contribution of Continental Europe was considerably below (11\% contribution to this tier of employment, against $36 \%$ overall). The contribution of Continental European countries to the expansion of the top two quintiles was proportional to its overall contribution to employment growth.

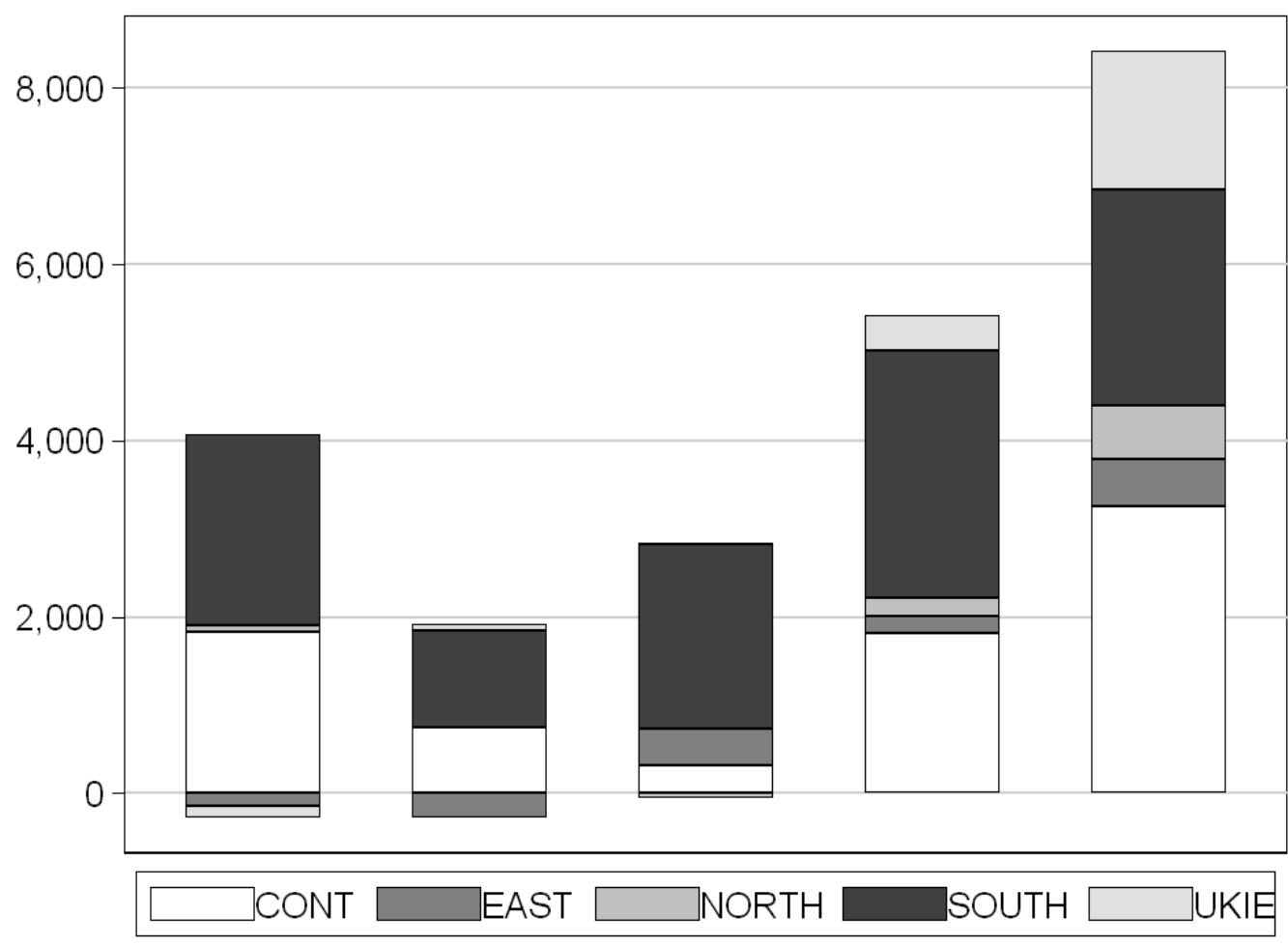

Figure 7.5: Patterns of employment growth in the EU, broken up by regions 


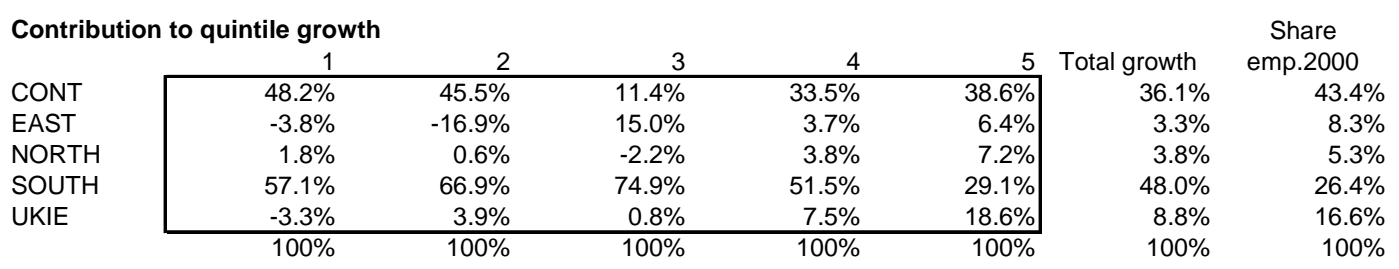

Table 7.I: Contribution to quintile growth of each region

2. Southern Europe. This group of countries made the biggest overall contribution to employment expansion in Europe between 1998 and 2007, accounting for almost one in every two new jobs: such contribution was far above its employment share in 2000, what means that this cluster expanded considerably its share in EU employment over this period. The contribution of the South to the EU patterns of job growth were quite different from those of Continental Europe: as can be seen in figure 7.5 , it contributed very strongly to the expansion of all quintiles, but most importantly to the third and fourth. The percentages of table 7.I show that where the contribution of the South was most significant for the EU patterns was in the third (a staggering three quarters of all employment created in this tier of the labour market came from Southern Europe), second (two thirds) and first quintiles (57\%). On the other hand, the contribution of this group of countries to the expansion of the quintile holding the $20 \%$ of best paid jobs was far below its overall contribution to employment ( $29 \%$, compared to $48 \%$ overall).

3. Eastern Europe. The contribution of the 7 Eastern European countries for which we have data was extremely thin $(3.3 \%)$, far below what would have corresponded to their share in employment (which was 8.3\% in 2000). Between 1998 and 2007, these countries were still suffering from a difficult economic transition, and although their economies were growing, their employment levels remained stagnant. Nevertheless, the contribution of this cluster to the European patterns of job growth was rather significant and different from that of other clusters. Its contribution to the expansion of the middle quintile (15\%) was completely disproportionate with respect to its overall contribution to employment growth (which was only 3\%). And, as a result of the large restructuring processes suffered during this period by these transition economies, this cluster had the biggest negative contributions of all European regions: Eastern Europe reduced by $17 \%$ the size of the second quintile, and by $4 \%$ the size of the quintile holding lowest paid jobs. 
4. Northern Europe. The contribution of Finland, Sweden and Denmark was unambiguously upgrading, contributing very little (less than what would have corresponded to their overall growth, as shown by table 7.I) to the expansion of the three bottom quintiles and most importantly (taking into account the small overall contribution of this cluster) to the expansion of the fifth quintile.

5. Isles. Finally, the contribution of the UK and Ireland was also strongly skewed to the top, even more than the contribution of Northern Europe. They hardly contributed at all to the growth of the three lowest quintiles, and their contribution to the top quintile was almost $20 \%$, despite the fact that these countries contributed by less than $9 \%$ to overall employment growth in the 23 EU countries covered here.

Figure 7.5 and table 7.I suggest that the regional grouping of countries (which, we must remember, is based on institutional similarities) is quite relevant for understanding the overall patterns of job growth in Europe. All European regions expanded significantly their share of relatively good jobs (what explains the overall upgrading pattern for the whole of the EU), but they had considerably different contributions, especially in the middle and bottom of the wage structure. But how do we know that such development is really associated to European regions, and not just a random result of the aggregation of countries into regions? Was the contribution of all countries within each cluster similar? Or did the contribution of each cluster depend on the impact of a few big countries? Are the clusters really relevant?

The answer to some of those questions has been partially given in previous chapters, when the analysis was done at the country level. Did all countries within the same European region share the same pattern of employment growth by job quality? Not really, or at least not very clearly. There seemed to be some link between polarization and continental Europe (the four clearest polarization cases were the Netherlands, France, Germany and Belgium); the three Scandinavian countries were put together within the group of upgrading countries; and most Southern European countries experienced some type of upgrading with strong growth of middle-quality jobs. But there were too many exceptions and ill-fittings to say that there was a clear link between the regions and the patterns of job growth. The position of Eastern European countries was totally unclear: apparently, they were located randomly across all typologies of job growth patterns (the Baltics were, in fact, simply inclassifiable); the characterization of 
the UK and Ireland was also unclear; Austria did not fit the polarization pattern at all, nor did Luxemburg (two Continental European countries). And the differences among Southern European countries in the patterns of job growth were considerable.

Table 7.II makes a further decomposition of the patterns of job growth in Europe, breaking up the contribution of each cluster into the contributions of each individual country. The difference between the results in this table and the analysis of chapter 5 is that, in this case, the perspective is strictly European. What is shown here is the contribution of each country to the EU patterns of job growth, instead of the national patterns of job growth defined in its own terms. All countries share the same job quality ranking, whereas in the previous chapters, we used nationally-specific wage and educational rankings to construct the quintiles. So this table should allow us to reconsider, from a more adequate perspective, the relevance of the clusters.

\begin{tabular}{|c|c|c|c|c|c|c|c|}
\hline Quintiles & Lowest & 2 & 3 & 4 & Highest & Overall growth & \\
\hline $\begin{array}{l}\text { EU (absolute growth, } \\
\text { thousands) }\end{array}$ & 3796.56 & 1639.16 & 2781.41 & 5423.83 & 8427.51 & 22068.46 & $\begin{array}{c}\text { Empl. Share } \\
2000 \\
\end{array}$ \\
\hline Continental & $48.2 \%$ & $45.5 \%$ & $11.4 \%$ & $33.5 \%$ & $38.6 \%$ & $36.1 \%$ & $43.4 \%$ \\
\hline AT & $3.5 \%$ & $8.9 \%$ & $2.9 \%$ & $-0.3 \%$ & $1.0 \%$ & $1.9 \%$ & $2.1 \%$ \\
\hline BE & $1.9 \%$ & $3.2 \%$ & $1.8 \%$ & $1.1 \%$ & $3.3 \%$ & $2.3 \%$ & $2.4 \%$ \\
\hline DE & $18.5 \%$ & $19.5 \%$ & $-10.0 \%$ & $15.7 \%$ & $12.7 \%$ & $12.1 \%$ & $20.9 \%$ \\
\hline FR & $14.8 \%$ & $8.4 \%$ & $13.3 \%$ & $13.0 \%$ & $18.1 \%$ & $15.0 \%$ & $13.3 \%$ \\
\hline LU & $0.1 \%$ & $0.0 \%$ & $0.2 \%$ & $0.1 \%$ & $0.3 \%$ & $0.1 \%$ & $0.1 \%$ \\
\hline $\mathrm{NL}$ & $9.5 \%$ & $5.3 \%$ & $3.3 \%$ & $4.0 \%$ & $3.3 \%$ & $4.7 \%$ & $4.5 \%$ \\
\hline$\overline{\text { East }}$ & $-3.8 \%$ & $-16.9 \%$ & $15.0 \%$ & $3.7 \%$ & $6.4 \%$ & $3.3 \%$ & $8.3 \%$ \\
\hline $\mathrm{CZ}$ & $-4.6 \%$ & $-4.8 \%$ & $3.3 \%$ & $2.5 \%$ & $1.5 \%$ & $0.4 \%$ & $2.7 \%$ \\
\hline EE & $0.2 \%$ & $-0.6 \%$ & $1.1 \%$ & $-0.2 \%$ & $0.3 \%$ & $0.2 \%$ & $0.3 \%$ \\
\hline HU & $-0.7 \%$ & $3.3 \%$ & $4.2 \%$ & $0.1 \%$ & $1.8 \%$ & $1.4 \%$ & $2.2 \%$ \\
\hline LT & $0.5 \%$ & $-6.0 \%$ & $1.0 \%$ & $-0.7 \%$ & $0.8 \%$ & $-0.1 \%$ & $0.8 \%$ \\
\hline LV & $-0.4 \%$ & $-3.2 \%$ & $1.9 \%$ & $0.9 \%$ & $0.6 \%$ & $0.4 \%$ & $0.5 \%$ \\
\hline SI & $0.1 \%$ & $-2.8 \%$ & $0.3 \%$ & $0.6 \%$ & $0.9 \%$ & $0.3 \%$ & $0.5 \%$ \\
\hline SK & $1.2 \%$ & $-2.8 \%$ & $3.2 \%$ & $0.5 \%$ & $0.6 \%$ & $0.7 \%$ & $1.2 \%$ \\
\hline North & $1.8 \%$ & $0.6 \%$ & $-2.2 \%$ & $3.8 \%$ & $7.2 \%$ & $3.8 \%$ & $5.3 \%$ \\
\hline DK & $-1.3 \%$ & $-2.2 \%$ & $-1.8 \%$ & $1.3 \%$ & $1.2 \%$ & $0.2 \%$ & $1.6 \%$ \\
\hline $\mathrm{FI}$ & $0.8 \%$ & $-0.9 \%$ & $0.1 \%$ & $0.8 \%$ & $2.5 \%$ & $1.2 \%$ & $1.4 \%$ \\
\hline SE & $2.3 \%$ & $3.7 \%$ & $-0.5 \%$ & $1.8 \%$ & $3.5 \%$ & $2.4 \%$ & $2.4 \%$ \\
\hline South & $57.1 \%$ & $66.9 \%$ & $74.9 \%$ & $51.5 \%$ & $29.1 \%$ & $48.0 \%$ & $26.4 \%$ \\
\hline CY & $0.4 \%$ & $1.0 \%$ & $0.6 \%$ & $0.3 \%$ & $0.3 \%$ & $0.4 \%$ & $0.2 \%$ \\
\hline ES & $45.8 \%$ & $50.1 \%$ & $54.4 \%$ & $20.4 \%$ & $15.2 \%$ & $29.3 \%$ & $8.9 \%$ \\
\hline GR & $2.8 \%$ & $-5.5 \%$ & $6.0 \%$ & $2.2 \%$ & $2.1 \%$ & $2.2 \%$ & $2.4 \%$ \\
\hline IT & $9.7 \%$ & $21.4 \%$ & $10.0 \%$ & $27.4 \%$ & $9.6 \%$ & $14.9 \%$ & $12.1 \%$ \\
\hline PT & $-1.6 \%$ & $-0.1 \%$ & $3.9 \%$ & $1.2 \%$ & $1.9 \%$ & $1.2 \%$ & $2.9 \%$ \\
\hline UK and Ireland & $-3.3 \%$ & $3.9 \%$ & $0.8 \%$ & $7.5 \%$ & $18.6 \%$ & $8.8 \%$ & $16.6 \%$ \\
\hline IE & $2.4 \%$ & $5.8 \%$ & $7.2 \%$ & $0.5 \%$ & $2.3 \%$ & $2.8 \%$ & $1.0 \%$ \\
\hline UK & $-5.8 \%$ & $-1.8 \%$ & $-6.4 \%$ & $7.0 \%$ & $16.3 \%$ & $6.0 \%$ & $15.6 \%$ \\
\hline
\end{tabular}

Table 7.II: Contribution to quintile growth of each region and country

Again, we will discuss each cluster one by one:

1. Continental Europe. Table 7.II shows that Germany, France, the Netherlands and Belgium all shared a polarizing contribution, similar to the overall contribution of this group. The German contribution is specially important, 
because it intensifies the polarization effect by strongly depressing the expansion of employment in the middle (in Germany, there was a net destruction of middle-paid jobs that accounted for $10 \%$ of the overall change of this tier). Austria and Luxemburg, on the other hand, did not contribute to polarization at all despite their belonging to this group: yet their overall effect is negligible because of their small size compared with the other countries in this group.

2. Southern Europe. This group seems much less homogeneous in its contribution to overall employment trends in Europe. First of all, the enormous quantitative importance of a single country for the contribution of this group is really striking. Spain accounts on its own for almost one third of all the expansion of employment between 1998 and 2007 in the 23 European countries of our sample. The contribution of Spain, therefore, has a disproportionate weight on the contribution of this cluster (the contribution of all the other countries together was almost half the contribution of Spain). But the contribution of Spain was, in fact, rather different from that of the other countries. Spain contributed mostly to the expansion of the bottom three quintiles (more than half of the growth of the three bottom quintiles in the EU came from Spain); Italy, to the expansion of the fourth European quintile (accounting for more than one fourth of it, nearly doubling its overall contribution to employment growth); Portugal to the third and fifth (destroying employment in the first and second) and Greece in the first and third (destroying employment in the second). So the overall contribution of this cluster seems more like the aggregation of rather disparate patterns (with one country totally dominating the result) than a coherent contribution from similar national developments.

3. Eastern Europe. The contribution from Eastern European countries is certainly not coherent, but there are some similarities. In all Eastern European countries there was strong destruction of employment in the bottom (which only happens here, and in some Southern European countries), and a strong expansion of the middle. So we could say that these countries can be clustered together because they are all very different from the rest, not so much because they really look like each other.

4. Northern Europe. Table 7.II shows that the developments in Northern Europe were not at all coherent. This is interesting because in the country-specific 
analysis all Nordic countries seemed relatively similar (all experiencing strong upgrading). When we use a single EU-wide wage ranking, some of those similarities disappear: in particular, Sweden looks somewhat polarized. This is because some of the jobs that expanded most in Sweden between 1998 and 2007 occupy a higher position in the wage ranking in this country than elsewhere (so that when we use the same ranking everywhere, Sweden looks more polarized). In any case, Denmark and Finland had a strongly upgrading contribution, whereas Sweden had a polarizing one: hence, there is no internal coherence in this group either.

5. Isles. The UK and Ireland look also rather incoherent, and as the UK is much bigger than Ireland, it dominates the aggregate contribution from this group of two: the strongly upgrading contribution of the Isles is totally a UK development. Ireland, at its more modest scale, contributed mostly to the expansion of the three lowest quintiles, in fact. The contribution of Ireland looks much more like Spain than the UK.

So only within the Continental group of countries there seems to be a coherent development in terms of job quality, leaving aside Austria and Luxemburg (Luxemburg is a perfectly reasonable outlier: it is too small and odd to expect similarity with anything; the Austrian case is more significant). The other groups have too much internal variability to be considered as different cases of the same model in terms of patterns of employment growth by job quality.

\section{Decomposing the EU patterns of job growth by sectors and regions}

In this last section, we will look at the sector composition of the EU patterns of job growth, and its interaction with the regional dimension. As happened with the previous section, some of these findings will seem familiar: after all, both the data and the basic method of decomposition are the same as those used in chapter 6 , so the results have to be at least coherent. There are, of course, some differences with the analysis of previous chapters that justify the addition of this section: all these differences can be summarized by saying that the perspective will be strictly transnational in this case. As in the rest of this chapter (and differently to previous chapters), the ranking of jobs will be based on the European average; the quintiles will be constructed for the whole of the EU(23); and 
the analysis will be carried out upside-down, from the European figures down to the regional results. Nevertheless, we will try to avoid repeating what we have already discussed in previous chapters, focusing on new information or, at least, on results which present the same information from a new angle.

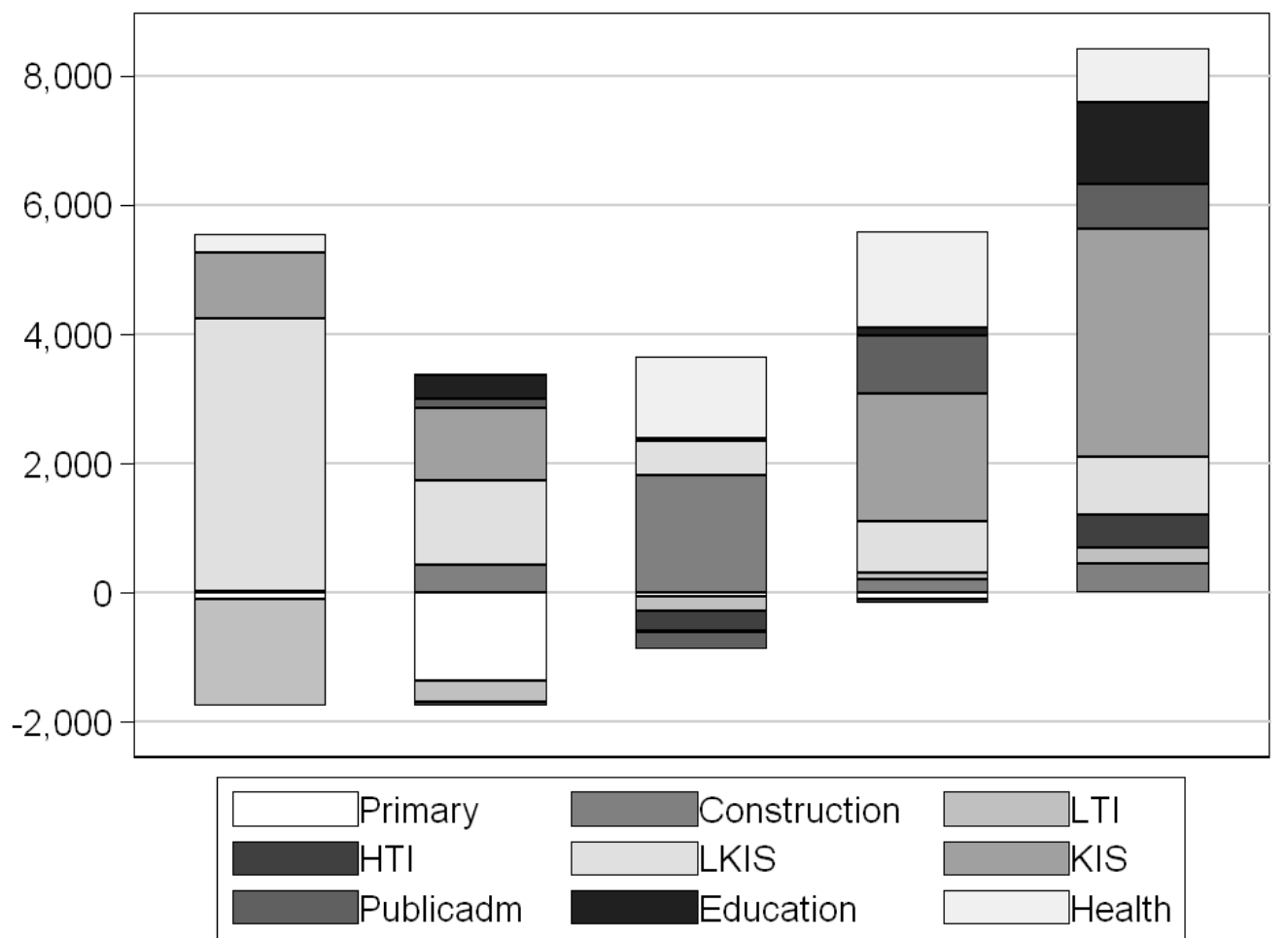

Figure 7.6: Patterns of employment growth in the EU, broken up by 9 sectors

\begin{tabular}{|c|c|c|c|c|c|c|c|}
\hline \multicolumn{7}{|c|}{ Contribution to quintile growth } & \multirow{2}{*}{$\begin{array}{c}\text { Share } \\
\text { emp.2000 }\end{array}$} \\
\hline & 1 & 2 & 3 & 4 & 5 & Total growth & \\
\hline Primary & $-2.4 \%$ & $-83.3 \%$ & $-1.9 \%$ & $-2.0 \%$ & $0.0 \%$ & $-7.3 \%$ & $5.1 \%$ \\
\hline Construction & $0.6 \%$ & $26.1 \%$ & $65.4 \%$ & $3.9 \%$ & $5.3 \%$ & $13.3 \%$ & $7.9 \%$ \\
\hline LTI & $-43.5 \%$ & $-20.1 \%$ & $-8.3 \%$ & $1.7 \%$ & $3.0 \%$ & $-8.5 \%$ & $13.7 \%$ \\
\hline HTI & $-0.1 \%$ & $-3.8 \%$ & $-11.0 \%$ & $-1.1 \%$ & $6.0 \%$ & $0.3 \%$ & $7.6 \%$ \\
\hline LKIS & $111.2 \%$ & $79.5 \%$ & $18.9 \%$ & $15.0 \%$ & $10.6 \%$ & $35.2 \%$ & $26.8 \%$ \\
\hline KIS & $26.7 \%$ & $69.0 \%$ & $-0.7 \%$ & $36.4 \%$ & $41.7 \%$ & $34.5 \%$ & $15.8 \%$ \\
\hline Publicadm & $0.0 \%$ & $8.7 \%$ & $-9.5 \%$ & $16.6 \%$ & $8.3 \%$ & $6.7 \%$ & $7.0 \%$ \\
\hline Education & $-0.3 \%$ & $22.8 \%$ & $2.0 \%$ & $2.3 \%$ & $14.9 \%$ & $8.1 \%$ & $6.8 \%$ \\
\hline Health & $7.8 \%$ & $1.2 \%$ & $45.1 \%$ & $27.3 \%$ & $10.1 \%$ & $17.7 \%$ & $9.3 \%$ \\
\hline & $100 \%$ & $100 \%$ & $100 \%$ & $100 \%$ & $100 \%$ & $100 \%$ & $100 \%$ \\
\hline
\end{tabular}

Table 7.III: Contribution to quintile growth of each sector

Figure 7.6 and table 7.III present a first breakdown of the overall EU patterns for the same 9 sectors used in chapter 6 for decomposing the national patterns. As happened with the overall EU patterns of job growth, these results provide an excellent summary of the country-level analysis of previous chapters, which clarifies what was general and what was particular in those earlier results. Looking at the two final columns of table 
7.III, we can see that there were two sectors that expanded considerably their employment share in Europe between 1998 and 2007: knowledge intensive services (KIS) and health (in both cases, their contributions to employment growth doubled their initial employment shares). Construction also expanded considerably, and to a lesser extent also personal and distributive services ${ }^{90}$ (LKIS) and education. All the other sectors contributed less than what corresponded to their initial shares, which implies a contraction in their relative importance. The Primary sector and Low Technology Industries (LTI) contracted even in absolute terms (that is, there was a net destruction of employment in these sectors between 1998 and 2007, which accounted for almost 2 million jobs in LTI and 1.6 million jobs in the Primary sector). High Technology Industries (HTI) did not destroy employment in net terms (although there were very large shifts across quintiles and regions, as we will see later), but it made no contribution whatsoever to the overall employment expansion ( $0.3 \%$ growth), which implies a significant contraction in its employment share. And finally, public administration expanded in absolute terms (by 1.5 millions), but such growth was in fact below its initial share, and therefore it contracted slightly in relative terms.

From a sector perspective, the characteristic asymmetrically polarized pattern of the European employment expansion that took place between 1998 and 2007 was the result of two main dynamics: the polarized nature of job growth in services and the contraction of middle and low paid employment in manufacturing and the primary sector. Such dynamics are clearly reflected in figure 7.6. Knowledge Intensive Services contributed enormously to the expansion of the fifth quintile (three and a half million more jobs), diminishing its contribution as it approached the middle quintile (to which it contributed negatively, in fact), and increasing it again, although much less strongly, in the lowest quintiles (it should be noted that despite being knowledge intensive, these services contributed with more than 1 million new jobs to the expansion of each of the two bottom quintiles). Personal and distributive services (LKIS) also had a somewhat polarized contribution, although much less pronounced and very strongly skewed to the bottom. In fact, nearly all the net expansion of low-paid employment was contributed by LKIS, as we can see in figure 7.6. The contribution of these two sectors together

\footnotetext{
${ }^{90}$ Although LKIS contributed the most to overall growth (35\%), this contribution was close to its initial share of employment (27\%), whereas the contribution of KIS was more than twice its initial share (35\% compared to $16 \%$ ) and health was a bit less than twice (18\% contribution compared with $9 \%$ initial share).
} 
(strongly upwards for KIS, strongly downwards for LKIS; in both cases somewhat polarized), which account for $70 \%$ of the overall expansion of employment, is what gives the EU pattern of job growth its polarized shape.

If it was only for services, the degree of polarization would be quite intense. The contribution of other sectors tended, therefore, to smooth the polarization effect of services. Most importantly, the middle and bottom of the wage structure were dragged down by the developments of the less dynamic (in terms of employment) sectors of the economy, which have traditionally been the driver of the mid-low paid segments of employment. Low Technology Industries, which still account for a large share of lowand mid-low paid jobs (first and second quintiles), contracted very importantly in Europe between 1998 and 2007, eliminating 1.6 million jobs in the lowest quintile, one quarter of a million jobs in the second lowest quintile and more than two hundred thousand jobs in the middle quintile. High technology Industries did not contract overall, but it nevertheless eliminated more than three hundred thousand jobs in the middle quintile. And the Primary sector still had a very significant contribution to the contraction of employment in the second quintile (1.3 million jobs destroyed in net terms), despite being such a marginal sector in terms of employment (it accounted for only $5 \%$ of overall EU employment in 2000).

Other significant developments were the expansion of high paid public employment (in the top two quintiles), especially in the health sector; and the expansion of employment in construction, which softened even further the polarization pattern of the service sector by closing the gap in the middle. As mentioned in the previous chapter, the expansion of construction over the last decade in Europe was clearly a one-off development, which has reversed very rapidly since 2007: an intensification of the polarization tendency in terms of overall EU job growth is therefore to be expected in the context of the current crisis.

So these are the sector dynamics underlying the EU patterns of employment growth by job quality. But the real objective of this section is to further breakdown such dynamics by their regional dimension. The expansion of the different sectors and their impact on the job quality structure took place within an economic space that, as we said earlier, is divided by (national) cultural boundaries but united by a joint (European) political and economic project. The policy of open borders for goods, services, capital and people could have some type of impact on the economic structure of the different countries, 
injecting some tendency towards integration that can involve some kind of panEuropean division of labour. If there is such a development, it should be reflected in our figures: in particular, we should see simultaneous expansions and contractions of employment of the same type (and quality) in different areas of Europe, as some areas concentrate the production of specific goods and services for the European market. If there is such a development, we should be able to evaluate also its implications for the geographical distribution of job quality across Europe. That is what we will try to do in the remainder of this chapter.

The nature of economic activity of the different sectors provides by itself, even before looking at any results, a partial answer to this question. European specialization requires European trade (as argued by Adam Smith a long time ago, the limits of the division of labour are given by the size of the market), but even within a highly developed single market as that of the EU, not all sectors are equally tradeable. Given the current state of technology and the practical lack of restrictions within the EU, the markets for agricultural and industrial products are already integrated to a large extent. But as we have seen earlier, these sectors account for a small and rapidly decreasing share of total employment, with the very large and heterogeneous service sector taking all the rest (around $70 \%$ of employment on average). And although services are very heterogeneous, one thing that they share in common is that they are much more difficult to trade than the products of the primary and secondary sectors.

Most services involve a direct and simultaneous interaction between the user and the producer, rather than an indirect interaction mediated through a product, as in the case of the primary and secondary sectors. Although the revolution in communication technologies is bringing new possibilities for the provision of services at a distance (medical services through the internet, for instance), so far it remains anecdotal except in a few very specific services subsectors (such as communications, for instance). On top of this, the degree of regulatory harmonization and formal openness to trade of services in the EU is much lower than in the case of agricultural and industrial goods: it is only very recently that the EU is taking concerted and decisive action in this respect (see OECD 2009 for a review). And finally, there is a large part of the services sector for which there is not even the will of an European integration (or if there is, it is of a totally different nature): public services. Although the EU itself is an example of integration of some types of public services (such as the administration of monetary 
policies), its size in terms of employment relative to national public services is absolutely negligible.

What we are trying to say is that, even before looking at any result, we can assume that the real potential for integration of the national employment structures into a hypothetical EU division of labour is rather limited. There is certainly some of that in the primary sector and in manufacturing, and we will see how that is reflected in our data. But most employment is concentrated in the service sector, which is much less integrated and much less capable of integrating. In the case of public services, it is simply absurd to look for the impact of European integration in terms of employment: so we will not include public administration, education nor health in the analysis of the following pages.

In the following pages, we will use the same five regional categories discussed in the previous section, which may seem odd considering that our findings suggested that there was too much internal variability within each region as to consider them really meaningful in terms of the patterns of job growth (except in the case of continental Europe, which seemed to be associated with the tendency towards polarization). Still, the regional categorization of the previous section remains a useful tool for simplifying an analysis that otherwise would become unmanageably complex (we will deal with 5 quintiles, 9 sectors and 23 countries), and we can understand it as a necessary first step of an attempt to evaluate the extent of geographic specialization.

We will discuss, one by one, the regional composition of each of the sector contributions to the overall patterns of job growth at the EU level. Hence, we will be doing a decomposition of a decomposition: each of the segments of figure 7.6 will be broken down by region. The analysis will be based on a graphical representation of absolute quintile growth (figures 7.7 to 7.12 ) and on a table breaking up the contribution of each sector to quintile growth by region (table 7.IV). The graphical representations require no further explanation: they follow the same principles as all charts used in this thesis (representing absolute growth by quintile, in this case corresponding to the contribution of each sector to overall EU quintile growth). Table 7.IV requires giving some more details. 


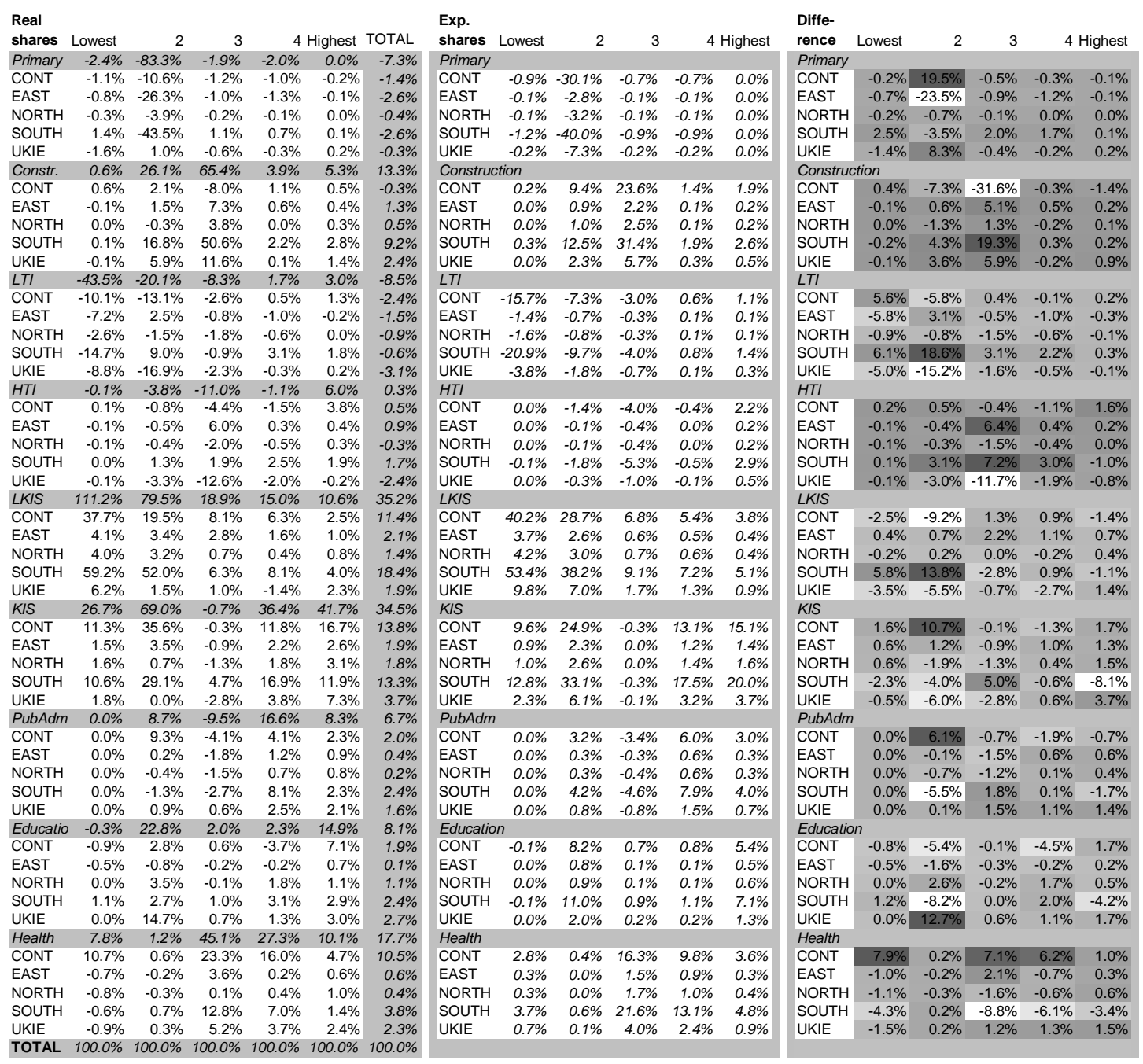

Table 7.IV: Decomposition of sector contributions to quintile growth by region

Table 7.IV includes three sub-tables. The first one (on the left), simply shows the percent contribution of each sector to overall quintile growth, broken up by region (therefore, it is just a regional decomposition of table 7.III). The second one (in the middle) shows a set of counterfactual values which represent how the regional composition of the sector contribution should have been if all regions had contributed equally, relative to their overall contribution. These "expected values" (expected under the assumption of no association between region and sector in the contribution to the patterns of job growth) are generated by multiplying the relative contribution of each sector to the growth of each quintile by the relative contribution of each region to overall job growth. The third sub-table within table 7.IV includes the raw difference between the real and expected values, with colour-coding of cells to identify more easily high and low values: this table will assist us in the identification of regional 
contributions which deviate from the general pattern of each sector contribution, and therefore suggest some type of relation between region and sector.

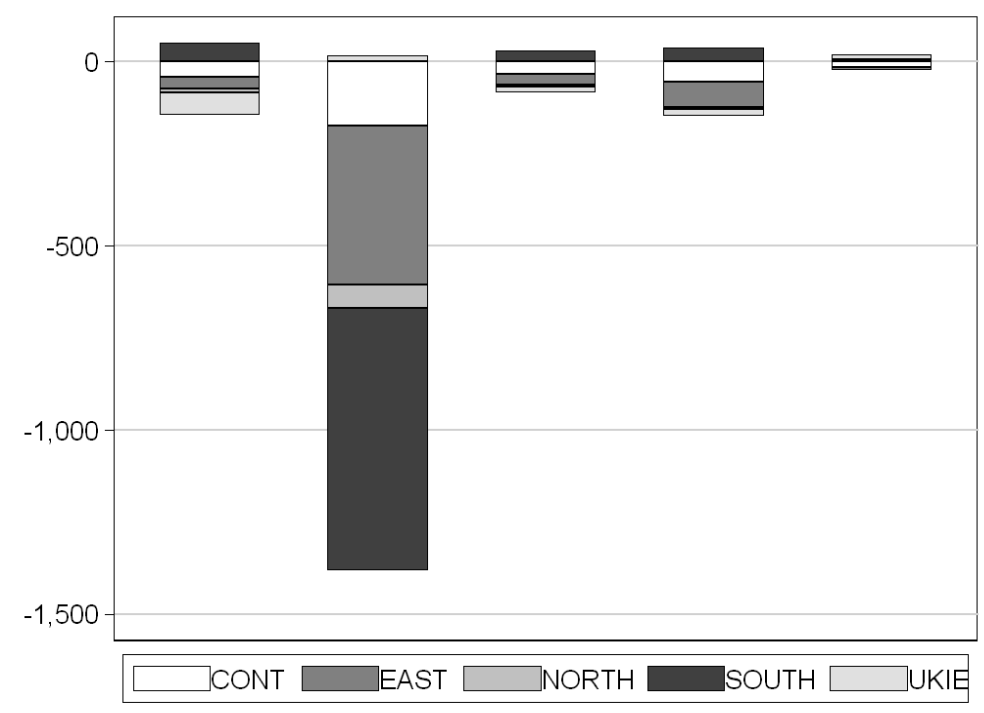

Figure 7.7: Contribution of PRIMARY SECTOR to quintile growth by region

1. The primary sector (fig. 7.7): as we have seen in previous pages, the contribution of the primary sector to the patterns of job growth was negative across the five quintiles, but strongly concentrated on the second lowest job quality tier at the EU level. Although this negative development was contributed by all European regions, it was disproportionately associated with Southern and Eastern European countries (which was in fact rather generalized within those two regions). Table 7.IV shows that in fact, the region with a highest negative contribution in relative terms was Eastern Europe, whereas the negative contribution from Continental Europe and the Isles was far below what would have corresponded to their overall employment contribution. What can we make of such development? It is surely more associated with a later industrialization of Southern and Eastern Europe than with anything else (that is, with a late introduction of labour-saving technologies in agricultural production). This development could also be related to a global rather than European division of labour (as agricultural production tends to become less competitive with economic development): but in fact, the Common Agricultural Policy has sheltered the European agricultural sector from external competition, so in terms 
of output there has not been a major reduction that could suggest a process of integration (or disappearance) of this sector into a global division of labour. ${ }^{91}$

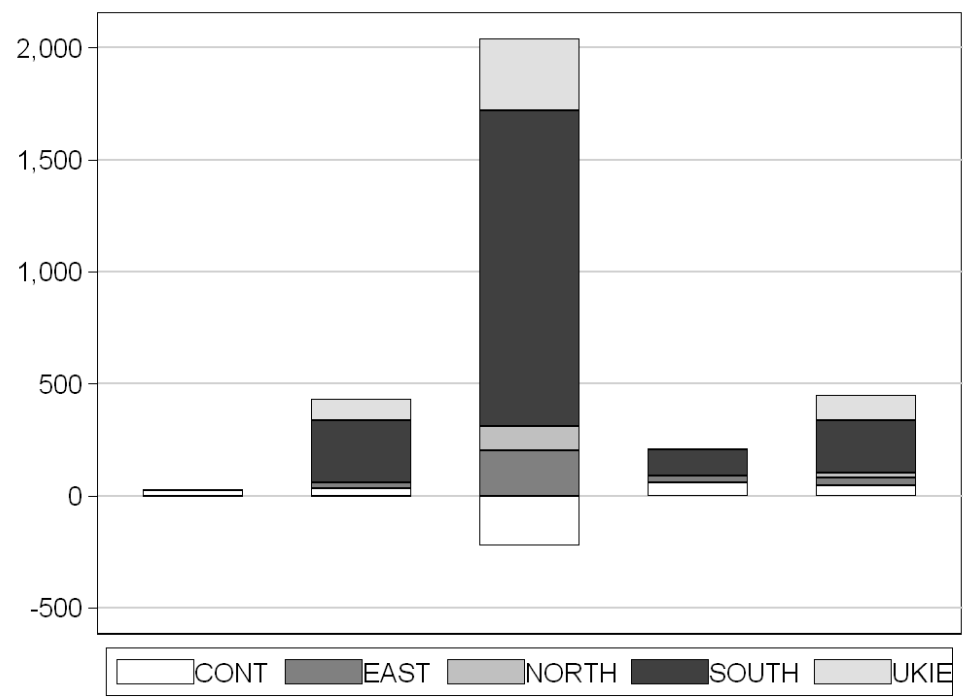

Figure 7.8: Contribution of CONSTRUCTION to quintile growth by region

2. Construction (fig. 7.8): the construction sector was responsible for most of the expansion of the third quintile. And as figure 7.8 shows, most of this contribution came from the South of Europe: although there was some expansion also in Eastern Europe and the Isles, it looks very small in comparison (and as shown by table 7.IV, it was more in line with their overall expansion of employment). A country-level analysis (not shown here, but available on request) shows that Spain in particular was responsible for most of this expansion of construction: but still, in most Mediterranean countries this expansion was above average. The development of this sector in Continental Europe was the complete opposite, destroying a significant amount of jobs in the middle quintile between 1998 and 2007. This negative contribution, in fact, can be reduced to a single country: Germany, where there were more than half a million jobs destroyed in net terms in construction between 1998 and 2007 (in other Continental countries, there was in fact a timid expansion of construction, although not big enough to imply an increase in its share). So in this sector there were clearly diverging developments between 1998 and 2007 across Europe: but the nature of activity in this sector does not allow to interpret such development

\footnotetext{
${ }^{91}$ While the share of labour force in agriculture declined in all European countries between 1995 and 2005 , the output value at producer prices of the agricultural industry was still raising in absolute terms (see Eurostat 2007a: 24)
} 
in terms of Europeanization (although it may be somewhat related to a specialization of Mediterranean Europe in touristic services, which probably contributed to the construction boom in those countries). The most plausible explanation of these diverging developments lies in the differential impact of the single European monetary policy, with the arrival of the Euro. On the one hand, the EU-wide interest rates were too low (below inflation) for Mediterranean countries and just about neutral for Continental Europe (especially Germany). On the other hand, these Mediterranean countries had never experienced such stable and favourable monetary conditions. All in all, this led to radically different outcomes in different regions, fuelling a huge construction boom from which we are still recovering in the Mediterranean while making little impact in Germany and other Continental economies. So again, no specialization: rather, different development paths, even if triggered by the same monetary policy.

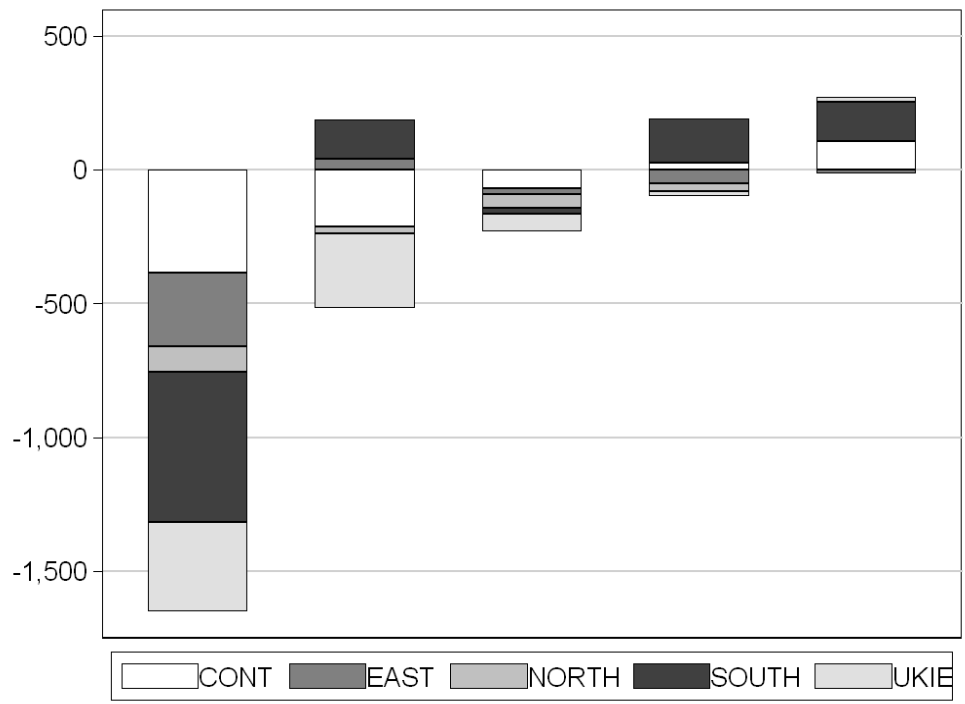

Figure 7.9: Contribution of LOW TECH INDUSTRIES to quintile growth by region

3. Low Technology Industries (LTI, fig. 7.9): we saw earlier that LTI were characterised by an intense destruction of employment, concentrated at the bottom of the wage structure. Where this destruction of low-paid, low-tech industrial jobs was more intense was in Southern Europe, which simultaneously created a smaller but significant amount of higher paid jobs in the same sector (second, fourth and fifth quintiles). Something similar, though less voluminous and less upgrading, happened in continental Europe. On the other hand, Eastern European countries destroyed large numbers of low-tech low-paid jobs without 
creating jobs in these sectors at any other pay level, and something similar (but at the second lowest rather than the lowest quintile) happened in the UK. The regions that created high-paid jobs in this sector simultaneously destroyed many more low-paid LTI jobs: this looks much more like the impact of an internal process of technical change than anything else. In fact, the sheer amount of employment destruction in this sector throughout Europe suggests that the key development of LTI, shared by all European regions, is one of progressive decadence (in terms of employment), probably driven by technical change and globalization (that is, the division of labour at a global rather than European scale). Still, there are some shifts in employment across regions and quintiles that could suggest some degree of Europeanization of Low Technology Industries, which would have benefited mostly the South of Europe (which contributed to the creation of more than 300.000 LTI jobs in the two highest quintiles) and Continental Europe (where it contributed to the creation of roughly 130.000 relatively well paid LTI jobs).

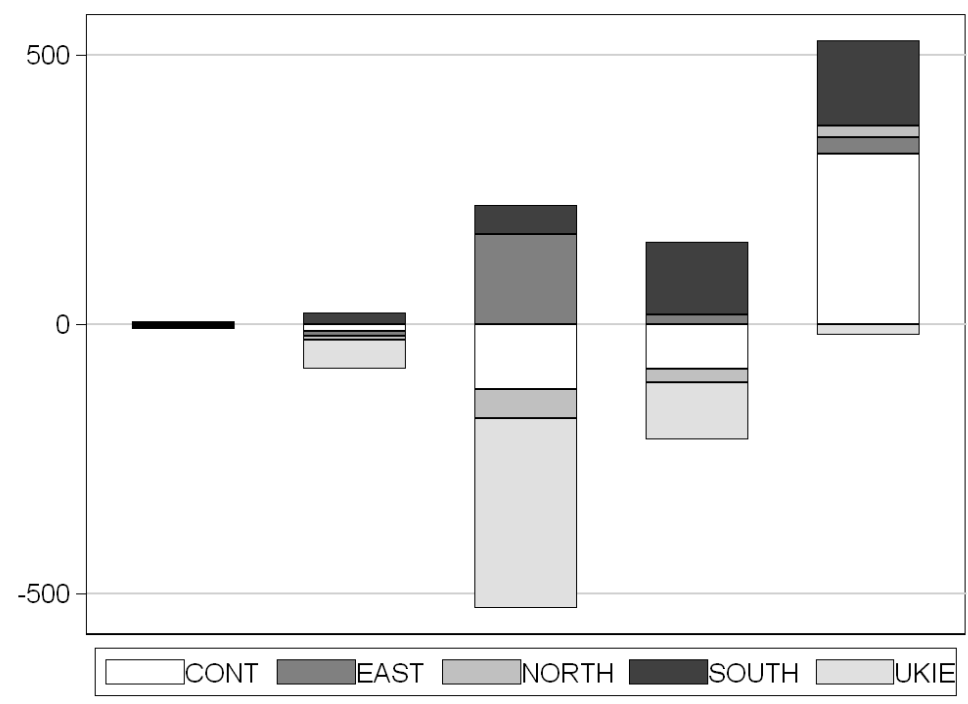

Figure 7.10: Contribution of HIGH TECH INDUSTRIES to quintile growth by region

4. High Technology Industries (HTI, fig. 7.10): despite its tiny contribution to overall employment growth, this sector is very interesting because it suffered very large shifts in employment across regions and quintiles. The UK and Ireland destroyed a very significant number of jobs in this particular sector across the five quintiles, but specially in the middle quintile (more than 300.000 jobs). There is no evidence of any job creation in this sector for this region, 
which suggests that the UK and Ireland were simply moving away from it. The developments in Nordic countries were very similar to the Isles, although at a smaller scale. On the other hand, in Southern Europe there was quite a lot of job creation in HTI, specially in the two upper quintiles (although table 7.IV shows that it was in the middle where the relative contribution from the South was more significant). In Eastern Europe there was also an important creation of HTI jobs, most of them within the middle quintile. And the development of this sector in Continental Europe suggests shifts upwards in the value chain: there was some job destruction in quintiles three and four, simultaneous with the creation of more than 350.000 jobs in the top quintile. So this is probably the case in which the evidence more clearly suggests some type of regional specialization, with some regions destroying many HTI low- and mid-paid jobs while other regions created many jobs of middle and high paid levels in the same sector. Still, the very limited impact of this sector on the overall European patterns of employment growth illustrates the limited relevance of this development for the division of labour at the European level.

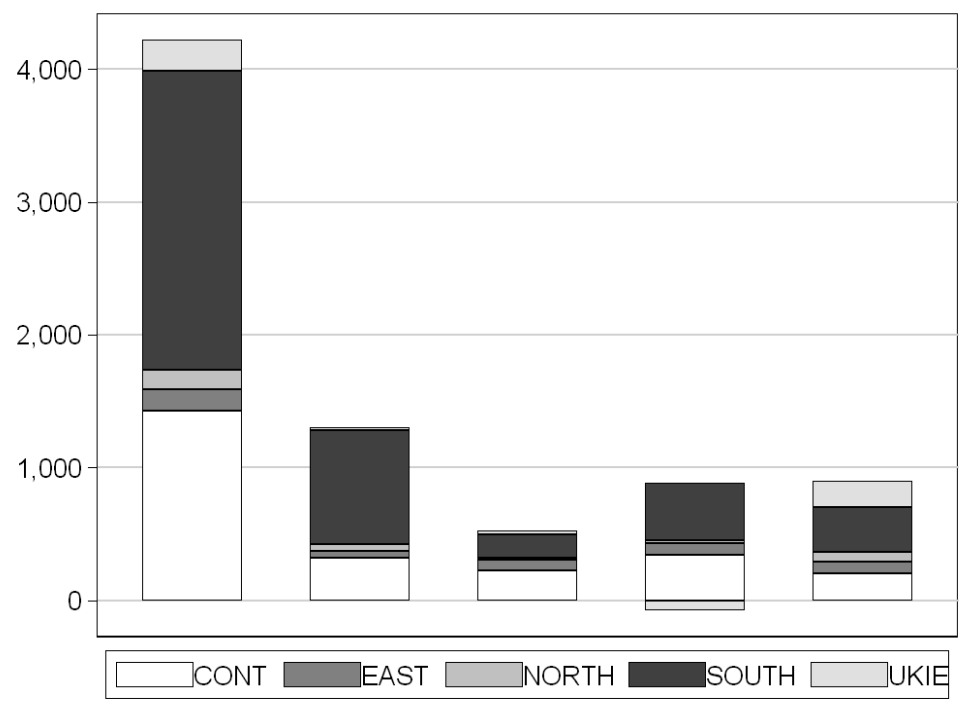

Figure 7.11: Contribution of LESS KNOWLEDGE INTENSIVE SERV. to quintile growth by region

5. (Private) Less Knowledge Intensive Services (LKIS, fig. 7.11): as we already mentioned in previous pages, the potential of services in general for integration within an European division of labour are, a priori, rather limited. This is especially true in the case of LKIS, which, we must recall, includes personal and distributive services: services that in most cases require a strict simultaneousness 
between production and consumption and the direct interaction between the producer and the consumer. It is nearly impossible to produce this kind of services in a different country from where it is consumed: therefore, except in areas which are very close to the border, there is little room for integration. Still, regional specialization and integration is not the only possible reason for the existence of different economic and employment structures: the general degree of economic development tends to be associated with different employment structures, as we saw in chapter 4, and there may be other cultural or even climatological reasons behind such differences. Southern Europe has the largest shares of employment in LKIS of Europe, and this peculiarity seems to intensify over time, as the expansion of this sector in Southern Europe was larger than could be expected according to the overall contribution of this region to the expansion of EU employment; and as we can see in table 7.IV, such contribution was more downwards biased than in any other region. Besides this Southern European peculiarity, there is not much more to highlight in the results for LKIS: this sector grew in absolute terms in all regions, and even if it was reasonable to expect some type of Europeanization of this sector (which it is not), our results would not support such hypothesis (there is no sign of employment transfers, and the differences in the patterns of growth are not large enough as to suggest specialization due to economic integration).

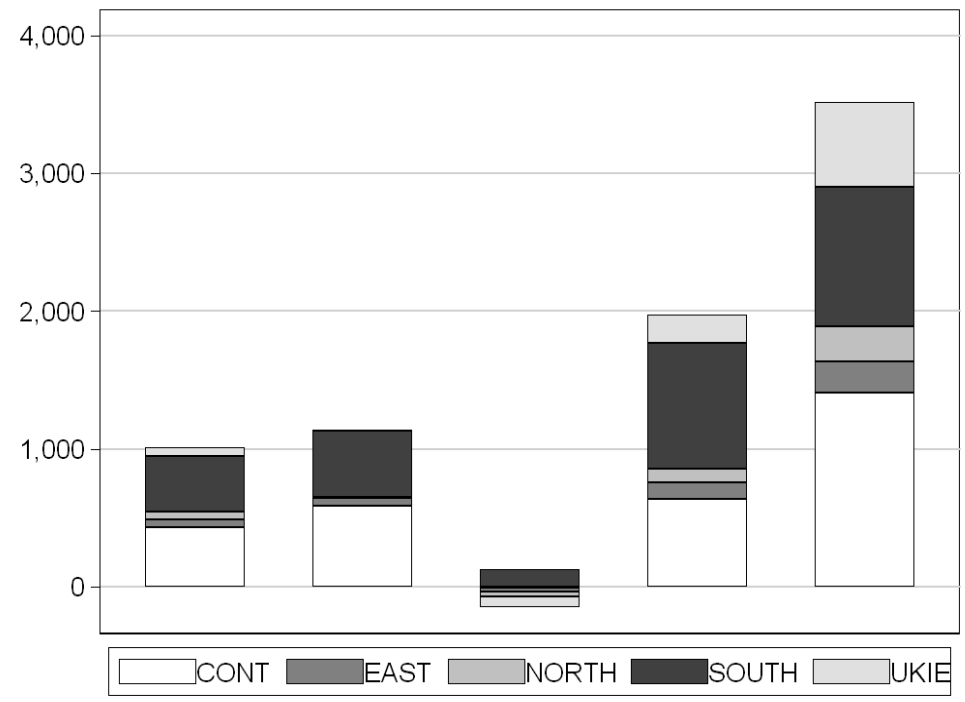

Figure 7.12: Contribution of KNOWLEDGE INTENSIVE SERVICES to quintile growth by region 
6. (Private) Knowledge Intensive Services (KIS, fig. 7.12): although this is also a category of services, and therefore in principle the potential for trade and hence a transnational integration is smaller than for manufacturing or agriculture, there have been recent technological developments which are rapidly changing this situation. In many activities that fall within this category, such as communications and financial services, new information technologies are allowing a degree of internationalization that can match or even surpass that of the most traditional activities producing material goods. The hypothesis of an Europeanization of this sector, with some regions specializing and absorbing European production and employment, is therefore a much more reasonable one. Do our results suggest any type of EU integration or specialization in this sector? Even more than in the case of LKIS, there was a generalized expansion of Knowledge Intensive Services across all Europe, and this expansion was strongly concentrated on the higher layers of employment everywhere, as we have repeatedly argued in previous pages. Yet, if we look at the deviations of the different regions from the overall patterns in table 7.IV, we can see some interesting results. The contribution from the South to the expansion of this particular sector was smaller than expected (that is, lower than its contribution to overall employment growth), and it was less concentrated on the top of the pay structure than elsewhere. The Isles, at a smaller scale, had the opposite type of contribution: much more upwards biased than any other region, and bigger than what would be expected according to the overall contribution of this region to EU employment growth. The other noteworthy result of table 7.IV regarding KIS is that Continental Europe had a larger than expected contribution to the two lowest quintiles (especially the second lowest), which shows that the polarization tendency of Continental Europe affects even this strongly upgrading sector. All in all, it seems that the expansion of this sector was too generalized across Europe, and too similar in its upgrading pattern, to sustain a hypothesis of a significant integration/regional specialization. Only in the case of the Isles (UK and Ireland), the very disproportionate contribution of this region to the European expansion of high paid Knowledge Intensive Services does suggest that this region has managed to specialize on this sector, absorbing a significant amount of KIS activity and employment at the European level (this is not surprising: the UK and Ireland have been very successful as centres of 
international financial services in the last two decades - though maybe not anymore -). Such development is surely important for this region: the progressive specialization of the Isles on these kind of activities at the European level is probably linked to the large expansion of high paid jobs in these two English-speaking countries between 1995 and 2007. But for the overall European patterns of job growth, this development has very limited impact, being diluted in much larger developments.

This last point could be a good summary for the whole section. We have looked for evidence of an Europeanization of the division of labour, or more precisely, of the kind of effects that such a development would have had for the structure of job quality. It has to be acknowledged that our approach is not really the most suited for answering such a question, as our data can only provide indirect evidence of an integration of employment structures (direct evidence would require studying directly trade flows in the different sectors). Still, if there would be such an Europeanization we should be able to see some of its effects with our job-based approach: we should see something like simultaneous positive and negative developments in different regions and job quality tiers (within the same sector). We could only see clear evidence of this type in the manufacturing sector, especially in High Tech Industries. Our results suggest that the South and East of Europe tended to concentrate the expansion of production activities in High Tech Industries, whereas the Isles and North moved away from this sector and in Continental Europe there were many new high paid jobs, probably in managerial, professional and technical activities, in this sector. This did have some impact on the job quality structures of the different countries, contributing to the expansion of good paid jobs in the Continent, the destruction of low and mid paid jobs in the Isles and North, and the creation of mid paid jobs in the South and East. But the relevance of these developments, when put in the context of the overall patterns of transformation of European employment structures, is very limited. Most of what happened between 1998 and 2007 to the European employment structure was the result of dynamics which were very similar across all European regions (such as the expansion of high paid jobs because of the move towards knowledge intensive services, or the contraction of mid and low paid jobs because of the collapse of the primary and secondary sectors), and therefore unrelated to the hypothetical formation of a European division of labour. 


\section{Conclusions}

The interest of this chapter derives mainly from the level at which the data was analysed. We have not included any new data or utilized any new analytical technique: we have just shifted our perspective by aggregating the data of the 23 countries into a single pool, representing a hypothetical (not to say chimerical) European labour market, which we have then studied with the same techniques used for individual countries in previous chapters (charting the patterns of employment expansion by job quality, and decomposing such patterns by sector and region). Our goal was to try to answer several questions that could only be answered by doing the analysis at this European level. We have provided several answers, some of them more conclusive than others: we will conclude by summarizing these answers one by one.

What kinds of jobs has the EU as a whole created in the last job expansion? We saw in the first section that between 1998 and 2007, the EU employment structure was both upgraded and polarized in terms of job quality. The expansion was largest in the top two job quality quintiles, so that there was an indisputable upgrading in the overall employment structure. The jobs that occupy mid-low positions in terms of median wages grew the least (quintiles 4 and 5 expanded three times more than quintiles 2 and 3 ). The lowest quintile, on the other hand, expanded considerably more than quintiles 2 and 3 (by almost 4 million in net terms, compared with nearly 2 million in each of the two subsequent quintiles), although much less than the jobs at the top two quintiles. Overall, the upgrading was more intense than the polarization, but both developments were significant.

How did the EU pattern of employment growth by job quality quintiles compare with the US pattern for a similar period? With all the precautions required in a comparison between what is really an integrated labour market and an artificial aggregation of 23 separate labour markets, we did this comparison in the second section of this chapter. The most remarkable outcome of such comparison is the similarity between the patterns of job growth of the two unions of states at both sides of the Atlantic: in both cases, the pattern of transformation was one of asymmetric polarization (or polarized upgrading), although the degree of upgrading was considerably bigger in Europe and the degree of polarization much more marked in the US. The similarity of patterns suggests a similarity of underlying dynamics which is confirmed by a comparison of our findings 
in this chapter and the findings of the American study which inspired this whole thesis. The expansion of high paid jobs was in both cases linked to an expansion of knowledge intensive services, the expansion of low paid jobs to personal and distributive services, and the gap in the middle to the secular decline of manufacturing employment (although there were differences too: in the American case High Tech Industries seemed to play a more significant role than in Europe, and in the US the expansion of the public sector was absent, at least from the analysis of Wright and Dwyer).

What was the contribution of each European region (or institutional country clusters) to the overall patterns of job growth? In answering this question, we simultaneously discussed the more general problem of whether the institutional country clusters often used for the analysis of European social and economic issues are relevant for studying the patterns of job growth. Our answer was, in broad terms, negative. In most cases, we found more variability within each cluster than across them, a finding that challenges the use of clusters for the analysis of the patterns of job growth for job quality (suggesting that these patterns are not linked to the big institutional families of Europe). There was one exception: just one, but very significant. The polarization tendency seemed to be rather clearly linked to Continental European countries (otherwise known as Conservative Welfare Regimes), with the single exceptions of Luxemburg and Austria.

And finally, is there any evidence of a geographic division of labour within Europe, and if there is, does it have implications for job quality? Even before carrying out any analysis, we could provide a partial negative answer to such question, simply by taking into account the different nature of the labour process of the different sectors: services lend themselves much less easily to international trade than manufacturing or agriculture, and the transformation of the European employment structure was mostly the result of developments within services. Anyway, we decomposed the contribution of each sector to the overall expansion of the five job quality tiers into the specific contribution of each region: any evidence of shifts in employment between regions, or at least of significantly different patterns of growth for the same sector would be taken as possible evidence of integration within an EU division of labour. Only in the manufacturing sector, especially in High Tech Industries, did we find some evidence of that kind (with the South and East absorbing most growth of middling jobs in High Tech Industries, and Continental Europe expanding its share of high-tech high-paid 
jobs). Other diverging developments did not seem plausibly linked to any ongoing integration within an European division of labour (for instance, the diverging developments in construction are more likely to be linked to the interaction between the EU monetary policy and local conditions; or in the primary sector, to the late industrialization of the South and East of Europe). And in most cases, the regional dynamics behind the sector contributions to the EU patterns of job growth were so similar that they simply suggest shared regional developments rather than any regional specialization within a hypothetical European division of labour. 


\section{Conclusions}

Each of the individual chapters of this thesis has its own concluding section with a detailed summary of the main results. In this final chapter of overall conclusions we will not repeat once again such a detailed summary of results, because it would be both tedious and unnecessary. Instead, we will concentrate on what we think are the key findings and on their implications with respect to the Social Sciences debate on the changing division of labour and job quality. For a detailed summary of the findings for each chapter, the interested reader is referred to the individual chapter conclusions.

\section{The debate on the effects of the changing division of labour on job quality}

In this thesis, I have tried to evaluate the nature of the recent evolution of European employment structures, from the perspective of job quality. Ultimately, it has been an attempt to contribute to the debate on the effects that technical and organizational change has on the conditions of work, a debate that goes back to the origins of contemporary political economy, and which remains at the core of the fields of sociology of work and labour economics.

Since the earliest contemporary analysis of the nature of work ${ }^{92}$ (with Adam Smith) until three or four decades ago, this debate was dominated by a profound pessimism which derived from the recognition of the contradictory impact of technical and organizational progress on the human condition. On the one hand, such technical and organizational change (for Adam Smith, the division of labour) was the ultimate source of economic growth, and hence of the massive expansion of the human capacity for

\footnotetext{
92 To avoid loading the conclusions with references, whenever our arguments are only summarizing the discussion of previous chapters we will not include any reference. When the arguments are new or based on literature which has not been discussed earlier, we will include the new references explicitly.
} 
survival and well-being that the Industrial Revolution was bringing about. On the other hand, these advances were produced at the expense of a continuous degradation of the condition of work: the proud independent producer of medieval guilds was transformed into a kind of brainless mechanical beast, which only provided his raw energy to production without even understanding the production process to which they contributed. Most classical economists and sociologists, including Adam Smith and Karl Marx, agreed that a continuous degradation of work was an inevitable consequence of technical and organizational progress, although of course they proposed radically different responses (a revolution that would abolish the division of labour in the case of Marx, a lukewarm programme of public education for industrial workers in the case of Adam Smith).

Such pessimism is slowly dispelled while, after the $2^{\text {nd }}$ World War, most advanced Western economies initiate a series of institutional reforms that transform them into more egalitarian and inclusive social systems. Despite a final wave of labour unrest in the 1960s and 1970s (which coincides with the publication of the swansong of the pessimist view described earlier, "Labour and Monopoly Capital" by Harry Braverman), it becomes more and more obvious that the labour landscape has changed radically since the $2^{\text {nd }}$ World War. It did not fit anymore, not even metaphorically, with the striking descriptions made by Engels of the conditions of the working class in England in the $19^{\text {th }}$ Century. Slowly, a new vision starts to dominate the debate on the impact of technical and organizational change on labour conditions, a vision that is much more optimistic than the previous one and which is linked to the debate on the coming of the Post-Industrial Society. It is precisely the enormous leap in productive human capacity derived from industrialization which, according to these new theories, has been slowly improving the conditions of work of the vast majority of the population, through two different ways. First, by reducing dramatically the amount of work necessary to produce the material goods required to support the population, which implies a gradual increase in the share of workers dedicated to the provision of immaterial services, leisure and well-being. Second, by the substitution of the most arduous industrial tasks by machines, and the transformation of the unskilled mass production worker into highqualified engineering workers whose main task is the design and supervision of machines. In both cases (increasing predominance of services, substitution of the mass industrial workers by smaller numbers of high-skilled engineers), the conditions of work 
are improved substantially, not only in extrinsic or compensatory aspects (such as wages, holidays or employment conditions), but also in the most intrinsic or anthropological aspects of work (autonomy, sociability, complexity). The profound pessimism of the classics is therefore abandoned and substituted by the somewhat messianic and naïve optimism of the theorists of Post-Industrialism (as illustrated by the titles of the main texts of this tradition: "The Great Hope of the $20^{\text {th }}$ Century", "The Coming of the Post-Industrial Society", etc.).

But the pendulum swings again when, around the mid-seventies, most advanced countries enter a crisis which affects first the economy and then the identity of the state itself. The political consensus that had dominated the previous three decades and built the Welfare State started to deteriorate, slow but steadily, until many of the previous social and labour policies are rolled back (in the eighties in the UK and the US, in the nineties elsewhere). Social inequalities start growing again, and many argue that the conditions of work and employment deteriorate over this period. Against this background, a more pessimistic view about the implications of technical and organizational change returns to the forefront of social sciences. It is, nevertheless, a more nuanced view, which in some ways can be understood as a synthesis between the pessimistic and optimistic views. According to this new synthesis, the structural evolution of the division of labour in advanced capitalist societies since the seventies is marked by polarization. The literature on this issue tends to focus on two explanatory factors, technology and international trade. On the one hand, the nature of technical change since the informational revolution tends to substitute workers that traditionally occupied intermediate positions (routine production and administrative tasks) while boosting the demand for labour at the two extremes of the skill-job quality hierarchy (knowledge intensive tasks and manual non-routine tasks). On the other hand, it is argued that the globalization of markets has also a destructive impact on the middle of the employment structure, especially on unskilled and semiskilled industrial jobs that can be equally performed in developing economies at a fraction of the cost. Some of these theories recognize the possibility that institutional and political factors (such as the decline of trade unionism or the shift towards neoliberal policies) may also have an important role in this polarization trend.

This thesis is an attempt to test empirically the main arguments of these three visions about the impact of technical and organizational change on job quality. For this purpose, 
we have applied a method which is still relatively new, which we called the "jobs" approach, to a sample of 23 European countries over a period of 13 years. It should not be a surprise if we say that our test is only partial and indirect, considering the large scale of the phenomenon we are studying here: but I hope that this dissertation has contributed to shedding some light on the shadows that still surround this subject, despite more than 200 years of debate.

In the final section of the theoretical chapter, we reformulated these three visions into five hypotheses, constructed as sets of propositions that could be empirically tested with our approach. These five hypotheses have guided all the empirical analysis of this thesis, and in the following pages we will use them here to summarize our main conclusions and to link them to the Social Sciences literature on this issue. But first, it is important to summarize the strategy followed for the empirical testing of these three dominating visions about the change in employment structures, demarcating scope and limitations of our analysis.

\section{Analytical strategy}

In this dissertation, we used an existing methodology which has been previously applied, with similar objectives, to study patterns of job growth in individual countries. We called this methodology the "jobs approach", and it basically consists in the following ideas: 1) using jobs rather than individuals as unit of analysis, defining jobs as specific occupations within specific sectors; 2) using a relative measure of job quality to group the jobs into ranked job quality tiers; 3) studying the change in the number of workers within each of those tiers over time.

In previous studies, the theoretical foundations of this approach were not fully developed, partly because in most cases the main concern of these studies was strictly empirical: to evaluate the nature of job creation over specific job expansions. In chapter 2, we tried to contribute to the development of these theoretical foundations by anchoring the jobs approach in the Marxian theory of the two-dimensional nature of the division of labour. Against Adam Smith (and we can add, against neoclassical economics, that still assume that all employment transactions are similarly governed by market mechanisms), Marx argued that the principles behind the division of labour within and across companies were fundamentally different: whereas within companies 
the coordinating principle is power and hierarchy, across companies it is the market which coordinates the allocation of work to the different units or tasks. A very similar idea lies behind the Coasean theory of the firm, which argues that the costs of using the price mechanism to organize labour within firms would be too high, hence the existence of a different mechanism for coordinating internal transactions, which is hierarchy and power. Our argument was that, because these two dimensions of the division of labour are behind the classifications of sector and occupation used to construct the jobs matrix, this matrix can be understood as a stylized structural representation of the division of labour in any society and point in time.

This implies a first delimitation of our analytic strategy: our evaluation of the three visions about the impact of the changing division of labour on job quality is limited to the analysis of quantitative changes in the employment structure (even if evaluated from a job quality perspective, as we will see later). In other words, our attention in this dissertation has been totally focused on studying the change in the quantities of labour which are allocated to the different tasks according to the vertical and horizontal classifications of the division of labour measured by the variables of occupation and sector. Because we are only looking at the change in the number of people within each cell in the jobs matrix (that is, within each specific combination of occupation and sector), we leave out everything that happens within those cells (for example, changes in the nature of work within each job) or outside the classification systems used (for instance, any changes which imply the creation of new types of economic activity). Of course, we do not leave those developments out of our analysis because we believe they are not important, but simply because our concern here is on the implications of structural change in labour markets.

But our objective is not to study structural change in the division of labour for its own sake, but from the perspective of job quality. Thus, to the quantitative jobs matrix (the matrix that holds employment levels by job, country and year), we added a qualitative element: the job quality rankings. These rankings are simply job quality indices measured at the job level, used to position all jobs within an economy in a job quality continuum. In previous studies using this same approach, the job quality rankings have been only based on the median hourly wages for each job: in this dissertation, we used the same type of measure for our primary job quality ranking, but to complement and enrich the analysis we added two further measures, one based on the average 
educational level of job-holders, and the other on the non-pecuniary attributes of each job.

This qualitative element and the way it was constructed implies a second important delimitation of our analysis: our vision of job quality was only partial, relative and static. Partial because we had to cope with the existing data sources at the European level, which simply do not allow for the construction of a single comprehensive job quality measure: hence the value of adding two more job quality indices, to reinforce our analysis. Relative because we only used these three measures as a way to rank the jobs according to their quality, from low to high: we believe that such approach is more adequate for comparing employment conditions across 23 very different economies (not to say that the use of a non-parametric, ordinal approach to job quality makes the analysis more robust to the data problems mentioned earlier). Finally, our vision of job quality was static because the rankings were unchanged for the whole period studied: although the relative structure of job quality may change slightly over a period of 13 years, using a fixed ranking allows to focus our attention on structural change, which was our real objective in this dissertation as explained earlier.

The quantitative and qualitative jobs matrices generated in the two earlier steps were finally merged into a single one (linked at the job level). The resulting information was then aggregated into quintiles, five groups of equal size in the year 2000 (in each country) and ranked from low to high job quality: the examination of the evolution in the number of workers across the quintiles over time is the basis of our empirical test of the three visions of change in the division of labour described earlier. Each of the three visions predicts a particular pattern of job growth, an image of change (to continue with the visual metaphor) which we contrasted with the empirical images of change given by the quintile change over time within each country.

This brings us to a third important delimitation: our test of the hypotheses of change in employment structures is superficial, in the sense that it does not really evaluate the causal mechanisms proposed by each hypothesis, but only whether their predicted effects on the structure of job quality conform to the empirical patterns of the 23 European countries of our sample. Still at this surface level, we took advantage of the possibility of decomposing the overall patterns by different levels of occupation, sector and employment status in order to refine our evaluation of the predictive capacity of the different hypotheses (because those hypotheses do not only predict the broad direction 
of change in employment structures, but also the occupation and sector dynamics behind).

A final important delimitation of our analysis concerns the time period covered. If in terms of geographic coverage this dissertation provides an important expansion of the scope of previous similar studies (which in most cases concerned just a single country), in terms of time scale this dissertation is somewhat limited by covering only 13 years, a period which can be considered as short-term for an evaluation of such a long-term phenomenon as the nature of structural change in the division of labour. This limitation was, in fact, imposed by the comparative nature of the exercise: at present, it is simply impossible to stretch the analysis any longer for an international comparison covering more than two or three countries. In some ways this limitation is more severe than the previous ones, because it is not the result of a conscious decision in order to focus our analysis, but of data limitations (without any doubt, it would have been better to cover a longer time span). Furthermore, it is difficult to assess or control its implications for our results (it is impossible to differentiate short-term from long-term changes). The only way to deal with this problem is to try and take it into account into the analysis as much as possible, which is what we have done. In the last section of these conclusions, we will come back to this issue.

Once the scope of our analysis has been delimited, we will discuss the empirical findings of this dissertation within the context of the main hypotheses derived from the social sciences literature on the implications of the changing division of labour on job quality.

\section{The hypothesis of the degradation of work}

In the final section of chapter 2, we summarized this hypothesis for our purposes as follows:

(Degradation of work hypothesis) The patterns of change in the jobs structure will be downgrading, either totally downgrading or polarizing with a very strong downgrading bias. This downgrading should be taking place across most sectors, in similar ways. By occupations, we should observe a strong destruction

of skilled and semi-skilled occupations, a simultaneous strong creation of 
unskilled occupations and some creation of technical and managerial jobs at the top of the wage structure.

In this case, our answer is categorical: according to our approach, for the period and countries analyzed, there has been no degradation of the jobs structure (in other words, there was no downwards-biased expansion of employment, with only two partial exceptions: Estonia and Slovakia). This finding, in fact, is not very surprising at all: most of the literature on this issue from the 1970s coincides that there is no empirical support for this hypothesis.

Nevertheless, we have to concede that the scope of our empirical approach (as delimited in the previous section) leaves out some of the most important elements of this hypothesis, as it was developed for instance by Harry Braverman in "Labour and Monopoly Capital”. It could be argued that the postulated process of degradation mainly concerns changes that take place within jobs (affecting their task content in ways that deskill and disempower workers), rather than changes in the composition of the labour market, which is our focus here. Insofar as the degradation of work arguments refer to changes which do not affect the structure of employment by sector and occupation, those arguments could be totally correct despite the results presented here. For instance, the countries studied in this thesis could have suffered between 1995 and 2007 a process of generalized deterioration of job quality, or even a deterioration that only affected the bottom of the employment structure, and our results would be totally unaffected (because we are blind to changes that take place within jobs, or changes across jobs that do not affect the relative position of each job against the rest). ${ }^{93}$

That said, it is also true that both Braverman and the classics did argue that technical and organizational change leads to a downgrading transformation of employment structures, in very similar terms as those used in this dissertation. Braverman argued that, even if technological change does reduce the overall number of industrial workers of all skill levels, the excess workers are absorbed into low-skilled, low-paid service occupations: "there has been therefore an immense shift of labour out of the traditional manufacturing, mining and construction, and transportation industries into the very

\footnotetext{
${ }^{93}$ In fact, all evidence suggests that there was not such a process of degradation of work in Europe even in this sense. In our previous work studying the evolution of working conditions in Europe between 1990 and 2005 (see European Foundation 2007; Parent-Thirion, Fernández-Macías, Hurley and Vermeylen 2008), we could not identify any generalized negative development except for the perception of work intensity, which seemed to increase more or less consistently over the years, and less clearly for the perception of autonomy at work.
} 
rapidly growing areas of real state, insurance, finance, services and wholesale and retail trade. But these rapidly growing fields of industry are precisely the low-wage portions of the economy, while the higher-wage sector is the stagnant or declining portion" (Braverman 1974: 323; see also chapter 17). We have seen in this dissertation that such arguments do not fit the empirical patterns of structural evolution of European labour markets: the overall sector dynamics that Braverman spoke about are broadly correct, but their implications for job quality are wrongly interpreted. The rapidly growing sectors were not, in most cases, the low-wage portions of the economy (nor the loweducated or the low-quality ones), but quite the opposite; and the stagnant or declining portion were not the higher-wage, but the middle and low paid segments of the labour market in most countries. So the empirical pattern of job growth is almost the complete opposite of what Braverman said.

\section{The upgrading hypotheses: the coming of the post-industrial society and skill- biased technological change}

There are two distinct theoretical traditions arguing for a generalized upgrading of the jobs structure as a result of technical and organizational change: the theories of the postindustrial society and the theories of skill-biased technological change (SBTC). The implications of these two theories for structural change as understood in this thesis are quite similar (although the post-industrial arguments focus more on sector dynamics, and the SBTC on shifts across occupations), but they differ considerably in tone, as we will see. The condensed arguments of these two theories for our purposes were summarized as follows:

(Post-industrialist hypothesis) The patterns of change in the jobs structure will be unambiguously upgrading. By sector, we should see a decrease in traditional industrial (and goods-producing) jobs of a low or mid-low quality, simultaneous with an increase in middling to high quality jobs in services and a strong increase in knowledge-intensive services of high quality. By occupations, we should see a decrease in unskilled and semi-skilled occupations, and a rapid growth in managerial, technical and professional occupations.

(SBTC hypothesis) The patterns of change in the jobs structure will be unambiguously upgrading. Across most sectors, we should see fast growth of 
highly-skilled (and highly paid) jobs, and stagnation or even destruction of middling and low-skilled jobs.

Of the three broad images of change that dominate the theoretical debate, this is the one for which we found a most universal empirical support, without any doubt. Practically all the European countries which we studied in this thesis, for the three indicators of job quality, experienced some type of upgrading in their employment structures: the relative growth of good jobs (those in the upper quintiles of the distribution) was in most cases higher than the relative growth of bad and middling jobs (which reduced their employment share in relative and in some cases even in absolute terms over the period). Even in the few countries which experienced a process of polarization in their employment structures, this polarization tended to be upwards-biased: that is, the expansion of good jobs was much larger than that of bad jobs, even if the bad jobs expanded more than middling jobs (the only exceptions being Netherlands and Slovakia, and only for the wage-based quintiles).

The sector and occupation dynamics that were behind this more or less generalized upgrading also coincide to a great extent with the arguments of post-industrial and SBTC theories. The sectors that pulled the expansion of good jobs were private knowledge-intensive services (a category which includes financial services, communications and research and development, among others), and health (where employment is primarily public in most European countries). On the other hand, the primary and secondary sectors pulled down jobs of middle and low quality. It is interesting to note that a similar development, according to the Wright and Dwyer 2003 paper that inspired this dissertation, is taking place at the other side of the Atlantic.

Nonetheless, there were some countries whose pattern of job expansion between 1995 and 2007 did not fit exactly the image of unambiguous upgrading predicted by the theories of post-industrialism and SBTC (there were some clear cases of polarization, and even some cases of centripetal job growth, with a relative expansion of middling jobs). Because of this reason, and also because the actual extent and type of upgrading varied considerably between countries, we argued that none of the three broad theories of change provides a universal answer to our problem: on the contrary, there seems to be some degree of indetermination in the patterns of job expansion, which has important implications that will be discussed later. 
But if we take the upgrading hypothesis in broad terms, as an upwards shift of the center of gravity of the employment structure, it is undeniable that most countries experienced such a process to some extent, an that the underlying sector and occupation dynamics also fit in broad terms the upgrading hypotheses. In this sense, the broad arguments of postindustrialist theories and SBTC are vindicated by our results: the idea that technical and organizational change boosts productivity and generates a surplus that contributes to the expansion of leisure and welfare activities, whose conditions of work tend to be above average; as well as the idea that technical change substitutes specifically for the low-mid segments of industrial employment, increasing the demand of skilled work in particular, seem like plausible explanations for the observed similarity in the European patterns of job growth.

We have to be careful with the interpretation of the wider implications of these results. In this sense, we feel much closer to the implicit somber interpretation of the theory of SBTC than with the somewhat naïve enthusiasm of some post-industrial theories. The theories of SBTC were originally an attempt to explain a not very pleasant development in the distribution of income in advanced capitalist economies since the 1970s: an unprecedented expansion, especially in Anglo-Saxon economies, of income inequalities. The core idea of SBTC is that technical change boosts the demand of skilled labour and depresses the demand of unskilled labour, which implies an upgrading transformation of employment structures as discussed earlier, but also a substantial expansion of wage inequality and/or unemployment. In a relatively free market economy, the bias in the relative demand for skilled vs. unskilled jobs would mean that the wages of the earlier are pushed up and the wages of the later are pushed down; in a regulated economy with minimum wages and strict employment protection, it can lead to an increase of unemployment (specifically for low-skilled workers; see Krugman 1994). Hence the paradox that the more or less generalized upgrading of the employment structure discussed earlier can be associated with a tendency towards higher inequality and social polarization, rather than with a generalized process of social progress and human flourishing as argued by post-industrialists. We would not like to push this argument too far, because we believe that social and employment policy can significantly change the wider implications of the upgrading pattern shown here. In other words, we disagree with the idea that technological change has the inevitable outcome of increasing inequality or increasing unemployment: good policies and regulations can help 
redistributing the benefits of technological change and neutralizing its negative social consequences (see Atkinson 2000). But what we would like to emphasize here is that the wider implications of the patterns of change we are discussing here are not straightforward: upgrading in the employment structure does not necessarily mean social progress, in fact it is more likely to be associated with social polarization.

\section{The polarization hypotheses: trade and technological explanations}

The polarization hypothesis has two main versions, one which focuses on trade and institutions and the other one on technological change. Although both versions predict a similarly polarized pattern of job growth, the sector and occupation dynamics postulated by each of them are rather different, so we will discuss them separately.

(Trade/institutional hypothesis) The patterns of change in the division of labour of advanced capitalist economies will tend towards polarization, although the institutional framework of each country can alter substantially the implications that this underlying trend will have for job quality. The patterns of transformation of the jobs structure should have a strong sector bias, because of the different openness to trade in the different industries.

This version of the polarization hypothesis is the earliest one, going back to the American debate on the "declining middle" of the 1980s. Although these theories also referred to technological change, it was mainly mentioned as an enabling factor for the key explanatory variable, which was the (increasing) degree of international trade. According to these theories, international trade has two types of impact on the employment structures of advanced capitalist economies, one which is direct and one which is indirect. The direct impact refers to the nature of trade-induced shifts in employment across regional blocs: the activities which are easily traded and whose labour costs are higher in developed economies tend to be shifted to the developing world. And those activities were precisely the basis of the middle of the advanced economies' employment structures: skilled and semi-skilled industrial jobs. Hence, the increase in international trade tends to empty the middle of the distribution. But the growing internationalization has also a second, indirect impact on the employment structures of advanced capitalist economies. The possibility of fleeing, or the threat of disinvestment, alters the balance of power between capital and labour that had existed 
since the $2^{\text {nd }}$ World War, giving capital the upper hand vis-à-vis workers and the state. The resulting rollback of some key elements of Post-War labour institutions (such as the role of trade unions, minimum wages or employment protection legislation) also has polarizing effects on the structure of the employment.

Do our results fit these arguments? Only to a very limited extent: the predicted polarization pattern was only visible in a handful of countries, and the postulated underlying dynamics were only partially confirmed even in those few countries. On the one hand, it is indisputable that the most internationalized sectors (especially, manufacturing industries) were stagnant or recessive in the most advanced European economies between 1995 and 2007. It is also true that in the particular case of High Technology Industries, we did find some indirect evidence that pointed towards the creation of a proto-European division of labour, with Central-Western Europe moving away from production but keeping the high-value added activities (a move up in the value chain which contributed to the upgrading of their employment structure), and Central-Eastern Europe absorbing labour-intensive production activities (contributing to the expansion of middle-paid jobs). But on the other hand, the significance of these developments is minor compared to developments that took place in the much larger service sector, less affected by international trade.

The main advantage of these theories, compared to the other theories discussed here, is that they explicitly consider the institutional framework as a mediating factor between the underlying mechanisms leading change in the division of labour (technical and organizational change) and job quality. This makes the predictions of this version of the polarization hypothesis less deterministic or universalistic, which fits much better the fundamental variability in the patterns of job growth that we found across our sample of 23 European countries. Although in fact, the mediating institutional factor proposed by this theory is not the most important one, according to our results. When Kuttner argued that institutional differences could lead to different outcomes of the same processes of technological change and internationalization, he mainly spoke about differences in wage structures. But in fact, we argued in chapter 5 that the differences found in the patterns of job growth across Europe are not mainly the result of the existence of different wage hierarchies, but the result of different types of structural change. Different European countries, in particular, seemed to differ in the (relative) quantities of low and mid paid jobs that they generated between 1995 and 2007. So we can infer 
that institutions do not matter only in terms of generating different wage hierarchies, but also in terms of generating different pathways of structural evolution in the division of labour. We will discuss this issue in more detail in the following section.

The second version of the polarization hypothesis is totally focused on technological factors:

(Task-Biased Technical Change, TBTC, hypothesis) The patterns of change in the jobs structure will tend towards outright polarization across most developed economies (as all are experiencing the same type of technological change). There should be some sector bias (the different types of tasks are differently distributed by sector), but the main driver of change should be occupational shifts in employment (the routine and cognitive content of tasks is mostly correlated with the vertical division of labour).

First of all, we have to say that the point made earlier for the trade version of the polarization hypothesis also applies here: only a handful of countries conform to the predicted pattern of structural change. That said, this version provides a more complete and plausible explanation for the polarization cases than the previous one. The main problem of the trade hypothesis is that it mostly applies to sectors which in practice account only for a small and shrinking proportion of employment, leaving out most of the service sector which is in fact the one driving the overall polarization pattern (in the cases where such pattern applied). In other words, outside the primary and secondary sectors there is even more polarization, and the trade hypothesis simply does not provide a clear answer for why that should be the case.

The TBTC theory classifies labour tasks in four big groups, according to whether they are routine or non-routine, and intellectual or manual. It argues that, in a similar way as mechanical technologies have been replacing routine manual workers since the Industrial Revolution, the new informational technologies allow the substitution of routine intellectual workers (clerks) for computers (as well as an even more efficient substitution of manual routine workers by programmable industrial machines). Routine manual and intellectual tasks (skilled and semi-skilled industrial workers, and clerks) constituted the bulk of the middle segments of employment until the 1970s: the technological revolution that took place in that decade and which found increasing application to production in the following two decades led to the stagnation and decadence of these types of employment, hence the gap in the middle. This explanation 
of the polarization patterns fits better our results than the trade-based one, because it not only predicts the collapse of middling industrial jobs, but also of service employment with middling salaries and working conditions which are not exposed to the effect of international trade. Our results show that the former (the centrifugal evolution of employment in services) was as important for the development of polarization as the latter. Also, as this theory predicts, the bottom of the employment structure, in those countries where there is polarization, grows mostly because of the expansion of personal and distributional services (that is, non-routine manual jobs). The top of the distribution also changes more or less as this theory predicts, but in this case the TBTC arguments do not differ from the previously discussed arguments of SBTC from which it derives.

But we are continuously emphasizing the fact that this theory provides a plausible explanation for the polarization pattern in those cases in which there is such a pattern. This is very important because it constitutes an obvious contradiction of the presumption that all countries are experiencing the same type of evolution because of the impact of the same type of underlying cause (task-biased technological change in this case). We have to say that in this, this theory does not differ too much from the other ones discussed here (except the trade/institutionalist arguments): there is a somewhat universalist, almost deterministic tone in their understanding of the evolution of the division of labour and its implications for job quality. In some empirical studies from the TBTC perspective (see Goos, Manning and Salomons 2009), this has lead to the claim that all European countries (except Portugal), as well as the US, have experienced the same polarization pattern of job growth during the nineties. In chapter 5 , we discussed with some detail why we believe the empirical support for such striking claims are very thin (they assume that the wage structures across all Europe are so similar that using the UK wage rankings for all countries does not make a difference, and they also define the job quality tiers in a surprisingly non-transparent way which amplifies the polarization effect); but even from a purely theoretical or aprioristic perspective, such claims seem quite implausible. It requires a truly platonic understanding of how employment structure changes over time to believe that a single pattern can characterize the evolution of employment structures of all European (15) economies (except Portugal) and the US. We know there are very important differences in the structure and development level of these economies, in their institutional 
frameworks, culture and values, even in the specific moment of the business cycle in which each economy was situated in the period 1995-2007. Even if we agree that all of these economies are experiencing more or less similar processes of technological change, how could it be that the change in the employment structures across all advanced economies (except Portugal) is only affected by that single universal factor? Even if such universal factor would generate similar tendencies in terms of the evolution of employment structures, would not these tendencies be distorted by the different economic structures, institutional frameworks, etc?

In chapter 5 of this dissertation, we presented copious evidence showing that the polarizing pattern did characterize the evolution of employment in some countries, but not in all of them. Because of the universal claims of the TBTC theory, this on its own implies a partial rejection of the TBTC hypothesis. We do not think that such hypothesis should be rejected completely, because it provides a plausible explanation of the patterns of job growth in those countries where polarization did happen: but it is obvious that this hypothesis should be reevaluated, because it cannot explain why in some cases there is polarization but not in others. We believe that the factors that are missing in this hypothesis and which makes it incomplete (as much as the other hypotheses) are related to institutions: the same institutions that systematically contradict most attempts to reduce processes of social change to a single universalist explanation, because they imply a fundamental indeterminacy, or non-inevitability, in any process of social change.

\section{Accounting for the plurality of patterns of employment growth}

In the previous pages, we have argued that although there are some broadly shared tendencies that fit better the upgrading hypothesis, there was an important plurality of patterns of job growth between 1995 and 2007 within our sample of 23 European countries, which none of the hypothesis can really explain. How do we interpret this outcome? Two main readings can be done. One would simply consider that the hypotheses were incorrectly specified: the plurality of patterns identified would be the result of a more complex set of factors, which could ultimately be reduced to a more complex, but still universal and deterministic explanatory model. Another reading would be that the identified plurality is the result of a fundamental indeterminacy of the processes of social change, indeterminacy which is itself the result of the capacity of 
human beings to direct - however successfully - its own destiny. The results of this thesis can not provide conclusive evidence for any of these two possible readings, but we believe that they do point in the second direction, because they suggest that behind the plurality of patterns there are institutional factors (which are, by definition, the result of conscious human action).

The first element that points to an institutional explanation is the fact that the polarizing countries experienced a simultaneous important process of destandardization of employment. The association between polarization and destandardization was not only one of simultaneity, but there was an obvious interaction: in the polarizing countries (which, we must remember, differed from the rest mainly because the bottom grew faster than the middle), the destandardization was strongly downwards-biased in terms of job quality (bad jobs became much more destandardized than good jobs, even if all the structure was affected to some extent). By definition, this process of destandardization has its origins in changes in labour regulation, and hence in mechanisms which are basically institutional. It must be noted that this argument is not necessarily contradictory with the TBTC explanation: it is perfectly reasonable to think that there may be some kind of underlying polarization tendency induced by technical factors as argued by TBTC, but which would not be able materialize into an expansion of low-paid jobs unless the institutional framework facilitates it (for instance, if there are minimum wages of a relatively high level, or high firing costs, low-paid jobs may not grow even if there is a "latent" demand of non-routine manual work).

The second element which points towards institutions is the geographic distribution of the different patterns of job growth across Europe. Although the usual institutional country clusters, in general terms, did not seem to be clearly associated with the variety of patterns of job growth, there was one significant exception which fulfilled the required internal homogeneity and external differentiation to be considered a meaningful cluster: the group of continental European countries (or conservative welfare model, or corporatist regimes). This group of countries included most of the polarization cases and only one country which did not fit the polarization pattern (Austria). ${ }^{94}$ The association between a country cluster, defined by its institutional

\footnotetext{
94 There was another exception which was Luxemburg, but this country is too atypical to be taken into account in this respect. As for the Austrian exception, there are some reasons to be suspicious about it, because there was a large reclassification of occupations that may have distorted the patterns of job
} 
similarities, and such a characteristic pattern of job growth clearly points to the importance of institutional factors.

It is, in fact, a surprising association, not exempt from irony, because it affects the countries that during the $90 \mathrm{~s}$ were subject to the fiercest criticism for the rigidity of their labour institutions, rigidity which (according to this criticism) was blocking the creation of low-paid jobs necessary to reduce unemployment to acceptable levels. As we know, these countries introduced important regulatory reforms, during the period studied here, in order to make their labour markets more flexible (see Zdrojewsky et al 2009; Ebbinghaus and Eichhorst 2006; or for a general review, Barbieri 2007): it seems impossible not to link such reforms with the simultaneous and intertwined processes of polarization and destandardization of employment discussed earlier. Whether these reforms were really successful (if the aim was expanding the low-paid segments of employment, they were) or necessary (what would have happened without these reforms?) is a different matter.

In any case, what we would like to emphasize, because we believe it is a useful contribution to the debate on the recent evolution of European employment structures, is that the plurality of patterns that we have found shows that there is nothing inevitable in this process. Universalist and deterministic explanations are not only likely to lead to defeatist and unassertive employment policies, but they actually do not fit the facts.

\section{The long view}

The period we have covered in this dissertation is very short, if we consider that our ultimate objective is to evaluate the implications of structural change in the division of labour. The problem of using a short period for studying a long-term issue is that, beyond our own common sense when interpreting the results, there is no way to differentiate between transitory and structural changes.

How can we know, then, whether our results can be extrapolated beyond the particular period that goes from 1995 to 2007? At most, we can do educated guesses. For instance, we know (now) that the large expansion of employment in construction that characterized several European countries between 1995 and 2007 (especially Spain and 
Ireland, but also most other Mediterranean and Eastern countries) was a fundamentally unsustainable development. After 2007, employment in this sector has collapsed dramatically in those same countries, and although they will eventually recover to some extent, it is very unlikely that they will ever go back to the high levels of 2007 . We can infer from this development (taking into account that construction jobs tend to be in the middle of the wage structure) that the pattern of job growth in some of these countries may become slightly more polarized (although it is difficult that it would alter radically the previous pattern, except maybe in the Spanish case). On the other hand, in Germany the construction cycle was completely deflationary between 1995 and 2007, which is not likely to continue either, and may tend to soften slightly the polarization trend of that country (although, again, it is unlikely that it would change significantly). The other sector whose growth was inflated because of the specific characteristics of the 19952007 business cycle is the finance sector, especially in the two English-speaking countries (UK and Ireland). This sector contributed importantly to the expansion of very well-paid jobs, especially in the two mentioned countries, from which we can infer that the tendency towards upgrading may be somewhat weakened.

But beyond these relatively small changes and some others that surely escape these sketchy considerations, it seems likely that the general trends observed for the 19952007 period will continue in relatively similar ways, for the same reasons that they have been going on until now. It seems likely that technical change and to a lesser extent international trade will keep on depressing, slowly but inexorably, the weight of the primary and secondary sectors in employment, which will keep on bringing down the lower and middle segments of employment. The services sector, and specifically services linked to knowledge, leisure and welfare provision are also likely to keep on expanding, making the share of relatively good jobs to grow as well. The expansion or not of low paid jobs, as we have also argued, is likely to depend on employment policies and regulations as much as on the impact of technical change (although eventually machines will be able to substitute for manual non-routine labour as well).

Anything can happen. But drawing from our results for the period 1995-2007, the most plausible prediction is that to a greater or lesser extent, the general upgrading trend will continue. However, as we said before, it would be totally wrong to interpret such tendency as an unambiguous proof of the inevitability of social progress. The existence of such a clear and more or less pervasive upgrading tendency can be very good news 
for the productive system and for highly qualified workers, but it is not necessarily good for low-skilled workers (who still exist) nor for those which are displaced by the march of technical and organizational progress ${ }^{95}$ (the workers affected by the net destruction of employment in the secularly receding sectors). For the latter, the upgrading trend can mean less employment opportunities and increasing competition, and consequently, decreasing wages, deteriorating working conditions and/or unemployment. In the context of the current recession, this possibility is becoming a very alarming reality. Since 2007, employment is being destroyed in many countries at much faster rates than it had grown in the 13 previous years. The return to the previous levels of employment will be slow, and the chances available for unskilled workers, assuming that the upgrading trend is likely to continue or even intensify, very bleak.

But as we argued in the previous point, there is nothing inevitable in social and economic change. The existence of a generalized pattern of upgrading in the employment structure shows that the potential of human society to provide for its needs keeps on expanding at a very fast pace. The benefits of such upgrading, which are the result of generations of human creativity and ingenuity applied to production, naturally belong to the whole of society, not only to those that have the chance to profit directly from them. There are always options for transformational public policy to make the implications of technical and organizational change truly beneficial for the whole society.

\footnotetext{
${ }^{95}$ A very similar point could be made with respect to long-term trends in productivity, which are in fact surely linked to the upgrading trends in the employment structure about which we are talking about.
} 


\section{BIBLIOGRAPHY}

-ACEMOGLU, D. (1999): "Changes in Unemployment and Wage Inequality: An Alternative Theory and Some Evidence", The American Economic Review, Vol. 89, No. 5 (Dec.), 1259-1278.

-ACEMOGLU, D. (2002): “Technical Change, Inequality, and the Labor Market”, Journal of Economic Literature, Vol. XL (March), 7-72.

-ALLEN, S.G. (1994): "Updated notes on the inter-industry wage structure", NBER working paper n. 4664.

-ARTS, W. and GELISSEN, J. (2002): "Three worlds of welfare capitalism or more? A state-of-the-art report", Journal of European Social Policy. 12(2), 137-158.

-ATKINSON, A.B. (2000): "The Changing Distribution of Income: Evidence and Explanations", in German Economic Review 1(1), 3-18.

-AUTOR, D.H., KATZ, L. and KEARNEY, M. (2006): "The polarization of the U.S. labour market", NBER Working Paper No. 11986.

-AUTOR, D.H., LEVY, F. and MURNANE, R.J. (2003): “The Skill Content of Recent Technological Change: An Empirical Exploration" in Quarterly Journal of Economics, 118(4), 1279-1334.

-BAUMOL, W.J. (1967): "Macroeconomics of unbalanced growth: The anatomy of urban crisis", in The American Economic Review, Vol. 57, No. 3, 415-426.

- BARBIERI, P. (2007): “Atypical employment and welfare regimes”, EqualSoc Policy Paper 1.

-BECK, U. (1992): Risk Society: Towards a New Modernity, Sage.

-BELL, D. (1976): The coming of post-industrial society: a venture in social forecasting, Harper.

-BLAUNER, R. (1964): Alienation and Freedom, Chicago University Press.

-BLUESTONE, B. and HARRISON, B. (1982): Deindustrialization of America, Basic Books.

-BRAVERMAN, H. (1998, originally 1974): Labor and Monopoly Capital, Monthly Review Press.

-CAHUC, P. and ZYLBERBERG, A. (2004): Labor Economics, MIT Press. 
-CASEY, B. (2004): “An Economy-Wide Perspective on Earnings Data in Ireland”, ESRI Quarterly Economic Perspective (Spring).

-CASTELLS, M. (2000): The Rise of the Network Society: Volume I: The Information Age, John Wiley and Sons.

-CHALMERS, D. and LODGE, M. (2003): "The Open Method of Co-ordination and the European Welfare State", ESRC Centre for Analysis of Risk and Regulation, Working Paper 11.

-CHARLIER, H. and FRANCO, A. (2001): "The EU Labour Force Survey on the way to convergence and quality", Proceedings of Statistics Canada Symposium 2001.

-CLARK, C. (1944): The Conditions of Economic Progress, Macmillan.

-COASE, R. (1996): “The Nature of the Firm" in PUTTERMAN, L. and KROZSNER (eds.): The Economic Nature of the Firm: A Reader. Cambridge University Press.

-COHEN, G.A. (2000): Karl Marx's theory of history: a defense, Princeton University Press.

-CRESPO, N. and FONTOURA, M. P. (2007): "Integration of CEECs into EU Market: Structural Change and Convergence", Journal of Common Market Studies, 45(3): 611-632.

-DEHEJIA, V.H. and SAMY, Y. (2006): "Labor Standards and Economic Integration in the European Union: An Empirical Analysis", CESIFO Working Paper 1746.

-DEW-BECKER, I. and GORDON, R.J. (2008): “The Role of Labour Market Changes in the Slowdown of European Productivity Growth", CEPR Discussion Paper No. 6722.

-DI MARTINO, V. (2003): Workplace violence in the health sector, ILO.

-DOERINGER, P. and PIORE, M. (1979): Internal labor markets and manpower analysis, Heath.

-DREW, E., EMEREK, R. AND MAHON, E. (1998): “Introduction”, in Women, work, and the family in Europe, Routledge.

-EBBINGHAUS, B. and EICHHORST, W. (2006): "Employment Regulation and Labor Market Policy in Germany, 1991-2005", in BLOSSFELD, H.P., IZA Discussion Papers n. 2505, Institute for the Study of Labour (IZA).

-EDGELL, S. (2006): The Sociology of Work: Continuity and Change in Paid and Unpaid Work, Pine Forge Press.

-EIRO (2006): "Conflict in education sector triggers strike action in Greece", Eironline, European Foundation for the Improvement of Living and Working Conditions.

-ELIAS, P. (1997): “Occupational Classification (ISCO-88): Concepts, Methods, Reliability, Validity and Cross-National Comparability”, OECD Labour Market and Social Policy Occasional Papers, No. 20, OECD Publishing.

-ELIAS, P. and BIRCH, M. (1994): ISCO 88 (COM). A Guide for Users, Institute for Employment Research, University of Warwick.

-EPUNET (2004): ECHP user guide, EPUNET. Downloaded from: http://epunet.essex.ac.uk/ECHP_USER_GUIDE_06-07-2004b.pdf 
-ESPING-ANDERSEN, G. (1999): Social Foundations of Postindustrial Economies, Oxford University Press.

-EUROPEAN COMMISSION (1993): White Paper on growth, competitiveness, and employment: The challenges and ways forward into the 21 st century, $\operatorname{COM}(93)$ 700 final.

-EUROPEAN COUNCIL (1999): Council Regulation (EC) No 530/1999 of 9 March 1999 concerning structural statistics on earnings and on labour costs, Official Journal of the EU L 063 , 12/03/1999 P. 0006 - 0010.

-EUROPEAN FOUNDATION FOR THE IMPROVEMENT OF LIVING AND WORKING CONDITIONS (2007): 15 years of Working Conditions in the EU: Charting the Trends, European Foundation for the Improvement of Living and Working Conditions.

-EUROSTAT (1996): NACE rev. 1, EUROSTAT. Downloaded from: http://cordis.europa.eu/cerif/ftp/nacerev1.doc

-EUROSTAT (2002): NACE rev. 1.1, EUROSTAT. Downloaded from: http://circa.europa.eu/irc/dsis/nacecpacon/info/data/en/NACE\%20Rev.\%202\%2 OIntroductory\%20guidelines\%20-\%20EN.pdf

-EUROSTAT (2005): Structure of Earnings Survey 2002 Metadata, EUROSTAT. Downloaded from: http://epp.eurostat.ec.europa.eu/cache/ITY SDDS/EN/earn ses esms.htm

-EUROSTAT (2006): Quality report of the European Labour Force Survey 2005, EUROSTAT. Downloaded from: http://epp.eurostat.ec.europa.eu/cache/ITY_OFFPUB/KS-RA-07-017/EN/KSRA-07-017-EN.PDF

-EUROSTAT (2007a): Agricultural Statistics Pocketbook 1995-2005, Eurostat.

-EUROSTAT (2007b): Structural Business Statistics Metadata, EUROSTAT. Downloaded from: http://europa.eu/estatref/info/sdds/en/sbs/sbs_base.htm

-EUROSTAT (2008): Comparative Final EU-SILC Quality Report 2005, EUROSTAT. Downloaded from: http://circa.europa.eu/Public/irc/dsis/eusilc/library?l=/quality_assessment/compa rative_quality_1/comparative_2005/_EN_1.0_\&a=d

-EUROSTAT (2009a): The European Union Labour Force Survey: main characteristics of the national surveys, EUROSTAT. Downloaded from: http://epp.eurostat.ec.europa.eu/cache/ITY_OFFPUB/KS-RA-09-002/EN/KSRA-09-002-EN.PDF.

-EUROSTAT (2009b): Comparative EU statistics on Income and Living Conditions: Issues and Challenges, Proceedings of the EU-SILC conference (Helsinki, 6-8 November 2006).

-FARBER, H. S. (1997): "Job Creation in the United States: Good Jobs or Bad", Princeton University Industrial Relations Section Working Paper 385.

-FELIX, B. (2006): "High tech industries and knowledge based services", Statistics in Focus 13/2006, Eurostat. 
-FENGER, H.J.M. (2007): "Welfare regimes in Central and Eastern Europe: Incorporating post-communist countries in a welfare regime typology", Contemporary Issues and Ideas in Social Sciences 3(2).

-FERNÁNDEZ-MACÍAS, E. (2003): "Job Instability and Political Attitudes Towards Work: Some Lessons from the Spanish Case", European Journal of Industrial Relations 9(2), 205-222.

-FERNÁNDEZ-MACÍAS, E. (2007): Recent changes in the Structure of Employment in the EU: Analytical Framework, European Foundation for the Improvement of Living and Working Conditions.

-FERNÁNDEZ-MACÍAS, E. and HURLEY, J. (2008): More and better jobs: Patterns of employment expansion in Europe (ERM Report 2008), European Foundation for the Improvement of Living and Working Conditions.

-FERNÁNDEZ-MACÍAS, E. and PETRAKOS, M. (2006): "Quality assurance in the 4th European Working Conditions Survey", Working Paper, European Foundation for the Improvement of Living and Working Conditions.

-FERRERA, M. (1996): “The 'Southern Model' of Welfare in Social Europe”, Journal of European Social Policy 6(1), 17-37.

-FOURASTIÉ, J. (1963): Le Grand Espoir du XXe Siecle, Gallimard.

-FRADE, C., DARMON, I., and LAPARRA, M. (2004): Precarious Employment in Europe: A Comparative Study of Labour Market related Risks in Flexible Economies, ESOPE project final report.

-FREEMAN, R. B. (2003): "Trade Wars: The Exaggerated Impact of Trade in Economic Debate," NBER working paper n. 10,000.

-FREEMAN, R. B. and RODGERS, W. (2004): “Jobless Recovery: Whatever Happened to the Great American Jobs Machine?" in CentrePiece Winter 2004

-GALLIE, D. (2007): “The quality of job tasks", EMPLOY State-of-the-art Report. Consulted through the internet: http://www.equalsoc.org/uploaded_files/regular/SOTAR_4_TheQualityofJobTa $\underline{\text { sks_DuncanGallie.pdf }}$

-GOOS, M. and MANNING, A. (2007): "Lousy and Lovely Jobs: the Rising Polarization of Work in Britain", in Review of Economics and Statistics (89)1, 118-133.

-GOOS, M., MANNING, A. and SOLOMONS, A. (2009): "The Polarization of the European Labor Market”, in American Economic Review, 99(2): 58-63.

-GORDON, D. M., R. EDWARDS and M. REICH (1986): Segmented work, divided workers, Cambridge University Press.

-GREGORY, M., SALVERDA, M. And SCHETTKAT, R. (2007): Services and Employment, Princeton University Press.

-GREEN, F. (2006): Demanding work: The paradox of job quality in the affluent economy, New Jersey: Princeton University Press.

-GRIMSHAW, D. and MIOZZO, M. (2006): "Knowledge intensive business services: understanding organizational forms and the role of country institutions", in 
GRIMSHAW, D. and MIOZZO, M. (ed.): Knowledge Intensive Business Services, Edgar Elgar.

-GROOT, W. and BRINK, H. M. (2000): "Overeducation in the labor market: a metaanalysis", in Economics of Education Review 19, 149-158.

-GUEST, D. (2002): "Perspectives on the study of work-life balance", in Social Science Information, Vol. 41, No. 2, 255-279.

-HARRISON, B. and BLUESTONE, B. (1988): The Great U-Turn, Basic Books.

-HATZICHRONOGLOU, T. (1997): "Revision of the High-Technology Sector and Product Classification”, OECD Science, Technology and Industry Working Papers, 1997/2, OECD Publishing.

-HOSPERS, G.J. (2004): Regional economic change in Europe: a neo-Schumpeterian vision, LIT Verlag.

-HUBER, E., and STEPHENS, J.D. (2001): Development and crisis of the welfare state: parties and policies in global markets, University of Chicago Press.

-HULL, F., FRIEDMAN, S. and ROGERS, T. (1982): “The Effect of Technology on Alienation from Work", Work and Occupations, vol. 9(1), pp. 31-57.

-HURLEY, J. (2006): Questionnaire translation process in the 4th European Working Conditions Survey, European Foundation for the Improvement of Living and Working Conditions.

-HURLEY, J. (2007): Minimum wages in Europe, European Foundation for the Improvement of Living and Working Conditions.

-ILG, R. (1996): “The Nature of Employment Growth, 1989-95”, Monthly Labor Review, June, pp. 29-36.

-ILG, R. and HAUGEN, S (2000): "Earnings and Employment Trends in the 1990s", Monthly Labor Review, March, pp. 21-33.

-ILO (1990): International Standard Classification of Occupations: ISCO-88, International Labour Office.

-INGLEHART, R. (2004): "Subjective well-being rankings of 82 societies", World Values Survey Working Paper.

-IRONMONGER (1998): “Towards a more comprehensive knowledge of all forms of work", paper presented at the Expert Group Meeting on Engendering Labor Force Statistics.

-JONES, E., and VERDUN, A. (2005): The political economy of European integration: theory and analysis, Routledge.

-KATTEL,R., REINERT, E.S. and SUURNA, M. (2009): "Industrial Restructuring and Innovation Policy in Central and Eastern Europe", Working Papers in Technology Governance and Economic Dynamics no. 23, Tallinn University of Technology.

-KIM, J. and MUELLER, C.W. (1978a): Introduction to factor analysis, Sage.

-KIM, J. and MUELLER, C.W. (1978b): Factor analysis: statistical methods and practical issues, Sage. 
-KRUEGER, A.B. and LAWRENCE, L. (1986): "Reflections on the inter-industry wage structure", NBER working paper $\mathrm{n} .1968$.

-KRUGMAN, P. and LAWRENCE, R. (1993): “Trade, jobs and wages”, NBER working papes n. 4478.

-KRUGMAN, P. (1994): "Past and Prospective Causes of High Unemployment", in Reducing Unemployment: Current Issues and Policy Options, Federal Reserve Bank of Kansas City,.

-KUTTNER, R. (1983): “The Declining Middle”, in The Atlantic Monthly (July) 60-72.

-KUZNETS, S (1971): Economic Growth of Nations, Harvard University Press.

-LITTEK, W. (2001): "Division of Labour" in International Encyclopedia of the Social \& Behavioral Sciences, Elsevier.

-LLORENTE, R. (2006): “Analytical Marxism and the division of labour”, in Science and Society, Vol. 70, No. 2, 232-251.

-MARGLIN, S.A. (1973): "What do bosses do? The Origins and Functions of Hierarchy in Capitalist Production", in Review of Radical Political Economics, Vol. 6, No. $2,60-112$.

-MARX, K. (1959, first published in 1932): Economic \& Philosophical Manuscripts of 1844, Progress Publishers of the Soviet Union. Consulted through the internet: http://www.marxists.org/archive/marx/works/1844/manuscripts/preface.htm.

-MARX, K. (1997, originally 1867): Capital, volume I, from Marx and Engels Collected Works, Progress Publishers of the Soviet Union. Consulted through the internet:

http://www.marxists.org/archive/marx/works/cw/volume35/index.htm.

-MAYER, T.F. (1994): Analytical Marxism, Sage.

-MITTAG, H.J. (2005): “Gross Earnings in Europe”, Statistics in Focus Series 12/2005, EUROSTAT.

-MUÑOZ DE BUSTILLO, R. and BONETE, R. (2009): Introduccion a la Union Europea: un análisis desde la economia, Alianza Editorial.

-MUÑOZ DE BUSTILLO, R. and FERNÁNDEZ-MACÍAS, E (2005): “Job satisfaction as an indicator of the quality of work", Journal of Socio-economics, vol. 34(5) pp. 656-673. 2005.

-MUÑOZ DE BUSTILLO, R., FERNÁNDEZ-MACÍAS, E., ANTÓN, J.I. (2008): El trabajo a tiempo parcial en España en el contexto de la Unión Europea, Ministerio de Trabajo e Inmigración.

-MUÑOZ DE BUSTILLO, R., FERNÁNDEZ-MACÍAS, E., ANTÓN, J.I. and ESTEVE, F. (2009): Indicators of Quality of the Working Environment, report for the European Parliament.

-NOLAN, A. and NOLAN, B. (2004):Ireland's Health Care System: Some Issues and Challenges, ESRI Working Papers 14.

-OECD (1994): Jobs Study, OECD.

-OECD (2001): Employment Outlook 2001, OECD.

-OECD (2003): Employment Outlook 2003, OECD. 
-OECD (2009): Economic Survey of the European Union 2009: Deepening the single market, OECD.

-PARENT-THIRION, A., FERNÁNDEZ-MACÍAS, E., HURLEY, J. AND VERMEYLEN, G. (2007): Fourth European Working Conditions Survey, European Foundation for the Improvement of Living and Working Conditions.

-PECK, J. (1996): Work-Place: The Social Regulation of Labour Markets, Guilford Press.

-POLAVIEJA, J. G. (2005): "Flexibility or polarization? Temporary employment and job tasks in Spain", Socio-Economic Review 3, 233-258.

-PUTTERMAN, L. and KROZSNER (1996): "The Economic Nature of the Firm: A New Introduction", in PUTTERMAN, L. and KROZSNER (eds.): The Economic Nature of the Firm: A Reader. Cambridge University Press.

-RICOY, C.J. (2003): "Marx on division of labour, mechanization and technical progress", European Journal of History of Economic Thought 10:1 47-79.

-ROTHENBACHER, F. (1997): "Public Sector Employment in Europe: Where Will the Decline End?", EURODATA Newsletter, issue 6, p. 1-11.

-RUBERY, J., FAGAN, C., GRIMSHAW, D. and SMITH, M. (2005): Women and European Employment, Routledge.

-SCHETTKAT, R. and YOCARINI, L. (1997): "The Shift to Services: A Review of the Literature", IZA Discussion Papers, No. 964.

-SCHWAB, K and SALA-I-MARTÍN, X. (eds.) (2009): Global Competitiveness Report, World Economic Forum.

-SHEPARD, J. (1977): “Technology, Alienation, and Job Satisfaction”, Annual Review of Sociology, vol. 3, pp. 1-21.

-SICHERMAN, N. (1991): "Overeducation in the labour market", Journal of Labor Economics, 9-2, pp. 101-122.

-SIEBERT, H. (1997): "Labor Market Rigidities: At the Root of Unemployment in Europe", Journal of Economic Perspectives 11(3), 37-54.

-SMITH, A. (1904, originally 1776): An Inquiry into the Nature and Causes of the Wealth of Nations, Methuen \& Co. Consulted through the internet: http://www.econlib.org/library/Smith/smWN.html.

-STANFORD, J. and VOSKO, L.F. (2004): Challenging the market: the struggle to regulate work and income, McGill-Queen's Press.

-STEHRER, R. and WARD, T. (2008): Recent Changes in the Jobs Structure of the EU: Technical Report, European Foundation for the Improvement of Living and Working Conditions.

-STEHRER, R., WARD, T. and FERNÁNDEZ-MACÍAS, E. (2009): Changes in the Structure of Employment in the EU and Their Implications for Job Quality, Vienna Institute for International Economic Studies Research Reports n. 354.

-SUHR, D.D. (2005): "Principal Component Analysis vs. Exploratory Factor Analysis", Proceedings of the Thirtieth Annual SAS Users Group International Conference. 
-SUN, G. (2005): “The Economics of Division of Labor from Xenophon to Hayek: A Review of Selected Literature", in SUN, G.: Readings in the Division of Labour, World Scientific Publishing.

-TAYLOR, F.W. (1911): The principles of scientific management, Harper \& Row. Consulted through the internet: http://www.marxists.org/reference/subject/economics/taylor/principles/index.ht $\underline{\mathrm{m}}$

-TITMUSS, R. (1958): Essays on the Welfare State, Allen \& Unwin.

-TRUBEK, D. and TRUBEK, L. (2005): "Hard and Soft Law in the Construction of SocialEurope: the Role of the Open Method of Coordination", in European Law Journal 11(3), 343-364.

-TSCHETTER, J. and LUKASIEWICZ, L. (1983): "Employment changes in construction: secular, cyclical, and seasonal", in Monthly Labour Review, March 1983 issue.

-UNESCO (2006): International Standard Classification of Education ISCED 1997, UNESCO.

-UNITED NATIONS STATISTICS DIVISION (1993): 1993 System of National Accounts, UN.

-UNITED NATIONS STATISTICS DIVISION (2002): International Standard Industrial Classification of All Economic Activities (ISIC) Revision 3.1, United Nations Statistical Papers Series M., n. 4, rev. 3.1.

-US COUNCIL OF ECONOMIC ADVISORS (1996): Job creation and employment opportunities: the United States labour market, 1993-1996, Office of the Chief Economist of the U.S.A., Washington DC.

-VALLAS, S. P. (1988): “New Technology, Job Content and Worker Alienation”, Work and Occupations, vol. 15(2), pp. 148-178.

-VAN DIJK, H. (1998): "Industrial decline and the development of the service economy", in NUÑEZ, C. and HAU, M. (eds.): De-industrialisation in Europe, 19th-20th centuries, Ediciones Universidad de Sevilla.

-VANDENBRANDE, T. et al (2006): Mobility in Europe. Analysis of the 2005 Eurobarometer survey on Geographical and labour market mobility, European Foundation for the Improvement of Living and Working Conditions.

-VASSILOPOULOS, J. (2006): “Greece: Sellout of teachers' strike paves way for massive government attacks", WSWS.ORG.

-VIOLANTE, G.L. (2008): "Skill-Biased Technical Change", in DARLAUF, S.N. and BLUME, L.E. (eds.): The New Palgrave Dictionary of Economics, Palgrave Macmillan.

-WALTER, N. (2002): “German Unity - 12 Years On”, Deutsche Bank Research Paper.

-WEISS, Y. and FERSHTMAN, C. (1992): "On the stability of occupational rankings", in Rationality and Society, vol. 4, n. 2, 221-233.

-WHITEHEAD, D. (2008): "Historical Trends in Work-Family: The Evolution of Earning and Caring", in KORABIK, K., LERO, D. and WHITEHEAD, D.: 
Handbook of Work-Family Integration: Theories, Perspectives and Best Practices, Academic Press

-WILKINSON, C. (2001): Fundamentals of Health at Work: the Social Dimensions, Taylor and Francis. London.

-WILSON, E. (1977): Women and the Welfare State, Tavistock.

-WORLD HEALTH ORGANIZATION (2000): World Health Report 2000, WHO.

-WRIGHT, E.O. and DWYER, R.E. (2000): "The American Jobs Machine: the trajectory of good and bad jobs in the 1960s and 1990s", Boston Review, December issue.

-WRIGHT, E.O. and DWYER, R.E. (2003): "Patterns of job expansions in the USA: a comparison of the 1960s and 1990s", in Socio-Economic Review (2003)1, 289325.

-WRIGHT, E.O. and DWYER, R.E. (2003): Methodological appendices to "Patterns of job expansions in the USA: a comparison of the 1960s and 1990s", working paper published on the web: www.ssc.wisc.edu/ wright/SERapnx.pdf.

-WDROJEWSKI, S., GRELET, Y. and VALLET, L. (2009): "Increasing employment instability in France? Young people's labour market entry and eary career since the 1990s", in BLOSSFELD, H.P., BUKODI, E. and KURZ, K. (ed.): Young workers, globalization and the labor market: comparing early working life in eleven countries, Elgar. 